UCRL-CR-122799

\title{
NASA BOEING 757 HIRF Test Series Low Power On-the-Ground Tests
}

\author{
Andrew J. Poggio \\ Steven T. Pennock \\ Richard A. Zacharias \\ Carlos A. Avalle \\ Lawrence Livermore National Laboratory \\ Hal L. Carney \\ NASA Langley Research Center
}

August 1, 1996

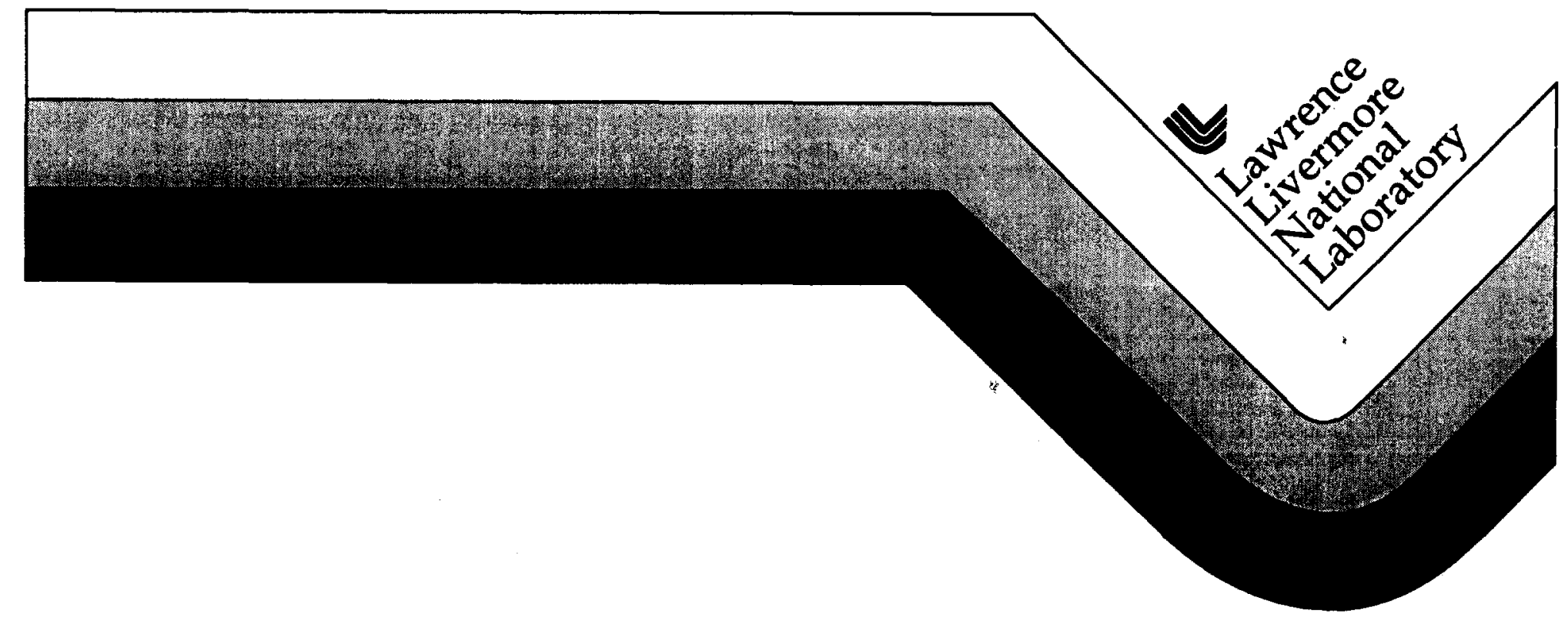




\section{DISCLAIMER}

This document was prepared as an account of work sponsored by an agency of the United States Government. Neither the United States Government nor the University of California nor any of their employees, makes any warranty, express or implied, or assumes any legal liability or responsibility for the accuracy. completeness, or usefulness of any information, apparafus, product, or process disclosed, or represents that its use would not infringe privately owned rights. Reference herein to any specific commercial products, process, or service by trade name. trademark, manufacturer, or otherwise, does not necessarily constitute or imply its endorsement, recommendation, or favoring by the United States Government or the University of California. The views and opinions of authors expressed herein do not necessarily state or reflect those of the United States Government or the University of California, and shall not be used for advertising or product endorsement purposes.

Work performed under the auspices of the U.S. Department of Energy by Lawrence Livermore National Laboratory under Contract W-7405-Eng-48. 


\section{Summary}

The data acquisition phase of a program intended to provide data for the validation of computational, analytical, and experimental techniques for the assessment of electromagnetic effects in commercial transports; for the checkout of instrumentation for following test programs; and for the support of protection engineering of airborne systems has been completed. Funded by the NASA Fly-ByLight/Power-By-Wire Program, the initial phase involved on-the-ground electromagnetic measurements using the NASA Boeing 757 and was executed in the LESLI Facility at the USAF Phillips Laboratory.

The major participants in this project were LLNL, NASA Langley Research Center, Phillips Laboratory, and UIE, Inc. The tests were performed over a five week period during September through November, 1994. Measurements were made of the fields coupled into the aircraft interior and signals induced in select structures and equipment under controlled illumination by RF fields. A characterization of the ground was also performed to permit ground effects to be included in forthcoming validation exercises.

This report and the associated test plan that is included as an appendix represent a definition of the overall on-the-ground test program. They include descriptions of the test rationale, test layout, and samples of the data. In this report, a detailed description of each executed test is provided, as is the data identification (data id) relating the specific test with its relevant data files. Samples of some inferences from the data that will be useful in protection engineering and EM effects mitigation are also presented.

The test plan which guided the execution of the tests, a test report by UIE Inc., and the report describing the concrete pad characterization are included as appendices. 


\section{Table of Contents}

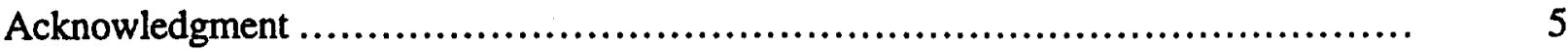

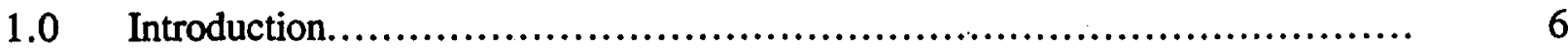

$2.0 \quad$ Objectives.................................................................. 6

2.1 The NASA B-757 HIRF Test Series ................................

2.2 On-the-Ground Test Objectives ................................... 7

3.0 Test Organization and Facilities ................................................ 7

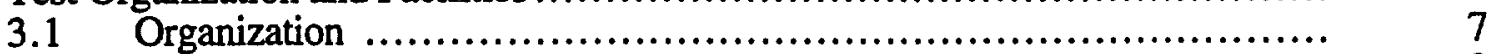

3.2 Test Aircraft ........................................................ 8

$3.3 \quad$ On-the-Ground Test Facility ...............................................

3.4 The On-The-Ground Test Series Coordinate Systems .................... 10

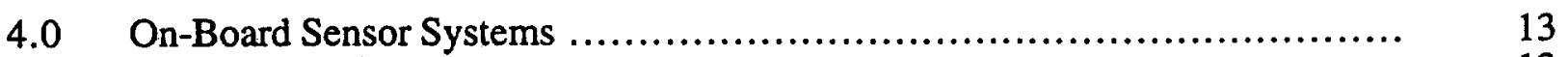

4.1 Cockpit Sensors ................................................. 13

4.2 Electronics Bay Sensors .......................................... 14

4.3 Cabin Sensors ........................................................ 16

4.4 Summary of Test Points and Measured Parameters ...................... 20

4.5 Sensor/Cable/Fiber Optic Transmitter Identifications ..................... 20

5.0 Creating the Electromagnetic Environment .................................... 22

5.1 Ground Plane ................................................. 22

6.0 The Low Power On-the-Ground Test Series .................................. 22

6.1. A Tabulation of the Executed Tests ................................. 22

6.2 Relating Data File Names to Experiment Test Numbers .................... 41

6.2.1 Interpreting a Data File Header ................................ 53

6.3 Pre- and Post-Aircraft Test Activities ............................... 54

6.3.1 Calibration Data for Sensor Systems ........................ 54

6.3.1.1 Calibration of the AD-60 Electric Field (D-Dot) Probe .. 55

6.3.2 Dipole Test Preparation ........................................ 56

6.3.3 Dipole Field Mapping ........................................ 59

6.3.4 Rhombic Field Mapping ....................................... 62

6.3.5 LESLI Pad Characterization ...................................... 64

6.4 Aircraft Test Series ................................................. 66

6.4.1 Ambient Noise Measurements ................................... . 66

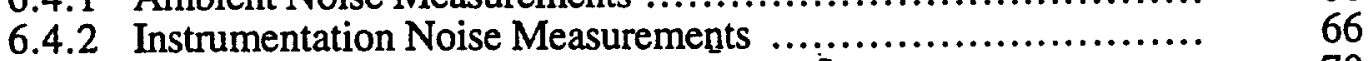

6.4.3 Dipole Tests ..................................................... 70

6.4.4 Test Series J \& K - Rhombic Tests.................................. 72

6.4.5 Test Series Z - Effects of System State........................... 80

6.4.6 Horizontal Polarization ....................................... 82

6.4.7 Test Series WF - Windshield Shielding Effects................... 84

$7.0 \quad$ Data Availability ......................................................... 85

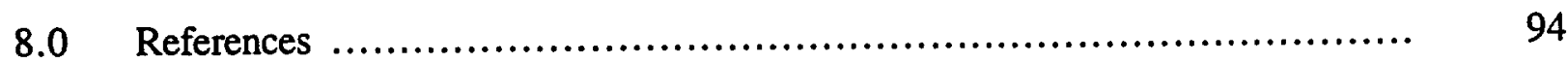

Appendix A NASA Boeing 757 HIRF Test Plan

Appendix B NASA B-757 Aircraft Test Report

Appendix C The Determination of the Constitutive Parameters of a Medium with Application to a Reinforced Concrete Pad 


\section{Figures}

Figure 3-1.

Figure 3-2.

Figure 3-3.

Figure 3-4.

Figure 3-5.

Figure 4-1.

Figure 4-2.

Figure 4-3.

Figure 4-4.

Figure 4-5.

Figure 4-6.

Figure 4-7.

Figure 4-8.

Figure 4-9.

Figure 4-10.

Figure 4-11.

Figure 6-1.

Figure 6-2.

Figure 6-3.

Figure 6-4.

Figure 6-5.

Figure 6-6.

Figure 6-7.

Figure 6-8.

Figure 6-9.

Figure 6-10.

Figure 6-11.

Figure 6-12.

Figure 6-13.

Figure 6-14.

Figure 6-15.

Figure 6-16.

Figure 6-17.

Figure 6-18.

Figure 6-19.

Figure 6-20.

Figure 6-21.

Figure 6-22.

Figure 6-23.

Figure 6-24.

Figure 6-25.

Figure 6-26.

Figure 6-27.

Figure 6-28.

Figure 6-29.

Figure 6-30.

Figure 6-31.

Figure 6-32.

Figure 6-33.
Organization for the execution of the On-the Ground Test Series

Sketch of the LESLI facility with the rhombic deployed and aircraft emplaced

LESLI Facility with rhombic radiator and ground plane coordinate system...

CW data acquisition and processing flow.

A Summary of the Coordinate System Needed in this Report....................

Cockpit sensors.

ATOPS box and pedestal in cockpit with coordinates

ATOPS box with conceptual representation of $E$ field sensors (d-dot).........

ATOPS box on pedestal in cockpit .

Electronics bay sensors

Electronics bay electric field (d-dot) sensor.

Cabin sensors

Electric field sensor (d-dot) on the EME rack and close-up.

The cabin long wire with dielectric supports (red) and coaxial cable feed .....

Current sensor on cable in cabin .............................................

Aircraft test system using two network analyzers............................

Calibration curves (magnitude only) for various AD-60 D-dot probes.........

Wooden stand for dipole testing .....

$172 \mathrm{MHz}$ dipole mounted on stand

The $172 \mathrm{MHz}$ dipole with balun and mount

Dipole field mapping coordinate system

Rhombic field map points.

$\mathrm{Pad}$ characterization wire layout.

Pad characterization wire layout - cross sectional view .......................

Ambient noise measurements for Test Series AN..........................

Instrumentation noise in measuring an electric field using $A D-60$ probe.......

Instrumentation noise in measuring voltage at a point.........................

Instrumentation noise in measuring current on a wire ..........................

Crosstalk signals evaluated during Test Series $\mathrm{C}$...........................

Dipole and reference locations.................................................

Dipole in position for aircraft test

B-757 on LESLI pad - overhead view

B-757 on LESLI pad - viewed from rhombic feedpoint

The effect of movement of a small conducting box on vertical electric field...

The effect of hatch cover state on vertical electric field in E-bay ................

Cockpit vertical electric field variation with angle of incidence..................

Climbing wire voltage with and without current probe........................

Cockpit vertical electric field for nose-on and side incidence....................

Cabin vertical electric field for nose-on and side incidence .....................

Repeatability check using corrected data ...................................

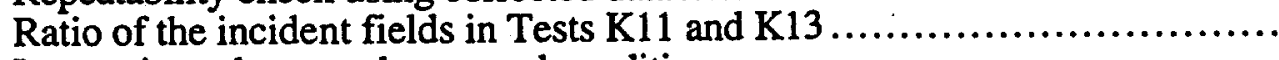

Long wire voltage under several conditions.

Ratio of axial E Field with seat at mid and forward positions

E-bay electric field dependency on circuit breaker state

LRU voltage dependency on circuit breaker state

E Field in Cockpit under horizontally polarized excitation

Voltage at select points under horizontal excitation.

Current on certain wires under horizontal excitation............................

Comparison of the vertical electric field in the cockpit with the windshield

uncovered and covered with aluminum foil. 


\section{Tables}

Table 4-1. Test Points, Measured Parameters and Locations........................... 20

Table 4-2. Test Point, Cable, Sensor and Fiber Optic Transmitter Designations............ 21

Table 6-1. Test Points and Measured Parameters .............................................

Table 6-2a. Xmit Ant ..............................................................................

Table 6-2b. Polarization ................................................................ 23

Table 6-2c. Probe or Sensor............................................................... 23

Table 6-3. Notes ................................................................. 24

Table 6-4. Test Series AN - Ambient Noise Measurements ............................... 26

Table 6-5. Test Series A - Dipole (Horizontal, 23 - 28 MHz) .......................... 27

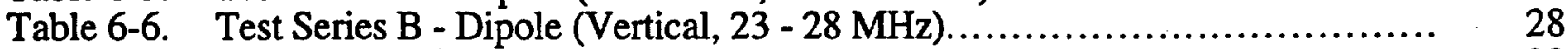

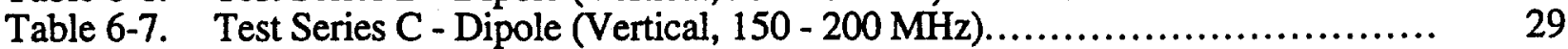

Table 6-8. Test Series D - Dipole (Horizontal, $150-200 \mathrm{MHz}$ ) ...................... 30

Table 6-9. Test Series E - Dipole (Vertical, $350-500 \mathrm{MHz}$ ).......................... 31

Table 6-10. Test Series F - Dipole (Horizontal, 350 - $500 \mathrm{MHz}$ ) ....................... 32

Table 6-11. Noise Floor Measurements ................................................ 33

Table 6-12. Test Series J - Rhombic (Nose Incident and Related Topics).................. 34

Table 6-13. Test Series K - Rhombic (Side Incident and Related Topics).................. 37

Table 6-14. Test Series Z - Rhombic (System State Effects - Circuit Breakers)........... 39

Table 6-15. Horizontal Polarization .................................................. 40

Table 6-16. Test Series WF - Window Shielded/Unshielded ................................ 40

Table 6-17. Sweep Designation Definitions.............................................. 41

Table 6-18. Data ID: Test Series AN - Ambient Noise Measurements ................... 42

Table 6-19. Data ID: Test Series A .................................................... 42

Table 6-20. Data ID: Test Series B ..................................................... 42

Table 6-21. Data ID: Test Series C ....................................................... 43

Table 6-22. Data ID: Test Series D .................................................... 43

Table 6-23. Data ID: Test Series E................................................. 44

Table 6-24. Data ID: Test Series F..................................................... 44

Table 6-25. Data ID: Noise Floor Measurements - Rhombic................................. 45

Table 6-26. Data ID: Test Series J - Rhombic (Nose Incident and Related Topics) ......... 46

Table 6-27. Data ID: Test Series K - Rhombic (Side Incident and Related Topics) .......... 49

Table 6-28. Data ID: Test Series Z - Rhombic (System State Effects - Circuit Breakers)... 51

Table 6-29. Data ID: Horizontal Polarization....................................... 52

Table 6-30. Data ID: Test Series WF - Window Shielded/Unshielded..................... 52

Table 6-31. A Typical Header for a Data File .............................................. 53

Table 6-32. Definition of Fields in Data File Header........................................ 53

Table 6-33. File Names for Calibration Data.................................................. 54

Table 6-34. AD-60 Serial Numbers and Configurations for Calibration...................... 55

Table 6-35. Dipole Field Map Locations.................................................. 60

Table 6-36. Data ID: Dipole Field Mapping ............................................. 61

Table 6-37. Rhombic Field Map Locations................................................... 62

Table 6-38. Rhombic Field Map Points (Common Mode, $0.3<$ frequency $<1000 \mathrm{MHz}$ ).. 63

Table 6-39. File Designation for Pad Characterization..................................... 65

Table 6-40. Frequency Table for Dipole Tests........................................ 70

Table 6-41. Dipole Test Series............................................................ 70

Table 6-42. Test Series and Available Data Files................................................... 86 


\section{Acknowledgment}

The authors are indebted to Felix Pitts and Charles Meissner of NASA Langley Research Center for their vision guidance and leadership of the Electromagnetic Environment (EME) portion of the NASA Fly-by-Light/Power-by-Wire (FBL/PBW) Program leading up to the execution of these tests. Significant contributions during the planning phase were also made by K. Peter Zaepfel and Peter Padilla of NASA LaRC. The bulk of the experimental work, including planning and execution, would not have been possible without the outstanding and continual technical support of John Millard and James Tucker of UIE, Inc., and Ly Dao and Donald Lin of the Phillips Laboratory. Also, the experimental team is indebted to NASA Operations for their support in facilitating aircraft usage in the tests. 


\subsection{Introduction}

The NASA Fly-by-Light / Power-by-Wire (FBL/PBW) program was initiated to develop technology for a future generation of commercial transport aircraft. Studies have shown that an all-electric aircraft (PBW) could have substantial benefits in terms of weight and maintenance costs over today's aircraft that rely on hydraulic and pneumatic controls. However, one major concern regarding the all-electric aircraft is the potential for conducted and radiated electromagnetic susceptibility of its electronic controls. A way to potentially alleviate this problem (and save weight at the same time) is to route all control and signal information over optical fiber control links (FBL) that are inherently easier to harden against the electromagnetic environment (EME).

Since electro-optic devices and electronic digital computers will still be required, a major objective of the FBL/PBW program is to develop tools and techniques for predicting the electromagnetic environment and for assessing the survivability of the modern aircraft using such equipment in the electromagnetic environment. The EME includes both externally generated high intensity radiated fields (HIRF) and internally-generated radiated or conducted emissions.

The EME effects assessment tools and techniques must ultimately be accepted by industry and the FAA regulatory body to be useful. Therefore, an important aspect of the program is to validate these tools and techniques to the satisfaction of this community. Here, validation is a demonstration of the utility, and a proof of the accuracy of the tool or technique during the execution of its intended role. As part of this exercise, a series of aircraft tests, specifically, low power on-the-ground tests at frequencies up to $1 \mathrm{GHz}$ and fly-by tests using three RF radiators in the HF, VHF and UHF ranges, have been conducted under NASA Langley Research Center sponsorship. This report describes the on-the-ground tests, which include those used for generating data for code validation as well as those which might support the development of protection engineering guidelines. It also contains a description of the output data, their format, and their identifying features.

\subsection{Objectives}

\subsection{The NASA B-757 HIRF Test Series}

The primary thrust of this aircraft test program, both on-the-ground and fly-by, is to develop a library or database of experimental results that were obtained using techniques as close to the "scientific method" as possible. This data can then be used for the validation of computational, analytical and experimental techniques for the assessment of electromagnetic effects in commercial transports and for the support of protection engineering of airborne systems. The on-the-ground tests provided a checkout of instrumentation for following test programs.

This report provides a roadmap for the test series and its associated data so that specific tests, test conditions, and the resulting data can be related and identified.

The data library is necessarily limited in size and scope by cost and time constraints, but nonetheless is expected to provide a reasonable database for validation exercises involving computer codes as well as analytical and experimental techniques. As an example, this exercise will "validate" the physics kernel of the code, its numerical algorithms, and its overall ability to provide the "right" answer. Of possibly equal importance, the data can help to establish the limits of applicability of a code, the validity of approximations and extensions which can enlarge its applicability, and the resources such as computer time and memory required to attain various levels of accuracy. Similar statements can be made regarding the analytical and experimental techniques. 


\subsection{On-the-Ground Test Objectives}

There were four objectives and four test series for the on-the-ground tests. The first test series generated data for the library which will be used directly for coupling code validation. For this objective, discrepancies and uncertainties between measurement and model were to be minimized or at least understood and quantized. These tests used dipole antennas, so the antenna can be included in the model, thus eliminating the uncertainty in the exact form of the wave entering the problem space.

The second test series exercised the equipment that will be used for the fly-by tests. The third test series provided more data for validation of the codes and for the development of protection engineering guidelines. This series also provided information to support the fly-by tests such as that relating to resonances near the fly-by frequencies and the coupling variation due to uncertainties in aspect angle or the position of interior objects. The fourth test series involved a continuing study of stirred frequency techniques.

The first and third test series are the primary subject of this report. The fly-by test equipment check and the stirred frequency or statistical electromagnetics tests are not described.

\subsection{Test Organization and Facilities}

\subsection{Organization}

The organization for the ground tests is shown in Figure 3-1. The Phillips Laboratory provided

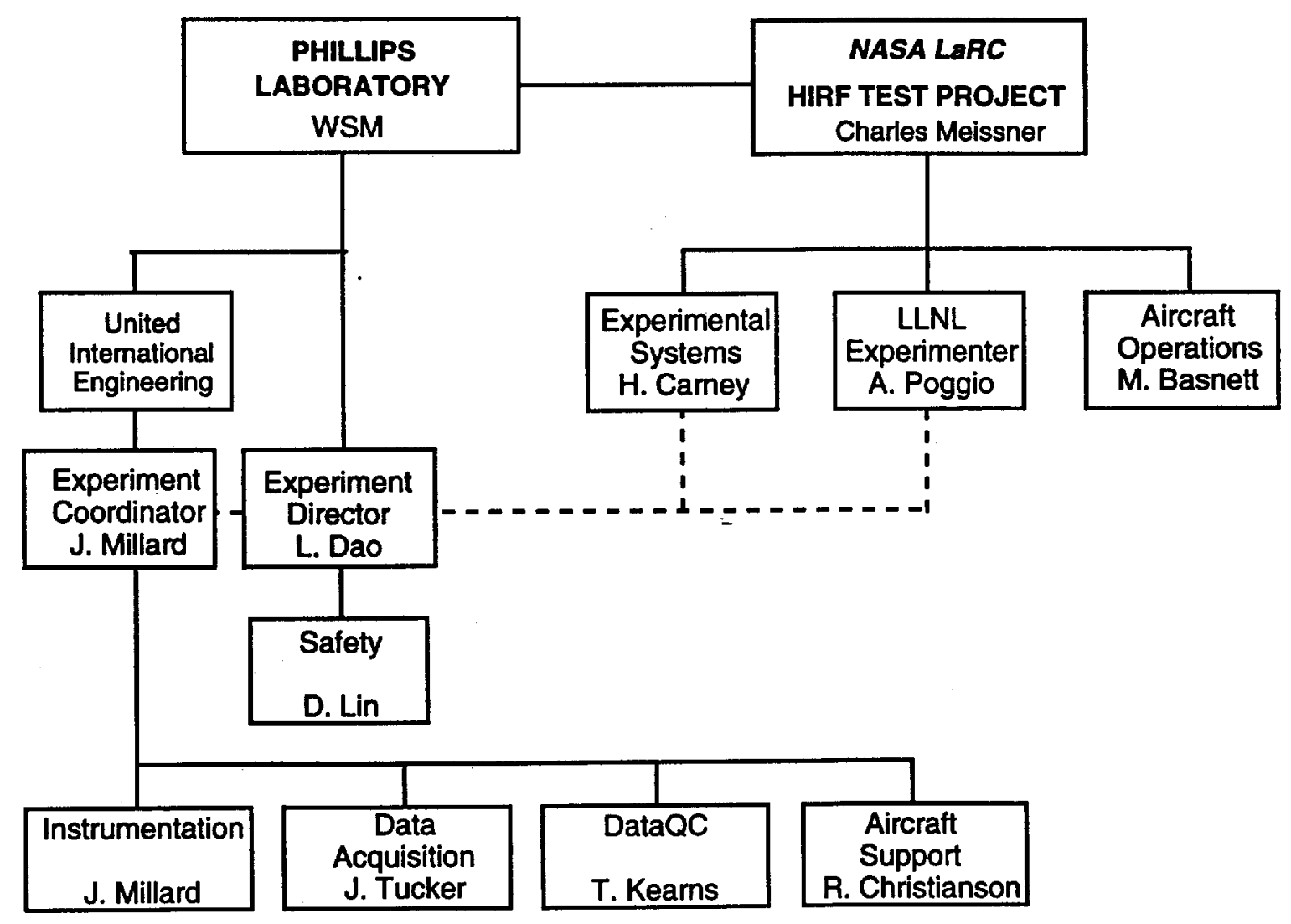

Figure 3-1. Organization for the execution of the On-the-Ground Test Series 
the test environment including the LESLI facility and its associated instrumentation, data processing of the data, and detailed test planning prior to execution. United International Engineering provided support in the execution of the tests and associated functions. LLNL was responsible for test planning and management of its execution. NASA was the funding agency with overall program management of FBL/PBW program as well as responsibility for development and emplacement of the sensors and other equipment on board the airplane. Figure 3-1 represents the various participants and lines of responsibility. The solid lines indicate the formal lines of responsibility while the dashed lines indicate the informal communication channel during the execution of the tests.

\subsection{Test Aircraft}

The primary test object for the HIRF Test Series was the NASA/Eastern (Boeing) 757-200. The onboard certificate identifies the aircraft as a Boeing 757-225, Serial Number 22191 with the 225 denoting the specific configuration requested by the original sole owner (Eastern Airlines). With the exception of the intrusion of the HIRF probes and data acquisition system, the aircraft was maintained in a typical airline passenger configuration (passenger seats, overhead baggage compartments, galleys and lavatories) during the duration of the test series. In the following, this aircraft is referred to as the B-757, NASA B-757, NASA 757, etc., for convenience.

\subsection{On-the-Ground Test Facility}

The on-the-ground tests were conducted at the U.S. Air Force Phillips Laboratory (PL) Large Electromagnetic System-Level Illuminator (LESLI) test facility. The LESLI facility is operated and maintained by the PL/WSR. This facility consists of concrete pad, a two-wire rhombic antenna supported by a non-conducting line attached to wooden poles, a source shack housing power amplifiers, and a data acquisition and control trailer (DAS). Figure 3-2 is a sketch of the aircraft in the LESLI facility with the rhombic radiators in the deployed configuration. These radiators were lowered and stowed during other test configurations, e.g., dipole tests to be described later.

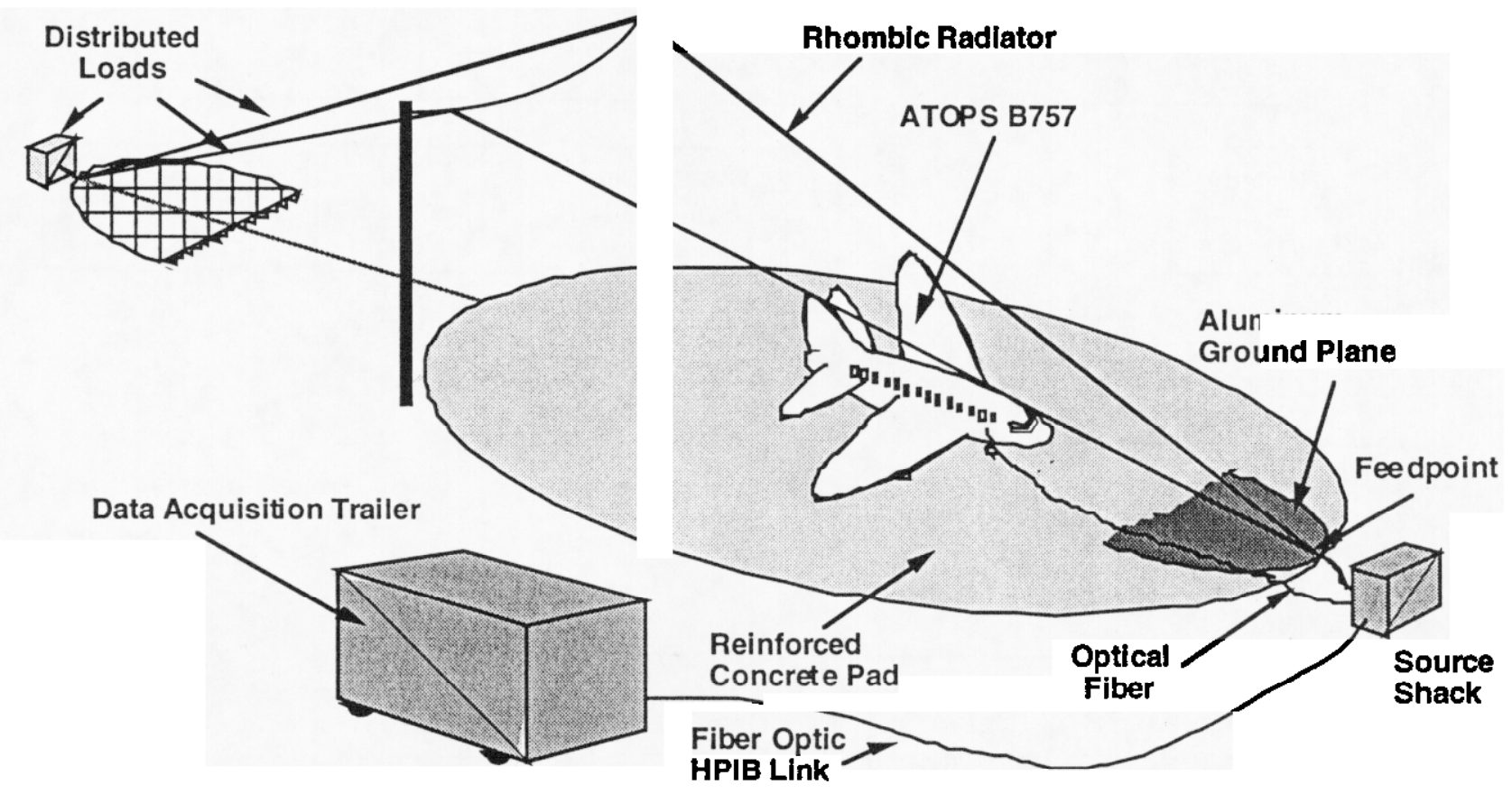

Figure 3-2. Sketch of the LESLI facility with the rhombic deployed and aircraft emplaced 
Figure 3-3 is an overhead plan view showing the LESLI facility, the circular concrete pad with 92 meter diameter, and the aluminum sheet that serves as a conducting ground plane in the near vicinity of the rhombic feedpoint, and the relative positions of the rhombic radiating wires as used during the on-the-ground test series. The insertion or feedpoint of the rhombic radiators was located about three meters from the edge of the concrete pad.

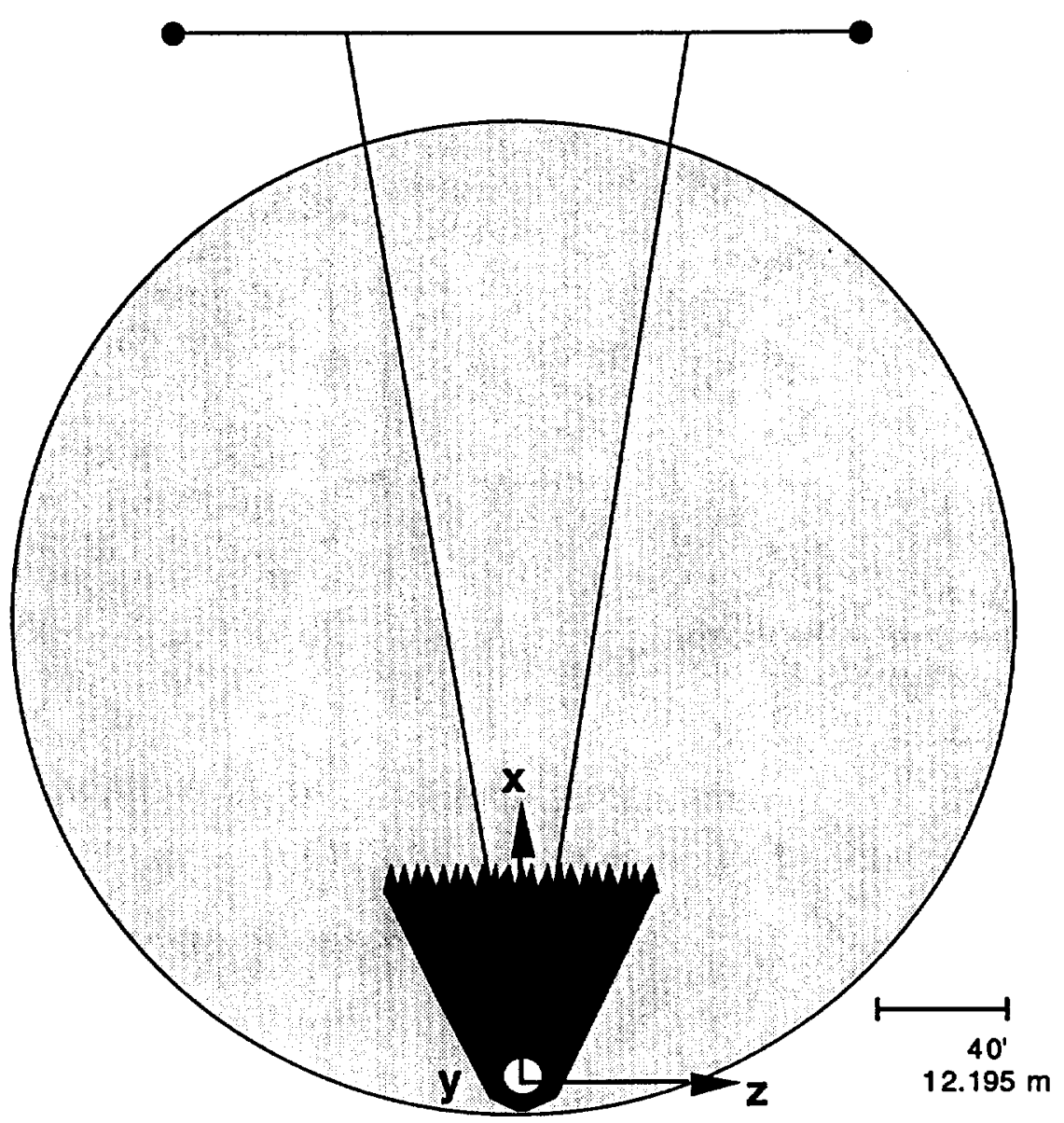

Figure 3-3. LESLI Facility with rhombic radiator and ground plane

All tests carried out in the LESLI made use of the data acquisition system in place in the facility. Since the tests were stepped CW over the frequency range of interest, vector automatic network analyzers (VANA) were used throughout. Figure 3-4 is an organizational layout of the data acquisition and processing system for a signal channel of data. In practice, a two channel system was employed.

A low-level RF signal was generated in the source shack by a VANA under computer control from the DAS. It was amplified to the desired level by one of the two power amplifiers in the source shack, and this high-level RF signal was used to generate the required fields in the test volume by driving the appropriate radiator (rhombic or dipole).

Responses to the generated fields were sensed by a probe (voltage or current) or field sensor. The resultant electrical signals were sent on Heliax, $50 \mathrm{ohm}$ coaxial cable to a fiber optic transmitter, 
converted to optical signals, transmitted to a fiber optic receiver in the source shack, converted back to electrical signals, and delivered to the data processing system for final processing, QC, and generation of hard and soft copies for the user.

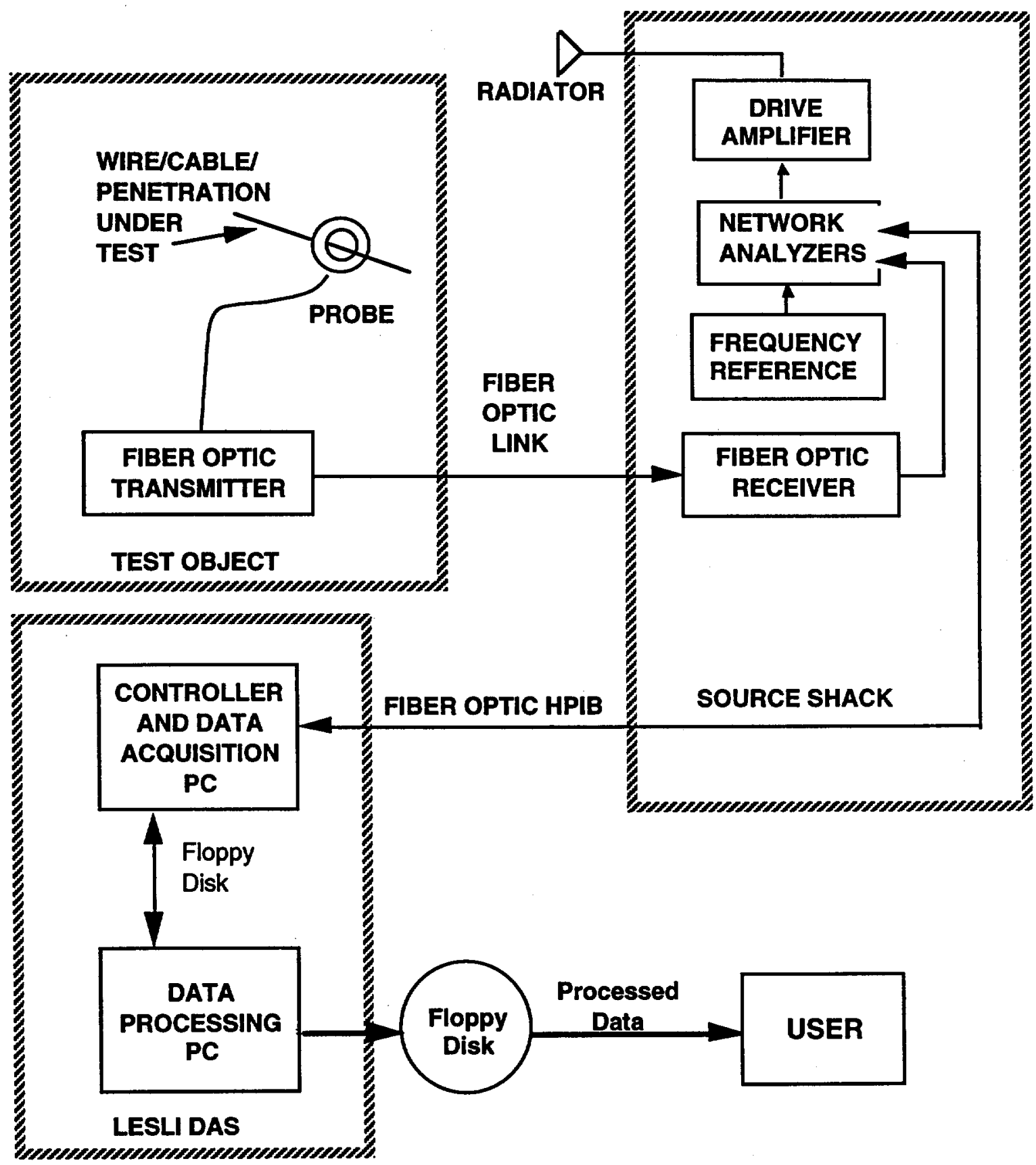

Figure 3-4. CW data acquisition and processing flow

3.4 The On-the-Ground Test Series Coordinate Systems Care should be taken throughout this report as the coordinate systems and their associated directions may different when referring to different measurements. This was a result of using pre-established coordinate systems for electromagnetic measurement facilities, geometry descriptions provided by airplane manufacturers, and lack of hard coordination between the teams performing the test planning and 
execution. The coordinate systems are summarized here to stress the importance of the issue rather than waiting for the section in which the particular measurement is described. The following can serve as a reference with details later in the text.

The main differences occur when referring to:

1) the rhombic radiator on the LESLI pad and locations on the pad,

2) dipole radiators in isolation as when measuring their radiated fields (field mapping),

3) the position of the dipole antenna and its associated reference sensor when measuring aircraft responses to dipole excitation

4) positions and locations of physical structures within the aircraft based on the aircraft manufacturers drawings and their datum

5) coordinated directions for field components within the aircraft.

Figure 3-5 is a summary of the systems used in defining the coordinates and directions in various parts of this report. The following is a brief description of the systems with an indication of the section in which the system is first referred.

Figure 3-5a is a repeat of Figure 3-3 and is an overhead plan of the LESLI facility showing the extent of the concrete pad, the aluminum ground plane, and the relative position of the rhombic radiating wires as used during the on-the-ground test series. (Sect. 3.3). The coordinate system is a righthand one in which positive " $X$ " is looking down the wires from the origin, in a westerly direction. Positive " $Y$ " is up (vertical), and positive " $Z$ " is orthogonal to " $X$ " in a northerly direction. Objects located on the test pad, e.g., the aircraft, were positioned with the respect to this system.

Figure 3-5b depicts the coordinate system for the dipole antennas standing alone, as when measuring their radiated fields. (Sect. 6.3.3). The origin is on the ground directly below the antenna center or feedpoint. The system is similar to the LESLI facility in that positive " $\mathrm{X}$ " is in a direction from the antenna towards the measurement point ( all field measurements were with positive " $X$ "), Positive " $Y$ " is up (vertical), and positive " $\mathrm{Z}$ " is orthogonal to " $\mathrm{X}$ " and " $\mathrm{Y}$ " in a right-hand sense.

Figure 3-5c shows the coordinate system which was used to position the dipole antennas for excitation of the aircraft. (Sect. 6.4.3). In this case, the system is with respect to the aircraft. The origin is a point on the test pad directly below the nose. In this right-hand system, the positive " $\mathrm{X}$ " direction is forward of the aircraft and parallel to the fuselage. The positive "Y" direction is up, and the positive " $Z$ " direction to starboard of the aircraft.

Figure 3-5d shows the coordinate directions used for defining the on-board measurements. (Sect. 4.1). These are used to define the components of the electric and magnetic fields.

Figure 3-5e shows the coordinate system used by the manufacturer in defining the geometry of the aircraft in their drawings. It is used here to define the location of physical objects in the aircraft when necessary. (Sect. 4.2). This information will be used in creating the computer models to be used in modeling. 


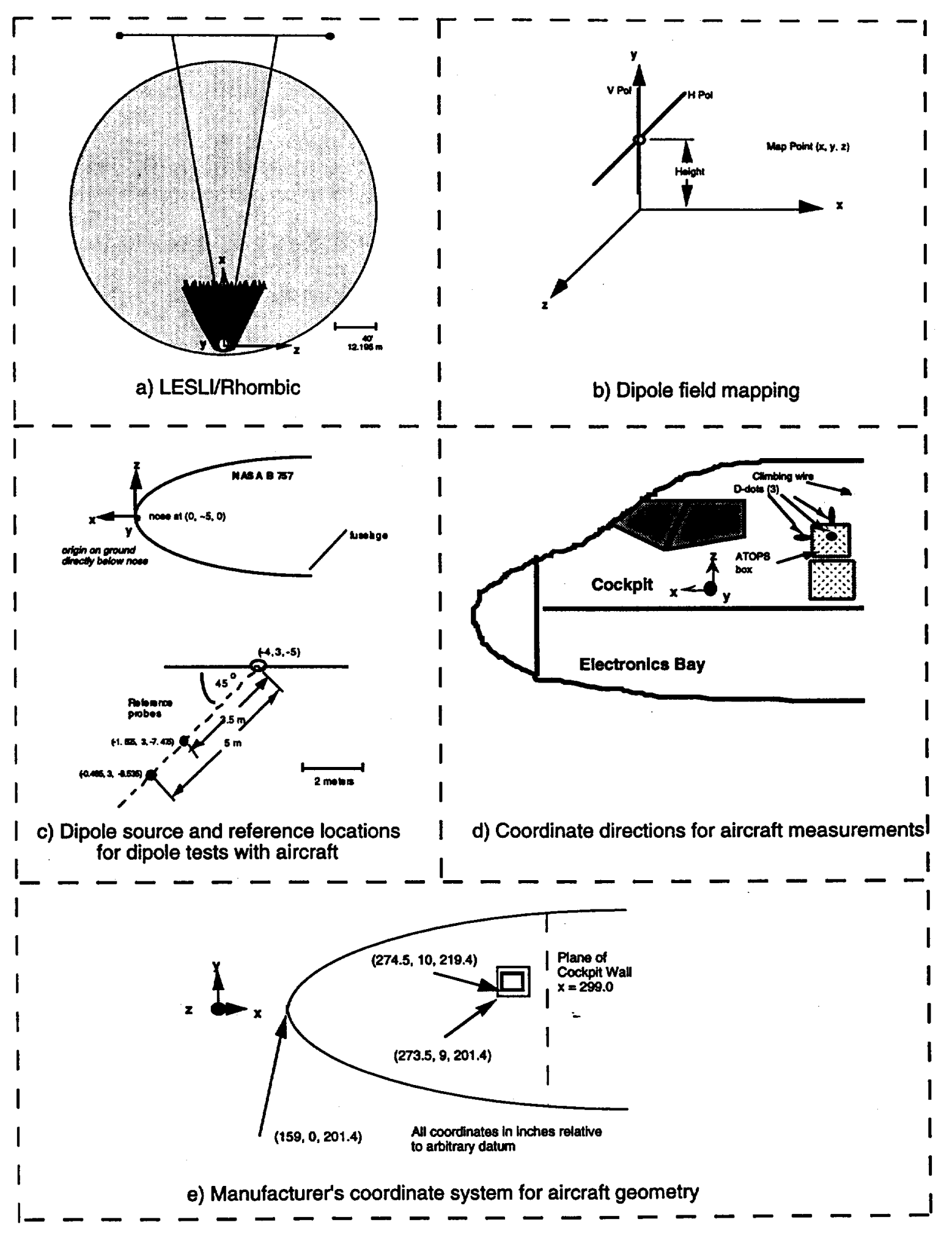

Figure 3-5. A summary of the coordinate systems used in this report 


\subsection{On-Board Sensor Systems}

The sensors for the on-the-ground tests were fitted into the aircraft prior to its arrival at the LESLI facility. Some of the sensors were removed for the flight to Kirtland AFB, and were reinstalled for the tests. The suite of 12 sensors were to sample fields, cable currents and voltages in three aircraft areas and the exterior of the fuselage. The internal measurements were in the cockpit, electronics bay (E-bay) and cabin, while the external measurement involved field sensing at a single point on the aircraft skin. During the remainder of this report, the external sensor is included in the cabin sensor suite.

\subsection{Cockpit Sensors}

Figure 4-1 depicts the various sensor locations in the cockpit. Also shown in Figure 4-1 is the coordinate system used in the definition of vector directions in the interior of the airplane. Note that this definition $(x, y, z)$ is different than that used for the definition of vector directions associated with the primary radiators, namely the rhombic and dipoles.

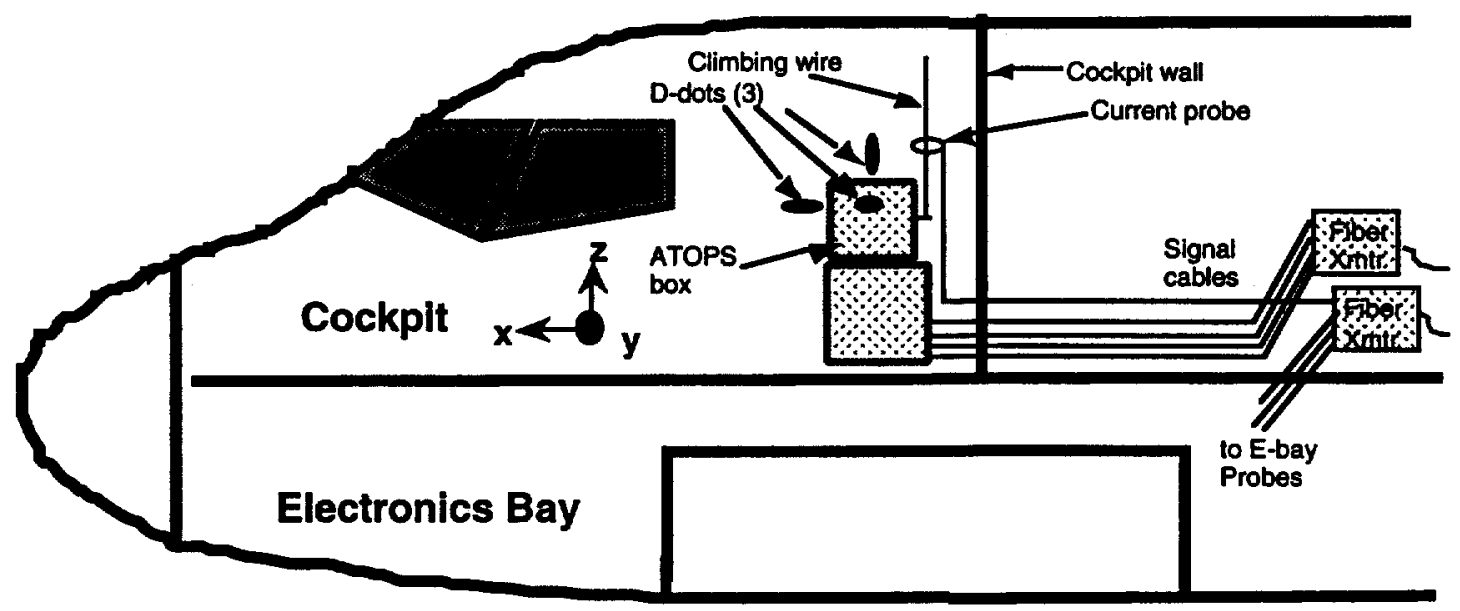

Figure 4-1. Cockpit sensors

There were five sensors in the cockpit. All of these sensors were mounted onto the same sensor box that was fabricated by NASA LaRC and is referred to as the ATOPS box. The box was a cube, 12 inches on a side, was constructed of welded aluminum and was mounted on a metal pedestal (14 inches square by 18 inches high). The ATOPS box had three orthogonal faces on which were placed Prodyn AD-60 D-dot sensors as well as a face with a feed-through connector to which was appended an external two foot long, AWG\#10 Bus Bar wire that was routed vertically. In the following, this wire will be referred to as the "climbing wire".

In addition, a Prodyn I-320 current probe was used on the climbing wire just outside of the box. This probe was also used to measure current on the starboard side window heater cable in a select test at point CP12 to be referred to later. The geometry of emplacement of the ATOPS box and its pedestal in the cockpit is shown in Figure 4-2 and a sketch of the ATOPS box with conceptual AD60 probes is shown in Figure 4-3. A picture of the ATOPS box emplaced in the cockpit is shown in Figure 4-4. 


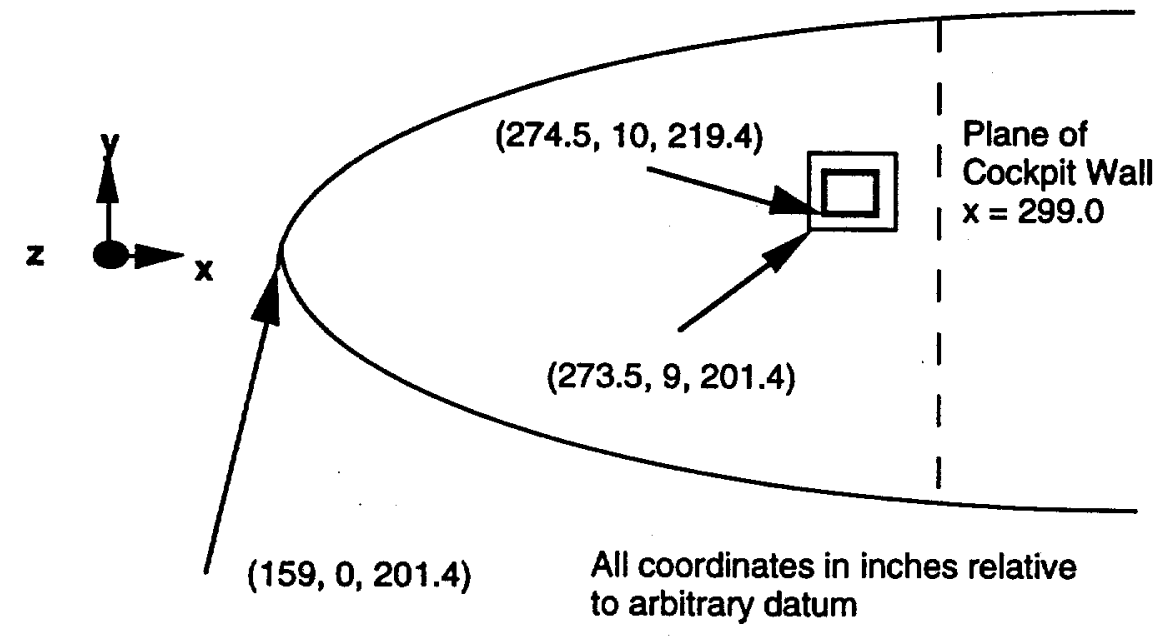

Figure 4-2. ATOPS box and pedestal in cockpit with coordinates

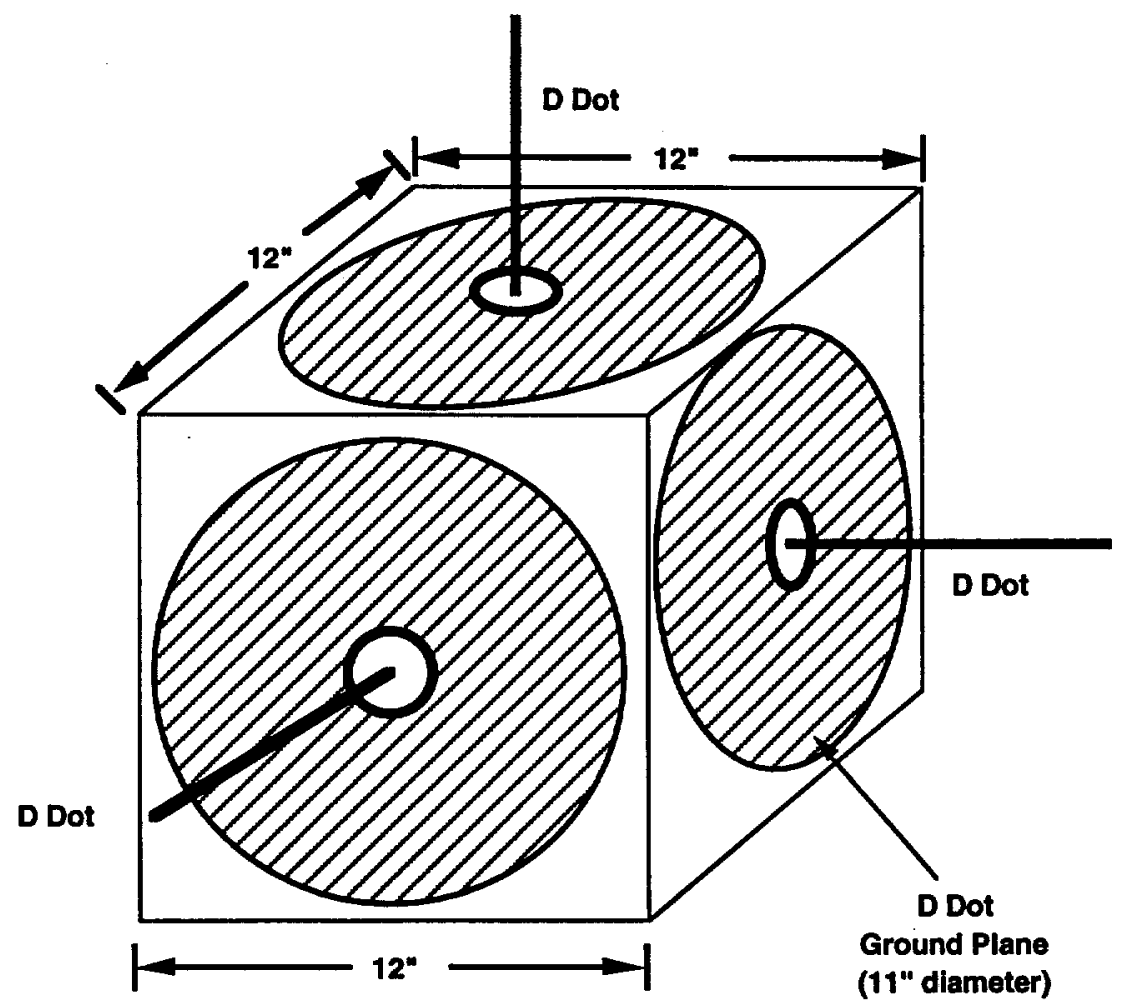

Figure 4-3. ATOPS box with conceptual representation of E field sensors (d-dot)

\subsection{Electronics Bay Sensors}

There were three sensors emplaced in the electronics bay as shown in Figure 4-5. A Prodyn AD-60 electric field probe was used to measure the vertical (z-directed) electric field near the floor within the bay (see Figure 4-6). A Prodyn I-320 current probe was placed on a power line within the E-bay which was used for energizing the cockpit windshield heater. The third measurement in the electronics bay was made inside a Collins VHF-700 transceiver box. In the remainder of this report, this unit will often be referred to as the LRU or RC-7 box or unit. The unit had been modified by LLNL to permit the measurement of the voltage on an internal power line pin. The unit was connected to the avionics bus during the low power RF stepped frequency tests. 


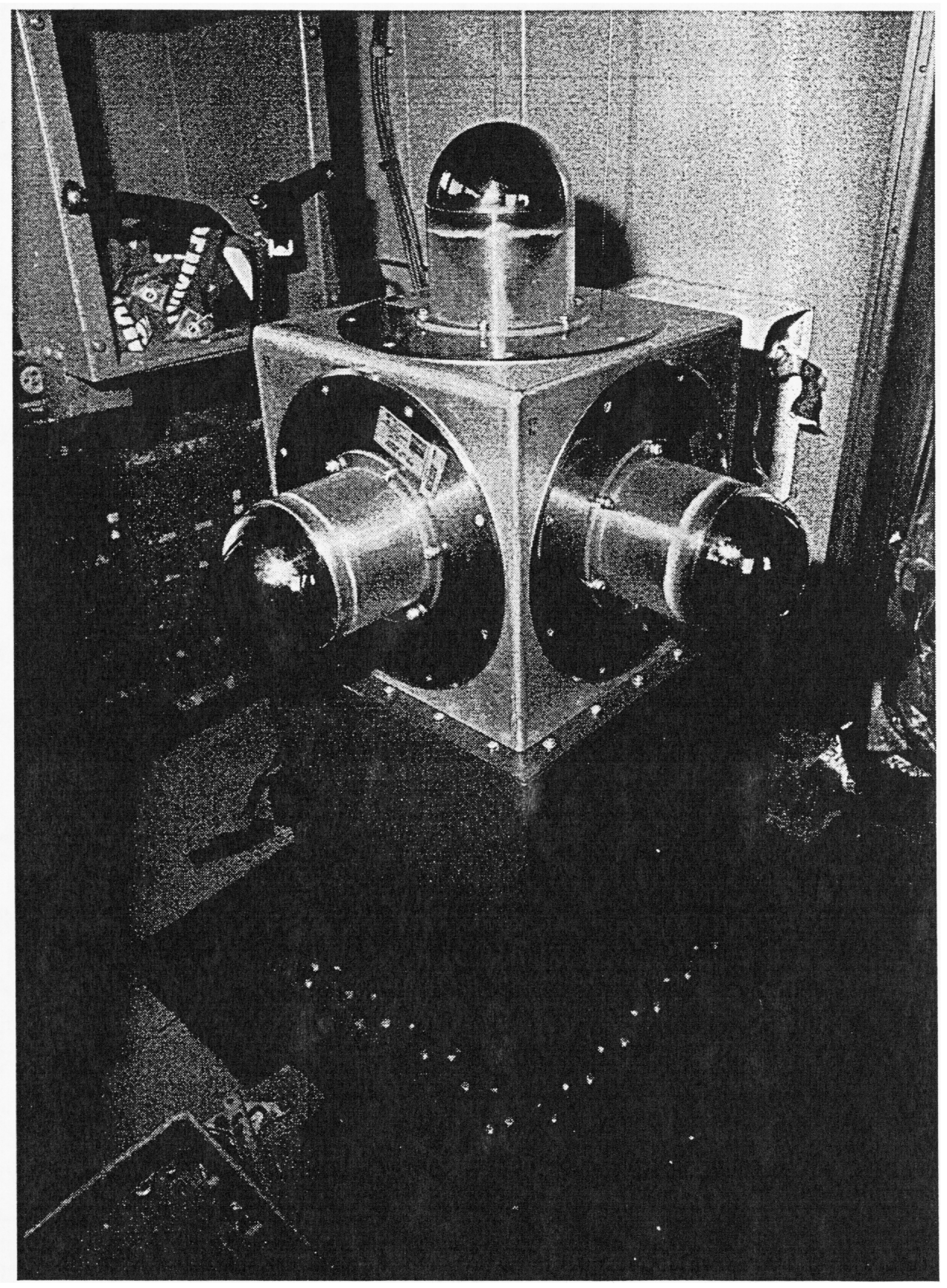

Figure 4-4. ATOPS box on pedestal in cockpit 


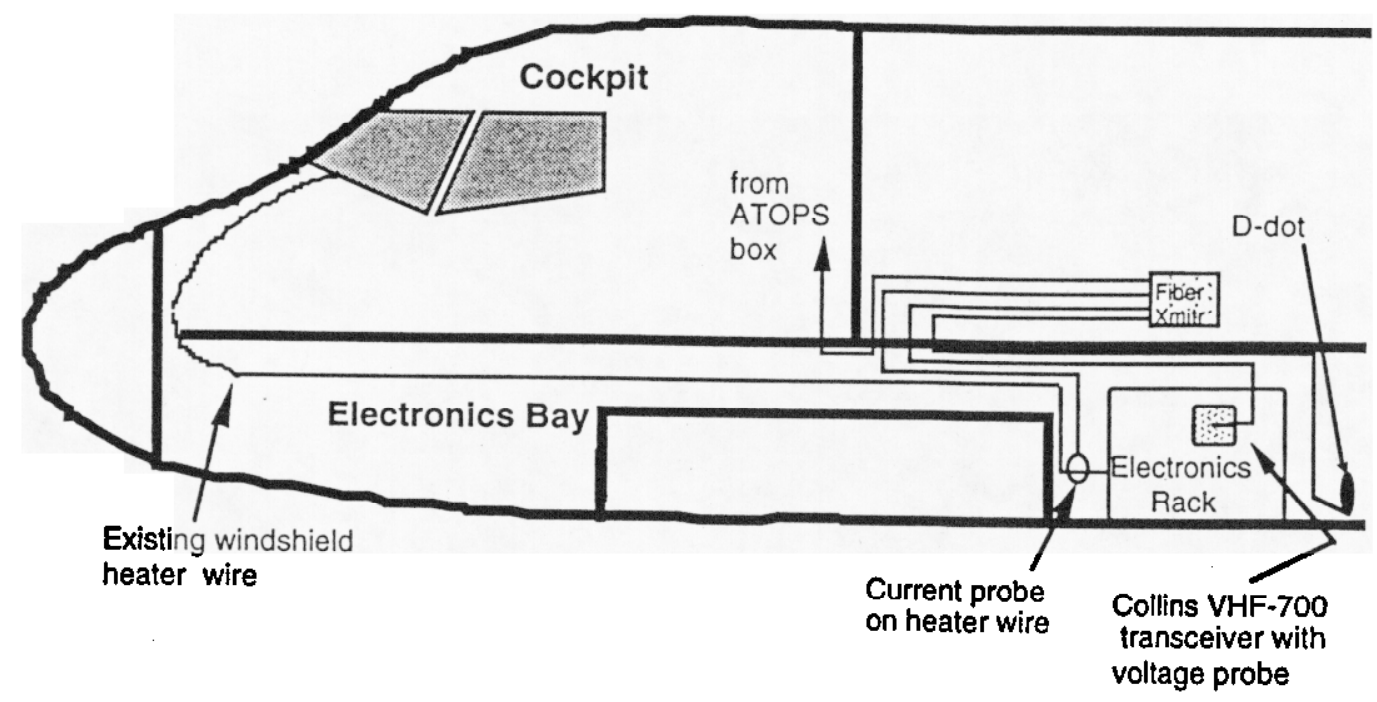

Figure 4-5. Electronics bay sensors

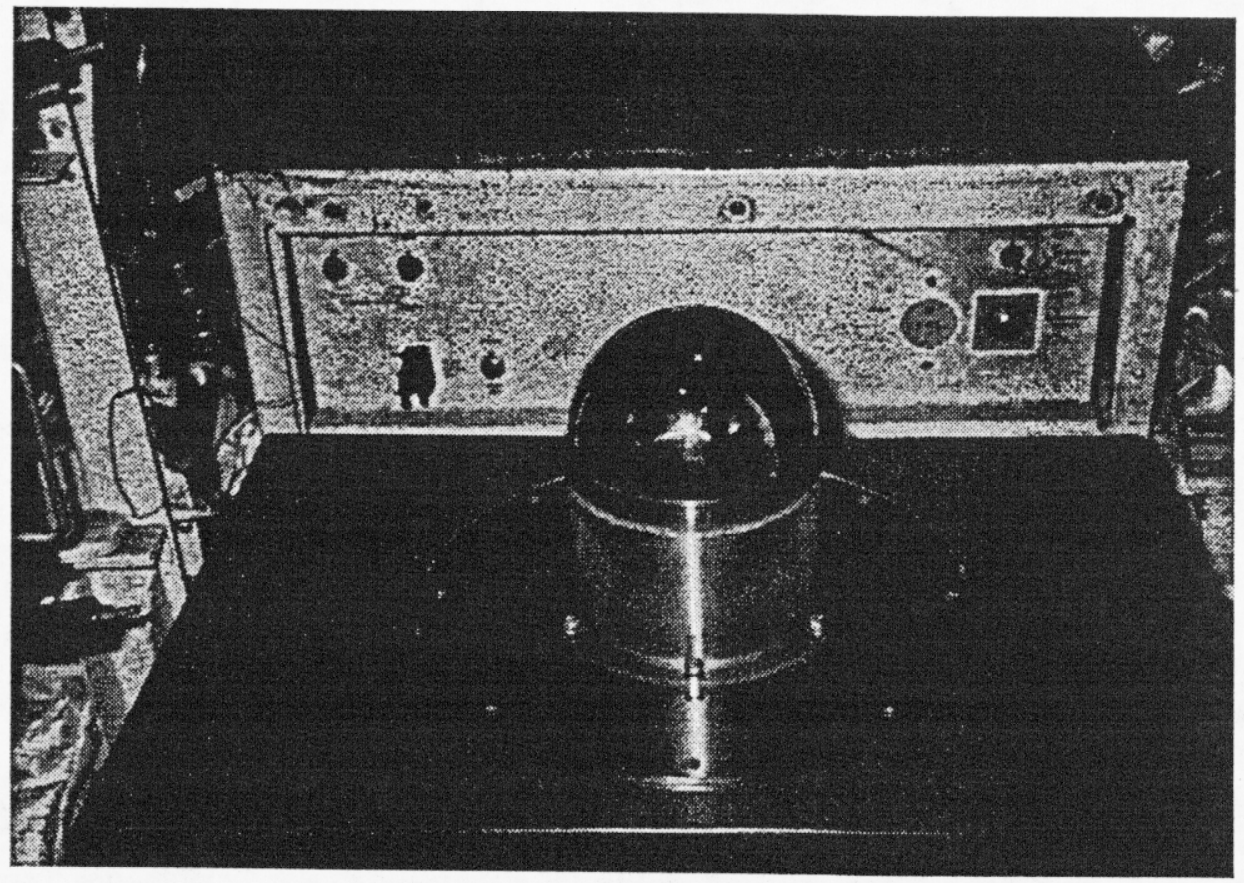

Figure 4-6. Electronics bay electric field (d-dot) sensor

\subsection{Cabin Sensors}

There were four sensors in the cabin area as shown in Figure 4-7. One of these was an AD-60 electric field sensor (D-dot sensor) mounted on top of the EME instrumentation rack (Figure 4-8). The EME rack and its power conditioning unit contained the data acquisition system for the fly-by experiments and was only used during the fly-by instrumentation checkout phase of the ground tests. This phase is not a subject of this report but suffice it to say that the system was not powered but otherwise was maintained in its fly-by configuration during the ground tests. 


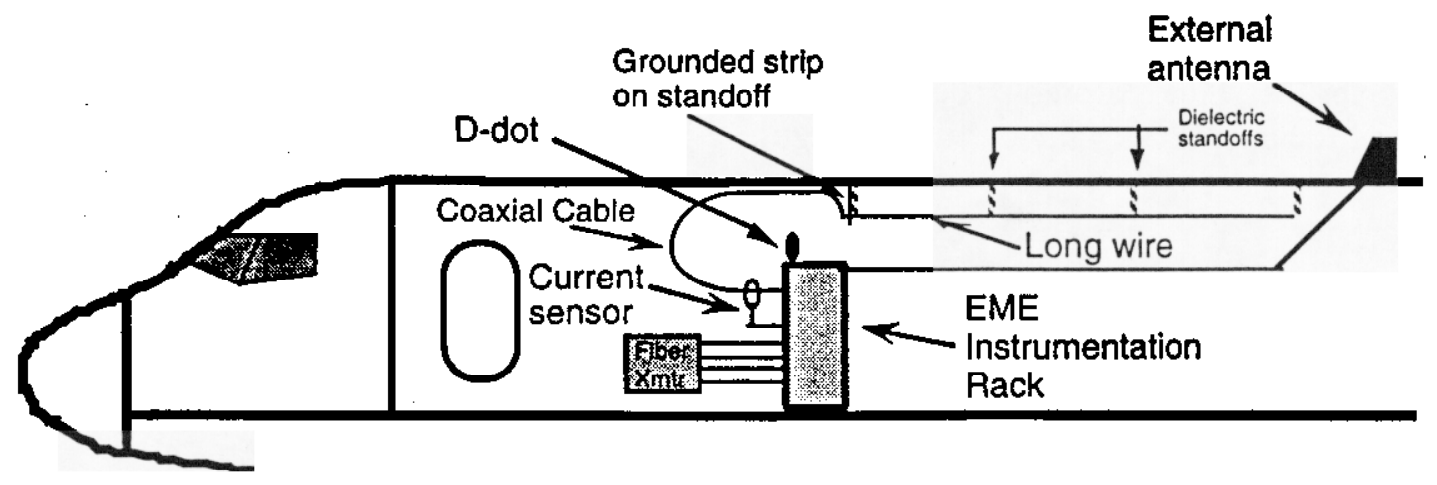

Figure 4-7. Cabin sensors
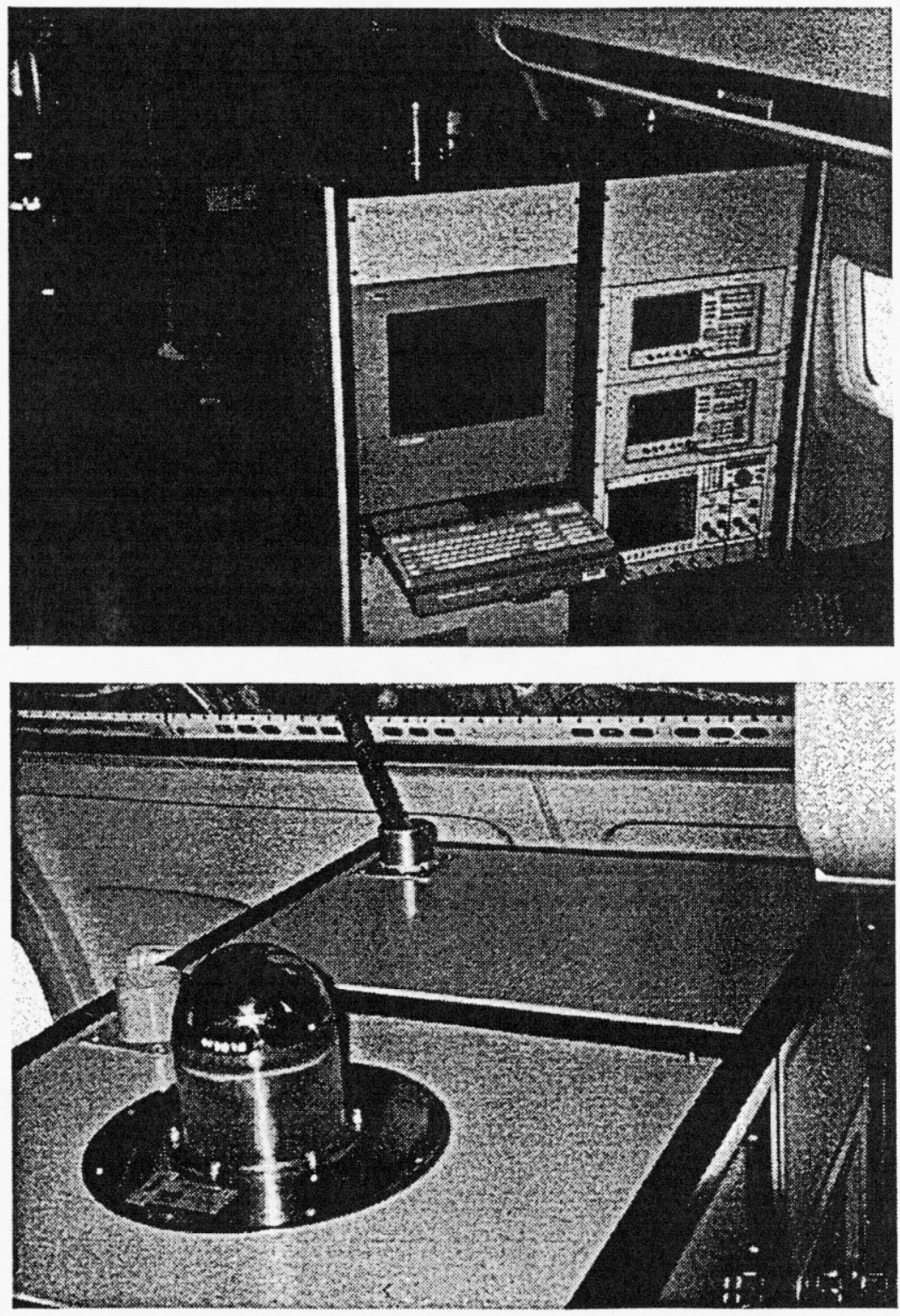

Figure 4-8. Electric field sensor (d dot) on the EME rack and close-up 
A second sensing device in the cabin was a long-wire that consisted of a 20 foot length of AWG\# 12 wire supported from the ceiling of the aircraft cabin by phenolic stand-offs. The stand-offs provided a 12 inch clearance from any metal aircraft parts. The picture in Figure 4-9a shows the supports (seen as vertical, dark colored structures spaced at uniform intervals) and the long wire (seen at the bottom of the supports). The picture in Figure 4-9b shows the first support that has an attached,

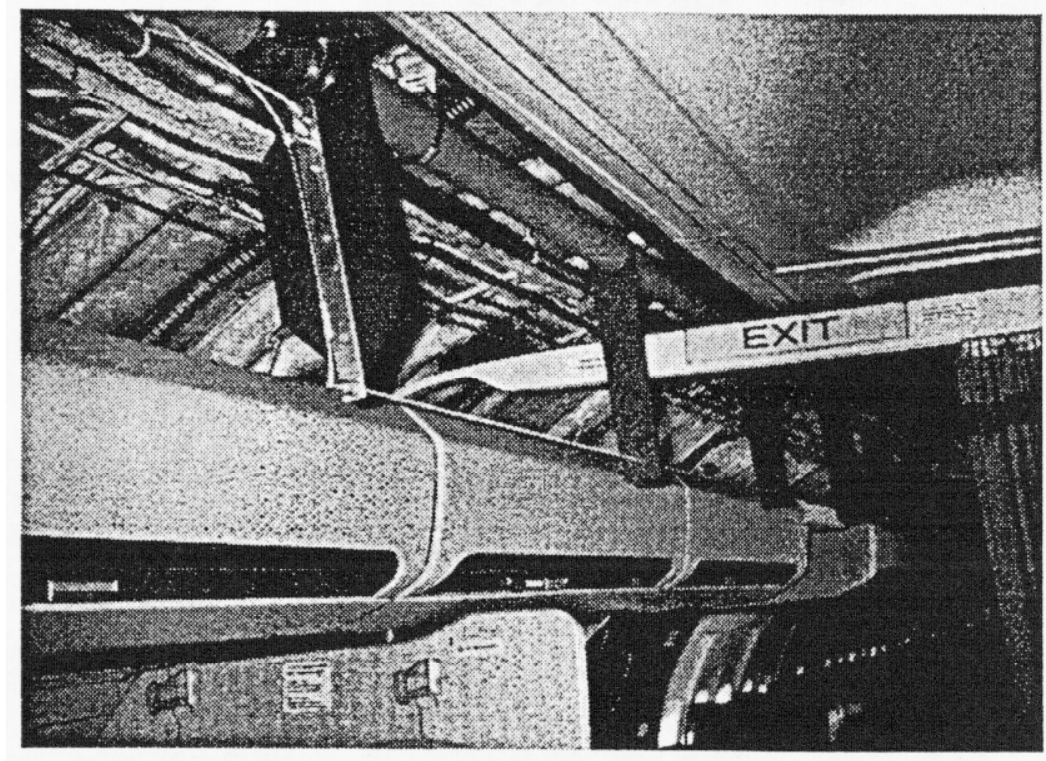

a) Long wire with dielectric supports and coaxial cable feed

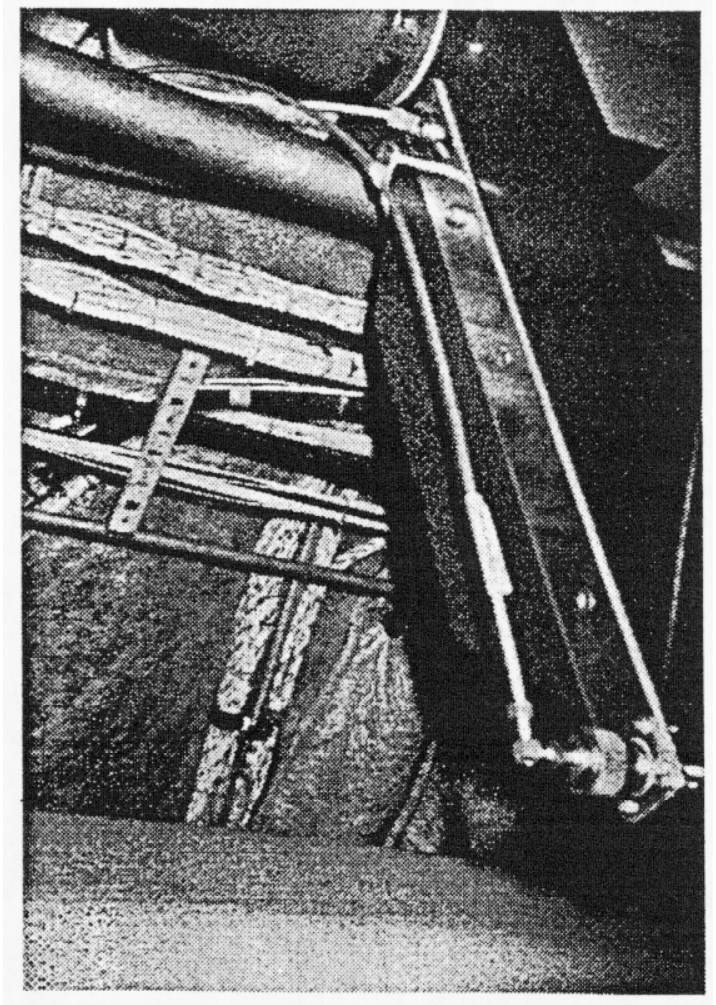

b) Long wire feed point

Figure 4-9. The cabin long wire with dielectric supports and coaxial cable feed 


\subsection{Summary of Test Points and Measured Parameters}

Table 4-1 summarizes the test point designation, the parameter being measured, and the location of each of the test points in the aircraft.

\begin{tabular}{|l|l|l|}
\hline \multicolumn{3}{|c|}{ Table 4-1. Test Points, Measured Parameters and Locations } \\
\hline $\begin{array}{c}\text { Test } \\
\text { Point }\end{array}$ & \multicolumn{1}{|c|}{$\begin{array}{c}\text { Measured } \\
\text { Parameter }\end{array}$} & \\
\hline CP1 & Vertical Field $E_{z}$ & E measured at ATOPS box in cockpit $_{z}$ mescription \\
\hline CP2 & Horiz.1 Field $E_{z}$ & $E_{z}$ (parallel to aircraft axis) measured at ATOPS box \\
\hline CP3 & Horiz.2 Field $E_{y}$ & $E_{y}$ (perpendicular to aircraft axis) measured at ATOPS box \\
\hline CP4 & Wire Current & Current on "climbing wire" at ATOPS box \\
\hline CP5 & Wire Voltage & Voltage at "climbing wire" \\
\hline CP12 & Wire Current & Current on side window heater wire \\
\hline EB1 & Vertical Field $E_{z}$ & $E_{z}$ (vertical) measured in E-bay \\
\hline EB2 & LRU Voltage & Voltage measured in power supply section of LRU (RC-7) \\
\hline EB3 & Cable Current & Current measured on power cable from windshield heater \\
\hline C1 & Vertical Field $E_{z}$ & E (vertical) measured atop the instrumentation rack in the cabin \\
\hline C2 & Wire Current & $\begin{array}{l}\text { Cable current on the coaxial cable connected to the "long wire" in } \\
\text { the cabin }\end{array}$ \\
\hline C3 & Voltage & Feedpoint voltage of the long wire in the cabin \\
\hline C4 & Field (Ext. Antenna) & Voltage measured at the feedpoint of the external blade antenna \\
\hline
\end{tabular}

\subsection{Sensor/Cable/Fiber Optic Transmitter Identifications}

The data acquisition system setup for the test series used the conceptual scheme of Figure 3-4, but for the purposes of expediency and efficiency employed two fiber optic transmitters (NanoFast)and two network analyzers (Hewlett Packard HP 8753C).This permitted simultaneous acquisition of two aircraft data channels. The block diagram for the system is shown in Figure 4-11. The sensors and their respective cabling to the F-O transmitters are not shown. The radiator and its reference in the figure were either the rhombic or dipole radiators with their associated reference probe.

The RF signal used in the tests was generated by using the RF output of the network analyzer and amplifying this signal to a level in the range of 100 to 200 watts. This was accomplished by passing the signal through one of two Amplifier Research RF amplifiers depending on the frequency range. For this test system, the ranges were defined as 0.3 to $100 \mathrm{MHz}$ (AR 250L220) and $100 \mathrm{MHz}$ to 1 GHZ (AR 200W/1000M7). This arrangement is shown in Figure 4-11. The two network analyzer measurement system involved the use of two F-O transmitters each having four input ports which could be remotely switched and a single output port.

Table 4-2 relates the test points, the variables measured, the location, the specific probe and its Heliax cable designation, and the designation of the F-O transmitter port to which the cable was attached. Retention of the information regarding the precise setup is important because the data recorded in the VANAs are, in effect, voltages or ratios of voltages at the VANA terminals.

Knowledge of the system setup permits the use of sensor and cable calibrations as well as fiber link characteristics, to back out the actual variable of interest. Thus, using the effective transfer function of the measurement system, one can gain access to a variable in a remote position (say an electric field strength) having measured a voltage at the terminals of the measurement system. Discussion of such calibration procedures is carried out in the discussion of pretest activities and the actual calibration data for the each element in the data acquisition system is identified. 
grounded metal strip on which is mounted a coaxial connector for the $50 \mathrm{ohm}$ semi-rigid coaxial cable: The feedthrough connector serves as the feedpoint for the long wire. A feedpoint voltage measurement was made on this cable as shown in Figure 4-9b. A current sensor to measure shield current was also installed on the semi-rigid coaxial cable at a position where the cable emerged from behind the wall panel at the floor of the cabin (Figure 4-10).

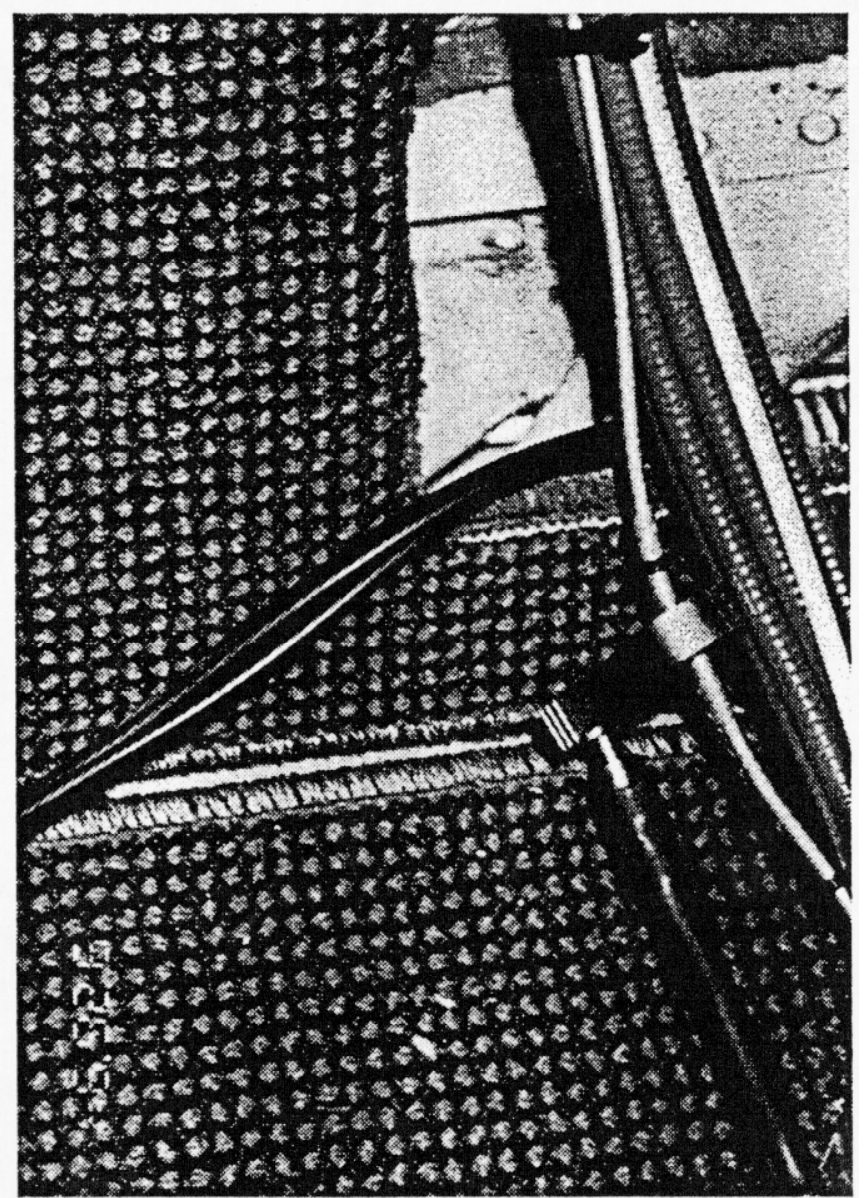

Figure 4-10. Current sensor on cable in cabin

The fourth sensor referred to as a cabin sensor was the VHF-L (left) antenna which is part of the normal complement of external antennas for the NASA aircraft . It was located on the upper surface of the fuselage. This antenna was primarily intended to provide a strong signal to be used as a trigger for the fly-by test instrumentation but also provided a measurement of the external field (perturbed by the aircraft). 


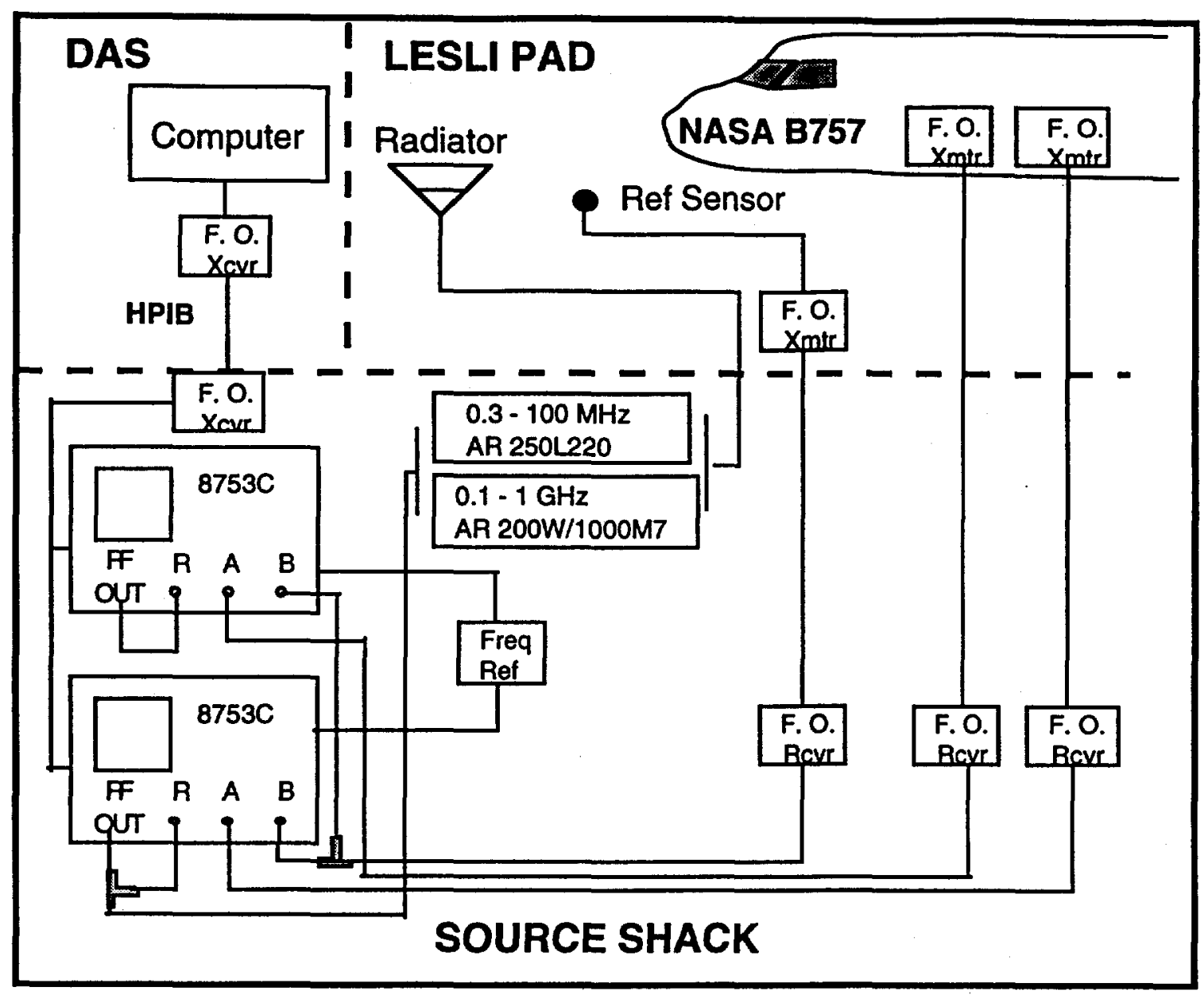

Figure 4-11. Aircraft test system using two network analyzers

\begin{tabular}{|c|c|c|c|c|c|c|}
\hline $\begin{array}{l}\text { Test } \\
\text { Point }\end{array}$ & $\begin{array}{l}\text { Measured } \\
\text { Parameter }\end{array}$ & Location & $\begin{array}{l}\text { Cable } \\
\text { Desig. }\end{array}$ & $\begin{array}{l}\text { Probel } \\
\text { Sensor }\end{array}$ & \begin{tabular}{|c|} 
XMTR \\
Input
\end{tabular} & Comments \\
\hline CP1 & Vertical Field $E_{z}$ & Cockpit & TIC3 & AD60 & NF50 & \\
\hline $\mathrm{CP2}$ & Horiz.1 Field $E_{x}$ & Cockpit & TIC1 & AD60 & NF20 & \\
\hline CP3 & Horiz.2 Field $E_{y}$ & Cockpit & T1C2 & AD60 & NF52 & \\
\hline CP4 & Wire Current & Cockpit & T1C4 & P132 & NF53 & \\
\hline $\mathrm{CP5}$ & Wire Voltage & Cockpit & T2C1 & APRB & NF51 & \\
\hline EB1 & Vertical Field $E_{z}$ & E-bay & T2C2 & AD60 & NF21 & \\
\hline EB2 & LRU Voltage & E-bay & T2C4 & APRB & NF22 & \\
\hline EB3 & Cable Current & E-bay & T2C3 & P134 & NF23 & \\
\hline $\mathrm{C} 1$ & Vertical Field $\mathrm{E}_{\mathrm{z}}$ & Cabin & T3C2 & AD60 & NF50 & Second Set \\
\hline $\mathrm{C} 2$ & Wire Current & Cabin & T3C4 & $\mathrm{P} 133$ & NF51 & Second Set \\
\hline $\mathrm{C} 3$ & Voltage & Cabin & T3C3 & APRB & NF20 & Second Set \\
\hline $\mathrm{C4}$ & Field (Ext. Antenna) & Cabin & T3C1 & APRB & NF21 & Second Set \\
\hline CP12 & $\begin{array}{l}\text { Wire Current (side } \\
\text { window heat wire) }\end{array}$ & Cockpit & TIC4 & $\overline{P 132}$ & NF53 & \\
\hline
\end{tabular}


In Table 4-2, the ports on the F-O transmitters are denoted NF\# with the 50 and 20 series denoting the two transmitters, respectively. Given the desire to record 12 channels, the cabling attachment to the F-O transmitters would have to be changed at least once during a series of tests. Thus ports NF20, NF21, NF50 and NF51 were used in each run with different cables attached. CP12 refers to a specific test which was executed during the program and which required the movement of one of the probes in the earlier series.

\subsection{Creating the Electromagnetic Environment}

The electromagnetic environment for the test object - the NASA B-757 - was generated in the LESLI facility. We have already discussed how the RF driver signal for radiators was produced - a VANA test signal was amplified in RF amplifiers to the 100 to 200 watt level. To produce the electromagnetic fields illuminating the B-757 as it sat on the LESLI concrete pad, the amplified RF signal was used to drive a radiator. For the purposes of this program, two separate radiators were used - the rhombic radiator as shown in Figures 3-2 and 3-3 and a dipole. In the following we describe the ground plane and the two distinct radiating structures.

\subsection{Ground Plane}

The concrete surface forming the pad in the LESLI facility was used in an unaltered state as the ground surface. It was originally planned that a conducting ground plane composed of interlocking metal plates would be used. This would permit accurate knowledge of the ground parameters and reduce the uncertainties in the aircraft's environment. A portable runway system owned by the US Army that could cover in excess of $15,000 \mathrm{sq}$. $\mathrm{ft}$. with approximately 1100 panels was located, inspected, and integrated into plans. However, military operational conflicts ultimately made such a ground plane unavailable and other plans for conducting ground planes proved impractical. Thus, the tests were reconfigured to use the unaltered concrete ground for which there was little information regarding constitutive parameters or the density and location of reinforcing materials. Since the parameters were unknown, and the concrete/aggregate/rebar/soil was not precisely describable, a measurement of the parameters was needed. A brief description of the method and the data is provided in Section 6.3.5 and the details are provided in NASA Contractor Report 4674 (Poggio et al., 1995).

\subsection{The Low Power On-the-Ground Test Series}

The tests conducted during the low power on-the-ground test program were governed by the test matrix presented in the test plan [Poggio et al., 1994] which is reproduced in Appendix A, for convenience. In that document the purpose of each test was defined and the test was described. Every attempt was made to adhere to that plan but in some cases tests were abandoned either because they were deemed not useful or because time restraints precluded their successful completion in view of other tests that were planned. The tests were not necessarily executed in the order presented in the test matrices but were carried out in a manner which maximized throughput by always striving for full usage of both channels in data acquisition and minimization of down-time for system reconfiguration.

\subsection{A Tabulation of the Executed Tests}

The following tables describe the tests which were executed. Table 6-1 contains the test point abbreviated name, the parameters measured at each test point, and a location description. 


\begin{tabular}{|l|l|l|}
\hline \multicolumn{3}{|c|}{ Table 6-1. Test Points and Measured Parameters } \\
\hline CP1 & Vert. Field & $E_{z}$ measured at ATOPS box in cockpit \\
\hline CP2 & Horiz.1 Field & $E_{z}$ (parallel to aircraft axis) measured at ATOPS box in cockpit \\
\hline CP3 & Horiz.2 Field & E $_{y}$ (transverse to aircraft axis) measured at ATOPS box in cockpit \\
\hline CP4 & Wire Current & Current on "climbing wire" at ATOPS box \\
\hline CP5 & Wire Voltage & Voltage at "climbing wire" \\
\hline EB1 & Field & E $_{\text {(vertical) measured in E-bay }}$ \\
\hline EB2 & LRU Voltage & Voltage measured in power supply section of LRU \\
\hline EB3 & Cable Current & Current measured on power cable from windshield heater \\
\hline C1 & Field & E (vertical) measured atop the instrumentation rack in the cabin \\
\hline C2 & Wire Current & $\begin{array}{l}\text { Cable current on the coaxial cable connected to the "long wire" in the } \\
\text { cabin }\end{array}$ \\
\hline C3 & Voltage & Feedpoint voltage of the long wire in the cabin \\
\hline C4 & Field & Voltage measured at the feedpoint of the external blade antenna \\
\hline CP12 & Wire Current & $\begin{array}{l}\text { Cockpit:; current measured on copilot's side window heater wire; uses } \\
\text { same channel as CP4; occasional use only }\end{array}$ \\
\hline
\end{tabular}

Tables 6-2 a, b, and c define the abbreviations used in the following tables.

\begin{tabular}{|c|c|}
\hline \multicolumn{2}{|c|}{ Table 6-2a. } \\
Xmit Ant \\
\hline $\mathrm{D}$ & Dipole \\
\hline $\mathrm{R}$ & Rhombic \\
\hline
\end{tabular}$\quad$\begin{tabular}{|c|c|}
\hline \multicolumn{2}{|c|}{ Table 6-2b. } \\
Polarization \\
\hline $\mathrm{V}$ & Vertical \\
\hline $\mathrm{H}$ & Horizontal \\
\hline
\end{tabular}

\begin{tabular}{|c|c|}
\hline \multicolumn{2}{|c|}{ Table 6-2c. $\quad$ Probe or Sensor } \\
\hline AD-60 & D-Dot or E field probe \\
\hline I-320 & Current Probe \\
\hline Ext. Antenna & External VHF blade antenna \\
\hline Climbing Wire & vertical wire on ATOPS Box \\
\hline Voltage Probe (also RC7) & voltage measurement in LRU \\
\hline Long Wire & voltage measurement in cabin \\
\hline
\end{tabular}


Table 6-3 contains a description of the meaning of the letter designators for the notes in the following tables.

\begin{tabular}{|c|c|}
\hline \multicolumn{2}{|r|}{ Table 6-3. Notes } \\
\hline $\bar{A}$ & Predictions of field coupling; transmit antenna in computational volume \\
\hline$\overline{\mathbf{B}}$ & Thin wire coupling \\
\hline $\mathbf{C}$ & Aircraft external field measurement \\
\hline$\overline{\mathrm{D}}$ & Typical coupling into a representative critical LRU in the E-bay \\
\hline E & Variations in coupling with frequency \\
\hline $\mathrm{G}$ & Predictions of field coupling; plane wave incident \\
\hline$\overline{\mathrm{G} 2}$ & E-bay hatch cover electrically sealed with conducting tape to assess leakage at seam \\
\hline G3 & E-bay hatch cover open to assess energy leakage \\
\hline$\overline{\mathrm{G} 4}$ & Negative pressure door sealed with conductive tape to assess change from normal state \\
\hline$\overline{\mathrm{H}}$ & Predictions of thin wire coupling; plane wave incident \\
\hline$\overline{\mathrm{I}}$ & Calibration of aircraft external field measurement, plane wave incident \\
\hline $\mathbf{J}$ & Typical coupling into a representative critical LRU in the E-bay, plane wave incident \\
\hline $\mathrm{J} 1$ & VHF circuit breaker pulled (out) \\
\hline $\mathrm{J} 2$ & VHF circuit breaker engaged (in) \\
\hline $\mathrm{K}$ & Repeatability \\
\hline$\overline{\mathbf{L}}$ & $\begin{array}{l}\text { Resolution element effects. "Small box" is a plastic box (3"x3" } \mathrm{x} 4 \text { ") wrapped in conducting } \\
\text { copper tape) located on the sensor box. }\end{array}$ \\
\hline$\overline{L 1}$ & Small box placed on lower port edge of the aft side of ATOPS Box with long axis parallel to floor \\
\hline$\overline{\mathrm{L} 2}$ & $\begin{array}{l}\text { Small box placed on upper starboard edge of the aft side of ATOPS Box with long axis } \\
\text { perpendicular to floor }\end{array}$ \\
\hline $\mathbf{M}$ & Stirring effects caused by people. Remove small box \\
\hline M1 & Pilot and copilot, personnel in seats $3 c, 3 d, 2 b$, and $3 b$ \\
\hline $\mathbf{N}$ & Cockpit Window model \\
\hline N1 & Current probe on heater wire at copilot's side window with cable toward window and forward \\
\hline $\mathrm{O}$ & $\begin{array}{l}\text { Effect of cable loads on cavity absorption. AWG \#20 wire } 3 \text { feet long with } 213 \Omega \text { resistor at } \\
\text { ground end on ATOPS Box. }\end{array}$ \\
\hline O1 & Free end of wire on pilot's seat \\
\hline$\overline{\mathbf{O} 2}$ & Free end of wire to top of jump seat \\
\hline$\overline{\mathbf{P}}$ & $\begin{array}{l}\text { Angular sensitivity. Movement to } 5^{\circ} \text { will be performed if measurements at } 10^{\circ} \text { indicate strong } \\
\text { sensitivity }\end{array}$ \\
\hline $\bar{Q}$ & Effect of aircraft static grounding wire \\
\hline $\overrightarrow{\mathbf{R}}$ & Repeatability (angular) \\
\hline S1 & Cockpit seats in mid position as compared to normal full forward in all other tests \\
\hline T & Measurement noise floors \\
\hline$\overline{\mathbf{U}}$ & Ambient noise measurement \\
\hline$\overline{\mathrm{V}}$ & $\begin{array}{l}\text { Instrumentation Noise Measurements: Strongest and weakest channels observed over the test } \\
\text { series are chosen. Probe is replaced by matched load and noise signals recorded. }\end{array}$ \\
\hline $\bar{X} 1$ & No cables connected to the other three ports of the respective $\mathrm{F}-\mathrm{O}$ transmitter to avoid crosstalk \\
\hline $\mathrm{Z}$ & \\
\hline
\end{tabular}

Tables 6-4 through 6-16 are a tabulation of the thirteen test series which were performed. Most of the data in these tables are extracted directly from the Test Plan, Appendix A. Also included in the tables are specific identifications of sensors, cables, date and time of acquisition, and remarks for each test for later reconstruction of the test environment, if necessary. 
A brief description of each table follows:

- Table 6-4. Test Series AN -Ambient noise measurements.

- Table 6-5. Test Series A - Dipole measurements, horizontal polarization from 23 to $28 \mathrm{MHz}$. NOTE: Tests A101 and A102 were added to the test matrix to better understand the effects of seat placement.

- Table 6-6. Test Series B - Dipole measurements, horizontal polarization from 23 to $28 \mathrm{MHz}$.

- Table 6-7 Test Series C - Dipole measurements, vertical polarization from 150 to $200 \mathrm{MHz}$. NOTE: Tests C104, 204, 404, and C109 were added to the test matrix to investigate the effects of cross-talk in the instrumentation

- Table 6-8 Test Series D - Dipole measurements, horizontal polarization from 150 to $200 \mathrm{MHz}$.

- Table 6-9 Test Series E - Dipole measurements, vertical polarization from 350 to $500 \mathrm{MHz}$. NOTE: Test E004 was a repeat measurement to increase the signal to noise response of the data.

- Table 6-10. Test Series F- Dipole measurements, horizontal polarization from 350 to $500 \mathrm{MHz}$. NOTE: Tests F008 and F010 were added to the test matrix to investigate the effects of crosstalk in the instrumentation.

- Table 6-11. Noise Floor Measurements- in which the sensors were replaced by a 50Q Load and the noise contributed by the instrumentation measured.

- Table 6-12. Test Series J - Rhombic measurements, nose-on from $0.3 \mathrm{MHz}$ to $1 \mathrm{GHz}$. NOTE: Test J05 was added to the test matrix as a vertical wire variation to investigate instrumentation loading, and Tests J026, J028, and J029 were added to investigate coupling into the aircraft with the cargo bay door sealed with copper tape.

- Table 6-13. Test Series K - Rhombic measurements, wing-on from 0-.3 MHz to $1 \mathrm{GHz}$. NOTE: Tests K16 through K22 (variations with people in various positions in the aircraft) were dropped because this experiment had been accomplished in Test Series J. Tests K007 and J024 were added to the test matrix after the aircraft returned to the nose-on orientation to investigate the effects of circuit breaker configurations.

- Table 6-14. Test Series Z - Rhombic measurements, nose-on from $0.3 \mathrm{MHz}$ to $1 \mathrm{GHz}$. NOTE: This entire test series was added to the test matrix to study various issues which arose during the test period.

- Table 6-15. Rhombic Horizontal Polarization - Rhombic in horizontal polarization measurements, nose-on from $0.3 \mathrm{MHz}$ to $1 \mathrm{GHz}$. NOTE: This entire test series was added to the test matrix to provide analysts with data pertaining to the aircraft's lowest order resonances.

- Table 6-16. Test Series WF - Rhombic measurements, nose-on from $0.3 \mathrm{MHz}$ to $1 \mathrm{GHz}$ with the aircraft windshield covered with aluminum foil. NOTE: This entire test series was added to study the effect of the $\mathrm{a} / \mathrm{c}$ windshield as a major POE. 
Table 6-4. Test Series AN - Ambient Noise Measurements

\begin{tabular}{|c|c|c|c|c|c|c|c|c|c|c|c|}
\hline $\begin{array}{c}\text { Test } \\
\#\end{array}$ & $\begin{array}{l}\text { Test } \\
\text { Point }\end{array}$ & $\begin{array}{l}\text { Measured } \\
\text { Parameter }\end{array}$ & $\begin{array}{c}\text { Probe or } \\
\text { Sensor }\end{array}$ & $\begin{array}{l}\text { Freq. } \\
\text { (MHz) }\end{array}$ & $\begin{array}{l}\text { Xmit } \\
\text { Ant. }\end{array}$ & Pol & Configuration & Notes & Cable ID & $\begin{array}{c}\text { Acquisition } \\
\text { DaterTime }\end{array}$ & Remarks \\
\hline AN1 & CP1 & Vert. Field & AD60 \#35 & $0.3 \cdot 1000$ & & & & $\bar{U}$ & TIC3/P5 & $9-23 / 10: 55$ & $\begin{array}{l}\text { REF1 }=\text { MGL-6 for } \mathrm{H}_{2} \\
\text { at }(-1.525,3,-7.475)\end{array}$ \\
\hline$\overline{\mathrm{AN} 2}$ & $\overline{\mathrm{CP} 2}$ & Horiz.l Field & $\overline{A D 60 ~ \# 32}$ & $0.3-1000$ & & & & $\bar{U}$ & T1Cl/GTC4 & $9-23 / 10: 55$ & $\begin{array}{l}\text { REF }=\text { MGL-6 for } \mathrm{H}_{2} \\
\text { at }(-1.525,3,-7.475)\end{array}$ \\
\hline$\overline{\text { AN3 }}$ & $\overline{\text { EB3 }}$ & Cable Current & $1-320 \# 34$ & $0.3-1000$ & & & & $\bar{U}$ & T2C3/P7 & $9-23 / 12: 45$ & $\begin{array}{l}\text { REF2 }=\mathrm{MGL}-6 \text { for } \mathrm{H}_{2} \\
\text { at }(-1.525,3,-7.475)\end{array}$ \\
\hline$\overline{\text { AN4 }}$ & $\overline{\mathrm{C} 4}$ & Field & Ext. antenna & $0.3-1000$ & & & & $\bar{U}$ & T3C1/P1 & $9-23 / 13: 30$ & $\begin{array}{l}\text { REF2 }=\text { MGL-6 for } \mathrm{H}_{2} \\
\text { at }(-1.525 ; 3,-7.475)\end{array}$ \\
\hline
\end{tabular}


Table 6-5. Test Series A - Dipole (Horizontal, 23 - 28 MHz)

\begin{tabular}{|c|c|c|c|c|c|c|c|c|c|c|c|}
\hline $\begin{array}{c}\text { Test } \\
\#\end{array}$ & $\begin{array}{c}\text { Test } \\
\text { Point }\end{array}$ & $\begin{array}{l}\text { Measured } \\
\text { Parameter }\end{array}$ & $\begin{array}{l}\text { Probe or } \\
\text { Sensor }\end{array}$ & $\begin{array}{l}\text { Freq. } \\
(\mathrm{MHz})\end{array}$ & $\begin{array}{l}\text { Xmit } \\
\text { Ant. }\end{array}$ & Pol & Configuration & Notes & Cable ID & $\begin{array}{l}\text { Acquisition } \\
\text { Date/Time } \\
\end{array}$ & Remarks \\
\hline$\overline{\mathrm{Al}}$ & $\mathrm{CPI}$ & Vert. Field & AD-60 \#35 & $23-28$ & $\bar{D}$ & $\overline{\mathrm{H}}$ & $\begin{array}{l}\text { Dipole } / / \text { to } \mathrm{a} / \mathrm{c} \\
\text { axis }\end{array}$ & $\bar{A}$ & TIC3/P5 & $9-26 / 14: 39$ & Note: Seats aft, see A101 \\
\hline A2 & $\mathrm{CP2}$ & Horiz.1 Field & AD-60 \#32 & $23-28$ & $\bar{D}$ & $\overline{\mathrm{H}}$ & $\begin{array}{l}\text { Dipole } / / \text { to a/c } \\
\text { axis }\end{array}$ & $\bar{A}$ & TICI/GTC4 & $9-26 / 14: 39$ & Note: Seats aft, see $\mathrm{Al} 102$ \\
\hline A3 & $\overline{\mathrm{CP3}}$ & Horiz.2 Field & $\mathrm{AD}-60 \# 36$ & $23-28$ & $\bar{D}$ & $\mathbf{H}$ & $\begin{array}{l}\text { Dipole } / / \text { to } a / c \\
\text { axis }\end{array}$ & $\bar{A}$ & TIC2/GTCl & $\begin{array}{l}9-261 \\
\text { 1. } 12: 45 \\
\text { 2. } 13: 07 \\
\end{array}$ & $\begin{array}{l}\text { 1. Repeat nu for jump at } \\
25 \mathrm{MHz} \\
\text { 2. Grad rise at } 75.5 \mathrm{MHz}\end{array}$ \\
\hline A4 & $\overline{\mathrm{CP} 4}$ & Wire Current & $1-320 \# 32$ & $23-28$ & D & $\mathbf{H}$ & $\begin{array}{l}\text { Dipole } / / \text { to } \mathrm{a} / \mathrm{c} \\
\text { axis }\end{array}$ & B & TIC4/GTC3 & $9-26 / 13: 56$ & \\
\hline$\overline{A 5}$ & $\overline{\mathrm{CP5}}$ & Wire Voltage & $\begin{array}{c}\text { Climbing Wire } \\
. \\
\end{array}$ & $23 \cdot 28$ & $\bar{D}$ & $\bar{H}$ & $\begin{array}{l}\text { Dipole } / / \text { to } \mathrm{a} / \mathrm{c} \\
\text { axis }\end{array}$ & $\mathbf{B}$ & T2C1/GTC2 & $9-26 / 15: 35$ & \\
\hline$\overline{A 6}$ & EB I & Field & AD-60 \#34 & $23 \cdot 28$ & D & $\mathrm{H}$ & $\begin{array}{l}\text { Dipole } / / \text { to a/c } \\
\text { axis }\end{array}$ & $\bar{A}$ & $\mathrm{~T} 2 \mathrm{C} 2 / \mathrm{P} 6$ & $9-26 / 12: 05$ & \\
\hline A7 & EB2 & LRU Voltage & RC7 & $23-28$ & $\bar{D}$ & $\mathbf{H}$ & $\begin{array}{l}\text { Dipole } / / \text { to a/c } \\
\text { axis }\end{array}$ & $\bar{D}$ & T2C4/GTC5 & $9-26 / 13: 07$ & $\begin{array}{l}\text { down to noise at about } \\
25.5 \mathrm{MHz} \& \text { back out at } \\
\text { about } 26.5 \mathrm{MHz} 22\end{array}$ \\
\hline A8 & EB3 & Cable Current & $1-320 \# 34$ & $23-28$ & $\bar{D}$ & $\mathbf{H}$ & $\begin{array}{l}\text { Dipole } / / \text { to a/c } \\
\text { axis }\end{array}$ & B & T2C3/P7 & $9-26 / 13: 56$ & \\
\hline A9 & $\overline{C l}$ & Field & AD-60 \#33 & $23-28$ & $\bar{D}$ & $\mathbf{H}$ & $\begin{array}{l}\text { Dipole } / / \text { to a/c } \\
\text { axis }\end{array}$ & $\bar{A}$ & T3C2/P2 & $9-26 / 10: 34$ & \\
\hline$\overline{A 10}$ & $\overline{\mathrm{C} 2}$ & Wire Current & $\mathrm{I}-320 \# 33$ & $23-28$ & $\bar{D}$ & $\bar{H}$ & $\begin{array}{l}\text { Dipole // to a/c } \\
\text { axis }\end{array}$ & B & $\mathrm{T} 3 \mathrm{C} 4 / \mathrm{P} 3$ & $9-26 / 10: 57$ & \\
\hline$\overline{A 11}$ & C3 & Voltage & Long Wire & $23-28$ & $\mathrm{D}$ & $\bar{H}$ & $\begin{array}{l}\text { Dipole } / / \text { to a/c } \\
\text { axis }\end{array}$ & B & T3C3/P4 & $\begin{array}{l}9-26 / 10: 34 \\
10: 57\end{array}$ & \\
\hline$\overline{A 12}$ & C4 & Field & Ext. Ant & $23-28$ & $\bar{D}$ & $\bar{H}$ & $\begin{array}{l}\text { Dipole } / / \text { to a/c } \\
\text { axis }\end{array}$ & $\mathrm{C}$ & $\mathrm{T} 3 \mathrm{Cl} / \mathrm{Pl}$ & $9-26 / 15: 35$ & \\
\hline$\overline{A 13}$ & $\begin{array}{l}\text { Weak } \\
\text { Signal } \\
\text { Line }\end{array}$ & LRU Voltage & RC7 & $23-28$ & $\mathrm{D}$ & $\mathrm{H}$ & $\begin{array}{l}\text { Dipole // to a/c } \\
\text { axis }\end{array}$ & $\bar{V}$ & T2C4/GTC5 & $9-26 / 16: 26$ & $\begin{array}{l}\text { Weak signal, } 50 \text { ohm load } \\
\text { on cable at RC7 }\end{array}$ \\
\hline A14 & $\begin{array}{l}\text { Strong } \\
\text { Signal } \\
\text { Line }\end{array}$ & Voltage & Long Wire & $23-28$ & $\bar{D}$ & $\bar{H}$ & $\begin{array}{l}\text { Dipole } / / \text { to } \mathrm{a} / \mathrm{c} \\
\text { axis }\end{array}$ & $\mathrm{v}$ & $\mathrm{T} 3 \mathrm{C} 3 / \mathrm{P} 4$ & $9-26 / 16: 08$ & $\begin{array}{l}\text { Strong signal, } 50 \mathrm{ohm} \text { on } \\
\text { cable at LW }\end{array}$ \\
\hline A101 & $\mathrm{CP1}$ & Field & AD-60 \#35 & $23-28$ & $\mathrm{D}$ & $\mathrm{H}$ & $\begin{array}{l}\text { Dipole } / / \text { to } \mathrm{a} / \mathrm{c} \\
\text { axis }\end{array}$ & $\mathrm{V}$ & T1C3/P5 & $9.26 / 15: 03$ & Seats forward \\
\hline A102 & CP2 & Field & AD-60 \#32 & $23-28$ & D & $\mathrm{H}$ & $\begin{array}{l}\text { Dipole } / / \text { to a/c } \\
\text { axis }\end{array}$ & $\bar{V}$ & T1C1/GTC4 & $9-26 / 15: 03$ & Seats forward \\
\hline
\end{tabular}


Table 6-6. Test Series B - Dipole (Vertical, 23 - $28 \mathrm{MHz}$ )

\begin{tabular}{|c|c|c|c|c|c|c|c|c|c|c|c|}
\hline $\begin{array}{l}\text { Test } \\
\#\end{array}$ & $\begin{array}{l}\text { Test } \\
\text { Point }\end{array}$ & $\begin{array}{l}\text { Measured } \\
\text { Parameter }\end{array}$ & $\begin{array}{l}\text { Probe or } \\
\text { Sensor }\end{array}$ & $\begin{array}{l}\text { Freq. } \\
\text { (MHz) }\end{array}$ & $\begin{array}{l}\text { Xmit } \\
\text { Ant. } \\
\end{array}$ & Pol & $\begin{array}{l}\text { Orientation / } \\
\text { Configuration } \\
\end{array}$ & Notes & Cable ID & $\begin{array}{l}\text { Acquisition } \\
\text { Date/Time }\end{array}$ & Remarks \\
\hline & & & & & & & & & & & \\
\hline B1 & $\mathrm{CPI}$ & Vert. Field & $A D-60 \# 35$ & $23-28$ & $\mathrm{D}$ & $\mathrm{V}$ & front, left of $a / c$ & $\mathrm{~A}$ & TIC3/P5 & $9-23 / 14: 20$ & \\
\hline B2 & CP2 & Horiz.1 Field & AD- $60 \# 32$ & $23-28$ & $\mathrm{D}$ & $\mathrm{V}$ & front, left of $a / c$ & $\mathrm{~A}$ & TICI/GTC4 & $9.23 / 15: 26$ & Copilot seat in aft post'n \\
\hline B3 & $\mathrm{CP3}$ & Horiz.2 Field & AD-60 \#36 & $23-28$ & $\mathrm{D}$ & $\mathrm{V}$ & front, left of a/c & $\mathrm{A}$ & $\mathrm{T} 1 \mathrm{C} 2 / \mathrm{GTCl}$ & $9-23 / 15: 05$ & \\
\hline B4 & CP4 & Wire Current & $1-320 \# 32$ & $23-28$ & $\mathrm{D}$ & $\mathbf{V}$ & front, left of $a / c$ & B & T1C4/GTC3 & $9.23 / 15: 26$ & \\
\hline B5 & CP5 & Wire Voltage & climbing wire & $23-28$ & $\mathrm{D}$ & $\bar{V}$ & front, left of $a / c$ & $\mathrm{~B}$ & T2C1/GTC2 & $9-23 / 14: 40$ & \\
\hline B6 & EBI & Field & AD-60 \#34 & $23-28$ & $\bar{D}$ & $\bar{V}$ & front, left of $a / c$ & $\mathbf{A}$ & $\mathrm{T} 2 \mathrm{C} 2 / \mathrm{P} 6$ & $9-26 / 8: 09$ & $\begin{array}{l}\text { App } 15.5 \mathrm{~dB} \text { dip at } 25.5 \\
\mathrm{MHz}\end{array}$ \\
\hline B7 & EB2 & LRU Voltage & RC7 & $23-28$ & $\bar{D}$ & $\mathrm{~V}$ & front, left of $a / c$ & $\mathrm{D}$ & T2C4/GTC5 & $9.23 / 15: 48$ & \\
\hline B8 & EB3 & Cable Current & $I-320 \# 34$ & $23-28$ & $\mathrm{D}$ & $\mathrm{V}$ & front, left of a/c & $\mathbf{B}$ & $\mathrm{T} 2 \mathrm{C} 3 / \mathrm{P} 7$ & $9-23 / 14: 30$ & \\
\hline B9 & $\mathrm{Cl}$ & Field & AD-60 \#33 & $23-28$ & $\bar{D}$ & $\mathrm{~V}$ & front, left of $a / c$ & $\mathrm{~A}$ & T3C2/P2 & 9-26/8:09 & \\
\hline B10 & $\mathrm{C} 2$ & Wire Current & I-320 \#33 & $23-28$ & D & $\bar{V}$ & front, left of $a / c$ & $\mathbf{B}$ & $\mathrm{T} 3 \mathrm{C} 4 / \mathrm{P} 3$ & $9-26 / 7: 45$ & \\
\hline B11 & $\mathrm{C} 3$ & Voltage & Long Wire & $23-28$ & D & $\mathrm{V}$ & front, left of $a / c$ & B & T3C3/P4 & $9-26 / 7: 45$ & \\
\hline B12 & $\mathrm{C} 4$ & Field & Ext. Ant. & $23-28$ & $\bar{D}$ & $\bar{V}$ & front, left of $a / c$ & $\mathrm{C}$ & $\mathrm{T} 3 \mathrm{Cl} 1 \mathrm{Pl}$ & $\begin{array}{c}9-23 / 14: 40 \\
15: 05\end{array}$ & \\
\hline B13. & $\mathrm{Cl}$ & Field & $A D-60 \# 33$ & $25.7-26$ & $\mathrm{D}$ & $\mathrm{V}$ & $\begin{array}{l}1 \% \mathrm{BW} \\
1000 \mathrm{pts}\end{array}$ & $\bar{E}$ & $\mathrm{~T} 3 \mathrm{C} 2 / \mathrm{P} 2$ & $9-26 / 8: 40$ & 1000 points \\
\hline B14 & $\begin{array}{l}\text { Weak } \\
\text { Signal } \\
\text { Line } \\
\end{array}$ & LRU Voltage & $\mathrm{RC7}$ & $23-28$ & $\bar{D}$ & $\bar{V}$ & front, left of $a / c$ & $\bar{V}$ & T2C4/GTC5 & $9-26 / 9: 00$ & $\begin{array}{l}50 \text { ohm load on cable at } \\
\text { RC7 }\end{array}$ \\
\hline B15 & $\begin{array}{l}\text { Strong } \\
\text { Signal } \\
\text { Line }\end{array}$ & Voltage & Long Wire & $23-28$ & $\bar{D}$ & $\mathrm{~V}$ & front, left of $a / c$ & $\mathrm{~V}$ & $\mathrm{~T} 3 \mathrm{C} 3 / \mathrm{P} 4$ & $9-26 / 9: 22$ & $\begin{array}{l}50 \text { ohm load on cable at } \\
\text { LW }\end{array}$ \\
\hline
\end{tabular}


Table 6-7. Test Series C - Dipole (Vertical, 150 - $200 \mathrm{MHz}$ )

\begin{tabular}{|c|c|c|c|c|c|c|c|c|c|c|c|}
\hline $\begin{array}{l}\text { Test } \\
\#\end{array}$ & $\begin{array}{l}\text { Test } \\
\text { Point } \\
\end{array}$ & $\begin{array}{l}\text { Measured } \\
\text { Parameter }\end{array}$ & $\begin{array}{l}\begin{array}{l}\text { Probe or } \\
\text { Sensor }\end{array} \\
\end{array}$ & $\begin{array}{l}\text { Freq. } \\
\text { (MHz) } \\
\end{array}$ & $\begin{array}{l}\text { Xmit } \\
\text { Ant. }\end{array}$ & Pol & $\begin{array}{l}\text { Orientation I } \\
\text { Configuration } \\
\end{array}$ & Notes & Cable ID & $\begin{array}{l}\text { Acquisition } \\
\text { Date/Time }\end{array}$ & Remarks \\
\hline & & & & & & & & & & & \\
\hline $\mathrm{Cl}$ & CPI & Vert. Field & AD-60 \#35 & $150-200$ & D & $\mathrm{V}$ & front, left of $a / c$ & A & $\mathrm{T} 1 \mathrm{C} 3 / \mathrm{P} 5$ & 9-27/9:31 & \\
\hline$\overline{C 2}$ & $\mathrm{CP2}$ & Horiz.1 Field & AD-60 \#32 & $150-200$ & D & $\bar{v}$ & front, left of $\mathrm{a} / \mathrm{c}$ & $\mathrm{A}$ & TIC1/GTC4 & $9-27 / 12: 55$ & \\
\hline $\mathrm{C} 3$ & $\mathrm{CP} 3$ & Horiz.2 Field & AD-60\#36 & $150-200$ & $\mathrm{D}$ & $\mathrm{V}$ & front, left of $a / c$ & $\mathrm{~A}$ & TIC2/GTCl & $9-27 / 10: 19$ & \\
\hline $\mathrm{C} 4$ & $\mathrm{CP} 4$ & Wire Current & $1-320 \# 32$ & $150-200$ & $\bar{D}$ & $\mathrm{~V}$ & front, left of $\mathrm{a} / \mathrm{c}$ & B & TIC4/GTC3 & $9-27 / 10: 47$ & \\
\hline $\mathrm{C5}$ & CP5 & Wire Voltage & climbing wire & $150-200$ & D & $\bar{v}$ & front, left of $\mathrm{a} / \mathrm{c}$ & B & T2C1/GTC2 & $9-27 / 9: 58$ & \\
\hline C6 & EBI & Field & $\mathrm{AD}-60 \# 34$ & $150 \cdot 200$ & $\bar{D}$ & $\bar{v}$ & front, left of $\mathrm{a} / \mathrm{c}$ & $\mathbf{A}$ & $\mathrm{T} 2 \mathrm{C} 2 / \mathrm{P} 6$ & $9-27 / 1: 15$ & \\
\hline $\mathrm{C} 7$ & EB2 & LRU Voltage & Voltage Probe & $150 \cdot 200$ & D & $\mathbf{v}$ & front, left of $\mathrm{a} / \mathrm{c}$ & D & T2C4/GTC5 & $9-27 / 10: 19$ & \\
\hline $\mathrm{C} 8$ & EB3 & Cable Current & $1-320 \# 34$ & $150-200$ & $\bar{D}$ & $\bar{v}$ & front, left of $\mathrm{a} / \mathrm{c}$ & B & $\mathrm{T} 2 \mathrm{C} 3 / \mathrm{P} 7$ & $9-27 / 10: 47$ & \\
\hline C9 & $\mathrm{Cl}$ & Field & $\mathrm{AD}-60 \# 33$ & $150-200$ & D & $\mathrm{V}$ & front, left of a/c & $\mathbf{A}$ & T3C2/P2 & $9-27 / 1: 38$ & \\
\hline $\mathrm{ClO}$ & $\mathrm{C} 2$ & Wire Current & $1-320 \# 33$ & $150-200$ & D & $\mathrm{v}$ & front, left of a/c & B & T3C4/P3 & $\begin{array}{c}9-27 / 12: 15, \\
1: 15 \\
\end{array}$ & \\
\hline$\overline{C l 1}$ & $\mathrm{C} 3$ & Voltage & Long Wire & $150-200$ & $\bar{D}$ & $\bar{v}$ & front, left of $\mathrm{a} / \mathrm{c}$ & $\mathrm{B}$ & $\mathrm{T} 3 \mathrm{C} 3 / \mathrm{P} 4$ & $9-27 / 9: 31$ & \\
\hline $\mathrm{C} 12$ & $\mathrm{C} 4$ & Field & Ext. antenna & $150-200$ & D & $\mathrm{V}$ & front, left of $a / c$ & C & $\mathrm{T} 3 \mathrm{C} 1 / \mathrm{Pl}$ & $9-28 / 9: 58$ & \\
\hline $\mathrm{C} 13$ & $\mathrm{Cl}$ & Field & AD-60 \#33 & $171-173$ & D & $\mathrm{V}$ & $\begin{array}{l}1 \% \mathrm{BW}, 1000 \\
\text { pts }\end{array}$ & $E$ & $\mathrm{~T} 3 \mathrm{C} 2 / \mathrm{P} 2$ & $9-27 / 1: 51$ & \\
\hline$\overline{C 14}$ & $\begin{array}{l}\text { Weak } \\
\text { Signal } \\
\text { Line }\end{array}$ & Wire Current & l $-320 \# 32$ & $150-200$ & $\bar{D}$ & $\mathrm{~V}$ & front, left of $a / c$ & $\mathrm{~V}$ & T104/GTC3 & $9-27 / 2: 25$ & $\begin{array}{l}50 \text { ohms on cable at } \\
\text { connection to I-320 }\end{array}$ \\
\hline$\overline{C 15}$ & $\begin{array}{l}\text { Strong } \\
\text { Signal } \\
\text { Line }\end{array}$ & Voltage & Long Wire & $150-200$ & $\bar{D}$ & $\bar{v}$ & front, left of $a / c$ & & T3C3/P4 & $9-27 / 2: 25$ & $\begin{array}{l}50 \text { ohms on cable at } \\
\text { connection to LW }\end{array}$ \\
\hline $\mathrm{C} 109$ & $\mathrm{C} 1$ & Field & AD-60 \#33 & $150-200$ & $\mathrm{D}$ & $\bar{v}$ & front, left of $a / c$ & & T3C2/P2 & $9-27 / 3: 12$ & $\begin{array}{l}\text { Path variation test- } \\
\text { compare to C9 }\end{array}$ \\
\hline C111 & $\mathrm{C3}$ & Voltage & Long Wire & $150-200$ & $\bar{D}$ & $\mathrm{v}$ & front, left of $a / c$ & & T3C3/P4 & $9-27 / 3: 12$ & $\begin{array}{l}\text { Path variation test- } \\
\text { compare to } \mathrm{Cl1}\end{array}$ \\
\hline $\mathrm{C} 104$ & $\overline{\mathrm{CP} 4}$ & Wire Current & I $320 \# 32$ & $150-200$ & $\mathrm{D}$ & $\bar{v}$ & front, left of $a / c$ & v & T104/GTC3 & $9-29 / 7: 33$ & $\begin{array}{l}\text { Study instrumentation } \\
\text { noise- } 50 \mathrm{ohm} \text { on input } 53 \\
\text { of F-O xmtr; possible } \\
\text { crosstalk }\end{array}$ \\
\hline C204 & $\mathrm{CP} 4$ & Wire Current & I $320 \# 32$ & $150-200$ & $\bar{D}$ & $\bar{v}$ & front, left of $a / c$ & V & T104/GTC3 & $9-28 / 7: 53$ & $\begin{array}{l}\text { More instrumentation } \\
\text { noise- } 50 \mathrm{ohm} \text { on input } 53 \\
\text { of F-O xmtr, disconnect } \\
\text { others }\end{array}$ \\
\hline C304 & $\overline{\mathrm{CP} 4}$ & Wire Current & $1320 \# 32$ & $150-200$ & $\overline{\mathrm{D}}$ & $\bar{v}$ & front, left of $a / c$ & $\bar{v}$ & T104/GTC3 & $9-28 / 8: 07$ & $\begin{array}{l}\text { Same as C204 but T1C4 } \\
\text { connected to NF53 \& 50 } \\
\text { ohm on sensor end of T1C4 } \\
\end{array}$ \\
\hline$\overline{C 404}$ & $\overline{\mathrm{CP} 4}$ & Wire Current & I $320 \# 32$ & $150 \cdot 200$ & $\bar{D}$ & $\bar{v}$ & front, left of $a / c$ & $\bar{v}$ & T104/GTC3 & $9-28 / 8: 21$ & $\begin{array}{l}\text { Rerun weak signal with no } \\
\text { cables attached to other } \\
\text { F-O ports }\end{array}$ \\
\hline
\end{tabular}


Table 6-8. Test Series D - Dipole (Horizontal, 150 - $200 \mathrm{MHz}$ )

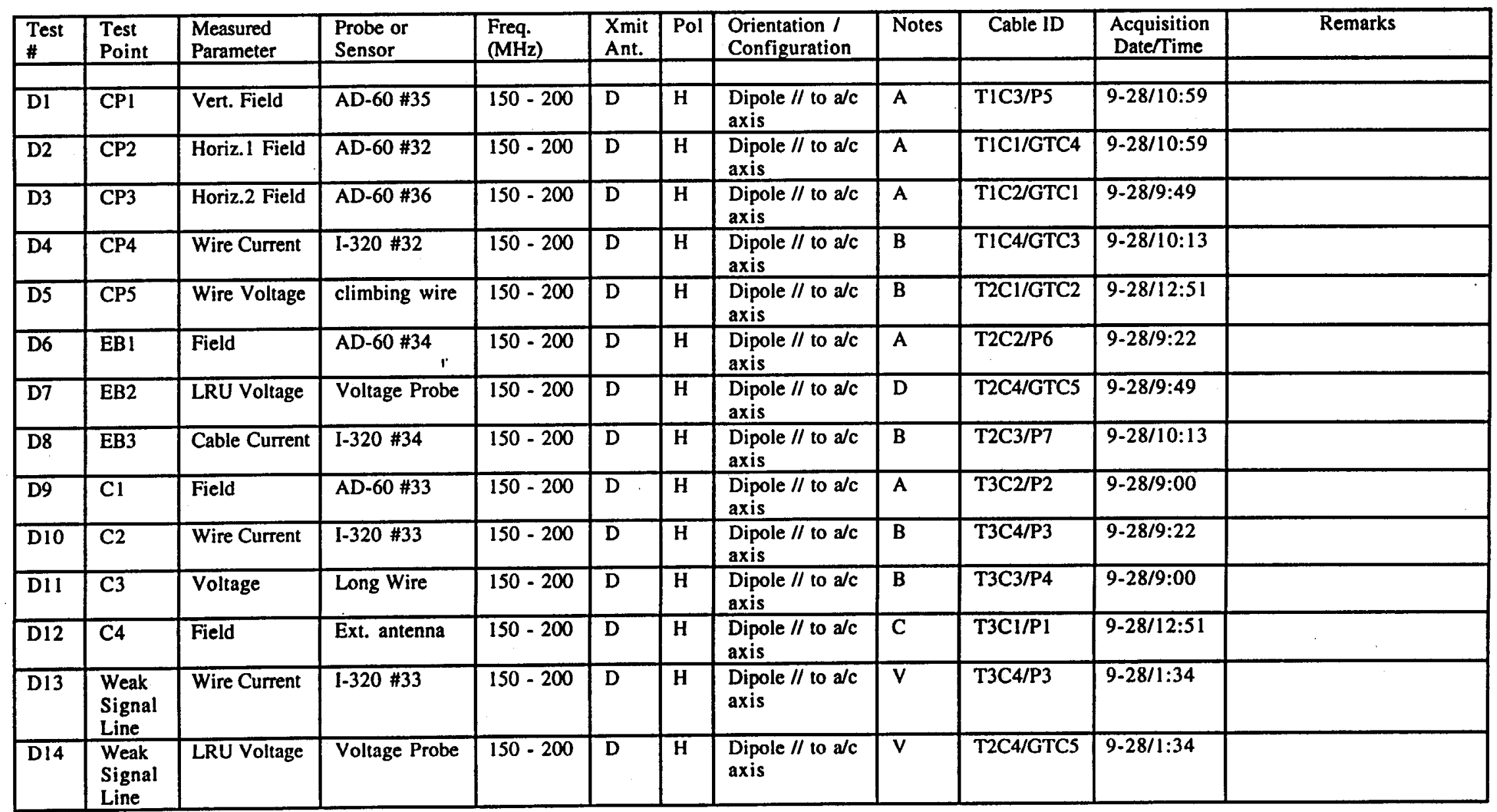


Table 6-9. Test Series E - Dipole (Vertical, 350 - 500 MHz)

\begin{tabular}{|c|c|c|c|c|c|c|c|c|c|c|c|}
\hline $\begin{array}{l}\text { Test } \\
\#\end{array}$ & $\begin{array}{l}\text { Test } \\
\text { Point }\end{array}$ & $\begin{array}{l}\text { Measured } \\
\text { Parameter }\end{array}$ & $\begin{array}{l}\text { Probe or } \\
\text { Sensor }\end{array}$ & $\begin{array}{l}\text { Freq. } \\
\text { (MHz) }\end{array}$ & $\begin{array}{l}\text { Xmit } \\
\text { Ant. }\end{array}$ & Pol & $\begin{array}{l}\text { Orientation / } \\
\text { Configuration }\end{array}$ & Notes & Cable ID & $\begin{array}{c}\text { Acquisition } \\
\text { Date/Time }\end{array}$ & Remarks \\
\hline & & & & & & & & & & & \\
\hline$\overline{E 1}$ & $\mathrm{CPI}$ & Vert. Field & $\mathrm{AD}-60 \# 35$ & $350-500$ & $\mathrm{D}$ & $\mathrm{V}$ & front, left of $\mathrm{a} / \mathrm{c}$ & A & T1C3/P5 & $9-29 / 1: 31$ & \\
\hline E2 & $\mathrm{CP2}$ & Horiz. 1 Field & $\mathrm{AD}-60 \# 32$ & $350-500$ & $\mathrm{D}$ & $\mathrm{V}$ & front, left of $a / c$ & $\mathrm{~A}$ & T1Cl/GTC4 & $9-29 / 1: 31$ & \\
\hline E3 & $\mathrm{CP} 3$ & Horiz.2 Field & $\mathrm{AD}-60 \# 36$ & $350-500$ & D & $\mathrm{V}$ & front, left of $\mathrm{a} / \mathrm{c}$ & $\mathbf{A}$ & T1C2/GTCl & $9-29 / 2: 48$ & \\
\hline $\mathbf{E A}$ & $\mathrm{CP4}$ & Wire Current & $\mathrm{I}-320 \# 32$ & $350-500$ & $\mathrm{D}$ & $\bar{v}$ & front, left of $a / c$ & B & T1C4/GTC3 & $9-29 / 11: 13$ & \\
\hline E5 & CP5 & Wire Voltage & climbing wire & $350-500$ & D & $\mathbf{V}$ & front, left of a/c & B & $\mathrm{T} 2 \mathrm{Cl} / \mathrm{GTC} 2$ & $9-29 / 10: 16$ & \\
\hline E6 & EB I & Field & AD-60 \#34 & $350-500$ & $\mathrm{D}$ & $\mathrm{V}$ & front, left of $\mathrm{a} / \mathrm{c}$ & $\mathbf{A}$ & $\mathrm{T} 2 \mathrm{C} 2 / \mathrm{P} 6$ & $9-29 / 10: 16$ & \\
\hline E7 & EB2 & LRU Voltage & Voltage Probe & $350-500$ & $\bar{D}$ & $\mathrm{~V}$ & front, left of a/c & D & T2C4/GTC5 & $9-29 / 10: 48$ & \\
\hline E8 & EB3 & Cable Current & $1-320 \# 34$ & $350-500$ & $\mathrm{D}$ & $\mathrm{V}$ & front, left of $\mathrm{a} / \mathrm{c}$ & $\mathrm{B}, \mathrm{XI}$ & $\mathrm{T} 2 \mathrm{C} 3 / \mathrm{P} 7$ & $9-29 / 8: 43$ & \\
\hline E9 & $\mathrm{Cl}$ & Field & AD-60 \#33 & $350-500$ & $\mathrm{D}$ & $\mathrm{V}$ & front, left of a/c & $\mathrm{A}$ & $\mathrm{T} 3 \mathrm{C} 2 / \mathrm{P} 2$ & $9-29 / 9: 30$ & \\
\hline E10 & $\mathrm{C2}$ & Wire Current & $1-320 \# 33$ & $350-500$ & D & $\mathrm{V}$ & front, left of $a / c$ & $\mathrm{~B}, \mathrm{X} \mathbf{1}$ & $\mathrm{T} 3 \mathrm{C} 4 / \mathrm{P} 3$ & $9-29 / 8: 43$ & \\
\hline E11 & $\mathrm{C3}$ & Voltage & Long Wire & $350-500$ & D & $\mathrm{V}$ & front. left of $a / c$ & $\mathrm{~B}$ & T3C3/P4 & $9-29 / 9: 30$ & \\
\hline E12 & $\mathrm{C4}$ & Field & Ext. antenna & $350-500$ & $\mathrm{D}$ & $\mathrm{V}$ & front, left of $\mathrm{a} / \mathrm{c}$ & $\mathrm{C}$ & $\mathrm{T} 3 \mathrm{Cl} / \mathrm{Pl}$ & $9-29 / 2: 09$ & \\
\hline E13 & $\mathrm{Cl}$ & Field & $\mathrm{AD}-60 \# 33$ & $427-432$ & D & $\mathrm{V}$ & $\begin{array}{l}1 \% \mathrm{BW}, 1000 \\
\text { pts }\end{array}$ & $\bar{E}$ & $\mathrm{~T} 3 \mathrm{C} 2 / \mathrm{P} 2$ & $9-29 / 11: 35$ & \\
\hline$\overline{E 004}$ & $\mathrm{CP4}$ & Wire Current & I-320 \#32 & $350-500$ & $\mathrm{D}$ & $\mathrm{V}$ & front, left of $a / c$ & $\mathbf{B}$ & T1C4/GTC3 & 9-29/2:09 & $\begin{array}{l}\text { Repeat E4 with gain to } 24 \\
\text { for better noise ratio }\end{array}$ \\
\hline
\end{tabular}


Table 6-10. Test Series F - Dipole (Horizontal, 350 - 500 MHz)

\begin{tabular}{|c|c|c|c|c|c|c|c|c|c|c|c|}
\hline $\begin{array}{l}\text { Test } \\
\#\end{array}$ & $\begin{array}{l}\text { Test } \\
\text { Point }\end{array}$ & $\begin{array}{l}\text { Measured } \\
\text { Parameter }\end{array}$ & $\begin{array}{l}\text { Probe or } \\
\text { Sensor }\end{array}$ & $\begin{array}{l}\text { Freq. } \\
\text { (MHz) }\end{array}$ & $\begin{array}{l}\text { Xmit } \\
\text { Ant. }\end{array}$ & Pol & $\begin{array}{l}\text { Orientation / } \\
\text { Configuration }\end{array}$ & Notes & Cable ID & $\begin{array}{l}\text { Acquisition } \\
\text { Date/Time }\end{array}$ & Remarks \\
\hline $\mathrm{Fl}$ & $\mathrm{CP1}$ & Vert. Field & $A D-60 \# 35$ & $350-500$ & $\bar{D}$ & $\bar{H}$ & $\begin{array}{l}\text { Dipole // to a/c } \\
\text { axis }\end{array}$ & $\bar{A}$ & T1C3/P5 & $9.28 / 2: 43$ & \\
\hline F2 & CP2 & Horiz.1 Field & $A D-60 \# 32$ & $350-500$ & D & $\mathbf{H}$ & $\begin{array}{l}\text { Dipole // to a/c } \\
\text { axis }\end{array}$ & A & T1C1/GTC4 & $9-28 / 2: 43$ & \\
\hline$\overline{F 3}$ & CP3 & Horiz.2 Field & AD-60 \#36 & $350-500$ & $\bar{D}$ & $\mathrm{H}$ & $\begin{array}{l}\text { Dipole // to a/c } \\
\text { axis }\end{array}$ & $\bar{A}$ & TIC2/GTCl & $9-28 / 3: 29$ & \\
\hline$\overline{F 4}$ & CP4 & Wire Current & $1-320 \# 32$ & $350-500$ & D & $\mathrm{H}$ & $\begin{array}{l}\text { Dipole // to a/c } \\
\text { axis }\end{array}$ & $\bar{B}$ & TIC4/GTC3 & $9-28 / 3: 52$ & \\
\hline$\overline{\text { F5 }}$ & CP5 & Wire Voltage & climbing wire & $350-500$ & $\mathrm{D}$ & $\mathrm{H}$ & $\begin{array}{l}\text { Dipole // to a/c } \\
\text { axis }\end{array}$ & B & T2Cl/GTC2 & $9-28 / 4: 40$ & \\
\hline$\overline{\text { F6 }}$ & EB1 & Field & $A D-60 \# 34$ & $350-500$ & $\bar{D}$ & $\mathrm{H}$ & $\begin{array}{l}\text { Dipole // to a/c } \\
\text { axis }\end{array}$ & $\bar{A}$ & $\mathrm{~T} 2 \mathrm{C} 2 / \mathrm{P} 6$ & $9-28 / 5: 04$ & \\
\hline F7 & EB2 & LRU Voltage & Voltage Probe & $350-500$ & D & $\mathrm{H}$ & $\begin{array}{l}\text { Dipole // to a/c } \\
\text { axis }\end{array}$ & $\bar{D}$ & T2C4/GTC5 & $9-28 / 3: 29$ & \\
\hline F8 & EB3 & \begin{tabular}{|l} 
Cable Current \\
\end{tabular} & $1-320 \# 34$ & $350-500$ & $\mathrm{D}$ & $\mathrm{H}$ & $\begin{array}{l}\text { Dipole // to a/c } \\
\text { axis }\end{array}$ & $\mathbf{B}$ & T2C3/P7 & $9-28 / 3: 52$ & \\
\hline F9 & $\mathrm{Cl}$ & Field & AD-60 \#33 & $350-500$ & $\bar{D}$ & $\mathrm{H}$ & $\begin{array}{l}\text { Dipole // to a/c } \\
\text { axis }\end{array}$ & $\bar{A}$ & T3C2/P2 & $9-28 / 5: 04$ & \\
\hline F10 & $\mathrm{C} 2$ & Wire Current & $\mathrm{I}-320 \# 33$ & $350-500$ & D & $\mathrm{H}$ & $\begin{array}{l}\text { Dipole // to a/c } \\
\text { axis }\end{array}$ & B & $\mathrm{T} 3 \mathrm{C} 4 / \mathrm{P} 3$ & $9-28 / 3: 07$ & \\
\hline$\overline{F 11}$ & $\mathrm{C3}$ & Voltage & Long Wire & $350-500$ & $\mathrm{D}$ & $\bar{H}$ & $\begin{array}{l}\text { Dipole // to a/c } \\
\text { axis }\end{array}$ & $\bar{B}$ & $\mathrm{~T} 3 \mathrm{C} 3 / \mathrm{P} 4$ & $9-28 / 4: 40$ & \\
\hline F12 & $\mathrm{C4}$ & Field & Ext. antenna & $350-500$ & $\bar{D}$ & $\mathrm{H}$ & $\begin{array}{l}\text { Dipole // to a/c } \\
\text { axis }\end{array}$ & C & $\mathrm{T} 3 \mathrm{Cl} / \mathrm{Pl}$ & $9-28 / 3: 07$ & \\
\hline$\overline{F 13}$ & C2 & Wire Current & $1-320 \# 33$ & $350-500$ & D & $\mathrm{H}$ & $\begin{array}{l}\text { Dipole // to a/c } \\
\text { axis }\end{array}$ & V & T3C4/P3 & $9-29 / 7: 19$ & \\
\hline F14 & EB3 & Cable Current & $1-320 \# 34$ & $350-500$ & $\bar{D}$ & $\mathrm{H}$ & $\begin{array}{l}\text { Dipole // to a/c } \\
\text { axis }\end{array}$ & $\bar{V}$ & $\mathrm{~T} 2 \mathrm{C} 3 / \mathrm{P} 7$ & $9-29 / 7: 19$ & \\
\hline F008 & EB3 & Cable Current & $\mathrm{I}-320 \# 34$ & $350-500$ & $\mathrm{D}$ & $\mathrm{H}$ & $\begin{array}{l}\text { Dipole // to a/c } \\
\text { axis }\end{array}$ & B & T2C3/P7 & $9-29 / 8: 00$ & $\begin{array}{l}\text { Repeat of } F 8 \text { because of } \\
\text { crosstalk }\end{array}$ \\
\hline F010 & $\mathrm{C} 2$ & Wire Current & $\begin{array}{r}1-320 \# 33 \\
i\end{array}$ & $350-500$ & $\bar{D}$ & $\mathrm{H}$ & $\begin{array}{l}\text { Dipole // to a/c } \\
\text { axis }\end{array}$ & B & $\mathrm{T} 3 \mathrm{C} 4 / \mathrm{P} 3$ & 9-29/8:00 & $\begin{array}{l}\text { Repeat of Flo because of } \\
\text { crosstalk }\end{array}$ \\
\hline
\end{tabular}


Table 6-11. Noise Floor Measurements - Rhombic

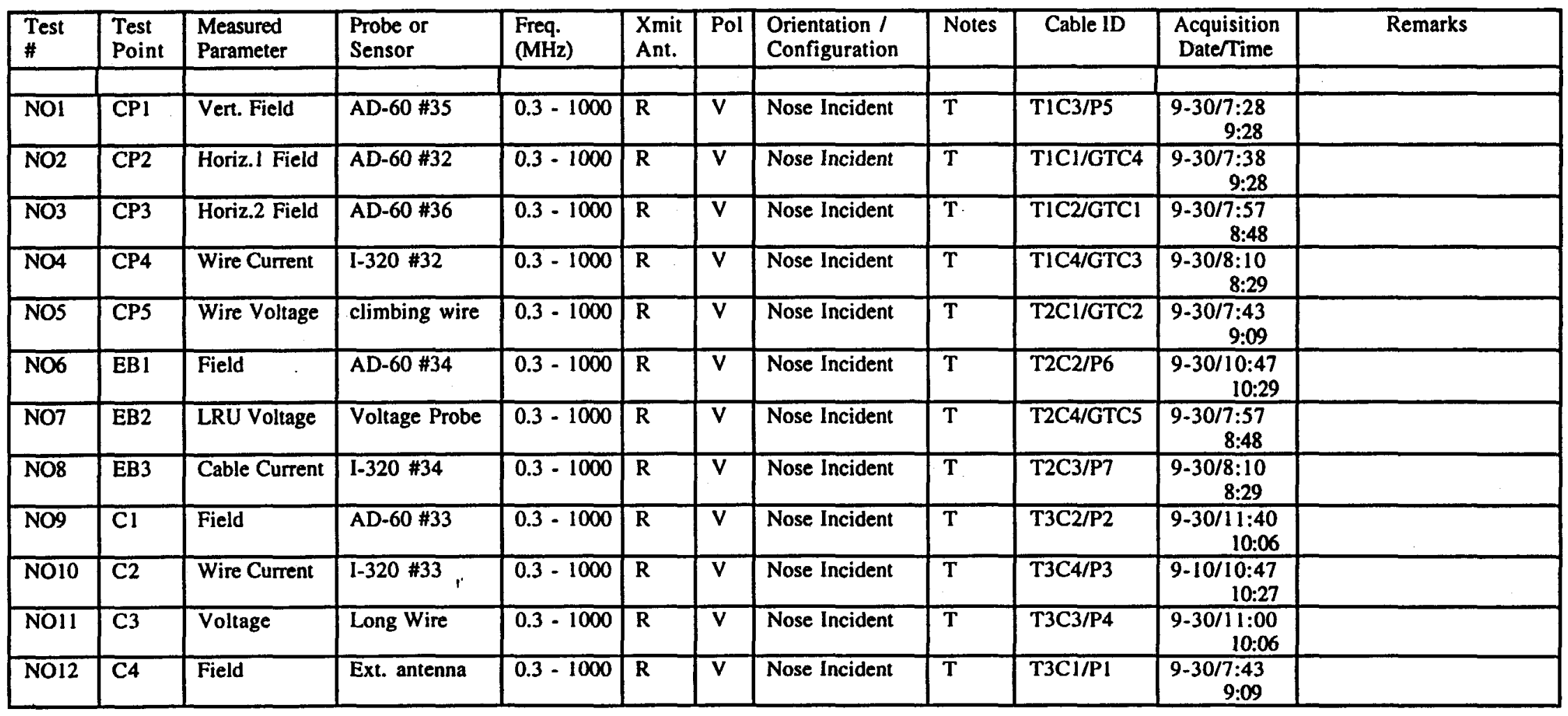


Table 6-12. Test Series J - Rhombic (Nose Incident and Related Tests)

\begin{tabular}{|c|c|c|c|c|c|c|c|c|c|c|c|}
\hline $\begin{array}{l}\text { Test } \\
\#\end{array}$ & $\begin{array}{l}\text { Test } \\
\text { Point }\end{array}$ & $\begin{array}{l}\text { Measured } \\
\text { Parameter }\end{array}$ & $\begin{array}{l}\text { Probe or } \\
\text { Sensor }\end{array}$ & $\begin{array}{l}\text { Freq. } \\
\text { (MHz) }\end{array}$ & $\begin{array}{l}\text { Xmit } \\
\text { Ant. }\end{array}$ & Pol & $\begin{array}{l}\text { Orientation / } \\
\text { Configuration }\end{array}$ & Notes & Cable ID & $\begin{array}{l}\text { Acquisition } \\
\text { Date/Time }\end{array}$ & Remarks \\
\hline $\mathrm{J} \mathbf{1}$ & $\overline{\mathrm{CP1}}$ & Vert. Field & $A D-60 \# 35$ & $0.3-1000$ & $\overline{\mathbf{R}}$ & $\mathrm{V}$ & $\begin{array}{l}\text { nose incident, } \\
\text { AM }\end{array}$ & $\bar{G}$ & T1C3/P5 & $\begin{array}{r}10-4 / 7: 34 \\
7: 18\end{array}$ & $\begin{array}{l}\text { Deleted - malfunction of } \\
\text { network analyzer; } \\
\text { see } \mathrm{J} 20\end{array}$ \\
\hline $\mathrm{J} 2$ & CP2 & Horiz.l Field & $\mathrm{AD}-60 \# 32$ & $0.3-1000$ & $\mathbf{R}$ & $\mathrm{V}$ & $\begin{array}{l}\text { nose incident, } \\
\text { AM }\end{array}$ & $G$ & T1C1/GTC4 & $\begin{array}{r}10-4 / 7: 34 \\
7: 18\end{array}$ & $\begin{array}{l}\text { Deleted - malfunction of } \\
\text { network analyzer; } \\
\text { see } \mathrm{J} 21\end{array}$ \\
\hline J3 & $\mathrm{CP3}$ & Horiz.2 Field & AD.60 \#36 & $0.3-1000$ & $\mathrm{R}$ & $\mathrm{V}$ & $\begin{array}{l}\text { nose incident, } \\
\text { AM }\end{array}$ & $G$ & TIC2/GTCl & $\begin{array}{r}10 / 4 / 7: 54 \\
8: 01\end{array}$ & $\begin{array}{l}\text { Deleted - malfunction of } \\
\text { network analyzer; } \\
\text { see } \mathrm{J} 22\end{array}$ \\
\hline $\mathrm{J} 4$ & CP4 & Wire Current & $1-320 \# 32$ & $0.3-1000$ & $\mathbf{R}$ & $\mathrm{V}$ & nose incident & $\mathrm{H}$ & TIC4/GTC3 & $\begin{array}{r}10-3 / 3: 21 \\
3: 08\end{array}$ & \\
\hline$\overline{J 5}$ & CP5 & Wire Voltage & climbing wire & $0.3-1000$ & $\overline{\mathbf{R}}$ & $\mathbf{V}$ & nose incident & $\mathrm{H}$ & T2C1/GTC2 & $\begin{array}{r}10-3 / 3: 57 \\
4: 28 \\
\end{array}$ & \\
\hline $\mathrm{J} 6$ & CPI & Vert. Field & $A D-60 \# 35$ & $0.3-1000$ & $\mathbf{R}$ & $\mathrm{V}$ & small box & $\mathrm{L}, \mathrm{Ll}$ & T1C3/P5 & $\begin{array}{r}10-4 / 1: 19 \\
1: 02\end{array}$ & \\
\hline J7 & $\mathrm{CP2}$ & Horiz.l Field & $A D-60 \# 32$ & $0.3-1000$ & $\mathbf{R}$ & $\mathrm{V}$ & small box & $\mathrm{L}, \mathrm{Ll}$ & TICI/GTC4 & $\begin{array}{r}10-4 / 1: 19 \\
1: 02\end{array}$ & \\
\hline $\mathrm{J} 8$ & $\overline{\mathrm{CP} 3}$ & Horiz.2 Field & AD-60 \#36 & $0.3-1000$ & $\mathbf{R}$ & $\mathrm{V}$ & small box & $\mathrm{L}, \mathrm{LI}$ & T1C2/GTCl & $\begin{array}{r}10-4 / 1: 40 \\
1: 48\end{array}$ & \\
\hline J9 & CP1 & Vert. Field & $\mathrm{AD}-60 \# 35$ & $0.3-1000$ & $\overline{\mathbf{R}}$ & $\bar{V}$ & move small box & L, L2 & T1C3/P5 & $\begin{array}{r}10-4 / 2: 22 \\
2: 09\end{array}$ & \\
\hline J10 & $\overline{\mathrm{CP2}}$ & Horiz.1 Field & $\mathrm{AD}-60 \# 32$ & $0.3-1000$ & $\overline{\mathbf{R}}$ & $\mathrm{V}$ & move small box & $\mathrm{L}, \mathrm{L2}$ & T1C1/GTC4 & $\begin{array}{r}10-4 / 2: 22 \\
2: 09 \\
\end{array}$ & \\
\hline J11 & $\overline{\mathrm{CP} 3}$ & Horiz.2 Field & AD-60 \#36 & $0.3-1000$ & $\overline{\mathbf{R}}$ & $\bar{v}$ & move small box & L, L2 & T1C2/GTCl & $\begin{array}{r}10-4 / 2: 40 \\
2: 49\end{array}$ & \\
\hline $\mathrm{J} 12$ & $\overline{\mathrm{CP1}}$ & Vert. Field & $A D-60 \# 35$ & $800-900$ & $\mathbf{R}$ & $\mathrm{V}$ & $\begin{array}{l}1 \% \mathrm{BW}, 1000 \\
\text { pts }\end{array}$ & $\bar{E}$ & T1C3/P5 & $10-3 / 4: 50$ & . \\
\hline $\mathrm{J13}$ & $\overline{\mathrm{CPI}}$ & Vert. Field & $A D-60 \# 35$ & $0.3-1000$ & $\overline{\mathbf{R}}$ & $\mathrm{V}$ & $\begin{array}{l}\text { person in left } \\
\text { seat }\end{array}$ & $\bar{M}$ & T1C3/P5 & $\begin{array}{r}10-4 / 8: 47 \\
8: 33\end{array}$ & \\
\hline J14 & $\overline{\mathrm{CP2}}$ & Horiz.l Field & $\mathrm{AD}-60 \# 32$ & $0.3-1000$ & $\mathbf{R}$ & $\mathrm{V}$ & $\begin{array}{l}\text { person in left } \\
\text { seat }\end{array}$ & $\bar{M}$ & T1C1/GTC4 & $\begin{array}{r}10-4 / 9: 47 \\
8: 33 \\
\end{array}$ & \\
\hline J15 & $\overline{\mathrm{CP} 3}$ & Horiz.2 Field & AD-60 \#36 & $0.3-1000$ & $\bar{R}$ & $\mathrm{~V}$ & $\begin{array}{l}\text { person in left } \\
\text { seat }\end{array}$ & $\bar{M}$ & T1C2/GTCl & $\begin{array}{r}10-4 / 9: 07 \\
9: 16\end{array}$ & \\
\hline J16 & $\mathrm{CP1}$ & Vert. Field & AD-60 \#35 & $0.3-1000$ & $\overline{\mathbf{R}}$ & $\mathrm{V}$ & $\begin{array}{l}\text { persons in both } \\
\text { seats }\end{array}$ & $\bar{M}$ & T1C3/P5 & $\begin{array}{r}10-4 / 9: 39 \\
9: 27\end{array}$ & \\
\hline $\mathrm{J17}$ & CP2 & Horiz.1 Field & AD-60 \#32 & $0.3-1000$ & $\overline{\mathrm{R}}$ & $\bar{v}$ & $\begin{array}{l}\text { persons in both } \\
\text { seats }\end{array}$ & $\bar{M}$ & TICI/GTC4 & $\begin{array}{r}10-4 / 9: 39 \\
9: 27\end{array}$ & \\
\hline$J 18$ & $\overline{\mathrm{CP3}}$ & Horiz.2 Field & AD-60 \#36 & $0.3-1000$ & $\bar{R}$ & $\mathrm{~V}$ & $\begin{array}{l}\text { persons in both } \\
\text { seats }\end{array}$ & $\mathbf{M}$ & T1C2/GTCl & $\begin{array}{r}10-4 / 9: 59 \\
10: 07 \\
\end{array}$ & \\
\hline
\end{tabular}









\begin{tabular}{|c|c|c|c|c|c|c|c|c|c|c|c|}
\hline $\begin{array}{l}\text { Test } \\
\#\end{array}$ & $\begin{array}{l}\text { Test } \\
\text { Point }\end{array}$ & $\begin{array}{l}\text { Measured } \\
\text { Parameter }\end{array}$ & $\begin{array}{l}\text { Probe or } \\
\text { Sensor }\end{array}$ & $\begin{array}{l}\text { Freq. } \\
\text { (MHz) }\end{array}$ & $\begin{array}{l}\text { Xmit } \\
\text { Ant. }\end{array}$ & Pol & $\begin{array}{l}\text { Orientation I } \\
\text { Configuration }\end{array}$ & Notes & Cable ID & $\begin{array}{l}\text { Acquisition } \\
\text { Date/Time }\end{array}$ & Remarks \\
\hline & & & & & & & & & & & \\
\hline $\mathrm{J} 40$ & $\overline{\mathrm{CP} 2}$ & Horiz.1 Field & AD-60 \#32 & $0.3-1000$ & $\overline{\mathbf{R}}$ & $\bar{v}$ & move $a / c 5^{\circ}$ off & $\bar{P}$ & TICI/GTC4 & $\begin{array}{r}10-6 / 10: 45 \\
11: 00\end{array}$ & \\
\hline J41 & $\overline{\mathrm{CP} 3}$ & Horiz.2 Field & AD-60 \#36 & $0.3-1000$ & $\overline{\mathbf{R}}$ & $\bar{v}$ & move $a / c 5^{\circ}$ off & $\bar{P}$ & TIC2/GTC1 & $\begin{array}{r}10-6 / 11: 20 \\
11: 13 \\
\end{array}$ & \\
\hline 5005 & CP5 & Wire Voltage & climbing wire & $0.3-1000$ & $\overline{\mathbf{R}}$ & $\bar{v}$ & nose incident & $\mathrm{H}$ & T2C1/GTC2 & \begin{tabular}{|r|}
$10-4 / 3: 42$ \\
$3: 51$
\end{tabular} & \\
\hline $\mathrm{J} 026$ & $\overline{\text { EBI }}$ & Field & AD-60 \#34 & $0.3-1000$ & $\begin{array}{l}\mathbf{R} \\
\end{array}$ & $\bar{v}$ & $\begin{array}{l}\text { nose incident; } \\
\text { negative press } \\
\text { door sealed }\end{array}$ & $\begin{array}{l}\text { G2, } \\
\text { G4 }\end{array}$ & \begin{tabular}{|l} 
T2C2/P6 \\
(a)
\end{tabular} & \begin{tabular}{|c|}
$10-11 / 7: 46$ \\
$8: 04$
\end{tabular} & \\
\hline J027 & $\overline{\text { EB2 }}$ & LRU Voltage & Voltage Probe & $0.3-1000$ & $\bar{R}$ & $\bar{v}$ & $\begin{array}{l}\text { nose incident; } \\
\text { negative press } \\
\text { door sealed }\end{array}$ & $\begin{array}{l}\text { G2, } \\
\text { G4 }\end{array}$ & T2C4/GTC5 & $\begin{array}{r}10-11 / 8: 27 \\
8: 16\end{array}$ & \\
\hline $\mathrm{J} 028$ & $\overline{\text { EB3 }}$ & Cable Current & $1-320 \# 34$ & $0.3-1000$ & $\overline{\mathrm{R}}$ & $\bar{v}$ & $\begin{array}{l}\text { nose incident; } \\
\text { negative press } \\
\text { door sealed }\end{array}$ & $\begin{array}{l}\text { G2, } \\
\text { G4 }\end{array}$ & T2C3/P7 & $\begin{array}{r}10-11 / 8: 58 \\
9: 19\end{array}$ & \\
\hline
\end{tabular}


Table 6-13. Test Series K - Rhombic (Side Incident and Related Tests)

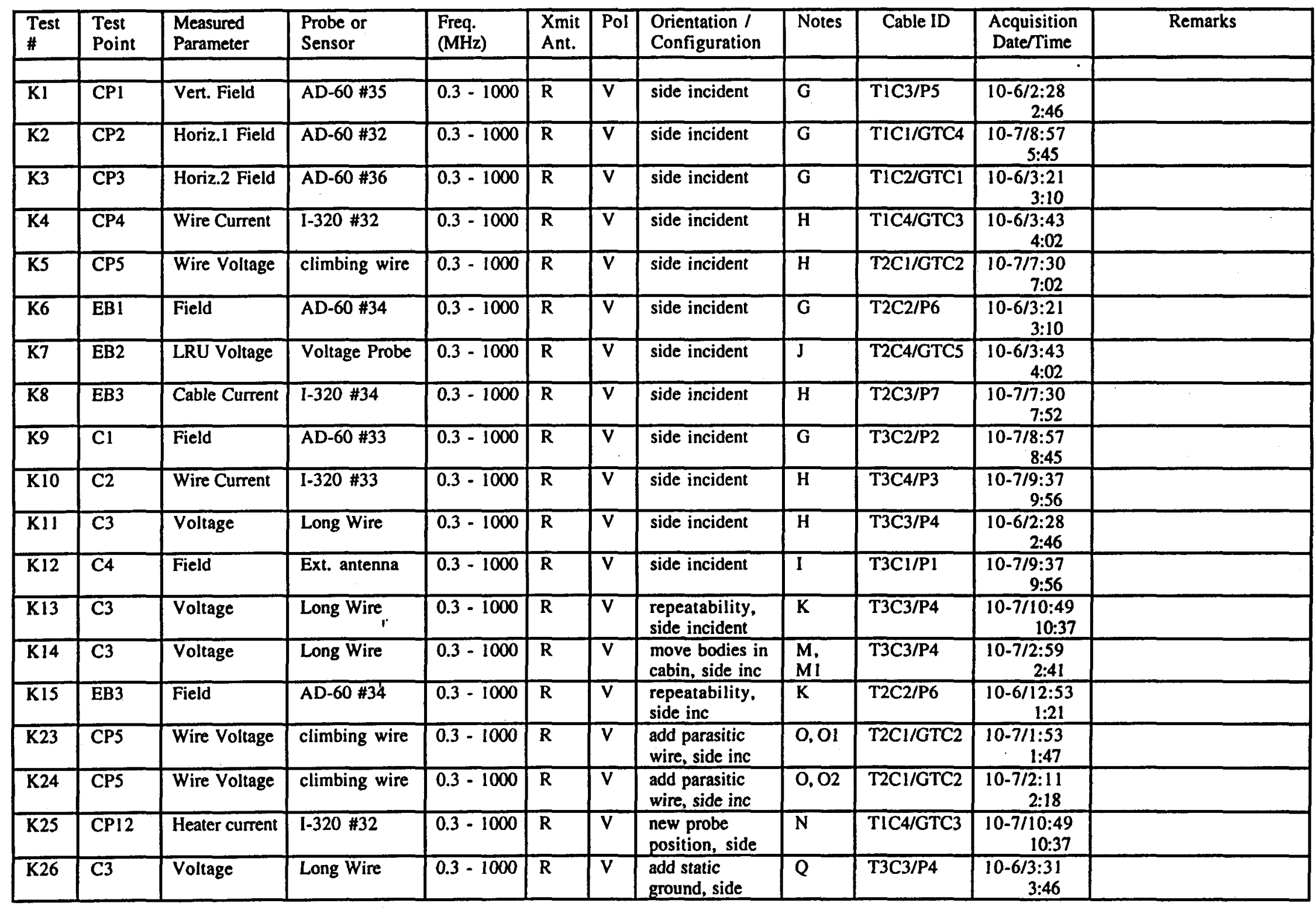




\begin{tabular}{|c|c|c|c|c|c|c|c|c|c|c|c|}
\hline $\begin{array}{l}\text { Test } \\
\#\end{array}$ & $\begin{array}{l}\text { Test } \\
\text { Point }\end{array}$ & $\begin{array}{l}\text { Measured } \\
\text { Parameter }\end{array}$ & $\begin{array}{l}\text { Probe or } \\
\text { Sensor }\end{array}$ & $\begin{array}{l}\text { Freq. } \\
\text { (MHz) }\end{array}$ & $\begin{array}{l}\text { Xmit } \\
\text { Ant. }\end{array}$ & Pol & $\begin{array}{l}\text { Orientation / } \\
\text { Configuration }\end{array}$ & Notes & Cable ID & $\begin{array}{c}\text { Acquisition } \\
\text { Date/Time }\end{array}$ & Remarks \\
\hline & & & & & & & & & & & \\
\hline K27 & $\overline{\mathrm{CPI}}$ & Vert. Field & AD-60 \#3 & $0.3-1000$ & $\overline{\mathbf{R}}$ & $\bar{V}$ & $\begin{array}{l}\text { retum to nose } \\
\text { incident }\end{array}$ & $\bar{R}$ & T1C3/P5 & $\begin{array}{c}1010 / 10: 02 \\
9: 51\end{array}$ & \\
\hline $\mathrm{K} 28$ & $\overline{\mathrm{CP} 2}$ & Horiz.1 Field & $\overline{A D}-60 \# 32$ & $0.3-1000$ & $\overline{\mathbf{R}}$ & $\bar{V}$ & $\begin{array}{l}\text { return to nose } \\
\text { incident }\end{array}$ & $\mathbf{R}$ & T1Cl/GTC4 & $\begin{array}{c}1010 / 10: 02 \\
9: 51\end{array}$ & \\
\hline$\overline{K 29}$ & $\overline{\mathrm{CP3}}$ & Horiz.2 Field & AD-60 \#36 & $0.3-1000$ & $\overline{\mathbf{R}}$ & $\mathrm{V}$ & $\begin{array}{l}\text { retum to nose } \\
\text { incident }\end{array}$ & $\bar{R}$ & TIC2/GTCl & $\begin{array}{c}10-10 / 9: 15 \\
9: 35 \\
\end{array}$ & \\
\hline $\mathrm{K} 30$ & $\mathrm{CPI}$ & Vert. Field & $\begin{array}{r}\mathrm{AD}-60 \# 35 \\
\text {. }\end{array}$ & $0.3-1000$ & $\mathbf{R}$ & $\mathrm{V}$ & $\begin{array}{l}5 \text { degrees from } \\
\text { nose-on }\end{array}$ & S1 & T1C3/P5 & $\begin{array}{r}10-10 / 7: 32 \\
7: 52 \\
\end{array}$ & \\
\hline $\mathrm{K} 31$ & $\overline{\mathrm{CP} 2}$ & Horiz.l Field & AD-60 \#32 & $0.3-1000$ & $\overline{\mathbf{R}}$ & $\bar{v}$ & $\begin{array}{l}5 \text { degrees from } \\
\text { nose-on }\end{array}$ & S1 & TICl/GTC4 & $\begin{array}{c}10-10 / 7: 32 \\
7: 52\end{array}$ & \\
\hline $\mathrm{K} 32$ & $\overline{\mathrm{CP3}}$ & Horiz.2 Field & AD-60 \#36 & $0.3-1000$ & $\overline{\mathbf{R}}$ & $\mathrm{V}$ & $\begin{array}{l}5 \text { degrees from } \\
\text { nose-on }\end{array}$ & SI & T1C2/GTCl & $\begin{array}{r}1010 / 8: 10 \\
8: 05 \\
\end{array}$ & \\
\hline K007 & $\overline{\text { EB2 }}$ & LRU Voltage & Voltage Probe & $0.3-1000$ & $\overline{\mathbf{R}}$ & $\bar{V}$ & side incident & $\bar{J}$ & T2C4/GTC5 & $\begin{array}{r}10-6 / 4: 41 \\
4: 29 \\
\end{array}$ & \\
\hline $\begin{array}{l}\text { J024 } \\
\text { brkr } \\
\text { in }\end{array}$ & EB2 & LRU Voltage & Voltage Probe & $0.3-1000$ & $\mathbf{R}$ & $\bar{V}$ & nose incident & J, J2 & T2C4/GTC5 & $\begin{array}{c}10-10 / 9: 15 \\
9: 35\end{array}$ & \\
\hline
\end{tabular}


Table 6-14. Test Series Z - Rhombic (System State Effects - Circuit Breakers)

\begin{tabular}{|c|c|c|c|c|c|c|c|c|c|c|c|}
\hline $\begin{array}{l}\text { Test } \\
\#\end{array}$ & $\begin{array}{l}\text { Test } \\
\text { Point }\end{array}$ & $\begin{array}{l}\text { Measured } \\
\text { Parameter }\end{array}$ & $\begin{array}{l}\text { Probe or } \\
\text { Sensor }\end{array}$ & $\begin{array}{l}\text { Freq. } \\
\text { (MHz) }\end{array}$ & $\begin{array}{l}\text { Xmit } \\
\text { Ant. }\end{array}$ & Pol & $\begin{array}{l}\text { Orientation / } \\
\text { Configuration }\end{array}$ & Notes & Cable ID & $\begin{array}{l}\text { Acquisition } \\
\text { Date/Time }\end{array}$ & Remarks \\
\hline$\overline{\mathrm{Z} 1}$ & $\mathrm{CP1}$ & Vert. Field & AD-60 \#35 & $0.3-1000$ & $\bar{R}$ & $\mathrm{~V}$ & nose incident & $\mathbf{G}$ & T1C3/P5 & & $\begin{array}{l}\text { Repeat of J1 (or J20) } \\
\text { Circuit breaker closed }\end{array}$ \\
\hline 22 & $\mathrm{CP2}$ & Horiz.1 Field & AD-60 \#32 & $0.3-1000$ & $\bar{R}$ & $\mathrm{~V}$ & nose incident & $G$ & TIC1/GTC4 & & $\begin{array}{l}\text { Repeat of J2 (or J21) } \\
\text { Circuit breaker closed }\end{array}$ \\
\hline 23 & $\overline{\mathrm{CP3}}$ & Horiz.2 Field & AD-60 \#36 & $0.3-1000$ & $\bar{R}$ & $\mathrm{~V}$ & nose incident & $G$ & $\mathrm{T1C2/GTC1}$ & & $\begin{array}{l}\text { Repeat of J3 (or J22) } \\
\text { Circuit breaker closed }\end{array}$ \\
\hline 24 & $\mathrm{CP} 4$ & Wire Current & $1-320 \# 32$ & $0.3-1000$ & $\overline{\mathbf{R}}$ & $\mathrm{V}$ & nose incident & $\mathrm{H}$ & TIC4/GTC3 & & $\begin{array}{l}\text { Repeat of J4 } \\
\text { Circuit breaker closed }\end{array}$ \\
\hline 25 & CP5 & Wire Voltage & climbing wire & $0.3-1000$ & $\overline{\mathrm{R}}$ & $\mathrm{V}$ & nose incident & $\mathrm{H}$ & T2C1/GTC2 & & $\begin{array}{l}\text { Repeat of J5 } \\
\text { Circuit breaker closed }\end{array}$ \\
\hline$\overline{26}$ & CPI2 & $\begin{array}{l}\text { Heater } \\
\text { Current }\end{array}$ & $\mathrm{I}-320 \# 32$ & $0.3-1000$ & $\bar{R}$ & $\mathrm{~V}$ & $\begin{array}{l}\text { new probe } \\
\text { position }\end{array}$ & $\mathrm{N}, \mathrm{N} 1$ & TIC4/GTC3 & & $\begin{array}{l}\text { Repeat of J19 } \\
\text { Circuit breaker closed }\end{array}$ \\
\hline$\overline{27}$ & EB1 & Field & $A D-60 \# 34$ & $0.3-1000$ & $\bar{R}$ & $\mathrm{~V}$ & nose incident & $G$ & $\mathrm{~T} 2 \mathrm{C} 2 / \mathrm{P} 6$ & & $\begin{array}{l}\text { Repeat of J23 } \\
\text { Circuit breaker closed }\end{array}$ \\
\hline$\overline{\mathbf{Z 8}}$ & $\overline{C l}$ & Field & AD-60 \#33 & $0.3-1000$ & $\overline{\mathrm{R}}$ & $\mathrm{V}$ & nose incident & G & T3C2/P2 & & $\begin{array}{l}\text { Repeat of J32 } \\
\text { Circuit breaker closed }\end{array}$ \\
\hline 29 & $\mathrm{C2}$ & Wire Current & $1-320 \# 33$ & $0.3-1000$ & $\overline{\mathbf{R}}$ & V & nose incident & $\mathbf{H}$ & T3C4/P3 & & $\begin{array}{l}\text { Repeat of J33 } \\
\text { Circuit breaker closed }\end{array}$ \\
\hline$\overline{210}$ & $\mathrm{C3}$ & Voltage & Long Wire & $0.3-1000$ & $\bar{R}$ & $V$ & nose incident & $\mathrm{H}$ & $\mathrm{T} 3 \mathrm{C} 3 / \mathrm{P} 4$ & & $\begin{array}{l}\text { Repeat of J34 } \\
\text { Circuit breaker closed }\end{array}$ \\
\hline 211 & $\overline{C P 5}$ & Wire Voltage & climbing wire & $0.3-1000$ & $\overline{\mathbf{R}}$ & $\mathrm{V}$ & $\begin{array}{l}\text { add parasitic } \\
\text { wire }\end{array}$ & 0,01 & T2C1/GTC2 & & $\begin{array}{l}\text { Repeat of K23 } \\
\text { Circuit breaker open; } \\
\text { compare to J5 }\end{array}$ \\
\hline $\mathrm{Z12}$ & $\overline{\mathrm{CP5}}$ & Wire Voltage & climbing wire & $0.3-1000$ & $\bar{R}$ & $\mathrm{~V}$ & $\begin{array}{l}\text { add parasitic } \\
\text { wire }\end{array}$ & 0,02 & T2C1/GTC2 & & $\begin{array}{l}\text { Repeat of K24 } \\
\text { Circuit breaker open; } \\
\text { compare to J5 }\end{array}$ \\
\hline 213 & $\mathrm{C3}$ & Voltage & Long Wire & $0.3-1000$ & $\overline{\mathrm{R}}$ & $\mathbf{V}$ & $\begin{array}{l}\text { add static } \\
\text { ground }\end{array}$ & $\mathbf{Q}$ & $\mathrm{T} 3 \mathrm{C} 3 / \mathrm{P} 4$ & & $\begin{array}{l}\text { Repeat of K26 } \\
\text { Circuit breaker open; } \\
\text { compare to J34 }\end{array}$ \\
\hline$\overline{\mathrm{Z} 14}$ & $\mathrm{CP2}$ & Horiz.1 Field & AD-60 \#32 & $0.3-1000$ & $\overline{\mathrm{R}}$ & $\mathrm{V}$ & $\begin{array}{l}\text { nose incident, } \\
\mathrm{AM}\end{array}$ & G & TICl/GTC4 & & $\begin{array}{l}\text { Repeat of } \mathrm{J} 2 \\
\text { Circuit breaker open }\end{array}$ \\
\hline
\end{tabular}


Table 6-15. Horizontal Polarization

\begin{tabular}{|l|l|l|l|l|l|l|l|l|}
\hline $\begin{array}{l}\text { Test } \\
\#\end{array}$ & $\begin{array}{l}\text { Test } \\
\text { Point }\end{array}$ & $\begin{array}{l}\text { Measured } \\
\text { Parameter }\end{array}$ & $\begin{array}{l}\text { Probe or } \\
\text { Antenna }\end{array}$ & $\begin{array}{l}\text { Freq. } \\
(\mathrm{MHz})\end{array}$ & $\begin{array}{l}\text { Xmit } \\
\text { Ant. }\end{array}$ & $\begin{array}{l}\text { Pol } \\
\text { Orientation 1 } \\
\text { Configuration }\end{array}$ & Notes \\
\hline HP1 & CP1 & Vert. Field & AD-60 D-Dot & $2-30$ & R & H & Nose-on & A \\
\hline HP2 & CP2 & Horiz.1 Field & AD-60 D-Dot & $2-30$ & R & H & Nose-on & A \\
\hline HP3 & CP3 & Horiz.2 Field & AD-60 D-Dot & $2-30$ & R & H & Nose-on & A \\
\hline HP4 & CP4 & Wire Current & I-320 Probe & $2-30$ & R & H & Nose-on & B \\
\hline HP5 & CP5 & Wire Voltage & climbing wire & $2-30$ & R & H & Nose-on & B \\
\hline HP6 & EB1 & Field & AD-60 D-Dot & $2-30$ & R & H & Nose-on & A \\
\hline HP7 & EB2 & LRU Voltage & Voltage Probe & $2-30$ & R & H & Nose-on & D \\
\hline HP8 & EB3 & Cable Current & I-320 Probe & $2-30$ & R & H & Nose-on & B \\
\hline HP9 & C1 & Field & AD-60 D-Dot & $2-30$ & R & H & Nose-on & A \\
\hline HP10 & C2 & Wire Current & I-320 Probe & $2-30$ & R & H & Nose-on & B \\
\hline HP11 & C3 & Voltage & Long Wire & $2-30$ & R & H & Nose-on & B \\
\hline HP12 & C4 & Field & Ext. antenna & $2-30$ & R & H & Nose-on & C \\
\hline HP13 & $\begin{array}{l}\text { Weak } \\
\text { Signal } \\
\text { Line }\end{array}$ & & & $2-30$ & R & H & Nose-on & V \\
\hline HP14 & $\begin{array}{l}\text { Strong } \\
\text { Signal } \\
\text { Line }\end{array}$ & & & & & & & \\
\hline
\end{tabular}

Table 6-16. Test Series WF - Window Shielded/Unshielded

\begin{tabular}{|c|c|c|c|c|c|c|c|c|c|c|c|}
\hline $\begin{array}{l}\text { Test } \\
\#\end{array}$ & $\begin{array}{l}\text { Test } \\
\text { Point }\end{array}$ & $\begin{array}{l}\text { Measured } \\
\text { Parameter }\end{array}$ & $\begin{array}{l}\text { Probe or } \\
\text { Sensor }\end{array}$ & $\begin{array}{l}\text { Freq. } \\
\text { (MHz) }\end{array}$ & $\begin{array}{l}\text { Xmit } \\
\text { Ant. }\end{array}$ & Pol & $\begin{array}{l}\text { Orientation / } \\
\text { Configuration }\end{array}$ & Notes & Cable ID & $\begin{array}{c}\text { Acquisition } \\
\text { Date/Time }\end{array}$ & Remarks \\
\hline & & & & & & & & & & & \\
\hline WF1 & $\overline{\mathrm{CP1}}$ & Vert. Field & $\mathrm{AD}-60 \# 35$ & $0.3-1000$ & $\mathbf{R}$ & $\bar{V}$ & nose-on & $\bar{Z}$ & T1C3/P5 & $\begin{array}{c}10-11 / 12: 57 \\
12: 33\end{array}$ & \\
\hline WF2 & $\mathrm{CP2}$ & Horiz.1 Field & AD-60 \#32 & $0.3-1000$ & $\overline{\mathrm{R}}$ & $\mathrm{V}$ & nose-on & $\mathbf{Z}$ & T1Cl/GTC4 & $\begin{array}{c}10-11 / 12: 57 \\
12: 33\end{array}$ & \\
\hline WF3 & $\mathrm{CP3}$ & Horiz.2 Field & AD-60 \#36 & $0.3-1000$ & $\bar{R}$ & $\bar{v}$ & nose-on & $\bar{Z}$ & T1C2/GTCl & $\begin{array}{c}10-11 / 1: 19 \\
1: 39 \\
\end{array}$ & \\
\hline WF4 & $\mathrm{CP} 4$ & Wire Current & $1-320 \# 32$ & $0.3-1000$ & $\bar{R}$ & $\bar{V}$ & nose-on & $\bar{Z}$ & T1C4/GTC3 & $\begin{array}{c}10-11 / 1: 59 \\
1: 52\end{array}$ & \\
\hline$\overline{\text { WF5 }}$ & $\mathrm{CP5}$ & Wire Voltage & climbing wire & $0.3-1000$ & $\bar{R}$ & $\bar{V}$ & nose-on & $\bar{Z}$ & T2Cl/GTC2 & $\begin{array}{c}10-11 / 2: 07 \\
2: 15 \\
\end{array}$ & \\
\hline WF6 & EB3 & Cable Current & $1-320$ & $0.3-1000$ & $\mathbf{R}$ & $\bar{V}$ & nose-on & $\bar{Z}$ & $\mathrm{~T} 2 \mathrm{C} 3 / \mathrm{P} 7$ & $\begin{array}{c}10-11 / 1: 19 \\
1: 39\end{array}$ & \\
\hline WF7 & $\mathrm{CP12}$ & Heater current & $1-320 \# 32$ & $0.3-1000$ & $\mathbf{R}$ & $\mathrm{V}$ & nose-on & $\bar{Z}$ & TIC4/GTC3 & $\begin{array}{c}10-11 / 2: 38 \\
2: 3 \cdot 1 \\
\end{array}$ & \\
\hline
\end{tabular}




\subsection{Relating Data File Names to Experiment Test Numbers}

The data collected during the course of the experimental program were recorded to data files with file names which allow the identification of a specific test from the test matrix (Tables 6-4 through 6-16). File names also identify characteristics retarding the sweep parameters. File names are in the form:

\section{a\#\#\#j\&\&\&}

where the first letter is a sweep designator and the number \#\# is a sweep number. The second letter represents a specific test series within the test matrix and the number \&\&\& identifies the test within the test series. Leading zeros have been omitted in Tables 6-4 through 6-16, except where they are used to indicate a test was repeated or added to the matrix.

The test team provided a sweep designation scheme, proved effective in other LESLI test efforts, which conveyed information regarding the frequency span and number of points in the sweep, as well as other details. The scheme is presented in Table 6-17.

\begin{tabular}{|c|c|c|c|c|c|}
\hline \multicolumn{6}{|c|}{ Table 6-17. Sweep Designation Definitions } \\
\hline $\begin{array}{c}\text { SWEEP } \\
\text { DESIGNATION }\end{array}$ & $F_{\text {min }}$ & $F_{\max }$ & $\begin{array}{c}\text { No. } \\
\text { POINTS }\end{array}$ & COMMENTS & $\begin{array}{c}\text { TEST } \\
\text { NUMBER }\end{array}$ \\
\hline$a$ & $300 \mathrm{kHz}$ & $100 \mathrm{MHz}$ & 932 & $\begin{array}{l}\text { NORMAL RHOMBIC } \\
\text { SWEEP }\end{array}$ & $\begin{array}{l}\mathrm{J} 1-\mathrm{J} 41 \\
\mathrm{~K} 1-\mathrm{K} 29\end{array}$ \\
\hline $\bar{b}$ & $100 \mathrm{MHz}$ & $1000 \mathrm{MHz}$ & 413 & $\begin{array}{l}\text { NORMAL RHOMBIC } \\
\text { SWEEP }\end{array}$ & $\begin{array}{r}\mathrm{J} 1-\mathrm{J} 41 \\
\mathrm{~K} 1-\mathrm{K} 29 \\
\end{array}$ \\
\hline $\bar{c}$ & $23 \mathrm{MHz}$ & $28 \mathrm{MHz}$ & 1000 & DIPOLESWEEP & $\begin{array}{c}\text { all } A \\
\text { all } B \text { exc B13 }\end{array}$ \\
\hline $\bar{d}$ & $25.7 \mathrm{MHz}$ & $26 \mathrm{MHz}$ & 1000 & DIPOLE SWEEP & B 13 \\
\hline e & $150 \mathrm{MHz}$ & $200 \mathrm{MHz}$ & 1000 & DIPOLE SWEEP & $\begin{array}{l}\text { all C exc C13 } \\
\text { all D }\end{array}$ \\
\hline f & $171 \mathrm{MHz}$ & $173 \mathrm{MHz}$ & 1000 & DPOLE SWEEP & $\mathrm{C} 13$ \\
\hline $\mathbf{g}$ & $350 \mathrm{MHz}$ & $500 \mathrm{MHz}$ & 1000 & DIPOLE SWEEP & $\begin{array}{l}\text { all } E \text { exc E13 } \\
\text { all } F\end{array}$ \\
\hline$h$ & $427 \mathrm{MHz}$ & $432 \mathrm{MHz}$ & 1000 & DIPOLE SWEEP & E13 \\
\hline$i$ & $25.85 \mathrm{MHz}$ & $25.85 \mathrm{MHz}$ & 1 & RHOMBIC DWELL & all $G$ \\
\hline $\mathrm{k}$ & $172 \mathrm{MHz}$ & $172 \mathrm{MHz}$ & 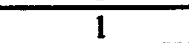 & RHOMBIC DWELL & all $\mathrm{H}$ \\
\hline 1 & $430 \mathrm{MHz}$ & $430 \mathrm{MHz}$ & 1 & RHOMBIC PULSE & all I \\
\hline$m$ & $950 \mathrm{MHz}$ & $959 \mathrm{MHz}$ & 1000 & RHOMBIC SWEEP & $\mathrm{J12}$ \\
\hline$n$ & $2 \mathrm{MHz}$ & $30 \mathrm{MHz}$ & 250 & $\begin{array}{l}\text { RHOMBIC SWEEP } \\
\text { DIFFERENTLAL }\end{array}$ & all HP \\
\hline $\bar{p}$ & $1 \mathrm{MHz}$ & $100 \mathrm{MHz}$ & 50 & $\begin{array}{c}\text { GROUND PLANE } \\
\text { CHARACTERIZATION }\end{array}$ & $\begin{array}{c}\text { Pad } \\
\text { Characterization }\end{array}$ \\
\hline $\bar{q}$ & $100 \mathrm{MHz}$ & $1 \mathrm{GHz}$ & 100 & $\begin{array}{l}\text { GROUND PLANE } \\
\text { CHARACTERIZATION }\end{array}$ & $\begin{array}{c}\text { Pad } \\
\text { Characterization }\end{array}$ \\
\hline
\end{tabular}

The designator " $\mathrm{m}$ " is used with test J12. Its data has the form "m\#\#012". This was an inadvertent oversight that could lead to confusion. The prefix " $\mathrm{m}$ " is usually used as a prefix to designate a merged file, i.e., a file that has been created by merging or appending data files for sweeps designated " $a$ " referring to the low frequency data sweep $(300 \mathrm{kHz}<f<100 \mathrm{MHz})$ and files for sweeps designated " $b$ " referring to the high frequency data sweep $(100 \mathrm{MHz}<\mathrm{f}<1 \mathrm{GHz})$. Thus, " $\mathrm{m}$ " is usually used as a prefix for files that correspond to data over the entire frequency range from $300 \mathrm{kHz}$ to $1 \mathrm{GHz}$ that is composed of parts acquired during different sweeps. Thus, the files "a\#汭\&\&" and " $\mathrm{b} \#$ \&\&\&\&" were merged to produce a single file covering $0.3 \mathrm{MHz}$ to

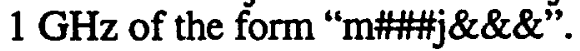

To facilitate the correspondence of data files to specific tests, a tabulation has been created which presents the executed test, by number and some identifying characteristics, and the files associated with that particular test. This information is contained in Tables 6-18 through 6-30. 
Table 6-18. Data ID: Test Series AN - Ambient Noise Measurements

\begin{tabular}{|c|c|c|c|c|c|c|c|c|}
\hline $\begin{array}{c}\text { Test } \\
\#\end{array}$ & $\begin{array}{c}\text { Test } \\
\text { Point }\end{array}$ & $\begin{array}{l}\text { Measured } \\
\text { Parameter }\end{array}$ & $\begin{array}{l}\text { Low Freq. Data } \\
(0.3-100 \mathrm{MHz})\end{array}$ & $\begin{array}{c}\text { Low Freq Reference } \\
(0.3-100 \mathrm{MHz})\end{array}$ & $\begin{array}{c}\text { High Freq Data } \\
(100-1000 \mathrm{MHz})\end{array}$ & $\begin{array}{c}\text { High Freq Reference } \\
(100-1000 \mathrm{MHz})\end{array}$ & $\begin{array}{c}\text { Merged Data } \\
(0.3-1000 \mathrm{MHz})\end{array}$ & $\begin{array}{l}\text { Merged Reference } \\
(0.3-1000 \mathrm{MHz}) \\
\end{array}$ \\
\hline & & & Data File Name & Data File Name & Data File Name & Data File Name & Data File Name & Data File Name \\
\hline & & & & & & & & \\
\hline AN1 & CP1 & Vert. Field & an00an01 & an00ref 1 & bn00an01 & bn00ref 1 & mn00ano1 & mn00refl \\
\hline$\overline{\mathrm{AN} 2}$ & $\mathrm{CP} 2$ & Horiz.1 Field & $\operatorname{an} 00$ an02 & an00ref 1 & bn00an02 & bn00ref 1 & mn00an02 & mn00refl \\
\hline AN3 & EB3 & Cable Current & an01an03 & an01ref 1 & bn01an03 & bn0lref1 & mn00an03 & mn0lrefl \\
\hline AN4 & $\mathrm{C4}$ & Field & an02an04 & an02refl & bn02an04 & bn02ref1 & $\mathrm{mn02an04}$ & mn02ref2 \\
\hline
\end{tabular}

Table 6-19. Data ID: Test Series A

\begin{tabular}{|l|l|l|l|l|}
\hline $\begin{array}{c}\text { Test } \\
\#\end{array}$ & $\begin{array}{c}\text { Test } \\
\text { Point }\end{array}$ & $\begin{array}{c}\text { Measured } \\
\text { Parameter }\end{array}$ & $\begin{array}{c}\text { Data } \\
(23-28 \mathrm{MHz})\end{array}$ & $\begin{array}{c}\text { Reference } \\
(23-28 \mathrm{MHz})\end{array}$ \\
\hline & & & Data File Name & Data File Name \\
\hline A1 & CP1 & Vert. Field & c059a001 & c059ref1 \\
\hline A2 & CP2 & Horiz.1 Field & c059a002 & c059ref1 \\
\hline A3 & CP3 & Horiz.2 Field & c056a003 & c056ref1 \\
\hline A4 & CP4 & Wire Current & c058a004 & c058ref1 \\
\hline A5 & CP5 & Wire Voltage & c061a005 & c061ref 1 \\
\hline A6 & EB1 & Field & c055a006 & c055ref2 \\
\hline A7 & EB2 & LRU Voltage & c056a003 & c056ref1 \\
\hline A8 & EB3 & Cable Current & c058a008 & c058ref1 \\
\hline A9 & C1 & Field & c053a009 & c053ref1 \\
\hline A10 & C2 & Wire Current & c054a010 & c054ref1 \\
\hline A11 & C3 & Voltage & c054a011 & c054ref1 \\
\hline A12 & C4 & Field & c061a012 & c061ref1 \\
\hline A13 & $\begin{array}{l}\text { Weak } \\
\text { Signal }\end{array}$ & LRU Voltage & c063a013 & c063ref2 \\
& Line & & & \\
\hline A14 & $\begin{array}{l}\text { Strong } \\
\text { Signal } \\
\text { Line }\end{array}$ & Voltage & c062a014 & c062ref2 \\
\hline A101 & CP1 & Field & c060a101 & c060ref1 \\
\hline A102 & CP2 & Field & c060a102 & c060ref1 \\
\hline
\end{tabular}

Table 6-20. Data ID: Test Series B

\begin{tabular}{|c|c|c|c|c|}
\hline $\begin{array}{c}\text { Test } \\
\#\end{array}$ & $\begin{array}{l}\text { Test } \\
\text { Point }\end{array}$ & $\begin{array}{l}\text { Measured } \\
\text { Parameter }\end{array}$ & $\begin{array}{c}\text { Data } \\
(23-28 \mathrm{MHz}) \\
\end{array}$ & $\begin{array}{c}\text { Reference } \\
(23-28 \mathrm{MHz})\end{array}$ \\
\hline & & & Data File Name & Data File Name \\
\hline$\overline{B 1}$ & CPI & Vert. Field & $\mathrm{c043b001}$ & c043refl \\
\hline$\overline{B 2}$ & CP2 & Horiz.1 Field & $\mathrm{co46c002}$ & c046ref 1 \\
\hline B3 & CP3 & Horiz.2 Field & $\mathrm{c045b003}$ & c045refl \\
\hline B4 & $\mathrm{CP4}$ & Wire Current & $\mathrm{c046b004}$ & c046ref 1 \\
\hline$\overline{B 5}$ & CP5 & Wire Voltage & $c 044 b 005$ & c044refl \\
\hline B6 & EB1 & Field & $\mathrm{c050b006}$ & c050refl \\
\hline B7 & EB2 & LRU Voltage & $\mathrm{c047b007}$ & c047ref2 \\
\hline B8 & EB3 & Cable Current & c043b008 & c043ref1 \\
\hline B9 & $\mathrm{Cl}$ & Field & c050b009 & c050ref1 \\
\hline $\mathrm{B} 10$ & $\mathrm{C} 2$ & Wire Current & $\mathrm{c} 049 \mathrm{~b} 010$ & c049refl \\
\hline B11 & $\mathrm{C3}$ & Voltage & $\mathrm{c} 049 \mathrm{~b} 011$ & c049refl \\
\hline $\mathrm{B} 12$ & $\mathrm{C4}$ & Field & $c 045 b 012$ & c045refl \\
\hline $\mathrm{B} 13$ & $\mathrm{C} 1$ & Field & d001b013 & d001refl \\
\hline$\overline{B 14}$ & $\begin{array}{l}\text { Weak } \\
\text { Signal } \\
\text { Line }\end{array}$ & LRU Voltage & c051b014 & c051ref2 \\
\hline B15 & $\begin{array}{l}\text { Strong } \\
\text { Signal } \\
\text { Line }\end{array}$ & Voltage & $\mathrm{c} 052 \mathrm{~b} 015$ & c052ref2 \\
\hline
\end{tabular}


Table 6-21. Data ID: Test Series C

\begin{tabular}{|c|c|c|c|c|}
\hline $\begin{array}{c}\text { Test } \\
\#\end{array}$ & $\begin{array}{l}\text { Test } \\
\text { Point }\end{array}$ & $\begin{array}{l}\text { Measured } \\
\text { Parameter }\end{array}$ & $\begin{array}{c}\text { Data } \\
(150-200 \mathrm{MHz})\end{array}$ & $\begin{array}{c}\text { Reference } \\
(150-200 \mathrm{MHz}) \\
\end{array}$ \\
\hline & & & Data File Name & Data File Name \\
\hline $\mathrm{Cl}$ & CPI & Vert. Field & e004c001 & c004refl \\
\hline $\mathrm{C} 2$ & $\mathrm{CP2}$ & Horiz.1 Field & $\mathrm{e} 010 \mathrm{c} 002$ & e010ref2 \\
\hline C3 & $\mathrm{CP3}$ & Horiz.2 Field & $e 007 c 003$ & e007ref1 \\
\hline $\mathrm{C4}$ & $\mathrm{CP4}$ & Wire Current & $e 009 c 004$ & e009refl \\
\hline $\mathrm{C5}$ & CP5 & Wire Voltage & $\mathrm{e} 006 \mathrm{c} 005$ & e006ref 1 \\
\hline C6 & EB1 & Field & $\mathrm{e} 012 \mathrm{c} 006$ & e012ref1 \\
\hline $\mathrm{C7}$ & EB2 & LRU Voltage & $\mathrm{e} 007 \mathrm{c007}$ & e007refl \\
\hline $\mathrm{C8}$ & EB3 & Cable Current & $e 009 \mathrm{c} 008$ & e009ref1 \\
\hline C9 & $\mathrm{Cl}$ & Field & $e 013 c 009$ & e013refl \\
\hline $\mathrm{ClO}$ & $\mathrm{C2}$ & Wire Current & $\mathrm{e} 012 \mathrm{c} 010$ & e012refl \\
\hline $\mathrm{Cll}$ & C3 & Voltage & $\mathrm{e} 004 \mathrm{c011}$ & c004ref I \\
\hline $\mathrm{Cl} 2$ & $\mathrm{C4}$ & Field & $\mathrm{e} 006 \mathrm{c} 012$ & e006refl \\
\hline $\mathrm{C} 13$ & $\mathrm{Cl}$ & Field & $\mathrm{f} 002 \mathrm{c013}$ & f002refl \\
\hline $\mathrm{C} 14$ & $\begin{array}{l}\text { Weak } \\
\text { Signal } \\
\text { Line }\end{array}$ & Wire Current & $c 015 c 014$ & c015refl \\
\hline $\mathrm{C} 15$ & $\begin{array}{l}\text { Strong } \\
\text { Signal } \\
\text { Line }\end{array}$ & Voltage & c015c015 & c0l5refl \\
\hline $\mathrm{Cl09}$ & $\mathrm{Cl}$ & Field & $e 016 \mathrm{c} 109$ & c016ref1 \\
\hline C111 & $\mathrm{C3}$ & Voltage & $\mathrm{e016c111}$ & c016ref 1 \\
\hline $\mathrm{C}_{104}$ & $\mathrm{CP} 4$ & Wire Current & $e 017 c 104$ & e017ref1 \\
\hline C204 & CP4 & Wire Current & $e 018 c 204$ & e018refl \\
\hline C304 & repeat? & Wire Current & $e 019 c 304$ & e019refl \\
\hline C404 & $\mathrm{CP} 4$ & Wire Current & $e 020 c 404$ & e020ref 1 \\
\hline
\end{tabular}

Table 6-22. Data ID: Test Series D

\begin{tabular}{|c|c|c|c|c|}
\hline $\begin{array}{c}\text { Test } \\
\#\end{array}$ & $\begin{array}{l}\text { Test } \\
\text { Point }\end{array}$ & $\begin{array}{l}\text { Measured } \\
\text { Parameter }\end{array}$ & $\begin{array}{c}\text { Data } \\
(150-200 \mathrm{MHz}) \\
\end{array}$ & $\begin{array}{c}\text { Reference } \\
(150-200 \mathrm{MHz}) \\
\end{array}$ \\
\hline & & & Data File Name & Data File Name \\
\hline$\overline{\mathrm{D} 1}$ & $\overline{C P 1}$ & Vert. Field & $e 027 d 001$ & e027ref1 \\
\hline$\overline{\mathrm{D} 2}$ & $\overline{C P 2}$ & Horiz.1 Field & $e 027 d 002$ & e027refl \\
\hline D3 & $\mathrm{CP3}$ & Horiz.2 Field & $e 024 d 003$ & e024refl \\
\hline D4 & CP4 & Wire Current & $e 025 d 004$ & c025refl 1 \\
\hline$\overline{D 5}$ & CP5 & Wire Voltage & e029d005 & e029ref1 \\
\hline D6 & EB1 & Field & $e 022 \mathrm{~d} 006$ & e022refl \\
\hline$\overline{\mathrm{D} 7}$ & EB2 & LRU Voltage & e024d007 & e024ref 1 \\
\hline$\overline{\mathrm{D} 8}$ & EB3 & Cable Current & $e 025 d 008$ & e025ref 1 \\
\hline D9 & $\mathrm{Cl}$ & Field & e021d009 & e021ref 1 \\
\hline D10 & $\mathrm{C2}$ & Wire Current & $e 022 d 010$ & e022refl \\
\hline D11 & C3 & Voltage & $e 021 d 011$ & e021refl \\
\hline D12 & $\mathrm{C4}$ & Field & $\mathrm{e} 029 \mathrm{~d} 012$ & e029ref 1 \\
\hline D13 & $\begin{array}{l}\text { Weak } \\
\text { Signal } \\
\text { Line }\end{array}$ & Wire Current & $e 031 d 013$ & e031ref 1 \\
\hline D14 & $\begin{array}{l}\text { Weak } \\
\text { Signal } \\
\text { Line }\end{array}$ & LRU Voltage & $e 031 d 014$ & c031ref 1 \\
\hline
\end{tabular}


Table 6-23. Data ID: Test Series E

\begin{tabular}{|c|c|c|c|c|}
\hline $\begin{array}{c}\text { Test } \\
\#\end{array}$ & $\begin{array}{l}\text { Test } \\
\text { Point } \\
\end{array}$ & $\begin{array}{l}\text { Measured } \\
\text { Parameter }\end{array}$ & $\begin{array}{c}\text { Data } \\
(350-500 \mathrm{MHz}) \\
\end{array}$ & $\begin{array}{c}\text { Reference } \\
(350-500 \mathrm{MHz}) \\
\end{array}$ \\
\hline & & & Data File Name & Data File Name \\
\hline & & & & \\
\hline El & CP1 & Vert. Field & $\mathrm{g} 024 \mathrm{e} 001$ & g024refl \\
\hline E2 & CP2 & Horiz.1 Field & $\mathrm{g} 024 \mathrm{e} 002$ & g024refl \\
\hline$\overline{E 3}$ & CP3 & Horiz.2 Field & $\mathrm{g} 022 \mathrm{e} 003$ & g022ref 1 \\
\hline $\mathbf{E} 4$ & $\mathrm{CP} 4$ & Wire Current & $\mathrm{g} 026 \mathrm{e} 004$ & g026ref 1 \\
\hline E5 & CP5 & Wire Voltage & $\mathrm{g} 020 \mathrm{e} 005$ & g020ref1 \\
\hline E6 & EB1 & Field & $8020 \mathrm{e} 006$ & g020ref 1 \\
\hline E7 & EB2 & LRU Voltage & $8022 \mathrm{e007}$ & g022ref 1 \\
\hline E8 & EB3 & Cable Current & $\mathrm{g} 014 \mathrm{e} 008$ & g014refl \\
\hline E9 & $\mathrm{Cl}_{1}$ & Field & g015e009 & g015ref 1 \\
\hline E10 & $\mathrm{C2}$ & Wire Current & $\mathrm{g} 014 \mathrm{e} 010$ & g014refl \\
\hline E11 & $\mathrm{C} 3$ & Voltage & g015e011 & g015refl \\
\hline E12 & $\mathrm{C4}$ & Field & $\mathrm{g} 026 \mathrm{e} 0.12$ & g026ref 1 \\
\hline E13 & $\mathrm{Cl}$ & Field & h001e013 & h001refl \\
\hline
\end{tabular}

Table 6-24. Data ID: Test Series F

\begin{tabular}{|c|c|c|c|c|}
\hline $\begin{array}{c}\text { Test } \\
\#\end{array}$ & $\begin{array}{l}\text { Test } \\
\text { Point } \\
\end{array}$ & $\begin{array}{l}\text { Measured } \\
\text { Parameter }\end{array}$ & $\begin{array}{c}\text { Data } \\
(350-500 \mathrm{MHz}) \\
\end{array}$ & $\begin{array}{c}\text { Reference } \\
(350-500 \mathrm{MHz}) \\
\end{array}$ \\
\hline & & & Data File Name & Data File Name \\
\hline F1 & CPI & Vert Fild & $g 002 f 001$ & \\
\hline F2 & $\mathrm{CP} 2$ & Horiz.1 Field & $\mathrm{g} 002 \mathrm{f} 002$ & g002ref 1 \\
\hline F3 & $\mathrm{CP} 3$ & Horiz.2 Field & $\mathrm{g} 004 \mathrm{f} 003$ & g004refl \\
\hline F4 & $\mathrm{CP4}$ & Wire Current & $\mathrm{g} 005 \mathrm{f} 004$ & g005ref 1 \\
\hline F5 & $\mathrm{CP5}$ & Wire Voltage & $\mathrm{g} 007 \mathrm{f} 005$ & g007refl \\
\hline F6 & EB1 & Field & g009f006 & g009refl \\
\hline$\overline{\text { F7 }}$ & EB2 & LRU Voltage & g004f007 & g004ref 1 \\
\hline F8 & EB3 & Cable Current & $\mathrm{g} 005 \mathrm{f} 008$ & g005refl \\
\hline F9 & $\mathrm{Cl}$ & Field & g009f009 & g009ref1 \\
\hline $\mathrm{F} 10$ & $\mathrm{C} 2$ & Wire Current & $\mathrm{g} 003 \mathrm{f} 010$ & g003ref 1 \\
\hline F11 & $\mathrm{C3}$ & Voltage & $\mathrm{g} 007 \mathrm{f} 011$ & g007ref 1 \\
\hline F12 & $\mathrm{C4}$ & Field & g003f012 & g003ref 1 \\
\hline F13 & $\mathrm{C2}$ & Wire Current & $\mathrm{g} 012 \mathrm{f0} 013$ & g012ref1 \\
\hline F14 & EB3 & Cable Current & $\mathrm{g} 012 \mathrm{f} 014$ & g012ref1 \\
\hline F008 & EB3 & Cable Current & g013f008 & g013ref1 \\
\hline F010 & $\mathrm{C2}$ & Wire Current & g013f010 & g013ref 1 \\
\hline
\end{tabular}


Table 6-25. Data ID: Noise Floor Measurements - Rhombic

\begin{tabular}{|c|c|c|c|c|c|c|c|c|}
\hline $\begin{array}{c}\text { Test } \\
\# \\
\end{array}$ & $\begin{array}{c}\text { Test } \\
\text { Point }\end{array}$ & $\begin{array}{l}\text { Measured } \\
\text { Parameter }\end{array}$ & $\begin{array}{l}\text { Low Freq Data } \\
(0.3-100 \mathrm{MHz})\end{array}$ & $\begin{array}{l}\text { Low Freq Reference } \\
(0.3-100 \mathrm{MHz})\end{array}$ & $\begin{array}{c}\text { High Freq Data } \\
(100-1000 \mathrm{MHz})\end{array}$ & $\begin{array}{c}\text { High Freq } \\
\text { Reference } \\
(100-1000 \mathrm{MHz}) \\
\end{array}$ & $\begin{array}{c}\text { Merged Data } \\
(0.3-1000 \mathrm{MHz})\end{array}$ & $\begin{array}{l}\text { Merged Reference } \\
(0.3-1000 \mathrm{MHz})\end{array}$ \\
\hline & & & Data File Name & Data File Name & Data File Name & Data File Name & Data File Name & Data File Name \\
\hline & & & & & & & & \\
\hline NOl & CP1 & Vert. Field & a100ni01 & a100ref 1 & b100ni01 & b100ref 1 & m100ni01 & m100refl \\
\hline $\mathrm{NO2}$ & CP2 & Horiz.1 Field & a 100ni02 & a100ref1 & b100ni02 & b100ref1 & m100ni02 & m100ref 1 \\
\hline NO3 & $\mathrm{CP3}$ & Horiz. 2 Field & a 102 ni03 & al02ref1 & b102ni03 & bl02refl & $\mathrm{m} 102 \mathrm{ni03}$ & m102ref 1 \\
\hline NO4 & CP4 & Wire Current & a 103ni04 & al03ref 1 & b103ni04 & b103ref1 & $\mathrm{ml03ni04}$ & m103refl \\
\hline NO5 & CP5 & Wire Voltage & a 101 ni05 & alolrefl & b101ni05 & blolref1 & m101ni05 & ml01refl \\
\hline NO6 & EMI & Field & a 105 ni06 & a105ref 1 & b105ni06 & bl05ref1 & mi05ni06 & m105ref1 \\
\hline NO7 & EM2 & LRU Voltage & a102ni07 & a102ref 1 & b102ni07 & b102refl & $\mathrm{m} 102 \mathrm{ni07}$ & m102refl \\
\hline NO8 & EM3 & Cable Current & al03ni08 & a103ref 1 & b103ni08 & bl03ref1 & $\mathrm{ml03ni08}$ & m103ref I \\
\hline NO9 & $\mathrm{Cl}$ & Field & a 104ni09 & al04ref1 & bl04ni09 & b104refl & mi04ni09 & m104ref 1 \\
\hline NOI0 & $\mathrm{C} 2$ & Wire Current & a 105 nilo & al05ref1 & blo5nilo & b105refl & m105nil0 & m105ref 1 \\
\hline NO11 & C3 & Voltage & a104nill & a 104ref 1 & b104ni11 & bl04refl & m104nil1 & m104ref 1 \\
\hline NO12 & $\mathrm{C4}$ & Field & a $10 \ln i 12$ & a101ref 1 & blolnil2 & bl01refl & m101nil2 & ml01refl \\
\hline
\end{tabular}


Table 6-26. Data ID: Test Series J - Rhombic (Nose Incident and Related Tests)

\begin{tabular}{|c|c|c|c|c|c|c|c|c|c|}
\hline $\begin{array}{c}\text { Test } \\
\#\end{array}$ & $\begin{array}{l}\text { Test } \\
\text { Point }\end{array}$ & $\begin{array}{l}\text { Measured } \\
\text { Parameter }\end{array}$ & $\begin{array}{c}\text { Low Freq Data } \\
(0.3-100 \mathrm{MHz})\end{array}$ & $\begin{array}{c}\text { Low Freq Reference } \\
(0.3-100 \mathrm{MHz})\end{array}$ & $\begin{array}{c}\text { High Freq Data } \\
(100-1000 \mathrm{MHz}) \\
\end{array}$ & $\begin{array}{c}\text { High Freq Reference } \\
(0.3-100 \mathrm{MHz})\end{array}$ & $\begin{array}{c}\text { Merged Data } \\
(0.3-1000 \mathrm{MHz}) \\
\end{array}$ & $\begin{array}{l}\text { Merged Reference } \\
(0.3-100 \mathrm{MHz}) \\
\end{array}$ & $\begin{array}{l}\text { Acquisition } \\
\text { Date/Time } \\
\end{array}$ \\
\hline & & & Data File Name & Data File Name & Data File Name & Data File Name & Data File Name & Data File Name & \\
\hline & & & & & & & & & \\
\hline JI & $\overline{\mathrm{CPI}}$ & Vert. Field & & DROPPED & & & & & DROPPED \\
\hline $\mathrm{J2}$ & $\mathrm{CP2}$ & Horiz.1 Field & & DROPPED & & & & & DROPPED \\
\hline J3 & $\mathrm{CP3}$ & Horiz.2 Field & & DROPPED & & & & & DROPPED \\
\hline J4 & $\overline{\mathrm{CP} 4}$ & Wire Current & a 109j004 & a 109ref1 & b109j004 & bl09ref1 & $\mathrm{ml09j004}$ & ml09refl & $\begin{array}{r}10-3 / 3: 21 \\
3: 08\end{array}$ \\
\hline$\sqrt{5}$ & CP5 & Wire Voltage & al11j005 & allirefl & b111j005 & b111refl & $\mathrm{m} 11 \mathrm{j} 005$ & ml11ref1 & $\begin{array}{r}10-3 / 3: 57 \\
4: 28\end{array}$ \\
\hline 56 & $\mathrm{CPI}$ & Vert. Field & al20j006 & a120refl & b/20j006 & b120refl & $\mathrm{ml20j006}$ & m120ref1 & $\begin{array}{r}10-4 / 1: 19 \\
1: 02 \\
\end{array}$ \\
\hline J7 & $\overline{\mathrm{CP} 2}$ & Horiz.1 Field & a120j007 & a120refl & b120j007 & b120ref1 & $\mathrm{m} 120 \mathrm{j} 007$ & m120refl & $\begin{array}{r}10-4 / 1: 19 \\
1: 02\end{array}$ \\
\hline 58 & $\overline{C P 3}$ & Horiz.2 Field & a $121 \mathrm{j} 008$ & al21refl & b121j008 & b121ref1 & $\mathrm{m} 121 \mathrm{j} 008$ & ml2lrefl & $\begin{array}{r}10-4 / 1: 40 \\
1: 48 \\
\end{array}$ \\
\hline J9 & $\mathrm{CPI}$ & Vert. Field & a122j009 & a122ref1 & b122j009 & b122ref 1 & $\mathrm{~m} 122 \mathrm{j} 009$ & $\mathrm{~m} 122 \mathrm{refl}$ & $\begin{array}{r}10-4 / 2: 22 \\
2: 09\end{array}$ \\
\hline $\mathrm{J10}$ & $\mathrm{CP2}$ & Horiz.1 Field & a122j010 & a122refl & b122j010 & b122refl & $\mathrm{m} 122 \mathrm{j} 010$ & m122ref I & $\begin{array}{r}10-4 / 2: 22 \\
2: 09\end{array}$ \\
\hline J11 & CP3 & Horiz.2 Field & a123j011 & a 123ref1 & b123j011 & b123refl & $\mathrm{m} 123 \mathrm{j011}$ & m123ref1 & $\begin{array}{r}10-4 / 2: 40 \\
2: 49\end{array}$ \\
\hline $\mathrm{J12}$ & CP1 & Vert. Field & $r$ & & $\mathrm{~b} 112 \mathrm{j} 012$ & b112ref1 & & & $10-3 / 4: 50$ \\
\hline $\mathrm{J13}$ & CP1 & Vert. Field & $\begin{array}{c}\text { Data Not } \\
\text { Available }\end{array}$ & Data Not Available & $\begin{array}{l}\text { Data Not } \\
\text { Available }\end{array}$ & Data Not Available & $\mathrm{ml16j013}$ & $\mathrm{ml16refl}$ & $\begin{array}{r}10-4 / 8: 47 \\
8: 33 \\
\end{array}$ \\
\hline $\mathrm{J14}$ & CP2 & Horiz.1 Field & $\begin{array}{l}\text { Data Not } \\
\text { Available }\end{array}$ & Data Not Available & $\begin{array}{c}\text { Data Not } \\
\text { Available }\end{array}$ & Data Not Available & $\mathrm{ml16j014}$ & m116refl & $\begin{array}{r}10-4 / 9: 47 \\
8: 33 \\
\end{array}$ \\
\hline 315 & $\mathrm{CP3}$ & Horiz..2 Field & $\begin{array}{c}\text { Data Not } \\
\text { Available } \\
\end{array}$ & Data Not Available & $\begin{array}{l}\text { Data Not } \\
\text { Available } \\
\end{array}$ & Data Not Available & $\mathrm{ml17j015}$ & mII7ref1 & $\begin{array}{r}10-4 / 9: 07 \\
9: 16 \\
\end{array}$ \\
\hline J16 & $\overline{C P I}$ & Vert. Field & $\begin{array}{l}\text { Data Not } \\
\text { Available }\end{array}$ & Data Not Available & $\begin{array}{l}\text { Data Not } \\
\text { Available }\end{array}$ & Data Not Available & $\mathrm{m} 118 \mathrm{j016}$ & $\mathrm{ml18 \text {refl }}$ & $\begin{array}{r}10-4 / 9: 39 \\
9: 27 \\
\end{array}$ \\
\hline $\mathrm{J17}$ & $\overline{\mathrm{CP} 2}$ & Horiz.I Field & $\begin{array}{l}\text { Data Not } \\
\text { Available }\end{array}$ & Data Not Available & $\begin{array}{l}\text { Data Not } \\
\text { Available }\end{array}$ & Data Not Available & $\mathrm{ml18j017}$ & $\mathrm{ml18ref1}$ & $\begin{array}{r}0-4 / 9: 39 \\
9: 27\end{array}$ \\
\hline $\mathrm{J} 18$ & CP3 & Horiz.2 Field & $\begin{array}{l}\text { Data Not } \\
\text { Available }\end{array}$ & Data Not Available & $\begin{array}{l}\text { Data Not } \\
\text { Available } \\
\end{array}$ & Data Not Available & $\mathrm{m} 119 \mathrm{j} 018$ & ml19refl & $\begin{array}{r}10-4 / 9: 59 \\
10: 07 \\
\end{array}$ \\
\hline J19 & $\mathrm{CP} 12$ & Heater current & al $25 j 019$ & a125refl & b125j019 & b125refl & $\mathrm{m} 125 \mathrm{j} 019$ & $\mathrm{ml25refl}$ & $\begin{array}{r}10-4 / 3: 21 \\
3: 15 \\
\end{array}$ \\
\hline
\end{tabular}




\begin{tabular}{|c|c|c|c|c|c|c|c|c|c|}
\hline $\begin{array}{c}\text { Test } \\
\# \\
\end{array}$ & $\begin{array}{l}\text { Test } \\
\text { Point } \\
\end{array}$ & $\begin{array}{l}\text { Measured } \\
\text { Parameter } \\
\end{array}$ & $\begin{array}{c}\text { Low Freq Data } \\
(0.3-100 \mathrm{MHz}) \\
\end{array}$ & $\begin{array}{c}\text { Low Freq Reference } \\
(0.3-100 \mathrm{MHz})\end{array}$ & $\begin{array}{c}\text { High Freq Data } \\
(100-1000 \mathrm{MHz}) \\
\end{array}$ & $\begin{array}{c}\text { High Freq Reference } \\
(0.3-100 \mathrm{MHz})\end{array}$ & $\begin{array}{c}\text { Merged Data } \\
(0.3-1000 \mathrm{MHz}) \\
\end{array}$ & $\begin{array}{c}\text { Merged Reference } \\
(0.3-100 \mathrm{MHz}) \\
\end{array}$ & $\begin{array}{l}\text { Acquisition } \\
\text { Date/Time }\end{array}$ \\
\hline & & & Data File Name & Data File Name & Data File Name & Data File Name & Data File Name & Data File Name & \\
\hline $\mathrm{J} 20$ & $\mathrm{CPI}$ & Vert. Field & a106j020 & a106ref 1 & b106j020 & b106refl & $\mathrm{ml06j020}$ & m106ref1 & $\begin{array}{r}10-3 / 1: 55 \\
1: 41\end{array}$ \\
\hline$\sqrt{21}$ & $\overline{\mathrm{CP} 2}$ & Horiz.l Field & a $106 \mathrm{j021}$ & a l06ref 1 & b106j021 & b106ref1 & $\mathrm{ml06j021}$ & m106refl & $\begin{array}{r}10-3 / 1: 55 \\
1: 41 \\
\end{array}$ \\
\hline$\sqrt{22}$ & $\overline{\mathrm{CP3}}$ & Horiz.2 Field & $\mathrm{a} 108 \mathrm{j} 022$ & al08refl & $\mathrm{b} 108 \mathrm{j} 022$ & b108ref 1 & $\mathrm{ml08 \textrm {j } 0 2 2}$ & m108refl & $\begin{array}{r}10-3 / 2: 34 \\
2: 54\end{array}$ \\
\hline $\mathbf{J 2 3}$ & EB I & Field & a $108 \mathrm{j} 023$ & a 108ref 1 & bl08j023 & b108ref1 & $\mathrm{ml08j023}$ & m108refl & $\begin{array}{r}10-3 / 2: 34 \\
2: 54\end{array}$ \\
\hline $\mathrm{J24}$ & EB2 & LRU Voltage & a $109 \mathrm{j} 024$ & a109refl & b109j024 & bl09ref1 & $\mathrm{ml09j024}$ & m109refl & $\begin{array}{r}10-3 / 3: 21 \\
3: 08\end{array}$ \\
\hline $\mathrm{J} 25$ & EB3 & Cable Current & a111j025 & allirefl & b111j025 & b111refl & $\mathrm{m} 111 \mathrm{j025}$ & ml11refl & $\begin{array}{r}10-3 / 3: 57 \\
4: 28 \\
\end{array}$ \\
\hline $\mathrm{J} 26$ & EBI & Field & al32j026 & Data Not Available & b132j026 & Data Not Available & $\mathrm{m} 132 \mathrm{j} 026$ & m132ref2 & $\begin{array}{r}10-5 / 9: 46 \\
10: 03 \\
\end{array}$ \\
\hline J27 & EB2 & LRU Voltage & a $131 \mathrm{j027}$ & Data Not Available & b131j027 & Data Not Available & $\mathrm{m} 131 \mathrm{j} 027$ & m13lref2 & $\begin{array}{c}10-5 / 9: 30 \\
9: 13\end{array}$ \\
\hline$J 28$ & EB3 & Cable Current & a $130 \mathrm{j} 028$ & Data Not Available & bI30j028 & Data Not Available & $\mathrm{m} 130 \mathrm{j} 028$ & m130ref2 & $\begin{array}{r}10-5 / 8: 44 \\
9: 02\end{array}$ \\
\hline J29 & EB1 & Field & a $127 \mathrm{j} 029$ & Data Not Available & b127j029 & Data Not Available & $\mathrm{m} 127 \mathrm{j029}$ & m127ref2 & $\begin{array}{r}10-4 / 4: 47 \\
4: 02 \\
\end{array}$ \\
\hline $\mathrm{J30}$ & EB2 & LRU Voltage & a128j030 & Data Not Available & $\mathrm{b} 128 \mathrm{j} 030$ & Data Not Available & $\mathrm{m} 128 \mathrm{j} 030$ & m128ref2 & $\begin{array}{r}10-5 / 7: 03 \\
7: 24\end{array}$ \\
\hline$\sqrt{31}$ & EB3 & Cable Current & a129j031 & Data Not Available & b129j031 & Data Not Available & $\mathrm{m} 129 \mathrm{j} 031$ & m129ref2 & $\begin{array}{r}10-5 / 8: 06 \\
7: 38 \\
\end{array}$ \\
\hline $\mathrm{J} 32$ & $\mathrm{Cl}$ & Field & a133j032 & a133ref1 & b133j032 & bl33ref 1 & $\mathrm{~m} 133 \mathrm{j} 032$ & m133refl & $\begin{array}{r}10-5 / 10: 56 \\
10: 37 \\
\end{array}$ \\
\hline J33 & $\mathrm{C2}$ & Wire Current & a $137 \mathrm{j} 033$ & al37refl & b137j033 & b137ref 1 & $\mathrm{~m} 137 \mathrm{j033}$ & mi37ref1 & $\begin{array}{r}10-5 / 1: 21 \\
1: 41 \\
\end{array}$ \\
\hline J34 & $\mathrm{C3}$ & Voltage & a133j034 & a133refl & $\mathrm{b} 133 \mathrm{j} 034$ & b133refl & $\mathrm{m133j034}$ & $\mathrm{m} 133 \mathrm{refl}$ & $\begin{array}{r}10-5 / 10: 56 \\
10: 37\end{array}$ \\
\hline $\mathrm{J35}$ & $\mathrm{C} 4$ & Field & a137j035 & al37refl & b137j035 & bl37refl & $\mathrm{m137j035}$ & m137refl & $\begin{array}{r}10-5 / 1: 21 \\
1: 41\end{array}$ \\
\hline 536 & $\mathrm{CPI}$ & Vert. Field & a $138 \mathrm{j036}$ & al38refl & bl38j036 & b138refl & $\mathrm{m} 138 \mathrm{j} 036$ & m138refl & $\begin{array}{r}10-6 / 5: 34 \\
8: 53 \\
\end{array}$ \\
\hline J37 & $\mathrm{CP2}$ & Horiz.1 Field & a138j037 & al38ref1 & b138j037 & bl38refl & $\mathrm{ml38j037}$ & m138refl & $\begin{array}{r}10-6 / 8: 34 \\
8: 53 \\
\end{array}$ \\
\hline$\sqrt{38}$ & $\mathrm{CP3}$ & Horiz.2 Field & al39j038 & al39refl & b139j038 & bl39refl & $\mathrm{m} / 39 \mathrm{j} 038$ & ml39refl & $\begin{array}{r}10-6 / 9: 23 \\
9: 17 \\
\end{array}$ \\
\hline J39 & CP1 & Vert. Field & a $140 \mathrm{j} 039$ & a 140refl & bl40j039 & bl40ref I & m140j039 & m140refl & $\begin{array}{r}10-6 / 10: 45 \\
11: 00 \\
\end{array}$ \\
\hline
\end{tabular}




\begin{tabular}{|c|c|c|c|c|c|c|c|c|c|}
\hline $\begin{array}{c}\text { Test } \\
\#\end{array}$ & $\begin{array}{c}\text { Test } \\
\text { Point } \\
\end{array}$ & $\begin{array}{l}\text { Measured } \\
\text { Parameter }\end{array}$ & $\begin{array}{c}\text { Low Freq Data } \\
(0.3-100 \mathrm{MHz})\end{array}$ & $\begin{array}{c}\text { Low Freq Reference } \\
(0.3-100 \mathrm{MHz})\end{array}$ & $\begin{array}{c}\text { High Freq Data } \\
(100-1000 \mathrm{MHz})\end{array}$ & $\begin{array}{c}\text { High Freq Reference } \\
(0.3-100 \mathrm{MHz})\end{array}$ & $\begin{array}{c}\text { Merged Data } \\
(0.3-1000 \mathrm{MHz}) \\
\end{array}$ & $\begin{array}{c}\text { Merged Reference } \\
(0.3-100 \mathrm{MHz}) \\
\end{array}$ & $\begin{array}{c}\text { Acquisition } \\
\text { Date/Time }\end{array}$ \\
\hline & & & Data File Name & Data File Name & Data File Name & Data File Name & Data File Name & Data File Name & \\
\hline & & & & & & & & & \\
\hline $\mathrm{J40}$ & $\mathrm{CP2}$ & Horiz.1 Field & a 140j040 & al40refl & b140j040 & b140refl & $\mathrm{m} 140 \mathrm{j} 040$ & m140refl & $\begin{array}{r}10-6 / 10: 45 \\
11: 00 \\
\end{array}$ \\
\hline J41 & $\mathrm{CP3}$ & Horiz.2 Field & a $141 \mathrm{j} 041$ & a141ref! & $\overline{b 141 j 041}$ & b14lrefl & $\mathrm{m} 141 \mathrm{j} 041$ & ml4lrefl & $\begin{array}{r}0-6 / 11: 10 \\
11: 13\end{array}$ \\
\hline $\mathrm{J} 005$ & CP5 & Wire Voltage & a 126j005 & a126refl & b126j005 & b126ref 1 & $\mathrm{m126j005}$ & mi26refl & $\begin{array}{r}10-4 / 3: 42 \\
3: 51\end{array}$ \\
\hline J026 & $\overline{\mathrm{EB} 1}$ & Field & al64j026 & Data Not Available & bl64j026 & Data Not Available & $\mathrm{ml64j026}$ & ml64ref2 & $10-11 / 7: 45$ \\
\hline $\mathrm{J027}$ & $\overline{\text { EB2 }}$ & LRU Voltage & a165j027 & Data Not Available & bl65j027 & Data Not Available & $\mathrm{m} 165 \mathrm{j} 027$ & ml65ref2 & $10-11 / 8: 27$ \\
\hline $\mathrm{J028}$ & EB3 & Cable Current & a $167 \mathrm{j028}$ & Data Not Available & b167j028 & Data Not Available & $\mathrm{ml67j028}$ & m167ref2 & $10-11 / 8: 58$ \\
\hline
\end{tabular}


Table 6-27. Data ID: Test Series K - Rhombic (Side Incident and Related Tests)

\begin{tabular}{|c|c|c|c|c|c|c|c|c|c|}
\hline $\begin{array}{c}\text { Test } \\
\#\end{array}$ & $\begin{array}{l}\text { Test } \\
\text { Point }\end{array}$ & $\begin{array}{l}\text { Measured } \\
\text { Parameter }\end{array}$ & $\begin{array}{c}\text { Low Freq Data } \\
(0.3-100 \mathrm{MHz})\end{array}$ & $\begin{array}{c}\text { Low Freq Reference } \\
(0.3-100 \mathrm{MHz})\end{array}$ & $\begin{array}{c}\text { High Freq Data } \\
(100-1000 \mathrm{MHz})\end{array}$ & $\begin{array}{c}\text { High Freq Reference } \\
(0.3-100 \mathrm{MHz})\end{array}$ & $\begin{array}{c}\text { Merged Data } \\
(0.3-1000 \mathrm{MHz})\end{array}$ & $\begin{array}{c}\text { Merged Reference } \\
(0.3-100 \mathrm{MHz})\end{array}$ & $\begin{array}{c}\text { Acquisition } \\
\text { Date/Time }\end{array}$ \\
\hline & & & Data File Name & Data File Name & Data File Name & Data File Name & Data File Name & Data File Name & \\
\hline$\overline{\mathrm{K} 1}$ & $\overline{\mathrm{CP1}}$ & Vert. Field & a $142 \mathrm{k} 001$ & a $142 \mathrm{refl}$ & b $142 \mathrm{k001}$ & b142ref1 & $\mathrm{ml42k001}$ & ml42refl & $\begin{array}{r}10-6 / 2: 28 \\
2: 46 \\
\end{array}$ \\
\hline$\overline{\mathrm{K} 2}$ & $\overline{\mathrm{CP} 2}$ & Horiz.1 Field & a149k002 & al49refl & bl49k002 & b149ref1 & $\mathrm{m149k002}$ & $\mathrm{ml49}$ refl & $\begin{array}{c}10-7 / 8: 57 \\
5: 45\end{array}$ \\
\hline$\overline{\mathrm{K} 3}$ & $\overline{\mathrm{CP} 3}$ & Horiz.2 Field & a $144 \mathrm{k} 003$ & a144refl & bl44k003 & b144ref1 & $\mathrm{m} 144 \mathrm{k} 003$ & m144refl & $\begin{array}{r}10-6 / 3: 21 \\
3: 10\end{array}$ \\
\hline$\overline{\mathrm{K} 4}$ & $\overline{\mathrm{CP} 4}$ & Wire Current & al45k004 & a145ref1 & b145k004 & bl45refl & $\mathrm{ml45k004}$ & m145refl & $\begin{array}{r}10-6 / 3: 43 \\
4: 02\end{array}$ \\
\hline$\overline{\mathrm{K} 5}$ & CP5 & Wire Voltage & a $147 \mathrm{k005}$ & a147refl & b147k005 & b147ref1 & m147k005 & $\mathrm{m} 147 \mathrm{refl}$ & $\begin{array}{r}10-7 / 7: 30 \\
7: 02\end{array}$ \\
\hline$\overline{\mathrm{K} 6}$ & EB1 & Field & a $144 \mathrm{k} 006$ & a)44ref I & b144k006 & b144ref1 & $\mathrm{m} 144 \mathrm{k} 006$ & $\mathrm{ml44refl}$ & $\begin{array}{r}10-6 / 3: 21 \\
3: 10\end{array}$ \\
\hline$\overline{\mathrm{K} 7}$ & $\overline{\mathrm{EB} 2}$ & LRU Voltage & al45k007 & al45refl & b 145k007 & bl45refl & $\mathrm{m} 145 \mathrm{k} 007$ & $\mathrm{~m} 145 \mathrm{refl}$ & $\begin{array}{r}10-6 / 3: 43 \\
4: 02\end{array}$ \\
\hline$\overline{K 8}$ & $\overline{\text { EB3 }}$ & Cable Current & a $147 \mathrm{k} 008$ & a147refl & b147k008 & b147ref 1 & m147k008 & m147refl & $\begin{array}{r}10-7 / 7: 30 \\
7: 52\end{array}$ \\
\hline$\overline{\text { K9 }}$ & $\mathrm{Cl}$ & Field & a $149 \mathrm{k009}$ & a149ref1 & b/49k009 & b/49refl & $\mathrm{ml49k009}$ & m149refl & $\begin{array}{r}10-7 / 8: 57 \\
8: 45\end{array}$ \\
\hline $\mathrm{K} 10$ & $\overline{C 2}$ & Wire Current & a151k010 & al51ref 1 & b151k010 & bl51refl & $\mathrm{m} 151 \mathrm{k} 010$ & m15lrefl & $\begin{array}{r}10-7 / 9: 37 \\
9: 56 \\
\end{array}$ \\
\hline$\overline{\mathrm{K} 11}$ & $\overline{C 3}$ & Voltage & a142kol1 & a 142refl & b142kol1 & b142ref1 & $\mathrm{m} 142 \mathrm{koll}$ & m142refl & $\begin{array}{r}10-6 / 2: 28 \\
2: 46\end{array}$ \\
\hline$\overline{K 12}$ & C4 & Field & a151k012 & al51 ref 1 & b151k012 & b151ref1 & $\mathrm{m} 151 \mathrm{k} 012$ & m15lrefl & $\begin{array}{r}10-7 / 9: 37 \\
9: 56 \\
\end{array}$ \\
\hline$\overline{K 13}$ & $\mathrm{C3}$ & Voltage & a $152 \mathrm{k} 013$ & a152ref1 & b152k013 & bls2refl & $\mathrm{m} 152 \mathrm{k} 013$ & $\mathrm{ml} 52 \mathrm{refl}$ & $\begin{array}{r}10-7 / 10: 49 \\
10: 37\end{array}$ \\
\hline$\overline{K 14}$ & C3 & Voltage & a $156 \mathrm{k} 014$ & Data Not Available & b 156k014 & Data Not Available & $\mathrm{m} 156 \mathrm{k014}$ & m156ref2 & $\begin{array}{r}10-7 / 2: 59 \\
2: 41\end{array}$ \\
\hline K15 & EB3 & Field & al53k015 & Data Not Available & b153k015 & Data Not Available & $\mathrm{m} 153 \mathrm{k} 015$ & $\mathrm{~m} 153 \mathrm{ref} 2$ & $\begin{array}{c}10-6 / 12: 53 \\
1: 21\end{array}$ \\
\hline $\mathrm{K} 23$ & CP5 & Wire Voltage & a154k023 & a154ref 1 & b154k023 & b154ref 1 & $\mathrm{m} 154 \mathrm{k} 023$ & ml54refl & $\begin{array}{r}10-7 / 1: 53 \\
1: 47 \\
\end{array}$ \\
\hline K24 & CP5 & Wire Voltage & al $55 \mathrm{k} 024$ & a 155refl & b155k024 & b155ref1 & $\mathrm{ml55k024}$ & mis5refl & $\begin{array}{r}10-7 / 2: 11 \\
2: 18\end{array}$ \\
\hline $\mathrm{K} 25$ & $\mathrm{CP} 12$ & Heater current & a152k025 & a152refl & b152k025 & bls2ref1 & $\mathrm{m} 152 \mathrm{k} 025$ & $\mathrm{~m} 152 \mathrm{refl}$ & $\begin{array}{r}10-7 / 10: 49 \\
10: 37 \\
\end{array}$ \\
\hline $\bar{K} 26$ & C3 & Voltage & a $158 \mathrm{k} 026$ & Data Not Available & b158k026 & Data Not Available & $\mathrm{m} 158 \mathrm{k} 026$ & m158ref2 & $\begin{array}{r}10-6 / 3: 31 \\
3: 46 \\
\end{array}$ \\
\hline
\end{tabular}




\begin{tabular}{|c|c|c|c|c|c|c|c|c|c|}
\hline $\begin{array}{c}\text { Test } \\
\#\end{array}$ & $\begin{array}{c}\text { Test } \\
\text { Point }\end{array}$ & $\begin{array}{l}\text { Measured } \\
\text { Parameter }\end{array}$ & $\begin{array}{c}\text { Low Freq Data } \\
(0.3-100 \mathrm{MHz})\end{array}$ & $\begin{array}{c}\text { Low Freq Reference } \\
(0.3-100 \mathrm{MHz})\end{array}$ & $\begin{array}{c}\text { High Freq Data } \\
(100-1000 \mathrm{MHz})\end{array}$ & $\begin{array}{c}\text { High Freq Reference } \\
(0.3-100 \mathrm{MHz})\end{array}$ & $\begin{array}{c}\text { Merged Data } \\
(0.3-1000 \mathrm{MHz}) \\
\end{array}$ & $\begin{array}{c}\text { Merged Reference } \\
(0.3-100 \mathrm{MHz})\end{array}$ & $\begin{array}{l}\text { Acquisition } \\
\text { Date/Time }\end{array}$ \\
\hline & & & Data File Name & Data File Name & Data File Name & Data File Name & Data File Name & Data File Name & \\
\hline & & & & & & & & & \\
\hline $\mathbf{K} 27$ & $\overline{\mathrm{CP1}}$ & Vert. Field & a162k027 & al62refl & b162k027 & bl62ref1 & $\mathrm{ml62k027}$ & m162refl & $\begin{array}{c}1010 / 10: 02 \\
9: 51\end{array}$ \\
\hline $\mathrm{K} 28$ & $\mathrm{CP2}$ & Horiz.1 Field & a162k028 & a162refl & b162k028 & bl62refl & $\mathrm{m} 162 \mathrm{k} 028$ & m162refl & $\begin{array}{c}1010 / 10: 02 \\
9: 51\end{array}$ \\
\hline K29 & $\mathrm{CP3}$ & Horiz.2 Field & a161k029 & al61refl & b161k029 & b161refl & $\mathrm{ml61k029}$ & ml61refl & $\begin{array}{c}10-10 / 9: 15 \\
9: 35\end{array}$ \\
\hline $\mathrm{K} 30$ & $\overline{\mathrm{CP} 1}$ & Vert. Field & a159k030 & a159refl & b159k030 & bl59refl & $\mathrm{m} 159 \mathrm{k} 030$ & m159refl & $\begin{array}{r}10-10 / 7: 32 \\
7: 52\end{array}$ \\
\hline $\mathrm{K} 31$ & $\overline{\mathrm{CP} 2}$ & Horiz.1 Field & a159k031 & al59ref1 & b159k031 & b159ref1 & $\mathrm{m} 159 \mathrm{k} 031$ & m159ref1 & $\begin{array}{r}10-10 / 7: 32 \\
7: 52\end{array}$ \\
\hline $\mathrm{K} 32$ & $\overline{\mathrm{CP3}}$ & Horiz.2 Field & a $160 \mathrm{k} 032$ & al60refl & b160k032 & bl60refl & $\mathrm{m} 160 \mathrm{k} 032$ & m160refl & $\begin{array}{r}1010 / 8: 10 \\
8: 05\end{array}$ \\
\hline K007 & EB2 & LRU Voltage & a 146k007 & Data Not Available & b/46k007 & Data Not Available & $\mathrm{ml46k007}$ & $\mathrm{ml46ref2}$ & $\begin{array}{r}10-6 / 4: 41 \\
4: 29\end{array}$ \\
\hline $\begin{array}{l}\text { J024 } \\
\text { brkr } \\
\text { in }\end{array}$ & $\overline{\mathrm{EB} 2}$ & LRU Voltage & a161j024 & al6lrefl & b161j024 & bl61refl & $\mathrm{ml61j024}$ & ml6lrefl & $\begin{array}{c}10-10 / 9: 15 \\
9: 35\end{array}$ \\
\hline
\end{tabular}


Table 6-28. Data ID: Test Series Z - Rhombic (System State Effects - Circuit Breakers)

\begin{tabular}{|c|c|c|c|c|c|c|c|c|}
\hline $\begin{array}{c}\text { Test } \\
\#\end{array}$ & $\begin{array}{c}\text { Test } \\
\text { Point } \\
\end{array}$ & $\begin{array}{l}\text { Measured } \\
\text { Parameter }\end{array}$ & $\begin{array}{l}\text { Low Freq Data } \\
(0.3-100 \mathrm{MHz})\end{array}$ & $\begin{array}{l}\text { Low Freq Reference } \\
(0.3-100 \mathrm{MHz})\end{array}$ & $\begin{array}{c}\text { High Freq Data } \\
(100-1000 \mathrm{MHz})\end{array}$ & $\begin{array}{c}\text { High Freq } \\
\text { Reference } \\
(0.3-100 \mathrm{MHz})\end{array}$ & $\begin{array}{c}\text { Merged Data } \\
(0.3-1000 \mathrm{MHz}) \\
\end{array}$ & $\begin{array}{l}\text { Merged Reference } \\
(0.3-100 \mathrm{MHz})\end{array}$ \\
\hline & & & Data File Name & Data File Name & Data File Name & Data File Name & Data File Name & Data File Name \\
\hline & & & & & & & & \\
\hline$\overline{Z 1}$ & $\mathrm{CPI}$ & Vert. Field & a173z001 & a173ref1 & b173z001 & b173refl & $\mathrm{m} 173 \mathrm{z} 001$ & m173ref1 \\
\hline $\mathbf{Z 2}$ & $\overline{\mathrm{CP} 2}$ & Horiz.1 Field & a173z002 & a173ref 1 & $\mathrm{~b} 173 \mathrm{z} 002$ & b173refl & $\mathrm{m} 173 \mathrm{z} 002$ & m173ref1 \\
\hline $\mathbf{Z 3}$ & CP3 & Horiz.2 Field & a 1742003 & al74refl & b1742003 & b174ref 1 & $\mathrm{~m} 174 \mathrm{z} 003$ & m174refl \\
\hline$\overline{\mathbf{Z A}}$ & CP4 & Wire Current & a1772004 & a177ref1 & b177z004 & b177refl & $\mathrm{m} 177 \mathrm{z} 004$ & m177ref I \\
\hline$\overline{\mathrm{ZS}}$ & CP5 & Wire Voltage & a $176 z 005$ & a176ref1 & b176z005 & b176ref1 & $\mathrm{m} 176 \mathrm{z} 005$ & m176refl \\
\hline$\overline{26}$ & $\mathrm{CP} 12$ & Heater current & a178z006 & a178ref 1 & b178z006 & b178ref 1 & $\mathrm{~m} 178 \mathrm{z} 006$ & m178ref I \\
\hline$\overline{\mathrm{Z7}}$ & EB 1 & Field & a1742007 & a174ref1 & b174z007 & bl74refl & $\mathrm{m} 174 \mathrm{z} 007$ & m174ref I \\
\hline$\overline{\mathbf{Z 8}}$ & $\mathrm{Cl}$ & Field & a 1792008 & a179refl & b179z008 & b179ref1 & $\mathrm{m} 179 \mathrm{z} 008$ & m179refl \\
\hline $\mathbf{Z 9}$ & $\mathrm{C} 2$ & Wire Current & a 180z,009 & al80ref1 & b180z009 & b180ref1 & $\mathrm{m} 180 \mathrm{z} 009$ & mi80refl \\
\hline $\mathrm{Z10}$ & C3 & Voltage & a $178 \mathrm{z} 010$ & a178ref1 & b178z010 & b178refl & $\mathrm{m} 178 \mathrm{z} 010$ & m178refl \\
\hline $\mathrm{Z11}$ & CP5 & Wire Voltage & a 1822011 & a182refl & b182z011 & b182refl & $\mathrm{m} 1822011$ & m182refl \\
\hline $\mathrm{Z12}$ & CP5 & Wire Voltage & a 1832012 & a183refl & b183z012 & bl83refl & $\mathrm{m} 183 \mathrm{z} 012$ & $\mathrm{mi}$ 183refl \\
\hline $\mathrm{Z13}$ & C3 & Voltage & a 1812013 & Data Not Available & b1812013 & Data Not Available & $\mathrm{m} 1812013$ & m181ref2 \\
\hline$\overline{\mathrm{Z} 14}$ & $\mathrm{CP2}$ & Horiz.1 Field & a 1842014 & al84ref1 & b1842014 & bl 84refl & $\mathrm{m} 184 \mathrm{z} 014$ & m184refl \\
\hline
\end{tabular}


Table 6-29. Data ID: Horizontal Polarization

\begin{tabular}{|c|c|c|c|c|}
\hline $\begin{array}{c}\text { Test } \\
\#\end{array}$ & $\begin{array}{c}\text { Test } \\
\text { Point }\end{array}$ & $\begin{array}{c}\text { Measured } \\
\text { Parameter }\end{array}$ & $\begin{array}{c}\text { Low Freq Data } \\
(0.3-100 \mathrm{MHz})\end{array}$ & $\begin{array}{c}\text { Low Freq Reference } \\
(0.3-100 \mathrm{MHz})\end{array}$ \\
\hline & & & Data File Name & Data File Name \\
\hline HP1 & CP1 & Vert. Field & n010hp01 & n010ref1 \\
\hline HP2 & CP2 & Horiz.1 Field & n010hp02 & n010ref1 \\
\hline HP3 & CP3 & Horiz.2 Field & n018hp03 & n018refl \\
\hline HP4 & CP4 & Wire Current & n017hp04 & n017refl \\
\hline HP5 & CP5 & Wire Voltage & n019hp05 & n019ref 1 \\
\hline HP6 & EB1 & Field & n018hp06 & n018ref1 \\
\hline HP7 & EB2 & LRU Voltage & n017hp07 & n017ref1 \\
\hline HP8 & EB3 & Cable Current & n019hp08 & n019ref1 \\
\hline HP9 & C1 & Field & n020hp09 & n020ref1 \\
\hline HP10 & C2 & Wire Current & n020hp10 & n020ref1 \\
\hline HP11 & C3 & Voltage & n020hp11 & n020ref1 \\
\hline HP12 & C4 & Field & n021 hp12 & n021 ref1 \\
\hline HP13 & $\begin{array}{c}\text { Weak } \\
\text { Signal } \\
\text { Line }\end{array}$ & Wire Current & n022hp13 & n022ref1 \\
\hline HP14 & $\begin{array}{c}\text { Strong } \\
\text { Signal } \\
\text { Line }\end{array}$ & $\begin{array}{c}\text { Voltage } \\
\text { Long Wire }\end{array}$ & n022hp14 & n022refl \\
\hline
\end{tabular}

Table 6-30. Data ID: Test Series WF - Window Shielded/Unshielded

\begin{tabular}{|c|c|c|c|c|c|c|c|c|c|}
\hline $\begin{array}{c}\text { Test } \\
\#\end{array}$ & $\begin{array}{l}\text { Test } \\
\text { Point }\end{array}$ & $\begin{array}{l}\text { Measured } \\
\text { Parameter }\end{array}$ & $\begin{array}{c}\text { Low Freq Data } \\
(0.3-100 \mathrm{MHz})\end{array}$ & $\begin{array}{c}\text { Low Freq Reference } \\
(0.3-100 \mathrm{MHz})\end{array}$ & $\begin{array}{c}\text { High Freq Data } \\
(100-1000 \mathrm{MHz})\end{array}$ & $\begin{array}{c}\text { High Freq Reference } \\
(0.3-100 \mathrm{MHz})\end{array}$ & $\begin{array}{c}\text { Merged Data } \\
(0.3-1000 \mathrm{MHz})\end{array}$ & $\begin{array}{c}\text { Merged Reference } \\
(0.3-100 \mathrm{MHz})\end{array}$ & $\begin{array}{l}\text { Acquisition } \\
\text { Date/Time }\end{array}$ \\
\hline & & & Data File Name & Data File Name & Data File Name & Data File Name & Data File Name & Data File Name & \\
\hline WF1 & $\mathrm{CP1}$ & Vert. Field & al68wf01 & al68ref1 & bl68wf01 & b168ref 1 & m168wf01 & m168ref1 & $1011 / 12: 57$ \\
\hline$\overline{W F 2}$ & $\overline{\mathrm{CP} 2}$ & Horiz.I Field & al $68 w f 02$ & a168refl & bl68wf02 & bl68refl & $\mathrm{m168wf02}$ & ml68refl & $\begin{array}{c}1011 / 12: 57 \\
12: 33\end{array}$ \\
\hline WF3 & $\overline{\mathrm{CP} 3}$ & Horiz. 2 Field & a) $69 w$ f03 & al69ref1 & bl69wf03 & b169refl & $m 169 w f 03$ & ml69refl & $\begin{array}{c}10-11 / 1: 19 \\
1: 39\end{array}$ \\
\hline WF4 & $\mathrm{CP4}$ & Wire Current & a170wf04 & al70refl & b170wf04 & bl70refl & $\mathrm{ml70wf04}$ & ml70refl & $\begin{array}{c}10-11 / 1: 59 \\
1: 52\end{array}$ \\
\hline WF5 & $\overline{\mathrm{CP5}}$ & Wire Voltage & a171wf05 & al71ref1 & b171wf05 & b17lrefl & $\mathrm{m} 171 \mathrm{wf05}$ & m171refl & $\begin{array}{c}10-11 / 2: 07 \\
2: 15\end{array}$ \\
\hline WF6 & EB3 & Cable Current & al69wf06 & al69ref 1 & b169wf06 & bl69refl & $\mathrm{ml69wf06}$ & ml69refl & $\begin{array}{c}10-11 / 1: 19 \\
1: 39\end{array}$ \\
\hline$\overline{W F 7}$ & $\mathrm{CP} 12$ & Heater current & al72wf07 & a172refl & b $172 w f 07$ & bl72refl & $\mathrm{ml72wf07}$ & m172refl & $\begin{array}{c}10-11 / 2: 38 \\
2: 31\end{array}$ \\
\hline
\end{tabular}




\subsubsection{Interpreting a Data File Header}

The data files, whose naming and subject have been described, contain the alpha numeric data specific to a given test. Each data file has a header which contains details of the test. Such a sample header is shown in Table 6-31 for test FMJ over the frequency range $0.3<\mathrm{f}<1000 \mathrm{MHz}$. The leading " $\mathrm{m}$ " refers to a merged file.

\section{Table 6-31. A Typical Header for a Data File}

M0997FMJ.COF TS :757T CS:A/B FC : LR AM:AMP6 AP:250 MT : EX NC: 12 ED :0 IN : N

D : 12SEP94 T : 1447 PI : ACD4 PO : 000 LI : NF50 LS : 18 E1 : B644 E2 : CO43 E3 : E4 : L1 : L2

OR : $000 \mathrm{C}: \mathrm{RM}$ BW : $10 \mathrm{Q}: 60 \mathrm{AV}: 1 \mathrm{PW}: 4.5 \mathrm{~W}: 0 \mathrm{M}: .03 \mathrm{CU}:$ YYYYYXX

ELECTRIC FIELD MEASUREMENT @ X=40, $Y=5, Z=8$.

FREQ

1370

C

(P, R, Error Log and Drive Name not shown in this example)

The meaning of the abbreviations defining the fields in this header are provided in Table 6-32.

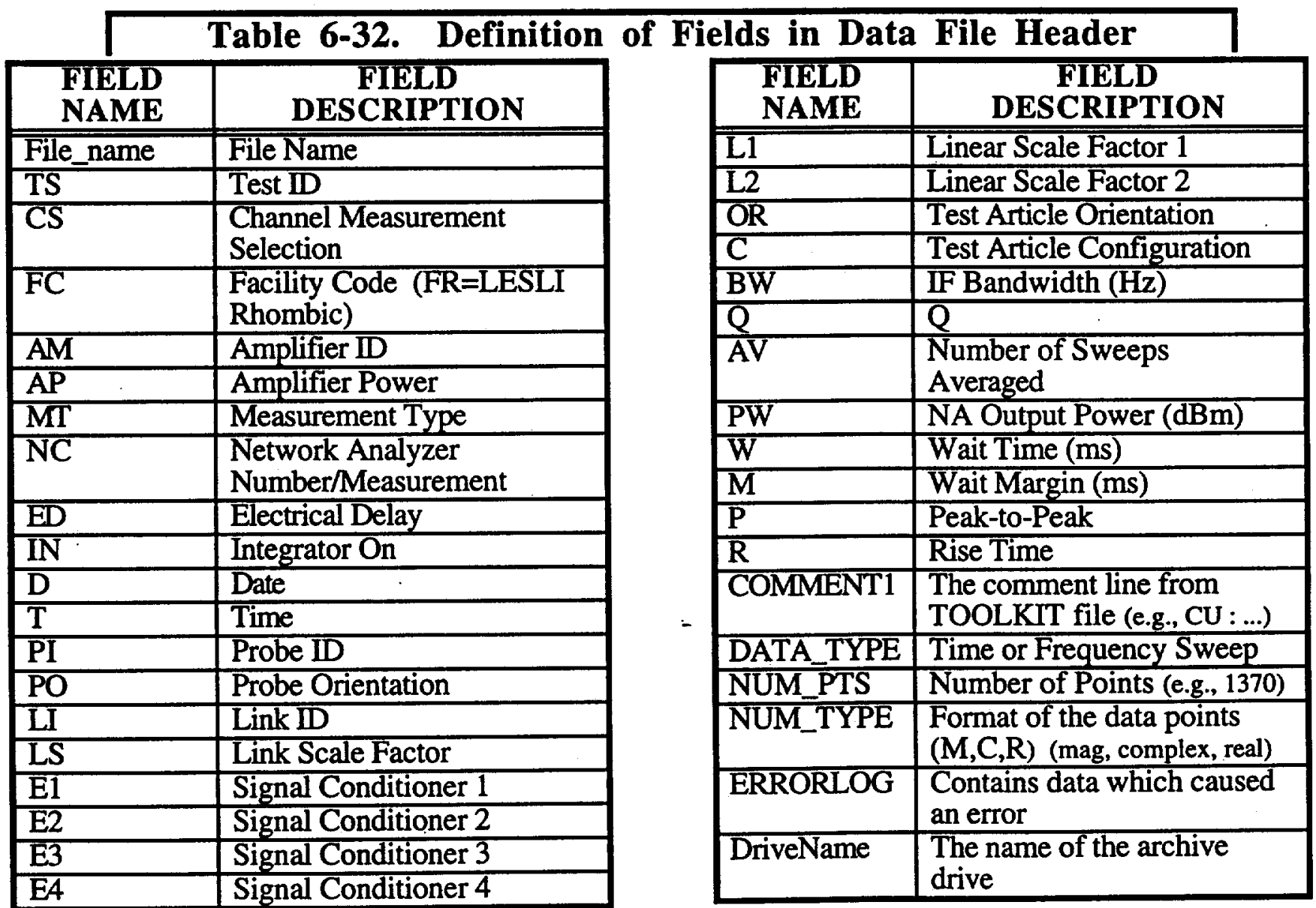

Note that for swept frequency measurements the frequency sample spacing may not be constant. Rather, the spacing is held constant only over the intervals between stopbands (frequency exclusion bands imposed by frequency allocation authorities). The scheme, which has little impact on this measurement program, is described in Randall (1993). 


\subsection{Pre- and Post-Aircraft Test Activities}

Prior to and after the tests involving use of the B-757, a number of additional experimental sequences were necessary. These involved preparation for the tests and calibrations.

\subsubsection{Calibration Data for Sensor Systems}

The cables and sensors were experimentally characterized, where possible. This was achieved using standard techniques, with some activities carried out at NASA LaRC and others at the Phillips Laboratory.

The AD-60 ground plane electric field probe, also referred to as a d-dot probe, was not experimentally characterized prior to the aircraft tests. Further information on the $A D-60$ calibration and its effects on the data found in the data files is provided in Section 6.3.1.1.

The data file names for the calibration data are listed in Table 6-33. File nistad60.cal is described in Section 6.3.1.1.

\begin{tabular}{|c|c|c|c|}
\hline \multicolumn{4}{|c|}{ TABLE 6-33. FILE NAMES FOR CALIBRATION DATA } \\
\hline File Name & Subject & File Name & Subject \\
\hline acd4.cal & Balanced E Field (ACD-4) & nf51.cal & NanoFast F-O Xmtr-Rcvr \\
\hline ad60.cal & Ground Plane E Field (AD-60) & nf52.cal & NanoFast F-O Xmtr-Rcvr \\
\hline aprb.cal & Direct Voltage Measurement & nf53.cal & NanoFast F-O Xmtr-Rcvr \\
\hline b643.cal & Balun & nf60.cal & NanoFast F-O Xmtr-Rcvr \\
\hline b644.cal & Balun & p132.cal & Prodyn Current Probe \\
\hline b657.cal & Balun & p133.cal & Prodyn Current Probe \\
\hline c027.cal & Cable & p134.cal & Prodyn Current Probe \\
\hline c043.cal & Cable & p135.cal & Prodyn Current Probe \\
\hline c044.cal & Cable & ps68.cal & Power Splitter \\
\hline c045.cal & Cable & t1c1.cal & Cable \\
\hline c046.cal & Cable & tlc2.cal & Cable \\
\hline c047.cal & Cable & tlc3.cal & Cable \\
\hline c050.cal & Cable & tlc4.cal & Cable \\
\hline c071.cal & Cable & t2c1.cal & Cable \\
\hline mgl6.cal & Balanced H Field (MGL-6) & t2c2.cal & Cable \\
\hline $\mathrm{mgl7.cal}$ & Ground Plane H Field (MGL-7) & t2c3.cal & Cable \\
\hline nf20.cal & NanoFast F-O Xmtr-Rcvr & t2c4.cal & Cable \\
\hline nf21.cal & NanoFast F-O Xmtr-Rcvr & t3c1.cal & Cable \\
\hline nf22.cal & NanoFast F-O Xmtr-Rcvr & t3c2.cal & Cable \\
\hline nf23.cal & NanoFast F-O Xmtr-Rcvr & t3c3.cal & Cable \\
\hline nf40.cal & NanoFast F-O Xmtr-Rcvr & t3c4.cal & Cable \\
\hline nf50.cal & NanoFast F-O Xmtr-Rcvr & nistad60.cal & $\mathrm{AD}-60$ refined \\
\hline
\end{tabular}




\subsubsection{Calibration of the AD-60 Electric Field (D-Dot) Probe}

During the planning stages for this program, it was intended that the terminal voltages at the AD-60 probes would be recorded and that the modeling activities would be expected to provide the probe terminal voltages. This is a challenge for modellers due to the scale of the probes relative to the rest of the aircraft. Given this difficulty and the value of having field variable information directly available, it was later decided to use probe transfer function (calibration) data to extract the field variable from the acquired data. The original calibration data were constructed by the data acquisition team using information provided by the manufacturer. An analytic function for the probe response, which involves a small number of parameters and is well established in the EMP community, was used to generate the calibration data as a function of frequency. These calibration data, presented in the calibration files as ad60.cal, were used throughout in correcting the data acquired during the tests. Well after the tests were executed and the raw data had been corrected and processed to provide the variables of interest prior to dissemination, a more accurate calibration became available.

NASA LaRC commissioned NIST to prepare calibration data for the AD-60. The intent was to obtain calibration data for the probe in its operating environment especially as the AD-60, a probe for vertical electric field measurement over an infinite ground plane, was operating over a ground plane that was rather limited in extent. The details of this calibration are provided in Johnk and Ondrejka (1996). During these calibration tests the probe, in various vertically oriented configurations over a ground plane, was illuminated by a vertically polarized plane (or very nearly plane) wave of known strength and the output voltage recorded as a function of frequency. The test system was a large conical antenna driven against a very large ground plane on which was mounted the probe being calibrated. Five AD-60 probes were used during the aircraft tests and each probe was identified by its serial number. During the calibration tests a different probe was used for each configuration, and the probe identification and its particular configuration are provided in Table 6-34.

\begin{tabular}{|c|l|}
\hline \multicolumn{2}{|c|}{ Table 6-34. AD-60 Serial Numbers and Configurations for Calibration } \\
\hline \hline Serial & \\
Number & \\
\hline 33 & D-dot mounted on a metal surface simulating top of EME rack in cabin \\
34 & (34n) D-dot mounted on the E Bay fixture with the narrow dimension facing the incident wave \\
34 & (34w) D-dot mounted on the E Bay fixture with the wide dimension facing the incident wave \\
35 & D-dot (vertical orientation) mounted on ATOPS box with pedestal \\
36 & D-dot measured on ground plane \\
37 & D-dot measured on ground plane \\
\hline
\end{tabular}

The entire set of probes was calibrated over the frequency range from $50 \mathrm{MHz}$ to $6 \mathrm{GHz}$. In addition, Probes $33,34,36$ and 37 were calibrated from $10 \mathrm{MHz}$ to $100 \mathrm{MHz}$ in order to extend the low frequency coverage. The ratio of output voltage at the probe terminals to incident electric field strength was recorded and presented as $20 \log$ (output voltage/incident field) which is, in effect, the transfer function (or an effective height) in $\mathrm{dB}$ relative to a meter.

Probes 33, 34, and 35 were calibrated in configurations similar, not identical, to the configurations in the aircraft. Probe number 33 was placed on a metal surface identical in size to the upper surface of the EME rack (see Figure 4-8) but the test configuration had this surface only about 3 inches above the ground plane. The entire rack was not included. Probe number 34 was calibrated in two configurations. The platform on which the AD-60 was mounted is shown in Figure 4-6 and the calibration tests were run with the platform in two different orientations relative to the incident plane wave. The vertical portions of the platform, approximately 4 inches in dimension, were solid in the narrow dimension and open in the wide dimension so that the platform looked like a foursided box mounted on a ground plane. In $34 \mathrm{n}$, the narrow portion of the platform faced the wave 
and in $34 w$, the wide portion of the platform faced the wave. Probe number 35 , the vertically oriented D-dot on the ATOPS box, was tested as configured in the aircraft. Probes 36 and 37 , which were used in the aircraft for the horizontally polarized field measurements on the ATOPS box, could not be calibrated in that configuration since horizontally polarized fields were not available. Rather, these probes were tested over the large ground plane of the test fixture used by NIST and are representative calibrations for the AD-60 probe in its intended configuration.

Figure 6-1 is a plot of the magnitude of the transfer function for each of the probes referred to as the response in $\mathrm{dB}$ as a function of frequency from $50 \mathrm{MHz}$ to $1 \mathrm{GHz}$. Also included in the figure is a curve labeled $\mathrm{AD}-60$ which represents the calibration used in correcting the raw data acquired in the tests to provide the corrected data in the files described in this report. The curves for probes 36 and 37 are similar to the AD-60 data that was used for correction (file ad60.cal) below $200 \mathrm{MHz}$ but differ by as much as $3 \mathrm{~dB}$ beyond $200 \mathrm{MHz}$. This suggests that the model and its parameters used for construction of the calibration curve may be suspect for frequencies above $200 \mathrm{MHz}$. The other differences observed in the figure may also be cause for concern as they indicate discrepancies as large as $5 \mathrm{~dB}$ above $200 \mathrm{MHz}$. Particular attention must be paid to the response of the probe on the ATOPS box below $100 \mathrm{MHz}$ where apparent standing waves are causing a behavior which deviates substantially from the expected behavior which is directly proportional to frequency.

The data for the AD- 60 probes in the various configurations represented in Table 6-34 and Figure 6-1 can be found in the set of calibration files and are denoted as nistad60.cal. The data for the various measurements using the $A D-60$ probe that are presented in this report and are provided in the numerous files can be refined by using the new calibration data. This is can be done by taking each data file for the desired variables which had used the original $A D-60$ data for correction purposes, extracting the original $A D-60$ calibration and then introducing the new $A D-60$ calibration information. This requires taking each file which involves measurements using the AD-60s, dividing by the ad60.cal file (the original AD- 60 calibration) and multiplying by the portion of the nistad60.cal file that relates to the particular probe involved in the measurement of interest. The operations must be performed for each frequency and should, as done with the original calibration data, be performed using complex arithmetic.

\subsubsection{Dipole Test Preparation}

Dipole antenna testing, which was introduced to permit modelers to explicitly include the radiating structure that created the electromagnetic environment in their computer models, was executed in the vicinity of three frequencies, namely, 25.85, 172, and $430 \mathrm{MHz}$. Ultimately, dipole testing in these frequency ranges was carried out both with and without the B-757. A wood support structure was assembled for the dipoles and is shown in Figures 6-2 and 6-3. The former is a drawing of the stand to scale and the latter a picture of the stand with a dipole mounted vertically and emanating from the balun on the square plate. Unfortunately, the dipole is not readily visible in this photograph but can be seen in Figure 6-4. The details of the structure and the mounting of the dipoles is contained in Appendix B.

The 25.85 MHz dipole was "tuned" using a process described in Appendix B where the dipole length was trimmed in an attempt to achieve a reasonably flat response, as indicated by a principal field component magnitude at broadside, across the 23 to $28 \mathrm{MHz}$ range. The other dipoles, for 172 and $430 \mathrm{MHz}$, had been trimmed prior to shipment to the Phillips Lab.

In each case, the balanced dipole was fed through a broadband, high power balun (Sabre Communications Corp.) driven by $50 \mathrm{ohm}$ coaxial cable. The trimmed lengths of the dipoles and the model numbers of the associated baluns for each frequency band were:

$25 \mathrm{MHz}$

$172 \mathrm{MHz}$

$430 \mathrm{MHz}$
5.817 meters

0.872 meters

0.349 meters
HDC $02 \mathrm{~A}$

HDC $12 \mathrm{~A}$

HDC $13 \mathrm{~A}$ 


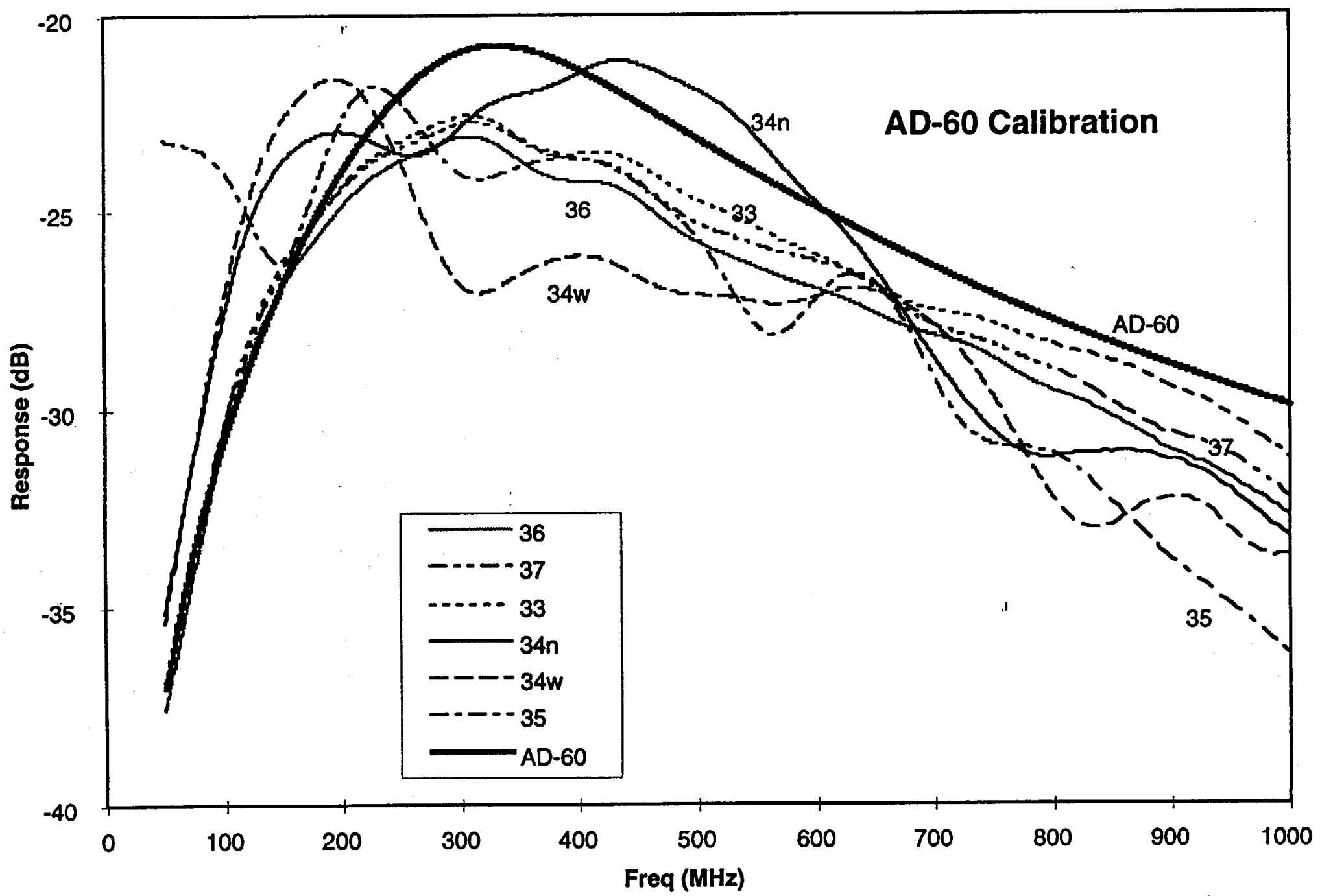

Figure 6-1. Calibration curves (magnitude only) for various AD-60 D-dot probes 


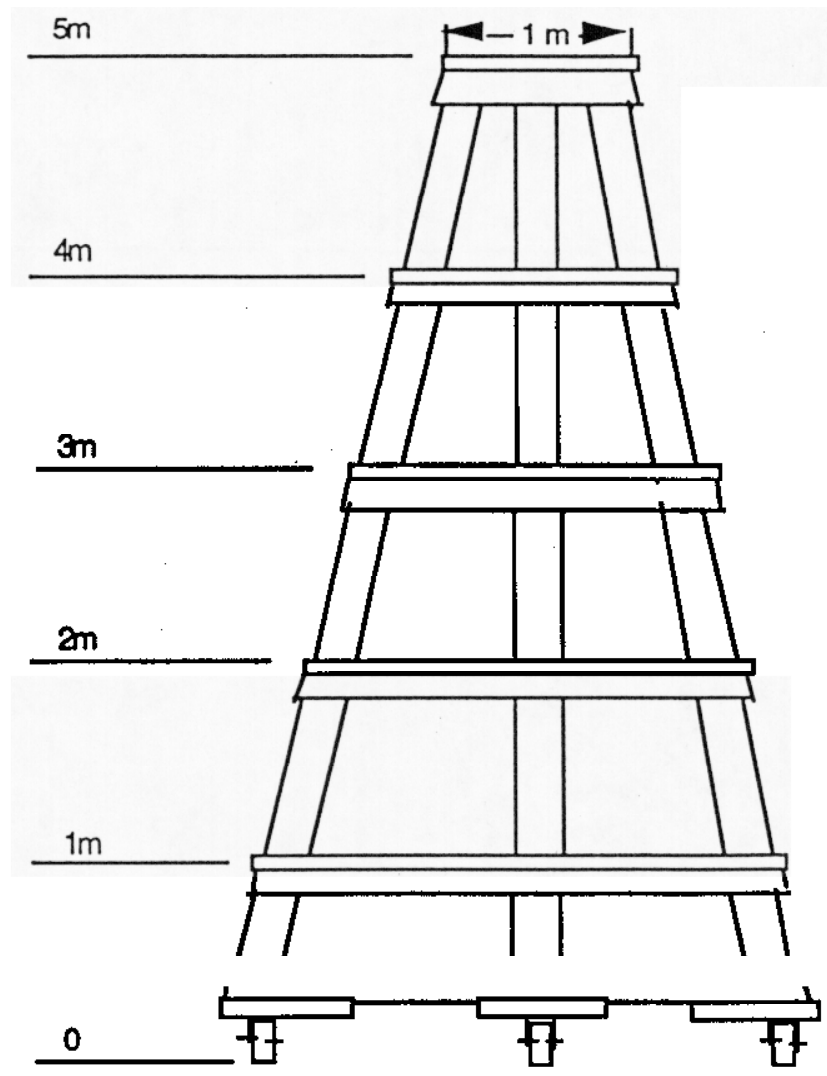

Figure 6-2. Wooden stand for dipole testing

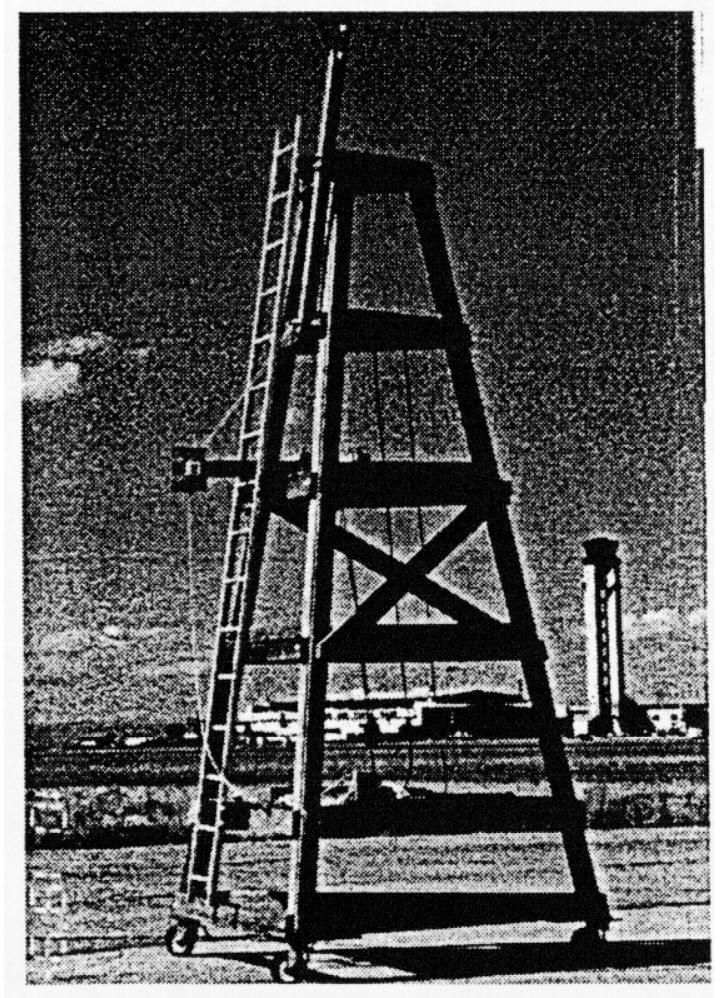

Figure 6-3. $172 \mathrm{MHz}$ dipole mounted on stand 
The $172 \mathrm{MHz}$ dipole with its associated balun mounted on its support structure is shown in Figure 6-4. Short sections of Plexiglas tubing (visible in the photograph) were used to support the dipole wires near the feedpoint.

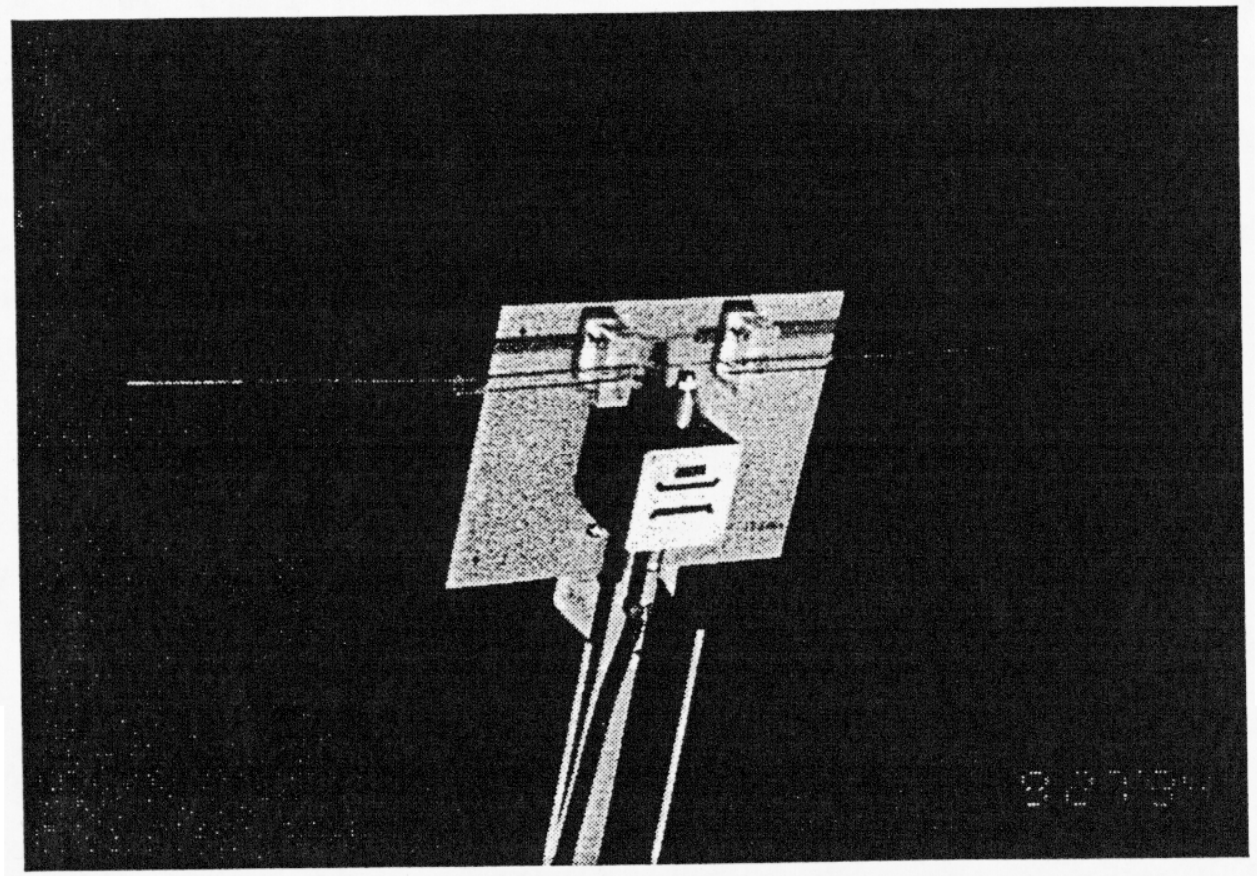

Figure 6-4. The $172 \mathrm{MHz}$ dipole with balun and mount

\subsubsection{Dipole Field Mapping}

Prior to performing the tests with the dipoles in the presence of the aircraft, a number of measurements, or field maps, were made of the dipole radiated fields. The data were collected on a number of days both prior to and after the airplane tests. These provide modelers with additional data for comparison and experimenters with an indication of proper performance. The coordinate system for the field mapping is shown in Figure 6-5. The orientation of this coordinate system is similar to that for the rhombic radiator in that the feedpoint is in the $\mathrm{x}=0$ plane and the $\mathrm{y}$ axis is vertical. The LESLI pad forms the $x-z$ plane. The height of the dipole feedpoint was 3 meters except for vertical polarization in the $25 \mathrm{MHz}$ range. For that case, the height of the feedpoint was 4 meters. In all cases, the dipole was located approximately 1.5 meters from the platform at its feedpoint. Since it was impossible, given the resources available, to measure either the input or radiated power for the dipoles, the fields were measured in all cases at two points, one of which was fixed for all cases (the reference sensor) at $(-2.5,3,0)$. Thus, the reference field was to provide a fixed datum for the mapping exercise and thus reduce the uncertainty due to the lack of accurate radiated power measurements. The reference would serve as a scaling factor for comparisons. The fields were measured at a number of points (listed in Table 6-35).

Table 6-36 summarizes the details of the dipole field mapping. It provides information on the map point, the dipole polarization, mapping point location, acquisition date, file names for the field components measured at that particular point and the references in each case. In the comments column one finds information regarding the probe used for measurement of the desired field (ACD4 for electric fields and MGL-6 for magnetic fields). In all cases, the reference field was measured with a MGL- 6 probe. The file names for the calibration data for the various probes are found in Table 6-33. The file names for magnetic fields are in italics. 


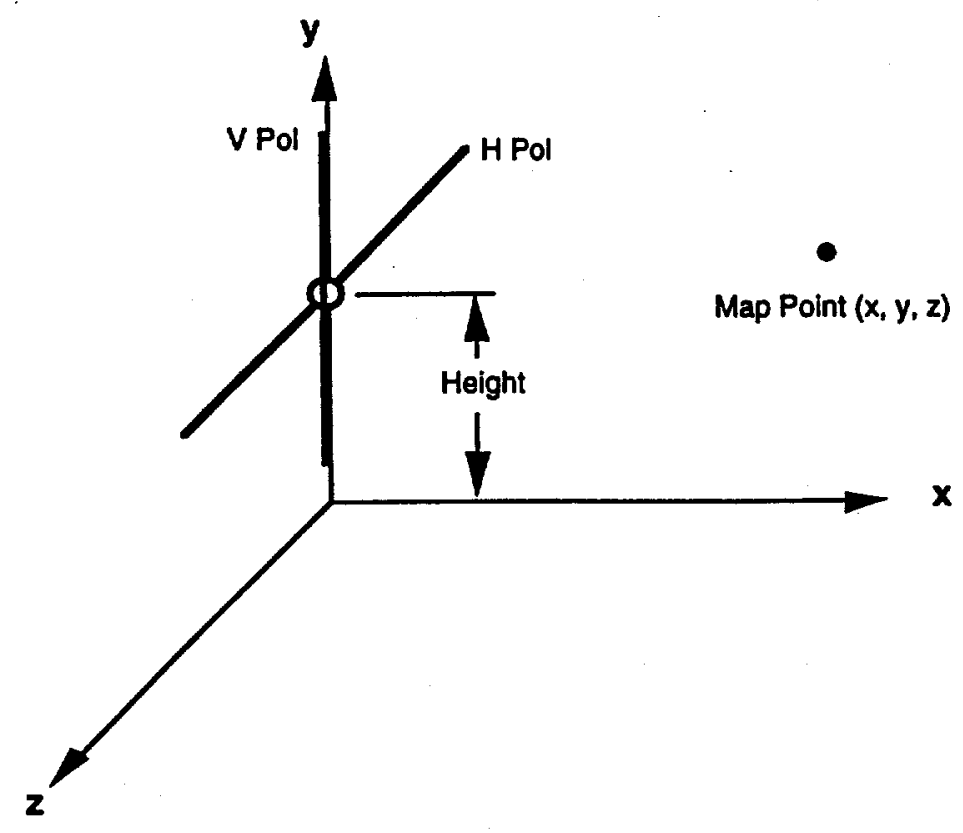

Figure 6-5. Dipole field mapping coordinate system

It should be noted that the reference field for the dipole measurements is always the dominant magnetic field of a dipole, i.e., it is the component of the magnetic field which, in the far zone, would be orthogonal to the dipole. In the tables and in the data headers it is referred to as $H_{z}$ but in all cases it should be interpreted as the component orthogonal to the dipole at the point at which the reference is measured. This comment holds for the dipole field mapping and the dipole tests that included the aircraft.

\begin{tabular}{|c|c|c|c|c|c|}
\hline \multicolumn{6}{|c|}{ Table 6-35. Dipole Field Map } \\
\hline $\begin{array}{c}\text { Freq } \\
\text { (MHz) }\end{array}$ & Pol & $\begin{array}{c}\text { X } \\
(\mathrm{m})\end{array}$ & $\begin{array}{c}\text { y } \\
(\mathrm{m})\end{array}$ & $\begin{array}{c}\text { Z } \\
(\mathrm{m})\end{array}$ & Fields \\
\hline 25 & V & 2 & 4 & 0 & E\&H \\
\hline 25 & V & 8 & 4 & 0 & E \\
\hline 25 & V & 8 & 2 & 0 & E \\
\hline 25 & H & 2 & 3 & 0 & E\&H \\
\hline 25 & H & 8 & 3 & 0 & E \\
\hline 25 & H & 8 & 2 & 0 & E \\
\hline 172 & V & 2 & 3 & 0 & E\&H \\
\hline 172 & V & 8 & 3 & 0 & E \\
\hline 172 & V & 8 & 2 & 0 & E \\
\hline 172 & H & 2 & 3 & 0 & E\&H \\
\hline 172 & H & 8 & 3 & 0 & E \\
\hline 172 & H & 8 & 2 & 0 & E \\
\hline 430 & V & 2 & 3 & 0 & E\&H \\
\hline 430 & V & 8 & 3 & 0 & E \\
\hline 430 & V & 8 & 2 & 0 & E \\
\hline 430 & H & 2 & 3 & 0 & E\&H \\
\hline 430 & H & 8 & 3 & 0 & E \\
\hline 430 & H & 8 & 2 & 0 & E \\
\hline 25 & H & 8 & 3 & 6 & E \\
\hline 25 & H & 8 & 3 & -6 & E \\
\hline 172 & H & 8 & 3 & 6 & E \\
\hline 172 & H & 8 & 3 & -6 & E \\
\hline 430 & H & 8 & 3 & 6 & E \\
\hline 430 & H & 8 & 3 & -6 & E \\
\hline
\end{tabular}


Table 6-36. Data ID: Dipole Field Mapping

\begin{tabular}{|c|c|c|c|c|c|c|c|c|c|c|c|c|c|}
\hline $\begin{array}{c}\text { MAP } \\
\text { POINT }\end{array}$ & $\begin{array}{c}\text { FREQ } \\
\text { MHz }\end{array}$ & POL & $\overline{\mathbf{X}}$ & $\mathbf{Y}$ & $\mathbf{Z}$ & $\begin{array}{c}\text { COMMENTS } \\
\text { (Probe ID and } \\
\text { Measured Field) }\end{array}$ & DATE & $\begin{array}{c}\mathbf{E}_{\mathrm{x}} \\
\mathbf{H}_{\mathbf{x}} \\
\text { file name }\end{array}$ & $\begin{array}{l}\text { Ref } \mathrm{H}_{2} \\
\text { file name }\end{array}$ & $\underset{\text { file name }}{\mathbf{H}_{\mathbf{y}}}$ & $\begin{array}{r}\text { Ref } \mathbf{H}_{2} \\
\text { file name }\end{array}$ & $\begin{array}{c}\mathbf{E}_{2} \\
\mathbf{H}_{2} \\
\text { file name }\end{array}$ & $\begin{array}{l}\text { Ref } \mathbf{H}_{2} \\
\text { file name }\end{array}$ \\
\hline DFMA & $\overline{25}$ & $\bar{V}$ & 2 & 4 & 0 & $\begin{array}{l}\text { ACD-4; E FIELD } \\
\text { MGL-6; H FIELD }\end{array}$ & $9 / 21$ & $\begin{array}{l}\text { c038dfma } \\
\text { c039dfma }\end{array}$ & $\begin{array}{l}\text { c038dref } \\
\text { c039dref }\end{array}$ & $\begin{array}{l}\text { c037dfma } \\
\text { c040dfma }\end{array}$ & $\begin{array}{l}\text { c037dref } \\
\text { c040dref }\end{array}$ & $\begin{array}{l}\text { c036dfma } \\
\text { c04ldfma }\end{array}$ & $\begin{array}{l}\text { c036dref } \\
\text { c04Idref }\end{array}$ \\
\hline$\overline{D F M B}$ & 25 & $\bar{v}$ & 8 & $\overline{4}$ & 0 & ACD-4; E FIELD & $9 / 21$ & c029dfmb & c029dref & $\mathrm{c031 \textrm {dfmb }}$ & c03Idref & c034dfmb & c034dref \\
\hline DFMC & 25 & $\overline{\mathrm{V}}$ & 8 & 2 & 0 & ACD-4; E FIELD & $9 / 21$ & $\mathrm{c030 \textrm {dfmc }}$ & c030ref & & & & \\
\hline DFMD & 25 & H & 2 & 3 & 0 & $\begin{array}{l}\text { ACD-4; E FIELD } \\
\text { MGL-6; H FIELD }\end{array}$ & $9 / 20$ & $\begin{array}{l}\text { c007dfmo } \\
\text { c008dfind }\end{array}$ & $\begin{array}{l}\text { c007dref } \\
\text { c008dref }\end{array}$ & $\begin{array}{l}\text { c005dfmd } \\
\text { c009dffind }\end{array}$ & $\begin{array}{l}\text { c005dref } \\
\text { c009dref }\end{array}$ & $\begin{array}{l}\text { c003dfmd } \\
\text { c010dfmd }\end{array}$ & $\begin{array}{l}\text { c003dref } \\
\text { co10dref }\end{array}$ \\
\hline$\overline{D F M E}$ & 25 & $\bar{H}$ & 8 & 3 & 0 & ACD-4; E FIELD & $9 / 20$ & & & c014dfme & c014ref & & \\
\hline DFMF & 25 & $\mathrm{H}$ & 8 & 2 & 0 & ACD-4;E FIELD & $9 / 20$ & c012dfmf & c012dref & $\mathrm{c018 \textrm {dfmf }}$ & col8dref & c020dfmf & c020dref \\
\hline DFMG & 172 & V & 2 & 3 & 0 & $\begin{array}{l}\text { ACD-4; E FIELD } \\
\text { MGL-6; H FIELD }\end{array}$ & $10 / 21$ & $\begin{array}{l}\text { e062dfmg } \\
\text { e063dfmg }\end{array}$ & $\begin{array}{l}\text { e062dref } \\
\text { e063dref }\end{array}$ & $\begin{array}{l}\text { e060dfmg } \\
\text { e065dfmg }\end{array}$ & $\begin{array}{l}\text { e060dref } \\
\text { e065dref }\end{array}$ & $\begin{array}{l}\text { c058dfmg } \\
\text { e066dfmg }\end{array}$ & $\begin{array}{l}\text { e058dref } \\
\text { e066dref }\end{array}$ \\
\hline DFMH & 172 & $\overline{\mathrm{v}}$ & 8 & 3 & 0 & ACD-4; E FIELD & $10 / 21$ & e054dfmh & e054dref & e055dfmh & e055dref & e057dfmh & e057dref \\
\hline DFMJ & 172 & H & 2 & 3 & 0 & $\begin{array}{l}\text { ACD-4; E FIELD } \\
\text { MGL-6; } H \text { FIELD }\end{array}$ & $10 / 20$ & $\begin{array}{l}\mathrm{e} 038 \mathrm{dfmj} \\
\mathrm{e032dfmj}\end{array}$ & $\begin{array}{l}\text { e038dref } \\
\text { e032dref }\end{array}$ & $\begin{array}{l}\text { e037dfmj } \\
\text { e033dfmj }\end{array}$ & $\begin{array}{l}\text { e037dref } \\
\text { e033dref }\end{array}$ & $\begin{array}{l}\text { c035dfmj } \\
\text { c034dfmi }\end{array}$ & $\begin{array}{l}\text { e035dref } \\
\text { e034dref }\end{array}$ \\
\hline DFMK & 172 & $\mathrm{H}$ & 8 & 3 & 0 & ACD-4; E FIELD & $10 / 21$ & e051dfmk & e0sidref & e050dfmk & e050dref & e049dfmk & e049dref \\
\hline DFML & 430 & $\mathrm{~V}$ & 2 & 3 & 0 & $\begin{array}{l}\text { ACD-4; E FIELD } \\
\text { MGL-6; H FIELD } \\
\end{array}$ & $10 / 20$ & $\begin{array}{l}\text { g029dfml } \\
\text { g034dfml }\end{array}$ & $\begin{array}{l}\text { g029dref } \\
\text { g034dref }\end{array}$ & $\begin{array}{l}\text { g031dfml } \\
\text { g035dfml }\end{array}$ & $\begin{array}{l}\text { g031dref } \\
\text { g035dref }\end{array}$ & $\begin{array}{l}\text { g033dfml } \\
\text { g036dfml }\end{array}$ & $\begin{array}{l}\text { g033dref } \\
\text { go36dref }\end{array}$ \\
\hline DFMM & 430 & $\mathrm{~V}$ & 8 & 3 & 0 & ACD-4;E FIELD & $10 / 20$ & $\mathrm{~g} 038 \mathrm{dfmm}$ & g038dref & g039dfmm & g039dref & g040dfmm & g040dref \\
\hline DFMN & 430 & H & 2 & 3 & 0 & $\begin{array}{l}\text { ACD-4; E FIELD } \\
\text { MGL-6; H FIELD } \\
\end{array}$ & $10 / 20$ & $\begin{array}{l}\text { g053dfmn } \\
\text { g058dfmn }\end{array}$ & $\begin{array}{l}\text { 8053dref } \\
\text { g058dref }\end{array}$ & $\begin{array}{l}\text { g054dfmn } \\
\text { g057dfmn }\end{array}$ & $\begin{array}{l}\text { 8054dref } \\
\text { g057dref }\end{array}$ & $\begin{array}{l}\text { g055dfmn } \\
\text { g056dfmn }\end{array}$ & $\begin{array}{l}\text { g055dref } \\
\text { g056dref }\end{array}$ \\
\hline DFMP & 430 & $\bar{H}$ & \begin{tabular}{|l|}
8 \\
\end{tabular} & 3 & 0 & ACD-4;E FIELD & $10 / 20$ & g043dfmp & g043dref & g042dfmp & g042dref & g041dfmp & g04ldref \\
\hline DFMQ & 25 & H & \begin{tabular}{|l|}
8 \\
\end{tabular} & 3 & 6 & ACD-4; EFIELD & $9 / 21$ & c028dfmg & c028dref & c027dfmq & c027dref & c025dfmg & c025dref \\
\hline DFMR & 25 & $\mathbf{H}$ & 8 & 3 & -6 & ACD-4; E FIELD & $9 / 21$ & $\mathrm{c021 \textrm {dfmr }}$ & c021dref & $\mathrm{c023 \textrm {dfmr }}$ & c023dref & $\mathrm{c024dfmr}$ & c024dref \\
\hline DFMS & 172 & $\mathrm{H}$ & 8 & $\overline{3}$ & 6 & ACD-4; E FIELD & $10 / 21$ & e044dfms & e044dref & e046dfms & e046dref & e048dfms & e048dref \\
\hline DFMT & 172 & $\mathrm{H}$ & 8 & 3 & -6 & ACD-4;EFIELD & $10 / 20$ & e039dfmt & e039dref & e041dfmt & e04Idref & e043dfmt & e043dref \\
\hline DFMU & 430 & $\mathbf{H}$ & 8 & 3 & 6 & ACD-4;E FIELD & $10 / 20$ & g051dfmu & g051dref & g049dfmu & g049dref & g048dfmu & g048dref \\
\hline$\overline{D F M W}$ & 430 & $\mathrm{H}$ & 8 & 3 & -6 & ACD-4;E FIELD & $10 / 20$ & g045dfmw & g045dref & g046dfmw & $g 046 d r e f$ & g047dfmw & g047dref \\
\hline
\end{tabular}

- The italicized file names are for magnetic fields 


\subsubsection{Rhombic Field Mapping}

The fields within the rhombic were also required in order to define the excitation of articles within the test volume for modeling exercises. Also, knowledge of the driving conditions would permit the understanding or interpretation of coupling phenomenology that takes place within the test articles. Estimates of the fields in the rhombic region had been achieved in the test plan using a simple quadripole transmission line model of the simulator. But, this was for TEM conditions and, over the broad frequency range encountered here, one could not expect that the TEM approximation was adequate. Nonetheless, this model was used to determine estimates of the field strengths involved for safety and detectability forecasting.

The fields using the rhombic radiator in the LESLI were measured at a number of points. Using the geometry and coordinates system of Figure 6-6, the fields were probed in the region near the anticipated location of the aircraft nose during the aircraft tests. The time available for the mapping was severely restricted and the number of points, as with the dipoles, were limited.

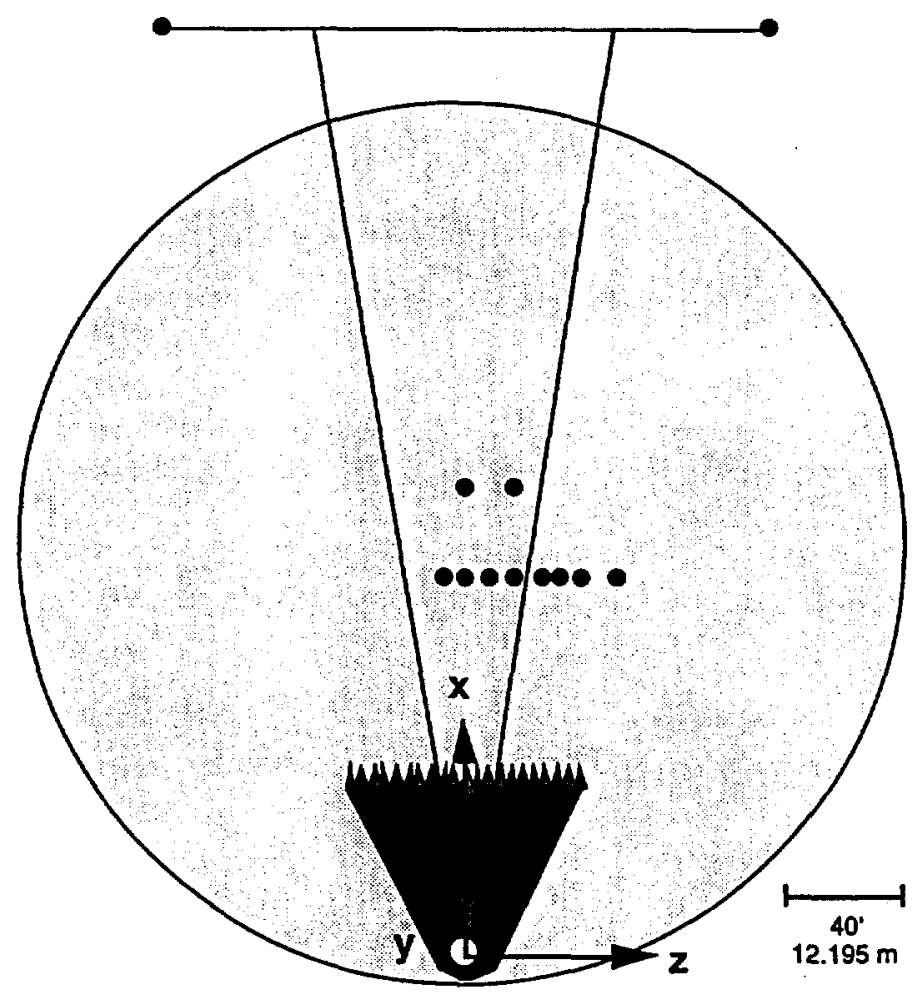

\begin{tabular}{|l|l|c|c|c|c|}
\hline \multicolumn{7}{|c|}{ Table 6-37. } \\
Locations \\
\hline Freq (MHz) & Pol & $\begin{array}{c}\text { X } \\
\text { (m) }\end{array}$ & $\begin{array}{c}\text { y } \\
\text { (m) }\end{array}$ & $\begin{array}{c}\text { z } \\
\text { (m) }\end{array}$ & Fields \\
\hline 0.3 to 1000 & V & 40 & 1 & 0 & E \\
\hline 0.3 to 1000 & V & 40 & 3 & 0 & E \\
\hline 0.3 to 1000 & V & 40 & 3 & 2 & E \\
\hline 0.3 to 1000 & V & 40 & 3 & 8 & E \\
\hline 0.3 to 1000 & V & 40 & 3 & 15 & E \\
\hline 0.3 to 1000 & V & 40 & 5 & -2 & E \\
\hline 0.3 to 1000 & V & 40 & 5 & 0 & E\&H \\
\hline 0.3 to 1000 & V & 40 & 5 & 2 & E \\
\hline 0.3 to 1000 & V & 40 & 5 & 5 & E\&H \\
\hline 0.3 to 1000 & V & 40 & 5 & 8 & E \\
\hline 0.3 to 1000 & V & 40 & 5 & 10 & E\&H \\
\hline 0.3 to 1000 & V & 40 & 5 & 12 & E \\
\hline 0.3 to 1000 & V & 40 & 5 & 15 & E \\
\hline 0.3 to 1000 & V & 50 & 3 & 0 & E \\
\hline 0.3 to 1000 & V & 50 & 3 & 5 & E \\
\hline
\end{tabular}

Figure 6-6. Rhombic field map points

Table 6-37 is a tabulation of the sample points for the rhombic mapping. The rhombic radiator was always driven in common mode thus giving rise to a very predominantly vertically polarized electric field. The dependability of the differential mode balun was questioned especially the constancy of its characteristics over the full frequency range from 0.3 to $1000 \mathrm{MHz}$. As a result, the rhombic was not mapped for the differential mode (predominant horizontal polarization). Furthermore, the differential mode was only used over a small frequency range for the purpose of collecting qualitative data on coupling phenomenology. The file names for the data for the rhombic field mapping have been tabulated in Table 6-38. The field map points have a 7FM prefix before a designator for each point. Again, the ACD-4 probe is a balanced electric field probe and the MGL6 is a balanced magnetic field probe. The reference fields are always the transverse component of the magnetic field at the groundplane on the centerline at a point 10 meters from the rhombic feedpoint. This field is measured with a ground plane magnetic field sensor, MGL-7. The file names for magnetic fields in Table 6-38 are in italics. 


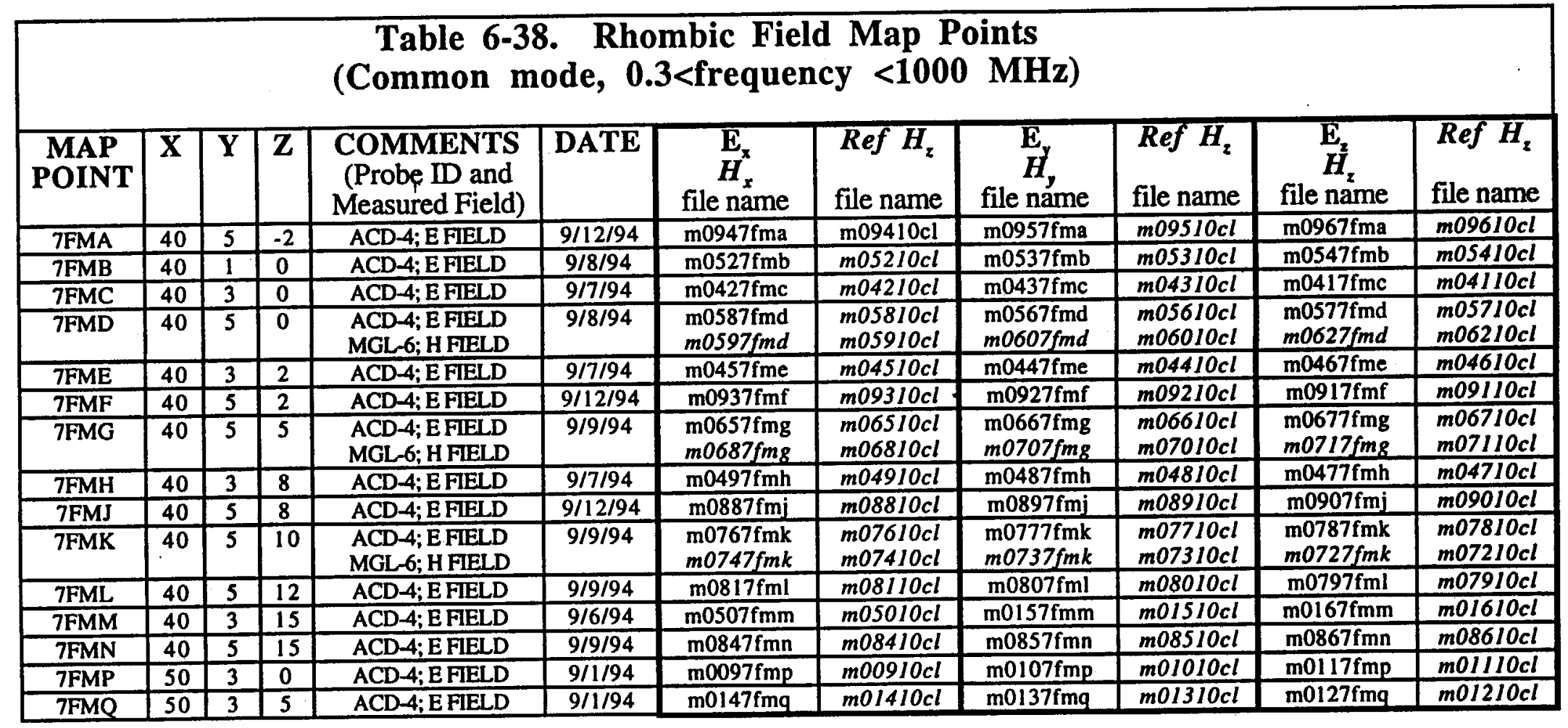




\subsubsection{LESLI Pad Characterization}

The LESLI pad required electromagnetic characterization in order to enable the modelers to include it in the definition of the electromagnetic environment. The evaluation of the constitutive parameters was to be performed under a number of constraints, namely,

- In order to limit costs, the measurement process was to be minimized in time extent without severely compromising the quality of the data.

- The measurement process was limited by the availability of the data acquisition system for only a one week period. This included assembly/disassembly, testing/dry runs, equipment downtime, and data acquisition.

- Construction details for the pad built over 40 years ago, were unavailable.

- The measurement techniques were to be completely noninvasive, namely, the pad could not be cored or probes emplaced below the surface.

The method chosen for the determination of the constitutive parameters is based on the theoretical solution for current propagation on a wire above a homogeneous half-space with arbitrary permittivity $\varepsilon$, and conductivity $\sigma$. A solution for the current distribution on an infinitely long wire over ground, with no limitations on the ground constants, had been developed by Chang et al. [1975], Olsen et al. [1978], and Kuester et al. [1978]. The solution of the inverse problem starting with measured current distributions allowed for the determination of the constitutive parameters as described in Poggio et al. [1995]. The report is included as Appendix C, for convenience.

Figure 6-7 shows the wire conductor on the LESLI pad. The current distributions on the wire were collected from approximately $1 \mathrm{MHz}$ to $1 \mathrm{GHz}$ at 90 points spaced $30 \mathrm{~cm}$ apart.

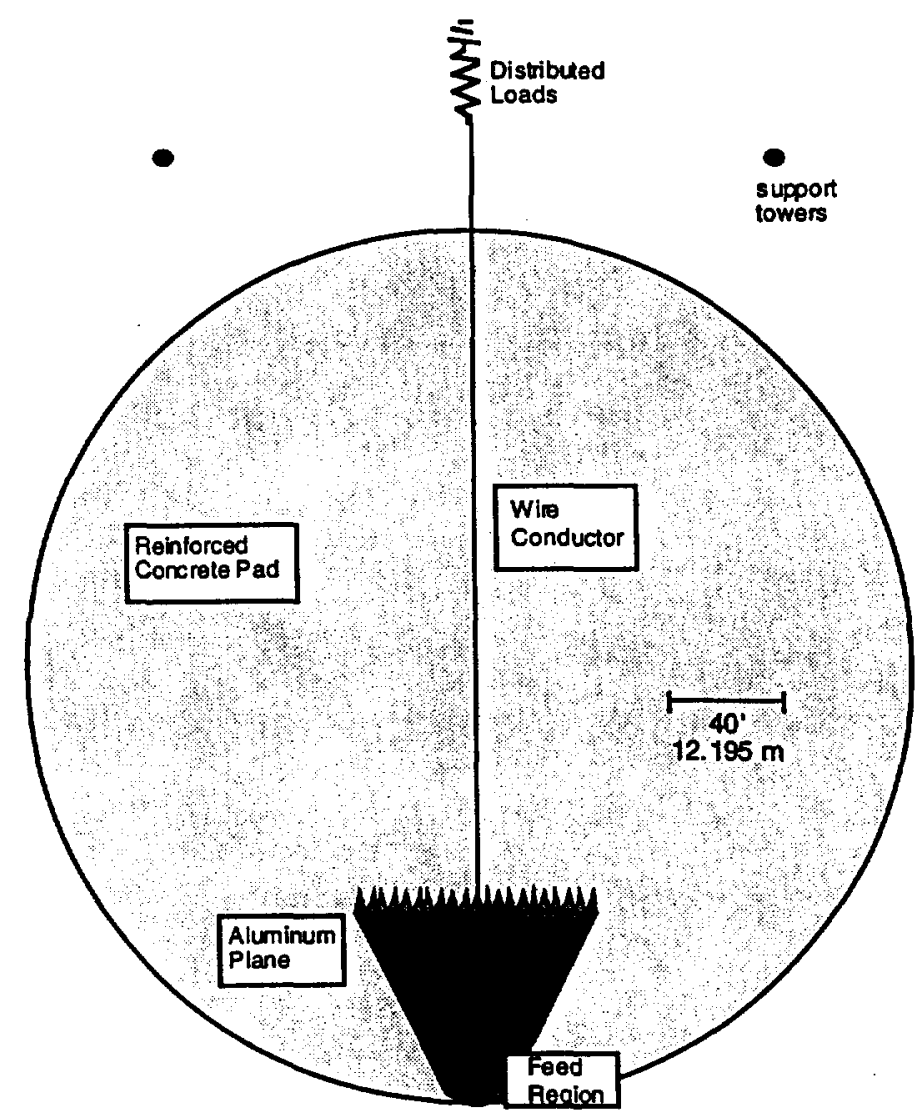

Figure 6-7. Pad characterization wire layout 
While the original plan called for another test set with the wire routed at another angle across the pad, time constraints did not permit this test.

Figure 6-8 is a cross section of the conducting wire on the pad showing the feedpoint, the aluminum plane, the wire, its terminating load, and the dielectric used to maintain its height above the pad.

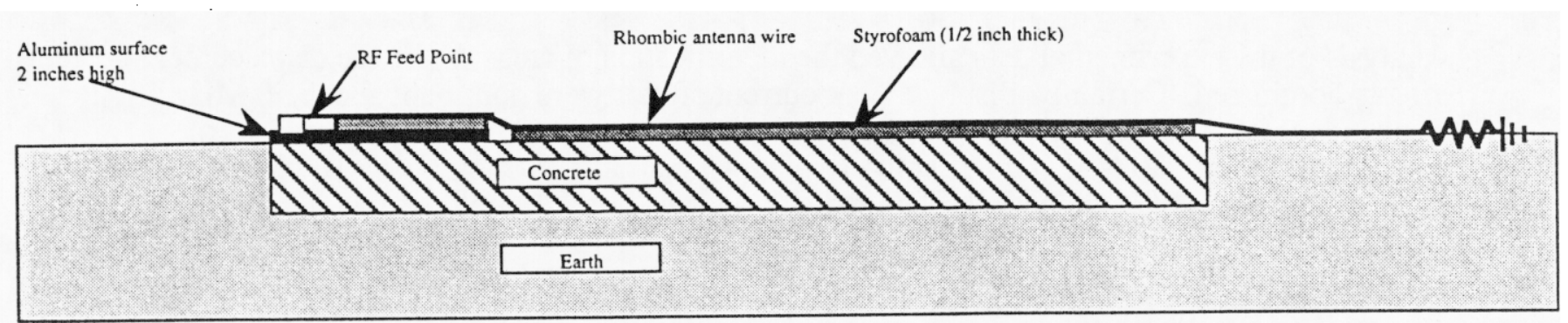

Figure 6-8. Pad characterization wire layout - cross sectional view

The data for the pad characterization are contained in numerous files. During the measurements, the first spatial point was used as a reference for measurements of every other spatial point.

Hence, each spatial point (gp02 through gp90) generated two merged files over the range 0.3 to $1000 \mathrm{MHz}$, one pertaining to the current at the spatial point and one for the reference (gp01). The file designations are defined in Table 6-39.

\begin{tabular}{|c|c|c|}
\hline Table 6-3 & File Desig & on for Pad Characterizatio \\
\hline file name & Freq. Range & Number \\
\hline p\#\#gp\&\& & $0.3-100 \mathrm{MHz}$ & $008 \leq$ sweep\# $\leq 131,02 \leq$ point $\& \leq 90$ \\
\hline q\#\#gp\&\& & $100-1000 \mathrm{MHz}$ & $008 \leq$ sweep $\# \leq 131,02 \leq$ point $\& \leq 90$ \\
\hline m\#\#gp\&\& & $0.3-1000 \mathrm{MHz}$ & $008 \leq$ sweep $\# \leq 131,02 \leq$ point $\& \leq 90$ \\
\hline p\#\#ref1 & $0.3-100 \mathrm{MHz}$ & $008 \leq$ sweep\# $\leq 131$ \\
\hline $\mathrm{q \# \# ref1}$ & $100-1000 \mathrm{MHz}$ & $008 \leq$ sweep\# $\leq 131$ \\
\hline $\mathrm{m \# \# ref1}$ & $0.3-1000 \mathrm{MHz}$ & $008 \leq$ sweep\# $\# 073$ \\
\hline w\#\#\#ref1 & $0.3-1000 \mathrm{MHz}$ & $070 \leq$ sweep\# $\leq 131$ \\
\hline
\end{tabular}

It was realized, as pad characterization data were being acquired, that the merged reference files for this experiment would have identical file names as the rhombic file names; e.g., M070REF1. For this reason, merged files for sweeps 070 and above were assigned the prefix "W" and processed accordingly. Note that the "M" and "W" files for sweeps 071 and 073 are identical. The merged data in this form were used in the analysis in Poggio et al. [1995].

Reasonably accurate estimates of attenuation and phase constants were obtained for frequencies between $10 \mathrm{MHz}$ and $126 \mathrm{MHz}$. Below this range, the spatial extent of sampling did not permit accurate estimates of the attenuation constant while beyond this frequency range, the sampling density and the limited support of the transmission line mode did not permit accurate estimates of the phase and attenuation constants, respectively.

Over the frequency range, the constants led to estimates of $\varepsilon_{\mathrm{r}}$ and $\sigma$ which were "reasonable" and within the range of physical realizability. Outside the range, the estimates for the constitutive parameters were decidedly less reliable. The estimates from the reliable range were used in a least 
squares fit to the Debye equations to yield expressions for the constitutive parameters as a function of $\omega=2 \pi f$ :

$$
\begin{aligned}
& \varepsilon_{\mathrm{r}}=4.2418+6.1943 /\left(1+1.129 \cdot 10^{-17} \cdot \omega^{2}\right) \\
& \sigma=4.416 \cdot 10^{-11} \cdot \omega /\left(1+1.354 \cdot 10^{-17} \cdot \omega^{2}\right)
\end{aligned}
$$

Analysis of the experimental data allowed the identification of areas where the displacement current effects dominated. Certainly, displacement current effects were dominant above $50 \mathrm{MHz}$ and it was evident from the reflection coefficient analysis that it was effectively independent of conductivity above $50 \mathrm{MHz}$ and effectively independent of frequency above $100 \mathrm{MHz}$. Thus concern regarding the conductivity was secondary as its impact would be felt only below $50 \mathrm{MHz}$.

\subsection{Aircraft Test Series}

The aircraft test series included a sequence of tests involving the aircraft that can be classified as noise measurements (ambient and noise floor) in Tests AN and Noise Floor; dipole measurements (three frequencies, vertical and horizontal dipoles) in Tests A through F; on-board instrumentation checkout using the rhombic radiator (beyond the scope of this report) in Tests $\mathrm{G}, \mathrm{H}$, and I; rhombic tests ( 0.3 to $1000 \mathrm{MHz}$, vertical polarization, nose-on and side incident) in Tests $\mathrm{J}$ and $\mathrm{K}$; some repeated tests to study sensitivities in Test Z; windshield covering effects ion Test WF; and the effects of horizontal polarization in limited tests using the rhombic in Test Horizontal Polarization. In the following, these tests will be briefly described with references to the tables appearing earlier in this section.

\subsubsection{Ambient Noise Measurements}

With the airplane in a nose-on configuration in the LESLI, several measurements were made of the signals that can be expected due to the environment. Figure 6-9 contains plots of the ambient noise signals measured at four test points. These ambient signals were taken at distinct instants and cannot be expected to completely characterize the ambient electromagnetic environment but can be used to provide an indication of the type of background that could be usually expected during the measurement runs. For these tests, two field components in the cockpit, the heater cable current in the E-bay and the external VHF antenna were measured over the entire rhombic frequency range with all sources in the facility in a disabled mode. The test conditions for these tests are described in Table 6-4 and the data identification is provided in Table 6-18.

In these tests, both the test probe and the reference probe (MGL-7 in the LESLI rhombic feedzone 10 meters from the feedpoint on its centerline) signals were measured using the VANAs. The VANAs recorded the reference probe signal and the ratio of the aircraft probe to the reference probe signals. The presence_of the FM radio signals is apparent in the AN4 measurement using the external antenna AN4. These measurements can be used to provide some indication of the level at which measurements can become unreliable due to interfering sources of signals in the environment.

\subsubsection{Instrumentation Noise Measurements}

Instrumentation noise measurements were made for stepped $\mathrm{CW}$ measurements in the rhombic. In the nose-on configuration, each data channel was, in turn, tested. The probe on each channel was removed and replaced by a matched load ( $50 \mathrm{ohms})$. Then under RF illumination in the rhombic, a complete sweep was executed and recorded for that channel. These data represent the signal in each recorded channel without any apparent input signal from a probe with all other channels in their normal configuration. The test conditions for these tests are described in Table 6-11 and the data identification is provided in Table 6-25. Figures 6-9,-10 and -11 present a representative instrumentation noise plot for voltage, current, and $\mathrm{E}$ field measurements involving AD-60 probes. 

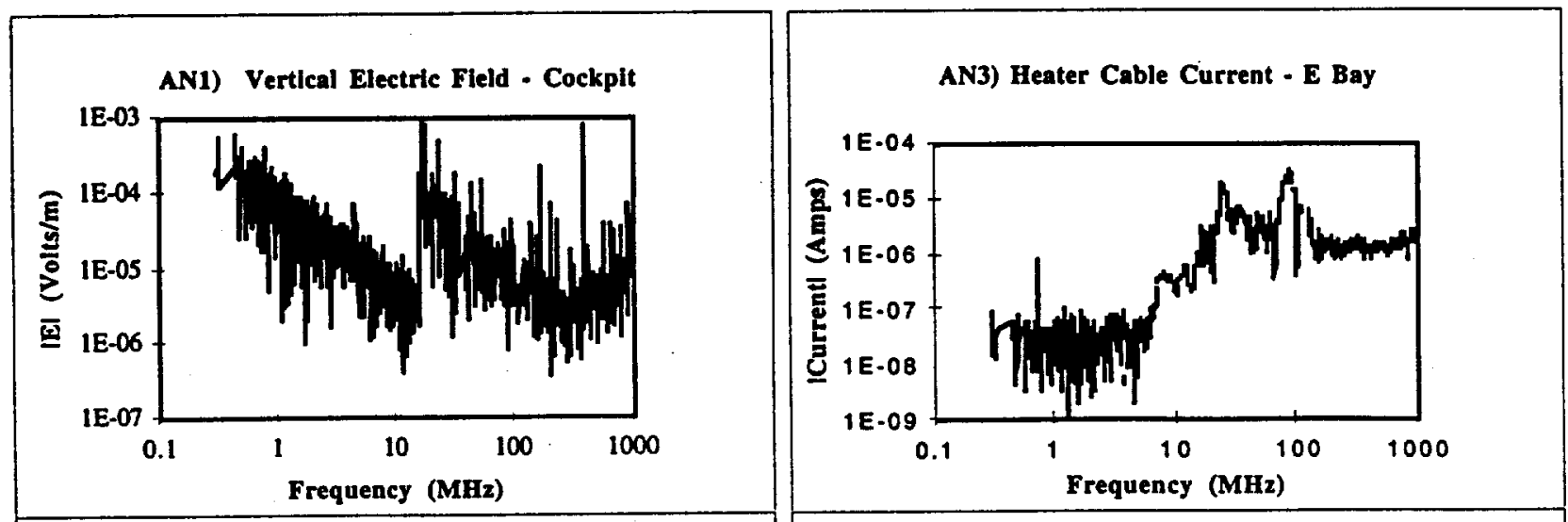

AN2) Axial Electric Field - Cockpit
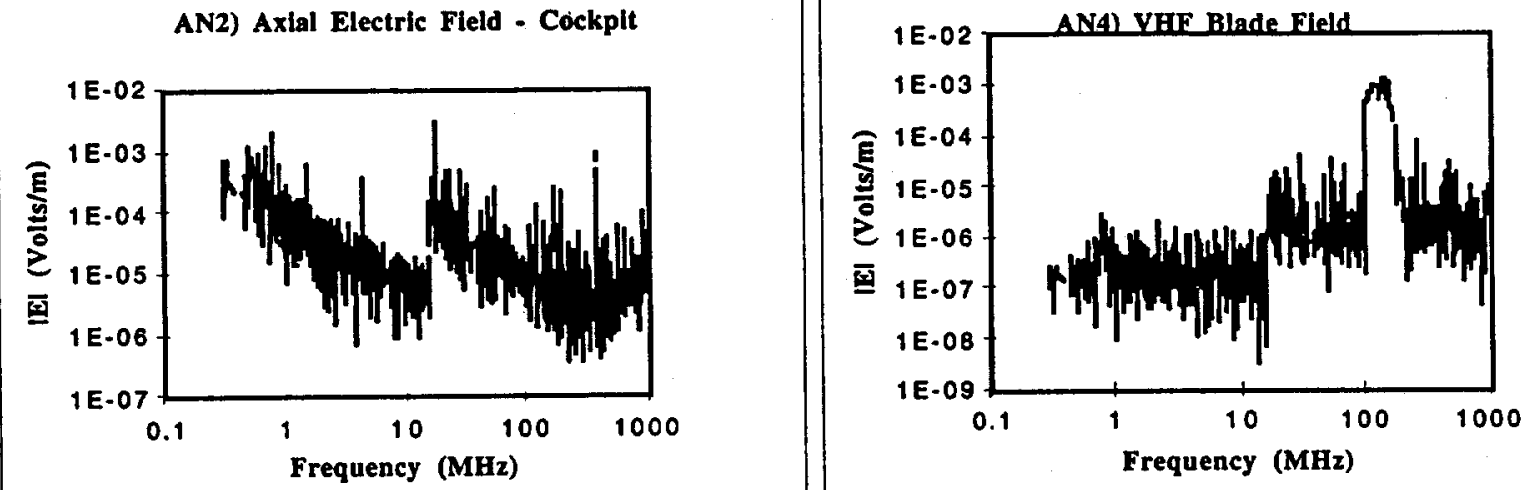

Figure 6-9. Ambient noise measurements for Test Series AN

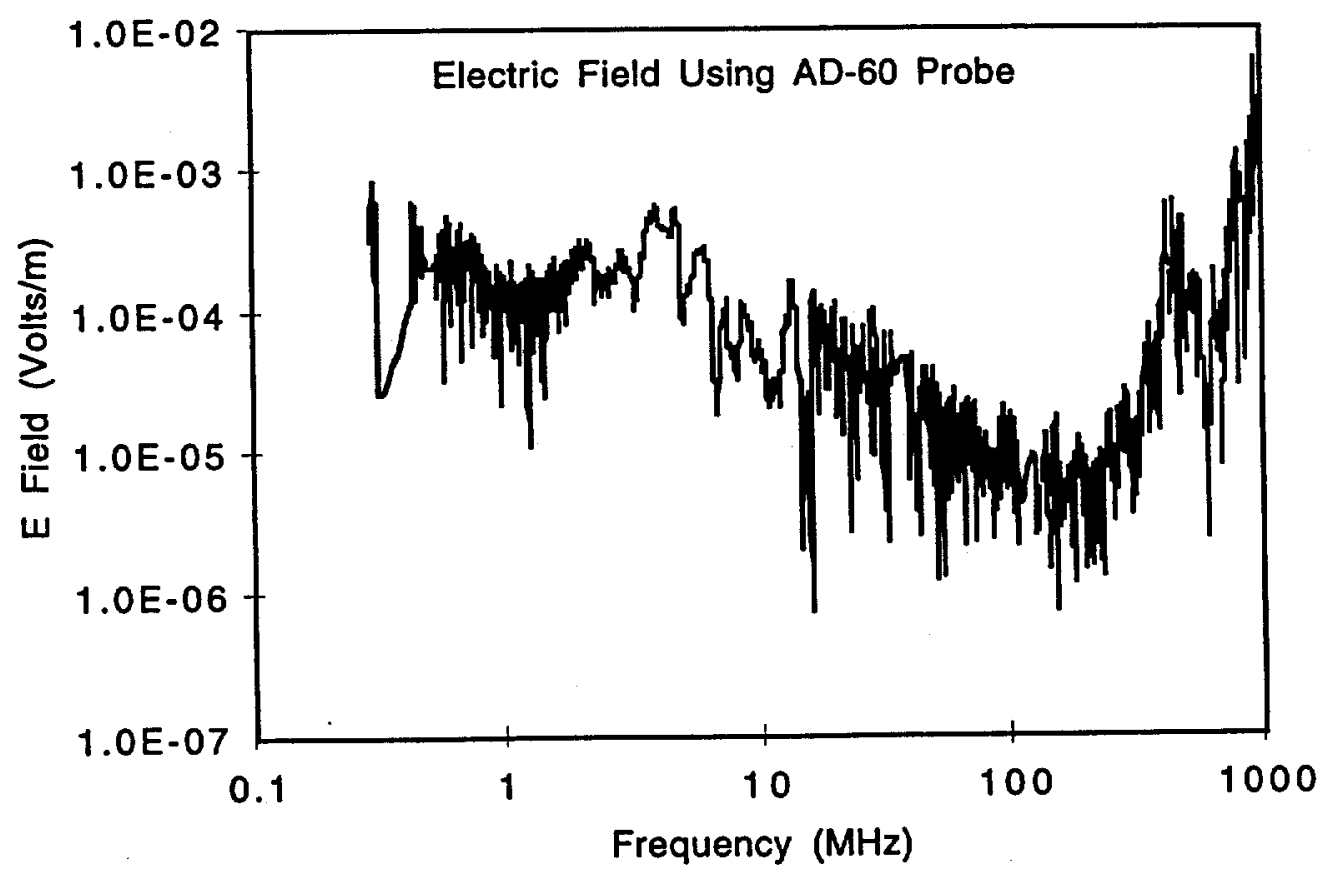

Figure 6-10 Instrumentation Noise in measuring an electric field using an AD-60 probe 


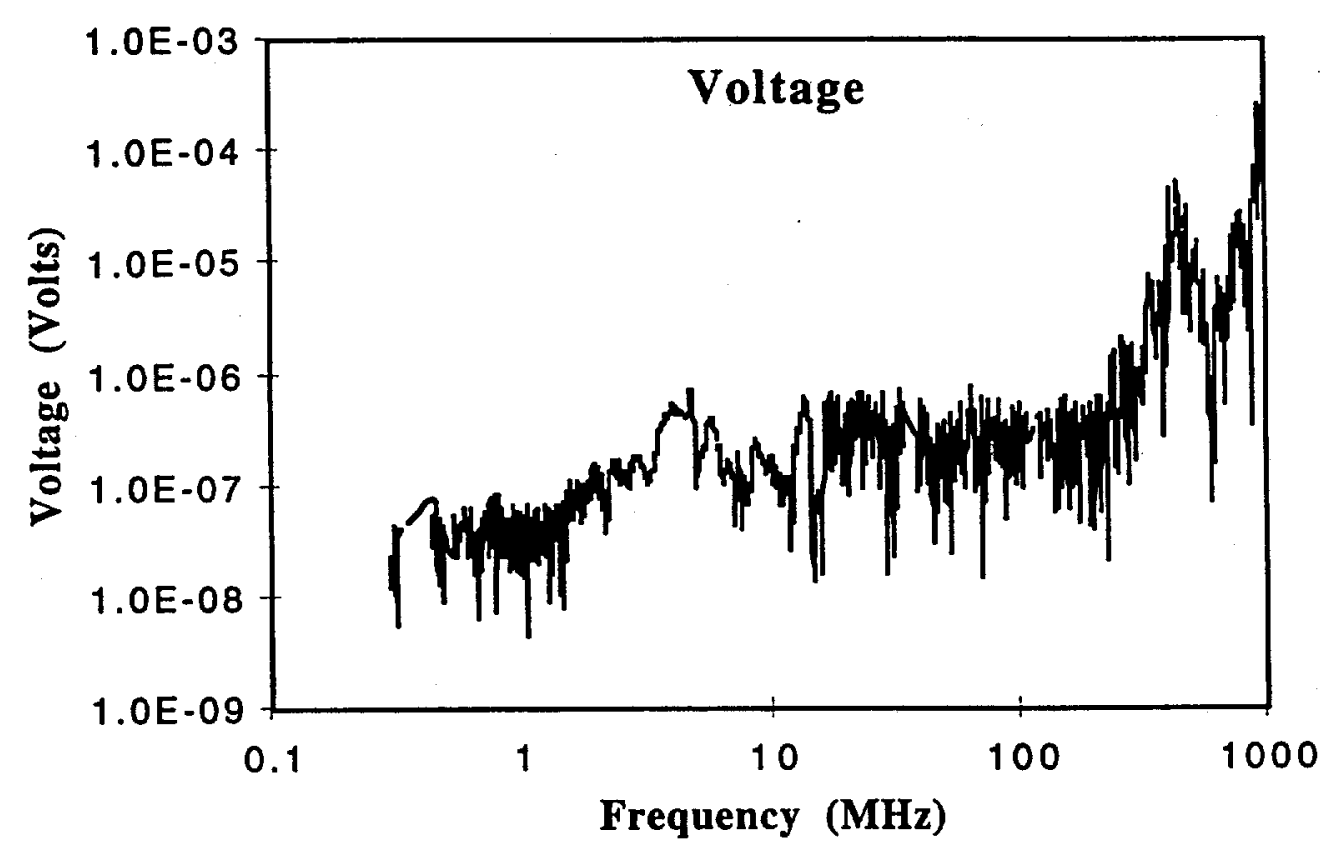

Figure 6-11 Instrumentation noise in measuring voltage at a point

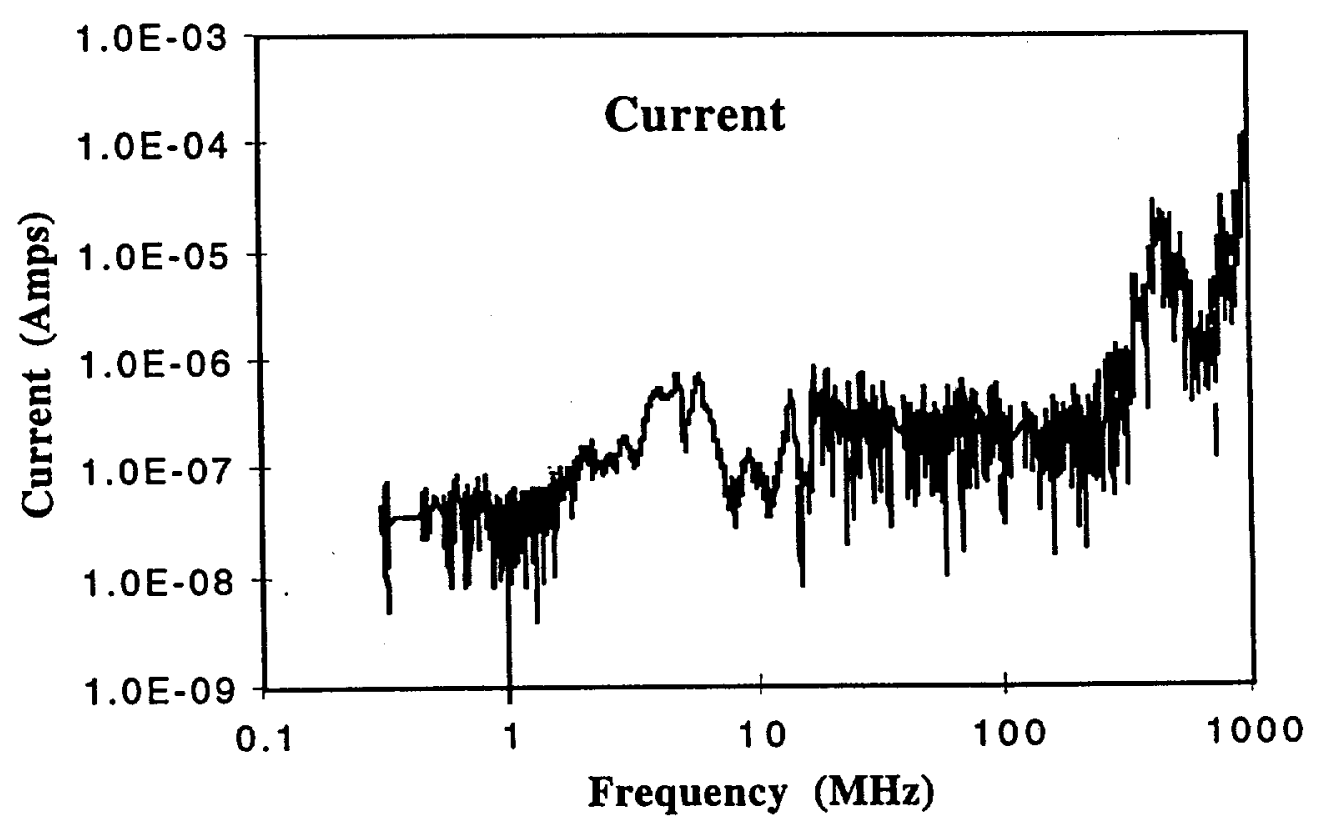

Figure 6-12 Instrumentation noise in measuring current on a wire 
This noise could be due to stray coupling of RF into the cables, crosstalk in the F-O devices and VANAs, and typical noise often experienced in electronic measuring equipment. A review of the data had indicated that the instrumentation noise characteristics were very similar for all channels measuring an identical variable. Hence, a single graphical description adequately conveys information on the instrumentation noise involved in measuring a variable. The external antenna measurement is considered a voltage measurement.

Similar tests were also conducted in the dipole series. There, the channels having the weakest signal and the strongest signal in each test series were determined. Then, in turn, the probe corresponding to each of these was replaced by a matched load and the signal in that channel was recorded. These tests were referred to as the weak signal line and strong signal line tests. Some observations in Test Series C led to concern with crosstalk as indicated in Millard [1995], a test report which is included as Appendix B, for convenience. In test $\mathrm{C14}$, the weak signal line test related to the $C 4$ test, coupling from some source onto the weak signal line was identified. That the measured weak signal was contaminated by a signal from a strong signal line was evidenced by two further measurements on the weak signal line, one with the strong signal probe attached and the weak signal input to the F-O link replaced by 50 ohms (Test C104) and the other with the weak signal input to the F-O link replaced by $50 \mathrm{ohms}$ and the others disconnected (Test C204). This is a manifestation of the crosstalk rejection capability of the NanoFast fiber optic links being limited to the 35 to $40 \mathrm{~dB}$ range. The test results are shown in Figure 6-13. Based on these observations

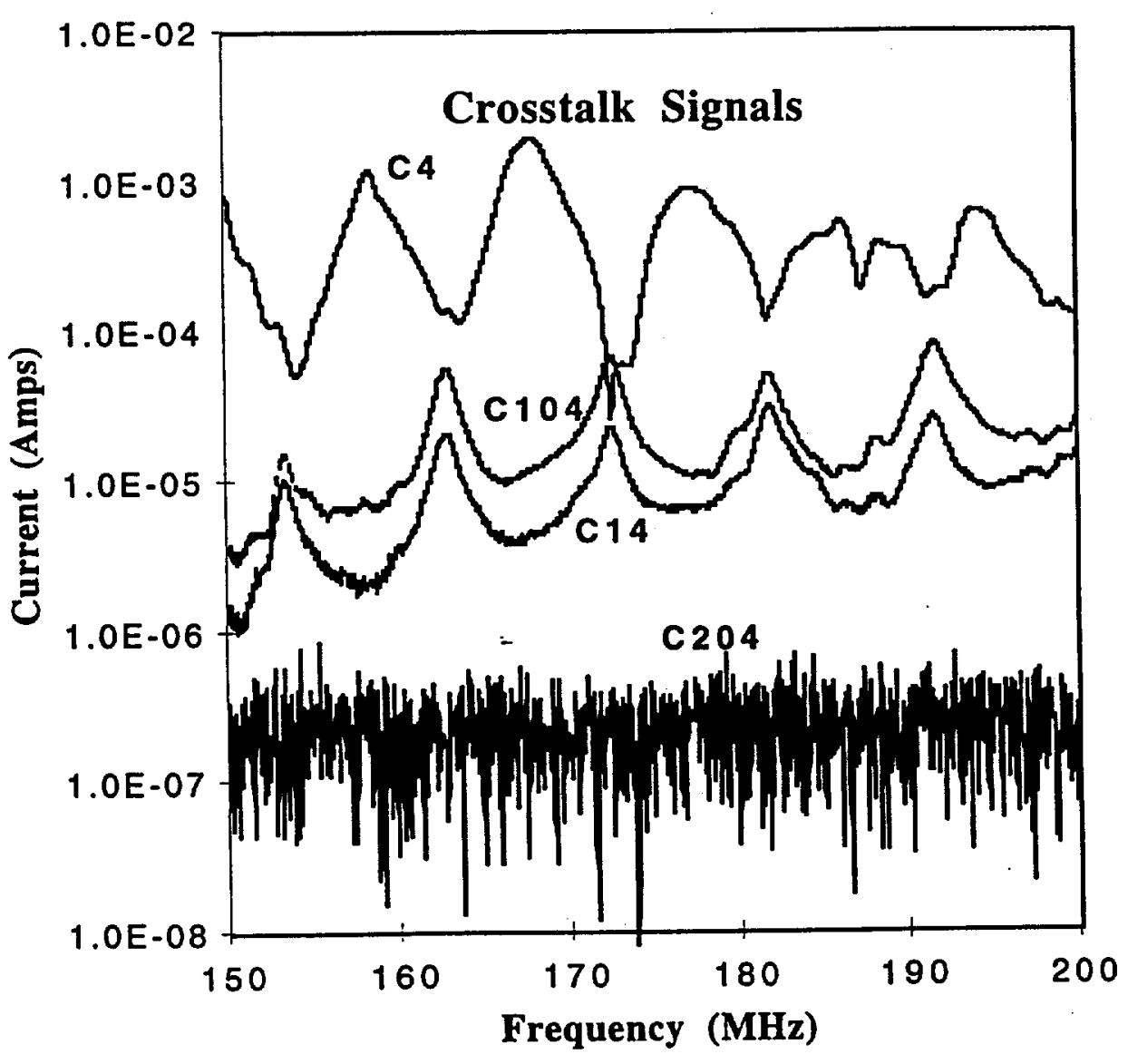

Figure 6-13. Crosstalk signals evaluated during Test Series C 
and the potential impact of crosstalk on the measurement of weak signals, operating procedures were changed for following tests so that weak signal measurements were made without any other channels being connected. Further study or the repeat of previous weak signal tests were not possible due to time and resource constraints.

\subsubsection{Dipole Tests}

The dipole tests were included to provide a test series employing a radiator that is not only well understood and characterized but is also readily included in the mathematical model space when using full wave computer models which are capable of self consistent solutions of Maxwell's Equations. The frequency ranges used in the dipole tests are provided in Table 6-40 as are skip bands, mandated by FAA and FCC as not useable. The number of frequencies at which data were acquired is also provided

\begin{tabular}{|c|c|c|c|c|}
\hline \multicolumn{6}{|c|}{ Table 6-40. Frequency Table for Dipole Tests } \\
\hline $\begin{array}{c}\text { Test } \\
\text { Freq } \\
\text { (MHz) }\end{array}$ & $\begin{array}{c}\text { Start } \\
\text { Freq } \\
(\mathrm{MHz})\end{array}$ & $\begin{array}{c}\text { Stop } \\
\text { Freq } \\
(\mathrm{MHz})\end{array}$ & $\begin{array}{c}\text { No. } \\
\text { Freq } \\
\text { Points }\end{array}$ & Skip Bands \\
\hline 25.85 & 23 & 28 & 1001 & 24.99 to $25.10,25.55$ to 25.67 \\
\hline 172.0 & 150 & 200 & 1001 & 162.375 to 162.575 \\
\hline 430.0 & 350 & 400 & 1001 & 401.6 to $402.2,406$ to 406.1 \\
\hline
\end{tabular}

The dipole tests are denoted as Tests $A$ through $F$ as shown in Table 6-41. The test conditions are found in Tables 6-5 through 6-10 and the data identification is found in Tables 6-19 through 6-24.

\begin{tabular}{|c|c|c|}
\hline \multicolumn{3}{|c|}{ Table 6-41. Dipole Test Series } \\
\hline Test Series Designator & $\begin{array}{c}\text { Freq Range } \\
(\mathrm{MHz})\end{array}$ & Orientation \\
\hline \hline A & $23-28$ & Horizontal, Dipole // to a/c axis \\
\hline B & $23-28$ & Vertical, left of a/c \\
\hline C & $150-200$ & Vertical, left of a/c \\
\hline D & $150-200$ & Horizontal, Dipole // to a/c axis \\
\hline E & $350-500$ & Vertical, left of a/c \\
\hline F & $350-500$ & Horizontal, Dipole // to a/c axis \\
\hline
\end{tabular}

- The location of the radiators relative to the aircraft is shown in Figure 6-14. The dipoles are mounted on a stand as shown previously in Figures 6-2 and 6-3. The geometrical arrangement is shown in Figure 6-14 with all coordinates in meters and the origin at a point on the ground plane directly below the nose of the aircraft, as shown. The center of the dipole source is located at $(-4,3,-5)$ for all frequencies except for the vertical polarization test near $25.85 \mathrm{MHz}$ when the center is raised to $(-4,4,-5)$. A reference probe (B dot sensor) is used in the measurements and is located 3.5 meters from the dipole source for all frequencies except for the measurements near $25.85 \mathrm{MHz}$ when the sensor is 5 meters from the dipole source. The coordinates are $(-1.525,3,-7.475)$ and $(-0.465,3,-8.535)$, respectively.

In all cases, the reference probe was oriented for response to the dominant magnetic field, that is, an orientation orthogonal to the dipole. In the data for the dipole tests and their field mappings, the reference field is denoted $\mathrm{H}_{z}$ and care should be exercised in its interpretation. 
The dipole tests were conducted over frequency ranges distributed about $25.85 \mathrm{MHz}$ (sometimes referred to as $25 \mathrm{MHz}$ range), $172 \mathrm{MHz}$, and $430 \mathrm{MHz}$. Figure 6-15 is a photograph of the 25 $\mathrm{MHz}$ dipole mounted on the stand with the reference probe (an MGL-6 balanced magnetic field
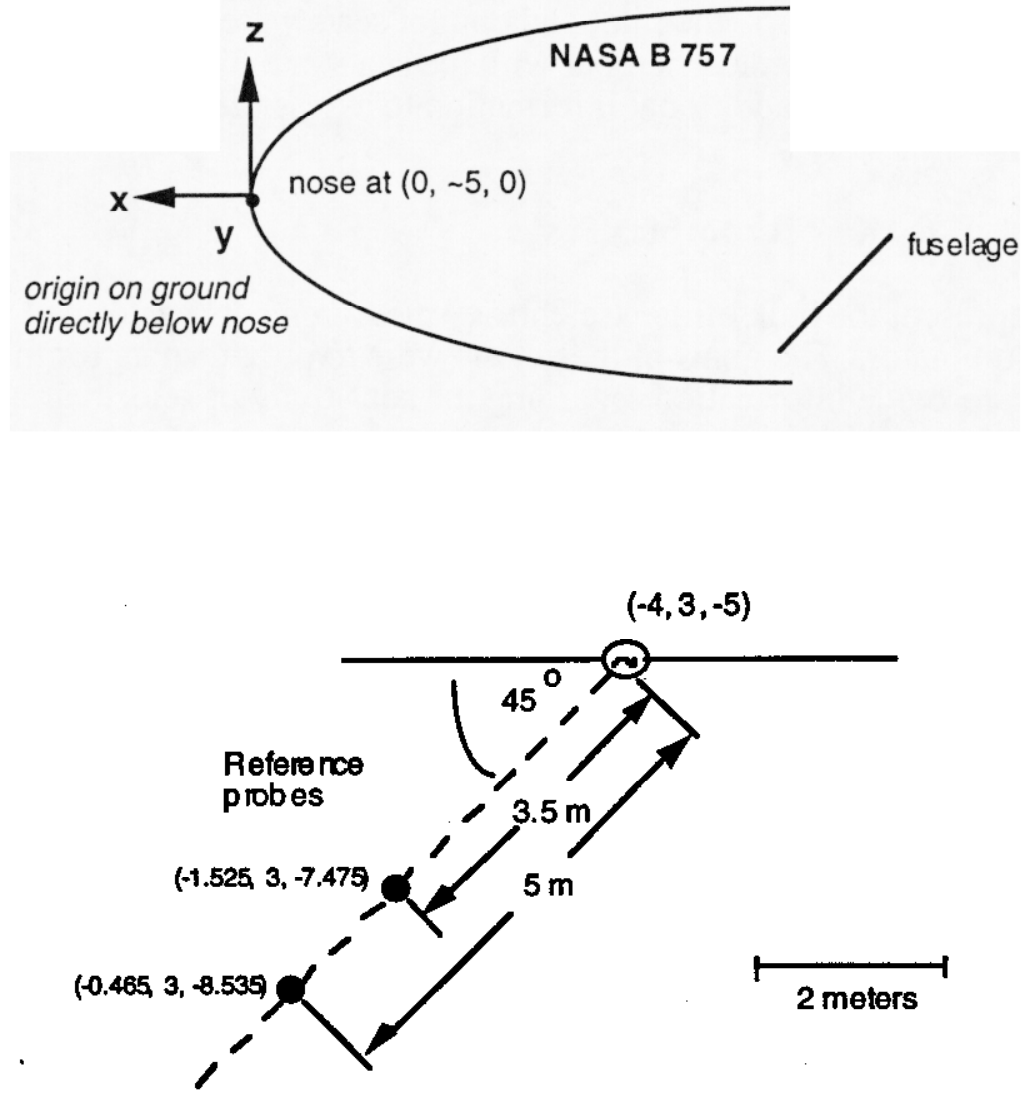

Figure 6-14. Dipole and reference locations

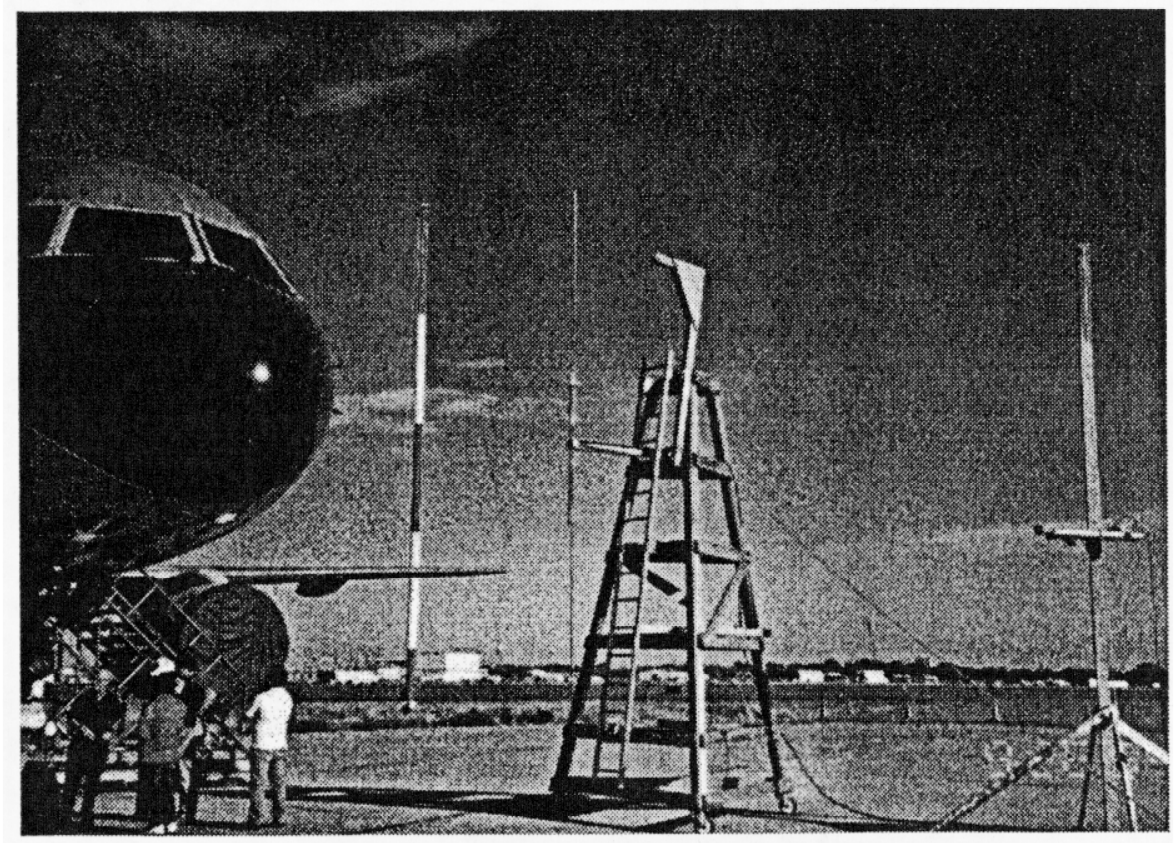

Figure 6-15. Dipole in position for aircraft test 
sensor) to the right in preparation for an aircraft test. The dipoles were fed by baluns to provide balanced currents on the dipole arms.

During the course of the dipole tests, the pilot and copilot seats were maintained in the fore position. Hence, tests A1 and A2 which were carried out with the seats in the aft position were repeated (tests A101 and A102). This provided additional information to assess the effect of seat position on the measured parameters.

\subsubsection{Test Series J \& K - Rhombic Tests}

Test Series J \& K represent the bulk of the "scientific" measurements performed within the LESLI with the rhombic illuminator. The goals of these tests were to provide data for the code validation exercise as well as to provide information regarding the sensitivity of select variables such as field, voltage and current at specific points to variations in system configuration, to repeated measurements at different times, and to angular orientation of the aircraft with respect to the incident electromagnetic field. The test conditions for the $\mathrm{J}$ series of tests are found in Table 6-12 while those for the $\mathrm{K}$ series are found in Table 6-13. The data ids for the $\mathrm{J}$ and $\mathrm{K}$ series are found in Tables 6-26 and 6-27, respectively.

Figures 6-15 and 6-16 are photographs of the B-757 in position on the LESLI pad. Figure 6-16 shows the B-757 in the nose-on configuration with its nose at a point 40 meters from the rhombic feedpoint. The leftmost pole in the photo is one of the support towers for the rhombic conductors. The other support tower is out of view to the left. All other poles were not used in this series. The aluminum ground plane is visible as are the source shack (at the apex of the aluminum plane) and the DAS trailer (lower right). In the background is the Kirtland Air Force Base and Albuquerque Airport facilities and runway.

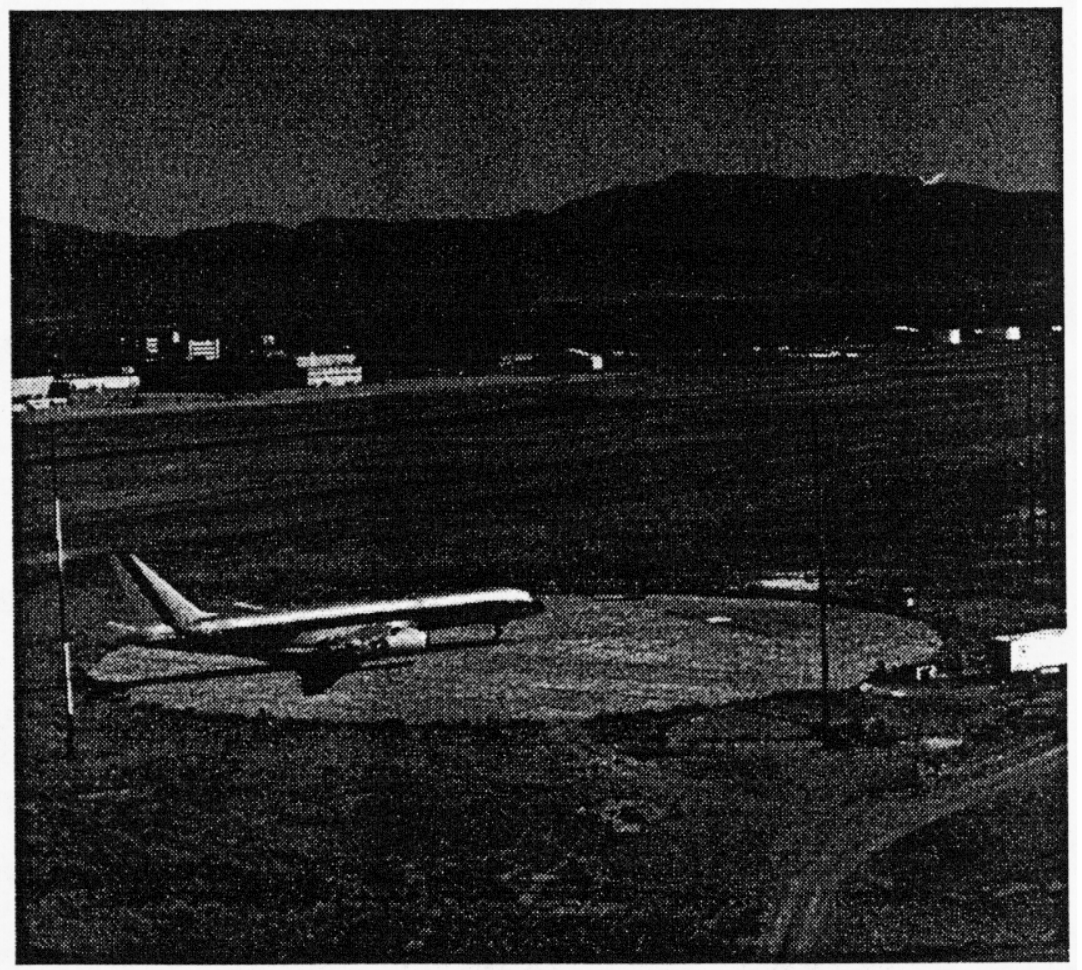

Figure 6-16. B-757 on LESLI pad - overhead view 
Figure 6-17 is a view of the B-757 from the rhombic feedpoint. Clearly visible is the balun used to convert the unbalanced RF signal from the coaxial line into a balanced common mode drive for the rhombic conductors. Also visible in the photo are the rhombic wires, the nylon steadying line running transverse to the facility, and the poles supporting the conductors. The cables routed along the aluminum ground plane are fiber optic cables used in the data acquisition system. They are routed to the aircraft and enter through a pressure relief flap on the right side of the aircraft (the left in the figure). The stairs in the photo are completely constructed of fiberglass and stayed in position during the tests.

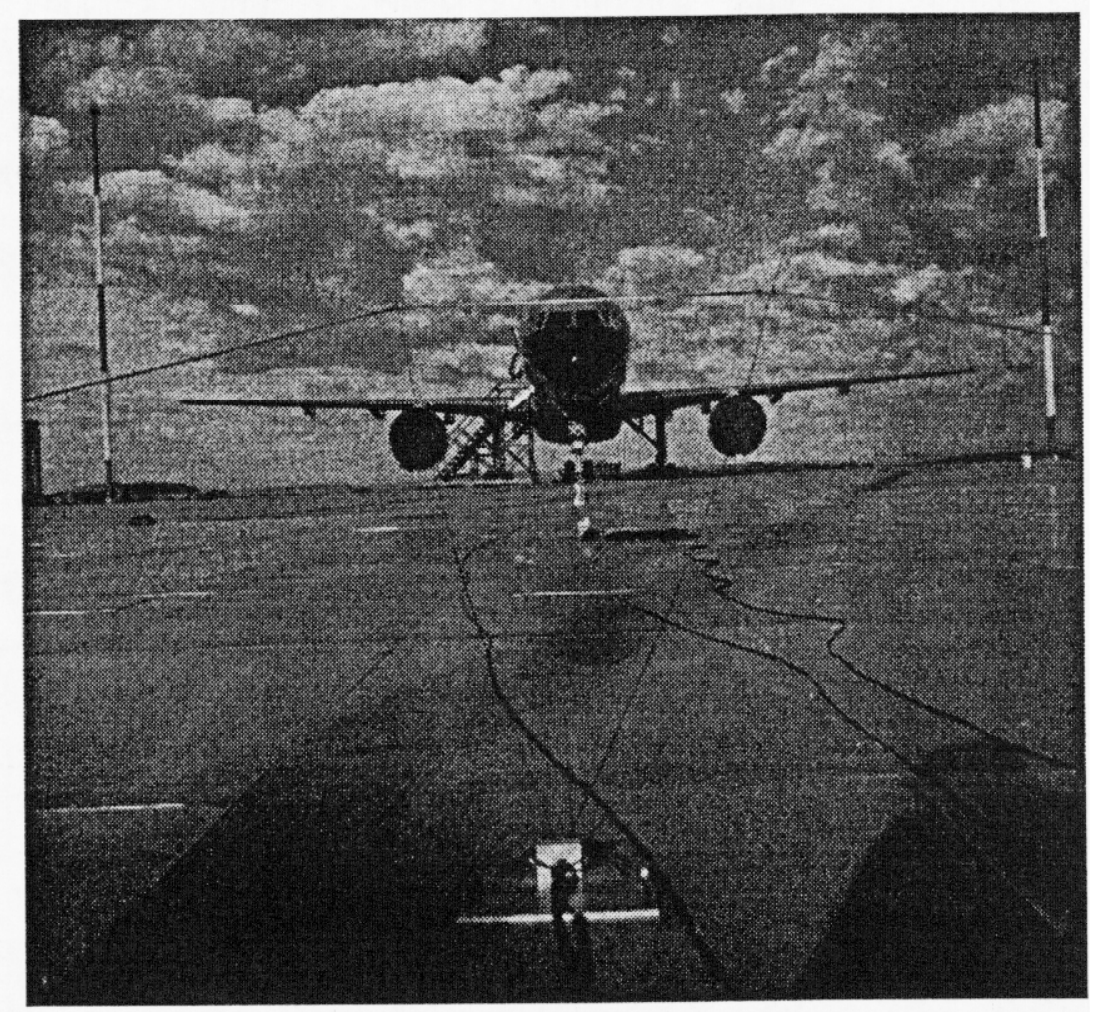

Figure 6-17. B-757 on LESLI pad - viewed from rhombic feedpoint

The data acquired during the rhombic tests were not scaled to a particular driving or incident field strength. Rather, a reference field measured at a reference probe on the rhombic centerline 10 meters from the feedpoint, was recorded for each data run during which a variable was measured. While the reference field did not vary significantly from run to run, it is provided to permit comparisons of the various runs (one need merely scale data to obtain identical reference fields or identical excitation conditions) or to allow an analyst to convert the reference field at the probe to an incident field at the aircraft.

Some general comments and observations regarding the J series follow:

- Test $\mathrm{J} 1, \mathrm{~J} 2$, and $\mathrm{J} 3$ were dropped after execution due to malfunctions in the network analyzer. Tests $\mathrm{J} 20, \mathrm{~J} 21$ and $\mathrm{J} 22$ are suitable replacements as they are identical in configuration and differ only in execution time. The intended repeatability text (data acquired in AM and PM) was not possible.

- Test J6 through J11 dealt with the movement of a conducting box ( a 3"x3"x4" plastic box covered with copper conducting tape) emplaced on the aft surface of the ATOPS box in the cockpit. The tests were intended to uncover the effects on the cockpit E fields of such movement. One would compare the condition of no box (J20, J21, J22) with (J6, J7, J8) and (J9, J10, J11). 
Figure 6-18 is an example of such a comparison showing the vertical electric field on the ATOPS box for no box, the box located on the lower portion of the rear (aft) surface of the ATOPS with its axis parallel to the floor, and the box on the upper portion of the aft surface with axis perpendicular to the floor.

- $\mathrm{J} 12$ is a test over $800<\mathrm{f}<900 \mathrm{MHz}$ looking at the field behavior over a narrow frequency range and providing information for later statistical EM studies.

- Test J13 through J19 were executed to uncover the effects of personnel presence and placement in the cockpit. In J13, J14 and J15 only a copilot was seated (right hand seat) at the half-way portion. In tests $\mathrm{J} 16, \mathrm{~J} 17$, and $\mathrm{J} 18$, both pilot and copilot were seated at the half-way position.

- Test J19 is a wire current measurement using the I-320 current probe (usually used to probe climbing wire current) to measure current on the heater wire for the copilot side window.

- Test J24 (voltage in LRU) was repeated later as Test J024. J24 had the circuit breaker for the VHF unit in the pulled state while J024 had the current breaker engaged (in).

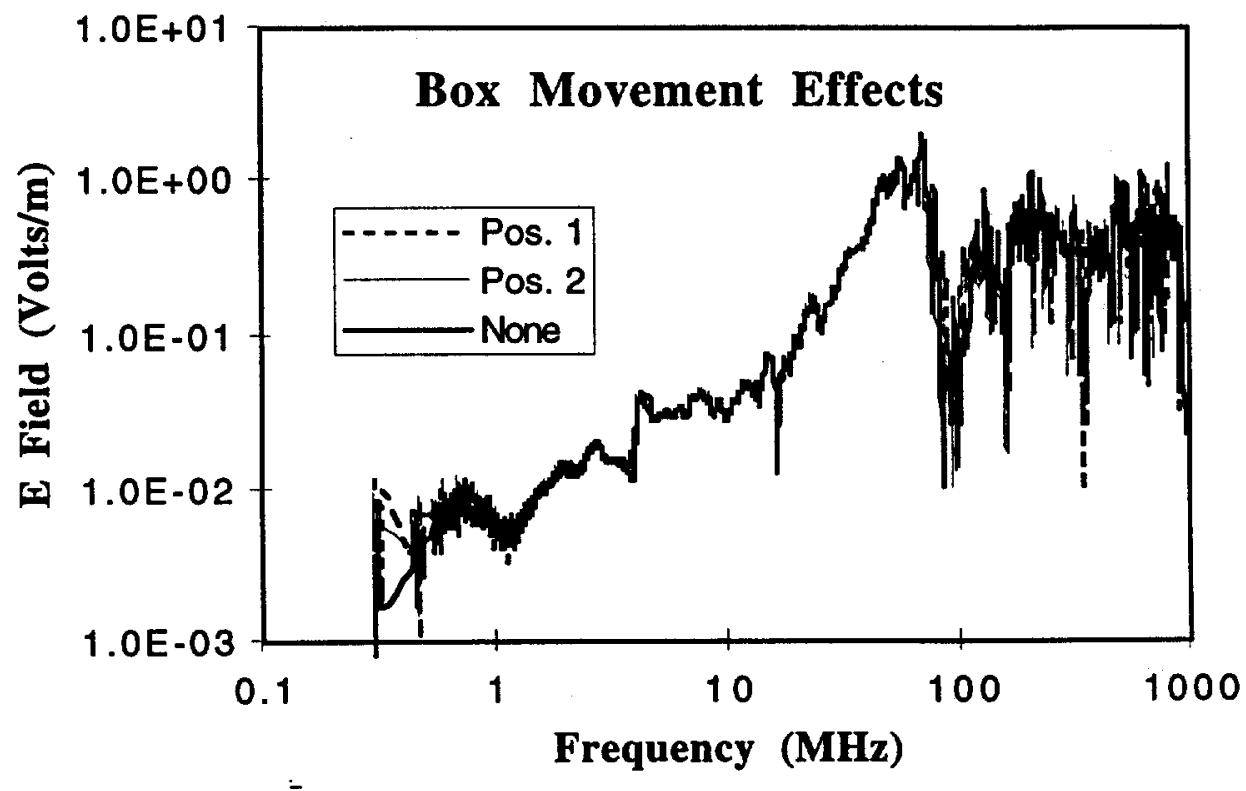

Figure 6-18. The effect of movement of a small conducting box on vertical electric field

- Tests J26 through J31 were executed to assess the effects of various states of the E-bay hatch cover. Figure 6-19 shows the vertical electric field for the normally closed state (J23, J24, J25), hatch sealed with conducting tape (J26, J27, J28), and the hatch open $(\mathrm{J} 29, \mathrm{~J} 30, \mathrm{~J} 31)$. 


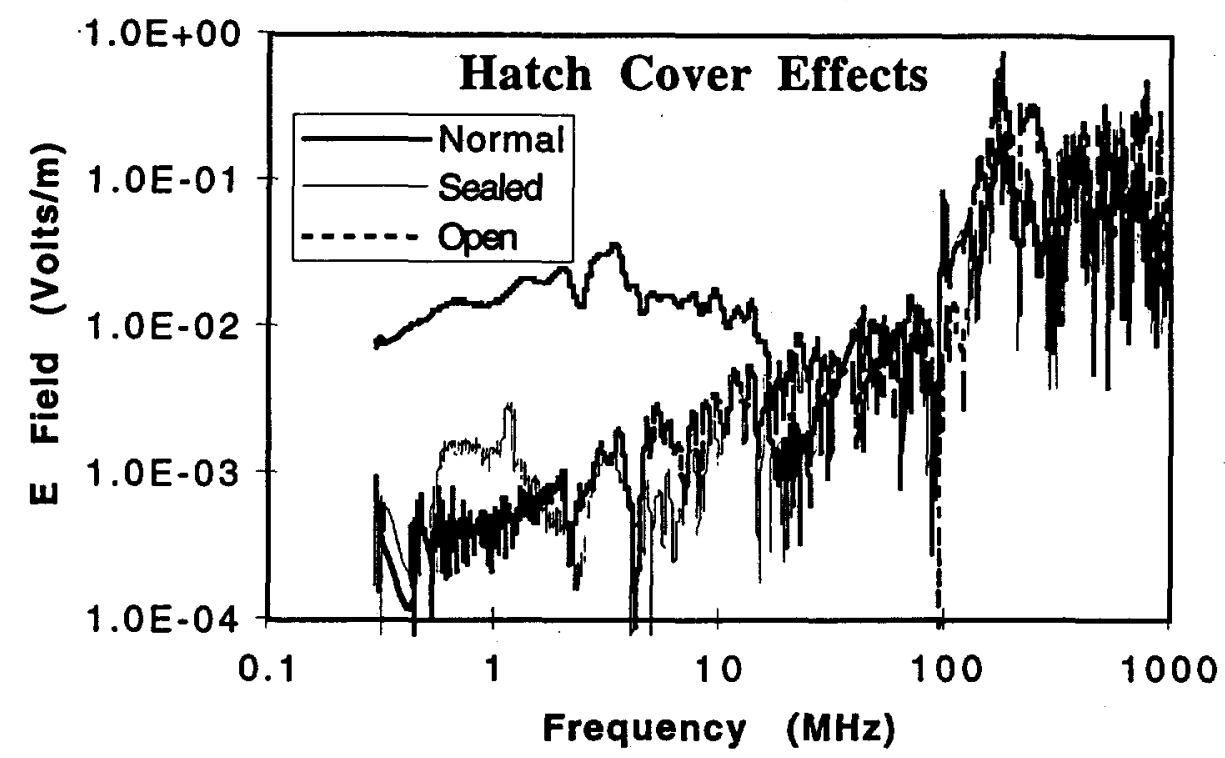

Figure 6-19. The effect of hatch cover state on vertical electric field in E-bay

- Tests J36, J37, and J38 were cockpit field measurements with the incident wave at 10 degrees from nose-on while Tests J39, J40, and J41 were at 5 degrees. The comparison in Figure 6-20 illustrates the sensitivity to angle of incidence. The pilot and copilot seats were full forward for the tests at 5 degrees. (they were repeated during the $\mathrm{K}$ series with the seats at mid position).

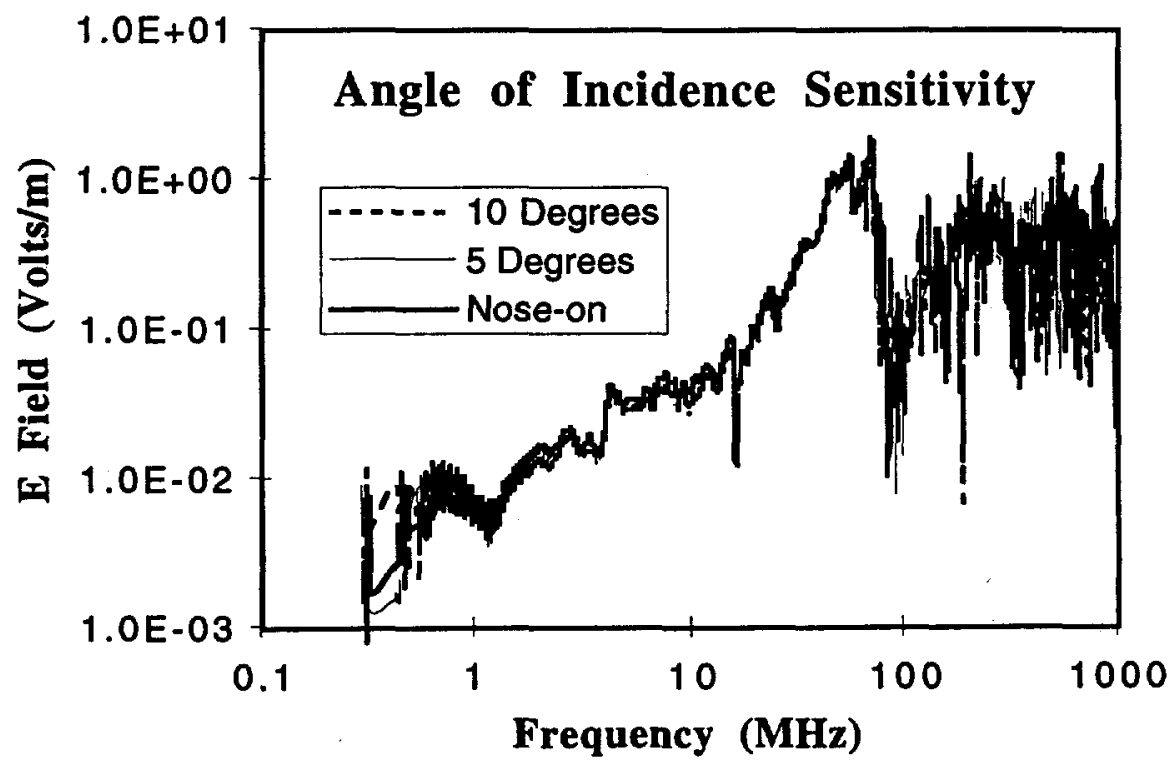

Figure 6-20. Cockpit vertical electric field variation with angle of incidence 
- Test J005 was a climbing wire voltage measurement similar to Test J5 except that the current probe was removed in order to determine the effect of the presence of the current probe during climbing wire voltage measurements. A comparison is shown on Figure 6-21.

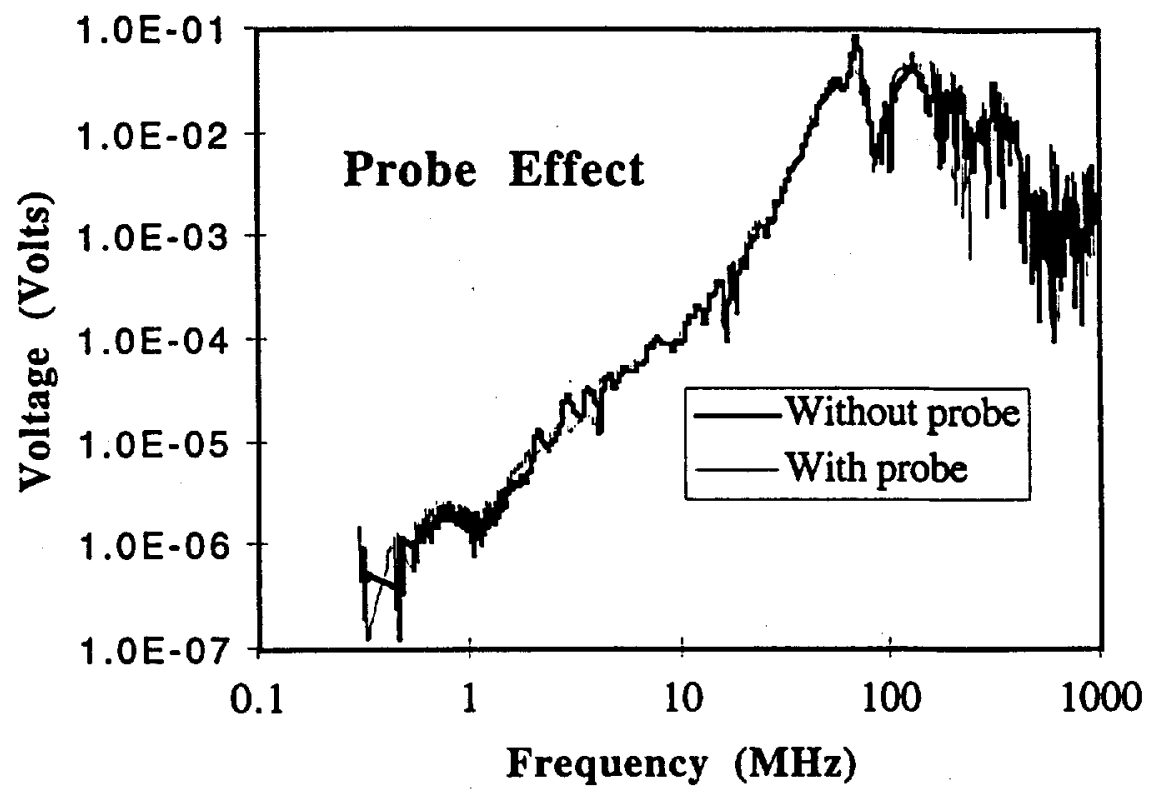

Figure 6-21. Climbing wire voltage with and without current probe

- Tests J026, J027, and J028 were repeats of the J26, J27, and J28 tests but with the pressure door on the right side of the fuselage which served as an exit port for the data acquisition cables sealed with conductive tape and aluminum foil.

Test Series K was primarily directed towards broadside incidence on the aircraft but also included some repeatability tests and repeats. Comments and observations on the $\mathrm{K}$ series follow.

- Tests K1 through K12 were to provide data for a direct comparison of variables for nose-on and side incidence. Corresponding tests in the $\mathrm{J}$ series and in the $\mathrm{K}$ series could be compared. Figures 6-22 and 6-23 are such comparisons for the vertical electric fields in the cockpit and cabin.

- Test $\mathrm{K} 13$ is a repeatability check of the results in K11. An example of such a comparison is presented in Figure 6-24 where repeatability is examined for the long wire base voltage in the cabin. In this case, the possible differences in driving field have been extracted. Recall that all comparisons so far have been made without extracting the differences that might be due to variations in the incident electric field. This was adequate for the qualitative comparisons that have been used as examples. A precise comparison would require that the differences in the driving field be extracted from the resulting data. To normalize the effect, i.e., to remove the impact of changes in results (E) due to changes in the excitation (Ref) for two tests, say test 1 and test 2, one need only perform $E_{1}=\left(R_{1} / R_{2}\right) E_{2}$. So, the results of Figure 6-24 are for identical excitation conditions and are a reasonable indication of the repeatability of the measurements. Figure 6-25 is a plot of the ratio of the incident fields $\left(R_{1} / R_{2}\right)$ and is representative of the ratios in other cases. It is indicative of the variability from test to test and highlights the need to normalize the data when performing quantitative comparisons. 


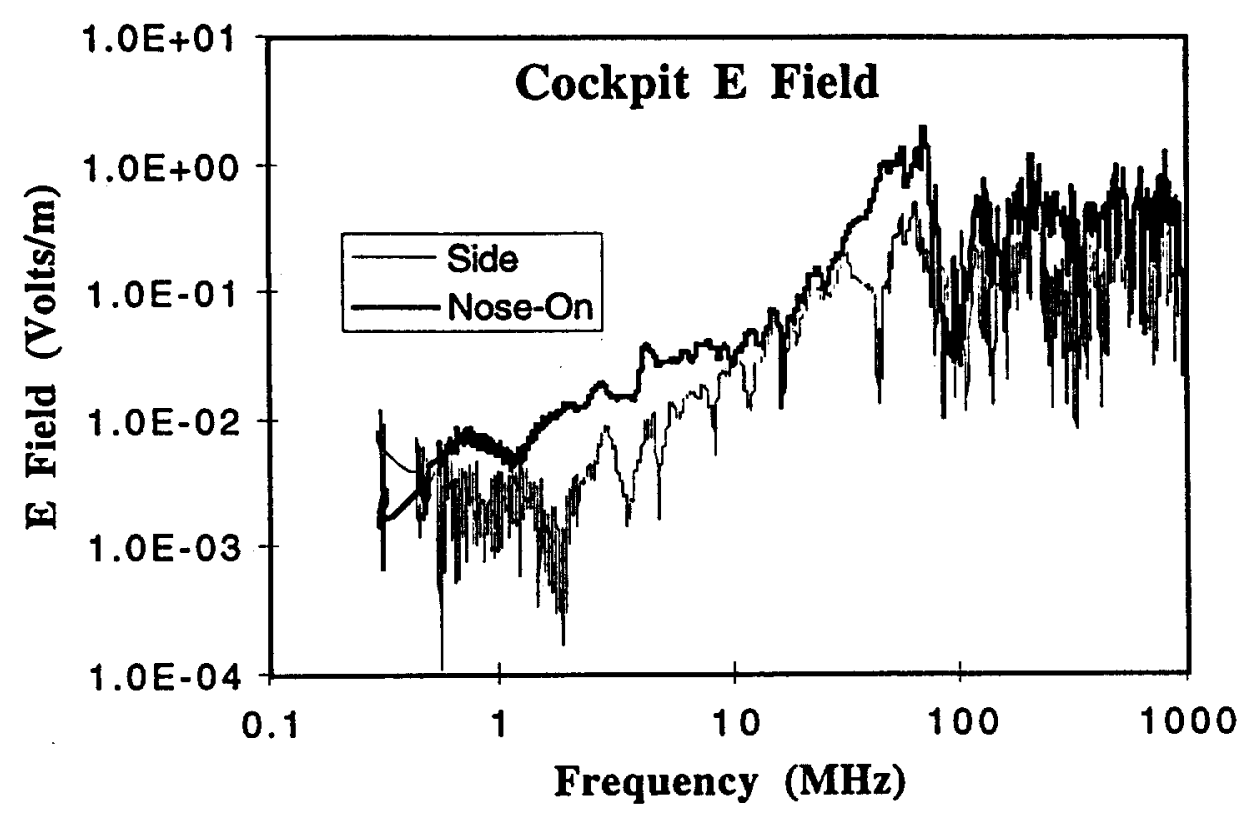

Figure 6-22. Cockpit vertical electric field for nose-on and side incidence

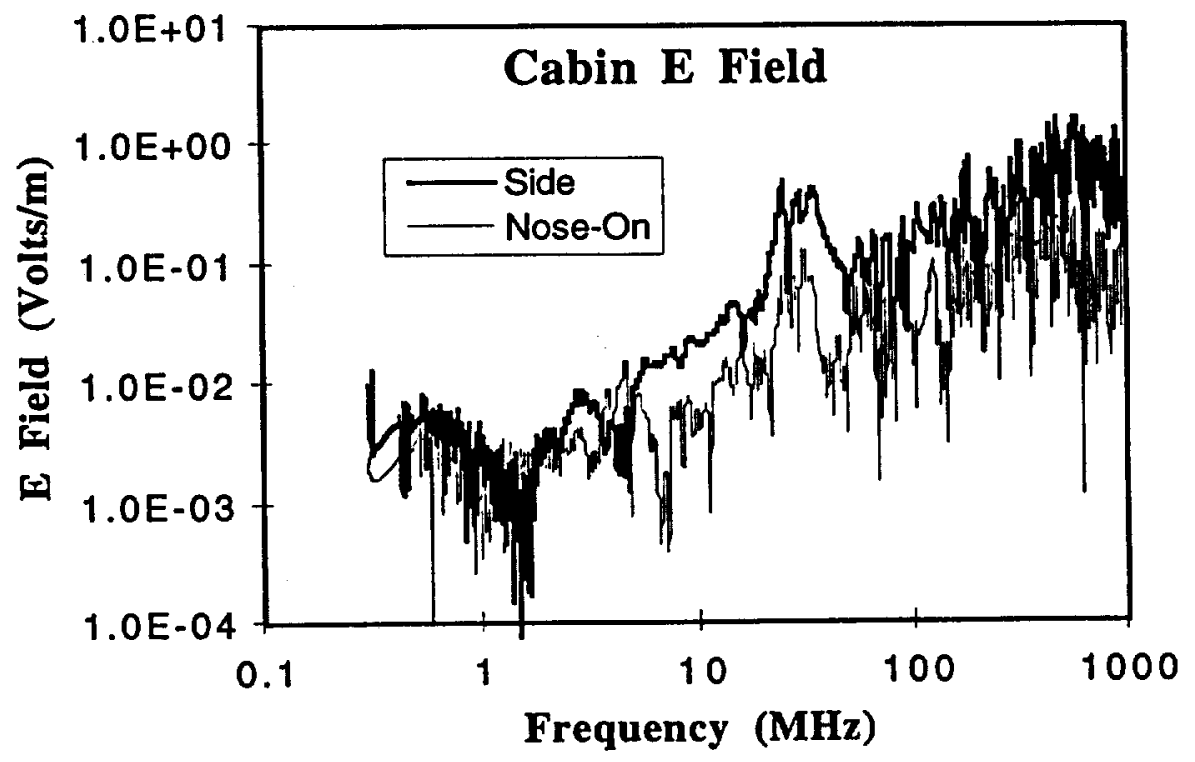

Figure 6-23. Cabin vertical electric field for nose-on and side incidence 


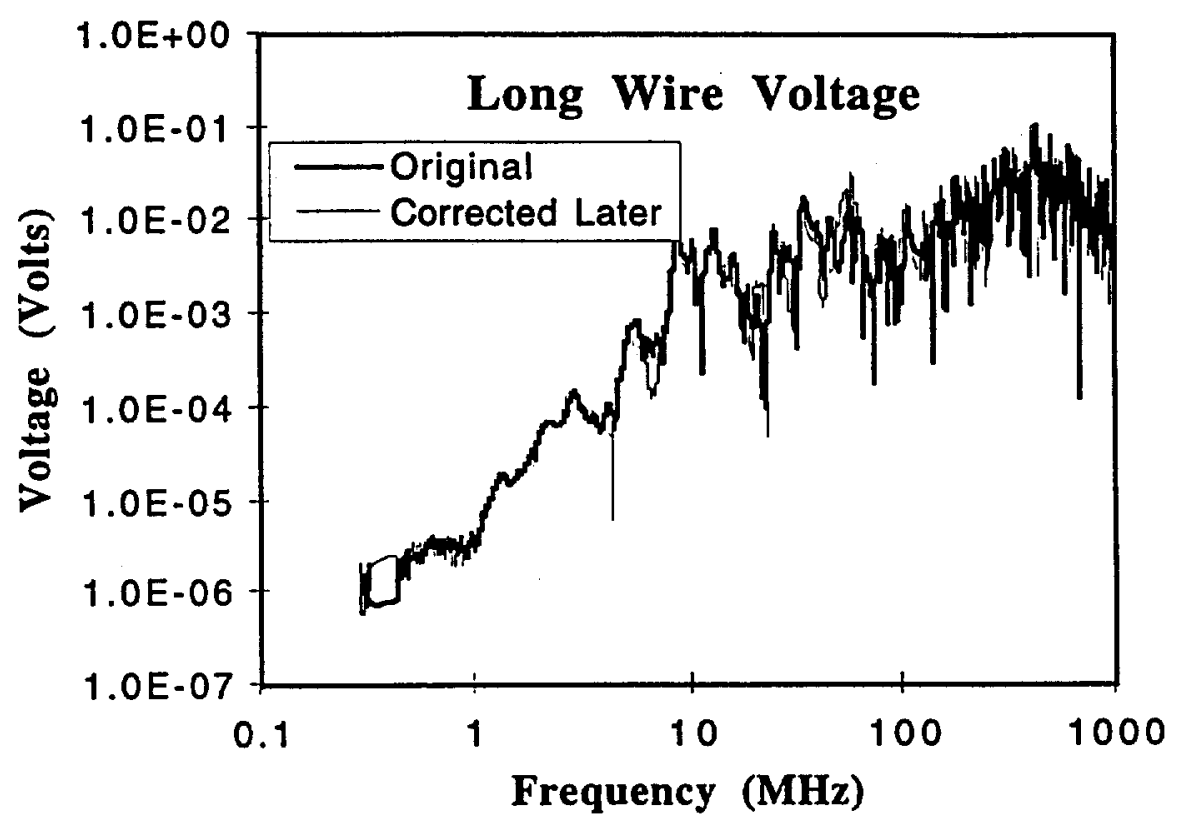

Figure 6-24. Repeatability check using corrected data

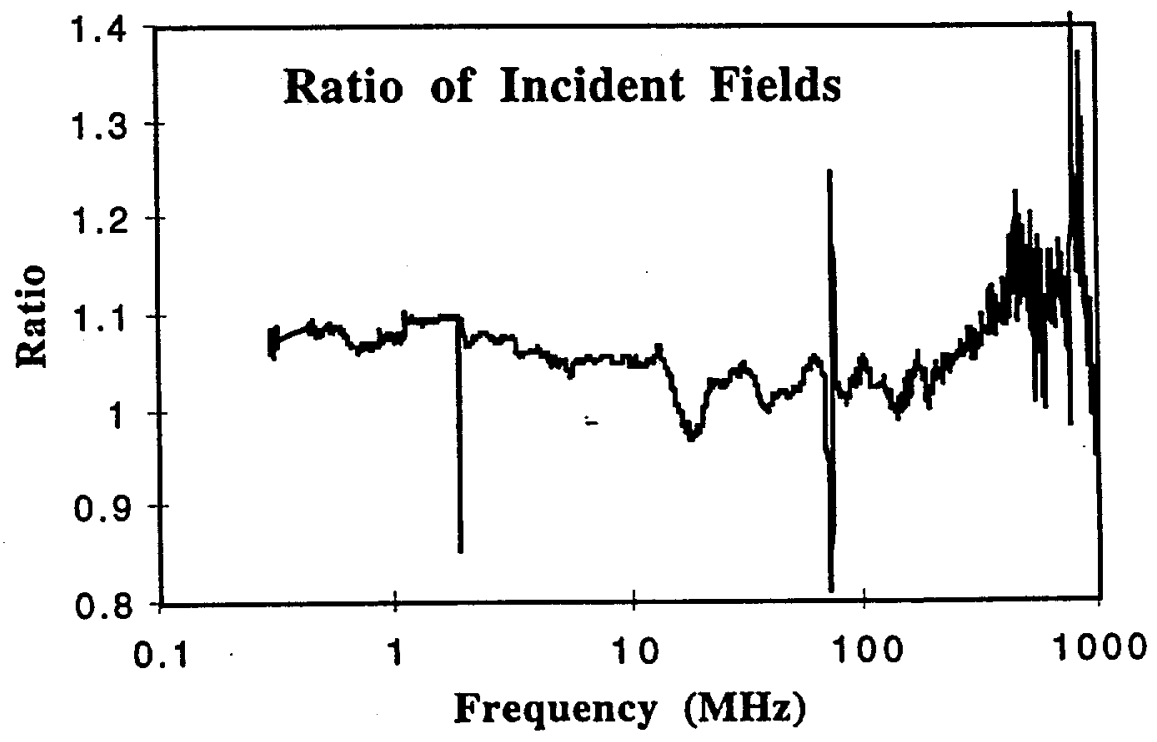

Figure 6-25. Ratio of the incident fields in Tests $\mathrm{K} 11$ and $\mathrm{K} 13$

- Test K14 was performed in order to evaluate the effect of people in the cabin of the aircraft. For this test, which measured the terminal voltage on the long wire, the pilot and copilot were in place 
in the cockpit and personnel were seated in cabin seats denoted $3 c, 3 d, 2 b$, and $3 b$. This result is compared to the result of Test K13 in Figure 6-26.

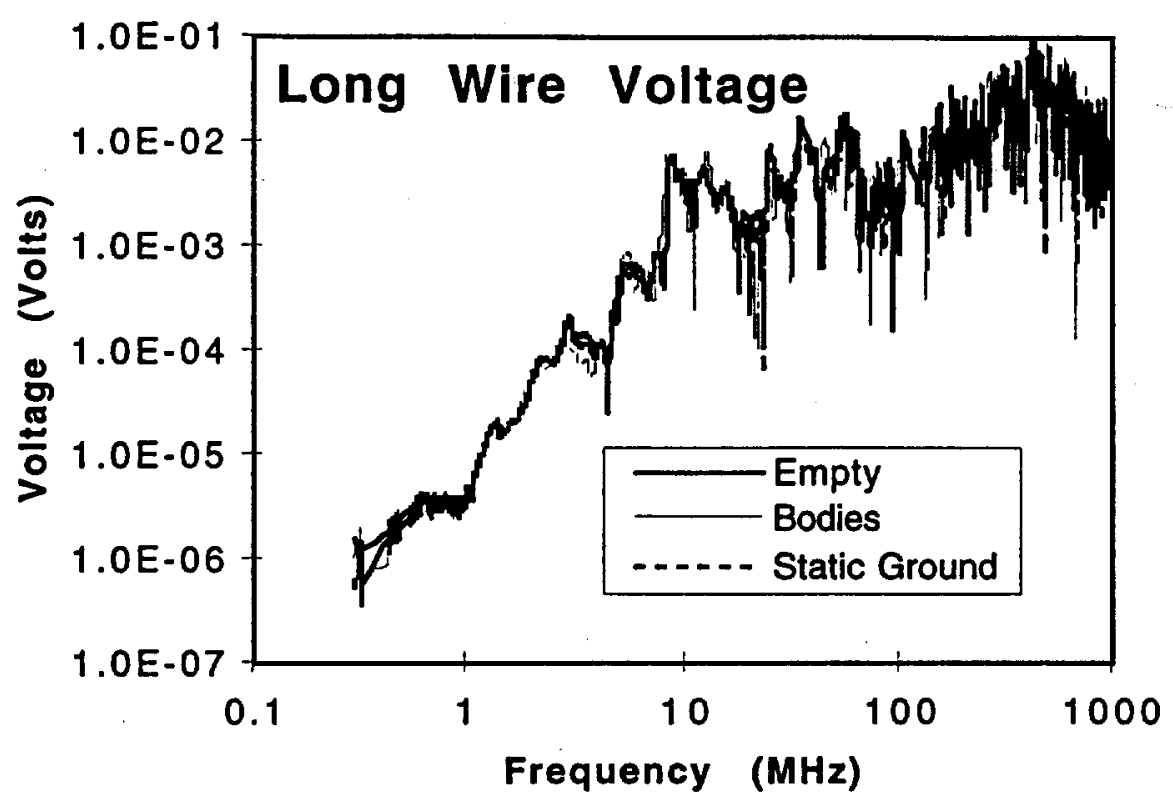

Figure 6-26. Long wire voltage under several conditions

- Test K26 was meant to evaluate the effect of a static ground on the terminal voltage of the cabin long wire. This static ground, which was ordinarily disconnected throughout the test program, was deliberately connected for this test. The grounding was effected by connecting a strap from the landing gear to a permanent ground hook at the LESLI facility. This result is also shown in Figure 6-26.

- Tests $\mathrm{K} 30, \mathrm{~K} 31$, and $\mathrm{K} 32$ were repeats of $\mathrm{J} 39, \mathrm{~J} 40$, and $\mathrm{J} 41$ but with the pilot and copilot seats in the mid position. During the J tests, the seats were in full forward position. Figure 6-27 shows a comparison of the cockpit axial field in the two cases in the form of a ratio of the two fields. The axial electric field probe is pointed directly at the back of the copilot seat and is in close proximity when the seat is at mid position. The size of the ratio emphasizes the importance of knowing configuration details.

- Tests K007 and J024 were performed to investigate the effect of the circuit breaker state on measurements. K007 was in a broadside incidence position and J024 was for nose on incidence. In both cases the breaker was engaged (in). 


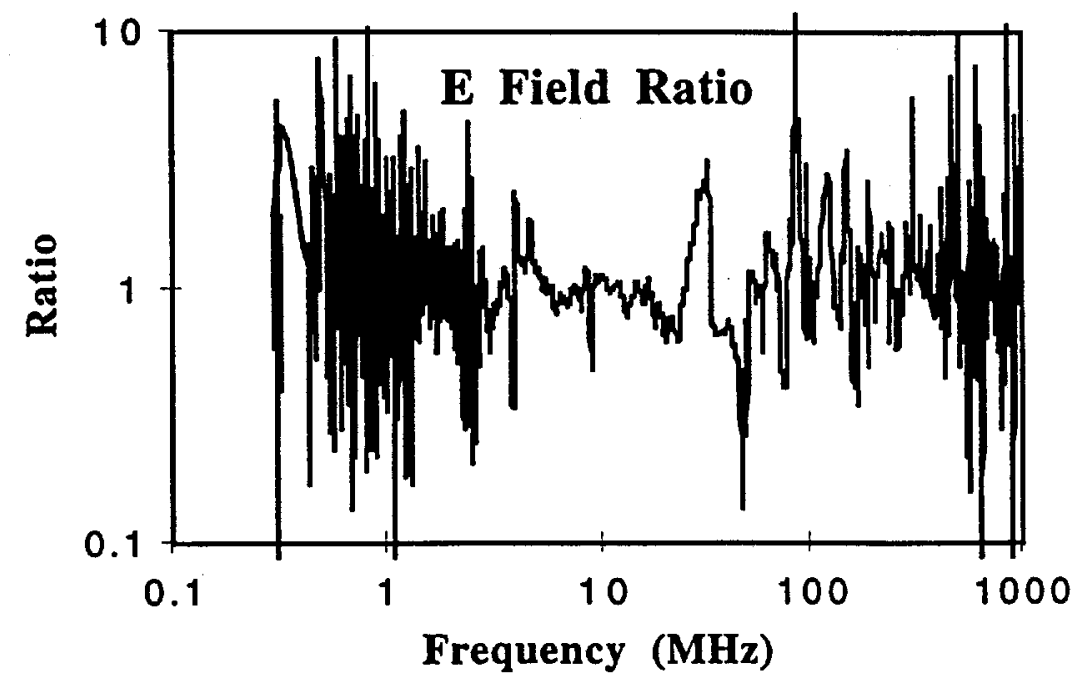

Figure 6-27. Ratio of axial $\mathrm{E}$ field with seat at mid and forward positions

\subsubsection{Test Series Z - Effects of System State}

During the execution of the on-the-ground tests, the configuration of the aircraft was maintained in a constant state except when deliberate modifications were introduced. Such test conditions are indicated throughout the test plan. During execution, circuit breakers were maintained in a known state. But, it was noted during a particular sequence that the state of a circuit breaker in the VHF transceiver power system was in an open condition while it had been expected to be in the closed condition as when the system was in operation. The effect of changes in this configuration state was a concern although future tests would also be executed in the breaker open state. A portion of Test Series $Z$ ( $Z 1$ through $Z 10$ ) was executed to assess the effects of changes in a single circuit breaker to the closed condition in a communication system power line. This circuit breaker was located in the cockpit panel and the transceiver was mounted in a rack in the E-bay thus indicating a power line for the system (and probably others) that was routed, at a minimum, through the cockpit and E-bay. Thus, this portion of Test Series $Z$ provides data that indicate the relative importance of knowing the state of such systems when performing such tests. Another portion of Test Series $Z$ was directed to evaluating the energy collecting capability of parasitic wires in the cockpit (Z11 and Z12), to assessing the effect of a connected static ground on the aircraft (Z13), and checking repeatability $(Z 14)$. The test conditions for Test Series $Z$ are found in Table 6-14 while the data identification is provided in Table 6-28.

The effects of the circuit breaker state were reasonably marked in the E-bay as seen in Figure 6-28 for the vertical electric field in the E-bay and Figure 6-29 for the voltage in the LRU (sensor RC7). The latter observation was made during the K Series where a special test referred to as "J024 brkr in" was performed as a repeat of Test J24 with the breaker out (open). The results are not totally surprising since the circuit breaker could open or complete the power line circuit to the LRU in question and could allow this power line to become a major source, in these tests, of conducted signals into the LRU. Note that in both cases the effects are most pronounced at low frequencies and even then are at low levels of the variables in question. 


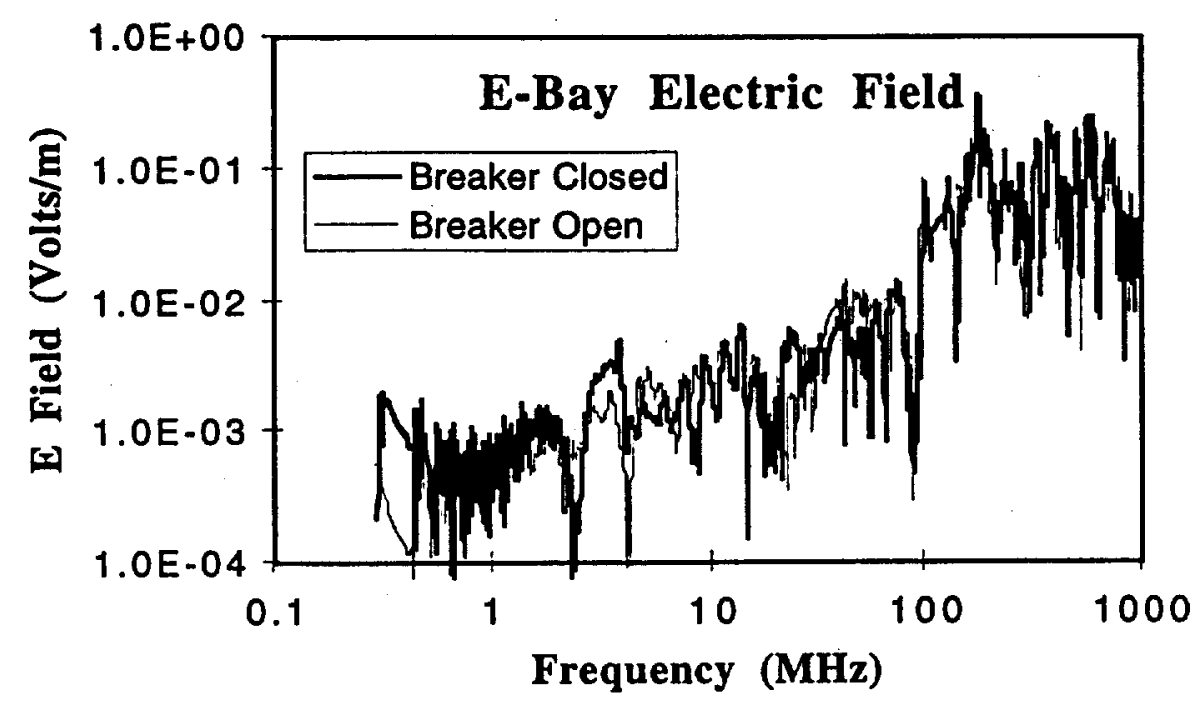

Figure 6-28. E-bay electric field dependency on circuit breaker state

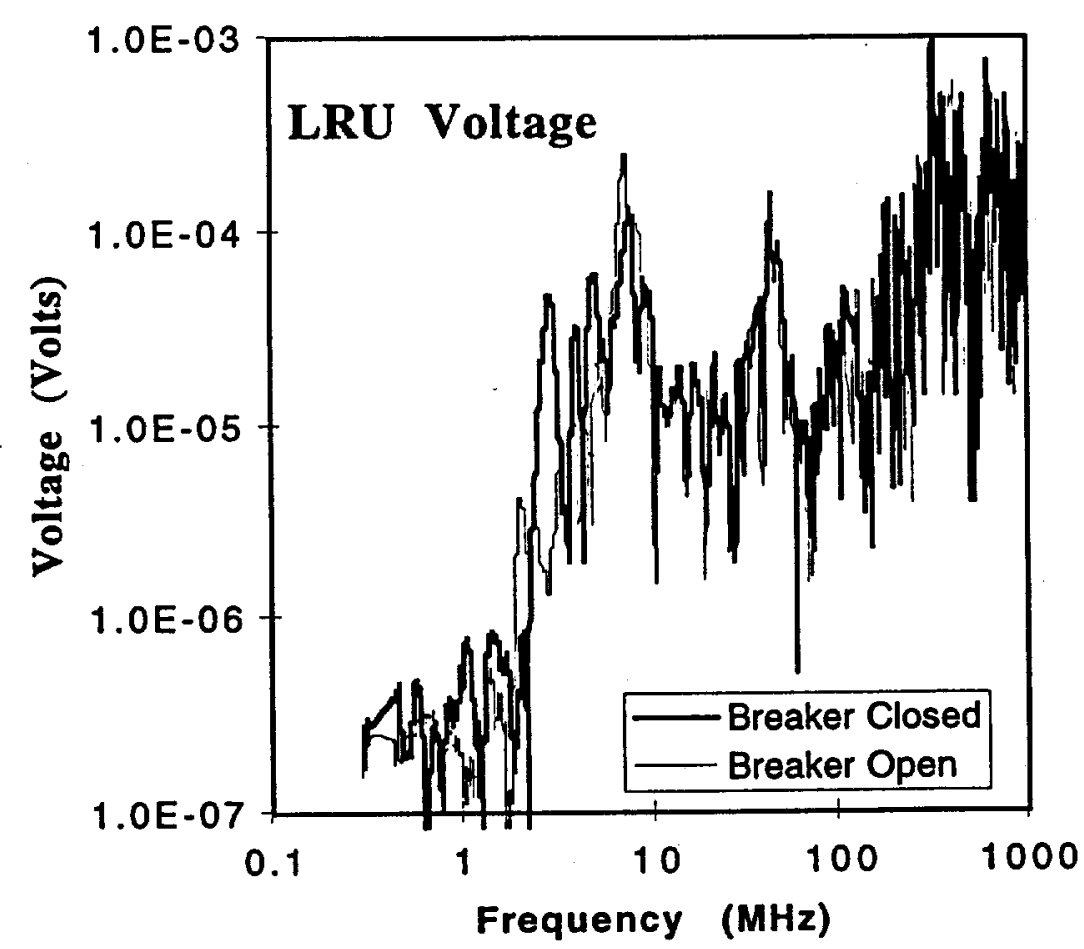

Figure 6-29. LRU voltage dependency on circuit breaker state 


\subsubsection{Horizontal Polarization}

Early planning of the experimental series did not include horizontal polarization due to the difficulties experienced by the Phillips Lab staff in earlier tests using the differential mode drive in the LESLI with the rhombic radiator. To generate the horizontal electric fields, a balun is used to provide the differential drive for the rhombic. The high power, high quality balun ordinarily used at LESLI for differential drive over a broad frequency range was inoperative. Also, the field behavior of the horizontal $\mathrm{E}$ field is quite poor in that the field is strongly horizontal (for pure differential mode current on the wires) only on the vertical plane of symmetry and is indeed vertical on the ground plane (if it were perfectly conducting) and at all points directly under the wires. The aircraft, in the nose-on configuration, has its fuselage illuminated by an electric field that has a predominantly horizontal. In response to requests by some analysts for qualitative measurements using horizontal polarization in the frequency range containing the lowest order resonances of the fuselage, a limited, somewhat ad hoc test series was conducted in the differential mode.

A substitute balun was procured albeit a rather narrowband device when compared to the sweep capability of the LESLI. The balun was rated by the manufacturer for operation in the 2.5 to 30 $\mathrm{MHz}$ range. This balun was not tested or characterized for the quality of its output in terms of the required relative phase difference in the current at its terminals and the stability of this quality over the frequency range. It was only tested for the spectral behavior of its impedance transformation characteristic. Time and resource constraints in conjunction with the relative value of the measurements given the concerns mentioned above led to a limited balun characterization. Overall then, the test conditions did not permit scientific quality measurements but only a qualitative "quick look" at typical responses in the twelve probes aboard the aircraft and a determination whether the fundamental aircraft mode is seen at the internal test points.

The reference field measurement was performed using a balanced magnetic field sensor (MGL-6) measuring the vertical component ( $y$-directed) of the magnetic field 3 meters above the pad and 15 meters from the feedpoint on the centerline i.e., at $(15,3,0)$.

The executed tests and the data identification for the Horizontal Polarization tests are found in Tables 6-15 and 6-29, respectively.

Some sample results for the tests are shown in Figures 6-29, 6-30, and 6-31. Several features of the data in these figures are notable. In Figure 6-30, it is apparent that the three components of the field in the cockpit are bounded on the topside by the transverse electric field. These fields exceed the other fields in the cabin and E-bay that are measured with an AD-60 probe. The voltage measurements in Figure 6-31 are dominated by the voltage at the feedpoint of the cabin long wire which is parallel to the fuselage with a length of 20 feet and suspended 12 inches below the upper inner surface of the fuselage. The LRU voltage measurement shows some sign of body resonances at low frequencies. Finally, it is seen in Figure 6-32 that the current in the E-bay on the windshield heater wire strongly dominates the current at other points. The magnitude of the dominance is remarkable and worthy of note to system designers as this current, which is probably excited in the cockpit, propagates effectively into the E-bay and can be the source of conducted and radiated coupling to systems in that enclosure. 


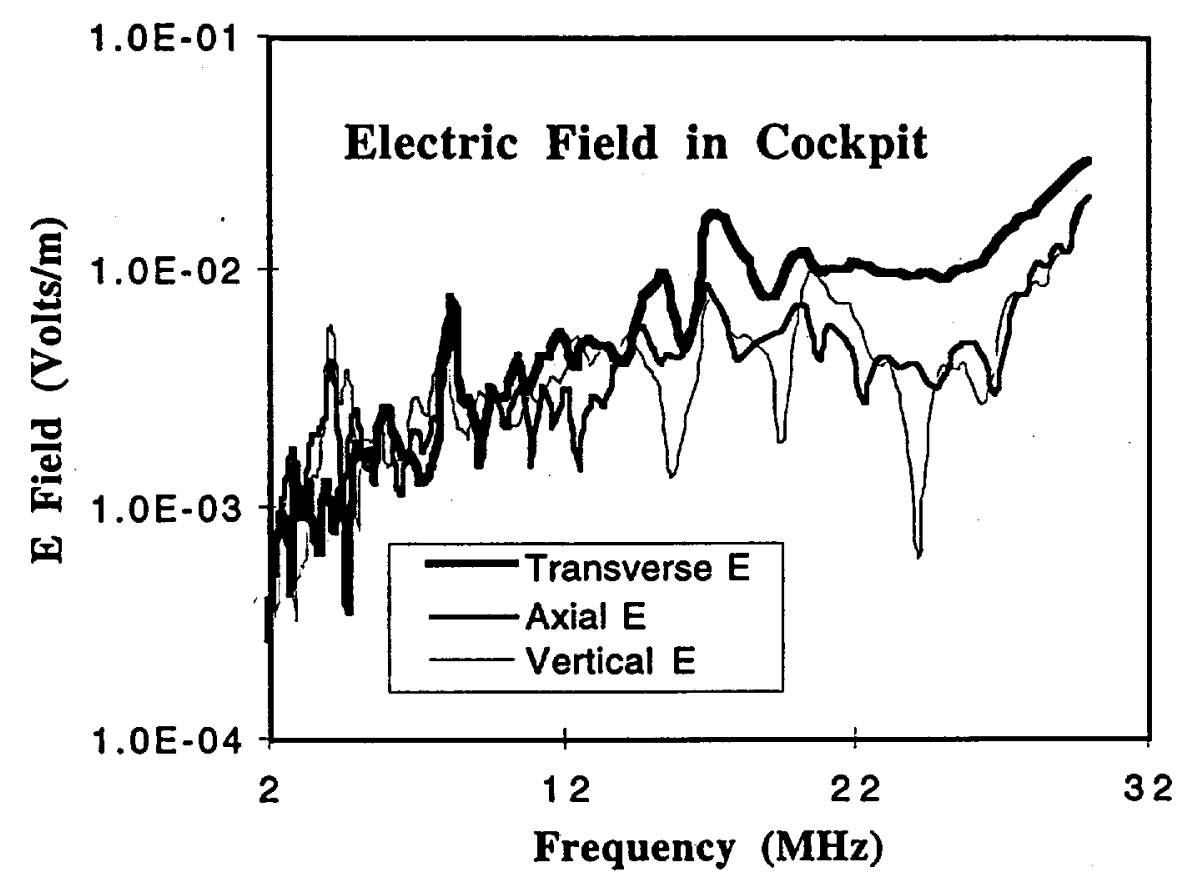

Figure 6-30. E Field in Cockpit under horizontally polarized excitation

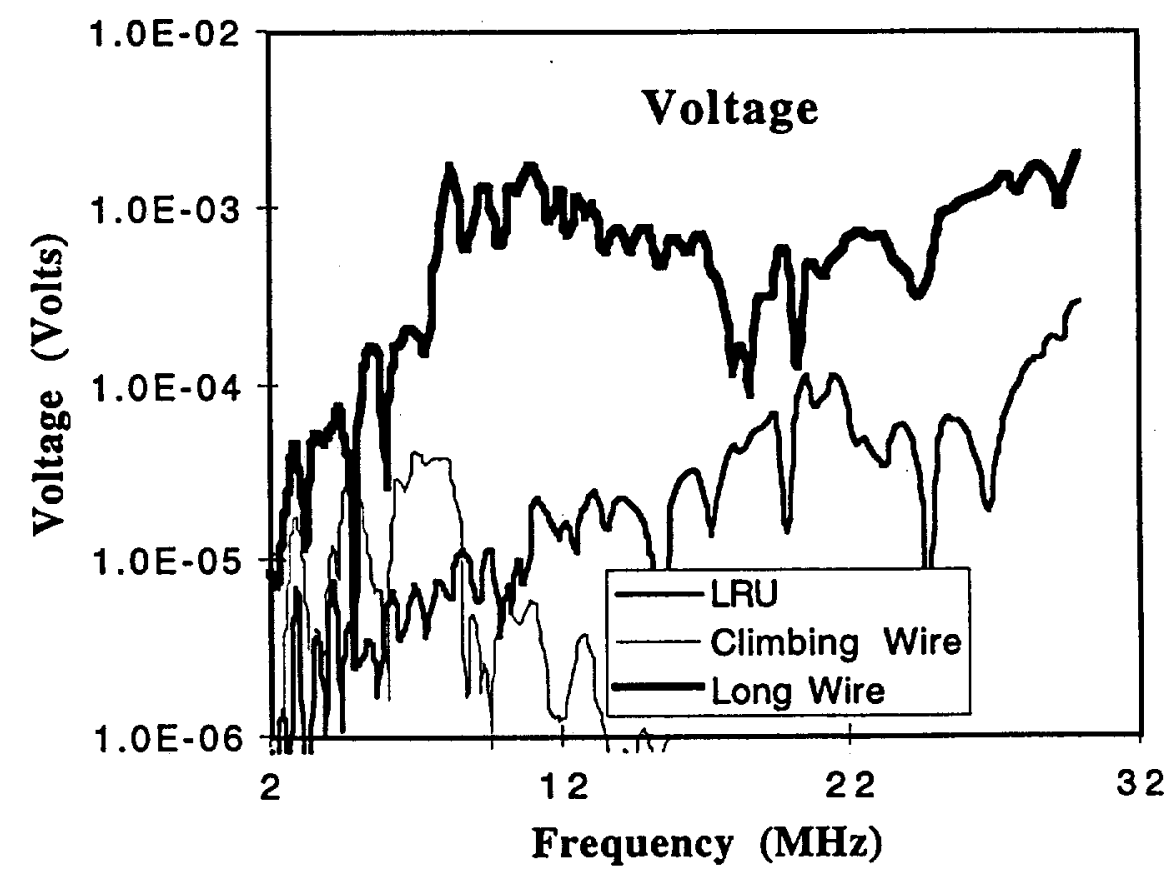

Figure 6-31. Voltage at select points under horizontal excitation 


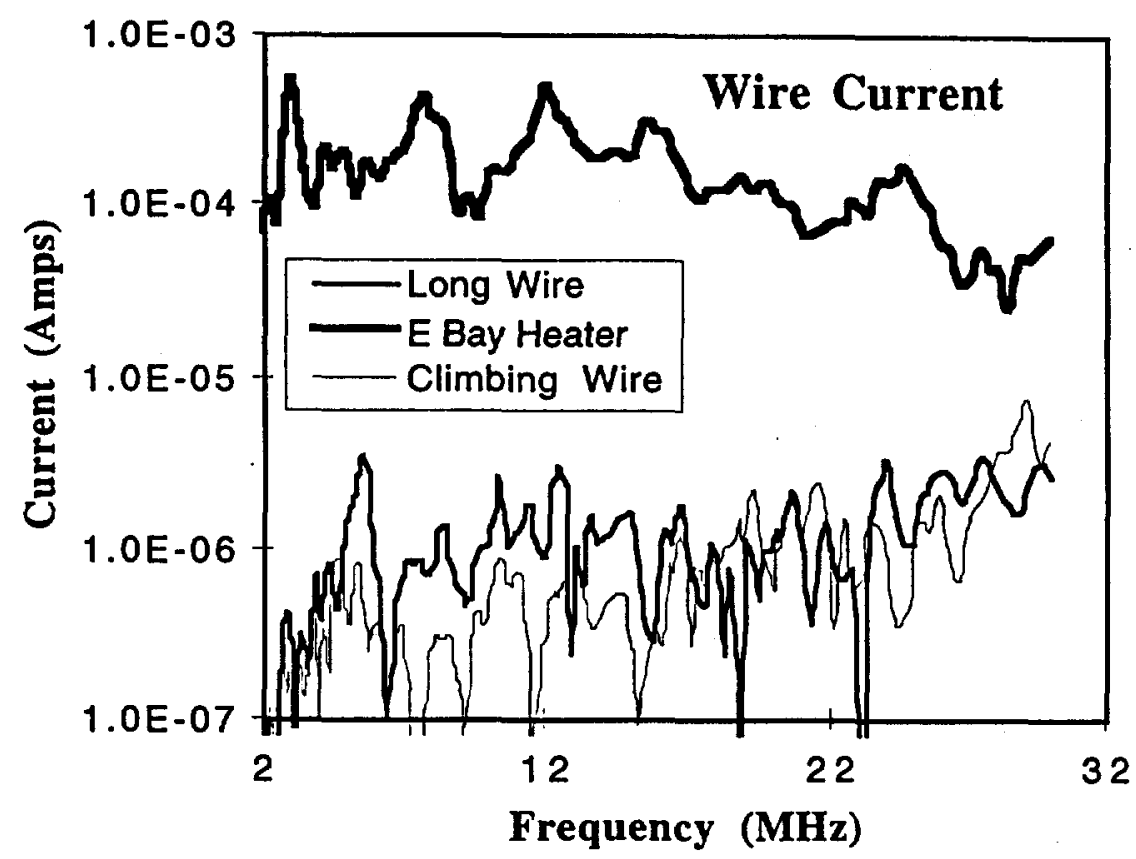

Figure 6-32. Current on certain wires under horizontal excitation

\subsubsection{Test Series WF - Windshield Shielding Effects}

The dominant port-of-entry (POE) for RF energy into the cockpit is often considered to be the aircraft windshield. To test this hypothesis, a test was suggested and executed wherein, in the nose-on configuration, the windshield of the cockpit was covered with aluminum foil and sealed with conducting copper tape. Data from the entire suite of probes were collected to enable comparison of measurements taken with and without the foil covering and estimation of the shielding afforded by the foil. The test conditions for the windshield covered test (Test Series WF - Window Shielded/Unshielded) are found in Table 6-16 while the data identification is found in Table 6-30.

Data comparisons are enabled by the data. For example, the effect of the aluminum foil shield can be assessed by comparing the results of tests in the WF Series and tests with the corresponding test conditions in Test Series J. As an example, one of the most marked differences in coupling in the two cases is illustrated by the vertical electric field in the cockpit. A comparison of the fields in the two cases is shown in Figure 6-33 where the differences are often $20 \mathrm{~dB}$ and should be an indicator to hardening engineers that reduction of this coupling path can yield significant benefits. The data have not been corrected for variations in the incident field. But, a comparison of the reference fields shows only small differences so that the resulting incident fields are sufficiently similar to allow qualitative comparisons. Of course, the reference fields are available so that corrections can be introduced to correct for the incident field variations. 


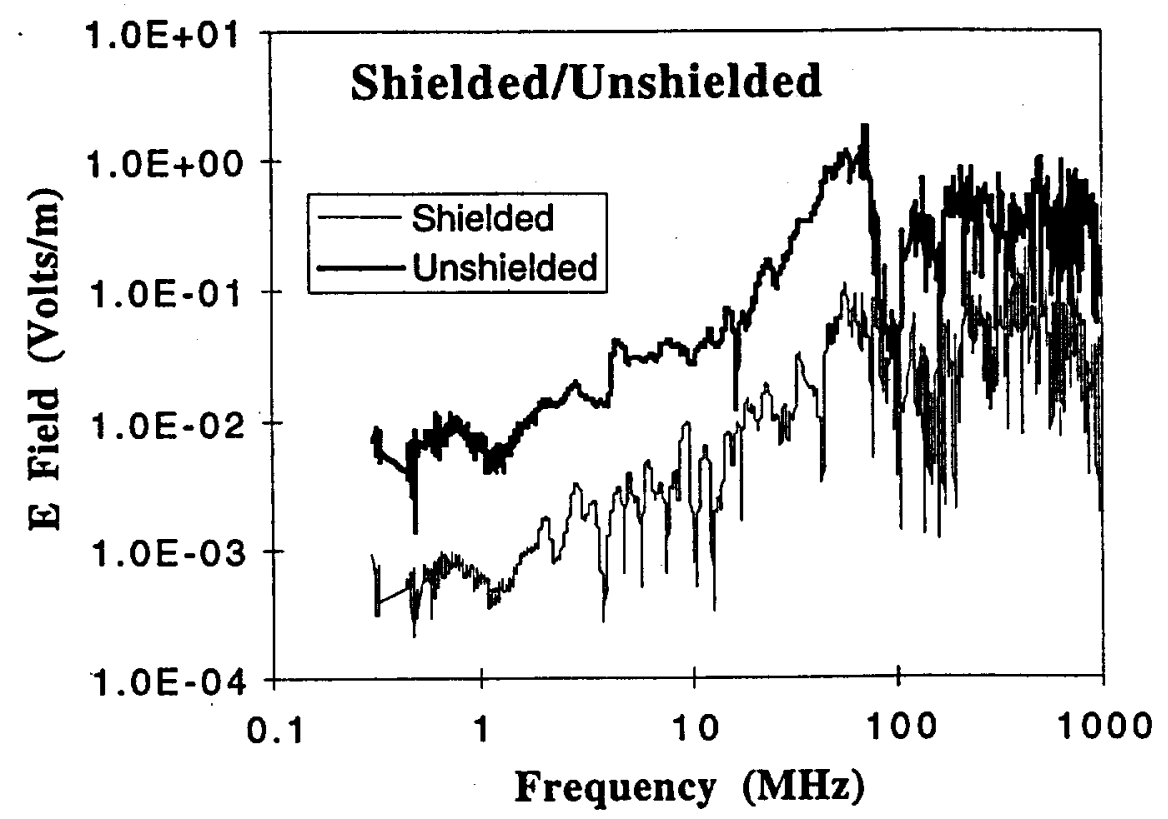

Figure 6-33. Comparison of the vertical electric field in the cockpit with the windshield uncovered and covered with aluminum foil

\title{
7.0 Data Availability
}

The complete data for the Low Power On-the-Ground Tests are available in digital format. The data files have been described in this document and the data ids provide a connection between the files and specific tests. A list of the available data files for each test series is found in Table 6-42.

The data in each file consists of a header as previously described and measured data, specifically, frequency and the real and imaginary part of the variable of interest. Arrangements for access to the data can be made through the funding organization NASA/LaRC which will also provide instructions on the steps required to electronically obtain the data. The point of contact is:

\author{
Karl J. Moeller :- \\ NASA Langley Research Center \\ Mail Code 490, Bldg. 1299 \\ Hampton, VA 23665-5225 \\ Voice: (804) 864-7673 \\ FAX: (804) 864-7975 \\ email: k.j.moeller@larc.nasa.gov
}


Table 6-42. Test Series and Available Data Files

\begin{tabular}{|c|c|c|c|c|c|c|}
\hline an & $\bar{a}$ & $\bar{b}$ & $\bar{c}$ & $\bar{d}$ & $\overline{\mathbf{e}}$ & $\overline{\mathbf{f}}$ \\
\hline $\begin{array}{l}\text { an00an01 } \\
\text { an00an02 } \\
\text { an00ref1 } \\
\text { an01an03 } \\
\text { an01ref1 } \\
\text { an02an04 } \\
\text { an02ref1 } \\
\text { bn00an01 } \\
\text { bn00an02 } \\
\text { bn00ref1 } \\
\text { bn01an03 } \\
\text { bn01ref1 } \\
\text { bn02an04 } \\
\text { bn02ref1 } \\
\text { mn00an01 } \\
\text { mn00an02 } \\
\text { mn00ref1 } \\
\text { mn01an03 } \\
\text { mn01ref1 } \\
\text { mn02an04 } \\
\text { mn02ref1 } \\
\text { mn02ref2 }\end{array}$ & $\begin{array}{l}\mathrm{c} 053 \mathrm{a} 009 \\
\mathrm{c} 053 \mathrm{ref} 1 \\
\mathrm{c} 054 \mathrm{a} 010 \\
\mathrm{c} 054 \mathrm{a} 011 \\
\mathrm{c} 054 \mathrm{ref} 1 \\
\mathrm{c} 055 \mathrm{a} 006 \\
\mathrm{c} 055 \mathrm{ref} 2 \\
\mathrm{c} 056 \mathrm{a} 003 \\
\mathrm{c} 056 \mathrm{a} 007 \\
\mathrm{c} 056 \mathrm{ref} 1 \\
\mathrm{c} 058 \mathrm{a} 004 \\
\mathrm{c} 058 \mathrm{a} 008 \\
\mathrm{c} 058 \mathrm{ref} 1 \\
\mathrm{c} 059 \mathrm{a} 001 \\
\mathrm{c} 059 \mathrm{a} 002 \\
\mathrm{c} 059 \mathrm{ref} 1 \\
\mathrm{c} 060 \mathrm{a} 101 \\
\mathrm{c} 060 \mathrm{a} 102 \\
\mathrm{c} 060 \mathrm{ref} 1 \\
\mathrm{c} 061 \mathrm{a} 005 \\
\mathrm{c} 061 \mathrm{a} 012 \\
\mathrm{c} 061 \mathrm{ref} 1 \\
\mathrm{c} 062 \mathrm{a} 014 \\
\mathrm{c} 062 \mathrm{ref} 2 \\
\mathrm{c} 063 \mathrm{a} 013 \\
\mathrm{c} 063 \mathrm{ref} 2\end{array}$ & $\begin{array}{l}\mathrm{c} 043 \mathrm{~b} 001 \\
\mathrm{c} 043 \mathrm{~b} 008 \\
\mathrm{c} 043 \mathrm{ref} 1 \\
\mathrm{c} 044 \mathrm{~b} 005 \\
\mathrm{c} 044 \mathrm{ref} 1 \\
\mathrm{c} 045 \mathrm{~b} 003 \\
\mathrm{c} 045 \mathrm{~b} 012 \\
\mathrm{c} 045 \mathrm{ref} 1 \\
\mathrm{c} 046 \mathrm{~b} 002 \\
\mathrm{c} 046 \mathrm{~b} 004 \\
\mathrm{c} 046 \mathrm{ref} 1 \\
\mathrm{c} 047 \mathrm{~b} 007 \\
\mathrm{c} 047 \mathrm{ref} 2 \\
\mathrm{c} 049 \mathrm{~b} 010 \\
\mathrm{c} 049 \mathrm{~b} 011 \\
\mathrm{c} 049 \mathrm{ref} 1 \\
\mathrm{c} 050 \mathrm{~b} 006 \\
\mathrm{c} 050 \mathrm{~b} 009 \\
\mathrm{c} 050 \mathrm{ref} 1 \\
\mathrm{c} 051 \mathrm{~b} 014 \\
\mathrm{c} 051 \mathrm{ref} 2 \\
\mathrm{c} 052 \mathrm{~b} 015 \\
\mathrm{c} 052 \mathrm{ref} 2 \\
\text { d001b013 } \\
\text { d001ref1 }\end{array}$ & $\begin{array}{l}\mathrm{e} 004 \mathrm{c} 001 \\
\mathrm{e} 004 \mathrm{c} 011 \\
\mathrm{e} 004 \mathrm{ref} 1 \\
\mathrm{e} 006 \mathrm{c} 005 \\
\mathrm{e} 006 \mathrm{c} 012 \\
\mathrm{e} 006 \mathrm{ref} 1 \\
\mathrm{e} 007 \mathrm{c} 003 \\
\mathrm{e} 007 \mathrm{c} 007 \\
\mathrm{e} 007 \mathrm{ref} 1 \\
\mathrm{e} 009 \mathrm{c} 004 \\
\mathrm{e} 009 \mathrm{c} 008 \\
\mathrm{e} 009 \mathrm{ref} 1 \\
\mathrm{e} 010 \mathrm{c} 002 \\
\mathrm{e} 010 \mathrm{ref} 2 \\
\mathrm{e} 012 \mathrm{c} 006 \\
\mathrm{e} 012 \mathrm{c} 010 \\
\mathrm{e} 012 \mathrm{ref} 1 \\
\mathrm{e} 013 \mathrm{c} 009 \\
\mathrm{e} 013 \mathrm{ref} 1 \\
\mathrm{e} 015 \mathrm{c} 014 \\
\mathrm{e} 015 \mathrm{c} 015 \\
\mathrm{e} 015 \mathrm{ref} 1 \\
\mathrm{e} 016 \mathrm{c} 109 \\
\mathrm{e} 016 \mathrm{c} 111 \\
\mathrm{e} 016 \mathrm{ref1} \\
\mathrm{e} 017 \mathrm{c} 104 \\
\mathrm{e} 017 \mathrm{ref} 1 \\
\mathrm{e} 018 \mathrm{c} 204 \\
\mathrm{e} 018 \mathrm{ref1} \\
\mathrm{e} 019 \mathrm{c} 304 \\
\mathrm{e} 019 \mathrm{ref} 1 \\
\mathrm{e} 020 \mathrm{c} 404 \\
\mathrm{e} 020 \mathrm{ref} 1 \\
\mathrm{f} 002 \mathrm{c} 013 \\
\mathrm{f} 002 \mathrm{ref} 1\end{array}$ & $\begin{array}{l}\text { e021dd009 } \\
\text { e021d011 } \\
\text { e021ref1 } \\
\text { e022d006 } \\
\text { e022d010 } \\
\text { e022ref1 } \\
\text { e024d003 } \\
\text { e024d007 } \\
\text { e024ref1 } \\
\text { e025d004 } \\
\text { e025d008 } \\
\text { e025ref1 } \\
\text { e027d001 } \\
\text { e027d002 } \\
\text { e027ref1 } \\
\text { e029d005 } \\
\text { e029d012 } \\
\text { e029ref1 } \\
\text { e031d013 } \\
\text { e031d014 } \\
\text { e031ref1 }\end{array}$ & $\begin{array}{l}\text { g014e008 } \\
\text { g014e010 } \\
\text { g014ref1 } \\
\text { g015e009 } \\
\text { g015e011 } \\
\text { g015ref1 } \\
\text { g020e005 } \\
\text { g020e006 } \\
\text { g020ref1 } \\
\text { g022e003 } \\
\text { g022e007 } \\
\text { g022ref1 } \\
\text { g023e004 } \\
\text { g023ref1 } \\
\text { g024e001 } \\
\text { g024e002 } \\
\text { g024ref1 } \\
\text { g026e004 } \\
\text { g026e012 } \\
\text { g026ref1 } \\
\text { h001e013 } \\
\text { h001ref1 }\end{array}$ & \begin{tabular}{|l} 
g002fo01 \\
g002f002 \\
g002ref1 \\
g003f010 \\
g003f012 \\
g003ref1 \\
g004f003 \\
g004f007 \\
g004ref1 \\
g005f004 \\
g005f008 \\
g005ref1 \\
g007f005 \\
g007f011 \\
g007ref1 \\
g009f006 \\
g009f009 \\
g009ref1 \\
g012f013 \\
g012f014 \\
g012ref1 \\
g013f008 \\
g013f010 \\
g013ref1
\end{tabular} \\
\hline
\end{tabular}


Table 6-42. Continued

\begin{tabular}{|c|c|c|c|c|c|c|}
\hline \multicolumn{2}{|c|}{ ni } & \multicolumn{5}{|c|}{$\mathbf{j}$} \\
\hline $\begin{array}{l}\text { a100ni01 } \\
\text { a100ni02 } \\
\text { a100ref1 } \\
\text { a101ni05 } \\
\text { a101ni12 } \\
\text { a101ref1 } \\
\text { a102ni03 } \\
\text { a102ni07 } \\
\text { a102ref1 } \\
\text { a103ni04 } \\
\text { a103ni08 } \\
\text { a103ref1 } \\
\text { a104ni09 } \\
\text { a104ni11 } \\
\text { a104ref1 } \\
\text { a105ni06 } \\
\text { a105ni10 } \\
\text { a105ref1 } \\
\text { b100ni01 } \\
\text { b100ni02 } \\
\text { b100ref1 } \\
\text { b101ni05 } \\
\text { b101ni12 } \\
\text { b101ref1 } \\
\text { b102ni03 } \\
\text { b102ni07 } \\
\text { b102ref1 } \\
\text { b103ni04 } \\
\text { b103ni08 } \\
\text { b103ref1 } \\
\text { b104ni09 } \\
\text { b104ni11 } \\
\text { b104ref1 } \\
\text { b105ni06 } \\
\text { b105ni10 } \\
\text { b105ref1 } \\
\text { m100ni01 } \\
\text { m100ni02 } \\
\text { m100ref1 } \\
\text { m101ni05 } \\
\text { m101ni12 } \\
\text { m101ref1 } \\
\text { m102ni03 } \\
\text { m102ni07 }\end{array}$ & $\begin{array}{l}\text { m102ref1 } \\
\text { m103ni04 } \\
\text { m103ni08 } \\
\text { m103ref1 } \\
\text { m104ni09 } \\
\text { m104ni11 } \\
\text { m104ref1 } \\
\text { m105ni06 } \\
\text { m105ni10 } \\
\text { m105ref1 }\end{array}$ & \begin{tabular}{|l} 
a106j020 \\
a106j021 \\
a106ref1 \\
a108j022 \\
a108j023 \\
a108ref1 \\
a109j004 \\
a109j024 \\
a109ref1 \\
a111j005 \\
a111j025 \\
a111ref1 \\
a120j006 \\
a120j007 \\
a120ref1 \\
a121j008 \\
a121ref1 \\
a122j009 \\
a122j010 \\
a122ref1 \\
a123j011 \\
a123ref1 \\
a125j019 \\
a125ref1 \\
a126j005 \\
a126ref1 \\
a127j029 \\
a128j030 \\
a129j031 \\
a130j028 \\
a131j027 \\
a132j026 \\
a133j032 \\
a133j034 \\
a133ref1 \\
a137j033 \\
a137j035 \\
a137ref1 \\
a138j036 \\
a138j037 \\
a138ref1 \\
a139j038 \\
a139ref1 \\
a140j039
\end{tabular} & $\begin{array}{l}\text { a140j040 } \\
\text { a140ref1 } \\
\text { a141j041 } \\
\text { a141ref1 } \\
\text { a161j024 } \\
\text { a161ref1 } \\
\text { a164j026 } \\
\text { a165j027 } \\
\text { a167j028 } \\
\text { b106j020 } \\
\text { b106j021 } \\
\text { b106ref1 } \\
\text { b108j022 } \\
\text { b108j023 } \\
\text { b108ref1 } \\
\text { b109j004 } \\
\text { b109j024 } \\
\text { b109ref1 } \\
\text { b111j005 } \\
\text { b111j025 } \\
\text { b111ref1 } \\
\text { b112j012 } \\
\text { b112ref1 } \\
\text { b120j006 } \\
\text { b120j007 } \\
\text { b120ref1 } \\
\text { b121j008 } \\
\text { b121ref1 } \\
\text { b122j009 } \\
\text { b122j010 } \\
\text { b122ref1 } \\
\text { b123j011 } \\
\text { b123ref1 } \\
\text { b125j019 } \\
\text { b125ref1 } \\
\text { b126j005 } \\
\text { b126ref1 } \\
\text { b127j029 } \\
\text { b128j030 } \\
\text { b129j031 } \\
\text { b130j028 } \\
\text { b131j027 } \\
\text { b132j026 } \\
\text { b133j032 }\end{array}$ & $\begin{array}{l}\text { b133j034 } \\
\text { b133ref1 } \\
\text { b137j033 } \\
\text { b137j035 } \\
\text { b137ref1 } \\
\text { b138j036 } \\
\text { b138j037 } \\
\text { b138ref1 } \\
\text { b139j038 } \\
\text { b139ref1 } \\
\text { b140j039 } \\
\text { b140j040 } \\
\text { b140ref1 } \\
\text { b141j041 } \\
\text { b141ref1 } \\
\text { b161j024 } \\
\text { b161ref1 } \\
\text { b164j026 } \\
\text { b165j027 } \\
\text { b167j028 } \\
\text { m106j020 } \\
\text { m106j021 } \\
\text { m106ref1 } \\
\text { m108j022 } \\
\text { m108j023 } \\
\text { m108ref1 } \\
\text { m109j004 } \\
\text { m109j024 } \\
\text { m109ref1 } \\
\text { m111j005 } \\
\text { m111j025 } \\
\text { m111ref1 } \\
\text { m116j013 } \\
\text { m116j014 } \\
\text { m116ref1 } \\
\text { m116ref2 } \\
\text { m117j015 } \\
\text { m117ref1 } \\
\text { m118j016 } \\
\text { m118j017 } \\
\text { m118ref1 } \\
\text { m118ref2 } \\
\text { m119j018 } \\
\text { m119ref1 }\end{array}$ & $\begin{array}{l}\text { m120j006 } \\
\text { m120j007 } \\
\text { m120ref1 } \\
\text { m121j008 } \\
\text { m121ref1 } \\
\text { m122j009 } \\
\text { m122j010 } \\
\text { m122ref1 } \\
\text { m123j011 } \\
\text { m123ref1 } \\
\text { m125j019 } \\
\text { m125ref1 } \\
\text { m126j005 } \\
\text { m126ref1 } \\
\text { m127j029 } \\
\text { m127ref2 } \\
\text { m128j030 } \\
\text { m128ref2 } \\
\text { m129j031 } \\
\text { m129ref2 } \\
\text { m130j028 } \\
\text { m130ref2 } \\
\text { m131j027 } \\
\text { m131ref2 } \\
\text { m132j026 } \\
\text { m132ref2 } \\
\text { m133j032 } \\
\text { m133j034 } \\
\text { m133ref1 } \\
\text { m137j033 } \\
\text { m137j035 } \\
\text { m137ref1 } \\
\text { m138j036 } \\
\text { m138j037 } \\
\text { m138ref1 } \\
\text { m139j038 } \\
\text { m139ref1 } \\
\text { m140j039 } \\
\text { m140j040 } \\
\text { m140ref1 } \\
\text { m141j041 } \\
\text { m141ref1 } \\
\text { m161j024 } \\
\text { m161ref1 }\end{array}$ & $\begin{array}{l}\mathrm{m} 164 \mathrm{j} 026 \\
\mathrm{~m} 164 \mathrm{ref} 2 \\
\mathrm{~m} 165 \mathrm{j} 027 \\
\mathrm{~m} 165 \mathrm{ref} 2 \\
\mathrm{~m} 167 \mathrm{j} 028 \\
\mathrm{~m} 167 \mathrm{ref} 2\end{array}$ \\
\hline
\end{tabular}


Table 6-42. Continued

\begin{tabular}{|c|c|c|c|c|c|c|}
\hline \multicolumn{3}{|c|}{$\bar{k}$} & \multicolumn{2}{|c|}{$\mathbf{Z}$} & $\overline{h p}$ & $\overline{\text { wf }}$ \\
\hline $\begin{array}{l}\text { a142k001 } \\
\text { a142k011 } \\
\text { a142ref1 } \\
\text { a144k003 } \\
\text { a144k006 } \\
\text { a144refl } \\
\text { a145k004 } \\
\text { a145k007 } \\
\text { a145ref1 } \\
\text { a146k007 } \\
\text { a147k005 } \\
\text { a147k008 } \\
\text { a147ref1 } \\
\text { a149k002 } \\
\text { a149k009 } \\
\text { a149refl } 1 \\
\text { a151k010 } \\
\text { a151k012 } \\
\text { a151ref1 } \\
\text { a152k013 } \\
\text { a152k025 } \\
\text { a152ref1 } \\
\text { a153k015 } \\
\text { a154k023 } \\
\text { a154ref1 } \\
\text { a155k024 } \\
\text { a155ref1 } \\
\text { a156k014 } \\
\text { a158k026 } \\
\text { a159k030 } \\
\text { a159k031 } \\
\text { a159ref1 } \\
\text { a160k032 } \\
\text { a160ref1 } \\
\text { a161k029 } \\
\text { a161ref1 } \\
\text { a162k027 } \\
\text { a162k028 } \\
\text { a162ref1 } \\
\text { b142k001 } \\
\text { b142k011 } \\
\text { b142ref1 } \\
\text { b144k003 } \\
\text { b144k006 }\end{array}$ & $\begin{array}{l}\text { b144ref1 } \\
\text { b145k004 } \\
\text { b145k007 } \\
\text { b145ref1 } \\
\text { b146k007 } \\
\text { b147k005 } \\
\text { b147k008 } \\
\text { b147ref1 } \\
\text { b149k002 } \\
\text { b149k009 } \\
\text { b149ref1 } \\
\text { b151k010 } \\
\text { b151k012 } \\
\text { b151ref1 } \\
\text { b152k013 } \\
\text { b152k025 } \\
\text { b152ref1 } \\
\text { b153k015 } \\
\text { b154k023 } \\
\text { b154ref1 } \\
\text { b155k024 } \\
\text { b155ref1 } \\
\text { b156k014 } \\
\text { b158k026 } \\
\text { b159k030 } \\
\text { b159k031 } \\
\text { b159ref1 } \\
\text { b160k032 } \\
\text { b160ref1 } \\
\text { b161k029 } \\
\text { b161ref1 } \\
\text { b162k027 } \\
\text { b162k028 } \\
\text { b162ref1 } \\
\text { m142k001 } \\
\text { m142k011 } \\
\text { m142ref1 } \\
\text { m144k003 } \\
\text { m144k006 } \\
\text { m144ref1 } \\
\text { m145k004 } \\
\text { m145k007 } \\
\text { m145ref1 } \\
\text { m146k007 }\end{array}$ & $\begin{array}{l}\text { m146ref2 } \\
\text { m147k005 } \\
\text { m147k008 } \\
\text { m147ref1 } \\
\text { m149k002 } \\
\text { m149k009 } \\
\text { m149ref1 } \\
\text { m151k010 } \\
\text { m151k012 } \\
\text { m151ref1 } \\
\text { m152k013 } \\
\text { m152k025 } \\
\text { m152ref1 } \\
\text { m153k015 } \\
\text { m153ref2 } \\
\text { m154k023 } \\
\text { m154ref1 } \\
\text { m155k024 } \\
\text { m155ref1 } \\
\text { m156k014 } \\
\text { m156ref2 } \\
\text { m158k026 } \\
\text { m158ref2 } \\
\text { m159k030 } \\
\text { m159k031 } \\
\text { m159ref1 } \\
\text { m160k032 } \\
\text { m160ref1 } \\
\text { m161k029 } \\
\text { m161ref1 } \\
\text { m162k027 } \\
\text { m162k028 } \\
\text { m162ref1 }\end{array}$ & \begin{tabular}{|l} 
a173ref1 \\
a173z001 \\
a173z002 \\
a174ref1 \\
a174z003 \\
a174z007 \\
a176ref1 \\
a176z005 \\
a177ref1 \\
a177z004 \\
a178ref1 \\
a178z006 \\
a178z010 \\
a179ref1 \\
a179z008 \\
a180ref1 \\
a180z009 \\
a181z013 \\
a182ref1 \\
a182z011 \\
a183ref1 \\
a183z012 \\
a184ref1 \\
a184z014 \\
b173ref1 \\
b173z001 \\
b173z002 \\
b174ref1 \\
b174z003 \\
b174z007 \\
b176ref1 \\
b176z005 \\
b177ref1 \\
b177z004 \\
b178ref1 \\
b178z006 \\
b178z010 \\
b179ref1 \\
b179z008 \\
b180ref1 \\
b180z009 \\
b181z013 \\
b182ref1 \\
b182z011
\end{tabular} & $\begin{array}{l}\text { b183ref1 } \\
\text { b183z012 } \\
\text { b184ref1 } \\
\text { b184z014 } \\
\text { m173ref1 } \\
\text { m173z001 } \\
\text { m173z002 } \\
\text { m174ref1 } \\
\text { m174z003 } \\
\text { m174z007 } \\
\text { m176ref1 } \\
\text { m176z005 } \\
\text { m177ref1 } \\
\text { m177z004 } \\
\text { m178ref1 } \\
\text { m178z006 } \\
\text { m178z010 } \\
\text { m179ref1 } \\
\text { m179z008 } \\
\text { m180ref1 } \\
\text { m180z009 } \\
\text { m181ref2 } \\
\text { m181z013 } \\
\text { m182ref1 } \\
\text { m182z011 } \\
\text { m183ref1 } \\
\text { m183z012 } \\
\text { m184ref1 } \\
\text { m184z014 }\end{array}$ & $\begin{array}{l}\text { n010hp01 } \\
\text { n010hp02 } \\
\text { n010ref1 } \\
\text { n017hp04 } \\
\text { n017hp07 } \\
\text { n017ref1 } \\
\text { n018hp03 } \\
\text { n018hp06 } \\
\text { n018ref1 } \\
\text { n019hp05 } \\
\text { n019hp08 } \\
\text { n019ref1 } \\
\text { n020hp09 } \\
\text { n020hp11 } \\
\text { n020ref1 } \\
\text { n021hp10 } \\
\text { n021hp12 } \\
\text { n021ref1 } \\
\text { n022hp13 } \\
\text { n022hp14 } \\
\text { n022ref1 } \\
\text { b171wf05 } \\
\text { b172ref1 } \\
\text { b172wf07 } \\
\text { m168ref1 } \\
\text { m168wf01 } \\
\text { m168wf02 } \\
\text { m169ref1 } \\
\text { m169wf03 } \\
\text { m169wf06 } \\
\text { m170ref1 } \\
\text { m170wf04 } \\
\text { m171ref1 } \\
\text { m171wf05 } \\
\text { m172ref1 } \\
\text { m172wf07 }\end{array}$ & \begin{tabular}{|l} 
a168ref1 \\
a168wf01 \\
a168wf02 \\
a169ref1 \\
a169wf03 \\
a169wf06 \\
a170ref1 \\
a170wf04 \\
a171ref1 \\
a171wf05 \\
a172ref1 \\
a172wf07 \\
b168ref1 \\
b168wf01 \\
b168wf02 \\
b169ref1 \\
b169wf03 \\
b169wf06 \\
b170ref1 \\
b170wf04 \\
b171ref1
\end{tabular} \\
\hline
\end{tabular}


Table 6-42. Continued

\begin{tabular}{|c|c|c|c|c|c|}
\hline $25 \mathrm{MHz} \mathrm{L}$ & pole Map & $172 \mathrm{MHz}$ & Tipole Map & $430 \mathrm{MHz}$ & Dipole Map \\
\hline $\begin{array}{l}\text { c003dfmd } \\
\text { c003dref } \\
\text { c005dfmd } \\
\text { c005dref } \\
\text { c007dfmd } \\
\text { c007dref } \\
\text { c008dfmd } \\
\text { c008dref } \\
\text { c009dfmd } \\
\text { c009dref } \\
\text { c010dfmd } \\
\text { c010dref } \\
\text { c012dfmf } \\
\text { c012dref } \\
\text { c014dfme } \\
\text { c014dref } \\
\text { c018dfmf } \\
\text { c018dref } \\
\text { c020dfmf } \\
\text { c020dref } \\
\text { c021dfmr } \\
\text { c021dref } \\
\text { c023dfmr } \\
\text { c023dref } \\
\text { c024dfmr } \\
\text { c024dref } \\
\text { c025dfmq } \\
\text { c025dref } \\
\text { c027dfmq } \\
\text { c027dref } \\
\text { c028dfmq } \\
\text { c028dref } \\
\text { c029dfmb } \\
\text { c029dref } \\
\text { c030dfmc } \\
\text { c030dref } \\
\text { c031dfmb } \\
\text { c031dref } \\
\text { c034dfmb } \\
\text { c034dref } \\
\text { c036dfma } \\
\text { c036dref } \\
\text { c037dfma } \\
\text { c037dref }\end{array}$ & $\begin{array}{l}\text { c038dfma } \\
\text { c038dref } \\
\text { c039dfma } \\
\text { c039dref } \\
\text { c040dfma } \\
\text { c040dref } \\
\text { c041dfma } \\
\text { c041dref }\end{array}$ & $\begin{array}{l}\text { e032dfmj } \\
\text { e032dref } \\
\text { e033dfmj } \\
\text { e033dref } \\
\text { e034dfmj } \\
\text { e034dref } \\
\text { e035dfmj } \\
\text { e035dref } \\
\text { e037dfmj } \\
\text { e037dref } \\
\text { e038dfmj } \\
\text { e038dref } \\
\text { e039dfmt } \\
\text { e039dref } \\
\text { e041dfmt } \\
\text { e041dref } \\
\text { e043dfmt } \\
\text { e043dref } \\
\text { e044dfms } \\
\text { e044dref } \\
\text { e046dfms } \\
\text { e046dref } \\
\text { e048dfms } \\
\text { e048dref } \\
\text { e049dfmk } \\
\text { e049dref } \\
\text { e050dfmk } \\
\text { e050dref } \\
\text { e051dfmk } \\
\text { e051dref } \\
\text { e054dfmh } \\
\text { e054dref } \\
\text { e055dfmh } \\
\text { e055dref } \\
\text { e057dfmh } \\
\text { e057dref } \\
\text { e058dfmg } \\
\text { e058dref } \\
\text { e060dfmg } \\
\text { e060dref } \\
\text { e062dfmg } \\
\text { e062dref } \\
\text { e063dfmg } \\
\text { e063dref }\end{array}$ & $\begin{array}{l}\text { e065dfmg } \\
\text { e065dref } \\
\text { e066dfmg } \\
\text { e066dref }\end{array}$ & $\begin{array}{l}\text { g029dfml } \\
\text { g029dref } \\
\text { g031dfml } \\
\text { g031dref } \\
\text { g033dfml } \\
\text { g033dref } \\
\text { g034dfml } \\
\text { g034dref } \\
\text { g035dfml } \\
\text { g035dref } \\
\text { g036dfml } \\
\text { g036dref } \\
\text { g038dfmm } \\
\text { g038dref } \\
\text { g039dfmm } \\
\text { g039dref } \\
\text { g040dfmm } \\
\text { g040dref } \\
\text { g041dfmp } \\
\text { g041dref } \\
\text { g042dfmp } \\
\text { g042dref } \\
\text { g043dfmp } \\
\text { g043dref } \\
\text { g045dfmw } \\
\text { g045dref } \\
\text { g046dfmw } \\
\text { g046dref } \\
\text { g047dfmw } \\
\text { g047dref } \\
\text { g048dfmu } \\
\text { g048dref } \\
\text { g049dfmu } \\
\text { g049dref } \\
\text { g051dfmu } \\
\text { g051dref } \\
\text { g053dfmn } \\
\text { g053dref } \\
\text { g054dfmn } \\
\text { g054dref } \\
\text { g055dfmn } \\
\text { g055dref } \\
\text { g056dfmn } \\
\text { g056dref }\end{array}$ & $\begin{array}{l}\text { g057dfmn } \\
\text { g057dref } \\
\text { g058dfmn } \\
\text { g058dref }\end{array}$ \\
\hline
\end{tabular}


Table 6-42. Continued

\begin{tabular}{|c|c|c|c|c|}
\hline \multicolumn{3}{|c|}{ Rhombic Field Map } & Calibration & \\
\hline $\begin{array}{l}\mathrm{m} 00910 \mathrm{cl} \\
\mathrm{m} 0097 \mathrm{fmp} \\
\mathrm{m} 01010 \mathrm{cl} \\
\mathrm{m} 01110 \mathrm{cl} \\
\mathrm{m} 0117 \mathrm{fmp} \\
\mathrm{m} 01210 \mathrm{cl} \\
\mathrm{m} 0127 \mathrm{fmq} \\
\mathrm{m} 01310 \mathrm{cl} \\
\mathrm{m} 0137 \mathrm{fmq} \\
\mathrm{m} 01410 \mathrm{cl} \\
\mathrm{m} 0147 \mathrm{fmq} \\
\mathrm{m} 01510 \mathrm{cl} \\
\mathrm{m} 0157 \mathrm{fmm} \\
\mathrm{m} 01610 \mathrm{cl} \\
\mathrm{m} 0167 \mathrm{fmm} \\
\mathrm{m} 04110 \mathrm{cl} \\
\mathrm{m} 0417 \mathrm{fmc} \\
\mathrm{m} 04210 \mathrm{cl} \\
\mathrm{m} 0427 \mathrm{fmc} \\
\mathrm{m} 04310 \mathrm{cl} \\
\mathrm{m} 0437 \mathrm{fmc} \\
\mathrm{m} 04410 \mathrm{cl} \\
\mathrm{m} 0447 \mathrm{fme} \\
\mathrm{m} 04510 \mathrm{cl} \\
\mathrm{m} 0457 \mathrm{fme} \\
\mathrm{m} 04610 \mathrm{cl} \\
\mathrm{m} 0467 \mathrm{fme} \\
\mathrm{m} 04710 \mathrm{cl} \\
\mathrm{m} 0477 \mathrm{fmh} \\
\mathrm{m} 04810 \mathrm{cl} \\
\mathrm{m} 0487 \mathrm{fmh} \\
\mathrm{m} 04910 \mathrm{cl} \\
\mathrm{m} 0497 \mathrm{fmh} \\
\mathrm{m} 05010 \mathrm{cl} \\
\mathrm{m} 0507 \mathrm{fmm} \\
\mathrm{m} 05210 \mathrm{cl} \\
\mathrm{m} 0527 \mathrm{fmb} \\
\mathrm{m} 05310 \mathrm{cl} \\
\mathrm{m} 0537 \mathrm{fmb} \\
\mathrm{m} 05410 \mathrm{cl} \\
\mathrm{m} 0547 \mathrm{fmb} \\
\mathrm{m} 05510 \mathrm{cl} \\
\mathrm{m} 05610 \mathrm{cl} \\
\mathrm{m} 0567 \mathrm{fmd}\end{array}$ & $\begin{array}{l}\text { m05710cl } \\
\text { m0577fmd } \\
\text { m05810cl } \\
\text { m0587fmd } \\
\text { m05910cl } \\
\text { m0597fmd } \\
\text { m06010cl } \\
\text { m0607fmd } \\
\text { m06210cl } \\
\text { m0627ffmd } \\
\text { m06310cl } \\
\text { m06510cl } \\
\text { m0657fmg } \\
\text { m06610cl } \\
\text { m0667fmg } \\
\text { m06710cl } \\
\text { m0677fmg } \\
\text { m06810cl } \\
\text { m0687fmg } \\
\text { m07010cl } \\
\text { m0707fmg } \\
\text { m07110cl } \\
\text { m0717fmg } \\
\text { m07210cl } \\
\text { m0727fmk } \\
\text { m07310cl } \\
\text { m0737fmk } \\
\text { m07410cl } \\
\text { m0747fmk } \\
\text { m07610cl } \\
\text { m0767fmk } \\
\text { m07710cl } \\
\text { m0777fmk } \\
\text { m07810 cl } \\
\text { m0787ffmk } \\
\text { m07910cl } \\
\text { m0797ffml } \\
\text { m08010cl } \\
\text { m0807fml } \\
\text { m08110cl }\end{array}$ & $\begin{array}{l}\text { m0857fmn } \\
\text { m08610cl } \\
\text { m0867fmn } \\
\text { m08810cl } \\
\text { m0887fmj } \\
\text { m08910cl } \\
\text { m0897fmj } \\
\text { m09010cl } \\
\text { m0907fmj } \\
\text { m09110cl } \\
\text { m0917fmf } \\
\text { m09210cl } \\
\text { m0927ffmf } \\
\text { m09310cl } \\
\text { m0937fmf } \\
\text { m09410cl } \\
\text { m0947fma } \\
\text { m09510cl } \\
\text { m0957fma } \\
\text { m09610cl } \\
\text { m0967fma } \\
\text { m09810cl } \\
\text { m0987fmd } \\
\text { m09910cl } \\
\text { m0997fmj }\end{array}$ & $\begin{array}{l}\text { acd4.cal } \\
\text { ad60.cal } \\
\text { aprb.cal } \\
\text { b643.cal } \\
\text { b644.cal } \\
\text { b657.cal } \\
\text { c027.cal } \\
\text { c043.cal } \\
\text { c044.cal } \\
\text { c045.cal } \\
\text { c046.cal } \\
\text { c047.cal } \\
\text { c050.cal } \\
\text { c071.cal } \\
\text { mg16.cal } \\
\text { mg17.cal } \\
\text { nf20.cal } \\
\text { nf21.cal } \\
\text { nf22.cal } \\
\text { nf23.cal } \\
\text { nf40.cal } \\
\text { nf50.cal } \\
\text { nf51.cal } \\
\text { nf52.cal } \\
\text { nf53.cal } \\
\text { nf60.cal } \\
\text { p132.cal } \\
\text { p133.cal } \\
\text { p134.cal } \\
\text { p135.cal } \\
\text { ps68.cal } \\
\text { t1c1.cal } \\
\text { t1c2.cal } \\
\text { t1c3.cal } \\
\text { t1c4.cal } \\
\text { t2c1.cal } \\
\text { t2c2.cal } \\
\text { t2c3.cal } \\
\text { t2c4.cal } \\
\text { t3c1.cal } \\
\text { t3c2.cal } \\
\text { t3c3.cal } \\
\text { t3c4.cal } \\
\text { nistad60.cal }\end{array}$ & \\
\hline
\end{tabular}


Table 6-42. Continued

\begin{tabular}{|c|c|c|c|c|c|c|}
\hline \multicolumn{7}{|c|}{ Ground Plane Characterization Data } \\
\hline $\mathrm{m008gp02}$ & $\mathrm{m031gp24}$ & m053gp15 & m078gp39 & m116gp76 & p022ref1 & p045ref1 \\
\hline m008ref1 & m031refl & m053ref1 & m079gp40 & m117gp77 & p023gp17 & p046gp08 \\
\hline m009gp03 & m032gp25 & m054gp16 & $\mathrm{m} 080 \mathrm{gp} 41$ & $\mathrm{~m} 119 \mathrm{gp} 78$ & p023ref1 & p046ref1 \\
\hline m009ref1 & m032ref1 & m054ref1 & m081gp42 & $\mathrm{m} 120 \mathrm{gp} 79$ & p025gp 18 & p047gp09 \\
\hline m010gp04 & m033gp26 & m055gp17 & $\mathrm{m} 082 \mathrm{gp} 43$ & $\mathrm{~m} 121 \mathrm{gp} 80$ & p025ref1 & p047ref1 \\
\hline m010refl & m033refl & m055refl & m083gp44 & $\mathrm{m} 122 \mathrm{gp} 81$ & p026gp 19 & p048gp10 \\
\hline m011gp05 & m034gp27 & m056gp18 & m084gp45 & $\mathrm{m} 123 \mathrm{gp} 82$ & p026ref1 & p048ref 1 \\
\hline m011ref1 & m034ref1 & m056ref1 & m085gp46 & $\mathrm{m} 124 \mathrm{gp} 83$ & $\mathrm{p} 027 \mathrm{gp} 20$ & p049gp 11 \\
\hline $\mathrm{m012gp06}$ & m035gp28 & m057gp19 & $\mathrm{m} 086 \mathrm{gp} 47$ & $\mathrm{~m} 125 \mathrm{gp} 84$ & p027refl & p049ref 1 \\
\hline m012ref1 & m035refl & m057ref1 & m087gp48 & $\mathrm{m} 126 \mathrm{gp} 85$ & p028gp21 & p050gp12 \\
\hline m013gp07 & m036gp29 & m058gp20 & m087ref1 & $\mathrm{m} 127 \mathrm{gp} 86$ & p028ref1 & p050ref1 \\
\hline m013ref1 & m036ref1 & m058ref1 & m088gp49 & $\mathrm{m} 128 \mathrm{gp} 87$ & p029gp22 & p051gp13 \\
\hline m014gp08 & m037gp30 & $\mathrm{m059gp21}$ & m088ref1 & m129gp88 & p029ref1 & p051ref1 \\
\hline m014ref1 & m037ref1 & m059ref1 & m089gp50 & m130gp89 & p030gp23 & p052gp14 \\
\hline m015gp09 & m038gp05 & $\mathrm{m060gp22}$ & m089ref1 & m131gp90 & p030ref1 & p052ref1 \\
\hline m015ref1 & m038ref1 & m060ref1 & m090gp51 & p008gp02 & p031gp24 & p053gp 15 \\
\hline m016gp10 & m039gp06 & $\mathrm{m} 061 \mathrm{gp} 23$ & m090ref1 & p008ref1 & p031ref1 & p053ref1 \\
\hline m016ref1 & m039ref1 & m061ref1 & m091gp52 & $\mathrm{p} 009 \mathrm{gp} 03$ & p032gp25 & p054gp16 \\
\hline m017gp11 & $\mathrm{m040gp02}$ & $\mathrm{m} 062 \mathrm{gp} 24$ & m091ref1 & p009ref1 & p032ref1 & p054ref1 \\
\hline m017ref1 & m040ref1 & m062refl & $\mathrm{m} 092 \mathrm{gp} 53$ & p010gp04 & p033gp26 & p055gp17 \\
\hline $\mathrm{m018gp12}$ & m04lgp03 & m064gp25 & m092ref1 & p010ref1 & p033refl & p055ref 1 \\
\hline m018ref1 & m041ref1 & m064ref1 & m093gp54 & p011gp05 & p034gp27 & p056gp18 \\
\hline m019gp13 & $\mathrm{m} 042 \mathrm{gp} 04$ & $\mathrm{~m} 065 \mathrm{gp} 26$ & m093ref1 & p011ref1 & p034refl & p056ref1 \\
\hline m019ref1 & m042ref1 & m065refl & m094gp55 & p012gp06 & p035gp28 & p057gp19 \\
\hline $\mathrm{m020gp14}$ & $\mathrm{m043gp05}$ & m066gp27 & m095gp56 & p012ref1 & p035refl & p057ref1 \\
\hline m020refl & m043ref1 & m066ref1 & m096gp57 & p013gp07 & p036gp29 & p058gp20 \\
\hline $\mathrm{m021gp15}$ & m044gp06 & m067gp28 & m097gp58 & p013ref1 & p036ref1 & p058ref1 \\
\hline m021ref1 & m044ref1 & m067ref1 & m098gp59 & p014gp08 & p037gp30 & p059gp21 \\
\hline $\mathrm{m022gp16}$ & m045gp07 & m068gp29 & m099gp60 & p014ref1 & p037ref1 & p059ref1 \\
\hline m022refl & m045ref1 & m068ref1 & m101gp61 & p015gp09 & p038gp05 & p060gp22 \\
\hline m023gp17 & m046gp08 & m069gp30 & $\mathrm{m} 102 \mathrm{gp} 62$ & p015ref1 & p038refl & p060ref1 \\
\hline m023ref1 & m046ref1 & m069ref1 & m103gp63 & p016gp 10 & p039gp06 & p061gp23 \\
\hline $\mathrm{m} 025 \mathrm{gp} 18$ & m047gp09 & $\mathrm{m} 070 \mathrm{gp} 31$ & m104gp64 & p016ref1 & p039ref1 & p061ref1 \\
\hline m025ref1 & m047ref1 & $\mathrm{mo}$ & m105gp65 & $\mathrm{p} 017 \mathrm{gp} 11$ & p040gp02 & p062gp24 \\
\hline m026gp19 & m048gp10 & m071gp32 & m106gp66 & p017ref1 & p040ref1 & p062ref1 \\
\hline m026ref1 & m048ref1 & m071ref1 & m107gp67 & p018gp12 & p04lgp03 & p063gp25 \\
\hline $\mathrm{m} 027 \mathrm{gp} 20$ & m049gp11 & m072gp33 & m108gp68 & p018ref1 & p041ref1 & p063ref1 \\
\hline m027ref1 & m049ref1 & m072ref1 & m109gp69 & p019gp13 & p042gp04 & p064gp25 \\
\hline m028gp21 & $\mathrm{m} 050 \mathrm{gp} 12$ & m073gp34 & $\mathrm{ml} 10 \mathrm{gp} 70$ & p019refl & p042ref1 & p064ref1 \\
\hline m028ref1 & m050refl & m073ref1 & $\mathrm{m} 111 \mathrm{gp} 71$ & p020gp14 & p043gp05 & p065gp26 \\
\hline m029gp22 & m051gp13 & m074gp35 & $\mathrm{m} 112 \mathrm{gp} 72$ & p020ref1 & p043ref1 & p065ref1 \\
\hline m029ref1 & m051ref 1 & m075gp36 & $\mathrm{ml13gp73}$ & p021gp15 & $\mathrm{p} 044 \mathrm{gp} 06$ & p066gp27 \\
\hline $\mathrm{m} 030 \mathrm{gp} 23$ & $\mathrm{~m} 052 \mathrm{gp} 14$ & $\mathrm{~m} 076 \mathrm{gp} 37$ & $14 \mathrm{gp} 74$ & p021ref1 & p044ref1 & p066ref1 \\
\hline m030ref1 & m052ref1 & m077gp38 & $\mathrm{m} 115 \mathrm{gp} 75$ & p022gp16 & p045gp07 & p067gp28 \\
\hline
\end{tabular}


Table 6-42. Continued

\begin{tabular}{|c|c|c|c|c|c|c|}
\hline \multicolumn{7}{|c|}{ Ground Plane Characterization Data (continued) } \\
\hline p067ref1 & p089ref1 & p111ref1 & q010refl & q033ref1 & q055ref1 & q078ref1 \\
\hline p068gp29 & p090gp51 & p112gp72 & q011gp05 & q034gp27 & q056gp18 & $\mathrm{q} 079 \mathrm{gp} 40$ \\
\hline p068ref1 & p090ref1 & p112ref1 & q011refl & q034ref1 & q056ref1 & g079ref1 \\
\hline p069gp30 & p091gp52 & p113gp73 & q012gp06 & q035gp28 & q057gp19 & $\mathrm{q} 080 \mathrm{gp} 41$ \\
\hline p069ref1 & p091ref1 & pl13ref1 & q012refl & q035ref1 & q057ref1 & q080ref1 \\
\hline p070gp31 & p092gp53 & p114gp74 & q013gp07 & q036gp29 & q058gp20 & q081 gp42 \\
\hline p070ref1 & p092ref1 & p114ref1 & q013ref1 & q036ref1 & q058ref1 & q081ref1 \\
\hline p071gp32 & p093gp54 & p115gp75 & q014gp08 & q037gp30 & q059gp21 & q082gp43 \\
\hline p071refl & p093refl & p115ref1 & q014refl & q037ref1 & q059ref1 & q082ref1 \\
\hline p072gp33 & p094gp55 & p1 16gp76 & q015gp09 & q038gp05 & $\mathrm{q} 060 \mathrm{gp} 22$ & q083gp44 \\
\hline p072refl & p094ref1 & p116ref1 & q015refl & q038ref1 & q060ref1 & q083ref1 \\
\hline p073gp34 & p095gp56 & p117gp77 & $\mathrm{q} 016 \mathrm{gp} 10$ & q039gp06 & g061gp23 & q084gp45 \\
\hline p073ref1 & p095ref1 & p117ref1 & q016ref1 & q039ref1 & q061ref1 & q084ref1 \\
\hline p074gp35 & p096gp57 & p119gp78 & q017gp11 & $\mathrm{q} 040 \mathrm{gp} 02$ & q062gp24 & q085gp46 \\
\hline p074ref1 & p096ref1 & p119refl & q017refl & q040ref1 & q062ref1 & q085ref1 \\
\hline p075gp36 & p097gp58 & p120gp79 & q018gp 12 & q041gp03 & q064gp25 & q086gp47 \\
\hline p075ref1 & p097ref1 & p120refl & q018ref1 & q041ref1 & q064ref1 & q086ref1 \\
\hline p076gp37 & p098gp59 & p121gp80 & q019gp 13 & $\mathrm{q} 042 \mathrm{gp} 04$ & q065gp26 & q087gp48 \\
\hline p076ref1 & p098ref1 & p121ref 1 & q019ref1 & q042ref1 & q065ref1 & q087ref1 \\
\hline p077gp38 & p099gp60 & $\mathrm{p} 122 \mathrm{gp} 81$ & q020gp 14 & q043gp05 & q066gp27 & q088gp49 \\
\hline p077ref1 & p099ref1 & p122ref1 & q020ref1 & q043ref1 & q066ref1 & q088refl \\
\hline p078gp39 & p100gp61 & p123gp82 & q021gp 15 & $\mathrm{q} 044 \mathrm{gp} 06$ & $\mathrm{q} 067 \mathrm{gp} 28$ & q089gp50 \\
\hline p078ref1 & pl00ref1 & p123ref1 & q021refl & q044ref1 & q067refl & q089ref1 \\
\hline p079gp40 & p101gp61 & p124gp83 & q022gp16 & q045gp07 & q068gp29 & q090gp51 \\
\hline p079refl & p101ref1 & p124ref1 & q022ref1 & q045ref1 & q068ref1 & q090ref1 \\
\hline p080gp41 & p102gp62 & p125gp84 & q023gp17 & q046gp08 & q069gp30 & q091gp52 \\
\hline p080ref1 & p102refl & p125ref1 & q023refl & q046ref1 & q069ref1 & q091ref1 \\
\hline p08 lgp42 & p103gp63 & p126gp85 & q025gp18 & q047gp09 & q070gp31 & q092gp53 \\
\hline p081ref1 & p103ref1 & p126ref1 & q025ref1 & q047ref1 & q070ref1 & q092ref1 \\
\hline p082gp43 & p104gp64 & p127gp86 & q026gp19 & q048gp10 & q071gp32 & q093gp54 \\
\hline p082refl & p104ref1 & p127ref1 & q026ref1 & q048ref1 & q071refl & q093ref1 \\
\hline p083gp44 & p105gp65 & p128gp87 & $\mathrm{q} 027 \mathrm{gp} 20$ & q049gp11 & q072gp33 & q094gp55 \\
\hline p083ref1 & p105ref1 & p128refl & q027ref1 & q049ref1 & q072ref1 & q094ref1 \\
\hline $4 g p 45$ & p106gp66 & p129gp88 & q028gp21 & q050gp12 & q073gp34 & q095gp56 \\
\hline p084ref1 & p106ref1 & p129ref1 & q028ref1 & q050ref1 & q073ref1 & q095ref1 \\
\hline p085gp46 & p107gp67 & p130gp89 & q029gp22 & q051gp 13 & q074gp35 & q096gp57 \\
\hline p085ref1 & p107ref1 & p130ref1 & q029ref1 & q051ref1 & q074ref1 & q096ref1 \\
\hline p086gp47 & p108gp68 & p131gp90 & $\mathrm{q} 030 \mathrm{gp} 23$ & q052gp14 & q075gp36 & q097gp58 \\
\hline p086ref1 & p108ref1 & ref1 & q030ref1 & q052ref1 & q075ref1 & q097ref1 \\
\hline p087gp48 & p109gp69 & q008gp02 & q031gp24 & q053gp15 & q076gp37 & q098gp59 \\
\hline p087ref1 & p109refl & q008ref1 & q031ref1 & q053ref1 & q076refl & q098ref1 \\
\hline p088gp49 & gp70 & q009gp03 & q032gp 25 & q054gp16 & q077gp38 & q099gp60 \\
\hline $8 \mathrm{ref1}$ & Oref1 & q009ref1 & q032ref1 & q054ref1 & q077ref1 & q099ref1 \\
\hline p089gp50 & p11lgp71 & q010gp04 & q033gp26 & q055gp17 & q078gp39 & q101gp61 \\
\hline
\end{tabular}


Table 6-42. Continued

Ground Plane Characterization Data (continued)

\begin{tabular}{ll}
\hline q101ref1 & q124ref1 \\
q102gp62 & q125gp84 \\
q102ref1 & q125ref1 \\
q103gp63 & q126gp85 \\
q103ref1 & q126ref1 \\
q104gp64 & q127gp86 \\
q104ref1 & q127ref1 \\
q105gp65 & q128gp87 \\
q105ref1 & q128ref1 \\
q106gp66 & q129gp88 \\
q106ref1 & q129ref1 \\
q107gp67 & q130gp89 \\
q107ref1 & q130ref1 \\
q108gp68 & q131gp90 \\
q108ref1 & q131ref1 \\
q109gp69 & w070ref1 \\
q109ref1 & w071ref1 \\
q110gp70 & w072ref1 \\
q110ref1 & w073ref1 \\
q111gp71 & w074ref1 \\
q111ref1 & w075ref1 \\
q112gp72 & w076ref1 \\
q112ref1 & w077ref1 \\
q113gp73 & w078ref1 \\
q113ref1 & w079ref1 \\
q114gp74 & w080ref1 \\
q114ref1 & w081ref1 \\
q115gp75 & w082ref1 \\
q115ref1 & w083ref1 \\
q116gp76 & w084ref1 \\
q116ref1 & w085ref1 \\
q117gp77 & w086ref1 \\
q117ref1 & w087ref1 \\
q119gp78 & w088ref1 \\
q119ref1 & w089ref1 \\
q120gp79 & w090ref1 \\
q120ref1 & w091ref1 \\
q121gp80 & w092ref1 \\
q121ref1 & w093ref1 \\
q122gp81 & w094ref1 \\
q122ref1 & w095ref1 \\
q123ref1 & w097ref1 \\
\end{tabular}




\subsection{References}

Chang D. C., R. G. Olsen, "Excitation of an Infinite Antenna Above a Dissipative Earth," Radio Science, Vol. 10, No. 8,9, pp. 823-831, August-September 1975.

Johnk R. T., A. R. Ondrejka, "Time-Domain Calibration of D-Dot Sensors," National Institute of Standards and Technology (NIST) Electromagnetic Fields Division report, to be published 1996.

Kuester E. F., D. C. Chang, R. G. Olsen, "Modal Theory of Long Horizontal Wire Structures Above the Earth, 2, Properties of Discrete Modes," Radio Science, Vol. 13, No. 4, pp. 615-623, July-August 1978.

Millard, J. W., "NASA B-757 Aircraft Test Report", United International Engineering, Inc., UIE-TR-94-0025, 15 December 1994.

Olsen, R. G., E. F. Kuester, D. C. Chang, "Modal Theory of Long Horizontal Wire Structures Above the Earth, 1, Excitation," Radio Science, Vol. 13, No. 4, pp. 605-613, July-August 1978.

Poggio, A. J., R. A. Zacharias, S. T. Pennock, C. A. Avalle, R. M. Sharpe, K. Kunz, "NASA FBL/PBW Program, NASA Boeing 757 HIRF Test Plan, Low Power On-theGround Tests", October 1994.

Poggio, A. J. , G. J. Burke, S. T. Pennock, "The Determination of the Constitutive Parameters of a Medium With Application to a Reinforced Concrete Pad", NASA Contractor Report 4674, June 1995.

Randall, R. N., "CWDAS Version 6.4 User's Manual”, UIE-TR-91-0012, R2, UIE, Inc., Albuquerque, New Mexico, September 1993. 


\section{Appendix A}

\section{NASA FBL / PBW Program NASA Boeing 757 HIRF TEST PLAN Low Power On-the-Ground Tests}

Andrew Poggio

Richard Zacharias

Steven Pennock

Carlos Avalle

Robert Sharpe

Karl Kunz

Lawrence Livermore National Laboratory

P. O. 808, L-153

Livermore, CA 94550

HIRF Test Project Manager: Charles Meissnèr

NASA Langley Research Center 


\section{Table of Contents}

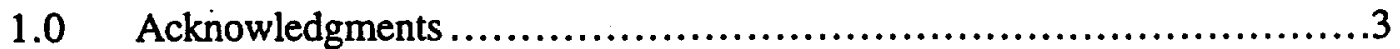

2.0 Introduction..........................................................

3.0 Background...........................................................

4.0 Test Program Objectives .............................................4

$5.0 \quad$ Test Aircraft ........................................................

6.0 Program Test Sequence ..............................................

7.0 Low-Power On-the-Ground Tests......................................5

7.1 On-the-Ground Test Objectives .....................................6

7.2 On-the-Ground Test Facility ....................................6

7.3 On-the-Ground Test Organization and Responsibilities..............6

7.4 Ground Plane ..................................................

7.5 Sensors..................................................... 14

7.6 Pretest Procedures ......................................... 18

7.7 Stepped CW Measurements: CWDAS with Dipole Antennas .....23

7.8 Fixed CW Measurements: On-Board Instruments with Rhombic. 29

7.9 Stepped CW Measurements: CWDAS with Rhombic Antenna.... 31

7.10 Stirred Frequency Tests............................................. 35

7.11 On-the-Ground Test Schedule................................... 42

7.12 Safety ........................................................... 44

7.13 Security ...................................................... 49

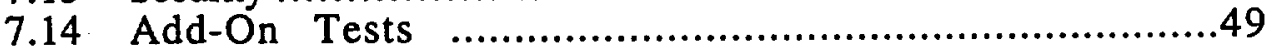

Appendix A Plane Wave On-the-Ground Test Objective......................53

Appendix B Power Densities for Aircraft Tests...............................56

Appendix C On-the-Ground Test Matrix..................................61 


\subsection{Acknowledgments}

This test plan was developed by the Lawrence Livermore National Laboratory (LLNL) in support of NASA's Fly-by-Light / Power-by-Wire program. The NASA Langley Research Center (LaRC) has responsibility for the HIRF effects and assessment methodology portions of the program. LLNL is tasked with developing modeling and analysis tools as well as with directing the aircraft tests to validate these tools. This test plan was written with input from Felix Pitts, K. Peter Zaepfel, Peter Padilla, and Charles Meissner of LaRC. Buddy Walton and Ly Dao of the USAF Phillips Laboratory and John Millard of United International Engineering, Inc. also provided valuable input.. This plan also borrows heavily from a test plan written by Howard LaValley of UIE.

\subsection{Introduction}

The NASA Fly-by-Light / Power-by-Wire (FBL/PBW) program was initiated to develop technology for a future generation of commercial transport aircraft. Studies have shown that an allelectric aircraft (PBW) could have substantial benefits in terms of weight and maintenance costs over today's aircraft that rely on hydraulic and pneumatic controls. However, one major concern regarding the all-electric aircraft is the potential for conducted and radiated electromagnetic susceptibility of its electronic controls. A way to potentially alleviate this problem (and save weight at the same time) is to route all control and signal information over optical fiber control links (FBL) that are inherently easier to harden against the electromagnetic environment (EME).

Since electro-optic devices and electronic digital computers will still be required, a major objective of the FBL/PBW program is to develop tools and techniques for assessing the survivability of the modern aircraft using such equipment in the electromagnetic environment. The EME includes both externally generated high intensity radiated fields (HIRF) and internally-generated radiated or conducted emissions. The ability to withstand internally-generated emissions is often referred to as electromagnetic compatibility (EMC).

The EME effects assessment tools and techniques developed under the program must ultimately be accepted by industry and the FAA regulatory body to be useful. Therefore, an important aspect of the program is that these tools and techniques must be validated to the satisfaction of this community. A series of aircraft tests are being planned for this purpose. Specifically, low power on-the-ground tests and fly-by tests will be conducted. This test plan describes, in detail, the onthe-ground tests which include those used for generating data for code validation as well as those which support the study of the stirred frequency technique. The test plan for the fly-by tests will be provided in a subsequent document.

\subsection{Background}

Full-threat tests of each make, model, and modification of each aircraft type would be far too expensive for the commercial aircraft community. Less expensive assessment methods usually rely on an EM stress-vs.-strength approach. In these methods, the EM stress that is applied to a component or subsystem is compared against the EM strength of the component or subsystem. The stress is measured as the current, voltage, power, or field that is received by or impressed upon an installed component or subsystem when the aircraft is exposed to the EME. The strength is the level of signal, expressed in the same units, the component or subsystem can withstand without upset or damage. There are several advantages to separating stress and strength. First, separating the stress estimate from the strength estimate allows the use of computer models, low power measurements, and/or similarity to estimate stress. These methods are much less expensive since they do not require the entire aircraft to be tested at full power. The strength can be measured 
with direct injection or radiation tests that involve only individual components or subsystems. Such tests inherently require less power and space to test than would full aircraft tests. This separation would allow the avionics manufacturers to test their boxes (strength tests) and the airframe manufacturers to test or analyze their airframes (stress tests) separately at reduced cost.

\subsection{Test Program Objectives}

The primary thrust of this aircraft test program is to develop a library or database of experimental results to be used in the evaluation of modeling codes. A second objective is to support the development of other assessment techniques such as mode-stirred chamber measurements. To support these objectives, it is also necessary to quantify measurement errors and to perform sensitivity studies to allow an estimation of the degree of agreement between measurement and model results that can reasonably be expected.

The data library will necessarily be limited in size and scope by cost and time constraints but will nonetheless provide a reasonable database for validation exercises involving computer codes that can be used for EME modeling activities. Here, the term validation is used to denote the determination whether a particular code is capable of producing accurate results for specific modeling tests in which it has been applied. Clearly, this will "validate" the physics kernel of the code, its numerical algorithms, and its overall ability to provide the "right" answer. Of possibly equal importance, the data might help to establish the limits of applicability of a code, the validity of approximations and extensions which can enlarge its applicability, and the resources such as computer time and memory required to attain various levels of accuracy.

A primary focus in this test plan is the validation of FDTD modeling of coupling into the airframe using a code such as TSAR. It will be tested extensively in its "intended " range where approximations other than discretization are not required (up to a few hundred $\mathrm{MHz}$ ) as well as in the range where approximations and special algorithms will be required to estimate stress. While a description of the approximations used to extend the domain of applicability of the code is beyond the scope of this plan, adequate attention has been paid to collecting appropriate data to allow for its use in future validation activities.

In many cases deterministic models are insufficient to predict the stress within an airframe. Statistical approaches to this problem are being considered at several institutions, and theoretical and experimental foundations are being developed. LLNL has been studying statistical electromagnetics and experimental techniques based on mode stirred chambers. At frequencies where the airframe is many wavelengths, the aircraft interior can be modeled as a complex highlyovermoded cavity having random internal fields. It is hypothesized that the power at a randomly selected point has a statistical distribution that is independent of the details of the shape of the cavity. If this assertion is validated, it may be possible to use a similarity concept to characterize the stress applied to the line replaceable units (LRUs) within the aircraft. That is, once the parameters of the distribution are known, the LRUs environment is statistically determined. Airframe testing or analysis would be required only to determine the parameters of the distribution of coupling into the airframe. The statistical distribution and its parameters would not be expected to change with modifications of the avionics or other changes inside the complex airframe cavity. LRUs could be tested in a mode-stirred chamber that develops an internal EME having the same statistical distribution. Validating these concepts is another test objective.

It can be expected that a comparison of the results generated by measurements and computer analysis will not result in perfect agreement. The experimental and analytical or computational modeling approaches each have their own uncertainties and sources of error so that neither can be assumed to be the "standard" against which all other results must be evaluated. There are numerous sources of systematic and random measurement error and modeling error. The measurement sample size will be insufficient to eliminate the random errors, and some amount of 
systematic errors will always exist in the measurements. Also, there will always be some amount of modeling error in the model. The intent here is to maintain an appreciation of these errors and to perform a comparison in the light of the fact that measurement and analysis may provide equally valid results given the errors associated with each process. So, in order to quantify some of the larger of these errors, a series of tests will be made. These tests will allow us to establish reasonable error bounds about the measurement and computer model predictions for use during our comparisons.

\subsection{Test Aircraft}

The primary test object is the NASA/Eastern (Boeing) 757-200. The onboard certificate identifies the aircraft as a Boeing 757-225, Serial Number 22191 with the 225 denoting the specific configuration requested by the original sole owner (Eastern Airlines). In the following, this aircraft is referred to as the B-757, NASA B-757, NASA 757, etc., for convenience.

\subsection{Program Test Sequence}

Aircraft flight tests are required to convince the community of the validity of effects assessment techniques for in-flight aircraft. However, such tests are difficult and expensive. Orientation and position parameters and incident field values are difficult to control or measure accurately in such a test. A series of on-the-ground tests to validate both modeling and mode-stirred measurement techniques under well-controlled conditions will precede the flight tests to provide data under better controlled conditions and to reduce the risks associated with the flight tests.

This test sequence is not being planned to certify the safety of an aircraft. Rather, it is being planned to validate tools and techniques for assessment. Hence, the test plan will have a somewhat different emphasis than a certification test would have. Here, it will not be necessary to measure or predict EM effects on each critical subsystem nor is it necessary to measure coupling into every section of the aircraft that contains critical equipment. A few representative subsystems in a few aircraft sections will suffice.

In the next section of this test plan, we concentrate on the first validation step, the low level on-theground measurements. The fly-by tests will be the subject of another test plan. Suffice it to say, the fly-by tests will include measurements associated with radiation from a UHF radar at Wallops Island, an antenna driven by a VHF source, and a VOA station in Greenville, NC. The on-theground tests have elements which are in preparation for the fly-by tests.

\subsection{Low-Power On-the-Ground Tests}

The low-power on-the-ground tests encompass a series of tests which are meant to achieve a set of objectives defined in Sect 7.1. The tests have been arranged to enable execution of the tests in priority order and the rationale will become clear during the course of this plan. An attempt was made to construct a plan paying due attention to the limited aircraft availability time, the need for maximizing data acquisition time, the requirements for system dry-runs, and the existence of priority in the test series. In light of such considerations, the following represents a test plan satisfying significant constraints yet striving for maximum value. It has been constructed using reasonably conservative time estimates, some slack time, and a prioritization that permits inclusion and exclusion of tests as time permits.

\subsection{On-the-Ground Test Objectives}


There are four objectives and four test series for the on-the-ground tests. The first test series will meet the first objective which is to generate data to be used directly for coupling code validation. For this objective, discrepancies and uncertainties between measurement and model must be minimized. These tests will use dipole antennas, so the antenna can be included in the model. This eliminates the uncertainty in the exact form of the wave entering the model problem space. The second test series will meet the second test objective for the on-the-ground tests which is to exercise the equipment that will be used for the fly-by tests. For these tests, the test signals should match those planned for the fly-by tests. Thus, rather than stepped CW measurements, these tests will be conducted with fixed-frequency $\mathrm{CW}$ or pulsed waveforms. The exact polarizations and orientations are not required. Only test points planned for the fly-by tests need be examined. The third test series will meet the third objective for the on-the-ground tests which is to prepare for the fly-by tests, as well as to provide more data for validation of the codes (objective one). We wish to know the coupling into the airframe so that any high-Q coupling resonances near the fly-by frequencies can be taken into account as well as the sensitivity of coupling to uncertainties in aspect angle or the position of interior objects. The fourth test series will involve a continuing study of stirred frequency techniques.

\subsection{On-the-Ground Test Facility}

These tests will be conducted at the US Air Force Phillips Laboratory (PL) Large Electromagnetic System-Level Illuminator (LESLI) test facility. The LESLI facility is operated and maintained by the PL/WSM. This facility consists of a concrete pad, a two-wire rhombic antenna, and an instrumentation trailer. Time has been allocated for assembly and checkout of the data acquisition system and for a dry-run of the complete experimental system.

In the preparation of this test plan, an attempt has been made to minimize time-consuming aircraft operations such as repositioning of the aircraft on the pad. For example, as presently configured, this plan requires initial placement of the NASA B-757 at nose-on incidence in the LESLI, i.e., an orientation of the aircraft with its axis parallel to the LESLI axis. Following the tests in this orientation, tests will be executed at $10^{\circ}$ and at angles less than $10^{\circ}$, if called for, and finally at broadside ( $90^{\circ}$ from nose-on).

The standard LESLI facility instrumentation will be used. This instrumentation is referred to as the CWDAS. The second test series will employ the LESLI rhombic antenna. PL will supply CW RF amplifiers which are part of the normal LESLI instrumentation while LLNL will supply a source capable of low power pulsed operation such as the HP 8360. The third test series will employ the LESLI rhombic antenna and its standard transmitters and instrumentation.

\subsection{On-the-Ground Test Organizations and Responsibilities}

\subsubsection{Test Organization}

The test support comes from many organizations. The NASA Langley Research Center (LaRC) is the funding organization for these tests. LaRC developed the on-board instrumentation. The Lawrence Livermore National Laboratory has responsibility for test planning and managing test execution. The Air Force Phillips Laboratory (PL) is located at Kirtland Air Force Base (KAFB). Emergency services, e.g., hospital, ambulance, and fire protection, are provided by the $377^{\text {th }}$ Air Base Wing (377 ABW), KAFB. The Advanced Weapons and Survivability Directorate (PLWS) within the PL is responsible for the LESLI facility. United International Engineering, Inc. (UIE) is a PL support contractor. The roles of each of these organizations is detailed below.

\subsubsection{Air Force - 377th Air Base Wing - Emergency and Security Services}


Air Force - 377th Air Base Wing will provide as required:
(a) hospital
(b) ambulance
(c) security
(d) fire protection (a $150 \mathrm{lb}$ power bottle aviation-type fire extinguisher on the pad satisfies NASA safety requirements in this regard)

\subsubsection{Lawrence Livermore National Laboratory: Experimenter}

LLNL is responsible for the following tasks:
(a) Defining test points, test sequence, test priorities
(b) Defining sensor types
(c) Defining test orientations, configurations and test frequencies
(d) Final data analysis
(e) Analytical predictions to compare to test results
(f) Final data preparation before introduction into a test data library
(g) Provide calibration factors for field (D dot) and voltage sensors used in the tests
(h) Provide pulsed RF source

\subsubsection{NASA Langley Research Center: Program Manager}

NASA has the following responsibilities:
(a) Provide the aircraft
(b) Provide required aircraft safety / quality control personnel to assure that the aircraft flight worthiness has not been compromised by the tests
(c) Provide a qualified ground crew who can power up aircraft systems, tow the aircraft to the required positions (or who can direct the PL/UIE aircraft ground crew to do this), etc. A $1 / 2$ day notice will be provided to PL/IE when towing is required.
(d) Provide on-board instrumentation responsive to experiment needs
(e) Provide personnel, as required, for set-up/operation of on-board instrumentation
(f) Provide calibration data for the installed coaxial cables and VHF antenna

\subsubsection{Air Force Phillips Laboratory/WSM: Test Facility}

PL/WSM, in conjunction with UIE, will provide the following:

(a) Provide and configure the LESLI test facility with rhombic antenna and calibrated instrumentation

(b) Provide an aircraft tug and driver to install the aircraft into the LESLI facility

-(c) Provide instrumentation personnel

(d) Provide safety assurance personnel, as required

(e) Provide data processing support for quick-look processed data during testing

(f) Calibrate current probes (current probes will be provided by NASA)

(g) Provide data Quality Control (QC) personnel to review the test data. 


\subsubsection{Functional Responsibilities}

The functional organization is shown in Figure 7.3-1.

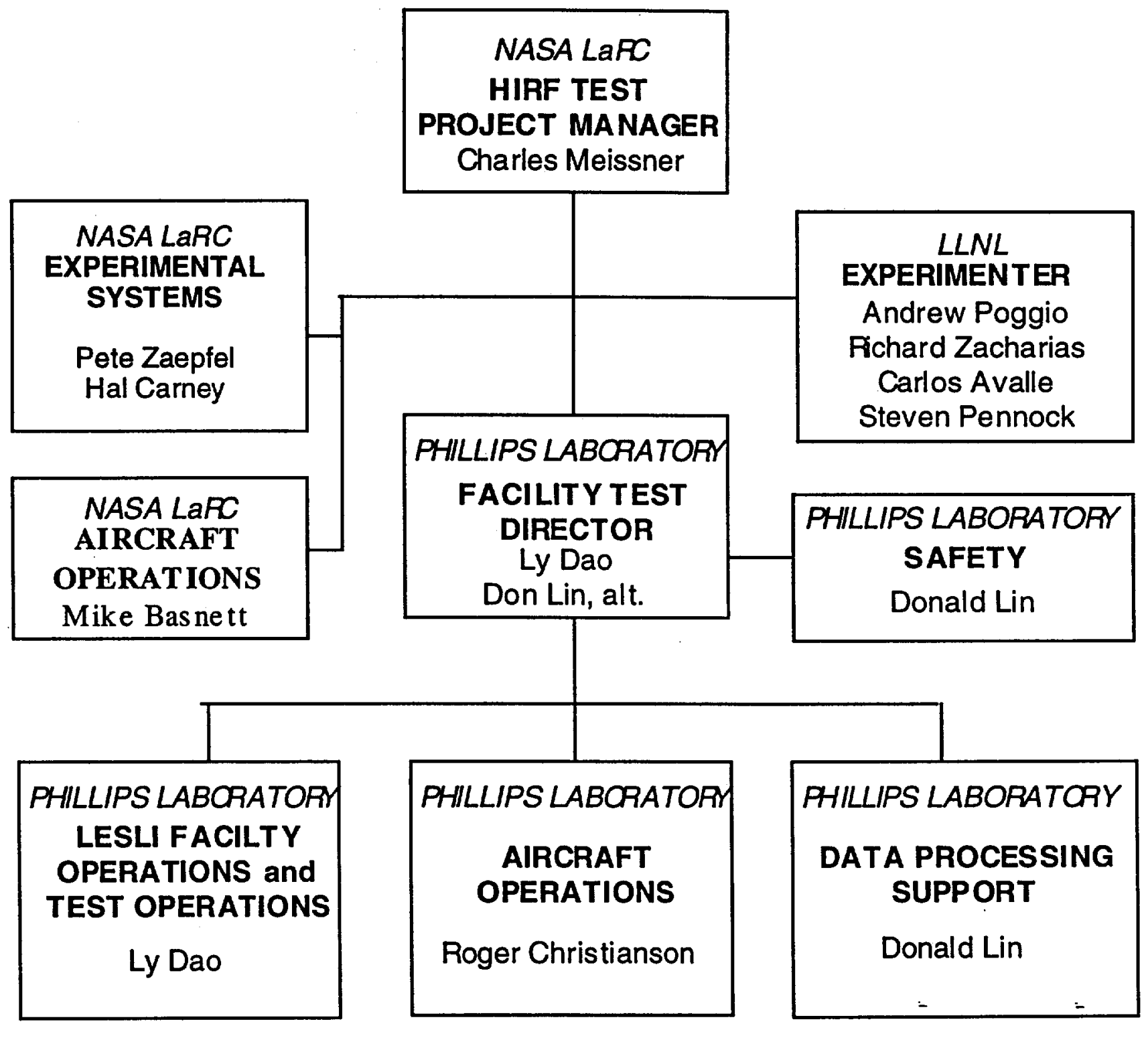

Figure 7.3-1. Functional organization.

\subsubsection{Experiment Test Director (LLNL)}

The Experiment Test Director will be Dr. Andrew Poggio. During testing, the LLNL Experiment Test Director, assisted by the PL Facility Test Director, will perform the test control function and will determine the experiment test sequence. 


\subsubsection{Facility Test Director (PL/WSM)}

The PL Facility Test Director, Mr. Ly Dao, is responsible for the implementation of the approved test plan and test safety plan. The responsibility of Facility Director will encompass coordination of all activities required to acquire data, including aircraft/instrumentation readiness, data acquisition system readiness, and coordination of $\mathrm{CW}$ sweep initiation.

\subsubsection{LESLI Facility Operations, Test Operations, and Support (PL/WSM supported by UIE)}

Facility Operations is responsible for simulator facility support, including facility operations, data acquisition system integration and check-out, instrumentation characterization, data acquisition system operation, and data translation. Test Support is responsible for fiber optic instrumentation installation and quick-look data analysis.

\subsubsection{Data Processing Support (PL/WSM supported by UIE)}

CW data will be uploaded to the LESLI processing PC where it will be corrected for instrumentation effects. Accepted data will be written to standard tapes for delivery to LLNL and the data will be archived in the PCSLEET database.

\subsubsection{Aircraft Operations (NASA LaRC)}

NASA LaRC is responsible for movement, operations, and maintenance of the NASA aircraft. PL/IE will support these activities during the tests. PL/UIE will also move the aircraft under NASA direction and provide other test support, as required.

\subsubsection{Test Operations Working Group (All)}

The Test Operations Working Group (TOWG), comprised of representatives from each organization, will meet daily during the test to insure test activity integration. Potential problems will be identified and an action item assigned for timely resolution.

\subsection{Ground Plane}

The concrete surface forming the pad in the LESLI facility will be used in an unaltered state as the ground surface. The aircraft will be positioned on this surface for the experiments. The LESLI is shown with and without the airplane in the scale drawings in Figures 7.4-1 through 7.4-4. The coordinate system for the ground plane is shown in Figure 7.4-1. The origin $(0,0,0)$ is located at the rhombic feedpoint. This right-handed coordinate system, denoted $x_{,}, z_{\vec{q}}$ is distinct from the coordinate system used for the interior of the aircraft that is fixed to the aircraft. 


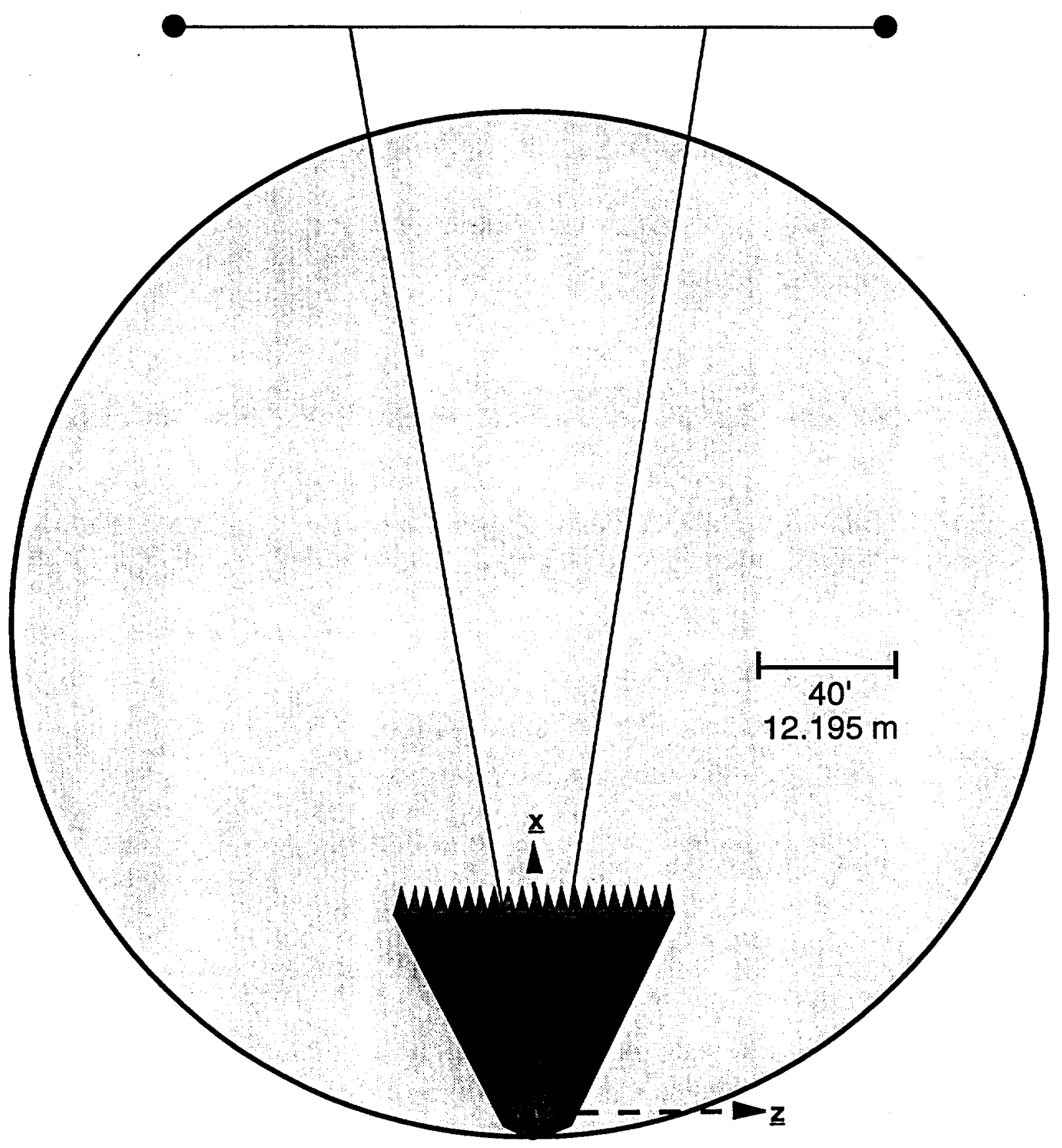

Figure 7.4-1. LESLI Facility with rhombic antenna and ground plane coordinate system 


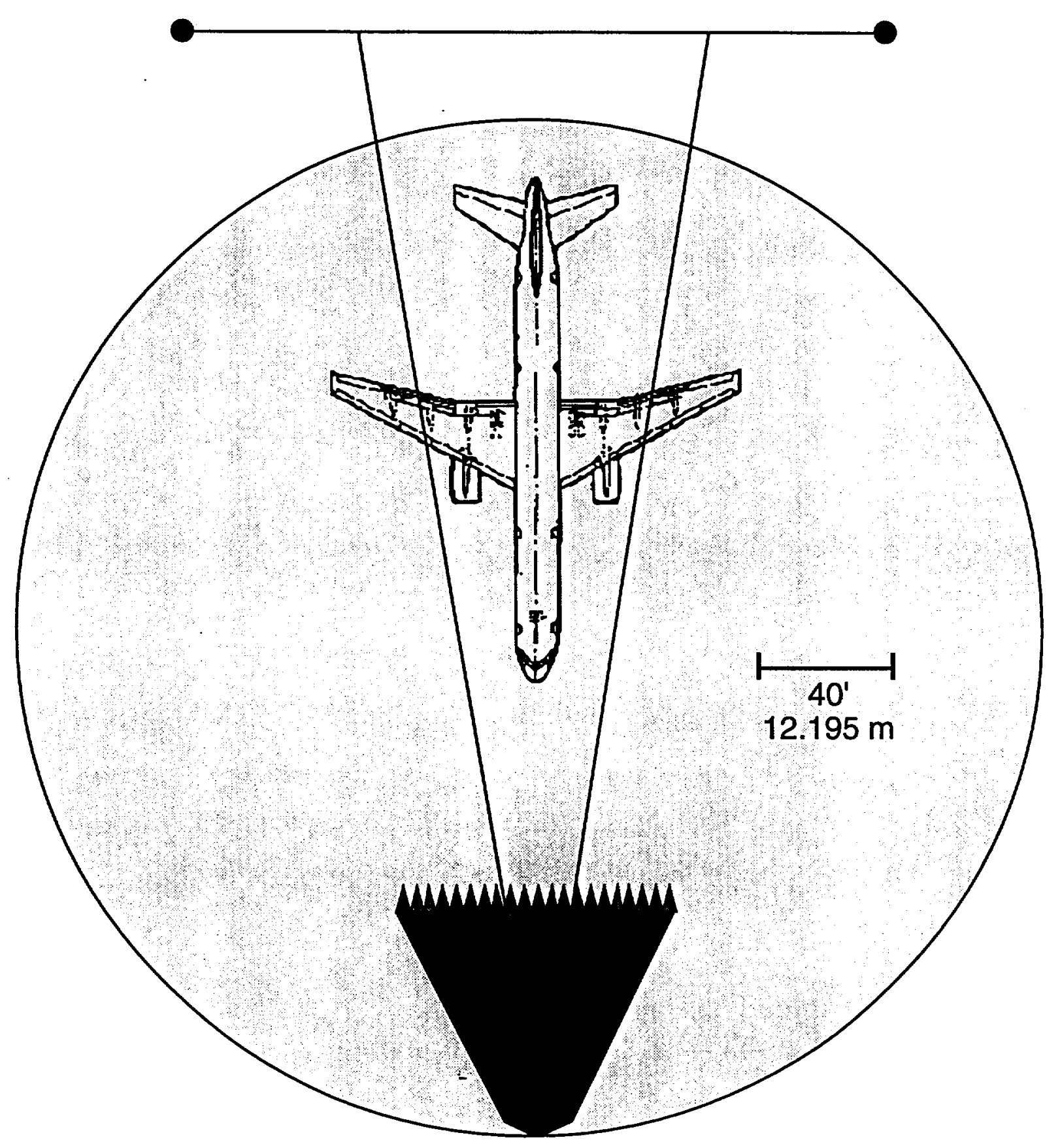

Figure 7.4-2. LESLI with B 757 nose-on incidence 


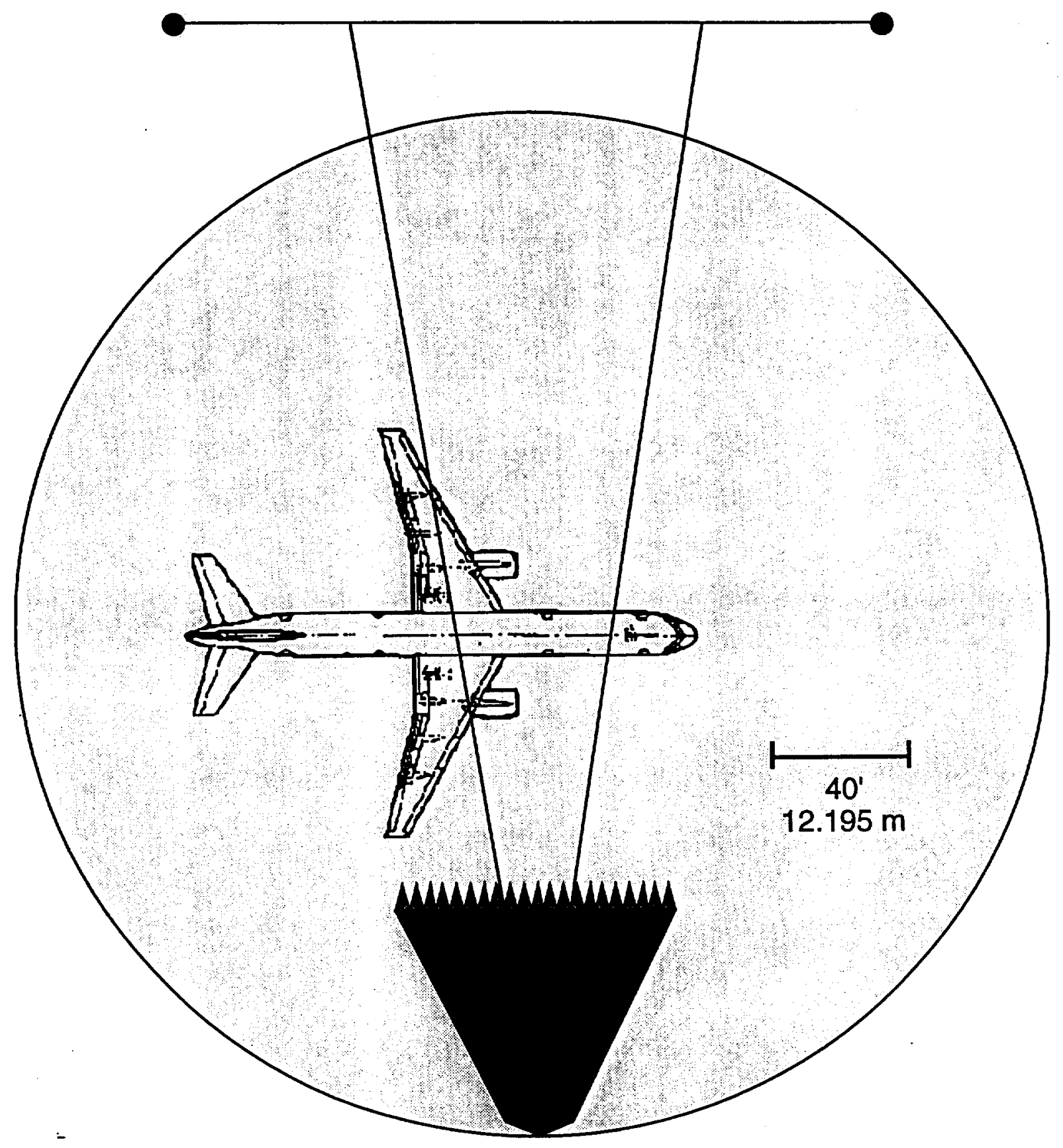

Figure 7.4-3 LESLI with B 757 broadside incidence 


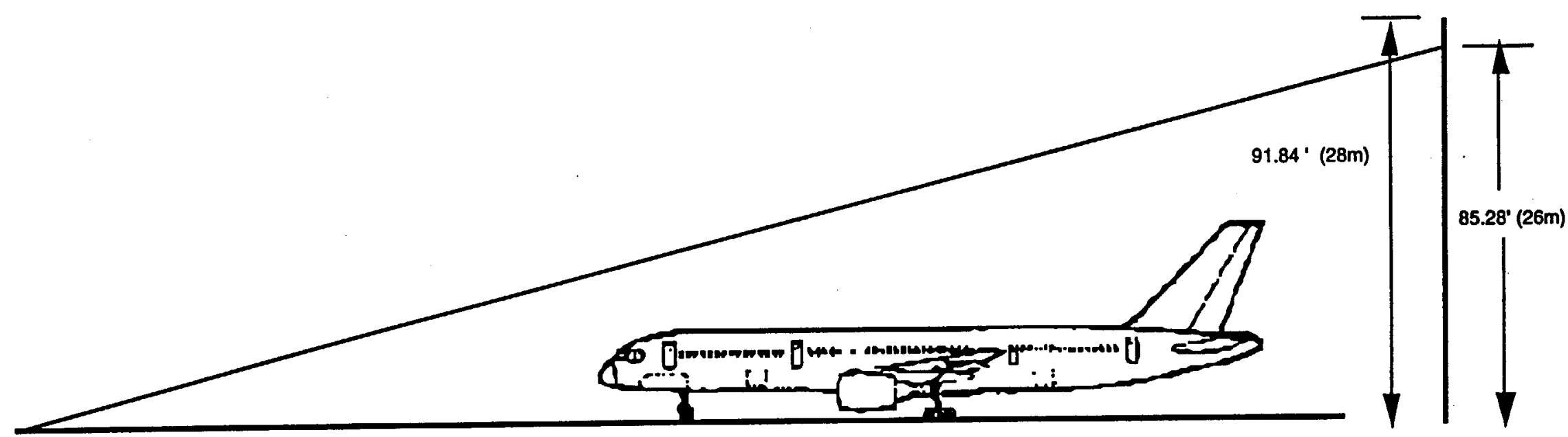

$\frac{7}{\omega}$

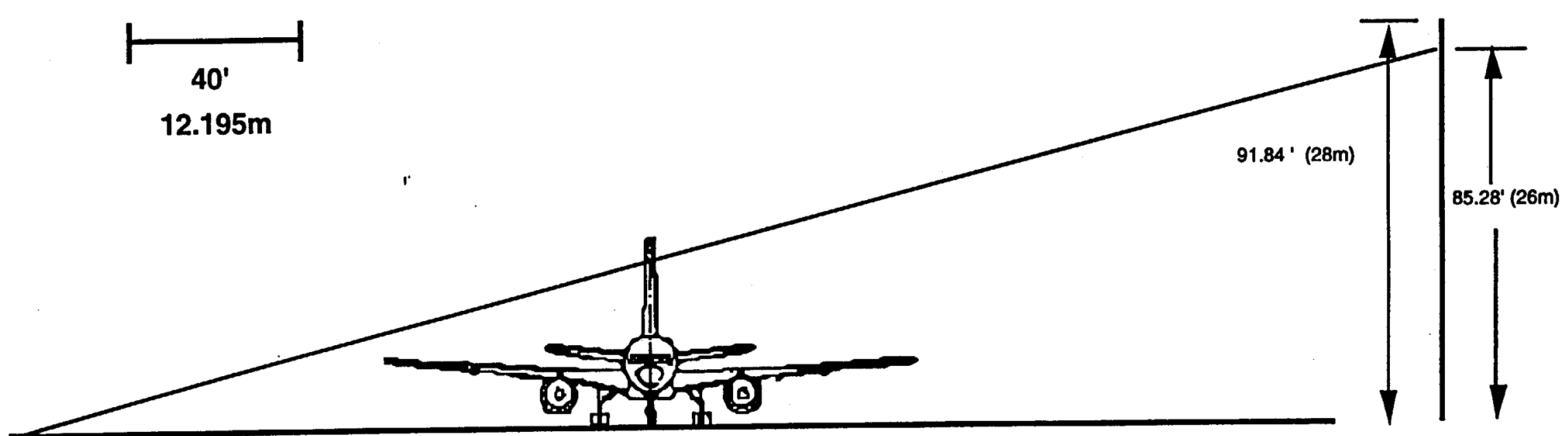

Figure 7.4-4 Side view of LESLI with B 757 


\subsection{Sensors}

The sensors for the on-the-ground tests will be fitted into the aircraft prior to its arrival at Kirtland. Some of the sensors will be removed for the flight to Kirtland, and installed for the tests. The suite of 12 sensors will sample fields and cable currents and voltages in three aircraft bays: the cockpit, the electronics bay, and the cabin. Figures 7.5-1, 7.5-2, and 7.5-3 show sensor locations.

There will be five sensors in the cockpit. All of these sensors will be mounted onto the same sensor box that will be fabricated by NASA LaRC. This box is also referred to as the ATOPS box. The box contains three orthogonal Prodyne AD-60 D-dot sensors and a wire that extends through the box on which a voltage measurement is made within the box. Thus there are four connectors on the box. In addition, a Prodyne I-320 current probe will be used on the bare wire just outside of the box.

The electronics bay will contain three sensors. A Prodyne AD-60 will be used to measure fields within the bay. A Prodyne I-320 current probe will be placed onto an existing aircraft line for the windshield heater within the bay. The third measurement in the electronics bay will be made on a Collins VHF-700 transceiver box. In the remainder of this plan, this unit will often be referred to as the RC-7 box or unit. The unit will have been previously modified to provide a probe to measure the voltage on an internal power line pin. The unit will be installed into an existing slot in the electronics rack in the electronics bay after the aircraft arrives at Kirtland. The box will thus be connected to the avionics bus for low RF power stepped frequency tests. The box will be installed after the aircraft is positioned for tests. The box will be removed for the on-board instrumentation tests where aircraft power would be applied and then reinstalled for later power-off tests. (See 7.8.1).

There will be four sensors in the cabin area. One of these will be an AD-60 D-dot mounted on top of the EME instrumentation rack. The second sensor will be a long-wire mounted along the ceiling of the cabin. This long wire will allow the measurement of small-signal low-frequency coupling. The long wire will terminate into a $50 \mathrm{ohm}$ cable across which voltage will be measured. A current sensor will also be installed onto the semi-rigid coaxial cable feeding the long wire to sense external shield current. The fourth cabin sensor will be the VHF-L (left) antenna which is part of the normal complement of external antennas for the NASA aircraft. This antenna will provide a measurement of the external field (perturbed by the aircraft) and will provide a strong signal to be used as a trigger for the fly-by tests.

LaRC will provide cables from each sensor that will mate to either the on-board instrumentation or will mate to fiber optic transmitters for the swept frequency measurements. 


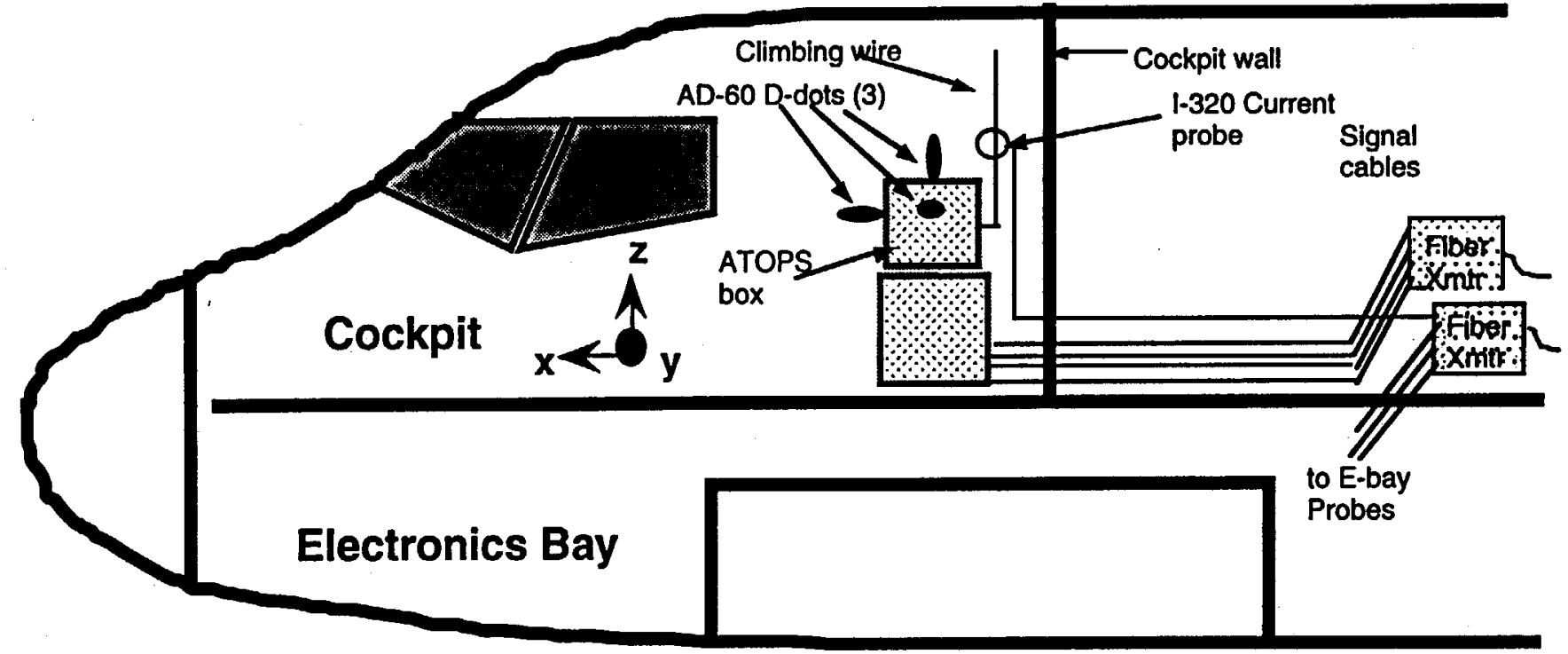

$\begin{array}{lllll}\text { Ch.\# } & \text { Probe } & \text { Parameter } & \text { Location } & \text { Fly-by } \\ & & & & \\ \text { T1C1 } & \text { AD-60 } & \text { Ex Field } & \text { ATOPS box } & \text { N } \\ \text { T1C2 } & \text { AD-60 } & \text { Ey Field } & \text { ATOPS box } & \text { N } \\ \text { T1C3 } & \text { AD-60 } & \text { Ez Field } & \text { ATOPS box } & \text { Y } \\ \text { T1C4 } & 1-320 & \text { Current } & \text { ATOPS box } & \text { N } \\ \text { T2C1 } & \text { Wire } & \text { Voltage } & \text { ATOPS box } & \text { N }\end{array}$

Figure 7.5-1. Cockpit sensors. 


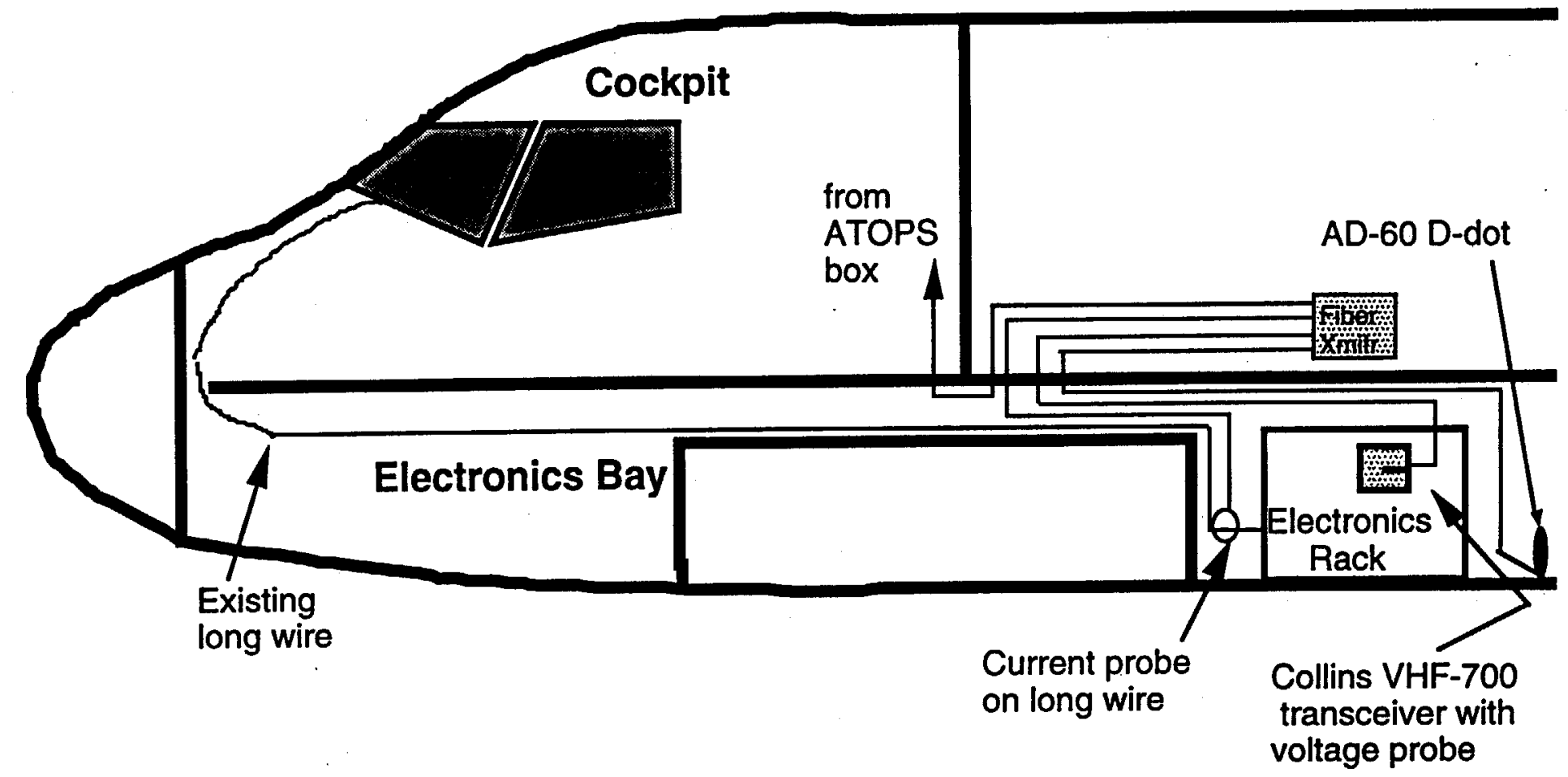

$\begin{array}{llllr}\text { Ch. \# } & \text { Probe } & \text { Parameter } & \text { Location } & \text { Fly-b } \\ \text { T2C2 } & \text { AD-60 } & \text { E Field } & \text { Elect. bay } & \text { Y } \\ \text { T2C3 } & \text { l-320 } & \text { Current } & \text { Existing wire } & \text { Y } \\ \text { T2C4 } 50 \mathrm{ohm} & \text { Voltage } & \text { RC-7 TCvr } & \text { N }\end{array}$

Figure 7.5-2. Electronics bay sensors 


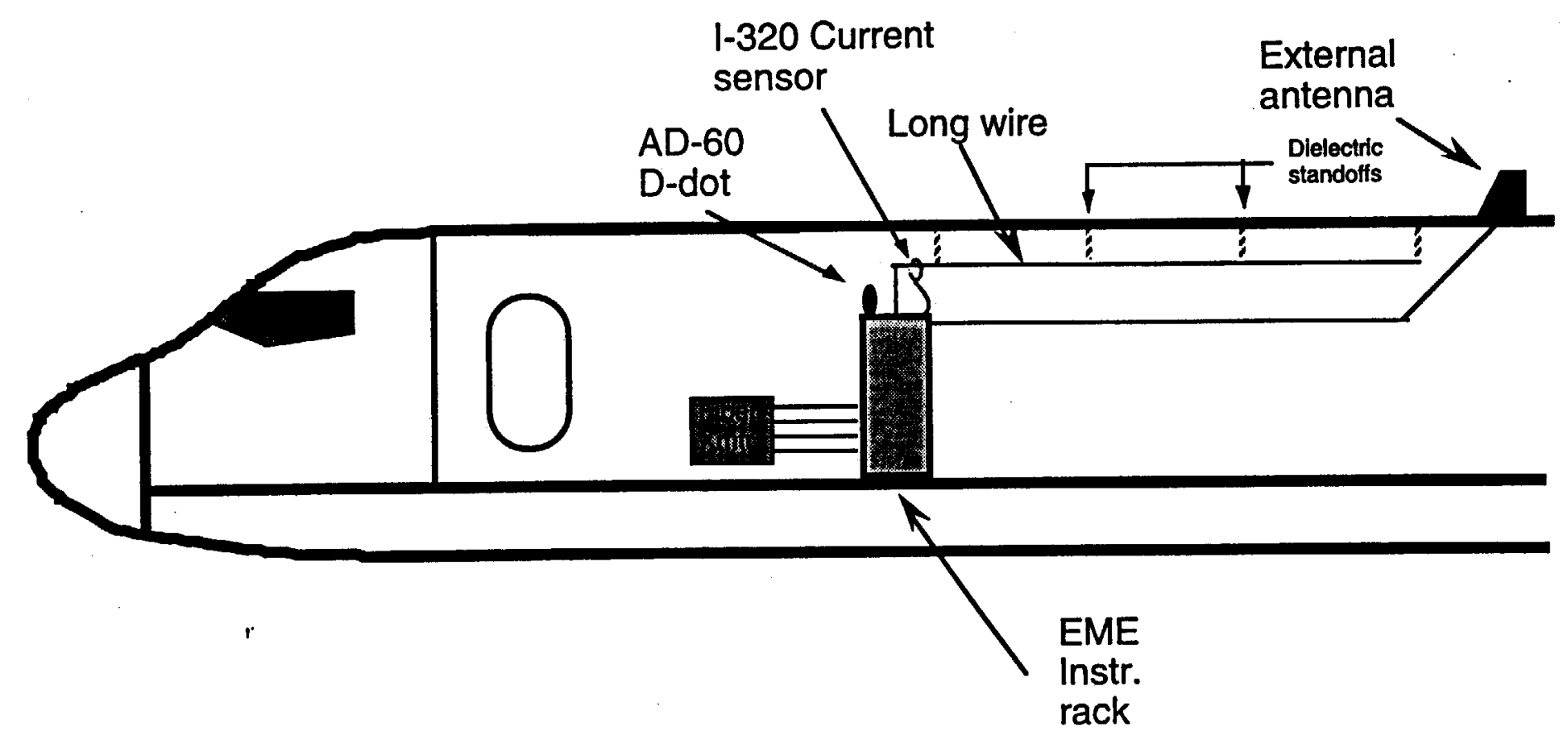

$\begin{array}{llllc}\text { Ch. \# } & \text { Probe } & \text { Parameter } & \text { Location } & \text { Fly-b } \\ & & & & \text { F } \\ \text { T3C1 } & \text { Ext. Ant. } & \text { Ext. Field } & \text { Fuselage } & \text { Y } \\ \text { T3C2 } & \text { AD-60 } & \text { Field } & \text { Equip rack } & \text { Y } \\ \text { T3C3 } & \text { Long Wire } & \text { Voltage } & \text { Ceiling } & \text { Y } \\ \text { T3C4 } & \text { I-320 } & \text { Current } & \text { Long wire } & \text { Y } \\ \text {, } & & & & \end{array}$




\subsection{Pretest Procedures}

\subsubsection{Characterization and Checkout}

7.6.1.1 Probe Characterization. Characterizations of the field mapping probes and current probes will be performed by the PL LESLI Facility Operation and Support Group prior to the start of the test. Probe characterizations shall be performed following the Continuous Wave Data Acquisition System (CWDAS) User's Guide. Figures 7.6-1 and 7.6-2 show the configurations used for the cable/attenuator/splitter, and current probe characterization. Current probes and cables will be characterized over the allowable CW logarithmic sweep frequencies in the $300 \mathrm{KHz}$ to $1 \mathrm{GHz}$ band as listed in the CWDAS User's Guide using Q's developed for the LESLI Field Map Testing. These data points will match those used for the rhombic antenna sweeps described in Section 7.9. For the narrowband sweeps using the dipole (described in Section 7.7) or narrowband rhombic sweeps, calibration data will be acquired in the same manner. Sensor characteristics will be provided by NASA/LLNL while calibration data for the cables installed aboard the aircraft will be provided by NASA.

7.6.1.2 Coaxial Instrumentation Cable Check-out. All external cables will be characterized using CWDAS by the Facility Support Group. Four sets of data characterizations will be done for each of the experiments described above. The data will be recorded in Toolkit format on PC-readable floppy disks and uploaded to the LESLI processing PC running MatLab. Cables will be visually inspected during the test. When acquired data are questionable, a cable characterization may be repeated as requested by the Test Director.

7.6.1.3 Link Characterization. Fiber optic data links will be characterized using CWDAS by Facility Support Group. The data will be uploaded to the LESLI processing PC running MatLab. The test setup used to characterize the fiber optic data links is shown in Figure 7.6-3. The links shall be characterized over a frequency range of $300 \mathrm{KHz}$ through $1 \mathrm{GHz}$.

7.6.1.4 Dry Runs. A series of dry runs will be executed to provide assurance that all elements of the experiment system are functioning properly. The dry runs will involve use of all available RF sources and amps, all antenna (illuminator) systems, selected sensors, and the data acquisition system that includes the instrumentation, computer control, acquisition and display functions. In effect, these runs will be "full-up" except for the presence of the NASA aircraft. It will test both the systems and the operational procedures in effect during the tests.

7.6.1.5 FAA Compatibility Tests Prior to the arrival of the aircraft, compatibility tests will be conducted to assure that the tests involving new frequency and antenna configurations cause no interference with operation of the FAA control tower. These tests will include all stepped frequency dipole test configurations (see Section 7.7) and all fixed CW and pulsed CW configurations (see Section 7.8). Power levels expected during the test sequences will be used. 


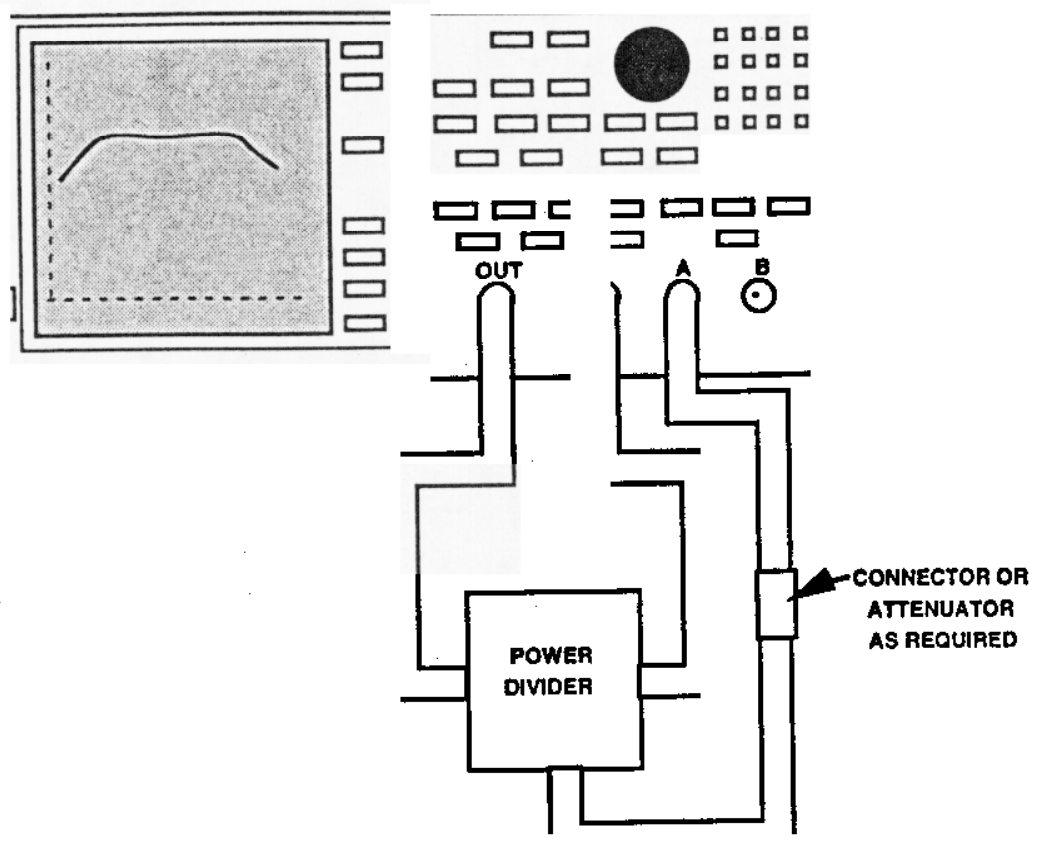

Figure 7.6-1. Cable / attenuator / splitter characterization test setup.

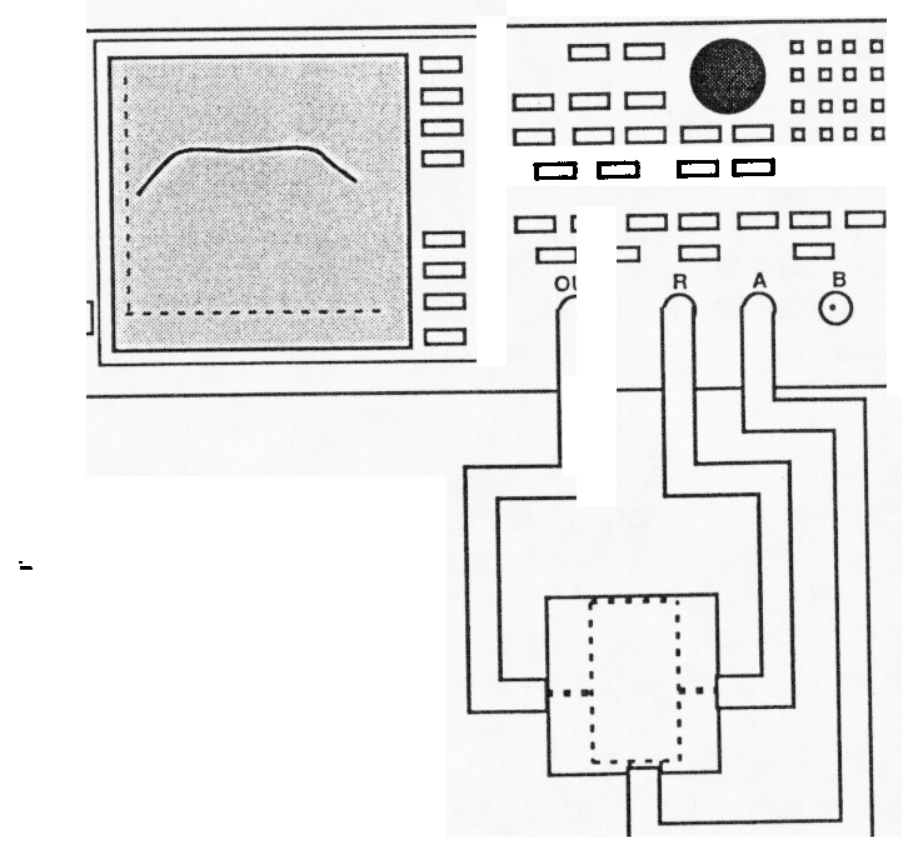

Figure 7.6-2. Probe characterization test setup. 


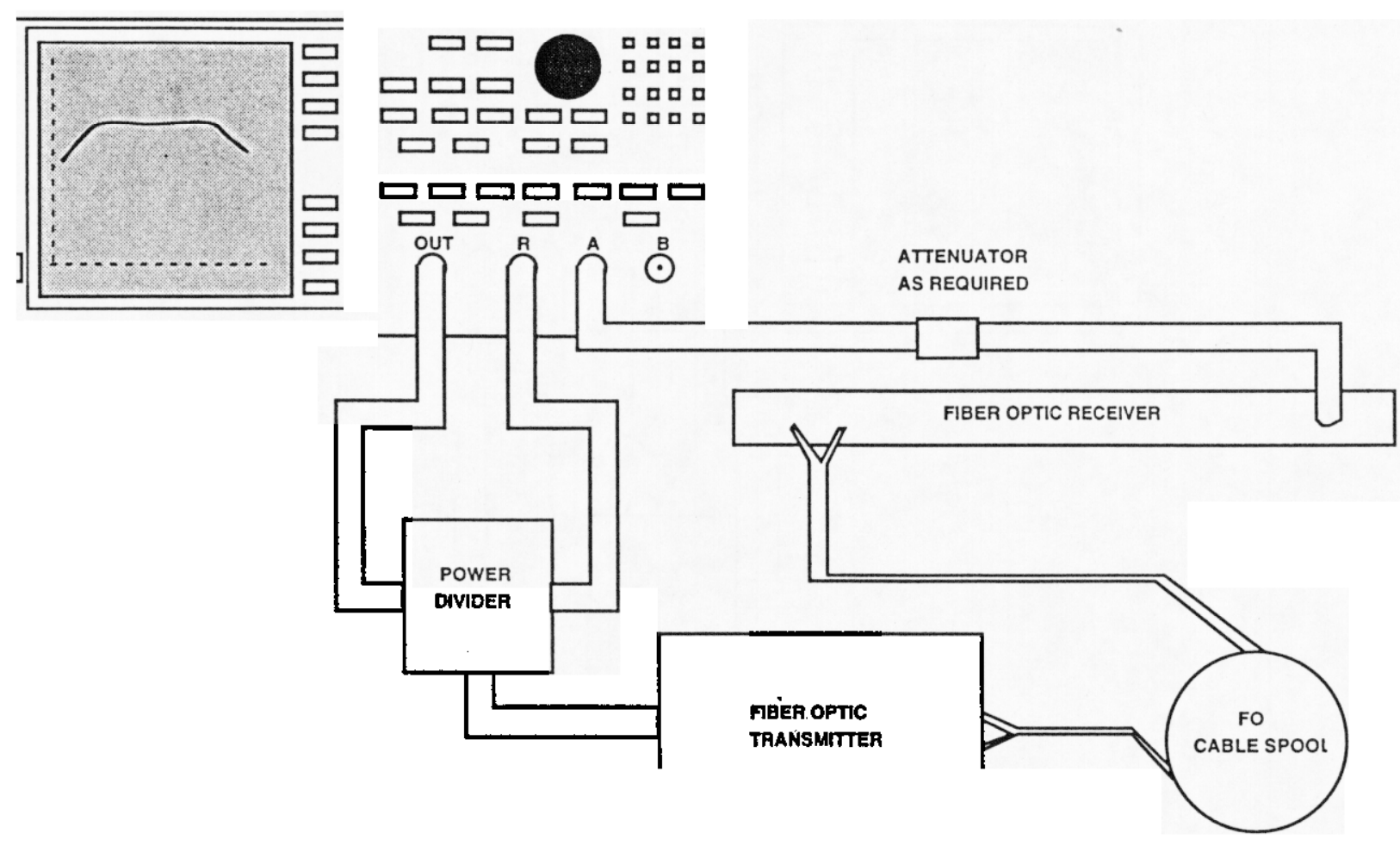

Figure 7.6-3. Fiber link characterization test setup.

\subsubsection{Field Mapping}

\subsubsection{Rhombic Antenna}

Prior to making swept $\mathrm{CW}$ measurements with the Rhombic antenna, field mapping measurements will be made to provide a baseline of the incident field wavefront. It is prohibitively expensive to make sufficient measurements to completely determine field components throughout the entire volume or over the closed surface of any computational volume for the numerical models which might be employed. (This is the primary reason for the dipole experiments described in Section 7.7.)

A model for the rhombic antenna exists that will relate the voltage incident to the antenna to the field at any point in space under the antenna. If this model were completely accurate and validated, no field mapping would be necessary. Since this is not the case, we must make measurements to validate it. However, we can use the model to greatly reduce the number of field map measurements required. Our plan is to make a few measurements to recalibrate the model, and then use the model to define the incident fields in the computer coupling models.

The field map locations are described in Table 7.6-1. Field measurements will be made in each of three orthogonal directions at each test point. B-field measurements will be made at each point and E- (or D-) field measurements will be made at select points. These are denoted E\&H in the table. Measurements will be made over the entire $300 \mathrm{KHz}$ to $1 \mathrm{GHz}$ band. The frequency sample spacing will be adjusted to match that of the stepped CW measurements described in Section 7.9. 
In Table 7.6-1, the coordinates are defined with the $\mathbf{x}$ axis along the center line of the rhombic illuminator, the $\mathbf{X}$ axis orthogonal to the ground plane in the vertical direction, and the $\underline{z}$ axis orthogonal to the $y-z$ plane and parallel to the ground plane. The origin is at the feed point and the system is right-handed. This coordinate system is shown in Figure 7.4-1.

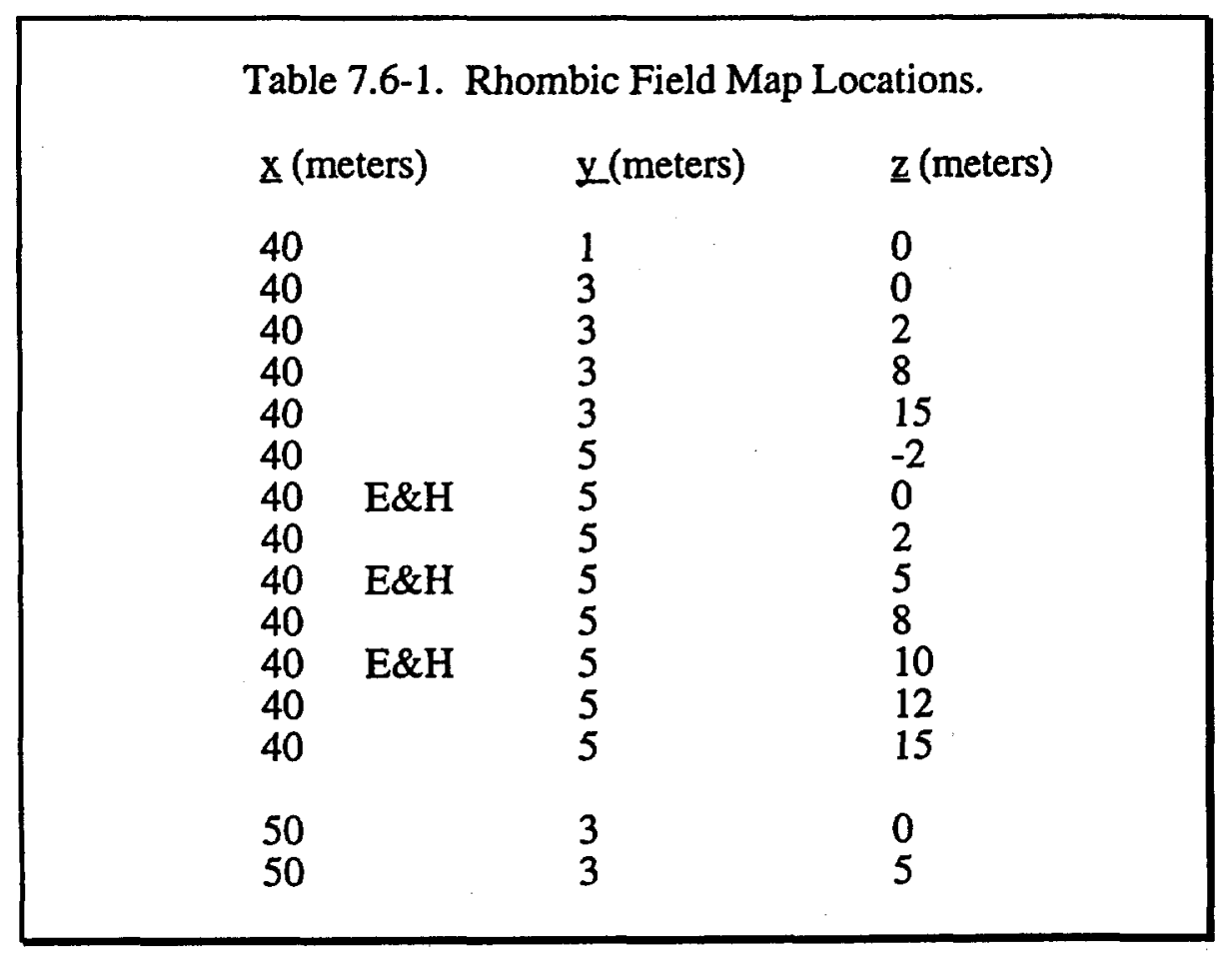

\subsubsection{Dipole Antennas}

Dipole antennas will also be used in certain tests. While these are to be included in the modeling volume and should, in principle, not require field mapping, it has been considered advisable to perform a limited mapping exercise. To this end, the dipole fields will be mapped as described in Table 7.6-2 with the field at each point measured for the three orthogonal directions. In some cases denoted by $\mathrm{E} \& \mathrm{H}$, both the $\mathrm{E}-$ and $\mathrm{H}$ - field will be measured. The coordinate system for these maps is indicated in Figure 7.6-4. The frequencies in the table define the approximate center frequency of a range of frequencies that is defined in the test matrices for the dipole tests. These frequency ranges will be extended above and below the center frequencies by an amount that will allow the structure of the spectrum in that vicinity to be investigated. The range may be limited by self-protection devices in the amplifiers which protect against high VSWRs at the transmitter output.

H Pol and V Pol refer to horizontal and vertical polarization, respectively, for the dipoles. For convenience, both orientations are shown in Figure 7.6-4. The height of the dipole feed point in the mapping exercises is 3 meters except for vertical polarization near $25 \mathrm{MHz}$ when it is 4 meters.

The coordinates in the table are relative to a point on the pad directly below the feed point of the dipoles. This point will be identical to that used during the dipole test sequence.

Original plans included use of a highly conducting plane composed of interlocked aircraft landing mats. Unavailability has required use of the concrete pad in an unaltered state. Hence, it will be necessary to characterize the pad from an electromagnetic standpoint. This will be performed after the NASA 757 test series is complete and will be the subject of another test plan. 


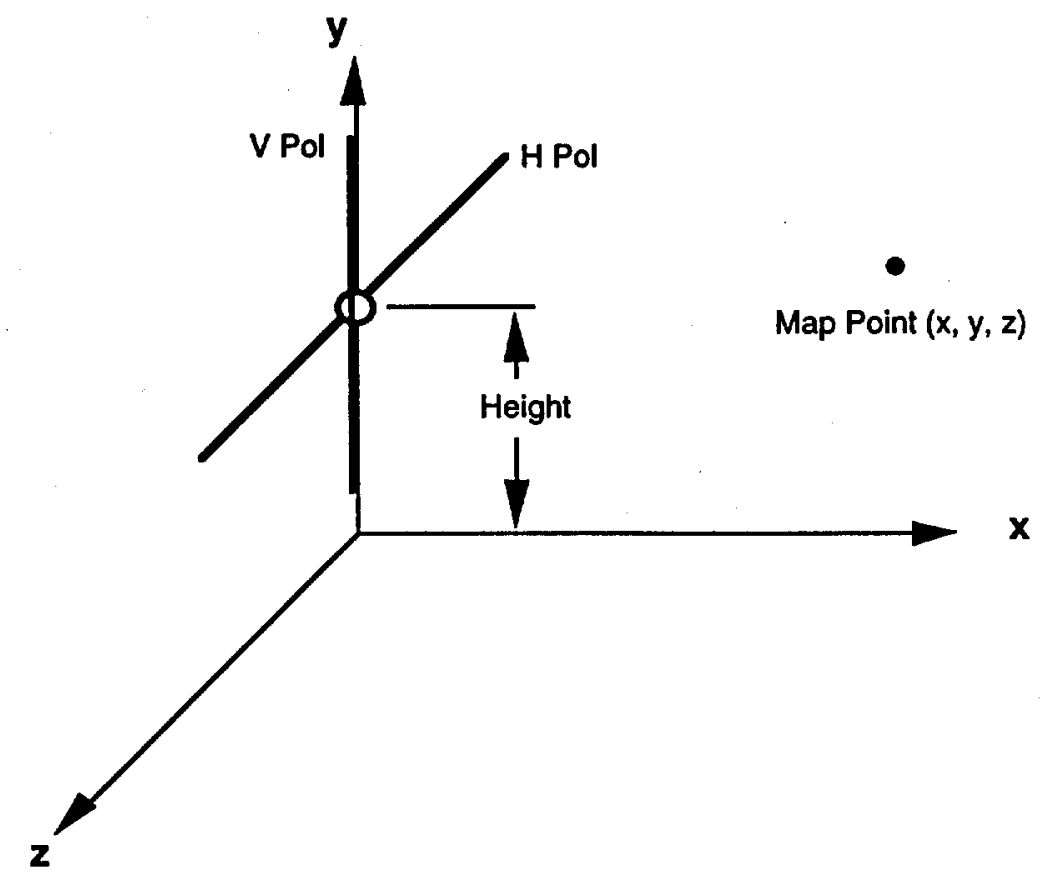

Figure 7.6-4 Dipole Field Mapping Coordinate System

\begin{tabular}{|c|c|c|c|c|c|}
\hline & & Table 7.6-2. & pole Field M & Cocations. & \\
\hline freq $(\mathrm{MHz})$ & pol & $\mathrm{x}$ (meters) & $\mathrm{y}$ (meters) & $\mathrm{z}$ (meters) & \\
\hline 25 & V & 2 & 4 & 0 & E\&H \\
\hline 25 & $\mathrm{~V}$ & 8 & 4 & 0 & \\
\hline 25 & V & 8 & 2 & 0 & \\
\hline 25 & $\mathrm{H}$ & 2 & 3 & 0 & E\&H \\
\hline 25 & $\mathrm{H}$ & 8 & 3 & 0 & \\
\hline 25 & $\mathrm{H}$ & 8 & 2 & 0 & \\
\hline 172 & V & 2 & 3 & 0 & $\mathrm{E} \& \mathrm{H}$ \\
\hline 172 & V & 8 & 3 & 0 & \\
\hline 172 & V & 8 & 2 & 0 & \\
\hline 172 & $\mathrm{H}$ & 2 & 3 & 0 & $\mathrm{E} \& \mathrm{H}$ \\
\hline 172 & $\mathrm{H}$ & 8 & 3 & 0 & \\
\hline 172 & $\mathrm{H}$ & 8 & 2 & 0 & \\
\hline 430 & $\mathrm{~V}$ & 2 & 3 & 0 & $E \& H$ \\
\hline 430 & $\mathrm{~V}$ & 8 & 3 & 0 & \\
\hline 430 & $\mathrm{~V}$ & 8 & 2 & 0 & \\
\hline 430 & $\mathrm{H}$ & 2 & 3 & 0 & $\mathrm{E} \& \mathrm{H}$ \\
\hline 430 & $\mathrm{H}$ & 8 & 3 & 0 & \\
\hline 430 & $\mathbf{H}$ & 8 & 2 & 0 & \\
\hline 25 & $\mathrm{H}$ & 8 & 3 & 6 & \\
\hline 25 & $\mathrm{H}$ & 8 & 3 & -6 & \\
\hline 172 & $\mathrm{H}$ & 8 & 3 & 6 & \\
\hline 172 & $\mathrm{H}$ & 8 & 3 & -6 & \\
\hline 430 & $\mathbf{H}$ & 8 & 3 & 6 & \\
\hline 430 & $\mathrm{H}$ & 8 & 3 & -6 & \\
\hline
\end{tabular}




\subsubsection{Aircraft Pretest Activities}

Before the aircraft arrives at KAFB, NASA LaRC will have installed some test probes and will have marked them with identification tags. Once the aircraft is positioned for the first test series, additional probes (including the RC-7 unit) will be installed by LaRC personnel. Not all probes are used for all test series. The sensors will remain installed in the same position for all test series, with a few exceptions which are noted in the test matrix.

The NASA aircraft will arrive at Kirtland AFB on September 18, 1994. The following paragraphs detail the expected pre-test activities.

7.6.3.1 Initial Aircraft Positioning. The aircraft will be towed from the runway to the LESLI facility and positioned under the antenna. The aircraft will be positioned appropriate for a test using the LESLI antenna with the aircraft cockpit facing the incident wave. However, at this time the LESLI two wire antenna will have been removed. NASA LaRC will be responsible for moving and repositioning the aircraft with support from PL UIE Aircraft Operations. LLNL will provide additional support for aircraft alignment. Facility Support will provide the required aircraft grounding equipment which will be installed during down times and after-hours only, not during the tests. The aircraft will remain in place through the dipole tests, unless otherwise directed by NASA LaRC.

\subsection{Stepped CW Measurements: CWDAS with Dipole Antennas}

\subsubsection{Probe Installation}

Two $300 \mathrm{KHz}$ to $1 \mathrm{GHz}$ Nanofast fiber optic data links will be employed for acquiring data from probes mounted in the aircraft.. Each link can support four inputs. Upon placement of fiber cables, the aircraft may be inspected by NASA and Aircraft Operations to ensure that all installations are done according to previously approved test modification packages and that the aircraft integrity has not been compromised.

\subsubsection{Dipole Antennas}

Dipole antennas will be used for these test series. The antennas will be simple thin-cylinder or thin-wire antennas mounted parallel to both the aircraft fuselage axis and ground for horizontal polarization or mounted perpendicular to the ground for vertical polarization. The geometrical arrangement is shown in Figure 7.7-1 with all coordinates in meters and the origin on the ground directly below the nose of the aircraft, as shown. The dipole source (its center) is located at $(-4,3$, -5 ) for all frequencies except for the vertical polarization test near $25.85 \mathrm{MHz}$ when the center is raised to $(-4,4,-5)$. A reference probe (B dot sensor) is used in the measurements and is located 3.5 meters from the dipole source for all frequencies except for the measurements near $25.85 \mathrm{MHz}$ when the sensor is 5 meters from the dipole source. The coordinates are $(-1.525,3,-7.475)$ and $(-0.465,3,-8.535)$, respectively. Details for the emplacement are found in Figure 7.7-1.

These thin-wire dipole antennas are not expected to have ultra-wide bandwidths. Thus, separate antennas will be required for each of the three fly-by frequencies. These frequencies are set at $25.850 \mathrm{MHz}, 172.0 \mathrm{MHz}$, and $430 \mathrm{MHz}$. The aircraft will be illuminated with both vertical and horizontal polarization using dipoles for both polarizations. The dipoles will be cut to halfwavelength resonance near each fly-by frequency. Stepped frequency measurements will be made over a band extending from $80 \%$ to $120 \%$ of each center frequency or as permitted by equipment VSWR restrictions. The starting frequency, frequency step size, and number of data points to be taken for each test series is shown in Table 7.7-1. 

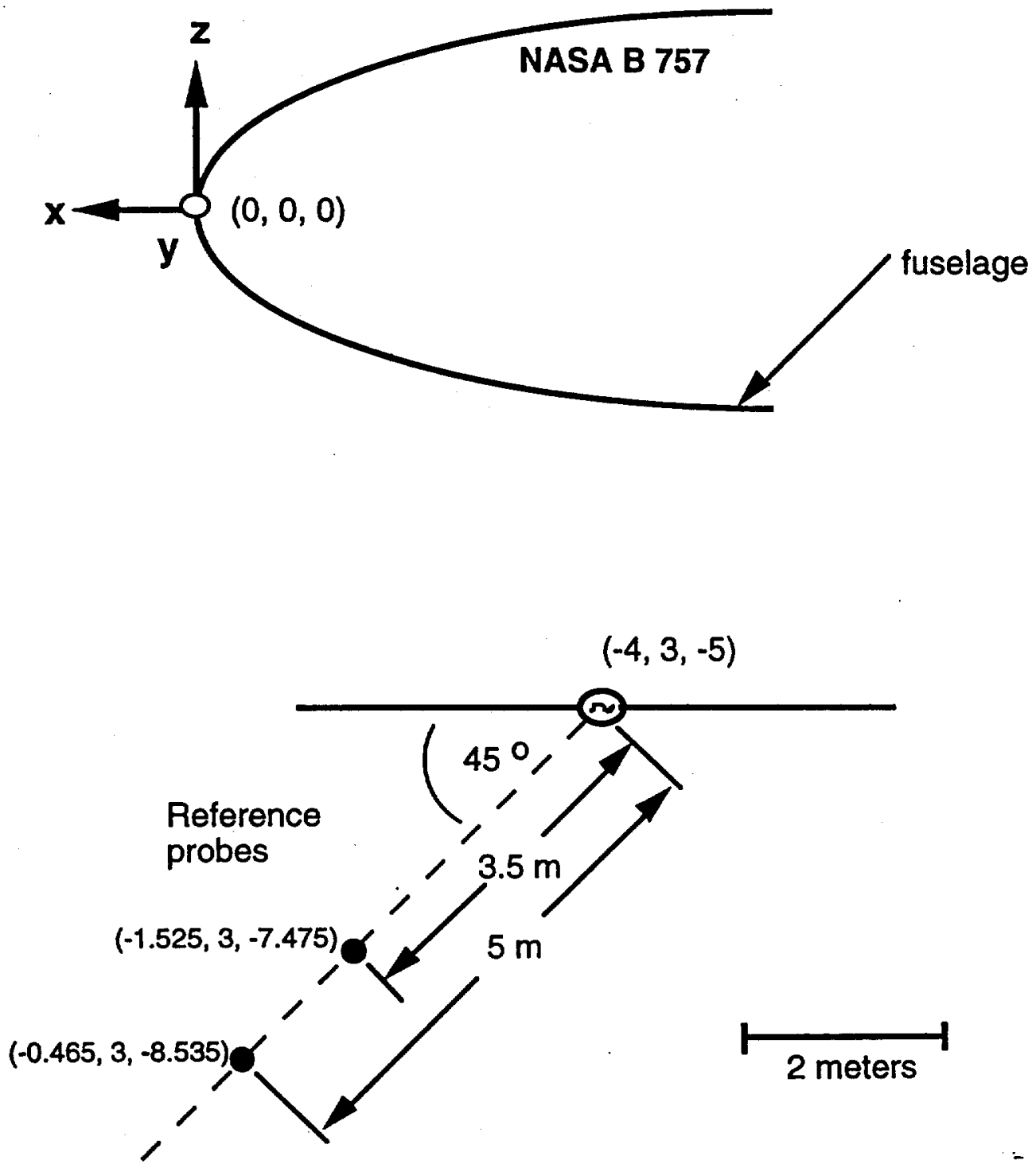

Figure 7.7-1. Dipole and reference probe locations for dipole tests 


\subsubsection{Dipole Test Operations}

This section outlines the specific operations which will be employed for the dipole tests. A block diagram of the instrumentation used for the dipole tests is shown in Figure 7.7-2.

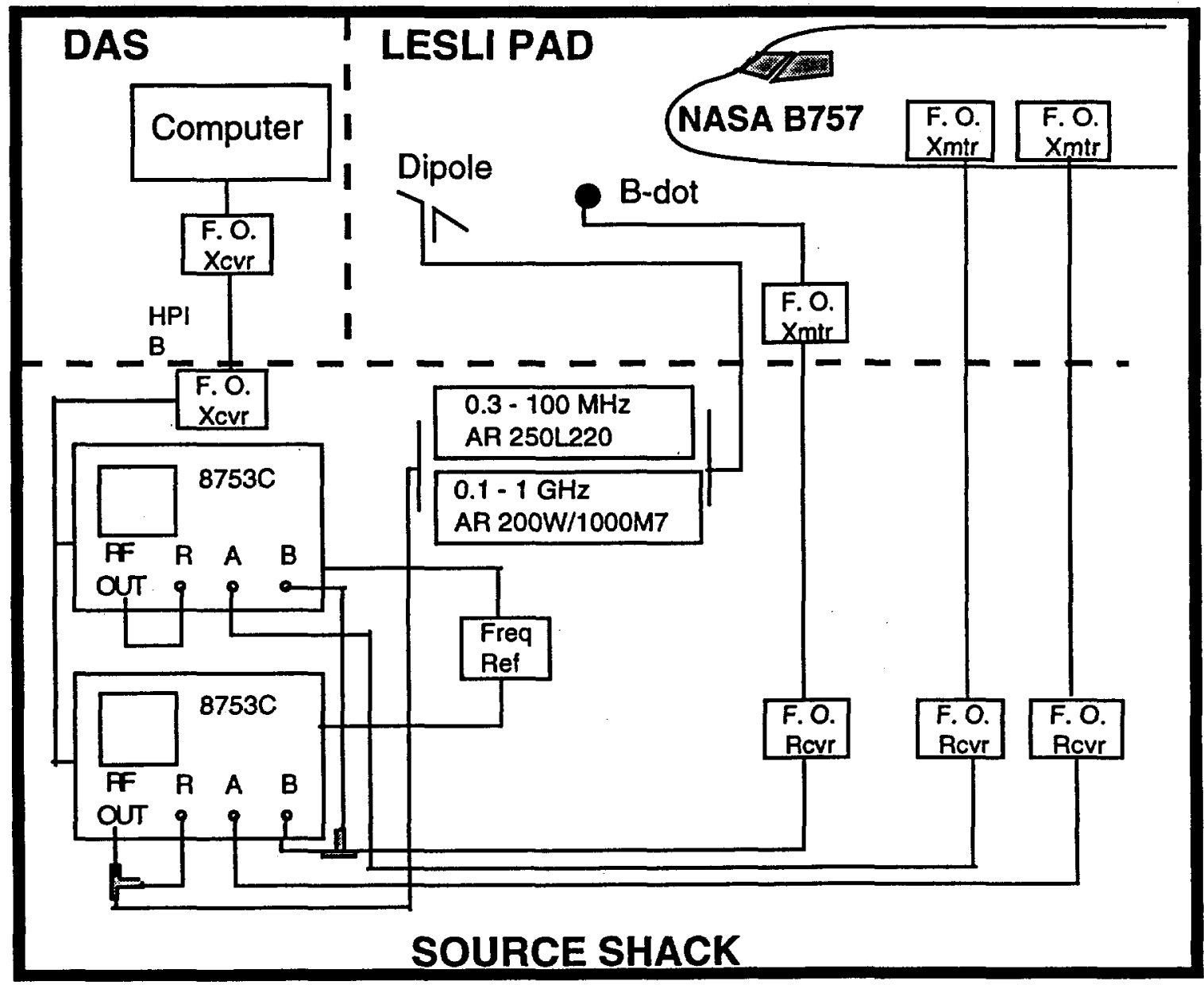

Figure 7.7-2. Dipole test instrumentation.

7.7.3.1 CW Procedures. The aircraft will be unpowered for these tests. The aircraft will be unmanned for this test series, although there is no RF hazard. The CW test points will be instrumented with the appropriate sensors as indicated on the detailed setup sheets. The probes will be connected to fiber optic transmitters; the outputs of the receivers will be connected to the network analyzer located in the source shack. On-board cables required to connect probes to the fiber transmitters will be supplied by LaRC. The network analyzers will be controlled by a control computer running the CWDAS software. CW sweeping will be accomplished using the FCC approved frequencies. Data acquisition (sweep) logs will be maintained by the Facility Operations Group.

Details of the data acquisition and processing to be accomplished are described in detail in the next subsection which also includes a detailed discussion of the acceptance criteria for data. Operationally, the Facility Test Director will follow the Test Point Matrix contained in this test plan and instrumentation setup sheets prepared by LLNL and Test Operations and Support. Facility Support will set up the appropriate DAS to acquire the requested test-points on any given sweep. When all are ready, the Facility Test Director will request that the sweep be initiated. The raw data 


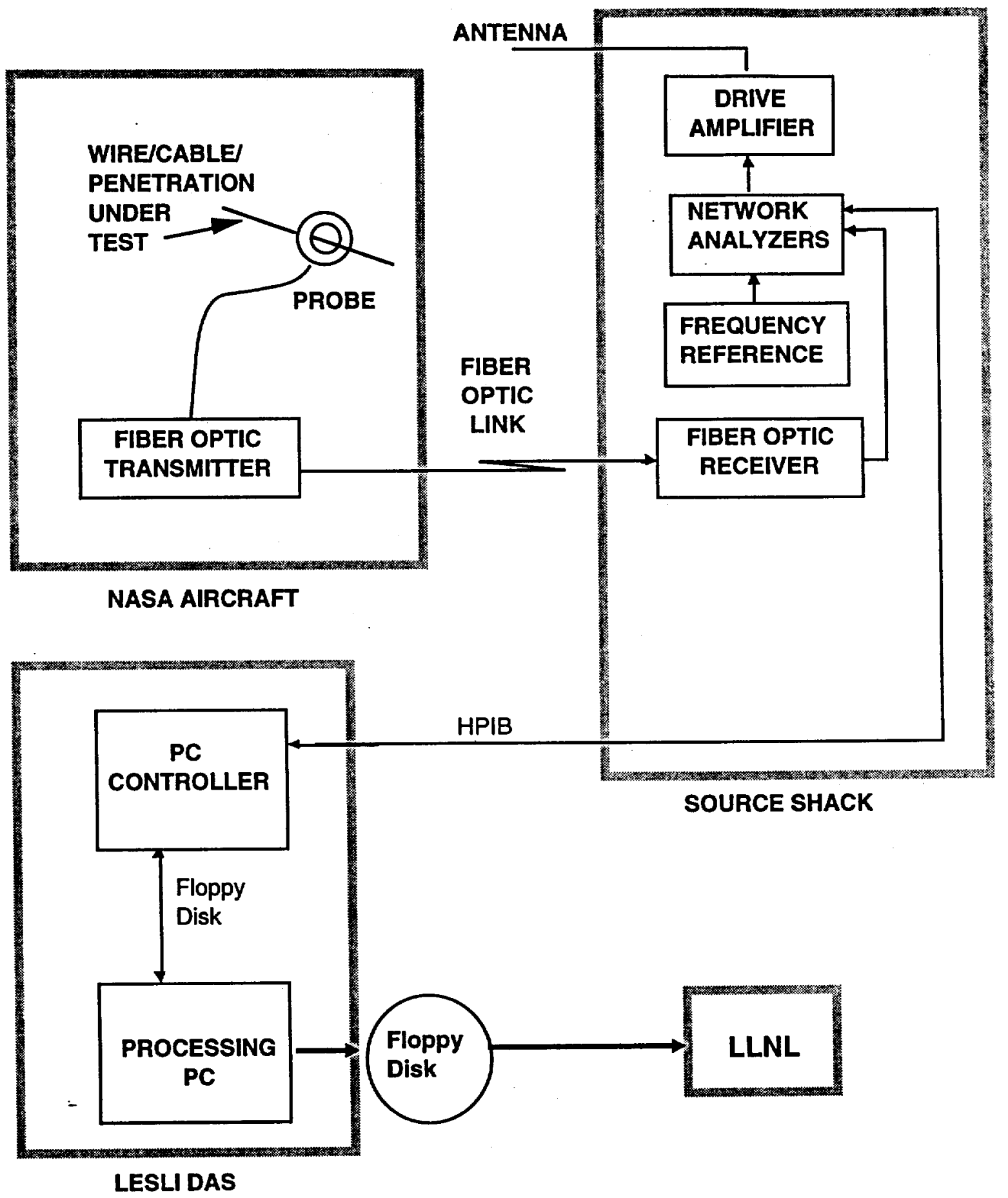

Figure 7.7-3. Example of $\mathrm{CW}$ data acquisition and processing flow. 
will be displayed on the in-DAS monitors for immediate QC. After data acceptance based on the "immediate-QC", the data will be uploaded for further processing. Data that has gone through final processing as well as the raw data may be plotted and provided to the Test Directors. Data, including characterization/calibration files, will be provided to LLNL on floppy disks Data will be downloaded and archived in PCSLEET.

The Test Operation and Support Group will inspect the installation of the probes, cables, and links each evening after the last test. These will be inspected to insure that they have been properly placed, that they match the required test points, and that the instrumentation log sheets are correct.

\subsubsection{CWDAS Data Acquisition, Processing, and Analysis}

\subsubsection{CWDATA}

The following sections describe the data acquisition, quality control and data processing procedures to be used for $\mathrm{CW}$ test data.

\subsubsection{Acquisition}

CW data acquisition will be accomplished using the CWDAS hardware and software configured in a two-network analyzer setup. The data flow is identical in all cases. Figure 7.7-3 shows the acquisition setup and data processing flow. Each test point will be connected to a recording channel on an HP 8753C Network Analyzer via a NanoFast fiber optic link. Test frequencies will be linearly spaced between skipbands but will have a logarithmic distribution over the broad frequency range and will use the start, stop, and frequency steps shown in Table 7.7-1. These may be modified by transmitter VSWR or FCC restrictions. Due to FCC restrictions, data will be acquired using the allowable transmit bands as listed in the CWDAS manual. Note that there are two skip bands in the $26 \mathrm{MHz}$ dipole band, one skip band in the $172 \mathrm{MHz}$ dipole band, and two skip bands in the $430 \mathrm{MHz}$ dipole band. Once a sweep has been completed, the data will be reviewed on the screen of the controlling personal computer. Instrumentation changes will be made and the data retaken as necessary. The acceptance criteria for the in-DAS QC of the CW data are listed in Table 7.7-2. Once accepted, the data, which include the measured variable at each test point and each frequency point, will be saved to the hard drive in the personal computer. The data will be periodically, at the direction of the Test Director, translated into TSA format and uploaded to the processing PC.

Table 7.7-1. Frequency tables for Dipole Tests $(\mathrm{MHz})$

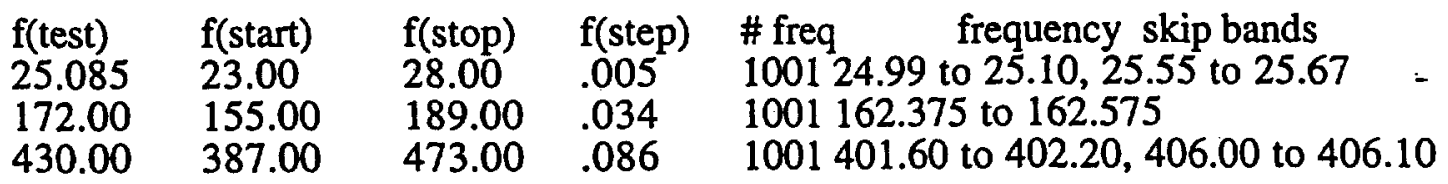

Table 7.7-2. In-DAS QC criteria for CW data.

1. Sufficient attenuation/gain has been used to prevent an overload or noise floor corruption of the network analyzer.

2. The peak of the recorded waveform is greater than $-10 \mathrm{dBm}$ for full scale screen deflection.

3. All header data is the same as that recorded on the data setup sheets.

\subsubsection{Processing in TSA}


As part of the uploading process into TSA, the data will be processed as described in Table 7.7-3.

Table 7.7-3. TSA processing steps for CW data.

1. Correct the data for probe/link, etc., frequency dependent and linear scale factors and save it as a .COF file.

2. Plot the following for use by QC:

Raw frequency (.RAW)

Corrected frequency (.COF)

The plots indicated in Table 7.7-3 will be used as they are available by Test Support/QC to ensure the quality of the processed data. Data sets returned for reprocessing will be documented on a User Change Request (UCR). Each UCR will identify the test point, the shot number, and what specific reprocessing is requested. Upon completion of the correction, UCRs will be filed in the original data folders. The QC criteria for TSA processing are listed in Table 7.7-4.

Table 7.7-4. TSA QC criteria for CW data.

1. Probes/links/etc. have been properly $\mathrm{u}$ folded from the data.

2. Final frequency dependent magnitude data look reasonable.

\subsubsection{Processing in PCSLEET}

Prior to the start og the test, alm of the characterization files will be loaded onto the LESLI processing PC as well as loaded knto PCSLEET. Field mapping and test data will be uploaded to the LESLI processing PC running MatLab for processing. Raw and processed data will be loaded into PCSLEET. During this process the header data and archive will be checked using the criteria listed in Table 7.7-5.

Table 7.7-5. PCSLEET Processing QC Criteria for CW Data

1. Header information is complete.

2. Only one data set is archived for each test point/test shot. (NOTE: Multiple sweeps may be taken at many test points. However, only one measurement will be archived for each test sweep.)

Note: Final data files will be immediately backed up by copying to an auxiliary storage medium such as copying to disk, the Boeing $720 \mathrm{~B}$ database or PC disk.

\subsubsection{Noise Measurements}

The noise environment will be characterized in order to quantify measurement quality, dynamic range, and error bounds. During these experiments, noise sources will be defined as arising from two sources, namely, the ambient environment and instrumentation.

The ambient noise will be established soon after the NASA 757 is in position on the pad and will be used as the standard for the entire test program. For the purposes of characterizing the ambient 
environment, four distinct measurements will be made. Using measurements made with two probes in the cockpit (vertical and transverse electric field probes), a cable current probe in the electronics bay, and an external VHF blade antenna on the airplane, a spectral map will be constructed of the noise environment.

Instrumentation noise for the dipole tests will be carried out for each specific frequency range and dipole polarization. The instrumentation noise measurements will be executed after each test sequence is performed. The strongest and weakest set of data in each sequence will be identified. Then, the corresponding probe will be removed, replaced by a matched load, and a data measurement sweep performed. The resulting data will provide an indication of the instrumentation noise at the lowest and highest gain settings for the data acquisition system.

\subsubsection{Test Matrix for Dipole Tests}

A detailed test matrix is presented in Appendix $\mathrm{C}$. The dipole tests are series $\mathrm{A}$ through $\mathrm{F}$. Tests are shown in prioritized order so that if insufficient time is allotted, the lower priority tests can be dropped. Estimates of the duration of each test are also shown in Appendix C.

\subsection{Fixed CW and Pulsed Measurements with On-Board Instrumentation}

This on-the-ground test series is intended to validate the instrumentation required for the fly-by tests. These tests would also allow a comparison between the power coupled to the aircraft interior both on and off the ground and would validate the modeling code's ability to predict this difference. In addition to determining whether the system can measure and record the RF signals, an equally important aspect of these tests is to determine whether the measurement system can measure the RF signals in the presence of noise generated by on board electronic systems.

These tests will be made with aircraft power supplied by the on-board auxiliary power unit (APU). If aircraft electronic systems can be powered (though not operating) when the aircraft is illuminated with RF, then all aircraft and experimental electronics systems will be powered to simulate the noise environment expected for the fly-by. If powering of the aircraft electronic systems (other than those associated with the power system) is not permissible, then the test series will necessarily proceed in two stages. Namely, with all electronic systems powered and no RF, the signals will be measured to provide an indication of the noise environment. Then, with all electronic systems off and RF on, the test series will be repeated. Clearly, this requires twice as many measurements as when allowed to have RF and all electronic systems powered simultaneously and somewhat compromises the test of the measurement system. The tests referred to in the preceding are denoted G, H, and I. Should a double set of runs be required, the noise background measurements will be referred to as $\mathrm{Gl}, \mathrm{H} 1$, and $\mathrm{Il}$.

The aircraft will be manned by LaRC ground crew and experimental systems operator personnei and by LLNL personnel. No upsets are expected for any installed aircraft hardware. Section 7.12.3 and Appendix B deal with the RF levels expected to be encountered.

Since this is not a certification test, there will be no need to test a large number of frequencies Three frequencies have been chosen to match those selected for the fly-by tests. The illumination will be provided by the two-wire rhombic antenna configured in its common-mode configuration for vertical polarization. A fixed-frequency CW RF source will drive the appropriate LESLI RF amplifier. The UHF source will also be operated in a pulsed mode to simulate the pulsed radar planned for the fly-by (Wallops Island ASRF Radar).

\subsubsection{Sensors}


The sensors for the on-the-ground on-board instrumentation check-out tests will be the same as those used for the fly-by tests. These are a subset of the sensors used for the on-the-ground tests described in Section 7.5. The suite of 7 sensors will consist of one in the cockpit, two in the electronics bay, and four in the cabin. The cockpit sensor will be the vertically polarized D-dot on the sensor box. The electronics bay sensors will consist of the D-dot and cable current probe. All four of the cabin probes described in Section 7.5 will be included in the fly-by sensor suite.

The modified Collins VHF-700 transceiver box (RC-7) within the electronics bay will be removed for these tests so that the unqualified box will not be on the aircraft bus when aircraft power is applied.

\subsubsection{Test Instrumentation}

The instrumentation for these tests will be quite different from the stepped frequency experiments. It consists of spectrum analyzers and oscilloscopes rather than a network analyzer. The instrumentation will be provided by NASA LaRC and will already be installed into the aircraft and checked out prior to the aircraft's arrival at PL. A block diagram of the instrumentation is shown in Figure 7.8-1.

\subsubsection{RF Instrumentation}

RF signals from antennas and sensors will be multiplexed to the RF instrumentation by switches. These switches will be computer controlled in order to select the proper antenna and sensor for each test. Amplitude or power measurements will be made in real time on each of two external or internal RF probe channels simultaneously. A 2-channel digitizing scope will be employed for absolute voltage measurements. A pair of spectrum analyzers will be used for power measurements.

Data acquisition and instrument control will be computer based. An automated system will be used to maximize data returned during the fly-by tests. There will be minimal operator intervention during simulated in-flight data collection; all sensors will be recorded within a few seconds.

\subsubsection{Set Up}

The aircraft will remain in the same location as for the previous set of dipole tests. The LESLI rhombic antenna will be installed for common mode, vertical polarization operation by Test Operations and Support. The source will be connected as shown in Figure 7.8-2.

Sensor cables will be disconnected from the fiber optic links by PL/UIE personnel and reconnected to the on-board instrumentation by LaRC or LLNL personnel once disconnected from the on-board FO transmitters.

\subsubsection{On-Board Equipment Test Procedure}

Aircraft power will be applied by the LaRC ground crew. The on-board instrumentation will be powered up by the LaRC experimental systems personnel. The aircraft will be manned during this test series. A block diagram of the on-board instrumentation set up is shown in Figure 7.8-1.

On-board personnel and transmitter source operators will be in communication via walkie talkies. Once the instrumentation has been determined to be operational, the on-board experiment director will request that RF power to the rhombic antenna be turned on. RF power will be applied for a period of 30 seconds, unless either the Experiment Test Director or Facility Test Director requests power to be shut off earlier. This time period is estimated as the dwell time at a particular frequency that is likely to be acceptable by the FAA. Earlier tests in the LESLI have always been 
stepped and dwells at a frequency in the millisecond range have been acceptable. During this 30 second period, the automated on-board instrumentation will collect data. This procedure will be repeated until the instrumentation is operating satisfactorily

LaRC personnel will be responsible for archiving any data collected during the on-board experiments. LLNL, PL, and UIE personnel will assist as needed.

\subsubsection{Test Matrix for On-Board Instrumentation Checkout}

A detailed test matrix is presented in Appendix C. The on-board check-out test are series $G$ through I. Tests are shown in prioritized order so that if insufficient time is allotted, the lower priority tests can be dropped. Estimates of the duration of each test are also shown in Appendix C.

\subsection{Stepped CW Measurements: CWDAS with Rhombic Antenna}

The instrumentation for this test series will be the same as described in Section 7.7, except that the rhombic antenna will be used instead of dipoles with a frequency range from $300 \mathrm{KHz}$ to $1 \mathrm{GHz}$.

\subsubsection{Set Up and Probe Installation}

A block diagram of the stepped CW measurements made with CWDAS and the Rhombic antenna is shown in Figure 7.9-1.

Probes will be disconnected from the on-board instrumentation and reconnected to the fiber optic transmitters by Test Support and Operations.

\subsubsection{Test Operations}

The NASA aircraft will be pre-positioned for the start of these tests. During the test sequence, the aircraft will be moved up to four times. Repositioning of the aircraft will be accomplished by LaRC and Aircraft Operations personnel.

The PL/WSM LESLI Data Acquisition System (DAS)/CWDAS will be used to acquire CW data. Acquired data will be uploaded to the LESLI processing PC running MatLab for processing and distribution. Data will also be downloaded and archived into the PCSLEET data base.

The aircraft will be manned during portions of this test. There is no RF hazard to on-board personnel associated with these tests (see Sect. 7-11 and Appendix B). The antenna is the twowire rhombic antenna described above.

The majority of the instrumentation resides in the Source Shack. The instrumentation will be controlled by a PC-based controller that resides in the LESLI DAS trailer. The LESLI DAS trailer is located off the side of the antenna. The Source Shack is located at the drive point of the antenna. With the exception of the NASA/LLNL provided probes, sensors, and cables, all of the required instrumentation will be provided by the Phillips Laboratory. 


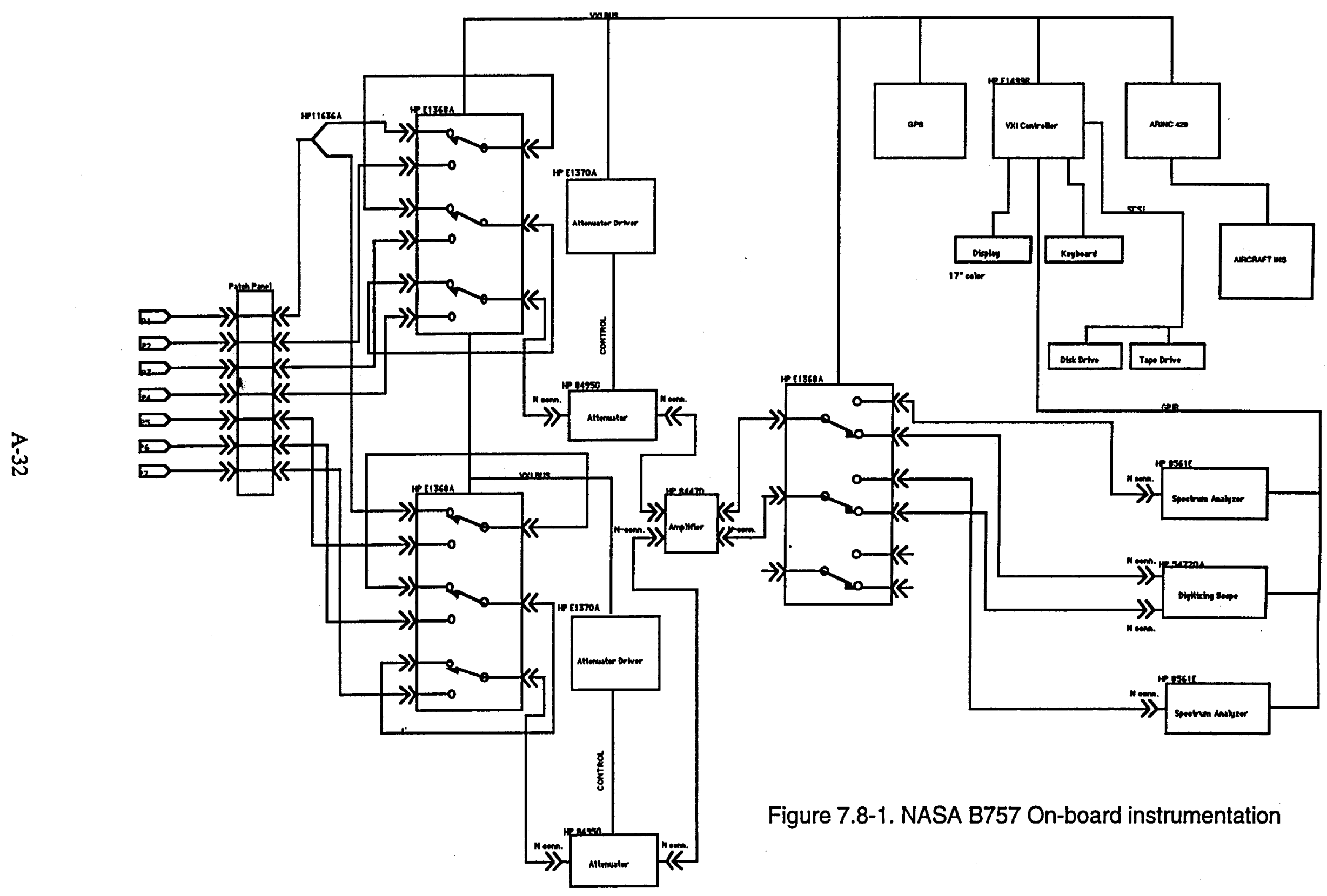




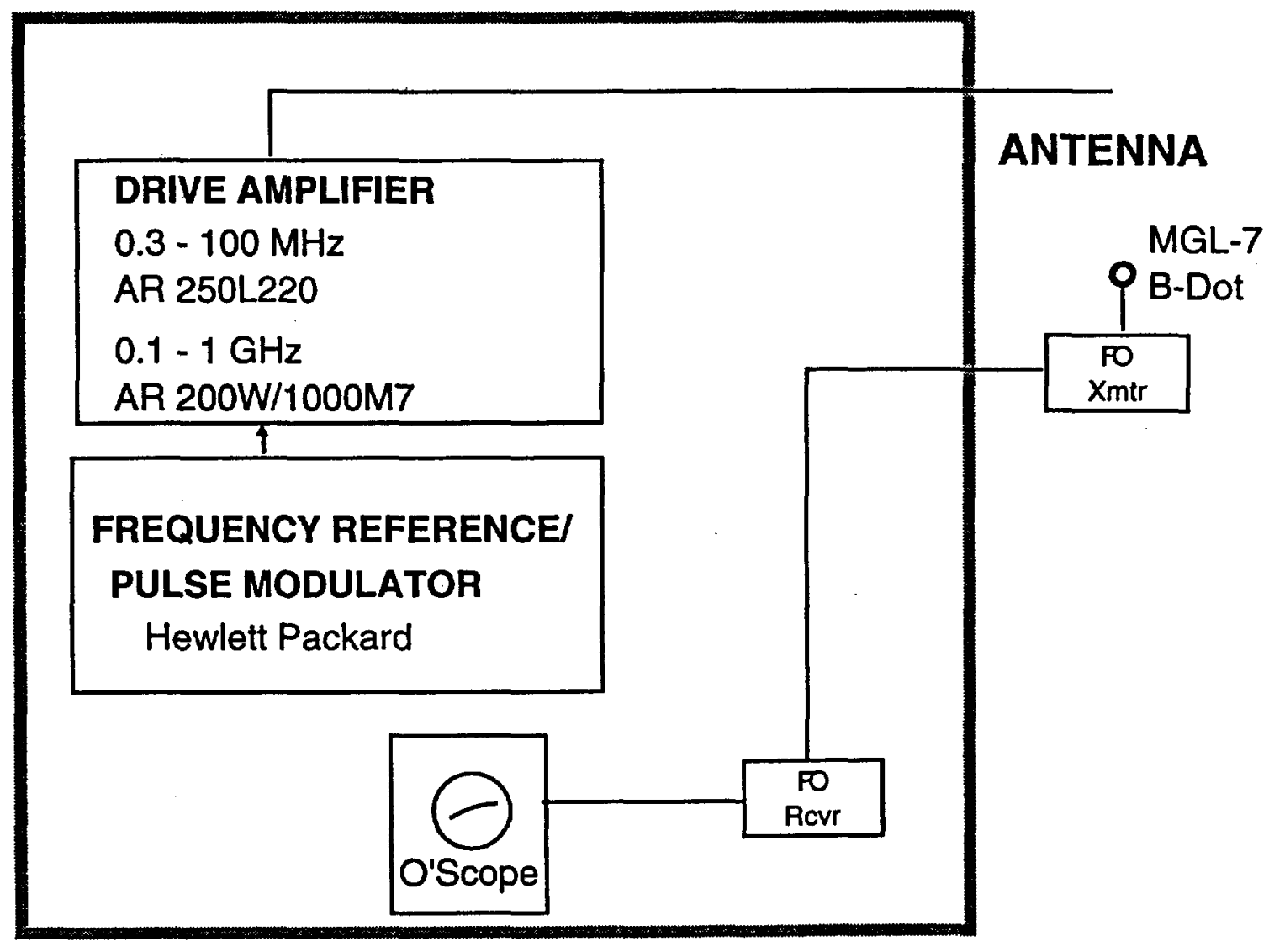

\section{SOURCE SHACK}

Figure 7.8-2. Source set up for on-board instrumentation check-out. 


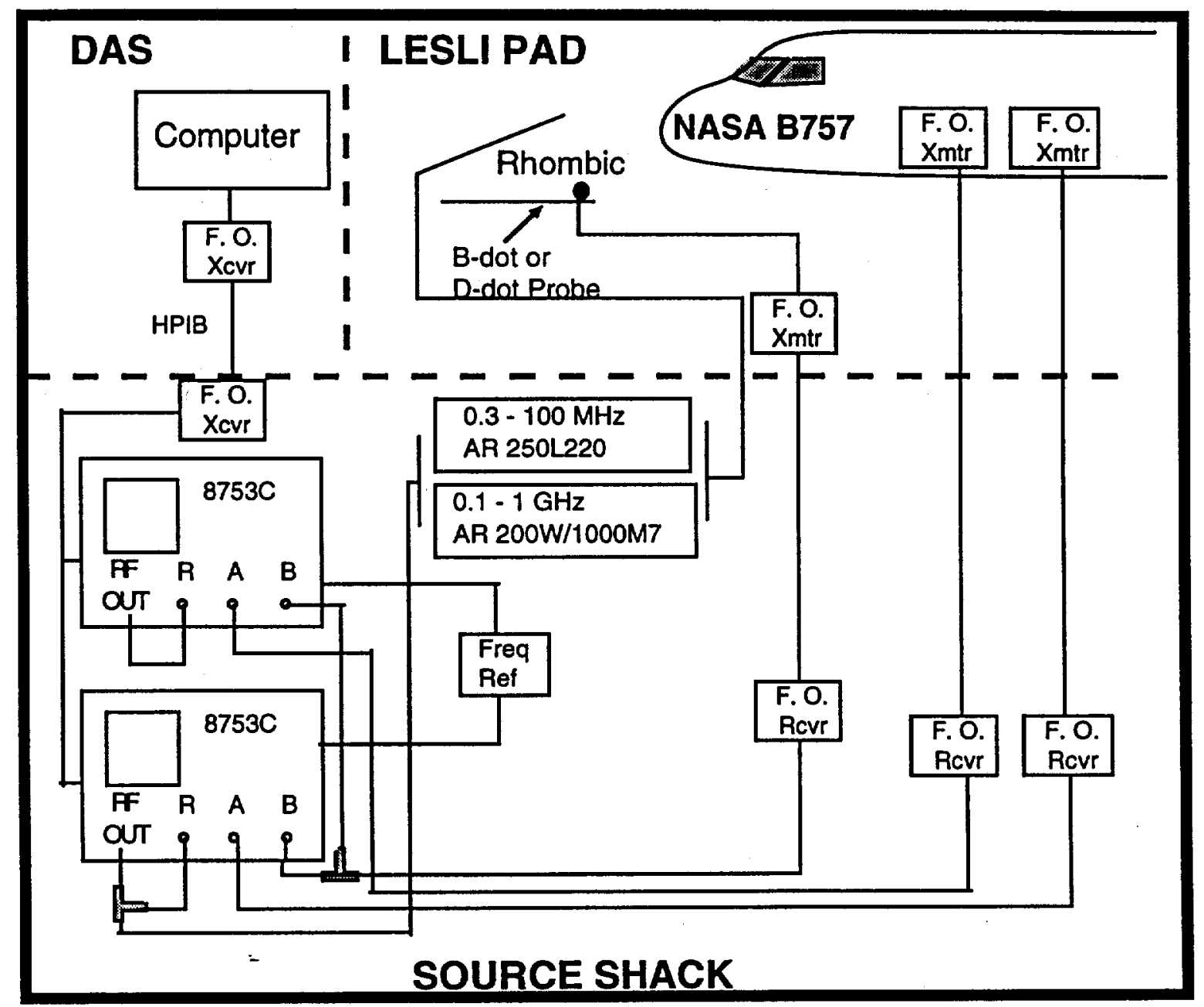

Figure 7.9-1. CWDAS instrumentation for stepped CW measurements with the rhombic antenna. 


\subsubsection{CWDAS Data Acquisition, Processing, and Analysis}

Data acquisition, processing, and analysis will follow the same procedures described in Section 7.7 .

\subsubsection{Instrumentation Noise Measurements for Stepped CW}

Instrumentation noise measurements for stepped $\mathrm{CW}$ measurements in the rhombic will be performed at the beginning of the rhombic test sequence. In the nose-on configuration, each data channel will, in turn, be tested. The probe on each channel will be removed and replaced by a matched load. Then under RF illumination in the rhombic, a complete sweep will be executed and recorded for that channel. The complete set of data will include a complete sweep for each channel and will represent the characterization of the noise floor for the measurements to be executed.

\subsubsection{Test Matrix for Stepped CW Measurements with Rhombic Antenna}

A detailed test matrix is presented in Appendix C. The stepped frequency tests with the rhombic antenna are series $\mathrm{J}$ and $\mathrm{K}$. Tests are shown in prioritized order so that if insufficient time is allotted, vhe lower priority tests can be dropped. Estimates of the duration of each test are also shown in Appendix C.

\subsection{Stirred Frequency Tests}

\subsubsection{Background}

As discussed in Section 4.0 it is hypothesized that the power density at a point in an aircraft cavity can have a statistical distribution that is dependent on the variation in excitation conditions (e.g., aspect angle) and the cavity losses. This hypothesis is based on the assuÔption the aircraft cavity is complex and large enough to be multimoded at the lowest frequency of interest. There is theoretical evidence that there exists statistical distribution function which describes the aircraft cavity electromagnetic environment (EME) for all possible excitation conditions and cavity configurations ${ }^{1}$. The theoretical distribution function is independent of the details of the shape or size of the aircraft cavity, the location of cable bundles, and the location of metal boxes such as avionics systems. Except for a normalization factor to account for specific external to internal coupling efficiencies and aircraft cavity losses, normally characterized by the quality factor $(Q)$, the statistical power density distribution is predicted to be independent of the particular cavity within the aircraft as well as the specific type of aircraft. These are very significant predictions which bear directly on how an aircraft's internal EME should be determined and how avionics systems should be tested.

The limited experimental data base currently available $e^{2}, 3,4$ supports this hypothesis and agrees with the postulated theoretical distribution function.

\footnotetext{
${ }^{1}$ Lehman, T. H., Paper presented at the Anechoic Chamber and Reverberation Chamber Operators Group Meeting, NSWCDD, Dahlgren, VA, Nov. 1992.

2 Hatfield, M.O., Freyer, G.J., Johnson, D.M., and Farthing, C.E., Electromagnetic Reverberation Characteristics of a Large Transport Aircraft, NSWCDD/TR-93/339, July 1994.

${ }^{3}$ Hill, D.A., Crawford, M. L., Johnk, R. T., Ondrejka, A.R., and Camell, D.G., Measurements of Shielding Effectiveness and Cavity Characteristics of Airplanes, NISTIR 5023, July 1994.

' Loughry, T. A., Hatfield, M.O., Freyer, G. J., Johnson, D. M., Ondrejka, A. R., and Johnk, R. T., Phase II Demonstration Test of the Electromagnetic Reverberation Characteristics of a Large Transport Aircraft, NSWCDD/TR, In Process.
} 
This test will provide additional data from a current operational passenger-configured aircraft.

\subsubsection{Specific Test Objectives}

1. Determine the statistical characteristics of the B-757 cavity EME.

2. Determine the cavity $Q$ by direct measurement of pulse decay time.

3. Determine cavity insertion loss.

4. Determine cavity-to-cavity coupling efficiency.

\subsubsection{Approach}

The characterization of cavity EME as described in this section will be performed with no power applied to any aircraft system. The test objectives will involve direct insertion of low power $(<1$ watt) signals into the aircraft cavities. The signals will be inserted into an aircraft cavity using a wide band horn transmit (TX) antenna. The power density in the cavity will be monitored by a similar wide band receive (RX) antenna. To achieve the desired statistical distribution data, an effective technique for cavity mode-mixing is required. The most common approach to date has been to use a mode-mixing technique, band limited white Gaussian noise (BLWGN), has been developed at the USAF Phillips Laboratory. This technique has been compared with mechanical mode-mixing in standard reverberation chamber (or mode stirred chamber) test facilities ${ }^{5}$. A primary objective of the Phase II demonstration test on a B-707, performed in March 1994, was to compare the BLWGN technique with mechanical mode-mixing for characterization of aircraft cavity EME. The analysis of the test data is still in process, but to date no unexpected results have been found.

A specific advantage of using BLWGN over mechanical mode-mixing is a significant reduction in test time. The demonstrated reduction in data acquisition time in the B-707 Phase II test was approximately a factor of 4.0 .

Figures 7.10-1 and 7.10-2 show the test instrumentation for characterizing the aircraft cavity EME using the BLWGN excitation technique. The control, stimulus, and measurement module, shown in Figure 7.10-1, contains the computer controller and plotter used to control the test equipment, acquire data and display data.

${ }^{5}$ Crawford, M.L., Loughry, T.A., Hatfield, M.O., and Freyer, G.J., Validation of Band Limited. White Guassian Noise Excitation of Reverberation Chambers and Verification of Applications te Radiated Susceptibility and Shielding Effectiveness Testing, NIST TR, In Process. 


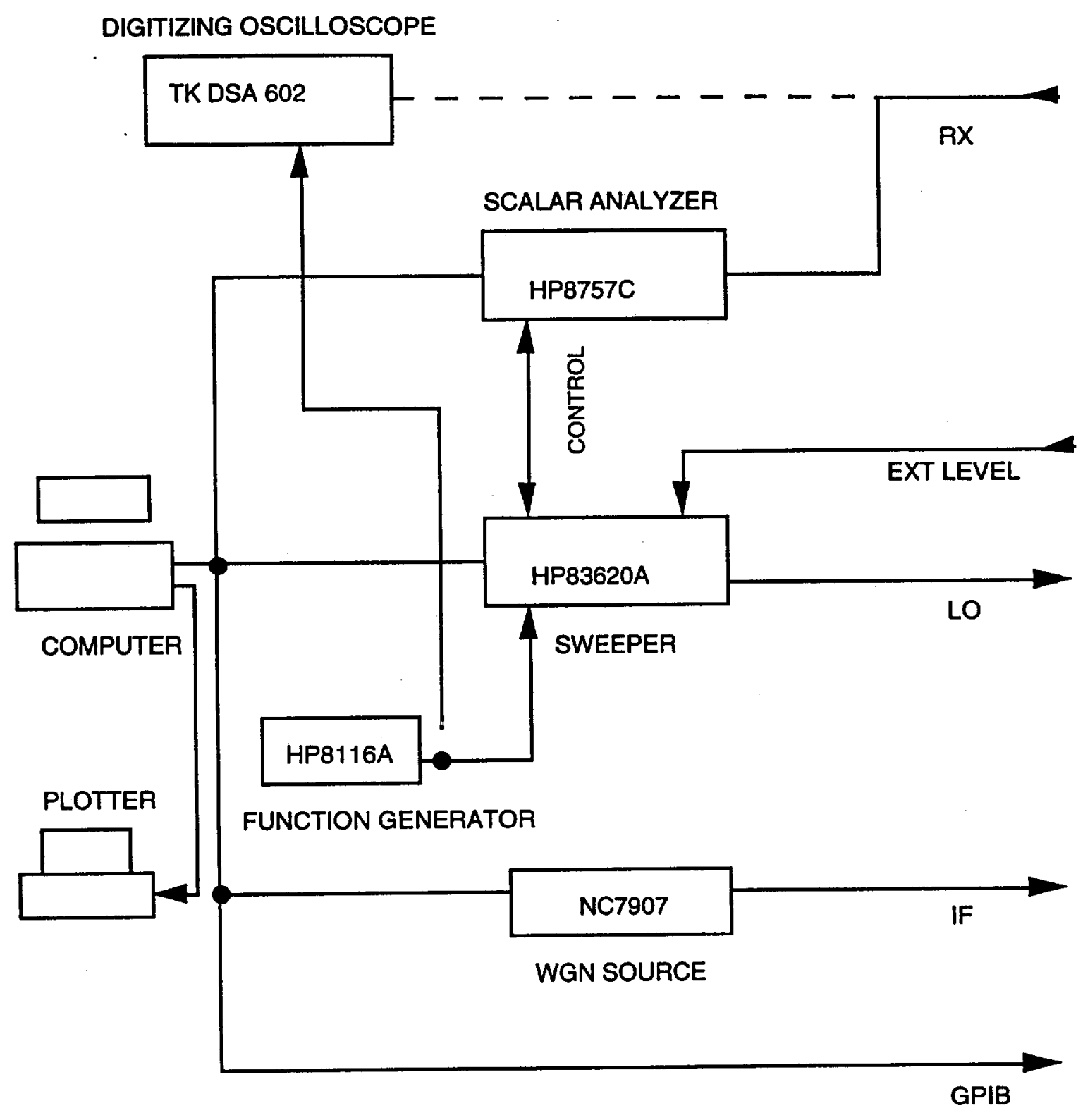

Figure 7.10-1. Control, stimulus and measurement module 


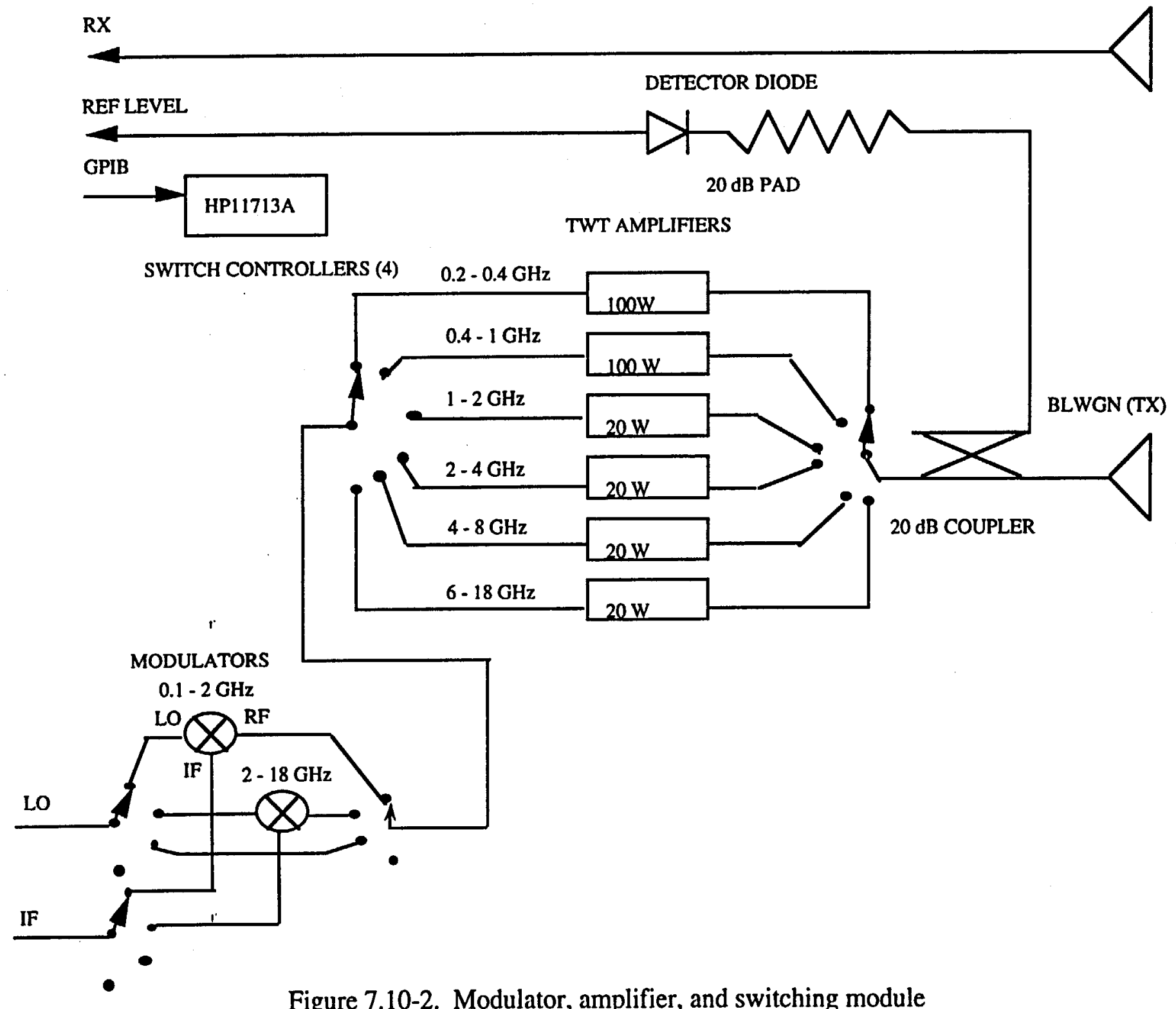

Figure 7.10-2. Modulator, amplifier, and switching module 
The sweeper generates the local oscillator signal which will cover the range 0.5 to $6 \mathrm{GHz}$ for this test. The WGN source generates noise at the proper bandwidth ( $50 \mathrm{MHz}$ for this test) as commanded by the computer.

For test objectives 1, 3, and 4 the function generator produces either a discrete frequency or a swept frequency continuous wave $(\mathrm{CW})$ signal. The scalar analyzer is used to measure the signals from the RX antenna.

For test objective 2, the function generator produces a discrete frequency, repetitively pulsed signal. A wide range of pulse repetition rates are available within the capabilities of the instrumentation system. Based on previous aircraft test experience, a $1 \mathrm{KHz}$ rep rate has been found to provide adequate accuracy and reasonable data acquisition times. A $1 \mathrm{KHz}$ rep rate will be used in this test for direct measurements of all aircraft cavity $Q$ values. The digitizing signal analyzer (DSA) is used to measure the pulse decay time of the signals from the RX antenna.

The modulator, amplifier and switching module, shown in Figure 7.10-2, performs the upconversion and amplification of the noise to produce the BLWGN signal. Part of the BLWGN signal is sampled and diode detected to provide an external level reference signal for the sweeper in Figure 7.10-1. The external level reference ensures that the BLWGN signal at the TX antenna remains constant despite varying amplifier gain and cable losses. The power radiated into an aircraft cavity will be controlled to be 1 watt or less. The switch controller selects the appropriate modulator and amplifier combination for the frequency being generated.

The transmit antenna locations and orientations are selected such that they are directed toward appropriate metal reflecting surfaces (e.g., the pressure hull, compartment walls, etc.) in each aircraft cavity. In no case will the TX antenna be directed toward aircraft electronic systems. Two TX antenna locations are defined for the cockpit and passenger cabin. Due to space limitations only one TX antenna location is defined for the electronics bay. These locations are identified in Figure 7.10-3 and described in Table 7.10-1.

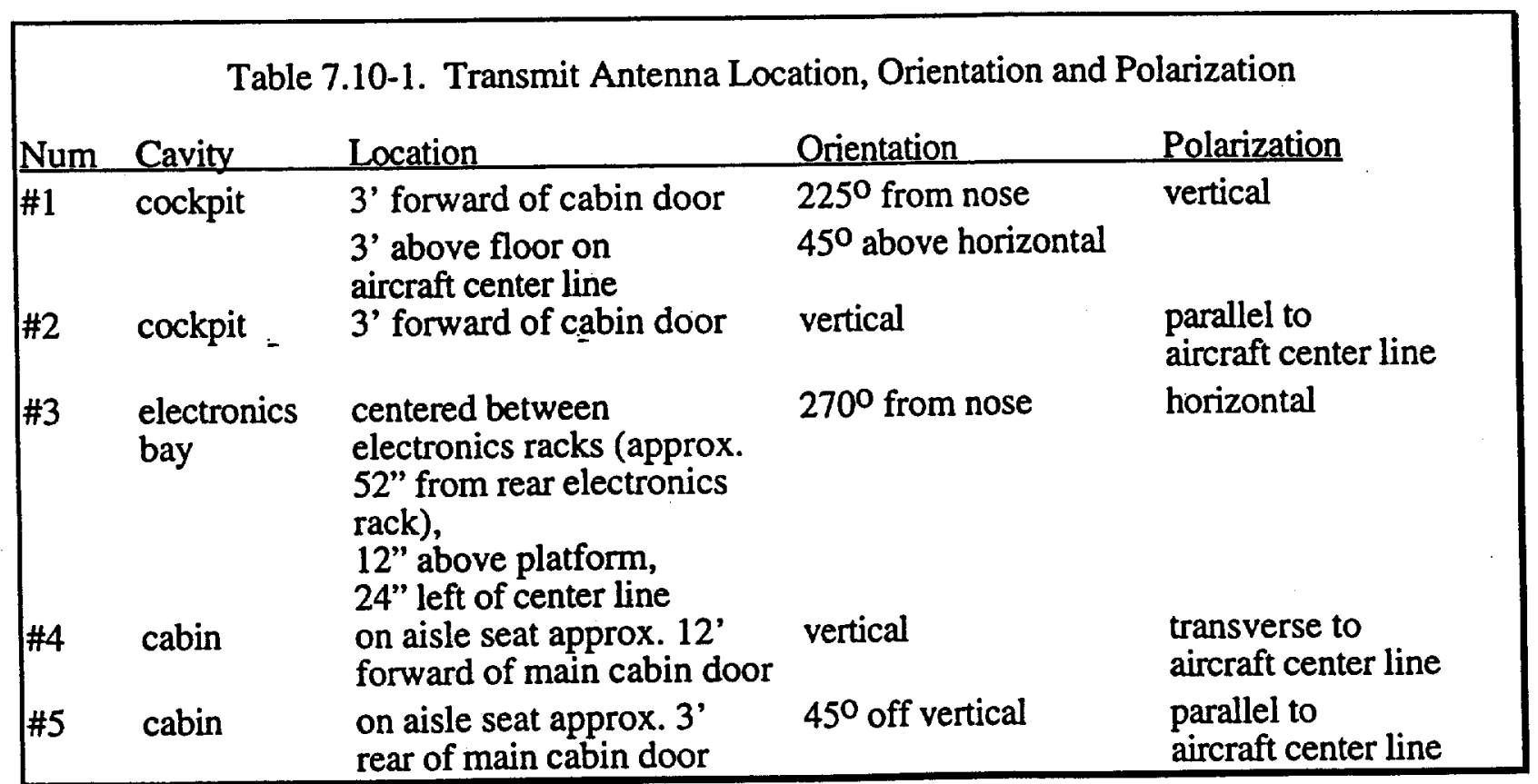

Several RX antenna locations will be used in each aircraft cavity. The RX antenna is passive and does not present any hazard to on board electronics systems. The RX antenna will be placed to 
obtain the desired aircraft cavity EM characteristics. The RX antenna will be located to avoid direct illumination by the TX antenna. A minimum of four RX antenna locations/orientations/polarizations will be sampled in each aircraft cavity. If test time permits, additional RX antenna locations/orientations/polarizations will be sampled in the cockpit and passenger cabin.

Coaxial cables will be attached to the TX and RX antenna feed points. These will be routed through the electronics bay or passenger cabin to the pressurization equalization port in the same manner as the sensor fiber optic cable for the primary test points. The coaxial cables installed for the cockpit ATOPS sensors will be used for the BLWGN TX and RX antennas.

Note that installation of all cables and placement of antennas will be coordinated with NASA aircraft operations personnel.

Sucoflex cable will be used from a point just inside the aircraft to the instrumentation cart located some distance from the aircraft. The Sucoflex cables have a highly attenuating cover which will minimize energy leakage into the aircraft. This approach will compensate for not using the standard isolating feed-throughs at the aircraft pressure hull.

The test matrix for the BLWGN excitation is given in Table C-22 in Appendix C.

\subsubsection{Test Organization}

The principal investigator on the BLWGN excitation test will be Capt. T. Loughry, PL/WSM. He will be assisted by SSgts. D. Little and C. VanZandt, also of PL/WSM. One or more of the following may also participate in this test: M. Hatfield, Naval Surface Warfare Center, Dahlgren Division; G. Freyer, Universal Systems, Inc.; and/or M. Johnson, Computer Science Corp. All of the above personnel have participated in at least one aircraft test using the BLWGN excitation technique.

All test instrumentation and data processing will be provided by Capt. Loughry. PL/WSM will designate a safety officer for the BLWGN test. All remaining organizational support function will be the same as on the primary test.

\subsubsection{Pretest Procedures}

Other than the aircraft survey which led to selection of the TX antenna locations, no special pretest activities are required.

The instrumentation, setup, and data acquisition and processing to be used for this test is the same as that employed on the recent B-707 test conducted by PL/WSM. All instrumentation and data acquisition software are available.

Calibration runs are performed on the "as installed" instrumentation, cables, and connectors.

\subsubsection{Safety}

During the BLWGN excitation test, all RF emisthons will be within the aircraft and the aircraft will not be manned, therefore no test unique personnel safety hazards exist.

The aircraft can remain grounded throughout the BLWGN test. 


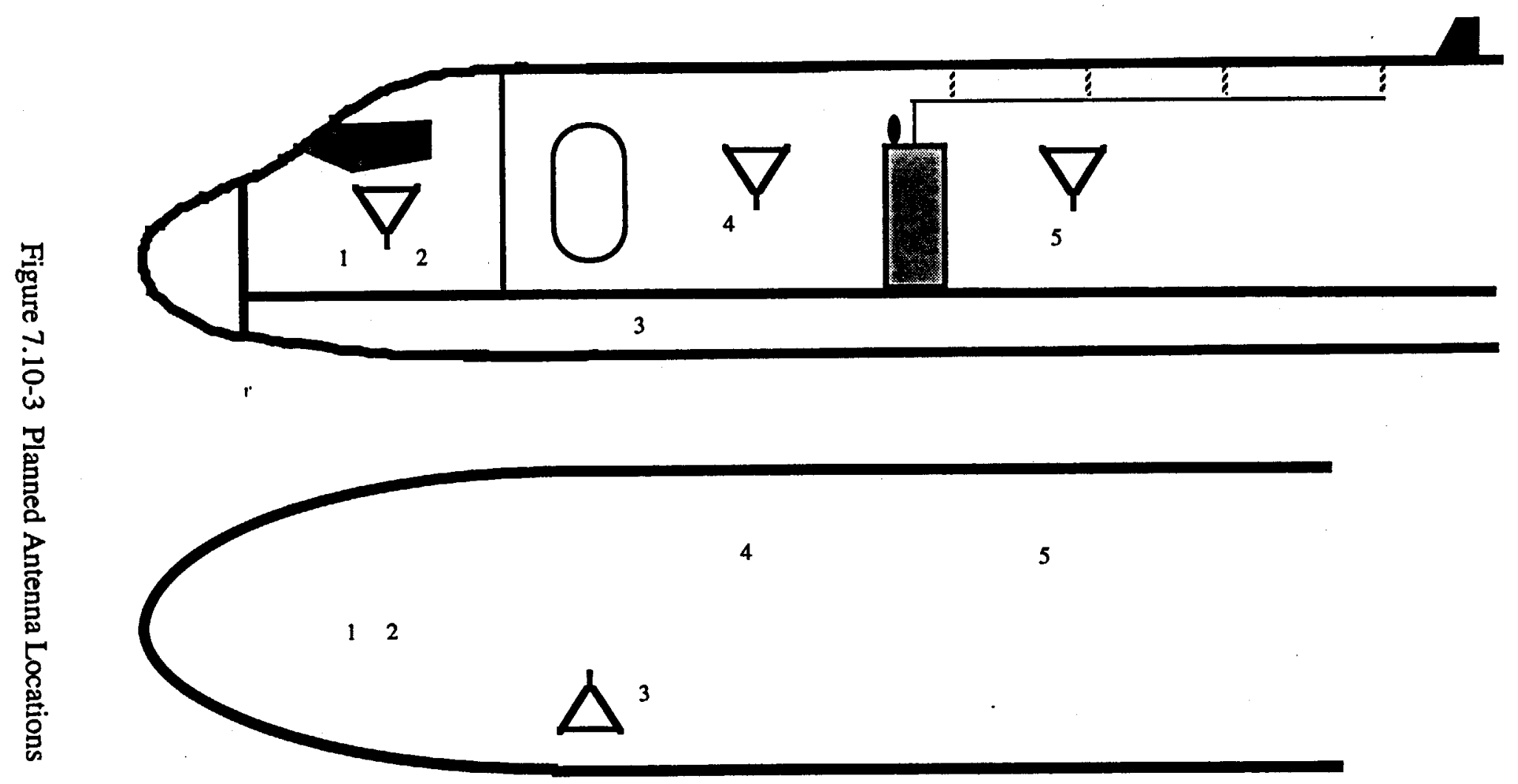

Figure 7.10-3 Planned Antenna Locations 
Note all test personnel access to the aircraft will be coordinated with the NASA aircraft operations personnel.

The safety issues and procedures for this test are the same as for the pertinent Sections of 7.12.

\subsubsection{Risk Analysis for Aircraft Electronic Systems}

If a directional TX antenna were pointed directly at an on board electronic system one meter from the antenna, the worst case field strength could be as high as $10 \mathrm{v} / \mathrm{m}$. Standard mode-mixing test procedures avoid direct illumination of test objects since this test configuration will bias the test results. In this test special care was taken in selecting TX antenna configurations to preclude direct illumination of any aircraft systems.

The mode-mixing accomplished through the BLWGN technique, in the ideal case, provides a uniform, isotropic, randomly polarized EME throughout the cavity. The power density in the cavity will depend on the $Q$ and volume of the cavity and the input power. Using the following equation derived from material in reference ${ }^{6}$

$$
\mathrm{E}(\mathrm{v} / \mathrm{m})=0.02 *\left(\mathrm{Q} * \lambda * \mathrm{P}_{\mathrm{in}}(\mathrm{w}) / \mathrm{Vol}\left(\mathrm{m}^{3}\right)\right)^{1 / 2}
$$

the estimated worst case power density is less than $14 \mathrm{v} / \mathrm{m}$. This value is based on the maximum input power of $1 \mathrm{w}$, on an estimate of the smallest test cavity volume in the B-757, and the maximum of all $Q^{*} \lambda$ values available from the $B-707$ tests. This last value was an isolated data point which was at least a factor of two higher than any other test data.

Since no power will be applied to any aircraft electronics system, these worst case estimates for the BLWGN test should present no risk to the on board electronics systems of the B-757.

\subsection{On-the-Ground Test Schedule}

The overall test schedule is presented in Figure 7.11-1. This schedule may be modified by the Test Director.

\subsubsection{Daily Test Schedule}

A typical test day for the stepped frequencies tests includes 8 hours of actual measurement time and additional time for preparation and stand-down of the site. A typical day will consist of the following:
a. Open site/site prep at 0630
b. Stand-up meeting at 0645
c. First measurement at 0715 hours
d. Lunch break 1100-1200
e. Last measurement at 1615
f. Meeting (TOWG) approximately 10 minutes after the last sweep
g. Data transfer complete/site secured by 1700

\footnotetext{
${ }^{6}$ Crawford, M. L., and Koepke, G.H., Evaluation and Use of a Reverberation Chamber for Performing Electromagnetic Susceptibility/Vulnerability Measurements, NBS TN 1092, 1986.
} 
Schedule for Phillips Lab Tests

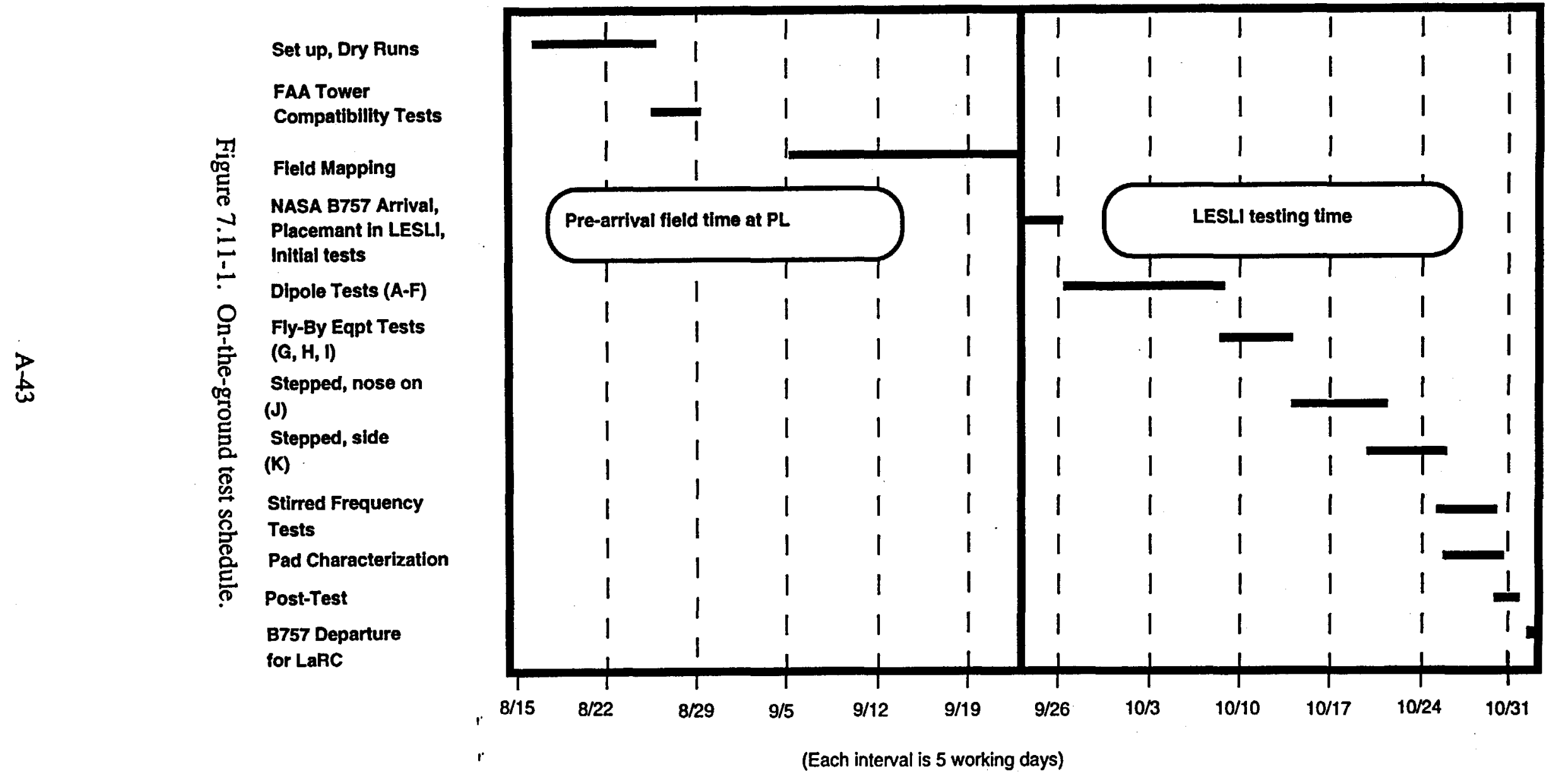




\subsection{Safety}

Performance of test operations in a hazardous manner can result in personnel injury, loss of life, or equipment damage. Serious mishaps can also affect meeting planned test objectives. This section summarizes data presented in more detailed hazards analysis and system safety program documentation and provides details for this specific test.

\subsubsection{Documents List}

The documents which define the applicable safety requirements and provide safety information are listed below:

(a) System Safety Program Plan, UIE-TR-88-0009, 22 Apr 1988

(b) Preliminary Hazards Analysis, UIE-TR-88-0010, 15 Apr 1988

(c) MIL-STD-882A, System Safety Program Requirements, 28 Jun 77

(d) Code of Federal Regulations, Title 29, Parts 1910 (Feb 83) and 1926 (Mar 83), (Occupational Safety and Health Act (OSHA))

(e) AFR 127 Series $(-2,-4,-7,-8,-12$, and -100$)$

(f) AFOSH 127 Series (as applicable)

(g) AFOSH 161 Series (-1 through -9 as applicable)

(h) AFPLR 127-3, Air Force Phillips Laboratory Regulation, Systems Safety Requirements

\subsubsection{Documentation Overview}

Document (a) is the System Safety Program Plan for the Boeing 720B. It establishes the basic system safety program for the maintenance and use of the Boeing $720 \mathrm{~B}$.

Document (b) is the Hazards Analysis for the Boeing 720B. It covers all of the potential hazards associated with the use of the Boeing $720 \mathrm{~B}$ and discusses the efforts to reduce all known hazards to acceptable safety levels.

Document $(c)$ is the source of the major safety requirements. It is required in the UIE contract.

Document (d), Code of Federal Regulations (Title 29, OSHA), is a broad and detailed delineation of general safety standards for industry. It covers a wide variety of occupations and working conditions to be considered in developing safe operating procedures.

Document (e), AFR 127 Series, provides information and guidelines on safety responsibility, safety program components, safety hazard abatement, and Department of Labor Inspections and Evaluations within the Air Force.

Document (f), AFOSH 127 Series, provides guidance for Air Force-wide and industrial safety accident prevention programs. It explains the hazards in and prescribes safety precautions for operating industrial equipment. It is designed to augment other Air Force publications and covers such subjects as the fundamentals of accident prevention, safety practices in construction and maintenance, health hazards and protection, fire protection, and safety in materials handling.

Document (g), AFOSH 161 Series, relates to health and environmental conditions such as radio frequency radiation, permissible exposure limits, carcinogenic substances, and other health subjects. 
Document (h), AFPLR 127-3, establishes a Technical Safety Committee within the Phillips Laboratory and describes safety review procedures. The document specifies that all experiments include an Emergency Action Plan which provides: (1) the general requirements and methods for alerting personnel of an emergency, and (2) the appropriate actions to be taken to respond to the emergency.

\subsubsection{Hazards Analysis}

The aircraft test environment during the tests, both under illumination during the dipole tests and the tests within the LESLI facility using the rhombic antenna, has been determined to be below the levels that might present a hazard to on-board electronic systems and to humans. Further discussion of this issue is found in Appendix B. In addition, an on board electric field sensor, such as a Narda 8616, will be used to provide a real time indication of field strengths when the sources are operational and personnel are present in the airplane.

Many hazards associated with the NASA aircraft are similar to those associated with the PL Boeing 720B aircraft noted in the document Preliminary Hazards Analysis, UIE-TR-88-0010, 15 Apr 1988 , which was prepared for the operation, maintenance, and use of a Boeing aircraft in EM testing experiments. The reader is directed to these documents for detailed discussions of the hazards and their mitigation to acceptable levels.

\subsubsection{Facility SAFETY PROGRAM}

Fundamental to a responsive and responsible safety program is implementation of the concept that anyone who detects an unsafe condition has the responsibility to alert the rest of the test team and immediately stop the hazardous operation(s). This section presents the safety program to be enforced for the facility.

\subsubsection{Safety Organization}

Ultimate responsibility for ensuring safety throughout the program resides in the Facility Test Director. Responsibility for implementation of all safety measures on-site will be delegated by the Facility Test Director to the designated Safety Officers as listed in Table 7.12-1. Each Test Safety Officer will ensure that the safety rules and procedures established by the PL are followed. The Facility Test Director will determine when conditions are safe for testing, including restart of test operations after an unsafe condition has been detected. The Facility Test Director will make this decision after consulting with each Safety Officer, who has direct access to the Facility Test Director. The responsibilities of each safety position are described in the following paragraphs.

\begin{tabular}{|llll|}
\hline \multicolumn{2}{c}{ Table $7.12-1$. Test safety assignments. } & \\
Title & Name & Office & Test Site \\
& & & \\
Facility Test Director: & Mr. L. Dao & $505-846-0995$ & $846-5998$ \\
NASA Aircraft Safety & Mr. M. Basnett & $804-864-3900$ & \\
Site Aircraft Safety: & Mr. R. Christianson & $505-846-1177$ & $822-4702 *$ \\
Site / Operation Safety: & Mr. Don Lin & $505-846-0995$ & $846-5998$ \\
* Pager Number & & & \\
\hline
\end{tabular}

7.12.3.3 Facility Test Director. The designated Facility Test Director has the overall authority to execute and implement those efforts required to achieve safe operation. He will be supported by 
specifically designated personnel in each of three areas: (1) Test Operations , (2) Aircraft Operations, and (3) Facilities.

7.12.3.4 Test Operations Safety. This individual is responsible for safe operations during test setup, preparation, and aircraft instrumentation.

7.12.3.5 Aircraft Operations Safety.(NASA and Site) These individuals are responsible for safe operations in and around the NASA aircraft while at the Phillips Lab.

7.12.3.6 Facility Safety. This individual is responsible for the safety of facility operations including data acquisition equipment, DAS operations, weather impacts, and general facility O\&M.

\subsubsection{Safety Procedures}

The procedures that are needed to ensure safe operations for the facility fall into three categories: (1) normal site safety operations, (2) NASA B-757 operations, and (3) procedures unique to test operations.

\subsubsection{Normal Safety Operations}

\subsection{Site Safety}

The Facility Support will set up safety barricades and signs for the test pads. A site safety briefing will be conducted prior to the start of work. Important considerations include:

(a) The use of physical barriers such as safety ropes and fences to limit personnel access into hazardous areas,

(b) Limits on the number of personnel allowed into the hazardous area,

(c) Establishment of functions, procedures and locations for personnel required in the hazardous area,

(d) Control of general personnel access to the facility from a safety standpoint,

(e) Control of personnel access to the site and in the site working volume during the direct drive and $\mathrm{CW}$ test operations,

(f) Provision of safety equipment and instructions for its use, and

(g) Regular inspection and checkout of safety equipment.

7.12.4.1.2 Area Signs. All hazardous areas require proper marking and identification so personnel not familiar with hazards in the area are made aware of the dangers. General guidelines for this type of activity are contained in both AFR 127-101 and OSHA Safety and Health Standards 29CFR 1910; 2206, revised Nov 7, 1978. A detailed discussion, therefore, of area posting requirements is not repeated in this supplement.

7.12.4.1.3 Access to Area. As one consequence of the security requirements of the test sites, access is limited to those personnel who have a definite need to be in the area. This supports safety considerations by limiting the number of people in the working areas. During times of hazardous operations, the Facility Test Director and Test Safety Officers will have complete control over the operations on the site and of access to the working areas. They will be able to suspend or terminate any test activity at their sole discretion, if in their judgment, safety is being compromised. Any visitors wishing to go within the controlled test area must get the permission of the Facility 
Test Director (or his delegate) prior to entering. PL will provide Contractors and Government organizations with any special site safety equipment that may be required such as hard hats, radiation detectors, etc.

7.12.4.1.4 Safety Equipment. The on-site safety equipment is divided into three classes:

(a) First aid equipment,

(b) Personal safety equipment, and

(c) Special safety equipment.

Adequate first aid equipment is currently located at the sites, and site personnel are trained in first aid techniques. Individuals performing hazardous operations, such as working on and around the test stands, will be required to use proper safety equipment to minimize hazards. Contractors and Government organizations which have personnel operating on the site will provide safety equipment for their personnel.

7.12.4.1.5 Personnel Safety Briefing. Safety procedures are only effective when adhered to. Each Test Safety Officer will ensure all test personnel receive a safety briefing prior to the start of testing at each test site. All personnel will be briefed in the following areas:

(a) Performance of daily tasks in a safe manner,

(b) Adherence to safety procedures for hazardous operations,

(c) Observance of all warning signs in the area,

(d) Continual examination of the site and test operations for potentially dangerous activities and conditions,

(e) Adherence to emergency procedures and regulations,

(f) Notification of proper personnel regarding safety matters,

(g) Adherence to safety requirements on the NASA aircraft, and

(h) Adherence to site safety requirements (e.g. hard hats, high voltage equipment, environmental hazards, etc.).

"Safety consciousness" is each individual's responsibility at all times, not just the responsibility of the Safety Officers.

Selected site and contractor personnel have been trained in areas of first aid, CPR, safety inspections and the proper use of special safety equipment. At least one person who is qualified to administer first aid will be physically present at the test site at all times.

\subsubsection{NASAB-757 Safety}

The NASA aircraft is a fully operational Boeing 757-200 aircraft. After the aircraft is towed, parked and properly oriented on the LESLI pad, it will be inspected by the Ground Crew Chief to ensure that it is properly and safely positioned. Personnel working on the NASA aircraft will adhere to NASA aircraft safety procedures. All personnel will be briefed on aircraft specific safety issues by the NASA aircraft Ground Crew Chief prior to work on or around the aircraft. The aircraft will be grounded at all times except when testing is being performed. The aircraft will be grounded prior to the entry or egress of personnel prior to or after RF illumination. A $150 \mathrm{lb}$ power bottle aviation-type fire extinguisher on the pad satisfies NASA safety requirements for fire hazards. 


\subsubsection{Test Unique Operations}

Operating procedures have been established at the EM test sites in the following areas: operations and maintenance, protective equipment, housekeeping, lighting and general safety practices. Under general safety practices there are restrictions concerning clothing, jewelry, intoxicants, tampering with equipment, personal conduct, electrical repairs, railings and toe boards, and the use and storage of hazardous material and equipment. Aircraft maintenance and test support personnel will comply with all safety requirements. It is the responsibility of the Facility Test Director to inform or to designate others to inform NASA, LLNL, or other visitors regarding these practices.

\subsubsection{Emergency Procedures}

If an emergency situation exists, all test activities will cease and the proper emergency personnel shall be contacted. All personnel will be briefed on access to emergency services.

\subsubsection{Medical Services}

The USAF Hospital at Kirtland AFB will provide emergency assistance and ambulance services for all on-site personnel.

\subsubsection{Fire Protection}

The $377 \mathrm{ABW}$ will provide fire protection services on an on-call basis. It is not expected that any on-site support will be required. Portable fire extinguishers will be required at the aircraft at all times. A $150 \mathrm{lb}$ power bottle aviation-type fire extinguisher on the pad will be required. Small portable fire-extinguishers will be located in trailers and office buildings for office/equipment fires.

\subsubsection{Accident Reporting}

Property and equipment damage, and personnel injury accident reporting procedures required by the Federal and State Governments, by the Government and its participating contractors and subcontractors/vendors will be complied with. Table 7.12-1 lists the personnel to be notified in case of any emergency or safety hazard.

\subsubsection{Safety Summary}

The Facility Safety Program consists of five major elements:

(a) The designation of a safety team with complete safety authority on the test site,

(b) The establishment and implementation of safety procedures for normal site operations, test preparation and testing operations,

(c) The acquisition and training for the proper utilization of required safety equipment. These include First Aid equipment, personal safety equipment, and any special safety equipment as may be required to minimize potential accidents,

(d) Personnel briefings stressing day-to-day safety practices, and specialized training for designated personnel in the areas of first aid and other safety matters, and

(e) The establishment and implementation of emergency procedures. 


\subsection{Security}

No classified documentation, equipment, or data will be handled during this test. Therefore accounting and control procedures for classified information are not required. There will, however, be base, facility, and test aircraft access and physical security requirements.

\subsubsection{Classified Information and Equipment}

No classified documentation, equipment, or data will be handled during this test.

\subsubsection{Base and Test Site Access}

\subsubsection{Base Access}

Kirtland AFB is a controlled access Air Force facility. In general, personnel entering base premises are required to show a vehicle entry pass. Contractors who routinely require access should follow the procedures in their On-Base Security Agreement for the issuance of entry passes.

\subsubsection{Test Site Access}

During test operations, the test site will be unrestricted to personnel who have base access. Appropriate signs will be displayed to require visitors to report to a central location at each test site. A roster of authorized personnel will be provided to all test participants at the start of the test. All test personnel will be charged with challenging individuals who are unfamiliar and are not on the roster. During off-testing hours, the test site will be locked and site security will be ensured by base security police within their normal patrolling routes.

\subsubsection{NASA Aircraft Access}

During test operations, access to the aircraft may be controlled by Aircraft Operations. Access will be permitted only to personnel who have been approved by Aircraft Operations or who are escorted by a person with escort authorization. During off-testing hours, the aircraft doors will be closed and the stairs and test stands may be pulled away from the aircraft. At the discretion of Aircraft Operations, the stairs and stands may be chained together to prevent unauthorized movement.

\subsection{Add-on Tests}

As time permits and necessity dictates, additional tests may be added to the program. At present, two major test have been indicated as needed for completeness.

\subsubsection{Pad Characterization}

As has already been mentioned, an electromagnetic characterization of the pad will be required. Specifically, the constitutive parameters of the pad must be measured to permit modelers to include the ground in simulations. Since the parameters are unknown, and the concrete/aggregate/rebar/soil is not precisely describable, a measurement of the parameters is needed. Since it is expected that the ground is inhomogeneous, a measurement scheme returning parameters averaged over a spatial extent will be made. These measurements will be made without the NASA 757.

To execute the tests, a long wire (the rhombic wire) will be spported a fixed height above the concrete pad on a styrofoam support strip and will be extended across the pad to the full extent of 
the wire. The height will be chosen to be as small as possible but consistent with the thicknesses of commercially available styrofoam panels. At this time the height is expected to be between $1 / 4$ and 3/4 inches. The wire will be driven against the aluminum source-region ground screen at the rhombic feed point. The wire will be extended in two separate directions (Cases 1 and 2) since the ground plane may be inhomogeneous with constitutive parameter variation as a function of angle with respect to the original rhombic axis. Overhead and cross sectional views of the wire placement are shown in Figures 7.14-1 and 7.14-2.

The wire will be driven against the aluminum plane and the current distribution will be measured by probing with an Prodyn I-320 current probe. The current will be probed at specified points along the wire so that estimates can be made of the attenuation and phase constants of the wave propagating along the wire. From this information, it is expected that the conductivity and permittivity of the pad can be extracted using the theory presented by Chang, Olsen and Kuester ${ }^{7,8,9}$. The test matrix for the pad characterization is presented in Table C-23.

\subsubsection{Horizontal Polarization Near Lowest Order Resonances}

A set of measurements at frequencies in the vicinity of the first body resonance of the B-757 has been suggested. Since the resonant wavelength for the fuselage is in the vicinity of 94 meters, the resonant frequency is approximately $3.2 \mathrm{MHz}$. The lowest order resonance associated with the wings falls above $4 \mathrm{MHz}$ while resonances associated with combinations of the wings and fuselage fall above 3.7 MHz. To make such measurements under horizontal polarization, it is expected that the LESLI rhombic will be used in its differential mode rather than attempting to use a $\lambda / 2$ dipole since a 47 meter dipole might be mechanically difficult to handle. A balun will be used at the rhombic feed point to ensure push-pull operation so that a horizontally polarized field can be generated. Due to the lack of a reliable broadband balun covering 0.3 to $1000 \mathrm{MHz}$ and questionable quality of the horizontal field, this was not included in the extensive set of rhombic measurements. Nonetheless, given the advisability of having horizontal drive and the inability to efficiently deal with a dipole at the 94 meter wavelength, it was decided to use a balun able to deal with a band possibly covering the lowest order resonances of the aircraft. A balun available to radio amateurs and advertised for 3.5 to $30 \mathrm{MHz}$ will be used and an attempt will be made to reach $3.2 \mathrm{MHz}$. The airplane will driven in its broadside configuration and measurements will be made at select test points. The decision as to which test points will be recorded will have to include factors such as priority and time available. The test matrix for this horizontal polarization test is provided in Table C-24.

\footnotetext{
${ }^{7}$ Chang, D. C. and Olsen, R. G., "Excitation of an Infinite Antenna Above a Dissipative Earth," Radio Science, Volume 10, No8,9, pp. 823-831, August-September, 1975.

${ }^{8}$ Kuester, E. F. , Chang, D. C. and Olsen, R. G. , " Modal Theory of Long Horizontal Wire Structures Above the Earth, 2, Properties of Discrete Modes", Radio Science, Vol 13, No. 4, pp 615-623, July-August 1978.

9 Olsen, R. G. , Kuester, E. F. and Chang, D. C. , Modal Theory of Long Horizontal Wire Structures Above the Earth, 1, Excitation", Radio Science, Vol. 13, No. 4, pp. 605-613, July-August 1978.
} 


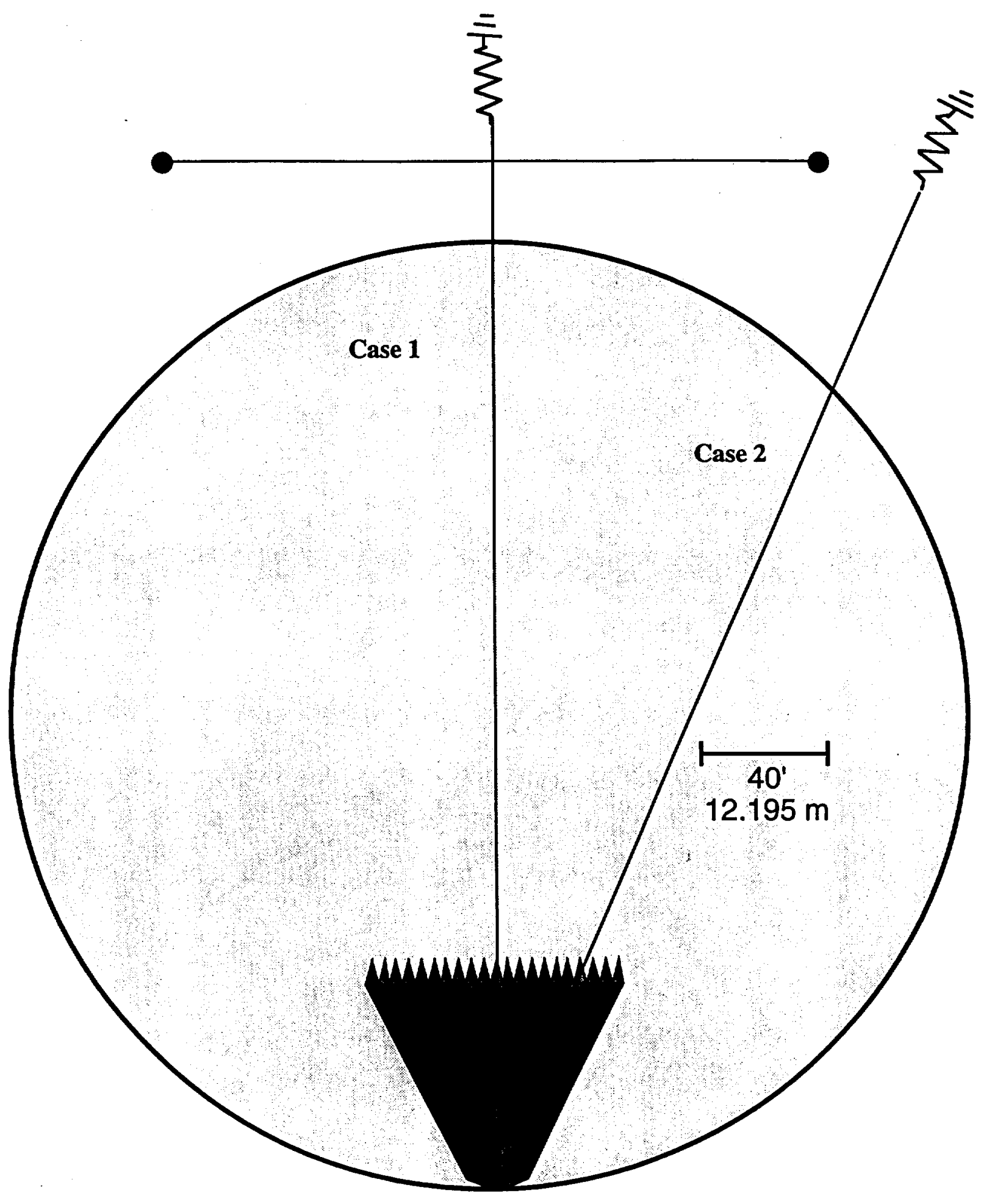

Figure 7.14-1 Pad Characterization Wire Layout for Two Cases 


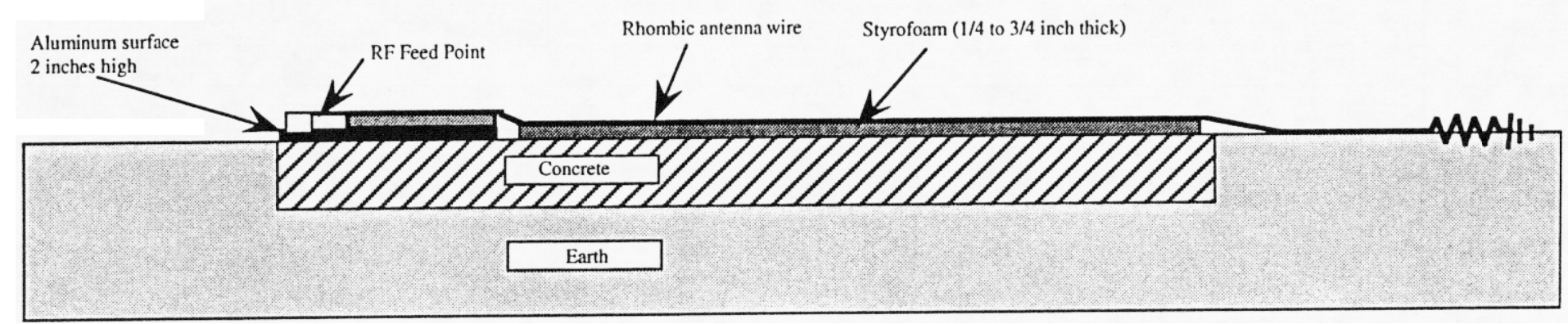

Figure 7.14-2 Pad Characterization Wire Layout - Cross Sectional View 


\section{Appendix A Plane Wave On-the-Ground Test Technical Objectives}

In this section we discuss some specific technical objectives of the on-the-ground low-level tests.

A primary objective of the tests is the collection of data for use in the validation of codes that are employed in the modeling of electromagnetic environment in aircraft and its effects on aircraftborne electronic systems. Of particular interest at this time is the validation of FDTD modeling of coupling into the airframe using a code such as TSAR. The data are also intended for inclusion in a library for use in validation exercises involving modeling tools based on the broad range of techniques and algorithms that are either presently available, in development or otherwise.

The term validation is used to denote the determination whether a particular code is capable of producing accurate results for specific modeling assessments in which it has been applied. Clearly, this will "validate" the physics kernel of the code, its numerical algorithms, and its overall ability to provide the "right" answer. In this particular program, the codes will be tested extensively in their "intended" range where approximations other than discretization are not required (perhaps up to hundreds of $\mathrm{MHz}$ ) as well as in the range where approximations and special algorithms will be required to estimate stress. While a description of the approximations used to extend the domain of applicability of the code is beyond the scope of this plan, adequate attention has been paid to collecting appropriate data to allow for its use in future validation activities.

During the course of collecting data for the validation exercise, we will also collect data to help us deal with some issues related to modeling, the impact of resolution or "graininess" in the model, random effects, repeatability, and robustness of the measured results. The following, while not totally inclusive, are some of these issues. Note that scheduling constraints may preclude some of the tests.

A.1 Body Resonance. A concern is whether the computer models can accurately account for coupling into the airframe at low frequencies, especially when the external airframe is in body resonance. At these low frequencies, the wavelength is very long with respect to windows and other apertures so that the major coupling path to the interior would likely be through wires or structures that electrically penetrate the airframe or through evanescent wave pickup onto wires or structures near apertures. The degree of coupling may depend heavily on small or seeminglyinnocuous features that could easily be missed in converting the physical structure into a computer model. Since we are emphasizing the FDTD model technique for low frequency coupling prediction, it is important that this capability be validated. This can be done by comparing predicted and measured field coupling at interior points in the airframe.

A.2 Thin Wires. Once the EM energy penetrates the airframe, it couples onto the wires and cables that connect the LRUs. (Even fiber-optically interfaced LRUs will have power cables that will pick up energy.) The basic FDTD technique would have difficulty modeling these cables because they are very thin with respect to the resolution element of the computer model. To overcome this problem, special algorithms have been developed that allow thin wires to be modeled. The performance of these algorithms has been validated in simpler geometries and will now be validated in an aircraft environment. This can be done by comparing predicted and measured bulk cable current coupling. Since the only interconnect cables in the FBL/PBW aircraft will be power cables (that are isolated from the airframe), the measurement and simulation will choose a cable that simulates the expected configuration - even if it has to be a dummy cable. This cable should have the correct type of bulkhead feedthrough and the correct type of airframe bonding, if any.

A.3 Thin Slots. Another feature that the basic FDTD model must often treat in a special way is a thin slot. Thin slots are analogous to thin wires - they require special algorithms. In the Boeing 757-200 test object, we feel that the coupling through windows will dominate and that coupling 
through thin slots such as door frame and hatch frame cracks will be negligible by comparison. However, this assertion, while very likely to be true for the test points of interest during these tests, should be validated. This can be checked by comparing coupling measurement with a suspected door frame or other thin slot initially open and then sealed with metallized tape. If the internal coupling doesn't change, the slot aperture coupling was negligible. The measurement should also be repeated with the door open, because if the door does not make good electrical contact around its perimeter, the coupling would be similar at resonant frequencies to that of an open door.

A.4 FDTD Resolution and the Effect of Randomly Located Features. When the airframe is modeled for the FDTD analysis, a resolution element is selected based on a combination of issues including the wavelength at the highest frequency of interest, the detail desired in the model, and/or the computer resources available. Metallic and dielectric structures that are small with respect to the size of the resolution element may be ignored. Some internal objects such as the crew, passengers, carry-on luggage, and other moveable cargo may be large, but their instantaneous positions cannot be described deterministically. The positions of "fixed" objects may vary from aircraft to aircraft over the fleet ensemble, so their positions must also be considered as randomly placed. In the computer model, these variations are either ignored or are described with representative structures in the model. The aggregate effect that these omitted or approximated structures have on coupling may be significant. It is important that the magnitude of the effect that these omitted or approximated features have on coupling be understood. In order to get an approximate estimate of the effect of these features a few measurements will be made where realistic representations of such objects of various sizes are moved around in random positions, or with someone in the cockpit to represent the pilot. The variations we see in these measured data will provide insight as to how accurately we can ever expect the predictions to approach reality.

A.5 Cockpit Window. The cockpit window is expected to be a significant coupling apertuse. However, this window is usually coated with an electrically conducting film that is connected by cable to a power supply to allow it to be heated for deicing. Therefore, the coupling through this aperture will depend strongly upon how this film is electrocally bonded to thg surrounding metal surface, if it is at all. If there is no bonding to the akrframe, this window might act like a large patch antenna, admitting frequencies above and below the aperture resonance that would be expected for an open window of the same size. If the film is bonded to the airframe in one location, the window would still accept frequencies only above its aperture resonancg. If the film is electrically opaque to the wave, then a thin slot algorithm may be required to describe it. We must validate that the model we use to represent this window is accurate. We can isolate the coupling through the window by making a measurement with the window open and then repeating it with the window sealed with metallic foil and tape. These measurements would allow us to investigate the computer model of the window. Similar data has been taken by the Phillips Laboratory that will provide a qualitative insight to cockpit coupling. This reduces the priority for this measurement. This low priority test will be placed at the end of the test schedule. Due to the compressed test schedule, this test will not likely occur.

A.6 Angular Sensitivity. When flight tests are ultimately made, the exact orientation of the aircraft at the time that the EME signal measurement is made will be estimated using on-board systems. There will be some error in these estimates. It is important to know how sensitive the measurements of coupling are to small changes in pitch, roll, and yaw. In the on-the-ground coupling tests, varying pitch and roll may be difficult. However, it is simple to vary yaw. Therefore, a series of measurements will be made with small changes in aw in order to quantify the error such warkations will have in coupling.

A.7 Measurement Repeatability. Measurement repeatability is an issue similar to angular variation. We can never expect to validate to an accuracy better than the random measurement error. To test this error, measurements of coupling into the airframe for a few select configurations and orientations will be repeated several times over the testing period. 
A.8 Effects of the Earth. One potential source of modeling error associated with on-theground tests that is not found in airborne tests is the effect of conductivity and dispersion in the soil or concrete upon which the aircraft sits. These values will change with the moisture content of the soil or pad. 


\section{Appendix B Power Densities and Field Strengths for Aircraft Tests}

\section{LESLI Rhombic Tests}

The aircraft will be manned during several tests, namely, during the fly-by instrumentation tests and during some tests which call for evaluation of sensitivity of the results to personnel deployment. The field strengths or power density in such tests must be below the levels which represent a hazard to humans. The IEEE C95.1-1991 / DoDI 6055.11 (Draft) standard for maximum permissible field exposure for personnel in a controlled environment is $1 \mathrm{~mW} / \mathrm{cm}^{2}$ over the frequency range from $300 \mathrm{KHz}$ to $1 \mathrm{GHz}$. This corresponds to an electric field strength of $61.4 \mathrm{~V} / \mathrm{m}$ for a plane wave field in free space. Neither LLNL, NASA, PL, or the older ANSI standard C95.1-1982 have exposure limits below $1 \mathrm{~mW} / \mathrm{cm}^{2}$ over any frequency band. Hence field levels below this level can be considered innocuous to humans.

Concern is also expressed for the safety of on-board equipment. While the vulnerability levels of this gear is not known at this time, field levels estimated below will allow the safety assessment to be performed.

The field within the LESLI rhombic, while not precisely describable by a transmission line model, has been approximately modeled in such a manner. ${ }^{10,11}$ The results have been found to be adequate in trials against experimental results. The following are some results from this analysis.

In a coordinate system with $\mathrm{x}$ along the axis of the rhombic, $\mathrm{z}$ transverse and along the ground plane, and y oriented perpendicular to the ground (see Fig 7.4-1 for help in this visualization)

$$
\begin{aligned}
& E_{y}=\operatorname{Sqrt}\left[1.87 \mathrm{~W} /\left(\pi Z_{c} / Z_{0}\right)\right](1 / b)[\exp (-j \omega r / c)] f_{c m}(z, y) \\
& E_{Z}=S q r t\left[1.87 \mathrm{~W} /\left(\pi Z_{c} / Z_{0}\right)\right](1 / b)[\exp (-j \omega r / c)] g_{c m}(z, y) \\
& \text { where } \\
& \begin{aligned}
f_{c m}= & b(y-b) /\left[(z-a)^{2}+(y-b)^{2}\right]+b(y-b) /\left[(z+a)^{2}+(y-b)^{2}\right] \\
& -b(y+b) /\left[(z-a)^{2}+(y+b)^{2}\right]-b(y+b) /\left[(z+a)^{2}+(y+b)^{2}\right]
\end{aligned} \\
& \mathrm{gcm}=\mathrm{b}(\mathrm{z}-\mathrm{a}) /\left[(\mathrm{z}-\mathrm{a})^{2}+(\mathrm{y}-\mathrm{b})^{2}\right]+\mathrm{b}(\mathrm{z}+\mathrm{a}) /\left[(\mathrm{z}+\mathrm{a})^{2}+(\mathrm{y}-\mathrm{b})^{2}\right] \\
& -b(z-a) /\left[(z-a)^{2}+(y+b)^{2}\right]-b(z+a) /\left[(z+a)^{2}+(y+b)^{2}\right] \\
& \mathrm{Z}_{\mathrm{C}}=\ln \left[\left[1+\left(1+(\mathrm{R} / \mathrm{b})^{\wedge} 2\right)^{1 / 2}-\mathrm{R} / \mathrm{b}\right] /\left[1-\left(1+(\mathrm{R} / \mathrm{b})^{\wedge} 2\right)^{1 / 2}+\mathrm{R} / \mathrm{b}\right]\right] /(4 \pi)+\ln \left[1+(\mathrm{b} / \mathrm{a})^{2}\right] /(8 \pi) \\
& \mathrm{Z}_{\mathrm{O}}=377 \mathrm{Ohms} \\
& \mathrm{W}=\text { Input Power } \\
& 1=\text { distance from source to max height of rhombic } \\
& a=\text { distance of wire from centerline at any } x \\
& b=\text { height of wire from ground at any } x \\
& \mathrm{R}=\text { wire radius } \\
& \mathrm{r}=\text { distance from source }
\end{aligned}
$$

Using these expressions, fields were calculated for points within the test volume. The specific quantities used were:

\footnotetext{
${ }^{10}$ Kehere, W. S.,Atchley, L., Engheta, N., Marin, L., Martinez, J. P., "Results of the Initial Field Mapping of the AFWL Hardness Surveillance Illuminator", Kaman Sciences Corp Report DC-TR-4088.430-2, January 1987. ${ }^{11}$ Darras, D., Zuffada, C., Marin, L., Atchley, L., "Theory of Operation of Achilles III and Performance Evaluation", Achilles Memos: Memo 11, AFWL.
} 


$$
\begin{aligned}
& \mathrm{W}=100 \text { watts (radiated power) } \\
& \mathrm{l}=100 \mathrm{~m} \\
& \mathrm{a}(\text { maximum })=16 \mathrm{~m} \\
& \mathrm{~b}(\text { maximum })=26 \mathrm{~m} \\
& \mathrm{R}=0.002 \mathrm{~m}
\end{aligned}
$$

The ranges of the parameters in the figures were chosen to approximately cover the spatial extent of the airplane and the intended test volume. The airplane will be located at least 40 meters from the source. Its fuselage radius is approximately 4.5 meters and its wingspan approximately 38 meters. Its fuselage height is less than 6.5 meters.

Figure B-1 shows the principal electric field (the $y$ component) for $x$ between 35 and 70 meters and $\mathrm{z}$ from 0 to 20 meters at the ground plane. The field never exceeds $3 \mathrm{~V} / \mathrm{m}$.

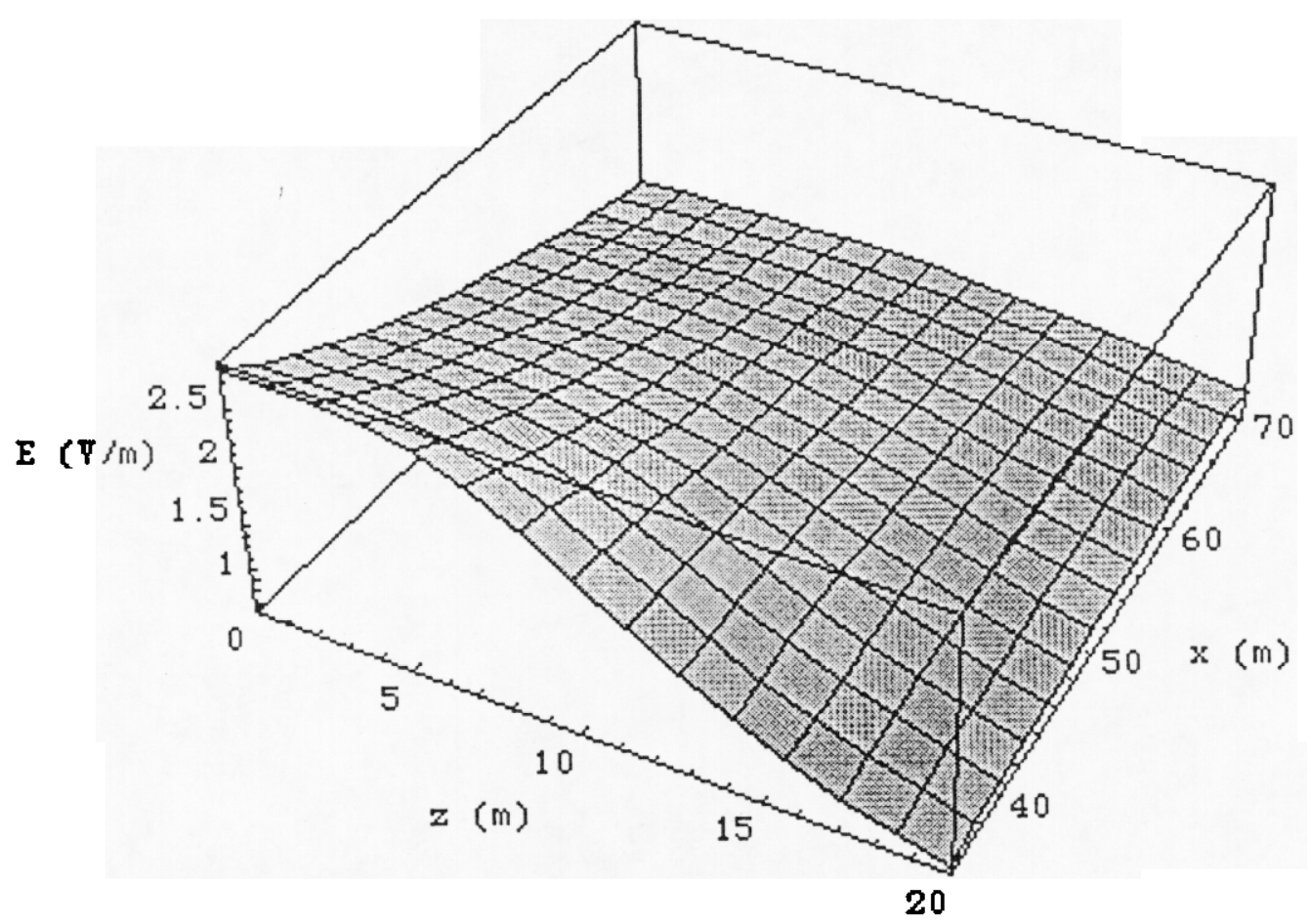

Fig B-1. Principal Electric Field at $y=0$

The corresponding electric field in the transverse plane ( $y-z$ plane) at $x=40$ meters is shown in Figure B-2. The peaking taking place near $y=4$ meters is due to proximity to the rhombic wire. Figure B-3 shows the same field component over a greater range of height and transverse position.

The cross polarized components of the field (the z-directed component) is also of concern. Physics requires this component to be zero along the ground plane ( for a perfect conductor) and symmetry requires it to be zero along the $z=0$ plane. Figures B-4 and B-5 show this transverse field in the transverse plane at $\mathrm{x}=40$ meters. At heights less than 5 meters the field is less than $1 \mathrm{~V} / \mathrm{m}$. 


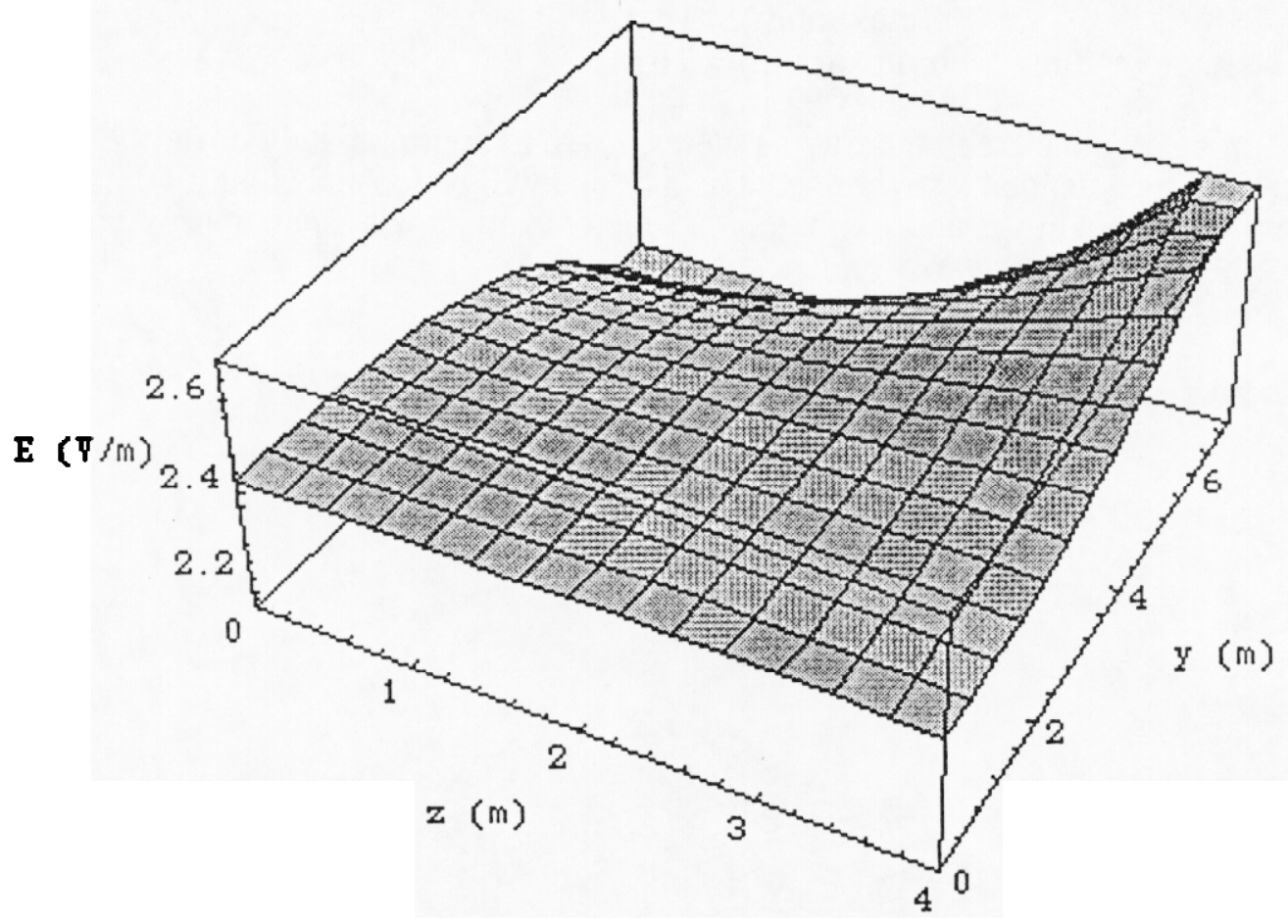

Figure B-2. Principal Electric Field at $x=40$ meters, $y$ less than 7 meters

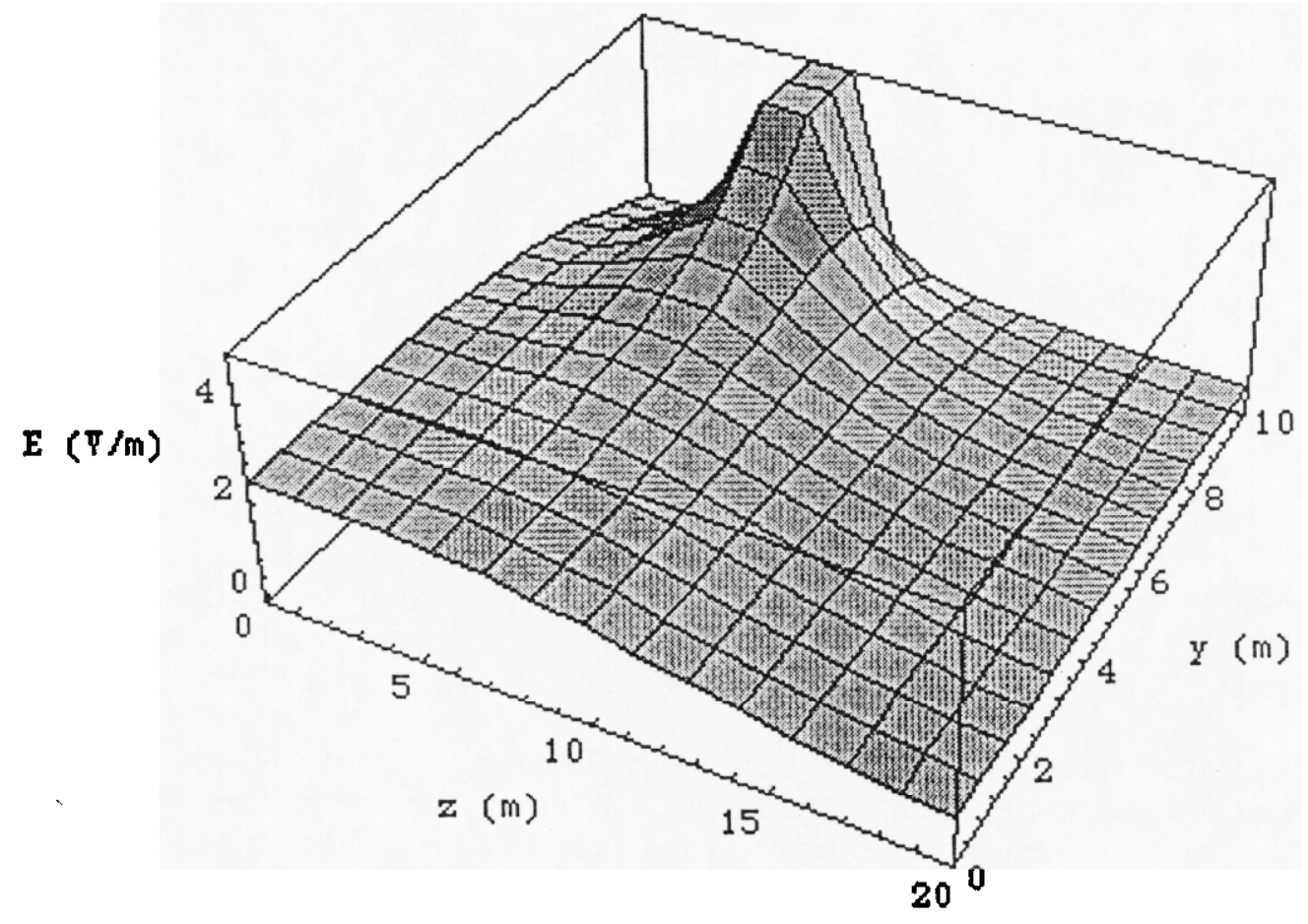

Figure B-3. Principal Electric Field at $\mathrm{x}=40$ meters, $\mathrm{y}$ less than 10 meters 


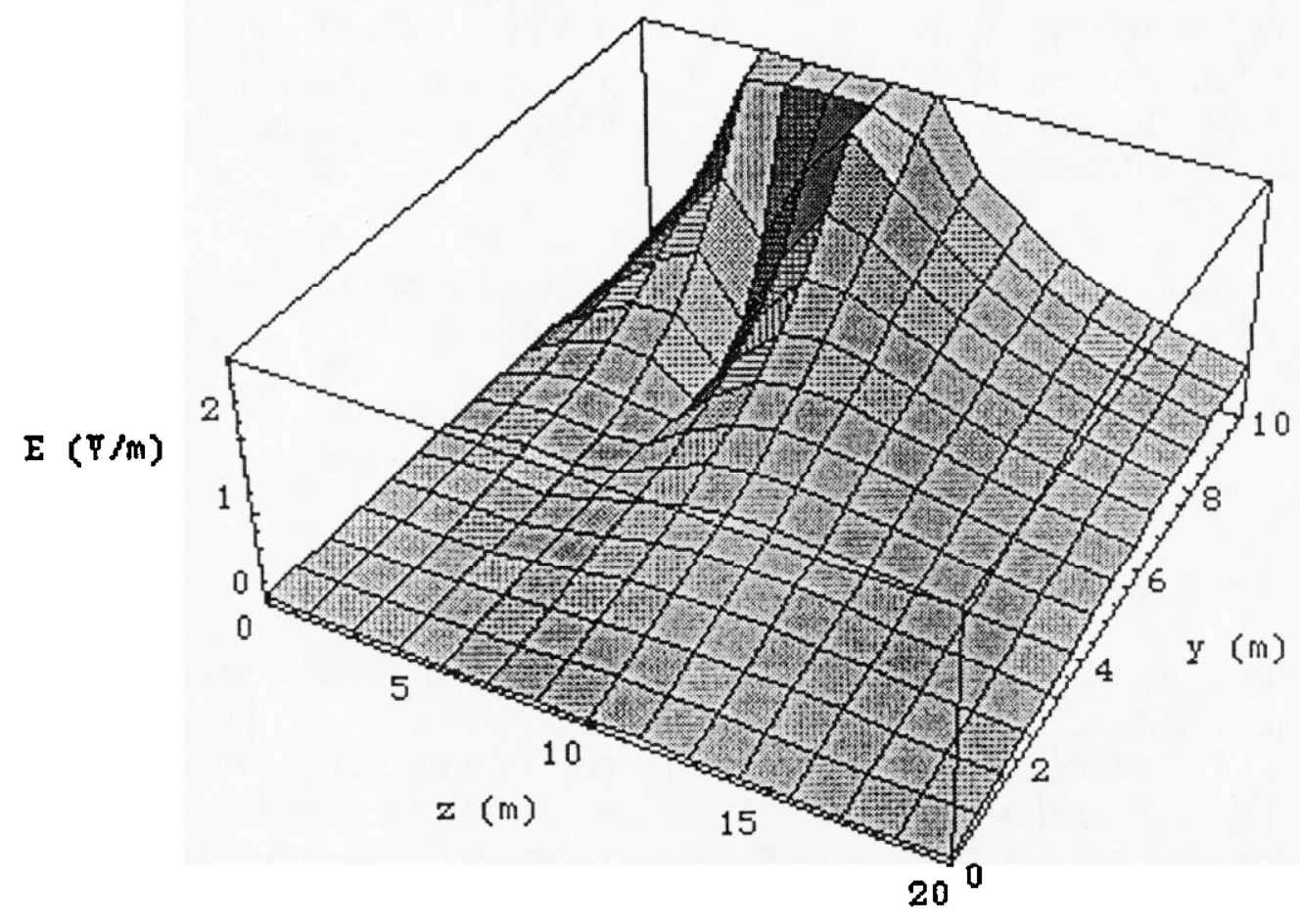

Figure B-4. Transverse electric field at $x=40$ meters

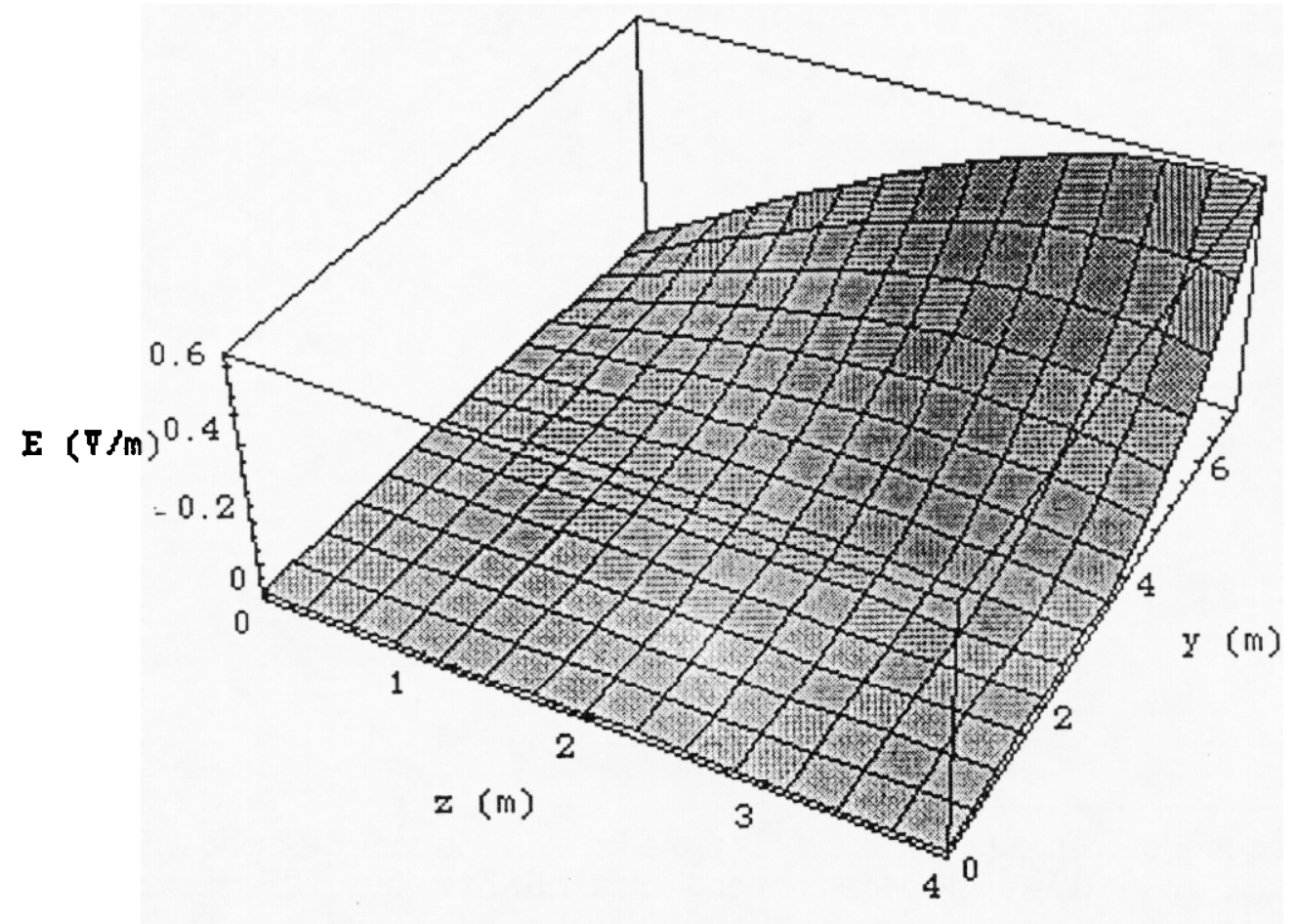

Figure B-5. Transverse electric field at $\mathrm{x}=40 \mathrm{~m}, \mathrm{z}<4 \mathrm{~m}, \mathrm{y}<7 \mathrm{~m}$ 
The field levels at the location of the airplane without its presence is thus below 5 volts per meter over the extent of the fuselage. This statement is true for either nose on or broadside incidence with the airplane in the positions shown in Figures 7.4-2 and 7.4-3. The equivalent power density is less than $0.007 \mathrm{~mW} / \mathrm{cm}^{2}$. Even allowing for inaccuracy in the model, this is well below the personnel safety standard by almost an order of magnitude in field ${ }^{12}$. Shielding provided by the aircraft will, of course, reduce the field levels.

\section{Dipole Tests}

The dipole tests will be performed with the dipole located at a point forward of the wing and outboard of the fuselage. Closest approach to the airplane is 10 meters. The dipoles will be very close to their half-wave resonances in the tests.

For horizontal polarization, the dipoles will be at a distance above the ground plane.

The incident power density in the direction of maximum gain is given by

$$
\mathrm{S}=\text { Gain } \times \text { Power Input / }\left(4 \pi \mathrm{R}^{2}\right)
$$

For a dipole in free space with gain of $1.64(2.15 \mathrm{dBi})$, a range of 5 meters, and a power input of 1 watt, the incident power density at the axis airplane is $0.0052 \mathrm{~W} / \mathrm{m}^{2}$ with the attendant electric field of $1.4 \mathrm{~V} / \mathrm{m}$. For a dipole over a ground plane, the peak gain can be twice as high as free space with peak incident power density of $0.021 \mathrm{~W} / \mathrm{m}^{2}$ and attendant electric field of $2.8 \mathrm{~V} / \mathrm{m}$. Hence, for a dipole over ground radiating 100 watts, the peak power density at 5 meters would be 2.1 $\mathrm{W} / \mathrm{m}^{2}$ and the peak electric field would be $28 \mathrm{~V} / \mathrm{m}$. These estimates are below safety thresholds.

${ }^{12}$ Further confidence in personnel safety is provided by measurements made within the facility. During the F-16 Pre Test Field Measurements in the LESLI facility, UIE reported on the field levels at various points on the test pad. For full-level, output power from the RF amps driving the rhombic with 100 watts, peak power density did not exceed $0.16 \mathrm{~mW} / \mathrm{cm}^{*} \mathrm{~cm}$ at a point $10 \mathrm{~m}$ from the feed on the centerline of the rhombic. ("Report of High Frequency Research Facility F-16 PreTest Field Measurements" United International Engineering, Inc., UIE-TR-92-0010, 22 June 1992. 


\section{Appendix C On-the-Ground Test Matrix}

This section contains an estimate of the time required to for the on-the-ground tests and a test matrix for low-power tests. These estimates are found in Tables $\mathrm{C}-1$ through C-8.

The test matrix is presented in Tables C-9 though C-24. Test Series A through F correspond to the dipole tests described in Section 7.7. Test Series $G$ through I correspond to the on-board instrumentation tests described in Section 7.8. Tests Series $\mathrm{J}$ and $\mathrm{K}$ correspond to the stepped frequency rhombic tests described in Section 7.9. Test Series PL, Pad Characterization, and Horizontal Polarization - Rhombic are described in Sections 7.10, 7.14.1, and 7.14.2, respectively. Each table contains the configurations, orientations, and test points for a test series with a given polarization and frequency band. Antenna configurations and/or instrumentation systems will be changed for each test series. This matrix also indicates a preferred order for the tests. The test will proceed such that the most time-consuming changes (such as moving the aircraft) occur less frequently, at the expense of making the less time-consuming changes (such as swapping probe locations) more often.

In all cases, the frequency ranges shown are the likely minimum. The ranges will be extended to include as much of the frequency range around the center frequency as possible

\section{Table C-1. Pre-aircraft field time at PL}

Set up data acquisition format, run-number code, etc.

Set up data acquisition instrumentation, antennas in required locations and check out equipment. Dry run tests.

Tests to assure FAA compatibility

Field map survey for Test Series J, K

1 day

Total pre-aircraft field time at $\mathrm{PL}$

5. days

2. days

10 . days

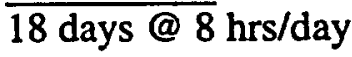

\section{Table C-2. Test Series A - F (three frequencies, vertical and horizontal dipoles, aircraft nose-incident orientation on ground plane)}

Fly In to $A B Q$, Aircraft Operations activities

Install non-flightworthy items, switch cable connections, cal cables

Position aircraft

Hook up instrumentation, fibers

RC-7 replaces installed NASA aircraft transceiver.

Initial tests - dynamic range, noise floor 12 tests

$26 \mathrm{MHz}$, vertical whip stepped measurements, setup, 12 tests:

$26 \mathrm{MHz}$, horizontal dipole stepped measurements, setup, 12 tests

$172 \mathrm{MHz}$, vertical dipole stepped measurements, setup, 12 tests

$172 \mathrm{MHz}$, horizontal dipole stepped measurements, setup, 12 tests

$430 \mathrm{MHz}$, vertical dipole stepped measurements, setup, 12 tests

$430 \mathrm{MHz}$, horizontal dipole stepped measurements, setup, 12 tests

Slack 4 hrs

Total 10 days @ 10 hr/day
1 day

1 day

$2.5 \mathrm{hrs}$

$1.5 \mathrm{hrs}$

$1 \mathrm{hr}$

1 day

1 day

1 day

1 day

1 day

1 day

1 day 
Table C-3 Test Series G, H, I

(On-board instrumentation check-out, rhombic, vertical, aircraft nose-incident)

Hook up sensors to on-board instrumentation,

$1 \mathrm{hr}$

Remove RC-7 box and install NASA aircraft transceiver $\quad 1 \mathrm{hr}$

Power up aircraft and instrumentation

Tests at 25.85 MHz, Debug, exercise equipment $4 \mathrm{hrs}$

Tests at $172.0 \mathrm{MHz}$, Debug, exercise equipment $4 \mathrm{hrs}$

Tests at $430.0 \mathrm{MHz}$, Debug, exercise equipment $4 \mathrm{hrs}$

Slack (large slack time to accommodate potential problems) $5 \mathrm{hrs}$

Reconnect sensors to fibers for stepped frequency tests, install RC-7 box

Install Rhombic Antenna

$10 \mathrm{hrs}$

4 hrs

Total 3.5 days @ $10 \mathrm{hr} /$ day

Table C-4 Test Series J $(0.3-1000 \mathrm{MHz}$, vertical, nose incident)

Reposition aircraft, $5^{\circ}$

Reposition aircraft, $2^{\circ}$ or $10^{\circ}$

Stepped measurements, 41 Tests

Total 4.0 days @ $10 \mathrm{hr} /$ day
$1.5 \mathrm{hrs}$

$1.5 \mathrm{hrs}$

$37 \mathrm{hrs}$

Table C-5 Test Series $\mathrm{K} \quad(0.3-1000 \mathrm{MHz}$, vertical, side incident)

Reposition aircraft to $90^{\circ}$

Stepped measurements, 30 tests

Remove fibers, $\mathrm{RC}-7$ box, etc.

Total 4.0 days @ $10 \mathrm{hr} /$ day
$1.5 \mathrm{hrs}$

$37 \mathrm{hrs}$

$1.5 \mathrm{hrs}$

\section{Table C-6 Post-Test Aircraft Time.}

Return aircraft to flightworthy state, QA inspection

Roll out aircraft, return to airport, fly out to LaRC

2 day

1 day

Total post-test aircraft time

$\overline{3 \text { days } @ 10 \mathrm{hrs} / \text { day }}$

Total aircraft test time

21.5 days @ $10 \mathrm{hr} . /$ day 
Table C-7 Post Aircraft Decommission

Clean up

2 days

Total post-aircraft field time

2 days@ 8 hrs/day

\section{Table C-8 Total Time Allocations}

Total Pre-Aircraft Field Time at PL

Test Series A-F

Test Series G, H, I

Test Series J

Test Series K

Post-Test Aircraft Time

Post Aircraft Decommission

18 days @ 8 hrs/day

10 days @ $10 \mathrm{hr} /$ day

3.0 days (up to $10 \mathrm{hr} /$ day)

4.0 days @ 10 hr/days

4.0 days @ $10 \mathrm{hr} /$ day

3.0 days@ $8 \mathrm{hrs} /$ day

2 days@8 hrs/day 


\section{Table C-9. Ambient Noise Measurements}

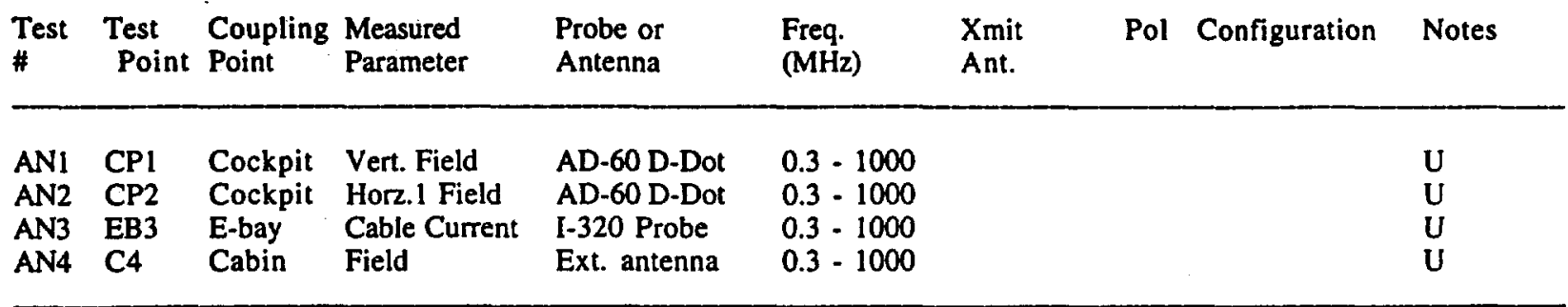

- Notes: This measurement set will investigate or validate:

U) Ambient noise measurement

\section{Table C-10. Test Series A}

$\begin{array}{llllllll}\text { Test } & \text { Test } & \text { Coupling } & \text { Measured } & \text { Probe or } & \text { Freq. } & \text { Xmit } & \text { Pol Configuration } \\ \# & \text { Point } & \text { Point } & \text { Parameter } & \text { Antenna } & \text { (MHz) } & \text { Ant. }\end{array}$

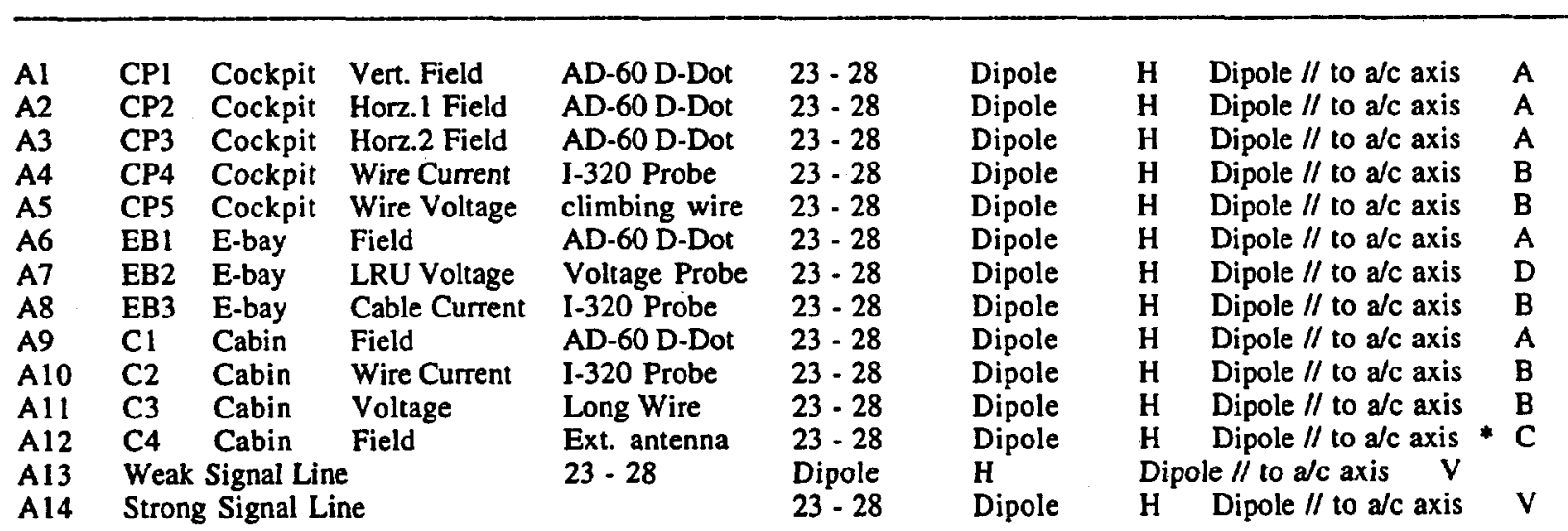

\footnotetext{
- Notes: This measurement set will investigate or validate:

A) FDTD predictions of field coupling; transmit antenna in computational volume

B) FDTD predictions of thin wire coupling; transmit antenna in computational volume

C) Calibration of aircraft external field measurement

D) Typical coupling into a representative critical LRU in the E-bay

V) Dipole Instrumentation Noise Measurements: Strongest and Weakest channels observed over the test series are chosen. Probe is replaced by matched load and noise signals recorded.

* Lower priority, skip if short of time
} 


\section{Table C-11. Test Series B}

\begin{tabular}{|c|c|c|c|c|c|c|c|c|c|}
\hline $\begin{array}{l}\text { Test } \\
\#\end{array}$ & $\begin{array}{c}\text { Test } \\
\text { Point }\end{array}$ & $\begin{array}{l}\text { Coupling } \\
\text { Point }\end{array}$ & $\begin{array}{l}\text { Measured } \\
\text { Parameter }\end{array}$ & $\begin{array}{l}\text { Probe or } \\
\text { Antenna }\end{array}$ & $\begin{array}{l}\text { Freq. } \\
\text { (MHz) }\end{array}$ & $\begin{array}{l}\text { Xmit } \\
\text { Ant. }\end{array}$ & Pol & Orientation / Config. & Notes \\
\hline $\begin{array}{l}\text { B1 } \\
\text { B2 } \\
\text { B3 } \\
\text { B4 } \\
\text { B5 } \\
\text { B6 } \\
\text { B7 } \\
\text { B8 } \\
\text { B9 } \\
\text { B10 } \\
\text { B11 } \\
\text { B12 } \\
\text { B13 } \\
\text { B14 } \\
\text { B15 }\end{array}$ & $\begin{array}{l}\text { CP1 } \\
\text { CP2 } \\
\text { CP3 } \\
\text { CP4 } \\
\text { CP5 } \\
\text { EB1 } \\
\text { EB2 } \\
\text { EB3 } \\
\text { C1 } \\
\text { C2 } \\
\text { C3 } \\
\text { C4 } \\
\text { C1 } \\
\text { Weak } \\
\text { Strong }\end{array}$ & $\begin{array}{l}\text { Cockpit } \\
\text { Cockpit } \\
\text { Cockpit } \\
\text { Cockpit } \\
\text { Cockpit } \\
\text { E-bay } \\
\text { E-bay } \\
\text { E-bay } \\
\text { Cabin } \\
\text { Cabin } \\
\text { Cabin } \\
\text { Cabin } \\
\text { Cabin } \\
\text { Signal Line } \\
\text { Signal Line }\end{array}$ & $\begin{array}{l}\text { Vert. Field } \\
\text { Horz.1 Field } \\
\text { Horz.2 Field } \\
\text { Wire Current } \\
\text { Wire Holtage } \\
\text { Field } \\
\text { LRU Voltage } \\
\text { Cable Current } \\
\text { Field } \\
\text { Wire Current } \\
\text { Voltage } \\
\text { Field } \\
\text { Field }\end{array}$ & $\begin{array}{l}\text { AD-60 D-Dot } \\
\text { AD-60 D-Dot } \\
\text { AD-60 D-Dot } \\
\text { I-320 Probe } \\
\text { climbing wire } \\
\text { AD-60 D-Dot } \\
\text { Voltage Probe } \\
\text { I-320 Probe } \\
\text { AD-60 D-Dot } \\
\text { I-320 Probe } \\
\text { Long Wire } \\
\text { Ext. antenna } \\
\text { AD-60 D-Dot }\end{array}$ & $\begin{array}{l}23-28 \\
23-28 \\
23-28 \\
23-28 \\
23-28 \\
23-28 \\
23-28 \\
23-28 \\
23-28 \\
23-28 \\
23-28 \\
23-28 \\
25.7-26 \\
23-28 \\
23-28\end{array}$ & $\begin{array}{l}\text { Dipole } \\
\text { Dipole } \\
\text { Dipole } \\
\text { Dipole } \\
\text { Dipole } \\
\text { Dipole } \\
\text { Dipole } \\
\text { Dipole } \\
\text { Dipole } \\
\text { Dipole } \\
\text { Dipole } \\
\text { Dipole } \\
\text { Dipole } \\
\text { Dipole } \\
\text { Dipole }\end{array}$ & $\begin{array}{l}V \\
V \\
V \\
V \\
V \\
V \\
V \\
V \\
V \\
V \\
V \\
V \\
V \\
V \\
V\end{array}$ & $\begin{array}{l}\text { front, left of } a / c \\
\text { front, left of } a / c \\
\text { front, left of } a / c \\
\text { front, left of } a / c \\
\text { front, left of } a / c \\
\text { front, left of } a / c \\
\text { front, left of } a / c \\
\text { front, left of } a / c \\
\text { front, left of } a / c \\
\text { front, left of } a / c \\
\text { front, left of } a / c \\
\text { front, left of } a / c \\
1 \% \text { BW, } 1000 \text { pts } \\
\text { front, left of } a / c \\
\text { front, left of } a / c\end{array}$ & $\begin{array}{l}\text { A } \\
\text { A } \\
\text { A } \\
\text { B } \\
\text { B } \\
\text { A } \\
\text { D } \\
\text { B } \\
\text { A } \\
\text { B } \\
\text { B } \\
\text { C } \\
\text { E } \\
\text { V } \\
\text { V }\end{array}$ \\
\hline
\end{tabular}

\footnotetext{
- Notes: This measurement set will investigate or validate:

A) FDTD predictions of field coupling; transmit antenna in computational volume

B) FDTD predictions of thin wire coupling; transmit antenna in computational volume

C) Calibration of aircraft external field measurement

D) Typical coupling into a representative critical LRU in the E-bay

E) Variations in coupling with frequency (frequency stirring)

V) Dipole Instrumentation Noise Measurements

* Lower priority, skip if short of time
}

\section{Table C-12. Test Series C}

\begin{tabular}{|c|c|c|c|c|c|c|c|c|c|}
\hline $\begin{array}{l}\text { Test } \\
\#\end{array}$ & $\begin{array}{l}\text { Test } \\
\text { Point }\end{array}$ & $\begin{array}{l}\text { Coupling } \\
\text { Point }\end{array}$ & $\begin{array}{l}\text { Measured } \\
\text { Parameter }\end{array}$ & $\begin{array}{l}\text { Probe or } \\
\text { Antenna }\end{array}$ & $\begin{array}{l}\text { Freq. } \\
\text { (MHz) }\end{array}$ & $\begin{array}{l}\text { Xmit } \\
\text { Ant. }\end{array}$ & Pol & Orientation / Config. & Notes \\
\hline $\begin{array}{l}\text { C1 } \\
\text { C2 } \\
\text { C3 } \\
\text { C4 } \\
\text { C5 } \\
\text { C6 } \\
\text { C7 } \\
\text { C8 } \\
\text { C9 } \\
\text { C10 } \\
\text { C11 } \\
\text { C12 } \\
\text { C13 } \\
\text { C14 } \\
\text { C15 }\end{array}$ & $\begin{array}{l}\text { CP1 } \\
\text { CP2 } \\
\text { CP3 } \\
\text { CP4 } \\
\text { CP5 } \\
\text { EB1 } \\
\text { EB2 } \\
\text { EB3 } \\
\text { C1 } \\
\text { C2 } \\
\text { C3 } \\
\text { C4 } \\
\text { C1 } \\
\text { Weak } \\
\text { Strong }\end{array}$ & $\begin{array}{l}\text { Cockpit } \\
\text { Cockpit } \\
\text { Cockpit } \\
\text { Cockpit } \\
\text { Cockpit } \\
\text { E-bay } \\
\text { E-bay } \\
\text { E-bay } \\
\text { Cabin } \\
\text { Cabin } \\
\text { Cabin } \\
\text { Cabin } \\
\text { Cabin } \\
\text { Signal Line } \\
\text { Signal Lin }\end{array}$ & $\begin{array}{l}\text { Vert. Field } \\
\text { Horz.1 Field } \\
\text { Horz.2 Field } \\
\text { Wire Current } \\
\text { Wire Voltage } \\
\text { Field } \\
\text { LRU Voltage } \\
\text { Cable Current } \\
\text { Field } \\
\text { Wire Current } \\
\text { Voltage } \\
\text { Field } \\
\text { Field } \\
\text { e } \\
\text { ne }\end{array}$ & $\begin{array}{l}\text { AD-60 D-Dot } \\
\text { AD-60 D-Dot } \\
\text { AD-60 D-Dot } \\
\text { I-320 Probe } \\
\text { climbing wire } \\
\text { AD-60 D-Dot } \\
\text { Voltage Probe } \\
\text { I-320 Probe } \\
\text { AD-60 D-Dot } \\
\text { I-320 Probe } \\
\text { Long Wire } \\
\text { Ext. antenna } \\
\text { AD-60 D-Dot } \\
\text { 150-200 }\end{array}$ & $\begin{array}{l}150-200 \\
150-200 \\
150-200 \\
150-200 \\
150-200 \\
150-200 \\
150-200 \\
150-200 \\
150-200 \\
150-200 \\
150-200 \\
150-200 \\
171-173 \\
\text { Dipole } \\
150-200\end{array}$ & $\begin{array}{l}\text { Dipole } \\
\text { Dipole } \\
\text { Dipole } \\
\text { Dipole } \\
\text { Dipole } \\
\text { Dipole } \\
\text { Dipole } \\
\text { Dipole } \\
\text { Dipole } \\
\text { Dipole } \\
\text { Dipole } \\
\text { Dipole } \\
\text { Dipole } \\
\text { V } \\
\text { Dipole }\end{array}$ & $\begin{array}{l}V \\
V \\
V \\
V \\
V \\
V \\
V \\
V \\
V \\
V \\
V \\
V \\
V \\
\text { front, } \\
\text { V }\end{array}$ & $\begin{array}{l}\text { front, left of } a / c \\
\text { front, left of } a / c \\
\text { front, left of } a / c \\
\text { front, left of } a / c \\
\text { front, left of } a / c \\
\text { front, left of } a / c \\
\text { front, left of } a / c \\
\text { front, left of } a / c \\
\text { front, left of } a / c \\
\text { front, left of } a / c \\
\text { front, left of } a / c \\
\text { front, left of } a / c \\
1 \% \text { BW, } 1000 \text { pts } \\
\text { left of } a / c \\
\text { front, left of } a / c\end{array}$ & $\begin{array}{l}\text { A } \\
\text { A } \\
\text { A } \\
\text { B } \\
\text { B } \\
\text { A } \\
\text { D } \\
\text { B } \\
\text { A } \\
\text { B } \\
\text { B } \\
\text { C } \\
\text { E } \\
\text { V } \\
\text { V }\end{array}$ \\
\hline
\end{tabular}

- Notes: This measurement set will investigate or validate:
A) FDTD predictions of field coupling; transmit antenna in computational volume
B) FDTD predictions of thin wire coupling; transmit antenna in computational volume
C) Calibration of aircraft external field measurement
D) Typical coupling into a representative critical LRU in the E-bay
E) Variations in coupling with frequency (frequency stirring)
V) Dipole Instrumentation Noise Measurements
* Lower priority, skip if short of time 


\section{Table C-13. Test Series D}

\begin{tabular}{|c|c|c|c|c|c|c|c|c|c|}
\hline $\begin{array}{l}\text { Test } \\
\#\end{array}$ & $\begin{array}{l}\text { Test } \\
\text { Point }\end{array}$ & $\begin{array}{l}\text { Coupling } \\
\text { Point }\end{array}$ & $\begin{array}{l}\text { Measured } \\
\text { Parameter }\end{array}$ & $\begin{array}{l}\text { Probe or } \\
\text { Antenna }\end{array}$ & $\begin{array}{l}\text { Freq. } \\
(\mathrm{MHz})\end{array}$ & $\begin{array}{l}\text { Xmit } \\
\text { Ant. }\end{array}$ & Pol & Orientation / Config. & Notes \\
\hline $\begin{array}{l}\text { D1 } \\
\text { D2 } \\
\text { D3 } \\
\text { D4 } \\
\text { D5 } \\
\text { D6 } \\
\text { D7 } \\
\text { D8 } \\
\text { D9 } \\
\text { D10 } \\
\text { D11 } \\
\text { D12 } \\
\text { D13 } \\
\text { D14 }\end{array}$ & $\begin{array}{l}\text { CP1 } \\
\text { CP2 } \\
\text { CP3 } \\
\text { CP4 } \\
\text { CP5 } \\
\text { EB1 } \\
\text { EB2 } \\
\text { EB3 } \\
\text { C1 } \\
\text { C2 } \\
\text { C3 } \\
\text { C4 } \\
\text { Weak } \\
\text { Strong }\end{array}$ & $\begin{array}{l}\text { Cockpit } \\
\text { Cockpit } \\
\text { Cockpit } \\
\text { Cockpit } \\
\text { Cockpit } \\
\text { E-bay } \\
\text { E-bay } \\
\text { E-bay } \\
\text { Cabin } \\
\text { Cabin } \\
\text { Cabin } \\
\text { Cabin } \\
\text { Signal Line } \\
\text { Signal Line }\end{array}$ & $\begin{array}{l}\text { Vert. Field } \\
\text { Horz.1 Field } \\
\text { Horz.2 Field } \\
\text { Wire Current } \\
\text { Wire Voltage } \\
\text { Field } \\
\text { LRU Voltage } \\
\text { Cable Current } \\
\text { Field } \\
\text { Wire Current } \\
\text { Voltage } \\
\text { Field } \\
\end{array}$ & $\begin{array}{l}\text { AD-60 D-Dot } \\
\text { AD-60 D-Dot } \\
\text { AD-60 D-Dot } \\
\text { I-320 Probe } \\
\text { climbing wire } \\
\text { AD-60 D-Dot } \\
\text { Voltage Probe } \\
\text { I-320 Probe } \\
\text { AD-60 D-Dot } \\
\text { I-320 Probe } \\
\text { Long Wire } \\
\text { Ext. antenna }\end{array}$ & $\begin{array}{l}150-200 \\
150-200 \\
150-200 \\
150-200 \\
150-200 \\
150-200 \\
150-200 \\
150-200 \\
150-200 \\
150-200 \\
150-200 \\
150-200 \\
150-200 \\
150-200\end{array}$ & $\begin{array}{l}\text { Dipole } \\
\text { Dipole } \\
\text { Dipole } \\
\text { Dipole } \\
\text { Dipole } \\
\text { Dipole } \\
\text { Dipole } \\
\text { Dipole } \\
\text { Dipole } \\
\text { Dipole } \\
\text { Dipole } \\
\text { Dipole } \\
\text { Dipole } \\
\text { Dipole }\end{array}$ & $\begin{array}{l}\mathbf{H} \\
\mathbf{H} \\
\mathbf{H} \\
\mathbf{H} \\
\mathbf{H} \\
\mathbf{H} \\
\mathbf{H} \\
\mathbf{H} \\
\mathbf{H} \\
\mathbf{H} \\
\mathbf{H} \\
\mathbf{H} \\
\mathbf{H} \\
\mathbf{H}\end{array}$ & 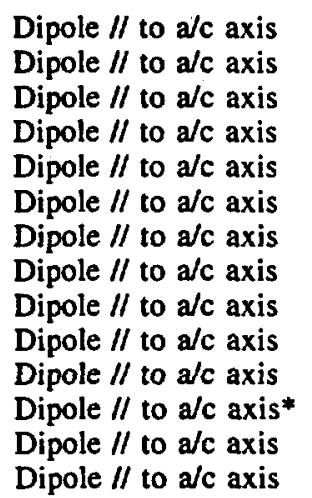 & $\begin{array}{l}\text { A } \\
\text { A } \\
\text { A } \\
\text { B } \\
\text { B } \\
\text { A } \\
\text { D } \\
\text { B } \\
\text { A } \\
\text { B } \\
\text { B } \\
\text { C } \\
\text { V } \\
\text { V }\end{array}$ \\
\hline
\end{tabular}

- Notes: This measurement set will investigate or validate:

A) FDTD predictions of field coupling; transmit antenna in computational volume

B) FDTD predictions of thin wire coupling; transmit antenna in computational volume

C) Calibration of aircraft external field measurement

D) Typical coupling into a representative critical LRU in the E-bay

V) Dipole Instrumentation Noise Measurements

* Lower priority, skip if short of time

\section{Table C-14. Test Series E}

\begin{tabular}{|c|c|c|c|c|c|c|c|c|c|}
\hline $\begin{array}{l}\text { Test } \\
\#\end{array}$ & $\begin{array}{l}\text { Test } \\
\text { Point }\end{array}$ & $\begin{array}{l}\text { Coupling } \\
\text { Point }\end{array}$ & $\begin{array}{l}\text { Measured } \\
\text { Parameter }\end{array}$ & $\begin{array}{l}\text { Probe or } \\
\text { Antenna }\end{array}$ & $\begin{array}{l}\text { Freq. } \\
(\mathrm{MHz})\end{array}$ & $\begin{array}{l}\text { Xmit } \\
\text { Ant. }\end{array}$ & Pol & Orientation / Config. & Notes \\
\hline $\begin{array}{l}\text { E1 } \\
\text { E2 } \\
\text { E3 } \\
\text { E4 } \\
\text { E5 } \\
\text { E6 } \\
\text { E7 } \\
\text { E8 } \\
\text { E9 } \\
\text { E10 } \\
\text { E11 } \\
\text { E12 } \\
\text { E13 } \\
\text { E14 } \\
\text { E15 }\end{array}$ & $\begin{array}{l}\text { CP1 } \\
\text { CP2 } \\
\text { CP3 } \\
\text { CP4 } \\
\text { CP5 } \\
\text { EB1 } \\
\text { EB2 } \\
\text { EB3 } \\
\text { C1 } \\
\text { C2 } \\
\text { C3 } \\
\text { C4 } \\
\text { C1 } \\
\text { Weak } \\
\text { Strong }\end{array}$ & $\begin{array}{l}\text { Cockpit } \\
\text { Cockpit } \\
\text { Cockpit } \\
\text { Cockpit } \\
\text { Cockpit } \\
\text { E-bay } \\
\text { E-bay } \\
\text { E-bay } \\
\text { Cabin } \\
\text { Cabin } \\
\text { Cabin } \\
\text { Cabin } \\
\text { Cabin } \\
\text { Signal Line } \\
\text { Signal Line }\end{array}$ & $\begin{array}{l}\text { Vert. Field } \\
\text { Horz.1 Field } \\
\text { Horz. } 2 \text { Field } \\
\text { Wire Current } \\
\text { Wire Voltage } \\
\text { Field } \\
\text { LRU Voltage } \\
\text { Cable Current } \\
\text { Field } \\
\text { Wire Current } \\
\text { Voltage } \\
\text { Field } \\
\text { Field } \\
\end{array}$ & $\begin{array}{l}\text { AD-60 D-Dot } \\
\text { AD-60 D-Dot } \\
\text { AD-60 D-Dot } \\
\text { I-320 Probe } \\
\text { climbing wire } \\
\text { AD-60 D-Dot } \\
\text { Voltage Probe } \\
\text { I-320 Probe } \\
\text { AD-60 D-Dot } \\
\text { I-320 Probe } \\
\text { Long Wire } \\
\text { Ext. antenna } \\
\text { AD-60 D-Dot }\end{array}$ & $\begin{array}{l}350-500 \\
350-500 \\
350-500 \\
350-500 \\
350-500 \\
350-500 \\
350-500 \\
350-500 \\
350-500 \\
350-500 \\
350-500 \\
350-500 \\
427-432 \\
350-500 \\
350-500\end{array}$ & $\begin{array}{l}\text { Dipole } \\
\text { Dipole } \\
\text { Dipole } \\
\text { Dipole } \\
\text { Dipole } \\
\text { Dipole } \\
\text { Dipole } \\
\text { Dipole } \\
\text { Dipole } \\
\text { Dipole } \\
\text { Dipole } \\
\text { Dipole } \\
\text { Dipole } \\
\text { Dipole } \\
\text { Dipole }\end{array}$ & $\begin{array}{l}V \\
V \\
V \\
V \\
V \\
V \\
V \\
V \\
V \\
V \\
V \\
V \\
V \\
V \\
V\end{array}$ & $\begin{array}{l}\text { front, left of } a / c \\
\text { front, left of } a / c \\
\text { front, left of } a / c \\
\text { front, left of } a / c \\
\text { front, left of } a / c \\
\text { front, left of } a / c \\
\text { front, left of } a / c \\
\text { front, left of } a / c \\
\text { front, left of } a / c \\
\text { front, left of } a / c \\
\text { front, left of } a / c \\
\text { front, left of } a / c \\
1 \% \text { BW, } 1000 \mathrm{pts} \\
\text { front, left of } a / c \\
\text { front, left of } a / c\end{array}$ & $\begin{array}{l}\text { A } \\
\text { A } \\
\text { A } \\
\text { B } \\
\text { B } \\
\text { A } \\
\text { D } \\
\text { B } \\
\text { A } \\
\text { B } \\
\text { B } \\
\text { C } \\
\text { E } \\
\text { V } \\
\text { V }\end{array}$ \\
\hline
\end{tabular}

\footnotetext{
- Notes: This measurement set will investigate or validate:

A) FDTD predictions of field coupling; transmit antenna in computational volume

B) FDTD predictions of thin wire coupling; transmit antenna in computational volume

C) Calibration of aircraft external field measurement

D) Typical coupling into a representative critical LRU in the E-bay

E) Variations in coupling with frequency (frequency stirring)

V) Dipole Instrumentation Noise Measurements* Lower priority, skip if short of time
} 
Table C-15. Test Series F

\begin{tabular}{|c|c|c|c|c|c|c|c|c|c|}
\hline $\begin{array}{l}\text { Test } \\
\#\end{array}$ & $\begin{array}{l}\text { Test } \\
\text { Point }\end{array}$ & $\begin{array}{l}\text { Coupling } \\
\text { Point }\end{array}$ & $\begin{array}{l}\text { Measured } \\
\text { Parameter }\end{array}$ & $\begin{array}{l}\text { Probe or } \\
\text { Antenna }\end{array}$ & $\begin{array}{l}\text { Freq. } \\
(\mathrm{MHz})\end{array}$ & $\begin{array}{l}\text { Xmit } \\
\text { Ant. }\end{array}$ & Pol & Orientation / Config. & Notes \\
\hline $\begin{array}{l}\text { F1 } \\
\text { F2 } \\
\text { F3 } \\
\text { F4 } \\
\text { F5 } \\
\text { F6 } \\
\text { F7 } \\
\text { F8 } \\
\text { F9 } \\
\text { F10 } \\
\text { F11 } \\
\text { F12 } \\
\text { F13 } \\
\text { F14 }\end{array}$ & $\begin{array}{l}\text { CP1 } \\
\text { CP2 } \\
\text { CP3 } \\
\text { CP4 } \\
\text { CP5 } \\
\text { EB1 } \\
\text { EB2 } \\
\text { EB3 } \\
\text { C1 } \\
\text { C2 } \\
\text { C3 } \\
\text { C4 } \\
\text { Weak } \\
\text { Strong }\end{array}$ & $\begin{array}{l}\text { Cockpit } \\
\text { Cockpit } \\
\text { Cockpit } \\
\text { Cockpit } \\
\text { Cockpit } \\
\text { E-bay } \\
\text { E-bay } \\
\text { E-bay } \\
\text { Cabin } \\
\text { Cabin } \\
\text { Cabin } \\
\text { Cabin } \\
\text { Signal Line } \\
\text { Signal Line }\end{array}$ & $\begin{array}{l}\text { Vert. Field } \\
\text { Horz.1 Field } \\
\text { Horz.2 Field } \\
\text { Wire Current } \\
\text { Wire Voltage } \\
\text { Field } \\
\text { LRU Voltage } \\
\text { Cable Current } \\
\text { Field } \\
\text { Wire Current } \\
\text { Voltage } \\
\text { Field }\end{array}$ & $\begin{array}{l}\text { AD-60 D-Dot } \\
\text { AD-60 D-Dot } \\
\text { AD-60 D-Dot } \\
\text { I-320 Probe } \\
\text { climbing wire } \\
\text { AD-60 D-Dot } \\
\text { Voltage Probe } \\
\text { I-320 Probe } \\
\text { AD-60 D-Dot } \\
\text { I-320 Probe } \\
\text { Long Wire } \\
\text { Ext. antenna }\end{array}$ & $\begin{array}{l}350-500 \\
350-500 \\
350-500 \\
350-500 \\
350-500 \\
350-500 \\
350-500 \\
350-500 \\
350-500 \\
350-500 \\
350-500 \\
350-500 \\
350-500 \\
350-500\end{array}$ & $\begin{array}{l}\text { Dipole } \\
\text { Dipole } \\
\text { Dipole } \\
\text { Dipole } \\
\text { Dipole } \\
\text { Dipole } \\
\text { Dipole } \\
\text { Dipole } \\
\text { Dipole } \\
\text { Dipole } \\
\text { Dipole } \\
\text { Dipole } \\
\text { Dipole } \\
\text { Dipole }\end{array}$ & $\begin{array}{l}\mathbf{H} \\
\mathbf{H} \\
\mathbf{H} \\
\mathbf{H} \\
\mathbf{H} \\
\mathbf{H} \\
\mathbf{H} \\
\mathbf{H} \\
\mathbf{H} \\
\mathbf{H} \\
\mathbf{H} \\
\mathbf{H} \\
\mathbf{H} \\
\mathbf{H}\end{array}$ & $\begin{array}{l}\text { Dipole } / / \text { to } \mathrm{a} / \mathrm{c} \text { axis } \\
\text { Dipole } / / \text { to } \mathrm{a} / \mathrm{c} \text { axis } \\
\text { Dipole } / / \text { to } \mathrm{a} / \mathrm{c} \text { axis } \\
\text { Dipole } / / \text { to } \mathrm{a} / \mathrm{c} \text { axis } \\
\text { Dipole } / / \text { to } \mathrm{a} / \mathrm{c} \text { axis } \\
\text { Dipole } / / \text { to } \mathrm{a} / \mathrm{c} \text { axis } \\
\text { Dipole } / / \text { to } \mathrm{a} / \mathrm{c} \text { axis } \\
\text { Dipole } / / \text { to } \mathrm{a} / \mathrm{c} \text { axis } \\
\text { Dipole } / / \text { to a/c axis } \\
\text { Dipole } / / \text { to } \mathrm{a} / \mathrm{c} \text { axis } \\
\text { Dipole } / / \text { to } \mathrm{a} / \mathrm{c} \text { axis } \\
\text { Dipole } / / \text { to a/c axis } \\
\text { Dipole } / / \text { to a/c axis } \\
\text { Dipole } / / \text { to a/c axis }\end{array}$ & $\begin{array}{l}\text { A } \\
\text { A } \\
\text { A } \\
B \\
B \\
\text { A } \\
\text { D } \\
B \\
\text { A } \\
B \\
B \\
\text { C } \\
\text { V } \\
\text { V }\end{array}$ \\
\hline
\end{tabular}

- Notes: This measurement set will investigate or validate:

A) FDTD predictions of field coupling; transmit antenna in computational volume

B) FDTD predictions of thin wire coupling; transmit antenna in computational volume

C) Calibration of aircraft external field measurement

D) Typical coupling into a representative critical LRU in the E-bay

V) Dipole Instrumentation Noise Measurements: Strongest and Weakest channels observed over the test series are chosen. Probe is replaced by matched load and noise signals recorded.

The frequency range shown is the likely minimum. The range will be extended to include as much of the 350 to $510 \mathrm{MHz}$ range as possible

* Lower priority, skip if short of time

\section{Table C-16. Noise Floor Measurements}

$\begin{array}{llllllll}\text { Test } & \text { Test } & \text { Coupling } & \text { Measured } & \text { Probe or } & \text { Freq. } & \text { Xmit } & \text { Pol Configuraation } \\ \# & \text { Point } & \text { Point } & \text { Parameter } & \text { Antenna } & (\mathrm{MHz}) & \text { Ant. }\end{array}$

\begin{tabular}{llllllllll}
\hline NO1 & CP1 & Cockpit & Vert. Field & AD-60 D-Dot & $0.3-1000$ & Rhombic & V & Nose Incident & T \\
NO2 & CP2 & Cockpit & Horz.1 Field & AD-60 D-Dot & $0.3-1000$ & Rhombic & V & Nose Incident & T \\
NO3 & CP3 & Cockpit & Horz.2 Field & AD-60 D-Dot & $0.3-1000$ & Rhombic & V & Nose Incident & T \\
NO4 & CP4 & Cockpit & Wire Current & I-320 Probe & $0.3-1000$ & Rhombic & V & Nose Incident & T \\
NO5 & CP5 & Cockpit & Wire Voltage & climbing wire & $0.3-1000$ & Rhombic & V & Nose Incident & T \\
NO6 & EB1 & E-bay & Field - & AD-60 D-Dot & $0.3-1000$ & Rhombic & V & Nose Incident & T \\
NO7 & EB2 & E-bay & LRU Voltage & Voltage Probe & $0.3-1000$ & Rhombic & V & Nose Incident & T \\
NO8 & EB3 & E-bay & Cable Current & I-320 Probe & $0.3-1000$ & Rhombic & V & Nose Incident & T \\
NO9 & C1 & Cabin & Field & AD-60 D-Dot & $0.3-1000$ & Rhombic & V & Nose Incident & T \\
NO10 C2 & Cabin & Wire Current & I-320 Probg & $0.3-1000$ & Rhombic & V & Nose Incident & T \\
NO11 & C3 & Cabin & Voltage & Long Wire & $0.3-1000$ & Rhombic & V & Nose Incident & T \\
NO12 & C4 & Cabin & Field & Ext. antenna & $0.3-1000$ & Rhombic & V & Nose Incident & T
\end{tabular}

- Notes: This measurement set will investigate or validate:

T) Measurement noise floors 


\section{Table C-17. Test Series G}

\begin{tabular}{|c|c|c|c|c|c|c|c|c|c|}
\hline $\begin{array}{l}\text { Test } \\
\#\end{array}$ & $\begin{array}{l}\text { Test } \\
\text { Point }\end{array}$ & $\begin{array}{l}\text { Coupling } \\
\text { Point }\end{array}$ & $\begin{array}{l}\text { Measured } \\
\text { Parameter }\end{array}$ & $\begin{array}{l}\text { Probe or } \\
\text { Antenna }\end{array}$ & $\begin{array}{l}\text { Freq. } \\
(\mathrm{MHz})\end{array}$ & $\begin{array}{l}\text { Xmit } \\
\text { Ant. }\end{array}$ & Pol & Orientation / Config. & Notes \\
\hline $\begin{array}{l}\text { G1 } \\
\text { G2 } \\
\text { G3 } \\
\text { G4 } \\
\text { G5 } \\
\text { G6 } \\
\text { G7 }\end{array}$ & $\begin{array}{l}\text { CP1 } \\
\text { EB1 } \\
\text { EB3 } \\
\text { C1 } \\
\text { C2 } \\
\text { C3 } \\
\text { C4 }\end{array}$ & $\begin{array}{l}\text { Cockpit } \\
\text { E-bay } \\
\text { E-bay } \\
\text { Cabin } \\
\text { Cabin } \\
\text { Cabin } \\
\text { Cabin }\end{array}$ & $\begin{array}{l}\text { Vert. Field } \\
\text { Field } \\
\text { Cable Current } \\
\text { Field } \\
\text { Wire Current } \\
\text { Voltage } \\
\text { Field }\end{array}$ & $\begin{array}{l}\text { AD-60 D-Dot } \\
\text { AD-60 D-Dot } \\
\text { 1-320 Probe } \\
\text { AD-60 D-Dot } \\
\text { 1-320 Probe } \\
\text { Long Wire } \\
\text { Ext. antenna }\end{array}$ & $\begin{array}{l}25.85 \\
25.85 \\
25.85 \\
25.85 \\
25.85 \\
25.85 \\
25.85\end{array}$ & $\begin{array}{l}\text { Rhombic } \\
\text { Rhombic } \\
\text { Rhombic } \\
\text { Rhombic } \\
\text { Rhombic } \\
\text { Rhombic } \\
\text { Rhombic }\end{array}$ & $\begin{array}{l}V \\
V \\
V \\
V \\
V \\
V \\
V\end{array}$ & $\begin{array}{l}\text { a/c nose incident } \\
a / c \text { nose incident } \\
a / c \text { nose incident } \\
a / c \text { nose incident } \\
a / c \text { nose incident } \\
a / c \text { nose incident } \\
a / c \text { nose incident }\end{array}$ & $\begin{array}{l}F \\
F \\
F \\
F \\
F \\
F \\
C, F\end{array}$ \\
\hline
\end{tabular}

- Notes: This measurement set will investigate or validate:

C) Calibration of aircraft external field measurement

F) On-board instrumentation check-out

\section{Table C-18. Test Series H}

\begin{tabular}{|c|c|c|c|c|c|c|c|c|c|}
\hline $\begin{array}{l}\text { Test } \\
\#\end{array}$ & $\begin{array}{l}\text { Test } \\
\text { Point }\end{array}$ & $\begin{array}{l}\text { Coupling } \\
\text { Point }\end{array}$ & $\begin{array}{l}\text { Measured } \\
\text { Parameter }\end{array}$ & $\begin{array}{l}\text { Probe or } \\
\text { Antenna }\end{array}$ & $\begin{array}{l}\text { Freq. } \\
(\mathrm{MHz})\end{array}$ & $\begin{array}{l}\text { Xmit } \\
\text { Ant. }\end{array}$ & Pol & Orientation / Config. & Notes \\
\hline $\begin{array}{l}\mathrm{H} 1 \\
\mathrm{H} 2 \\
\mathrm{H} 3 \\
\mathrm{H} 4 \\
\mathrm{H} 5 \\
\mathrm{H} 6 \\
\mathrm{H} 7\end{array}$ & $\begin{array}{l}\text { CP1 } \\
\mathrm{EB} 1 \\
\mathrm{EB3} \\
\mathrm{C} 1 \\
\mathrm{C} 2 \\
\mathrm{C} 3 \\
\mathrm{C} 4\end{array}$ & $\begin{array}{l}\text { Cockpit } \\
\text { E-bay } \\
\text { E-bay } \\
\text { Cabin } \\
\text { Cabin } \\
\text { Cabin } \\
\text { Cabin }\end{array}$ & $\begin{array}{l}\text { Vert. Field } \\
\text { Field } \\
\text { Cable Current } \\
\text { Field } \\
\text { Wire Current } \\
\text { Voltage } \\
\text { Field }\end{array}$ & $\begin{array}{l}\text { AD-60 D-Dot } \\
\text { AD-60 D-Dot } \\
\text { I-320 Probe } \\
\text { AD-60 D-Dot } \\
\text { I-320 Probe } \\
\text { Long Wire } \\
\text { Ext. antenna }\end{array}$ & $\begin{array}{l}172.0 \\
172.0 \\
172.0 \\
172.0 \\
172.0 \\
172.0 \\
172.0\end{array}$ & $\begin{array}{l}\text { Rhombic } \\
\text { Rhombic } \\
\text { Rhombic } \\
\text { Rhombic } \\
\text { Rhombic } \\
\text { Rhombic } \\
\text { Rhombic }\end{array}$ & $\begin{array}{l}V \\
V \\
V \\
V \\
V \\
V \\
V\end{array}$ & $\begin{array}{l}a / c \text { nose incident } \\
a / c \text { nose incident } \\
a / c \text { nose incident } \\
a / c \text { nose incident } \\
a / c \text { nose incident } \\
a / c \text { nose incident } \\
a / c \text { nose incident }\end{array}$ & $\begin{array}{l}\text { F } \\
\text { F } \\
\text { F } \\
\text { F } \\
F \\
F \\
C, F\end{array}$ \\
\hline
\end{tabular}

- Notes: This measurement set will investigate or validate:

C) Calibration of aircraft external field measurement

F) On-board instrumentation check-out

\section{Table C-19. Test Series I}

\begin{tabular}{|c|c|c|c|c|c|c|c|c|c|}
\hline $\begin{array}{l}\text { Test } \\
\#\end{array}$ & $\begin{array}{l}\text { Test } \\
\text { Point }\end{array}$ & $\begin{array}{l}\text { Coupling } \\
\text { Point }\end{array}$ & $\begin{array}{l}\text { Measured } \\
\text { Parameter }\end{array}$ & $\begin{array}{l}\text { Probe or } \\
\text { Antenna }\end{array}$ & $\begin{array}{l}\text { Freq. } \\
(\mathrm{MHz})\end{array}$ & $\begin{array}{l}\text { Xmit } \\
\text { Ant. }\end{array}$ & Pol & Orientation / Config. & Notes \\
\hline $\begin{array}{l}\text { I1 } \\
12 \\
13 \\
14 \\
15 \\
16 \\
17\end{array}$ & $\begin{array}{l}\text { CP1 } \\
\text { EB1 } \\
\text { EB3 } \\
\text { C1 } \\
\text { C2 } \\
\text { C3 } \\
\text { C4 }\end{array}$ & $\begin{array}{l}\text { Cockpit } \\
\text { E-bay } \\
\text { E-bay } \\
\text { Cabin } \\
\text { Cabin } \\
\text { Cabin } \\
\text { Cabin }\end{array}$ & $\begin{array}{l}\text { Vert. Field } \\
\text { Field } \\
\text { Cable Current } \\
\text { Field } \\
\text { Wire Current } \\
\text { Voltage } \\
\text { Field }\end{array}$ & $\begin{array}{l}\text { AD-60 D-Dot } \\
\text { AD-60 D-Dot } \\
\text { I-320 Probe } \\
\text { AD-60 D-Dot } \\
\text { I-320 Probe } \\
\text { Long Wire } \\
\text { Ext. antenna }\end{array}$ & $\begin{array}{l}430 \text { pulsed } \\
430 \text { pulsed } \\
430 \text { pulsed } \\
430 \text { pulsed } \\
430 \text { pulsed } \\
430 \text { pulsed } \\
430 \text { pulsed }\end{array}$ & $\begin{array}{l}\text { Rhombic } \\
\text { Rhombic } \\
\text { Rhombic } \\
\text { Rhombic } \\
\text { Rhombic } \\
\text { Rhombic } \\
\text { Rhombic }\end{array}$ & $\begin{array}{l}V \\
V \\
V \\
V \\
V \\
V \\
V\end{array}$ & $\begin{array}{l}a / c \text { nose incident } \\
a / c \text { nose incident } \\
a / c \text { nose incident } \\
a / c \text { nose incident } \\
a / c \text { nose incident } \\
a / c \text { nose incident } \\
a / c \text { nose incident }\end{array}$ & $\begin{array}{l}\text { F } \\
\mathbf{F} \\
\mathbf{F} \\
\mathbf{F} \\
\mathbf{F} \\
\mathbf{F} \\
\mathrm{C}, \mathrm{F}\end{array}$ \\
\hline
\end{tabular}

- Notes: This measurement set will investigate or validate:

C) Calibration of aircraft external field measurement

F) On-board instrumentation check-out

F) Measure aircraft $Q$ 
Table C-20. Test Series J

$\begin{array}{lllllll}\text { Test } & \begin{array}{l}\text { Test } \\ \text { Point }\end{array} & \begin{array}{l}\text { Coupling Measured } \\ \text { Point }\end{array} & \begin{array}{l}\text { Probe or } \\ \text { Parameter }\end{array} & \begin{array}{l}\text { Freq. } \\ \text { Antenna }\end{array} & \begin{array}{l}\text { Xmit } \\ \text { Ant. }\end{array}\end{array} \quad$ Pol Orientation / Config. Notes

\begin{tabular}{|c|c|c|c|c|c|c|c|c|c|}
\hline J 1 & CP1 & Cockpit & Vert. Field & AD-60 D-Dot & $0.3-1000$ & Rhombic & $\mathrm{v}$ & nose incident, $\mathrm{AM}$ & G \\
\hline J2 & CP2 & Cockpit & Horz.1 Field & AD-60 D-Dot & $0.3-1000$ & Rhombic & $\mathrm{V}$ & nose incident, AM & G \\
\hline J3 & CP3 & Cockpit & Horz.2 Field & AD-60 D-Dot & $0.3-1000$ & Rhombic & $\mathrm{v}$ & nose incident, $\mathrm{AM}$ & G \\
\hline J4 & CP4 & Cockpit & Wire Current & 1-320 Probe & $0.3-1000$ & Rhombic & $\mathrm{v}$ & nose incident & H \\
\hline J5 & CP5 & Cockpit & Wire Voltage & climbing wire & $0.3-1000$ & Rhombic & $\mathrm{v}$ & nose incident & H \\
\hline J 6 & CP1 & Cockpit & Vert. Field & AD-60 D-Dot & $0.3-1000$ & Rhombic & $\mathrm{V}$ & small box & L \\
\hline J7 & CP2 & Cockpit & Horz.1 Field & AD-60 D-Dot & $0.3-1000$ & Rhombic & $\mathrm{V}$ & small box & $\mathbf{L}$ \\
\hline $\mathrm{J} 8$ & CP3 & Cockpit & Horz.2 Field & AD-60 D-Dot & $0.3-1000$ & Rhombic & $\mathrm{v}$ & small box & L \\
\hline J9 & CPI & Cockpit & Vert. Field & AD-60 D-Dot & $0.3-1000$ & Rhombic & $\mathrm{v}$ & move sensor box & $\mathrm{L}$ \\
\hline $\mathrm{J} 10$ & CP2 & Cockpit & Horz.1 Field & AD-60 D-Dot & $0.3-1000$ & Rhombic & $\mathrm{v}$ & move sensor box & L \\
\hline J11 & $\mathrm{CP} 3$ & Cockpit & Horz.2 Field & AD-60 D-Dot & $0.3-1000$ & Rhombic & $\mathrm{v}$ & move sensor box & $\mathrm{L}$ \\
\hline $\mathrm{J} 12$ & CP1 & Cockpit & Vert. Field & AD-60 D-Dot & $800-900$ & Rhombic & $\mathrm{v}$ & $1 \% \mathrm{BW}, 1000$ pts & \\
\hline $\mathrm{J} 13$ & CP1 & Cockpit & Vert. Field & AD-60 D-Dot & $0.3-1000$ & Rhombic & $\mathrm{v}$ & person in left seat & n \\
\hline J 14 & CP2 & Cockpit & Horz.1 Field & AD-60 D-Dot & $0.3-1000$ & Rhombic & $\mathrm{v}$ & person in left seat & $\mathrm{M}$ \\
\hline J15 & CP3 & Cockpit & Horz.2 Field & AD-60 D-Dot & $0.3-1000$ & Rhombic & $\mathrm{V}$ & person in left seat & $M$ \\
\hline$J 16$ & CPI & Cockpit & Vert. Field & AD-60 D-Dot & $0.3-1000$ & Rhombic & $\mathrm{v}$ & in both seats & $M$ \\
\hline $\mathrm{J} 17$ & CP2 & Cockpit & Horz.1 Field & AD-60 D-Dot & $0.3-1000$ & Rhombic & $\mathrm{V}$ & persons in both seats & $M$ \\
\hline $\mathrm{J} 18$ & CP3 & Cockpit & Horz.2 Field & AD-60 D-Dot & $0.3-1000$ & Rhombic & $\mathrm{v}$ & persons in both seats & $\mathbf{M}$ \\
\hline $\mathrm{J} 19$ & $\mathrm{CP} 12$ & Cockpit & Heater Current & I-320 Probe & $0.3-1000$ & Rhombic & $\mathbf{v}$ & new probe position & $\mathrm{v}$ \\
\hline $\mathrm{J} 20$ & CP1 & Cockpit & Vert. Field & AD-60 D-Dot & $0.3-1000$ & Rhombic & v & repeatability, PM & $k$ \\
\hline $\mathrm{J} 21$ & CP2 & Cockpit & Horz.1 Field & AD-60 D-Dot & $0.3-1000$ & Rhombic & V & repeatability, PM & $\mathrm{K}$ \\
\hline $\mathrm{J} 22$ & CP3 & Cockpit & Horz.2 Field & AD-60 D-Dot & $0.3-1000$ & Rhombic & $\mathrm{v}$ & repeatability, PM & $k$ \\
\hline $\mathrm{J} 23$ & EB1 & E-bay & Field & AD-60 D-Dot & $0.3 \cdot 1000$ & Rhombic & $\mathrm{v}$ & nose incident & $\mathbf{G}$ \\
\hline J24 & EB2 & E-bay & LRU Voltage & Voltage Probe & $0.3-1000$ & Rhombic & $\mathrm{v}$ & nose incident & $\mathbf{J}$ \\
\hline $\mathrm{J} 25$ & EB3 & E-bay & Cable Current & I-320 Probe & $0.3-1000$ & Rhombic & $\mathrm{v}$ & nose incident & $\mathbf{H}$ \\
\hline $\mathrm{J} 26$ & EB1 & E-bay & Field & AD-60 D-Dot & $0.3-1000$ & nbic & $\mathrm{v}$ & nose in & G2 \\
\hline $\mathrm{J} 27$ & EB2 & E-bay & LRU Voltage & Voltage Probe & $0.3-1000$ & Rhombic & $\mathrm{v}$ & nose incident & G2 \\
\hline $\mathrm{J} 28$ & EB3 & E-bay & Cable Current & Probe & $0.3-1000$ & & v & nose & G2 \\
\hline$J 29$ & EB 1 & E-bay & Field & AD-60 D-Dot & $0.3-1000$ & Rhombic & $\mathrm{v}$ & nose incident & G3 \\
\hline $\mathrm{J} 30$ & EB2 & E-bay & LRU Voltage & Voltage Probe & $0.3-1000$ & & $\mathrm{~V}$ & nose $\mathrm{i}$ & G3 \\
\hline $\mathrm{J} 31$ & EB3 & E-bay & Cable Current & 1-320 Probe & $0.3-1000$ & Rhombic & $\mathrm{v}$ & nose incident & G3 \\
\hline $\mathrm{J} 32$ & $\mathrm{Cl}$ & Cabin & Field & AD-60 D-Dot & $0.3-1000$ & Rhombic & $\mathrm{V}$ & nose incident & 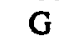 \\
\hline J33 & C2 & Cabin & Wire Current & I-320 Probe & $0.3-1000$ & Rhombic & $\mathrm{v}$ & nose incident & H \\
\hline J34 & C3 & Cabin & Voltage & Long Wire & $0.3-1000$ & Rhombic & $\mathrm{v}$ & nose incident & H \\
\hline $\mathrm{J} 35$ & $\mathrm{C} 4$ & Cabin & Field & Ext. antenna & $0.3-1000$ & Rhombic & $\mathrm{v}$ & nose incident & I \\
\hline J36 & CPI & Cockpit & Vert. Field & AD-60 D-Dot & $0.3-1000$ & Rhombic & $\mathbf{v}$ & move $\mathrm{a} / \mathrm{c} 10^{\circ}$ off axis & $\mathbf{P}$ \\
\hline $\mathrm{J} 37$ & CP2 & Cockpit & Horz.1 Field & AD-60 D-Dot & $0.3-1000$ & Rhombic & $\mathrm{v}$ & move $a / c 10^{\circ}$ off axis & $\mathbf{P}$ \\
\hline $\mathrm{J} 38$ & CP3 & Cockpit & Horz. 2 Field & AD-60 D-Dot & $0.3-1000$ & Rhombic & $\mathrm{v}$ & move $a / c 10^{\circ}$ off axis & $\mathbf{P}$ \\
\hline J39 & CP1 & Cockpit & Vert. Field & AD-60 D-Dot & $0.3-1000$ & Rhombic & V & move a/c $5^{\circ}$ off & $\mathbf{P}$ \\
\hline $\mathrm{J} 4 \mathrm{C}$ & $\mathrm{CP} 2$ & Cockpit & Horz.1 Field & AD-60 D-Dot & $0.3-1000$ & Rhombic & V & move a $/ \mathrm{c} 5^{\circ}$ off & $\mathbf{P}$ \\
\hline $\mathrm{J} 4$ & $\mathrm{CP} 3$ & Cockpit & Horz.2 Field & AD-60 D-Dot & $0.3-1000$ & Rhombic & $\mathrm{v}$ & move $\mathrm{a} / \mathrm{c} 5^{\circ}$ off & $\mathbf{P}$ \\
\hline
\end{tabular}

- Notes: This measurement set will investigate or validate: -

E) Variations in coupling with frequency (frequency stirring)

G) FDTD predictions of field coupling; plane wave incident

G2) E Bay hatch cover electrically sealed with conducting tape to assess leakage at seam

G3) E Bay hatch cover open to assess energy leakage

H) FDTD predictions of thin wire coupling; plane wave incident

I) Calibration of aircraft external field measurement, plane wave incident

J) Typical coupling into a representative critical LRU in the E-bay, plane wave incident

K) Repeatability

L) FDTD resolution element effects. "Small box" refers to emplacement of a small metallic box (dimensions order of resolution element size) near the sensor box. Movement of sensor box may be impossible if rigidly bonded to airframe

M) Stirring effects caused by people: Remove small box

N) Cockpit Window model

P) Angular sensitivity. Movement to $5^{\circ}$ will be performed if measurements at $10^{\circ}$ indicate strong sensitivity 
Table C-21. Test Series K

\begin{tabular}{|c|c|c|c|c|c|c|c|c|c|}
\hline est & $\begin{array}{l}\text { Test } \\
\text { Point }\end{array}$ & $\begin{array}{l}\text { Coupling } \\
\text { Point }\end{array}$ & $\begin{array}{l}\text { Measured } \\
\text { Parameter }\end{array}$ & $\begin{array}{l}\text { Probe or } \\
\text { Antenna }\end{array}$ & $\begin{array}{l}\text { Freq. } \\
\text { (MHz) }\end{array}$ & $\begin{array}{l}\text { Xmit } \\
\text { Ant. }\end{array}$ & Pol & Orientation / Config. & Notes \\
\hline $\begin{array}{l}\text { K1 } \\
\text { K2 } \\
\text { K4 } \\
\text { K5 } \\
\text { K6 } \\
\text { K7 } \\
\text { K8 } \\
\text { K9 } \\
\text { K10 } \\
\text { K11 } \\
\text { K12 } \\
\text { K13 } \\
\text { K14 } \\
\text { K15 } \\
\text { K16 } \\
\text { K17 } \\
\text { K18 } \\
\text { K19 } \\
\text { K20 } \\
\text { K21 } \\
\text { K22 } \\
\text { K23 } \\
\text { K24 } \\
\text { K25 } \\
\text { K26 } \\
\text { K27 } \\
\text { K28 } \\
\text { K29 }\end{array}$ & $\begin{array}{l}\text { CP1 } \\
\text { CP2 } \\
\text { CP3 } \\
\text { CP4 } \\
\text { CP5 } \\
\text { EB1 } \\
\text { EB2 } \\
\text { EB3 } \\
\text { C1 } \\
\text { C2 } \\
\text { C3 } \\
\text { C4 } \\
\text { C3 } \\
\text { C3 } \\
\text { EB3 } \\
\text { CP1 } \\
\text { CP1 } \\
\text { CP2 } \\
\text { CP3 } \\
\text { CP1 } \\
\text { CP2 } \\
\text { CP3 } \\
\text { CP5 } \\
\text { CP5 } \\
\text { CP12 } \\
\text { C3 } \\
\text { CP1 } \\
\text { CP2 } \\
\text { CP3 }\end{array}$ & $\begin{array}{l}\text { Cockpit } \\
\text { Cockpit } \\
\text { Cockpit } \\
\text { Cockpit } \\
\text { Cockpit } \\
\text { E-bay } \\
\text { E-bay } \\
\text { E-bay } \\
\text { Cabin } \\
\text { Cabin } \\
\text { Cabin } \\
\text { Cabin } \\
\text { Cabin } \\
\text { Cabin } \\
\text { E-bay } \\
\text { Cockpit } \\
\text { Cockpit } \\
\text { Cockpit } \\
\text { Cockpit } \\
\text { Cockpit } \\
\text { Cockpit } \\
\text { Cockpit } \\
\text { Cockpit } \\
\text { Cockpit } \\
\text { Cockpit } \\
\text { Cabin } \\
\text { Cockpit } \\
\text { Cockpit } \\
\text { Cockpit }\end{array}$ & $\begin{array}{l}\text { Vert. Field } \\
\text { Horz.1 Field } \\
\text { Horz.2 Field } \\
\text { Wire Current } \\
\text { Wire Voltage } \\
\text { Field } \\
\text { LRU Voltage } \\
\text { Cable Current } \\
\text { Field } \\
\text { Wire Current } \\
\text { Voltage } \\
\text { Field } \\
\text { Voltage } \\
\text { Voltage } \\
\text { Field } \\
\text { Vert. Field } \\
\text { Vert. Field } \\
\text { Horz.1 Field } \\
\text { Horz.2 Field } \\
\text { Vert. Field } \\
\text { Horz.1 Field } \\
\text { Horz.2 Field } \\
\text { Wire Voltage } \\
\text { Wire Voltage } \\
\text { Heater current } \\
\text { Voltage } \\
\text { Vert. Field } \\
\text { Horz.1 Field } \\
\text { Horz.2 Field }\end{array}$ & $\begin{array}{l}\text { AD-60 D-Dot } \\
\text { AD-60 D-Dot } \\
\text { AD-60 D-Dot } \\
\text { I-320 Probe } \\
\text { climbing wire } \\
\text { AD-60 D-Dot } \\
\text { Voltage Probe } \\
\text { I-320 Probe } \\
\text { AD-60 D-Dot } \\
\text { 1-320 Probe } \\
\text { Long Wire } \\
\text { Ext. antenna } \\
\text { Long Wire } \\
\text { Long Wire } \\
\text { AD-60 D-Dot } \\
\text { AD-60 D-Dot } \\
\text { AD-60 D-Dot } \\
\text { AD-60 D-Dot } \\
\text { AD-60 D-Dot } \\
\text { AD-60 D-Dot } \\
\text { AD-60 D-Dot } \\
\text { AD-60 D-Dot } \\
\text { climbing wire } \\
\text { climbing wire } \\
\text { I-320 Probe } \\
\text { Long Wire } \\
\text { AD-60 D-Dot } \\
\text { AD-60 D-Dot } \\
\text { AD-60 D-Dot }\end{array}$ & $\begin{array}{l}0.3-1000 \\
0.3-1000 \\
0.3-1000 \\
0.3-1000 \\
0.3-1000 \\
0.3-1000 \\
0.3-1000 \\
0.3-1000 \\
0.3-1000 \\
0.3-1000 \\
0.3-1000 \\
0.3-1000 \\
0.3-1000 \\
0.3-1000 \\
0.3-1000 \\
800-900 \\
0.3-1000 \\
0.3-1000 \\
0.3-1000 \\
0.3-1000 \\
0.3-1000 \\
0.3-1000 \\
0.3-1000 \\
0.3-1000 \\
0.3-1000 \\
0.3-1000 \\
0.3-1000 \\
0.3-1000 \\
0.3-1000\end{array}$ & $\begin{array}{l}\text { Rhombic } \\
\text { Rhombic } \\
\text { Rhombic } \\
\text { Rhombic } \\
\text { Rhombic } \\
\text { Rhombic } \\
\text { Rhombic } \\
\text { Rhombic } \\
\text { Rhombic } \\
\text { Rhombic } \\
\text { Rhombic } \\
\text { Rhombic } \\
\text { Rhombic } \\
\text { Rhombic } \\
\text { Rhombic } \\
\text { Rhombic } \\
\text { Rhombic } \\
\text { Rhombic } \\
\text { Rhombic } \\
\text { Rhombic } \\
\text { Rhombic } \\
\text { Rhombic } \\
\text { Rhombic } \\
\text { Rhombic } \\
\text { Rhombic } \\
\text { Rhombic } \\
\text { Rhombic } \\
\text { Rhombic } \\
\text { Rhombic }\end{array}$ & $\begin{array}{l}V \\
V \\
V \\
V \\
V \\
V \\
V \\
V \\
V \\
V \\
V \\
V \\
V \\
V \\
V \\
V \\
V \\
V \\
V \\
V \\
V \\
V \\
V \\
V \\
V \\
V \\
V \\
V \\
V\end{array}$ & $\begin{array}{l}\text { side incident } \\
\text { side incident } \\
\text { side incident } \\
\text { side incident } \\
\text { side incident } \\
\text { side incident } \\
\text { side incident } \\
\text { side incident } \\
\text { side incident } \\
\text { side incident } \\
\text { side incident } \\
\text { side incident } \\
\text { repeatability } \\
\text { move bodies in cabin } \\
\text { repeatability } \\
\text { 1\% BW, 1000 pts } \\
\text { person in left seat } \\
\text { person in left seat } \\
\text { person in left seat } \\
\text { persons in both seats } \\
\text { persons in both seats } \\
\text { persons in both seats } \\
\text { add parasitic wire } \\
\text { add parasitic wire } \\
\text { new probe position } \\
\text { add static ground } \\
\text { retum to nose incident } \\
\text { return to nose incident } \\
\text { return to nose incident }\end{array}$ & $\begin{array}{l}\text { G } \\
G \\
G \\
H \\
H \\
G \\
J \\
H \\
G \\
H \\
H \\
I \\
K \\
M \\
K \\
E \\
M \\
M \\
M \\
M \\
M \\
M \\
O \\
O \\
N \\
Q \\
R \\
R \\
R\end{array}$ \\
\hline
\end{tabular}

- Notes: This measurement set will investigate or validate:

E) Variations in cowpling with frequency (frequency stirring)

G) FDTD predictions of field coupling; plane wave incident

H) FDTD predictions of thin wire coupling; plane wave incident

I) Calibration of aircraft external field measurement, plane wave incident

J) Typical coupling into a repsesentative critical LRU in the E-bay, plane wave incident

K) Repeatability (instrumentation)

M) Stirring effects caused by people. Remove small box

O) Effect of cable loads on cavity absorption

P) Angular sensitivity

Q) Effect of ground wire

R) Repeatability (angular)

Skip if included in previous test series 


\section{Table C-22. Test Series PL}

$\begin{array}{lll}\text { TX Ant } & \begin{array}{l}\text { Rx Ant } \\ \text { location }\end{array} & \text { Comments } \\ \text { location } & \end{array}$

\section{B) CAVITYO}

$\# 1$

\#3

\#4 use DSA $602,100 \mathrm{MHz}$ steps to $1 \mathrm{GHz}$, $200 \mathrm{MHz}$ steps from $1 \mathrm{GHz}$ to $6 \mathrm{GHz}$ use DSA $602,100 \mathrm{MHz}$ steps to $1 \mathrm{GHz}$, $200 \mathrm{MHz}$ steps from $1 \mathrm{GHz}$ to $6 \mathrm{GHz}$ use DSA $602,100 \mathrm{MHz}$ steps to $1 \mathrm{GHz}$, $200 \mathrm{MHz}$ steps from $1 \mathrm{GHz}$ to $6 \mathrm{GHz}$

\section{C) CAVITY TO CAVITY COUPLING

$\# 1$ four locations in cabin \\ four locations in cockpit \\ four locations in cabin \\ four locations in cockpit \\ four locations in electronics bay}

\section{Table C-23. Test Series Pad Characterization}

\begin{tabular}{|c|c|c|c|c|}
\hline Measurement & Probe & $\begin{array}{l}\text { Frequencies } \\
\text { Range }\end{array}$ & Increment & Positions \\
\hline \multicolumn{5}{|c|}{ Case 1 and Case 2} \\
\hline Current & Prodyn I-320 & $\begin{array}{c}1 \text { to } 15 \mathrm{MHz} \\
20 \text { to } 100 \mathrm{MHz} \\
110 \text { to } 1000 \mathrm{MHz} \\
=\end{array}$ & $\begin{array}{l}2 \mathrm{MHz} \\
5 \mathrm{MHz} \\
10 \mathrm{MHz}\end{array}$ & $\begin{array}{l}\text { Pairs: Starting } 22.5 \mathrm{~m} \text { from feed } \\
\mathrm{P}_{\mathrm{n}}=(22.5+\Delta \mathrm{r}(2 \mathrm{n}-2), 22.5+\Delta \mathrm{r}(2 \mathrm{n}-1)) \\
\text { for } \mathrm{n}=1 \text { to } 45 \text { with } \Delta \mathrm{r}=30 \mathrm{~cm}\end{array}$ \\
\hline
\end{tabular}




\section{Table C-24. Horizontal Polarization - Rhombic}

\begin{tabular}{|c|c|c|c|c|c|c|c|c|c|}
\hline $\begin{array}{l}\text { Test } \\
\#\end{array}$ & $\begin{array}{l}\text { Test } \\
\text { Point }\end{array}$ & $\begin{array}{l}\text { Coupling } \\
\text { Point }\end{array}$ & $\begin{array}{l}\text { Measured } \\
\text { Parameter }\end{array}$ & $\begin{array}{l}\text { Probe or } \\
\text { Antenna }\end{array}$ & $\begin{array}{l}\text { Freq. } \\
\text { (MHz) }\end{array}$ & $\begin{array}{l}\text { Xmit } \\
\text { Ant. }\end{array}$ & Pol & Orientation / Config. & Notes \\
\hline $\begin{array}{l}\text { HP1 } \\
\text { HP2 } \\
\text { HP3 } \\
\text { HP4 } \\
\text { HP5 } \\
\text { HP6 } \\
\text { HP7 } \\
\text { HP8 } \\
\text { HP9 } \\
\text { HP10 } \\
\text { HP11 } \\
\text { HP12 } \\
\text { HP13 } \\
\text { HP14 }\end{array}$ & $\begin{array}{l}\text { CP1 } \\
\text { CP2 } \\
\text { CP3 } \\
\text { CP4 } \\
\text { CP5 } \\
\text { EB1 } \\
\text { EB2 } \\
\text { EB3 } \\
\text { C1 } \\
\text { C2 } \\
\text { C3 } \\
\text { C4 } \\
\text { Weak } \\
\text { Strong }\end{array}$ & $\begin{array}{l}\text { Cockpit } \\
\text { Cockpit } \\
\text { Cockpit } \\
\text { Cockpit } \\
\text { Cockpit } \\
\text { E-bay } \\
\text { E-bay } \\
\text { E-bay } \\
\text { Cabin } \\
\text { Cabin } \\
\text { Cabin } \\
\text { Cabin } \\
\text { Signal Line } \\
\text { Signal Line }\end{array}$ & $\begin{array}{l}\text { Vert. Field } \\
\text { Horz.1 Field } \\
\text { Horz.2 Field } \\
\text { Wire Current } \\
\text { Wire Voltage } \\
\text { Field } \\
\text { LRU Voltage } \\
\text { Cable Current } \\
\text { Field } \\
\text { Wire Current } \\
\text { Voltage } \\
\text { Field } \\
\end{array}$ & $\begin{array}{l}\text { AD-60 D-Dot } \\
\text { AD-60 D-Dot } \\
\text { AD-60 D-Dot } \\
\text { I-320 Probe } \\
\text { climbing wire } \\
\text { AD-60 D-Dot } \\
\text { Voltage Probe } \\
\text { I-320 Probe } \\
\text { AD-60 D-Dot } \\
\text { I-320 Probe } \\
\text { Long Wire } \\
\text { Ext. antenna }\end{array}$ & $\begin{array}{l}3-30 \\
3-30 \\
3-30 \\
3-30 \\
3-30 \\
3-30 \\
3-30 \\
3-30 \\
3-30 \\
3-30 \\
3-30 \\
3-30 \\
3-30 \\
3-30\end{array}$ & $\begin{array}{l}\text { Rhombic } \\
\text { Rhombic } \\
\text { Rhombic } \\
\text { Rhombic } \\
\text { Rhombic } \\
\text { Rhombic } \\
\text { Rhombic } \\
\text { Rhombic } \\
\text { Rhombic } \\
\text { Rhombic } \\
\text { Rhombic } \\
\text { Rhombic } \\
\text { Rhombic } \\
\text { Rhombic }\end{array}$ & $\begin{array}{l}\mathbf{H} \\
\mathbf{H} \\
\mathbf{H} \\
\mathbf{H} \\
\mathbf{H} \\
\mathbf{H} \\
\mathbf{H} \\
\mathbf{H} \\
\mathbf{H} \\
\mathbf{H} \\
\mathbf{H} \\
\mathbf{H} \\
\mathbf{H} \\
\mathbf{H}\end{array}$ & $\begin{array}{l}\text { Side incident } \\
\text { Side incident } \\
\text { Side incident } \\
\text { Side incident } \\
\text { Side incident } \\
\text { Side incident } \\
\text { Side incident } \\
\text { Side incident } \\
\text { Side incident } \\
\text { Side incident } \\
\text { Side incident } \\
\text { Side incident } \\
\text { Side incident } \\
\text { Side incident }\end{array}$ & $\begin{array}{l}\text { A } \\
\text { A } \\
\text { A } \\
\text { B } \\
\text { B } \\
\text { A } \\
\text { D } \\
\text { B } \\
\text { A } \\
\text { B } \\
\text { B } \\
\text { C } \\
\text { V } \\
\text { V }\end{array}$ \\
\hline
\end{tabular}

- Notes: This measurement set will investigate or validate:

A) FDTD predictions of field coupling

B) FDTD predictions of thin wire coupling

C) Calibration of aircraft external field measurement

D) Typical coupling into a representative critical LRU in the E-bay

V) Dipole Instrumentation Noise Measurements

* Lower priority, skip if short of time 


\section{Appendix B}

UNITED INTERNATIONAL ENGINEERING, INC.

2201 BUENA VISTA DRIVE S.E., SUITE 207

ALBUQUERQUE, NEW MEXICO 87106

(505) 242-9200

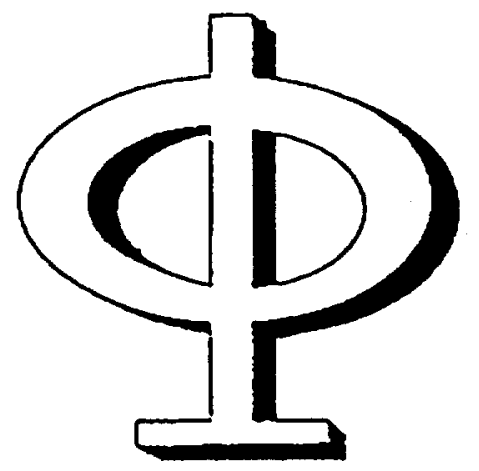

NASA B-757 Aircraft Test Report

EMP Development, Analysis, and Testing (EMPDAT)

Program

Contract F29601-88-C-0001

Contracting Office:

Phillips Laboratory/PKWA

Technical Office:

Phillips Laboratory/WSM

Subtask:

03-18

CDRL:

A016

UIE-TR-94-0025

15 DECEMBER 1994 


\section{FOREWORD}

This NASA B-757 Aircraft Test Report, UIE-TR-94-0025, has been prepared in accordance with Subtask 03-18, Contract Data Requirements List A016. This report is submitted by UIE, Inc., 2201 Buena Vista Drive S.E., Suite 207, Albuquerque, New Mexico 87106 to the Phillips Laboratory/WSM, 3550 Aberdeen S.E., Kirtland Air Force Base, New Mexico 87117-5776.

Prepared By:

John W. Millard

Principal Investigator

SS 03-18

Approved By:

Howard N. LaValley

UIE EMPDAT Program Manager 


\section{CONTENTS}

Section Page

1.0 INTRODUCTION 1

1.1 BACKGROUND 1

$\begin{array}{ll}1.2 \text { ORGANIZATION } & 2\end{array}$

1.3 EXPERIMENTS 3

1.4 SCHEDULE 4

1.4.1 Scheduling: As-Planned/As-Accomplished 4

1.4.2 Daily Test Schedule 5

2.0 PRE-TEST ACTIVITIES

2.1 DETAILED TEST PLANNING

2.1.1 CW Sweep Designation Definitions 7

2.1.2 Field Map Point Identification 8

2.1.3 Instrumentation Planning $\quad 11$

2.2 DIPOLE ANTENNA FABRICATION AND CHECKOUT 12

2.2.1 Dipole Antenna Fabrication $\quad 12$

2.2.2 Dipole Antenna Checkout $\quad 15$

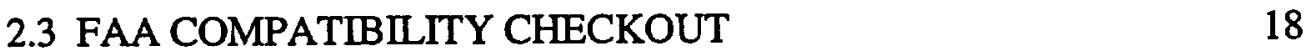

2.4 DIPOLE ANTENNA FIELD MAPPING 20

- 2.5 RHOMBIC ÄNTENNA FIELD MAPPING 21

$\begin{array}{ll}2.6 \text { AIRCRAFT INSTRUMENTATION } & 23\end{array}$

$\begin{array}{ll}3.0 \text { TEST ACTIVITIES } & 24\end{array}$

3.1 DIPOLE MEASUREMENTS 24

3.1.1 Configuration 24

3.1.2 Measurement Procedures 25

$\begin{array}{ll}\text { 3.1.3 Skip Bands } & 26\end{array}$ 
CONTENTS (Concluded)

Section

Page

3.2 ON-BOARD INSTRUMENTATION CHECKOUT SUPPORT 26

3.3 COMMON MODE RHOMBIC MEASUREMENTS 28

3.3.1 Aircraft Orientations and Configurations 29

3.3.2 Measurement Procedures 30

3.3.3 Skip Bands 31

3.3.4 Configuration Issues 31

3.4 DIFFERENTIAL MODE RHOMBIC MEASUREMENTS 33

3.4.1 Configuration 33

3.4.2 Measurement Procedures 34

3.5 GROUND PLANE CHARACTERIZATION MEASUREMENTS 35

3.5.1 Configuration 35

3.5.2 Measurement Procedures 37

3.5.3 Skip Bands 38

$\begin{array}{ll}3.6 \text { CROSSTALK } & 38\end{array}$

$\begin{array}{ll}\text { REFERENCES } & 42\end{array}$ 


\section{FIGURES}

Number/Title

Page

2-1. LESLI Five-Meter Test Stand. 13

2-2. Dipole Antenna Mount 14

2-4. $25 \mathrm{MHz}$ Antenna Tuning, Initial Response. 17

2-5. $25 \mathrm{MHz}$ Antenna Tuning, Final Response. 18

2-7. Short Rhombic Antenna Field Map Points. 21

2-8. Ten Sweep Overlay of 10CL. 22

3-1. Dipole Antenna Position. 24

3-3. Onboard Instrumentation Check. 27

3-4. B-757 Nose-on Under the Short Rhombic Antenna in LESLI 29

3-5. Rhombic Instrumentation. 30

3-6. Test Point EB2 Overlay Plots. 32

3-7. Test Point CP4 Instrumentation Noise; Other Inputs Connected. 40

3-8. Test Point CP4 Instrumentation Noise; Other Inputs Disconnected. 40 


\section{TABLES}

Number/Title

Page

2-1. Sweep Designation Definitions

8

2-2. Common Mode Rhombic Field Map Points

9

2-3. Dipole Field Map Points

10

2-4. Test Point/Transmitter Selection

12

3-1. Ground Plane Characterization Points 


\subsection{INTRODUCTION}

\subsection{BACKGROUND}

The Phillips Laboratory (PL), by agreement with the NASA Langley Research Center (LaRC), provided support to test a NASA Boeing 757 (B-757) aircraft in its Large

Electromagnetic System-Level Illuminator (LESLI) facility. The purpose of the test series was to acquire low level $\mathrm{CW}$ data to validate computer modeling codes and to provide an estimation of the aircraft response to electromagnetic threats expected in a future fly-by test program.

As stated in the General Test Plan provided by Lawrence Livermore National Laboratory (LLNL), "NASA FBL/PBW Program, NASA Boeing 757 HIRF Test Plan, Low Power on-the-Ground Tests" (Ref. 1),

The NASA Fly-by-Light/Power-by-Wire program was initiated to develop technology for a future generation of commercial transport aircraft. Studies have shown that an all-electric aircraft (PBW) could have substantial benefits in terms of weight and maintenance costs over today's aircraft that rely on hydraulic and pneumatic controls. However, one major concern regarding the all-electric aircraft is the potential for conducted and radiated electromagnetic susceptibility of its electronic controls. A way to potentially alleviate this problem (and save weight at the same time) is to route all control and signal information over optical fiber control links (FBL) that are inherently easier to harden against the electromagnetic environment (EME).

Since electro-optic devices and electronic digital computers will still be required, a major objective of the FBL/PBW program is to develop tools and techniques for assessing the survivability of the modern aircraft using such equipment in the electromagnetic environment. The EME includes both externally generated high intensity radiated fields (HIRF) and internally-generated radiated or conducted emissions. The ability to withstand internally-generated emissions is often referred to as electromagnetic compatibility (EMC). 
The EME effects assessment tools and techniques developed under the program must ultimately be accepted by industry and the FAA regulatory body to be useful. Therefore, an important aspect of the program is that these tools and techniques must be validated to the satisfaction of this community. A series of aircraft tests are (sic) being planned for this purpose. Specifically, low power on-the-ground tests and fly-by tests will be conducted....

The primary thrust of this aircraft test program is to develop a library or data base of experimental results to be used in the evaluation of modeling codes. A second objective is to support the development of other assessment techniques such as mode-stirred chamber measurements. To support these objectives, it is also necessary to quantify measurement errors and to perform sensitivity studies to allow an estimation of the degree of agreement between measurement and model results that can be reasonably expected.

This report describes the on-the-ground tests performed at the LESLI facility to generate the data which will be used for computer modeling code validation and measurement/model result comparison. Most of the data to support mode-stirred chamber techniques were acquired in a parallel effort by the PL and a report of this aspect of the test program will be provided separately.

\subsection{ORGANIZATION}

Support for the NASA B-757 CW test came from several organizations. The NASA Langley Research Center is the funding organization which also developed and installed the on-board instrumentation. The Lawrence Livermore National Laboratory was responsible for test planning and test execution management. The Phillips Laboratory (PL/WSM), supported by United International Engineering (UIE), provided the test environment which included the LESLI facility, instrumen-tation, data acquisition, data reduction, aircraft support, and detailed test planning. Figure 1-1 depicts the key participants in the test. 


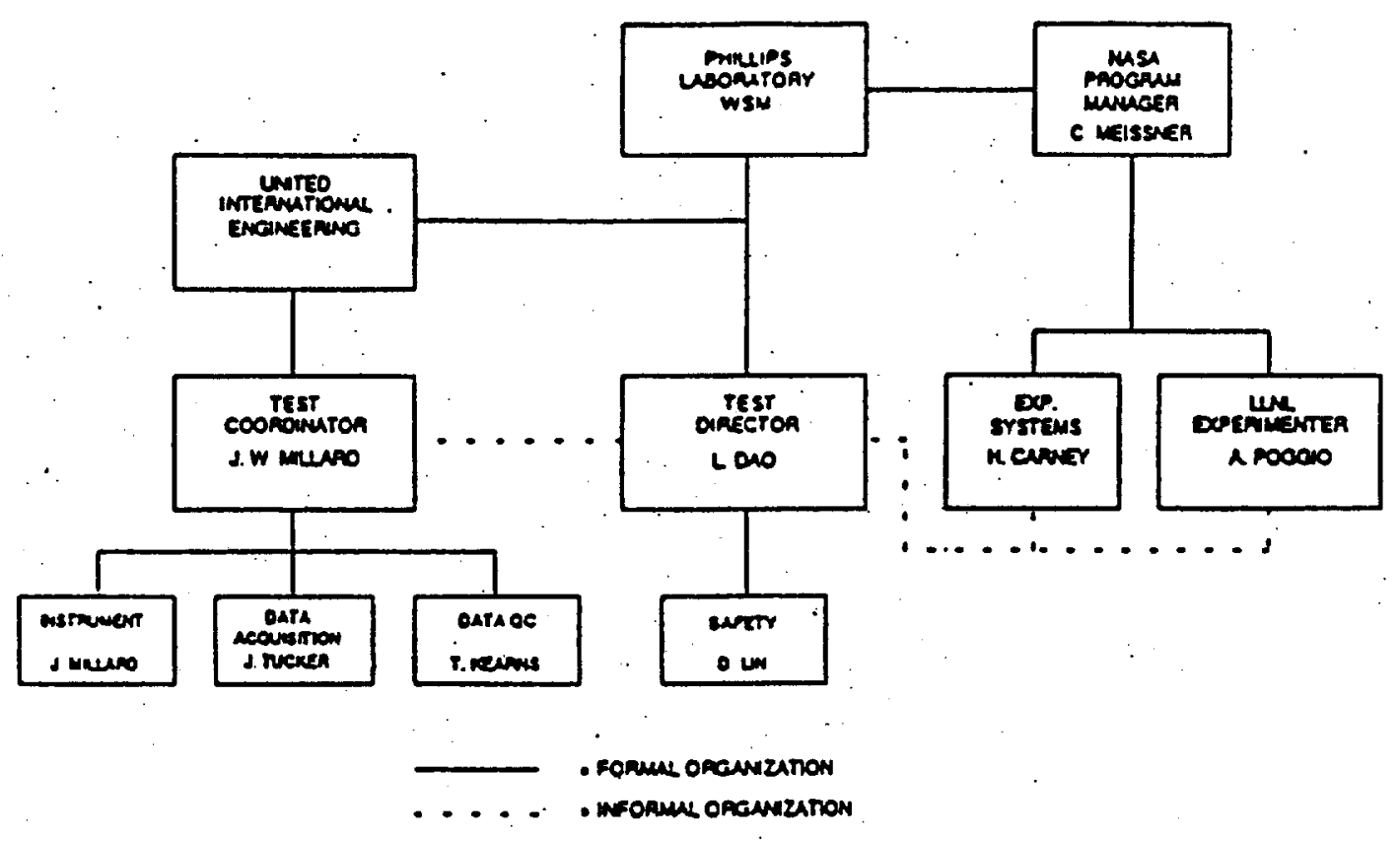

Figure 1-1. B-757 Test Organization.

\subsection{EXPERIMENTS}

Reference 1 discussed in detail the experiments which were performed during the B-757 on-the-ground test. They are summarized as follows:

1. Experiments in which the aircraft was excited by dipole antennas driven by stepped CW around three center frequencies $(25.85 \mathrm{MHz}, 172 \mathrm{MHz}$, and $430 \mathrm{MHz}$ ) and in two polarizations (vertical and horizontal). The three center frequencies represent the frequencies of the threats against which the aircraft is to fly. 
2. Experiments to check out the on-board instrumentation in which the aircraft was excited by the common mode rhombic antenna dwelling at $25.85 \mathrm{MHz}$ and $172 \mathrm{MHz}$ and pulsed with a burst of modulated $430 \mathrm{MHz} \mathrm{CW}$.

3. Experiments to assess the broadband response of the aircraft when it was excited by the common mode rhombic antenna driven by stepped $\mathrm{CW}$ from $300 \mathrm{kHz}$ to $1 \mathrm{GHz}$. The excitation was provided with the aircraft nose on, side on, and with orientation variations.

4. Experiments to assess the aircraft's resonant response at $3 \mathrm{MHz}$ when excited by the differential mode (horizontal polarization) rhombic antenna driven by stepped $\mathrm{CW}$ from $2 \mathrm{MHz}$ to $30 \mathrm{MHz}$.

5. An experiment to determine characteristics of the concrete ground plane in which one leg of the rhombic antenna was laid out over the ground plane on a styrofoam pad and current measurements made along its length as it was driven from $300 \mathrm{kHz}$ to $1 \mathrm{GHz}$.

\subsection{SCHEDULE}

\subsubsection{Scheduling: As-Planned/As-Accomplished}

The NASA Boeing 757 Low Power On-the-Ground Test was a dynamic program. The original plan called for the test of a Boeing 737 aircraft. When a B-757 was substituted, major impacts on test planning and scheduling resulted. Experiments had to be modified and the aircraft arrival date was not determined until a few weeks before the start of the test. As the data were reviewed, new experiments were indicated and added to the schedule. Consequently, the schedule changed frequently over the course of program development. Attempts to document all the changes would produce confusion rather than clarification. Figure 1-2 illustrates the original test schedule as determined on 1 August 1994 compared to that which was accomplished. 


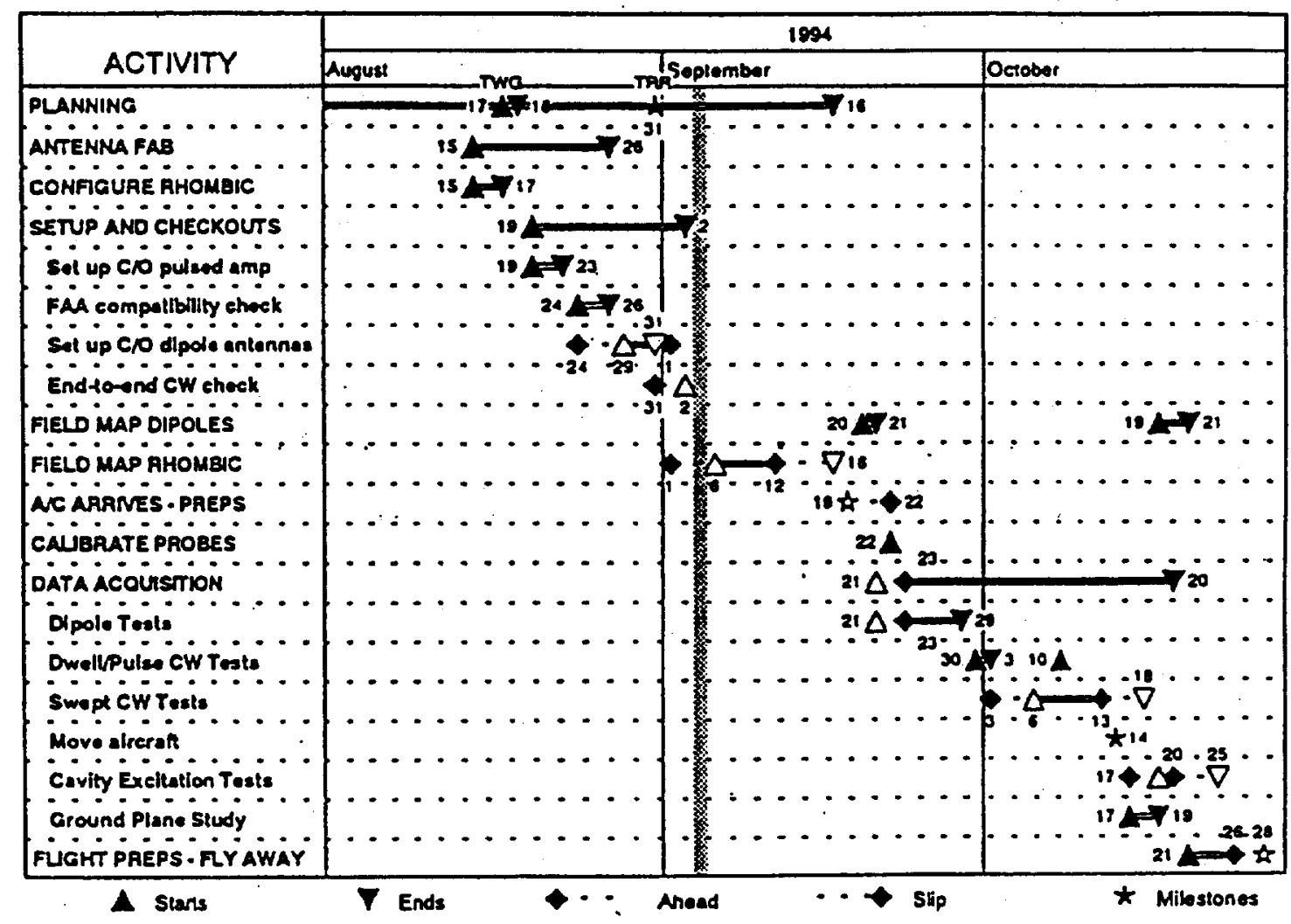

Figure 1-2. NASA B-757 Test Schedule.

\subsubsection{Daily Test Schedule}

The NASA B-757 test plan, Reference 1, called for a test day which provided for eight hours of data acquisition, an hour at the start of each day for preparation, an hour at the end of the day to secure the site, and a one-hour lunch break. Considering the hours of sunrise and sunset during August through October, it was decided to open the site at 0630 in the morning and have it secured by 1730 . Figure 1-3 shows the daily schedule as planned and the parties responsible for activity accomplishment. The daily schedule was actually more flexible than depicted. Frequently, data acquisition started before 0730 and lunch was taken when a convenient break occurred around 1100. On several occasions, data continued to be acquired past 1630 to complete a configuration. 


\begin{tabular}{|c|c|c|c|c|c|c|c|c|c|c|c|c|c|}
\hline ACTINTY & $+\infty$ & 0700 & Toen & 0000 & 1000 & 1100 & 1200 & 1300 & 1400 & 1500 & 1000 & 11700 & RESPONSIBLE \\
\hline ОРЕн झ्ञTE & $\star$ & & & & & & & & & & & & FACHmES \\
\hline monumo mern & & & & & & & & & & & & & ace : \\
\hline CONAOUnE ETR & & & & & & & & & & & & & EACILTIES \\
\hline CONFGURE IST & & & . & & & & & & & & & & mese \\
\hline INSTRUMEMT & & & & & & & & . & & & & & INSTRUMENTATION \\
\hline PREPANE DAS & & & & & & & & & & & & & Data aco \\
\hline ACOURAE DATA & & 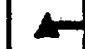 & & & & & & & & & 7 & & |OATAMCOANST' \\
\hline LUNCH & & & & & & & & & & & & & ale \\
\hline paocess onta & : & & & & & & & & & & L & 7 & OATa Aco \\
\hline SEcunt site & & & & & & & & & & & 2 & $\boldsymbol{T}$ & FACILTESS \\
\hline SEcuAn TST & & & & & & & & & & & 4 & $\boldsymbol{\nabla}$. & mese \\
\hline CLOSE ETE & & & & & & & & & & & & $\star$ & FACUTIES \\
\hline
\end{tabular}

Figure 13. NASA B-757 Dally Teat Schedule. 


\subsection{PRE-TEST ACTIVITIES}

\subsection{DETAILED TEST PLANNING}

Reference 1, while a good general test plan for the NASA B-757 test, is not suffi-ciently detailed to implement at the LESLI site. For example, it does not provide test point identification in a format that lends itself to entering in a CWDAS header, nor does it provide or contain all of the information required to properly prepare instrumentation setups. Therefore, a significant amount of detailed test planning was required by the Phillips Laboratory before the arrival of the aircraft. This section of the report describes those activities.

\subsubsection{CW Sweep Designation Definitions}

Reference 1 requires 15 separate and distinct $C W$ sweep types which vary the $F_{M \mathbb{N}}, F_{M A X}$, and $\mathrm{Q}$ (number of points) parameters. Previous tests at LESLI have used the sweep designations $A, B, C$, and D in the CWDAS header to represent the $300 \mathrm{kHz}$ to $100 \mathrm{MHz}$, $100 \mathrm{MHz}$ to $1 \mathrm{GHz}, 1 \mathrm{GHz}$ to $2 \mathrm{GHz}$, and $2 \mathrm{GHz}$ to $3 \mathrm{GHz}$ amplifiers, respectively. Since there is another field in the header for amplifier identification, this procedure has always been redundant. Sweep designations for the B-757 test were determined by the sweep parameters. Since the sweep number appears as half the data file name, a data reviewer can immediately determine which parameters were used. The value of " $Q$ " was determined for each sweep required by the test plan and Table 2-1 was prepared for use throughout the test. Sweep designations "A" and "B" are the normal LESLI sweeps, sweep designations " $\mathrm{J}$ " and " $\mathrm{K}$ " represent dwelling at $25.85 \mathrm{MHz}$ and $172 \mathrm{MHz}$, respectively, and sweep designation " $\mathrm{L}$ " is the pulse at $430 \mathrm{MHz}$. 
Table 2-1. Sweep Designation Definitions.

\begin{tabular}{|c|c|c|c|c|c|}
\hline $\begin{array}{c}\text { SWEEP } \\
\text { DESIGNATION }\end{array}$ & $F_{\text {MIN }}$ & $F_{\text {max }}$ & $\begin{array}{c}\text { No. } \\
\text { POINTS }\end{array}$ & $\bar{Q}$ & COMMENTS \\
\hline$A$ & $300 \mathrm{kHz}$ & $100 \mathrm{MHz}$ & 932 & 60 & $\begin{array}{l}\text { NORMAL RHOMBIC } \\
\text { SWEEP }\end{array}$ \\
\hline $\bar{B}$ & $100 \mathrm{MHz}$ & $1000 \mathrm{MHz}$ & 413 & $\overline{82}$ & $\begin{array}{l}\text { NORMAL RHOMBIC } \\
\text { SWEEPP }\end{array}$ \\
\hline $\bar{C}$ & $23 \mathrm{MHz}$ & $28 \mathrm{MHz}$ & 1000 & 2502 & DIPOLE SWEEP \\
\hline $\bar{D}$ & $25.7 \mathrm{MHz}$ & $26 \mathrm{MHz}$ & 1000 & 5580 & DIPOLE SWEEP \\
\hline $\bar{E}$ & $150 \mathrm{MHz}$ & $200 \mathrm{MHz}$ & 1000 & 1595 & DIPOLE SWEEP \\
\hline $\bar{F}$ & $171 \mathrm{MHz}$ & $173 \mathrm{MHz}$ & 1000 & 42700 & DIPOLE SWEEP \\
\hline$\overline{\mathbf{G}}$ & $350 \mathrm{MHz}$ & $500 \mathrm{MHz}$ & 1000 & 1282 & DIPOLE SWEEP \\
\hline$\overline{\mathrm{H}}$ & $427 \mathrm{MHz}$ & $432 \mathrm{MHz}$ & 1000 & 42650 & DIPOLE SWEEP \\
\hline $\mathrm{J}$ & $25.85 \mathrm{MHz}$ & $25.85 \mathrm{MHz}$ & 1 & N/A & RHOMBIC DWELL \\
\hline $\bar{K}$ & $172 \mathrm{MHz}$ & $172 \mathrm{MHz}$ & 1 & N/A & RHOMBIC DWELL \\
\hline $\bar{L}$ & $430 \mathrm{MHz}$ & $430 \mathrm{MHz}$ & 1 & N/A & RHOMBIC PULSE \\
\hline $\bar{M}$ & $950 \mathrm{MHz}$ & $959 \mathrm{MHz}$ & 1000 & 52700 & RHOMBIC SWEEP \\
\hline $\bar{N}$ & $2 \mathrm{MHz}$ & $30 \mathrm{MHz}$ & 250 & 35 & RHOMBIC SWEEP DIFF. \\
\hline$\overline{\mathbf{P}}$ & $1 \mathrm{MHz}$ & $100 \mathrm{MHz}$ & 2 & 50 & GROUND PLANE CHAR. \\
\hline $\bar{Q}$ & $100 \mathrm{MHz}$ & $1 \mathrm{GHz}$ & 18 & 100 & GROUND PLANE CHAR. \\
\hline
\end{tabular}

\subsubsection{Field Map Point Identification}

Two field map efforts were required; a map of the common mode rhombic antenna had been included in the original plan, and field mapping of the dipole antennas was later levied as an additional task.

Table 7.6-1 of Reference 1 identifies field map locations for the rhombic antenna. Locations were defined according to the LESLI coordinate system in which the " $\mathrm{X}$ " direction is along the axis of the antenna, " $\mathrm{Y}$ " is up, and " $\mathrm{Z}$ " is to the right when facing the antenna termination from the source. The associated text provided that the antenna be swept from 300 $\mathrm{kHz}$ to $1 \mathrm{GHz}$. Table 2-2 was created during the detailed planning process 
to provide names for each of the points for data acquisition and processing purposes.

Table 2-2. Common Mode Rhombic Field Map Points.

\begin{tabular}{|c|c|c|c|c|c|}
\hline MAP POINT & $\bar{x}$ & $\bar{Y}$ & $\mathbf{Z}$ & COMMENTS & ACQUIRED \\
\hline 7FMA & 40 & 5 & -2 & ACD-4; THREE COMPONENTS & $9 / 12 / 94$ \\
\hline 7FMB & 40 & 1 & 0 & ACD-4; THREE COMPONENTS & $9 / 8 / 94$ \\
\hline 7FMC & 40 & 3 & 0 & ACD-4; THREE COMPONENTS & $9 / 7 / 94$ \\
\hline 7FMD & 40 & 5 & 0 & $\begin{array}{l}\text { ACD-4, MGL-6; THREE COMPONENTS } \\
\text { EACH }\end{array}$ & $9 / 8 / 94$ \\
\hline 7FME & 40 & 3 & 2 & ACD-4; THREE COMPONENTS & $9 / 7 / 94$ \\
\hline 7FMF & 40 & 5 & 2 & ACD-4; THREE COMPONENTS & $9 / 12 / 94$ \\
\hline 7FMG & 40 & 5 & 5 & $\begin{array}{l}\text { ACD-4, MGL-6; THREE COMPONENTS } \\
\text { EACH }\end{array}$ & $9 / 8 / 94$ \\
\hline 7FMH & 40 & 3 & 8 & ACD-4; THREE COMPONENTS & $9 / 7 / 94$ \\
\hline$\overline{7 F M J}$ & 40 & 5 & 8 & ACD-4; THREE COMPONENTS & $9 / 12 / 94$ \\
\hline 7FMK & 40 & 5 & 10 & $\begin{array}{c}\text { ACD-4, MGL-6; THREE COMPONENTS } \\
\text { EACH }\end{array}$ & $9 / 9 / 94$ \\
\hline 7FML & 40 & 5 & 12 & ACD-4; THREE COMPONENTS & 9/9/94 \\
\hline 7FMM & 40 & 3 & 15 & ACD-4; THREE COMPONENTS & $9 / 6 / 94$ \\
\hline 7FMN & 40 & 5 & 15 & ACD-4; THREE COMPONENTS & 9/9/94 \\
\hline 7FMP & 50 & 3 & $\overline{0}$ & ACD-4; THREE COMPONENTS & $9 / 1 / 94$ \\
\hline 7FMQ & 50 & 3 & 5 & ACD-4; THREE COMPONENTS & $9 / 1 / 94$ \\
\hline
\end{tabular}

The dipole field map locations are contained in Table 7.6-2 of Reference 1. The coordinate system for this effort was with respect to the dipole antennas. In a system similar to the Rhombic, the positive " $\mathrm{X}$ " direction was in a line perpendicular to the dipole when the dipole was in the horizontal orientation. The " $\mathrm{Y}$ " direction was up, and " $\mathrm{Z}$ " to the right when looking from the antenna in a positive " $\mathrm{X}$ " direction. Table 2-3 was developed to name each of the points. As can be seen in the table, only the center frequency was provided in Reference 1. After bandwidth experimentation during antenna checkout, and through informal liaison 
with LLNL, it was decided to field map the dipoles over the same frequency ranges used for aircraft tests, sweep designations C, E, and G from Table $2-1$.

Table 2-3. Dipole Field Map Points.

\begin{tabular}{|c|c|c|c|c|c|c|c|}
\hline $\begin{array}{l}\text { MAP } \\
\text { POINT }\end{array}$ & $\begin{array}{l}\text { FREQ. } \\
\mathrm{MHz}\end{array}$ & POL & $\bar{x}$ & $\mathbf{Y}$ & $\bar{Z}$ & COMMENTS & ACQUIRED \\
\hline DFMA & 25 & $\mathbf{V}$ & 2 & 4 & $\overline{0}$ & $\begin{array}{l}\text { ACD-4, MGL-6; THREE } \\
\text { COMPONENTS EACH }\end{array}$ & $9 / 21 / 94$ \\
\hline DFMB & 25 & $\overline{\mathbf{V}}$ & 8 & 4 & 0 & ACD 4 ; THREE COMPONENTS & $9 / 21 / 94$ \\
\hline DFMC & 25 & $\bar{V}$ & 8 & 2 & 0 & ACD-4; THREE COMPONENTS & $9 / 21 / 94$ \\
\hline DFMD & 25 & $\overline{\mathrm{H}}$ & 2 & 3 & 0 & $\begin{array}{l}\text { ACD 4, MGL-6; THREE } \\
\text { COMPONENTS EACH }\end{array}$ & $9 / 20 / 94$ \\
\hline DFME & $\overline{25}$ & $\bar{H}$ & 8 & 3 & $\mathbf{0}$ & ACD-4; THREE COMPONENTS & $9 / 20 / 94$ \\
\hline DFMF & 25 & $\mathbf{H}$ & 8 & 2 & $\mathbf{0}$ & ACD-4; THREE COMPONENTS & $9 / 20 / 94$ \\
\hline DFMG & 172 & $\overline{\mathbf{V}}$ & $\overline{2}$ & 3 & 0 & $\begin{array}{l}\text { ACD-4, MGL-6; THREE } \\
\text { COMPONENTS EACH }\end{array}$ & $10 / 21 / 94$ \\
\hline DFMH & 172 & $\overline{\mathbf{V}}$ & 8 & 3 & 0 & ACD4; THREE COMPONENTS & $10 / 21 / 94$ \\
\hline DFMN & 172 & $\bar{H}$ & 2 & 3 & 0 & $\begin{array}{l}\text { ACD -4, MGL-6; THREE } \\
\text { COMPONENTS EACH }\end{array}$ & $10 / 20 / 94$ \\
\hline DFMK & $\overline{172}$ & $\bar{H}$ & $\overline{8}$ & 3 & $\mathbf{0}$ & ACD-4; THREE COMPONENTS & $10 / 21 / 94$ \\
\hline$\overline{D F M L}$ & 430 & $\bar{v}$ & 2 & 3 & 0 & $\begin{array}{l}\text { ACD4, MGL-6; THREE } \\
\text { COMPONENTS EACH }\end{array}$ & $10 / 20 / 94$ \\
\hline DFMM & 430 & $\bar{V}$ & 8 & 3 & $\overline{0}$ & ACD-4; THREE COMPONENTS & $10 / 20 / 94$ \\
\hline DFMN & 430 & $\bar{H}$ & 2 & 3 & $\overline{0}$ & $\begin{array}{l}\text { ACD-4, MGL-6; THREE } \\
\text { COMPONENTS EACH }\end{array}$ & $10 / 20 / 94$ \\
\hline DFMP & 430 & $\bar{H}$ & 8 & 3 & 0 & ACD-4; THREE COMPONENTS & $10 / 20 / 94$ \\
\hline$\overline{\text { DFMQ }}$ & 25 & $\bar{H}$ & 8 & 3 & 6 & ACD-4; THREE COMPONENTS & $9 / 21 / 94$ \\
\hline DFMR & $\overline{25}$ & $\bar{H}$ & 8 & 3 & -6 & ACD-4; THREE COMPONENTS & $9 / 21 / 94$ \\
\hline DFMS & 172 & $\bar{H}$ & 8 & 3 & $6-$ & ACD-4; THREE COMPONENTS - & $10 / 21 / 94$ \\
\hline$\overline{\text { DFMT }}$ & 172 & $\mathbf{H}$ & 8 & 3 & -6 & ACD-4; THREE COMPONENTS & $10 / 20 / 94$ \\
\hline DFMU & 430 & $\bar{H}$ & 8 & 3 & 6 & ACD-4; THREE COMPONENTS & $10 / 20 / 94$ \\
\hline DFMW & 430 & $\mathbf{H}$ & 8 & 3 & -6 & ACD-4; THREE COMPONENTS & $10 / 20 / 94$ \\
\hline
\end{tabular}




\subsubsection{Instrumentation Planning}

Twelve basic test points were created in the NASA B-757 and data on these points were taken in many different orientations and configurations. A cable from each point was routed to the internal instrumentation console located in the forward, starboard side of the first class compartment. Each cable could be easily discon-nected from the internal instrumentation and connected to an external system.

The original plan called for the PL to provide three multi-input (four), high frequency, NanoFast fiber optic links for routing data to the LESLI CW data acquisition system. Each point could be permanently instrumented, requiring re-instrumentation only to change batteries or configuration and, most importantly, ensuring the test point maintained the same instrumentation setup.

As it turned out, only two links were available when the test started, so a plan had to be devised to maximize link efficiency and to minimize the number of times re-instrumentation would be required. Appendix $\mathrm{C}$ of Reference 1 contains the B-757 on-the-ground test matrix and includes all of the required variations. After a review of Appendix $C$, it was apparent the Test Series J was the key for instrumentation. This test series was to be taken in the nose-on configuration under the common mode rhombic antenna and included many variations on a few key points.

Table 2-4 was developed as the heart of the instrumentation plan. As can be seen in the table, each cockpit and E-bay point was instrumented simultaneously for baseline and variation data acquisition; therefore, only two points had to be changed on each transmitter to effect a complete reinstrumentation. This scheme also permitted acquisition of variation data in the cabin without additional changes to the setup. 
Table 2-4. Test Point/Transmitter Selection.

\begin{tabular}{|c|c|c|c|c|c|}
\hline TEST POINT & LOCATION & CABLE DESIG. & $\begin{array}{l}\text { PROBE } \\
\text { SENSOR }\end{array}$ & $\begin{array}{l}\text { XMTR } \\
\text { INPUT }\end{array}$ & COMMENTS \\
\hline CP1 & COCKPIT & T1C3 & AD60 & NF50 & \\
\hline CP2 & COCKPIT & T1C1 & AD60 & NF20 & \\
\hline$\overline{C P 3}$ & COCKPIT & T1C2 & $\overline{A D 60}$ & NF52 & \\
\hline$\overline{C P 4}$ & COCKPIT & T1C4 & P132 & NF53 & \\
\hline$\overline{\text { CP5 }}$ & COCKPIT & T2C1 & APRB & NF51 & \\
\hline CP12 & COCKPIT & T1C4 & P132 & NF53 & $\begin{array}{l}\text { MOVE PROBE FOR TWO } \\
\text { MEASUREMENTS }\end{array}$ \\
\hline EB1 & E-BAY & T2C2 & AD60 & NF21 & \\
\hline EB2 & E-BAY & T2C4 & $\overline{\text { APRB }}$ & NF22 & \\
\hline$\overline{\text { EB3 }}$ & E-BAY & $\overline{T 2 C 3}$ & P134 & NF23 & \\
\hline $\mathrm{CI}$ & CABIN & $\mathrm{T} 3 \mathrm{C2}$ & AD60 & NF50 & $\begin{array}{l}\text { AFTER COCKPIT } \\
\text { MEASUREMENTS }\end{array}$ \\
\hline$\overline{C 2}$ & CABIN & T3C4 & P133 & NF51 & $\begin{array}{l}\text { AFTER COCKPIT } \\
\text { MEASUREMENTS }\end{array}$ \\
\hline C3 & CABIN & T3C3 & $\overline{A P R B}$ & NF20 & $\begin{array}{l}\text { AFTER COCKPIT } \\
\text { MEASUREMENTS }\end{array}$ \\
\hline $\mathrm{C4}_{4}$ & $\overline{\text { CABIN }}$ & T3C1 & APRB & NF21 & $\begin{array}{c}\text { AFTER E-BAY } \\
\text { MEASUREMENTS }\end{array}$ \\
\hline
\end{tabular}

\subsection{DIPOLE ANTENNA FABRICATION AND CHECKOUT}

\subsubsection{Dipole Antenna Fabrication}

Reference 1 required three dipole antennas, having center frequencies of $25.85 \mathrm{MHz}, 172 \mathrm{MHz}$, and $430 \mathrm{MHz}$ respectively, to be mounted three meters above the ground in both horizontal and vertical orientations for excitation of the aircraft. A new support structure was constructed for the effort. It was designed as a trun-cated pyramid with instrumentation platforms at one meter intervals ranging from one meter to five meters above the ground. The triangular-shaped base was chosen to provide stability on the cambered surface of the concrete LESLI test pad. The pyramid shape was also chosen to enhance stability and to make it easier for personnel to instrument on each platform (one can lean into the structure while performing tasks on one of the higher platforms). The structure members were built from untreated redwood and each platform was made of exterior 
grade plywood. A fiberglass ladder was provided for personnel access. The stand was mounted on locking wheels to ease moving about the test facility. Figure 2-1 is a drawing of the new test stand.

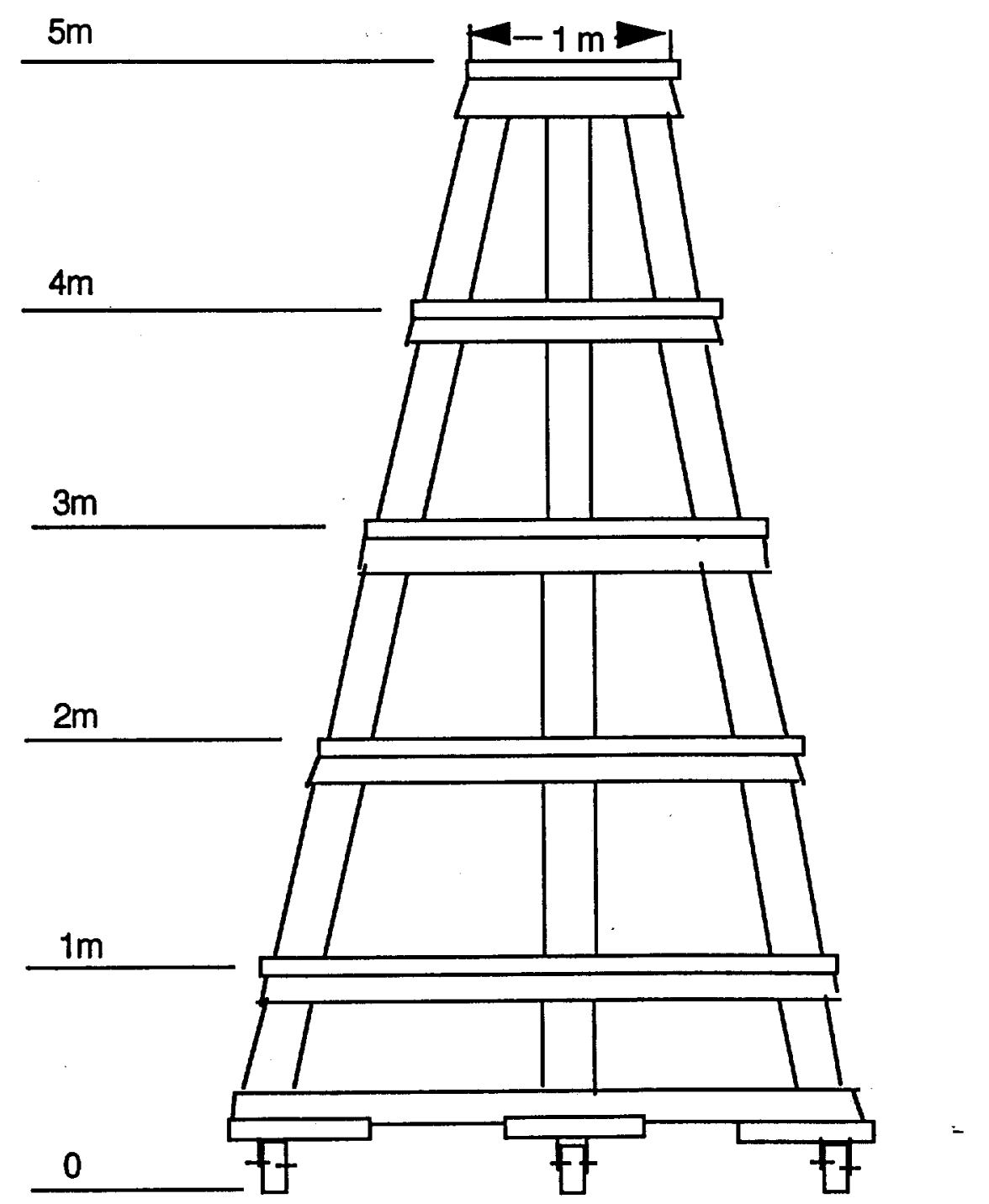

Figure 2-1 LESLI Five-Meter Test Stand

Preliminary test planning called for the PL to fabricate the three dipole antennas using baluns provided by LLNL. As the $172 \mathrm{MHz}$ and $430 \mathrm{MHz}$ antennas were relatively short, LLNL fabricated them in Livermore, with appropriate balun interfaces and mounting structures, and delivered them to the LESLI site. The $25.85 \mathrm{MHz}$ antenna, almost 20 feet in length, required a bit of design effort, including the basic antenna material which 
had to interface with the provided balun, and a robust support to keep it steady in the wind, avoid damaging the balun, and suspend it as far away from the test stand as practical. Ten foot lengths of one-half inch copper pipe were chosen as the antenna material because it is light in weight, yet stiff enough to minimize bending and sagging.

Figure 2-2 is a sketch of the antenna mounting which was designed and fabricated. An 8' 4" by 4" fiberglass " $\mathrm{I}$ " beam provided the main support. Attachment to the test stand was made by means of $2 " \mathrm{X}$ 4" wooden clamps

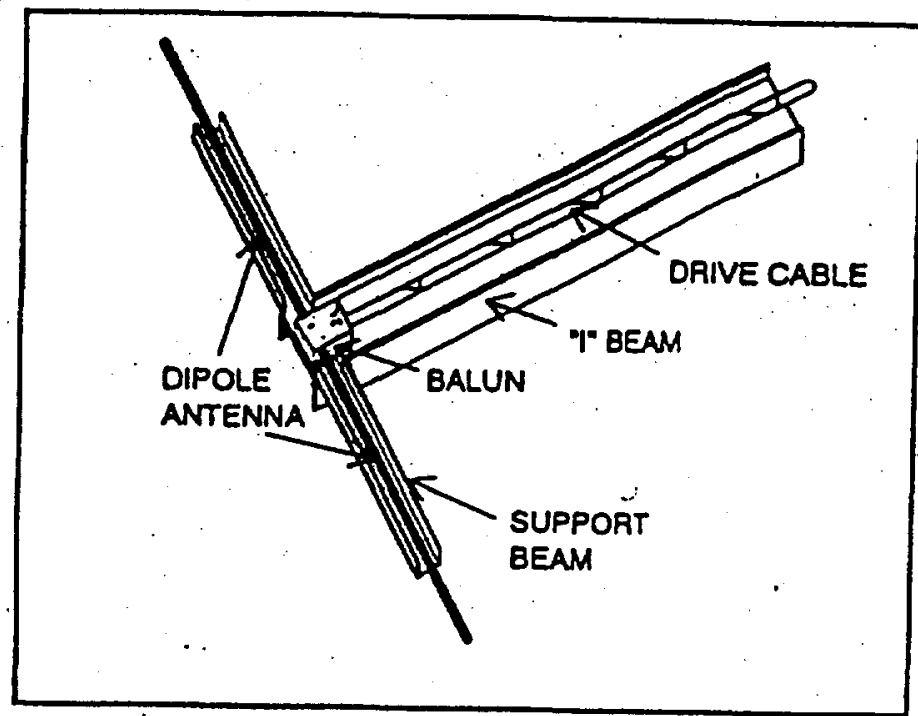

Figure 2-2. Dipole Antenna Mount which secured the . beam when the antenna was in either the horizontal or vertical orientation. An eight foot length of fiberglass " $U$ " channel support beam was fixed to the " $I$ " beam with a rabbit joint and a cutout was provided to accommodate the balun. The "I" beam, support beam, and balun were all bolted together with $1 / 4 \times 20$ machine screws. The balun came equipped with a standard " $N$ " connector for attachment of the drive cable and $6 \mathrm{~mm} \times 100$ metric studs for antenna leg attachment. Two short pieces of half-inch brass round stock were acquired and drilled and tapped for a $6 \mathrm{~mm} \mathrm{X} 100$ thread. They were soldered in one end of each $10^{\prime}$ copper pipe. In this way, a smooth interface was provided by threading each leg of the antenna directly onto the balun.

Additional antenna support, not shown in the sketch, was provided by placing styrofoam spacers between the antenna legs and support beam to keep from applying torque to the balun studs and by stiffening the whole cross member with nylon tie wraps around the 
support beam and antenna legs in several locations. When the antenna was mounted in the horizontal orientation, lengths of parachute cord were tied from the ends of the support beam to the test stand to eliminate sag and torque on the balun studs.

This system worked well in practice. It required two people to mount the antenna on the test stand but, once the " $\mathrm{I}$ " beam was partially clamped, it was possible to easily and precisely position the antenna over the desired spot on the ground. Since the baluns for the other two antennas had the same mounting hole arrangement, their mounting hardware was removed and the "I" beam, without the support beam, was also used to mount them.

\subsubsection{Dipole Antenna Checkout}

\subsubsection{High Frequency Antennas}

The initial checkout of the 172

$\mathrm{MHz}$ and $430 \mathrm{MHz}$ antennas

was made to verify the performance of the antennas

when installed on the test stand and to determine if the antennas could be driven at 200 watts from twenty percent below to twenty percent above the center frequency without VSWR difficulties. Figure $2-3$ is the instrumentation setup which

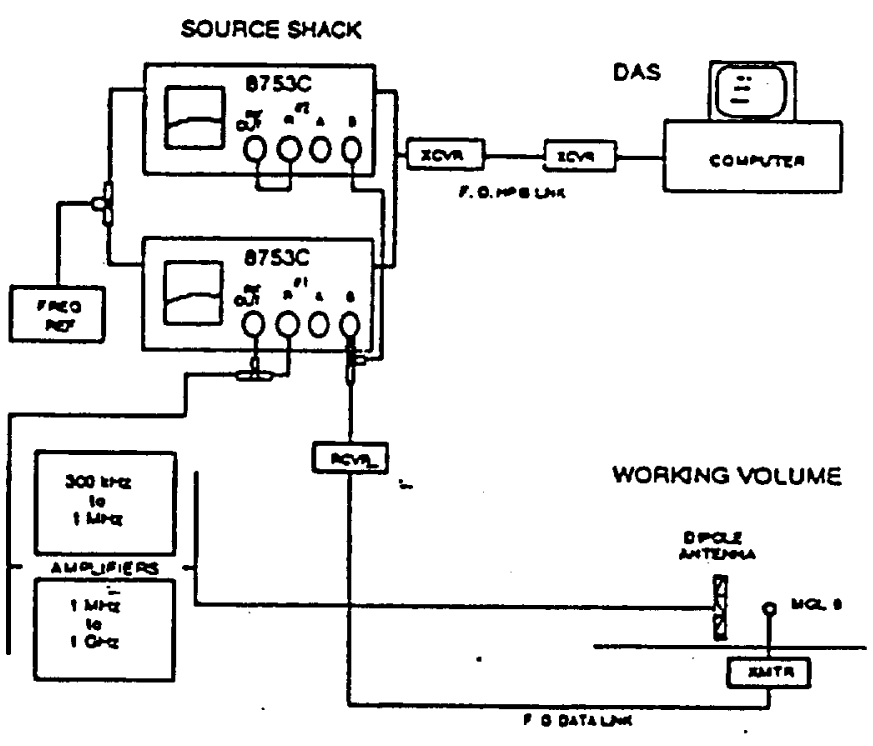

Figure 2-3. Checkout Instrumentation Setup was used for the checkout. Each antenna, in turn, was mounted on the one-meter platform of the test stand in a horizontal orientation. An MGL-6, B-dot, sensor was mounted on the opposite side of the platform about 1.5 meters away from the antenna and oriented for the primary magnetic field. The drive cable from the high frequency amplifier in the Source Shack was connected to the antenna and the MGL-6 was connected to a high fre- 
quency NanoFast fiber optic link which transferred the sensor response data to both network analyzers in the Source Shack, and then to the DAS via a fiber optic HPIB link. The checkout of each antenna was performed in three basic steps:

1. The high frequency amplifier was driven at $-6 \mathrm{dBm}(9 \mathrm{~dB}$ below that required for a match with the low frequency amplifier when it has a 200 watt output) with a " $Q$ " resulting in about 100 points in a sweep from a frequency ten percent below the center frequency to a frequency ten percent above the center frequency of the antenna. The data were reviewed in the DAS for reasonability and to verify agreement between the two network analyzers in the Source Shack.

2. The amplifier was driven at $-6 \mathrm{dBm}$ with a " $Q$ " resulting in about 200 points from a frequency twenty percent below the center frequency to a frequency twenty percent above the center frequency of the antenna. The data were reviewed in the DAS to verify that \pm 20 percent was the useable bandwidth of the antenna.

3. The amplifier was driven at $3 \mathrm{dBm}$ with a " $Q$ " resulting in 1000 points over the \pm 20 percent bandwidth of the antenna. The data were reviewed in the DAS to verify amplifier performance and saved and processed for later review by LLNL.

An LLNL representative was on site for the antenna checkout and requested that the antennas also be checked in the vertical orientation and that cross-polarization data be acquired as well. These data were acquired, saved, processed, and delivered to LLNL. 


\subsubsection{Tuning and Checkout of the $25 \mathrm{MHz}$ Antenna}

Paragraph 2.2.1 discusses fabrication of the $25.85 \mathrm{MHz}$ dipole antenna. It was necessary to tune the antenna following fabrication. The theoretical length of a half-wave dipole antenna at that frequency is a little over 19 feet. Each leg of the antenna was trimmed to 9' $9^{\prime \prime}$ and the antenna, with a total length of $19^{\prime} 11^{\prime \prime}$, was mounted on the three-meter platform of the test stand, extending away from it by about a meter in the horizontal orientation. An MGL-6 magnetic field sensor was mounted on the other side of the platform in the primary magnetic field orientation. The low frequency amplifier was driven at $-3 \mathrm{dBm}$ with a " $\mathrm{Q}$ " resulting in 1000 points from a frequency twenty percent below the center frequency to a frequency twenty percent above the center frequency of the antenna. The data were noisy and the amplifier drive was increased to the maximum, $4.5 \mathrm{dBm}$. The data, as reviewed in the DAS, had peaks around $22 \mathrm{MHz}$ and at about 26.75 and $28.75 \mathrm{MHz}$. Figure $2-4$ is the processed data for this sweep.

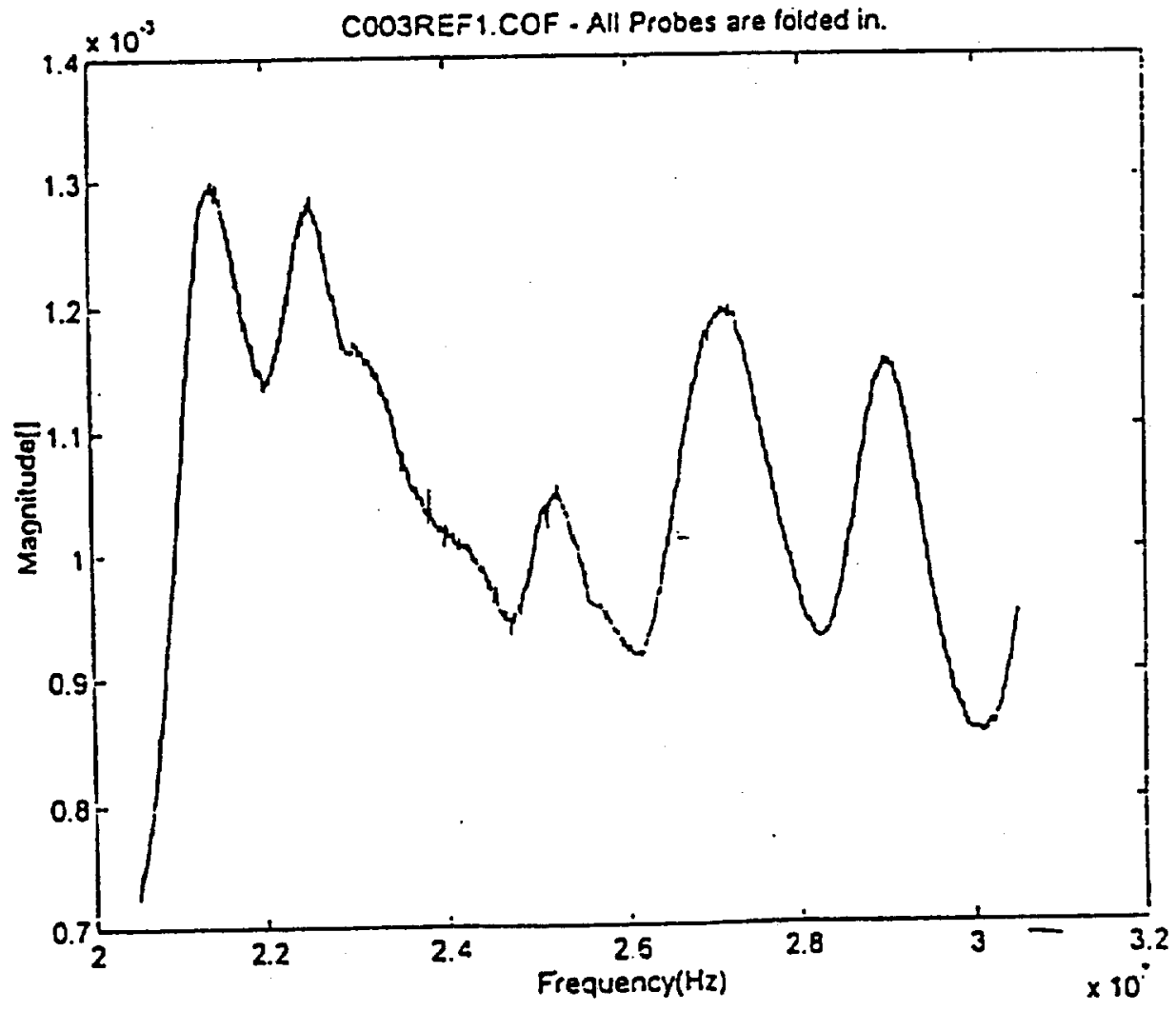

Figure 24. $25 \mathrm{MHx}$ Antenna Tuning, Initial Response. 
Three inches of copper pipe were trimmed from each leg of the antenna and the sweep was repeated. There was, as observed in the DAS, some gain above $23 \mathrm{MHz}$, but the basic structure of the antenna output was the same. Two more inches were trimmed from each end of the antenna, resulting in a total antenna length of $19^{\prime} 1 "$. Another sweep was taken. This time, although a slight gain above $23 \mathrm{MHz}$ was observed, the lower end of the response was going down. Since no LLNL representative was on site to review the data, it was decided to cease trimming the antenna at this point until the data could be reviewed. Subsequently, LLNL reviewed the data and concurred that the final trimming had produced the optimum antenna/balun combination. Figure 2-5 is the processed data.

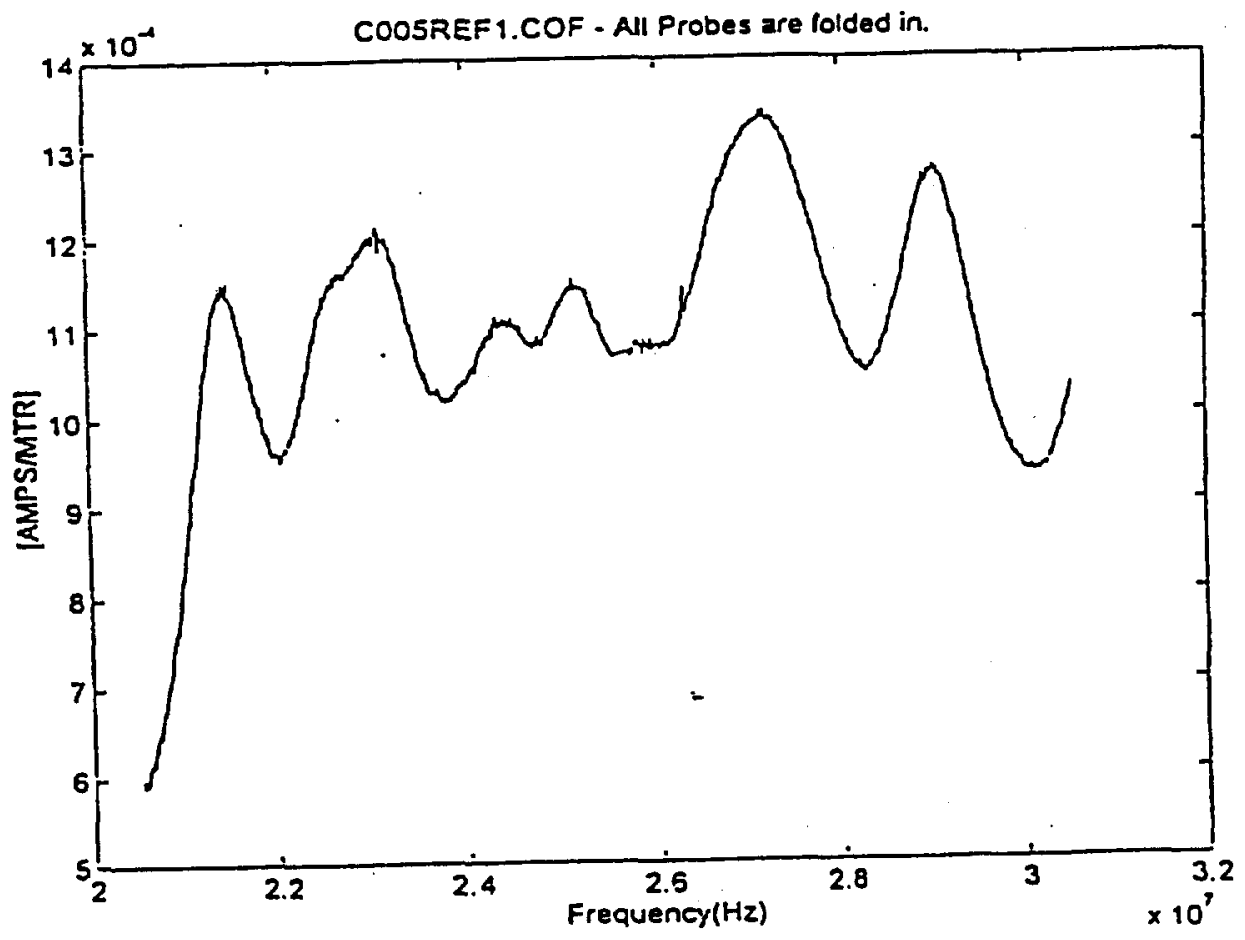

Figure 2-5. 25 $\mathrm{MH}_{2}$ Antenna Tuning, Final Response.

\subsection{FAA COMPATIBILITY CHECKOUT}

A unique aspect of the NASA B-757 test program was a requirement to transmit $430 \mathrm{MHz}$ $\mathrm{CW}$ in a pulsed mode from the rhombic antenna to simulate a radar against which the aircraft will be flown. LLNL provided an HP8665A signal generator to provide a 430 MHz source and an HP8112A pulse generator to convert the source into a pulsed mode. 
The output of the pulse generator drove the PL high frequency amplifier into the common mode rhombic antenna.

An obvious concern with this technique was that harmonics of $430 \mathrm{MHz}$ would be produced during the pulse generation process which would be amplified and were likely to be in skip bands imposed by the FAA. Accordingly, an experiment was devised to investigate possible FAA interference. Figure 2-6 is the instrumentation setup which was used for the experiment. The HP8665A and the HP8112A were configured in the Source Shack along with a DSA 602 oscilloscope to monitor the response of the LESLI reference sensor. A Tektronics 2712 spectrum analyzer was first used in the Source Shack and then set

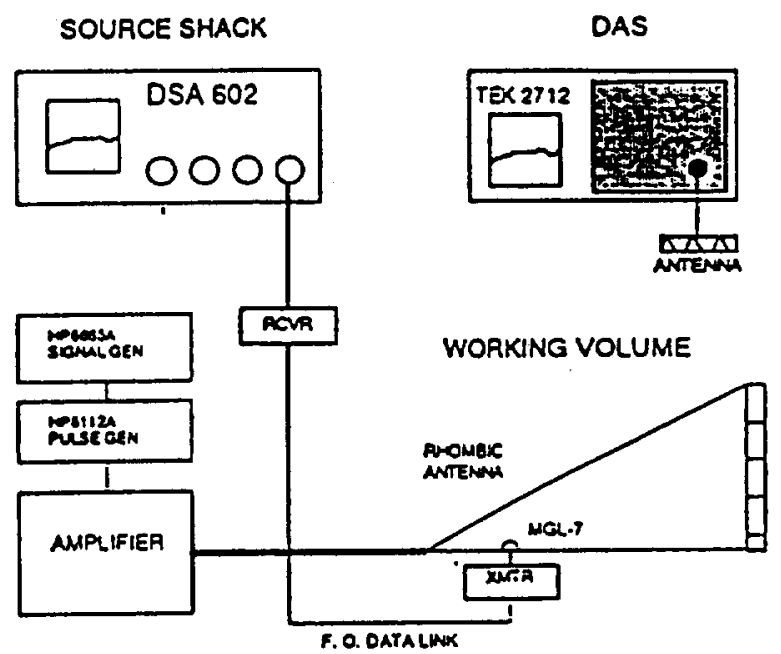

Figure 2.6. FAA Check Instrumentation up in the DAS to monitor the output of the rhombic antenna. The experiment was conducted in three steps.

1. First, the output of the amplifier was input to the spectrum analyzer through $60 \mathrm{~dB}$ of attenuation to verify proper operation and check the level of produced harmonics. The third harmonic at $1290 \mathrm{MHz}$ was $36 \mathrm{~dB}$ below the primary frequency; this equates to about $13 \mathrm{~mW}$ into the antenna when radiating. This harmonic was close the airport TACAN frequency and close attention was paid to it during the remainder of the experiment.

2. The second procedure drove the amplifier into the rhombic antenna, starting at $-6 \mathrm{dBm}$ into the amplifier and increasing the amplifier input in $3 \mathrm{~dB}$ steps until $3 \mathrm{dBm}$ was achieved. The LESLI reference sensor and rhombic antenna spectrum were monitored during this process. No harmonics were detected by the spectrum analyzer at any power level. 
3. Finally, a series of pulse trains, thirty seconds in duration, was made every ten minutes for one hour. It was thought that the actual test procedure would be similar when the onboard instrumentation was being checked in this mode.

The FAA reported no interference during the compatibility check, and it was decided to conduct the onboard experiment at the appropriate time.

\subsection{DIPOLE ANTENNA FIELD MAPPING}

As can be seen in Figure 1-2, field mapping of the dipole antennas was conducted in two phases. The $25 \mathrm{MHz}$ antenna was mapped on 20 and 21 September, immediately following its tuning and checkout (described in Paragraph 2.2.2.2). The other two dipole antennas were mapped on 19 October through 21 October after the LESLI aircraft data acquisition and ground plane data acquisition activities had been completed and the B-757 had been moved to Pad 4 for cavity experiments.

Table 2-3 lists the dipole field map points and provides their locations with respect to the antenna. The table also includes the date on which data for each point were acquired. General measurement procedures are described in Paragraph 3.1, above. Except for the $25 \mathrm{MHz}$ antenna in the vertical orientation on the four-meter platform, the antennas were mounted on the three-meter platform about $1.5 \mathrm{~m}$ from the edge. The reference sensors were mounted behind the antennas on the platform. Their coordinates (Table 2-3) were $X$ $=-2.5, Y=3$, and $\mathrm{Z}=0$ on the three-meter platform and $\mathrm{X}=-2.5, \mathrm{Y}=4$, and $\mathrm{Z}=0$ on the four-meter platform.

\subsection{RHOMBIC ANTENNA FIELD MAPPING}

Field mapping of the rhombic antenna was conducted from 1 September through 12 September and was completed ahead of schedule. Table 2-2 lists the field map points and indicates the date on which each was acquired. Figure 2-7 is a scale drawing of the LESLI test pad with the field map points indicated. 


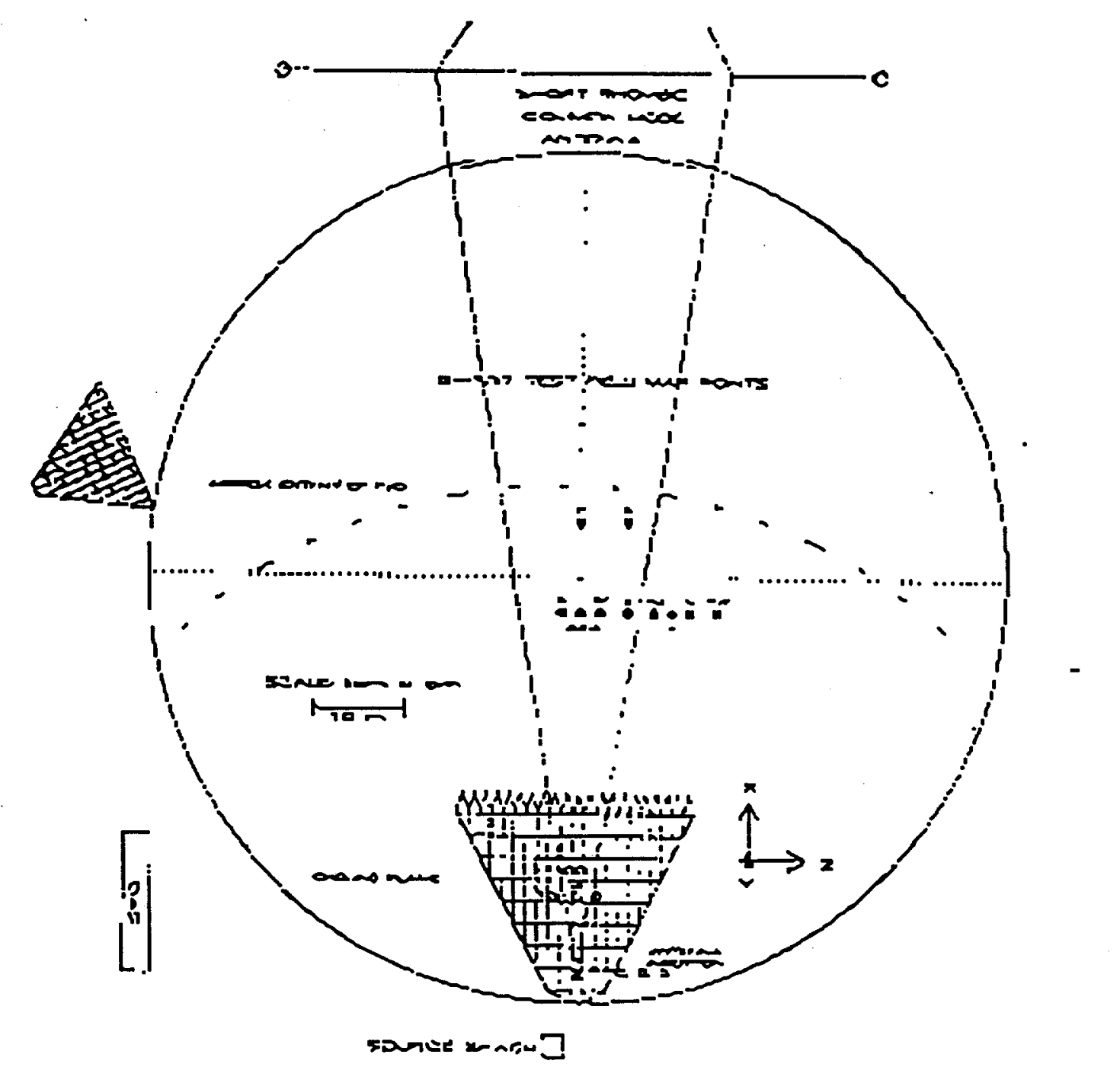

Figure 2.7. Short IRhowbje Anlarat Field Midy Pointh

Because all work with the dipoles was performed in the center of the test pad, a 55 meter foam-flex coaxial cable was used to drive the antennas. A shorter cable was used to drive the rhombic antenna. DAS QC observed that the reference data were bad above $600 \mathrm{MHz}$ on the first sweep of the field map effort with the high frequency amplifier. After an investigation and troubleshooting, it was determined that one of the drive cable connectors had a cracked solder joint. The connector was repaired and the high frequency data appeared to be correct. The low frequency response, however, exhibited a spike at about $65 \mathrm{MHz}$. The cause of the spike was never determined; since it appears in both the ground 
plane data from the MGL-7 reference sensor at 10CL and in the field data, it is deemed to be real. Figure 2-8 is a ten-sweep overlay of $10 \mathrm{CL}$ measurements.

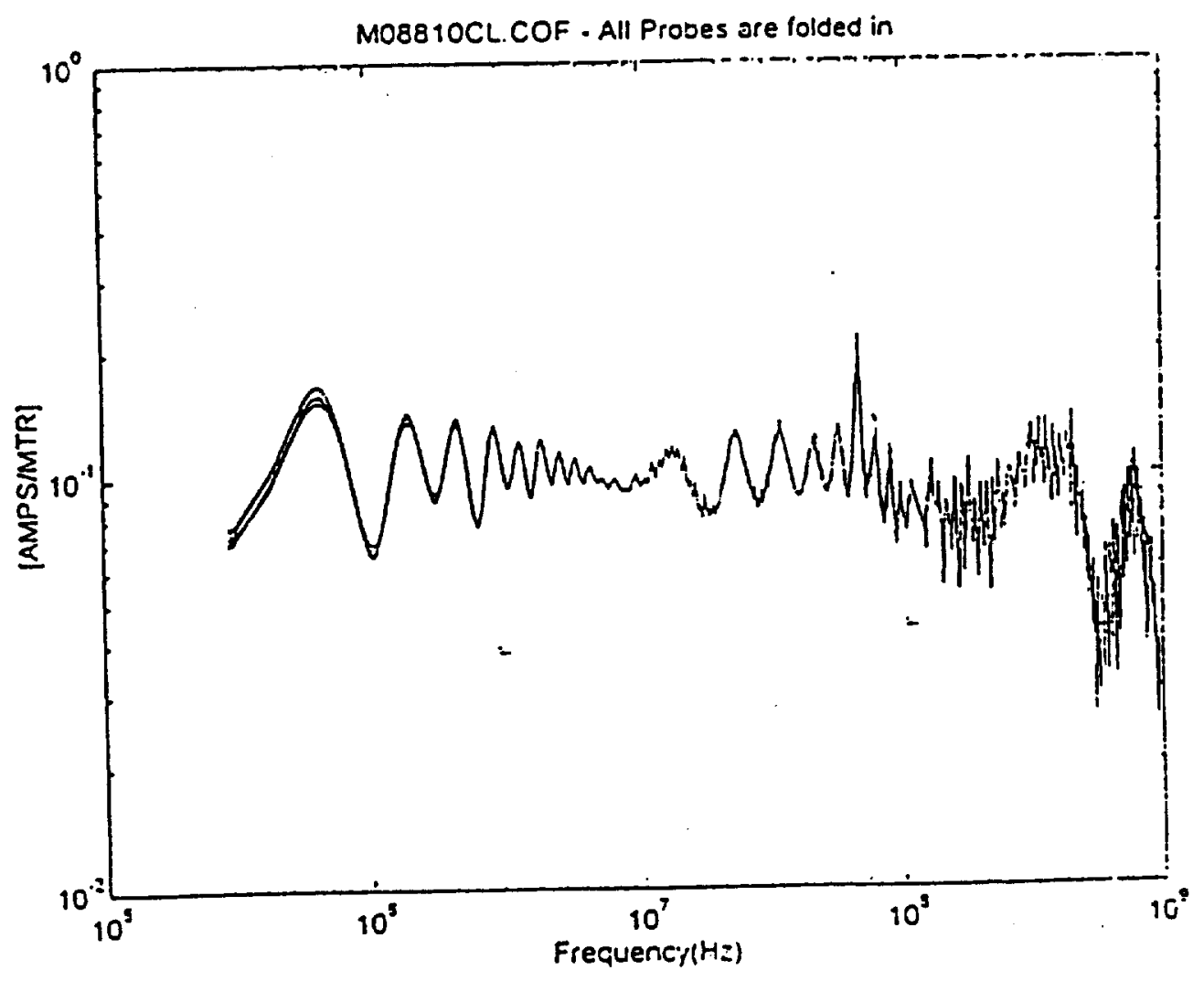

Figure 2-8. Ten Sweep Overlay of 10CL.

\subsection{AIRCRAFT INSTRUMENTATION}

The aircraft arrived on 22 September, was positioned in the nose-on orientation on the LESLI pad, and was prepared for testing. The NASA crew installed the sensor box in the cockpit, the Collins VHF radio in the E-Bay, and connected the external antenna to the instrumentation console in the cabin (see Figures 7.2.1 through 7.2.3 of Reference 1). The PL team routed the NanoFast fibers into the aircraft via an overpressure vent in the EBay and instrumented two fiber optic links. 
The CWDAS program was booted up and several sweeps taken without the amplifiers being energized to verify the instrumentation was operating properly.

Paragraph 7.5 of Reference 1 discusses the probes and sensors which were used throughout the B-757 test. Three of these were Prodyne I-320 current probes. These probes were removed from the aircraft and characterized in the DAS. The resultant .CAL files were put in the calibration library for future data processing and the probes reinstalled in the aircraft.

The final aspect of preparation was to move the test stand into place, rig the $25 \mathrm{MHz}$ antenna in the horizontal orientation, and position the reference sensor. 


\subsection{TEST ACTIVITIES}

\subsection{DIPOLE MEASUREMENTS}

Dipole measurement activity started ahead of schedule on 23 September and was completed on 29 September. Measurements included aircraft ambient noise and aircraft response data for each of the three antennas in both the horizontal and vertical orientations. The measurement matrix for the dipole tests is contained in Tables C-9 through C-15 of Appendix C to Reference 1.

\subsubsection{Configuration}

The B-757 was positioned on the LESLI pad in the nose-on orientation, with the nose 40 meters from the rhombic antenna insertion point, for the later rhombic antenna tests. The dipole antennas were located in relation to the aircraft as shown by the coordinate system in Figure 3-1. Since the dipole antennas were to be positioned in the modeling space for the modeling effort, it was necessary to have them

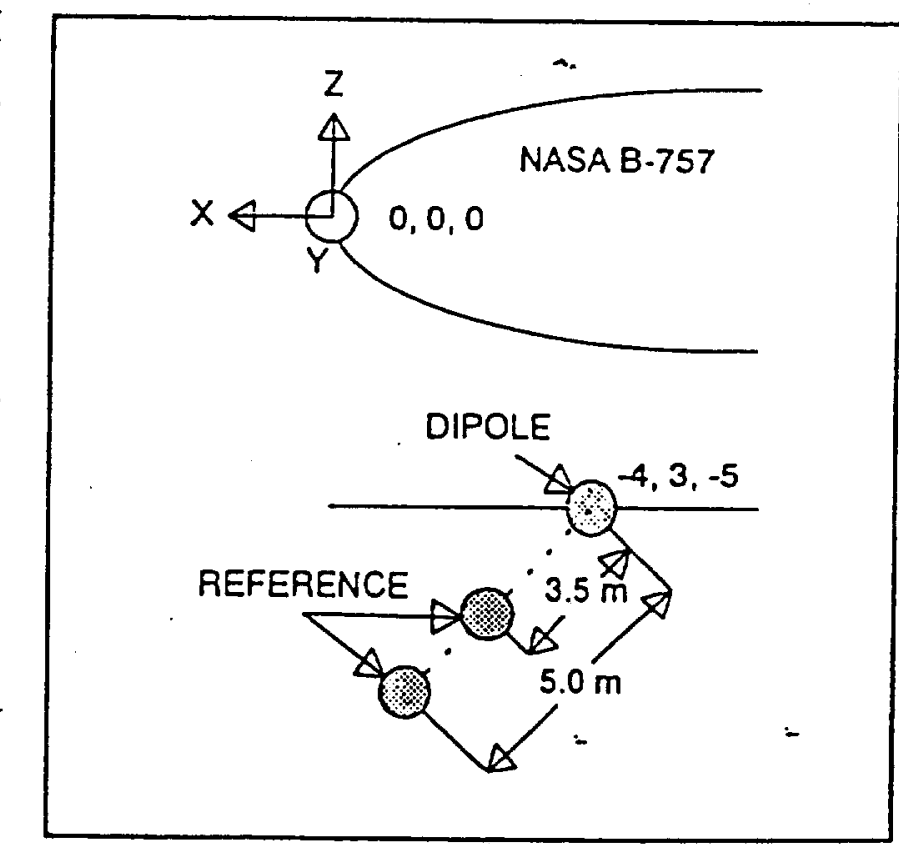

Figure 3-1. Dipole Antenna Position.

relatively close to the aircraft when data were acquired for comparison. The dipoles were placed five meters from the centerline of the aircraft and four meters back from the nose. With the exception of the 25 $\mathrm{MHz}$ antenna in the vertical orientation, the center of each antenna balun was three meters above the ground. The vertical $25 \mathrm{MHz}$ dipole was positioned four meters in the air to reduce ground effects on the lower leg. These antenna placements provided maximum 
coupling to the cockpit and E-bay with significant coupling to the cabin area. The external VHF antenna, located on the dorsal aircraft surface at the forward end of the cabin, received significant energy, as well.

The MGL- 6 reference sensor was instrumented on another test stand and positioned on a line forty-five degrees from a line parallel to the aircraft centerline as measured from the dipole antenna. The reference was 3.5 meters from the dipole for the $172 \mathrm{MHz}$ and 430 $\mathrm{MHz}$ antennas, and 5.0 meters away for the $25 \mathrm{MHz}$ antenna. The reference was positioned at a height of three meters in all cases.

\subsubsection{Measurement Procedures}

Figure 3-2 diagrams the instrumentation setup which was used for the dipole tests.

All the instrumentation, including NanoFast fiber optic link gain settings, was under computer control by the CWDAS program via a fiber optic HPIB link between the DAS and the Source Shack.

- $\quad$ Two HP8753C network analyzers were used to acquire

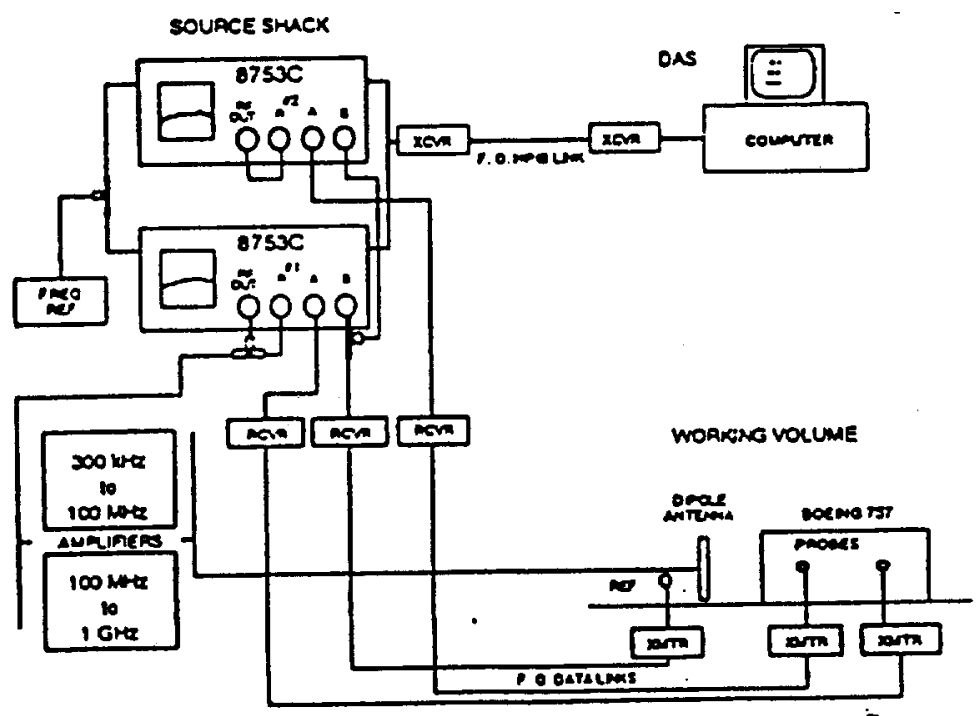

Figure 3-2. Dipole Instrumentation. two channels of data on one sweep. Network analyzer \#1 provided the drive to either the low frequency $(300 \mathrm{kHz}$ $100 \mathrm{MHz}$ ) amplifier or the high frequency (100 MHz $-1 \mathrm{GHz})$ amplifier which was under manual control by the Source Shack Operator. The network analyzers were synchronized by a $10 \mathrm{MHz}$ frequency reference. 
As discussed in Paragraph 2.6, two fiber optic links were installed in the aircraft. The inputs to each transmitter were instrumented as listed in Table 2-4; the fibers were routed to their receivers in the Source Shack and connected to the "A" input of either network analyzer \#1 or \#2.

A unique feature of this program was the treatment of the reference data. It was desired to take the ratio of the data to the reference and multiply it by the reference during processing to preserve phase information and eliminate sweep-to-sweep variations. The output of the reference NanoFast receiver was sent through a power divider into the " $B$ " input of each network analyzer to acquire ratio data on both channels. The resultant $6 \mathrm{~dB}$ loss through the divider was accounted for in the processing.

The DAS operator acquired data according to the procedures in Sections 4.0 and 9.0 of Reference 2. The data were processed in accordance with Paragraph 7.9.2 of Reference 1 and delivered to LLNL.

\subsubsection{Skip Bands}

Table 2-1 lists the frequency ranges which were used for the dipole tests. There was a requirement levied by the FAA and FCC that the dipole antennas not radiate over certain, specified frequency bands. These bands are termed "skip bands" and are listed in Reference 3. CWDAS was programmed to omit these frequencies when controlling

- network analyzer \#1. Within each range listed in Table 2-1, there were frequencies which were not transmitted.

\subsection{ON-BOARD INSTRUMENTATION CHECKOUT SUPPORT}

The rhombic antenna was rigged in the common mode on the afternoon of 29 September. Instrumentation noise measurements (measurements in which the probe or sensor is replaced by a fifty-ohm load) were taken for all of the installed data paths pursuant to 
Table 2-4 and Table C-16 of Reference 1. The data were processed, QC'd, and delivered to LLNL for review and use in their analyses.

Paragraphs 1.3, and 7.8 of Reference 1 indicate one of the test objectives was to validate performance of the onboard instrumentation in low-level steady state and pulsed CW environments to predict appropriate settings for the future fly-by test. Five days were scheduled for the experiment because the NASA team had not exercised their equipment under field conditions and had no pre-experiment predictions. This procedure had never been performed at LESLI either, so it was decided to do a dry run of the steady state aspect of the experiment while waiting for the LLNL representative to arrive and set up the pulsed mode.

This was a power-on experiment.

The aircraft remained in the nose-on orientation under the common mode rhombic antenna and, on the afternoon of 30 September, the aircraft was powered by means of its APU. Communications were established between the NASA operator in the aircraft and the Source Shack operator via handheld radios. The Test Director stationed himself in the DAS to monitor the spectrum analyzer and

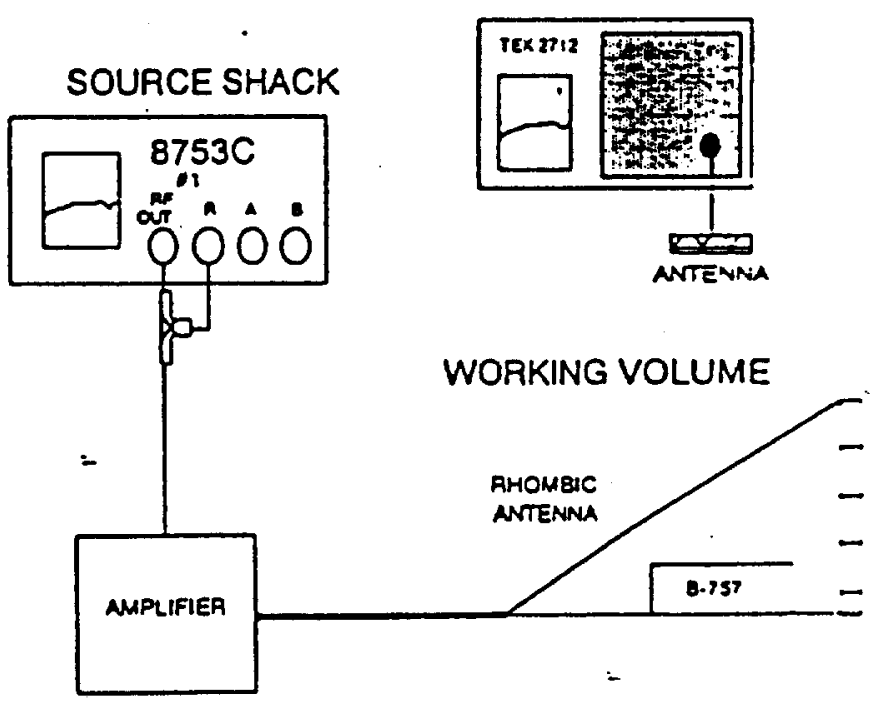

Figure 3-3. Onboard Instrumentation Check to communicate with the FAA, should that become necessary. The Source Shack operator put Network Analyzer \#1 on local control and set it up to output a $25.85 \mathrm{MHz}$ sine wave to the amplifier (Figure 3-3). The NASA operator got his system up and running and requested that the amplifier be turned on. The Source Shack operator turned on the amplifier for about thirty seconds, resulting in a steady state $25.85 \mathrm{MHz}$ output from the rhombic antenna which was monitored in the DAS. 
The NASA operator made adjustments to his equipment during the thirty second period. After a series of these antenna excitations, he was satisfied that his equipment was properly adjusted. The procedure was repeated for $172 \mathrm{MHz}$ and $430 \mathrm{MHz}$ with similar positive results.

Data were acquired for Test Series G (25.85 MHz) and Test Series H (172 MHz) of Reference 1 on the morning of 3 October. Since the experiments went so well and we were ahead of schedule, instrumentation noise measurements were also acquired at the two frequencies in addition to the test plan requirements.

The LLNL representative configured the pulse-mode instrumentation for $430 \mathrm{MHz}$ (see Figure 2-6). Communications were established between the aircraft and the Source Shack, as above. After a number of pulses were attempted, it was deter-mined that the pulse generator, with the 200W LESLI amplifier driving the rhombic antenna, did not provide adequate signal levels inside the aircraft. The ambient noise was so high that the NASA instrumentation triggered on the noise rather than on the $430 \mathrm{MHz}$ pulse. Though somewhat disappointing, this was a valuable result because it provided NASA with the opportunity to acquire band-pass filtering and/or additional amplification in their instrumentation suite and check it out before the fly-by test.

\subsection{COMMON MODE RHOMBIC MEASUREMENTS}

Measurements under the common mode rhombic antenna began on 3 October and were completed on 13 October. Measurements included all the tests listed in Tables C-20 and C-21 of Appendix $\mathrm{C}$ to Reference 1 and some experiments which were added as the data were reviewed and issues arose which had to be resolved. These included a repeat of the ten degree off-axis measurements (Tests J36, J37, and J38 of Table C-20 to Reference 1), a repeat of the E-Bay sealing experiment (Tests J26, J27, and J28 of Table C-20 to Reference 1), a repeat of the parasitic wire experiment (Tests K23 and K24 of Table C-21 to Reference 1), and a number of measurements to investigate the effects of configuration differences (see Paragraph 3.3.4). 


\subsubsection{Aircraft Orientations and Configurations}

The NASA test team was responsible for aircraft configuration and UIE positioned the B757 in its various orientations as directed by the test plan. Figure $3-4$ is a scale drawing of the LESLI pad with the B-757 in the nose-on orientation for the Test Series J measurements. The coordinate system is indicated in the figure.

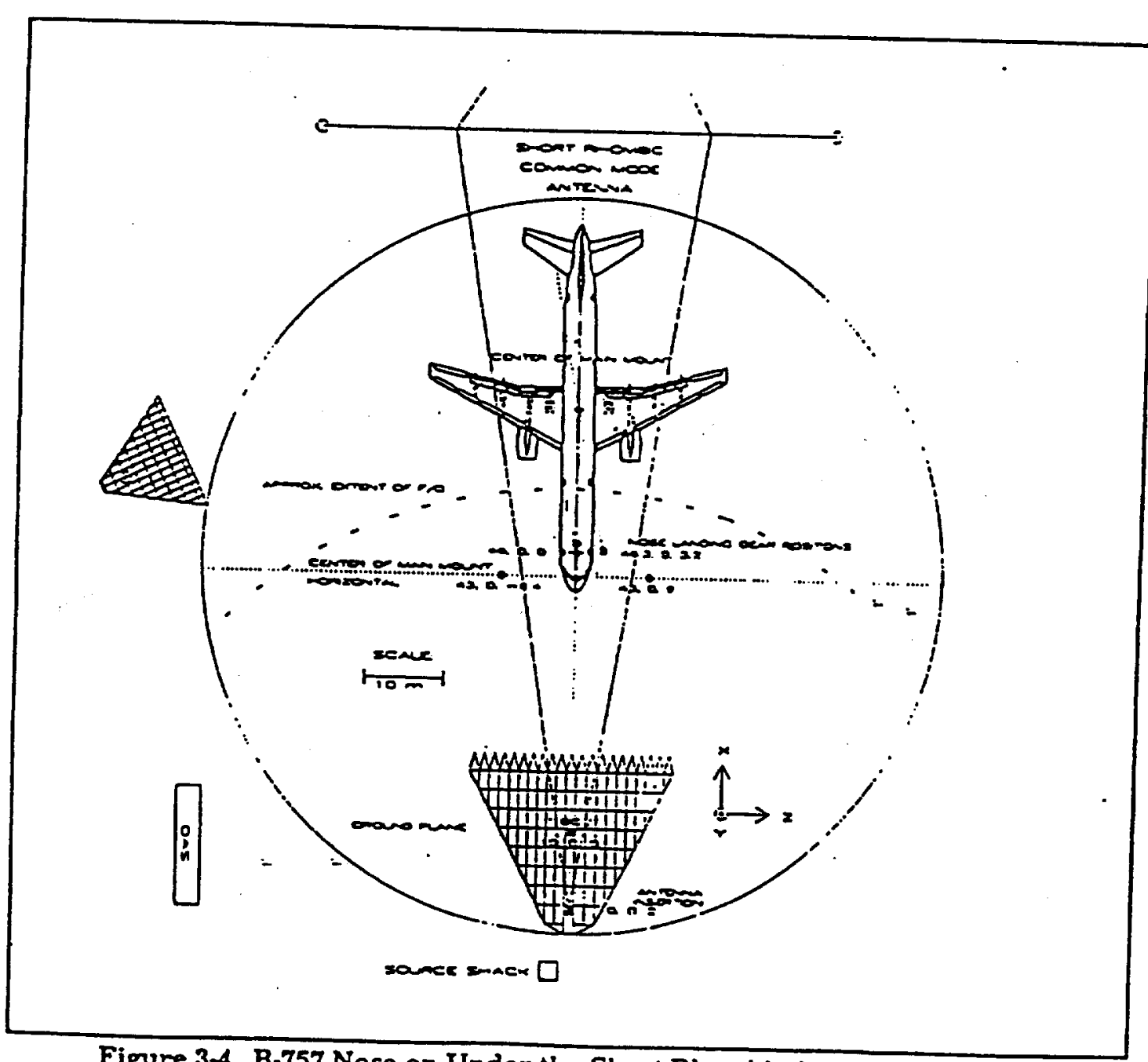

Figure 3-4. B-757 Nose-on Under the Short Rhombic Antenna in LESLI.

The aircraft was spotted on the rhombic antenna centerline with the nose 40 meters from the antenna insertion point $(X=40)$. Other orientations were with the aircraft rotated counter-clockwise about the main landing gear at five and ten degrees. For the Test Series $\mathrm{K}$ measurements, the $\mathrm{B}-757$ was positioned perpendicular to the antenna centerline, forty 
three meters from the antenna insertion point $(X=43)$, and with the nose fifteen meters in the positive " $Z$ " direction.

All the B-757 data under the short rhombic antenna were acquired with the power off. No attempt was made to simulate a power-on condition using circuit breaker settings, jumpers, etc. Settings of circuit breakers were important to test results. Their positions were verified by NASA before the start of each test day. It was also found that the positions of the pilot and co-pilot seats and armrests were important, so the seats were configured in the mid-point stop position with the inboard armrest up for all baseline tests. Except for the variation tests laid out in Tables C-20 and C-21 of Appendix C to Reference 1 , all data were acquired with doors and hatches shut, no personnel in the aircraft, and with the static ground off.

\subsubsection{Measurement Procedures}

Figure 3-5 shows the instrumentation setup used for the rhombic antenna tests. With the exception of the antenna and the reference sensor, it was identical to the setup used for acquisition of dipole antenna data. The antenna, of course, was the rhombic configured in the

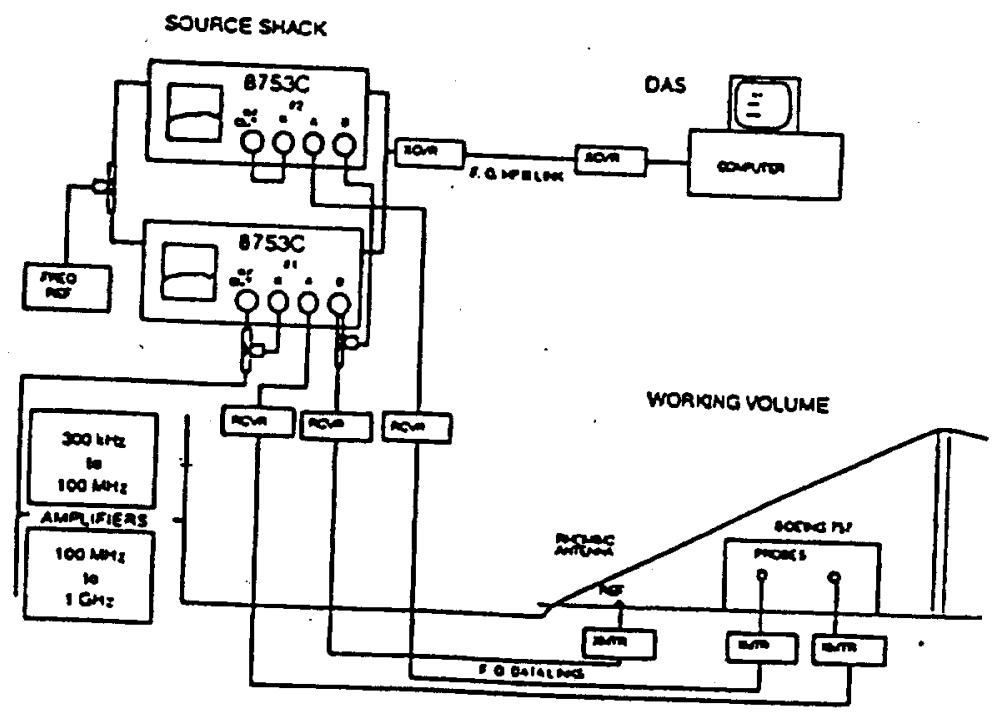

Figure 3-5. Rhombic Instrumentation. common mode. The reference sensor was an MGL-7 positioned on the metal ground plane, ten meters from the antenna insertion on the center line of the antenna. 
Data acquisition and processing procedures for the common mode rhombic measurements were identical to those described in Paragraph 3.1.2.

\subsubsection{Skip Bands}

Paragraph 3.1.3 discusses the requirement to avoid transmitting from the dipole antennas on certain frequencies. The same requirement was levied on the rhombic antenna. The skip bands are listed in Reference 3.

\subsubsection{Configuration Issues}

Intentional variations in aircraft configuration were an important aspect of the test and are discussed in Reference 1. They included positioning and moving people in the cockpit and cabin, inserting and moving a small metallic box in the cockpit, introducing a small antenna in the cockpit, opening the E-bay door and electromagnetically sealing the E-bay door, covering the windshield with aluminum foil, and connecting the static ground to the aircraft. Data were acquired for all these variations.

Configuration control of a system-level test object like the B-757 is not an easy task; two cases of unintentional configuration variations were observed during the testing of the B757. A Collins VHF radio was modified with internal instrumentation and installed in place of the existing radio in the E-bay upon aircraft arrival. The Collins radio could not be powered because of the modifications. The standard procedure was to pull the circuit breaker to the radio, located in the cockpit, before powering the aircraft to run daily functional checks.

In Test Series J, the breaker was found to be pulled during a configuration inspection. It was determined the breaker had been in the pulled condition during the previous day's data acquisition. The test point in the VHF radio (EB2) measured the electrical power cable to the box, so it became apparent the status of the breaker was likely to be important. The appropriate measurement was repeated and Figure 3-6 is an overlay of the two plots. 


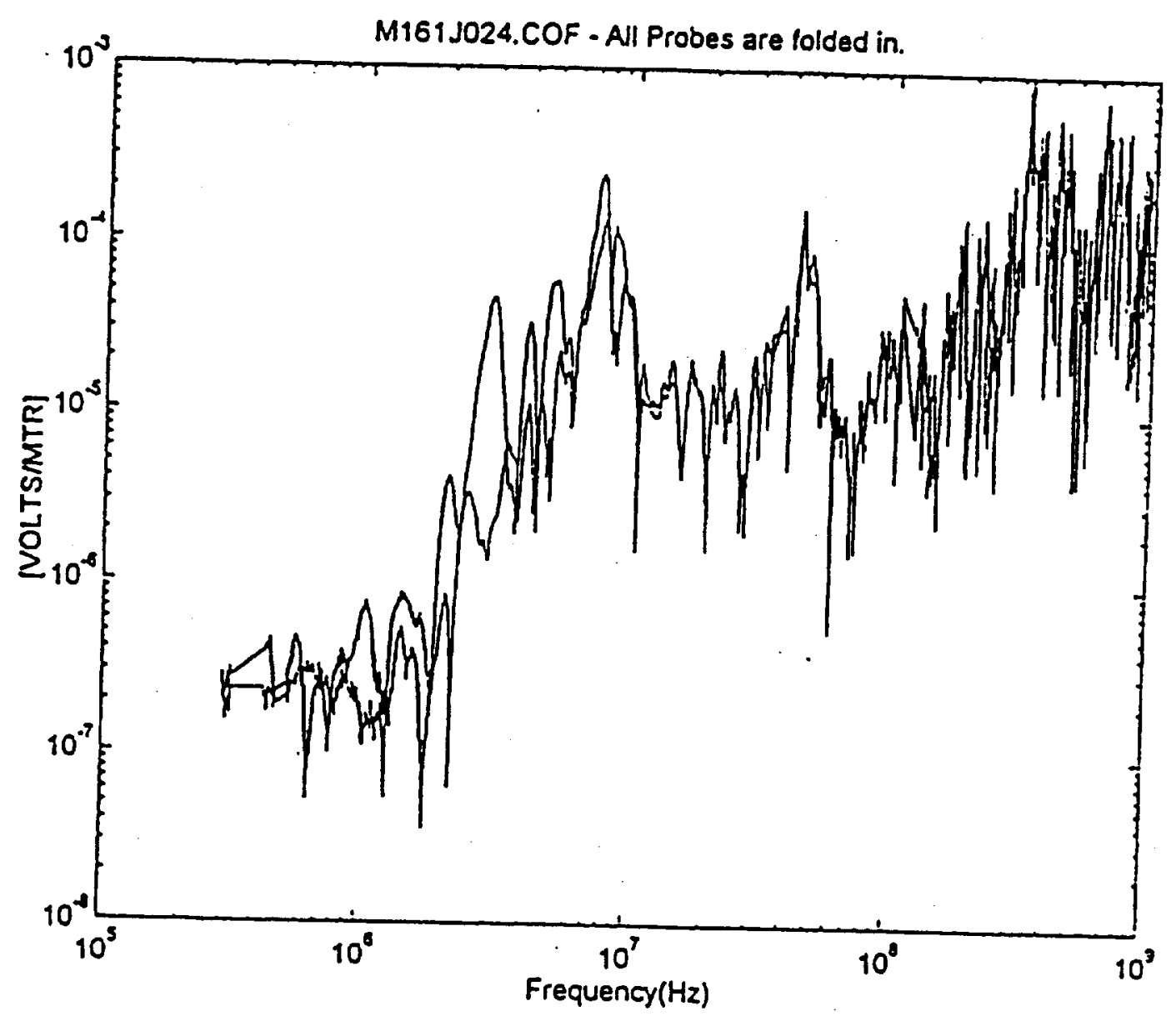

Figure 3:6. Test Point EB2-Overlay Plots.

As evidenced by the figure, the status of the VHF radio circuit breaker made a significant difference in the response of test point EB2 at the frequencies from about $1 \mathrm{MHz}$ to 10 $\mathrm{MHz}$, but had little effect at the higher frequencies. This is not an unexpected result considering the circuit breaker was located in the cockpit and the test point was in the Ebay. All the measurements on this point were repeated when the aircraft returned to the nose-on orientation during Test Series K.

The second out-of-configuration condition occurred during the five-degree rotation experiment in Test Series J. Differences in the raw data from the cockpit which seemed to be excessive in view of the small change in orientation were observed during DAS QC. A configuration check revealed the pilot and co-pilot seats to be in the full forward position rather than the half-way stop. An initial plan was to repeat the five degree rotation 
measurements as part of Test Series J but, upon review of the processed data, LLNL determined the ten-degree rotation to be more important. A repeat of this orientation was accomplished.

\subsection{DIFFERENTIAL MODE RHOMBIC MEASUREMENTS}

A new requirement was identified by LLNL to investigate the response of the aircraft to a horizontal E-field at $3 \mathrm{MHz}$. After several options were explored, it was decided to use the LESLI rhombic antenna in the differential mode.

\subsubsection{Configuration}

LLNL provided a $200 \mathrm{~W}$ balun with an appropriate interface for the rhombic antenna. It was specified from $2.5 \mathrm{MHz}$ to $100 \mathrm{MHz}$, though it had not been tested. The rhombic antenna was lowered on 11 October and configured for the differential mode. During the hoisting process, the north wire became fouled in its caribiner which caused the hoisting line to be pulled partly off its sheave. As a result, the hoisting line was chafed along a two-foot length. The antenna design engineer was consulted and indicated there was a significant safety margin designed into the hoisting gear. He considered the hoisting line to be serviceable but recommended both lines be replaced when convenient since they have been exposed to the elements for quite a few years.

When hoisting was again attempted on 12 October, the south antenna wire parted at one of the termination resistors. Investigation determined the extension wires had not been connected between the drive end and the termination end of the antenna when converting the antenna to differential mode. The resistor was repaired, the extension wires inserted, and the antenna was hoisted without further incident.

This experiment was the first opportunity to use the rhombic antenna in the differential mode since it was fabricated three years ago. From calculations and experience with the long wire rhombic antenna in differential mode, it was known the E-field is primarily 
horizontal on the test pad, but there is a significant vertical component near the antenna wires. Accordingly, an issue was raised as to the type and location for a reference sensor. After some discussion it was decided to continue using the MGL-7 magnetic ground plane sensor located at $10 \mathrm{CL}$. It proved to be adequate for LLNL's needs, though it was not the best choice. If future efforts with the rhombic antenna in the differential mode are anticipated, a stand should be designed and fabricated to mount an MGL-6 magnetic free field sensor over the ground plane. The stand should be designed to allow precise replication of the positioning of the sensor.

The aircraft was oriented nose-on, on the antenna center line, with the nose forty meters from the antenna insertion point as it had been for the common mode measurements (see Figure 3-4). It was configured as it had been for the baseline common mode measurements (all doors and hatches closed, no personnel on board, static ground off, etc).

\subsubsection{Measurement Procedures}

As mentioned above, the antenna balun was rated from $2.5 \mathrm{MHz}$ to $100 \mathrm{MHz}$ and LLNL was interested in data near $3 \mathrm{MHz}$, specifically from as low as feasible to $30 \mathrm{MHz}$. Since the amplifier had internal VSWR protection, a sweep was attempted from $2 \mathrm{MHz}$ to 30 MHz. It was accomplished successfully and LLNL decided data should be acquired with 250 points in that frequency range. Sweep designation " $N$ " in Table 2-1 was the result. Table C-24 of Reference 1 lists the points for which measurements were made under the differential mode rhombic antenna. Data acquisition and processing procedures were as described in Paragraph 3.1.2.

\subsection{GROUND PLANE CHARACTERIZATION MEASUREMENTS}

As mentioned, a major objective of the B-757 test was to acquire data for validation of coupling code models. Because the concrete pad on which the aircraft was positioned is an imperfect conductor, modelers required the constitutive parameters of the pad to include 
the ground in model simulations. This, in turn, generated a requirement for an electromagnetic characterization of the pad.

LLNL developed a plan to acquire the requisite data by sweeping one leg of the rhombic antenna which was positioned at a fixed height ( _ inch) above the concrete. Wire currents were measured along the antenna wire at fixed intervals.

\subsubsection{Configuration}

LLNL acquired strips of $1 / 2$ inch, closed cell, styrofoam, eight inches wide. Two layers of the material were laid on the concrete pad along the centerline of the antenna from $X=$

20 meters to $X=60$ meters (Figure $3-4$ ), and secured to the concrete with duct tape. The rhombic antenna was lowered and one leg disconnected. Both arms of the tensioning gear were connected to the remaining antenna wire and adjusted so the wire was positioned over the centerline of the antenna. The wire was stretched hand tight and the west end was secured to a stake driven into the ground just off the concrete pad. The termination end was permitted to remain on the ground in the position it assumed when the antenna was lowered. Cutouts were made in the styrofoam at the test point locations (Table 3-1) to accommodate current probes and keep the wire straight during instrumentation. The wire was tightened with the turnbuckles of the tensioning gear to rest evenly on the styrofoam along its length and conform to the camber of the pad.

\subsubsection{Measurement Procedures}

Reference 1 required current measurements to be taken every thirty centimeters along the antenna wire from $X=22.5$ meters to $X=49.2$ meters. Table 3-1 was created to identify the resultant ninety test points (GP01 through GP90). 
Table 3-1. Ground Plane Characterization Points.

\begin{tabular}{|c|c|c|c|c|c|c|c|}
\hline TEST POINT (GPnn) & $\bar{x}$ & $\bar{Y}$ & $\mathbf{Z}$ & TEST POINT (GPnn) & $\bar{x}$ & $\bar{Y}$ & $\bar{Z}$ \\
\hline 1 & 22.50 & 0 & 0 & 46 & 36.00 & 0 & 0 \\
\hline 2 & 22.80 & 0 & 0 & 47 & 36.30 & 0 & 0 \\
\hline 3 & 23.10 & 0 & 0 & 48 & 36.60 & 0 & 0 \\
\hline 4 & 23.40 & 0 & 0 & 49 & 36.90 & 0 & 0 \\
\hline 5 & 23.70 & 0 & 0 & 50 & 3720 & 0 & 0 \\
\hline 6 & 24.00 & 0 & 0 & 51 & 37.50 & 0 & 0 \\
\hline 7 & 24.30 & 0 & 0 & 5 & 37.80 & 0 & 0 \\
\hline 8 & 24.60 & 0 & 0 & 53 & 38.10 & 0 & 0 \\
\hline 9 & 24.90 & 0 & 0 & 54 & 38.40 & 0 & 0 \\
\hline 10 & 25.20 & 0 & 0 & 56 & 38.70 & 0 & 0 \\
\hline 11 & 25.50 & 0 & 0 & 56 & 39.00 & 0 & 0 \\
\hline 12 & 25.80 & 0 & 0 & 57 & 39.30 & 0 & 0 \\
\hline 13 & 26.10 & 0 & 0 & 58 & 39.60 & 0 & 0 \\
\hline 14 & 26.40 & 0 & 0 & 59 & 39.90 & 0 & 0 \\
\hline 15 & 26.70 & 0 & 0 & $\boldsymbol{\omega}$ & 40.20 & 0 & 0 \\
\hline 16 & 27.00 & 0 & 0 & 61 & 40.50 & 0 & 0 \\
\hline 17 & 27.30 & 0 & 0 & Q & 40.80 & 0 & 0 \\
\hline 18 & 27.60 & 0 & 0 & (3) & 41.10 & 0 & 0 \\
\hline 19 & 27.90 & 0 & 0 & 64 & 41.40 & 0 & 0 \\
\hline 20 & 28.20 & 0 & 0 & 65 & 41.70 & 0 & 0 \\
\hline 21 & 28.50 & 0 & 0 & 6 & 42.00 & 0 & 0 \\
\hline 2 & 28.80 & 0 & 0 & 67 & 42.30 & 0 & 0 \\
\hline$\overline{23}$ & 29.10 & 0 & 0 & $\overline{68}$ & 42.60 & 0 & 0 \\
\hline 24 & 29.40 & 0 & 0 & $\boldsymbol{\theta}$ & 42.90 & 0 & 0 \\
\hline 25 & 29.70 & 0 & 0 & 70 & 43.20 & 0 & 0 \\
\hline 26 & 30.00 & 0 & 0 & 71 & 43.50 & 0 & 0 \\
\hline 27 & 30.30 & 0 & 0 & 72 & 43.80 & 0 & 0 \\
\hline 28 & 30.60 & 0 & 0 & 73 & 44.10 & 0 & 0 \\
\hline 29 & 30.90 & 0 & 0 & 74 & 44.40 & 0 & 0 \\
\hline 30 & 31.20 & 0 & 0 & 75 & 44.70 & 0 & 0 \\
\hline 31 & 31.50 & 0 & 0 & 76 & 45.00 & 0 & 0 \\
\hline 32 & 31.80 & 0 & 0 & 77 & 45.30 & 0 & 0 \\
\hline 33 & 32.10 & 0 & 0 & 78 & 45.60 & 0 & 0 \\
\hline 34 & 32.40 & 0 & 0 & 79 & 45.90 & 0 & 0 \\
\hline 35 & 32.70 & 0 & 0 & 80 & 4620 & 0 & 0 \\
\hline 36 & 33.00 & 0 & 0 & 81 & 46.50 & 0 & 0 \\
\hline 37 & 33.30 & 0 & 0 & 82 & 46.80 & 0 & 0 \\
\hline 38 & 33.60 & 0 & 0 & 83 & 47.10 & 0 & 0 \\
\hline 39 & 33.90 & 0 & 0 & 84 & 47.40 & 0 & 0 \\
\hline 40 & 34.20 & 0 & 0 & 86 & 47.70 & 0 & 0 \\
\hline 41 & 34.50 & 0 & 0 & 86 & 48.00 & 0 & 0 \\
\hline 42 & 34.80 & 0 & 0 & 87 & 48.30 & 0 & 0 \\
\hline 43 & 35.10 & 0 & 0 & 88 & 48.60 & 0 & 0 \\
\hline 44 & 35.40 & 0 & 0 & 89 & 48.90 & 0 & 0 \\
\hline 45 & 35.70 & 0 & 0 & 90 & 49.20 & 0 & 0 \\
\hline
\end{tabular}

The initial setup for acquiring the ground plane characterization data was identical to

Figure 3-5; i.e., a two-network analyzer setup in which two measurements would be made on each sweep. One-meter coaxial cables were chosen to connect Prodyne 1320 current probes to NanoFast high frequency fiber optic links. The cables were positioned orthogonal to the antenna wire. This arrangement minimized both coupling to the 
instrumentation cable shields and interference from the NanoFast transmitters, while keeping the cables short enough to prevent high frequency loss.

A dry run was conducted on 17 October. To preserve the required phase information, the first point, GP01, was designated as Reference and all the data were acquired with respect to that point. Accordingly, GP01 was connected to input B of both network analyzers and GP02 and GP03 were connected to network analyzers \#1 and \#2, respectively. A sweep was taken with the high frequency amplifier (sweep parameters "P" and "Q" of Table 2-1 pertain to the ground plane characterization experiment) and electrical delay was introduced into each data path so the electrical difference between the data path and the reference path was zero. Thus, the phase differences between the paths were zero and phase differences in data would be between the data point and the reference.

The phase information between the two network analyzers did not match well and it was apparent that future analysts could become confused between the two. A decision was made to use a single network analyzer and a single data set per sweep. The electrical delay calculated to produce zero phase between GP01 and GP02 was used for all measurements. The dry run was continued by instrumenting the first thirty points, in turn, acquiring high (low) frequency data, changing amplifiers, and acquiring low (high) frequency data. This process was time consuming and introduced the additional risk of damaging an amplifier connector, but it did have the advantage of acquiring both the sweeps without an instrumentation change.

The data were processed as described in Paragraph 3.1.2 and, upon review, the LLNL representative decided it was more important to acquire the data quickly and safely. Accordingly, on 18 October, all the low frequency and one third of the high frequency data were acquired. The remaining high frequency data were obtained on 19 October. 


\subsubsection{Skip Bands}

The LESLI antenna had never before been used in the configuration required for the ground plane characterization effort. It was unlikely there would be any emissions beyond the site; however, the rhombic skip bands (Reference 3 ) were used to be safe.

\subsection{CROSSTALK}

The multi-input NanoFast fiber optic links have a crosstalk rejection of only $30-40 \mathrm{~dB}$. This fact has been recognized for some time and has been accounted for in high level pulse testing by instrumenting like-measurements on the same transmitter; e.g., all wire measurements or all bulk cable measurements. In this way, no single transmitter input coupled its response in a dominate fashion to other inputs.

The NASA B-757 test was the first to make extensive use of the NanoFast multi-input capability at LESLI and problems with crosstalk were not anticipated in the low level environment. Each dipole test series ( $A$ through $F$ ) had a requirement to.acquire instrumentation noise for a test point with a strong response and a test point with a weak response. As used at the LESLI facility, instrumentation noise is a measurement taken with the test point's sensor or probe replaced by a 50 load and the antenna excited in the normal manner. All of the other instrumentation, including cables attached to other fiber optic inputs, remains in the same configuration. One is thus able to determine the effects of the instrumentation in making measurements.

After a DAS review of the responses for all the baseline measurements during Test Series $\mathrm{C}(172 \mathrm{MHz}$ dipole antenna in vertical mode.), Test Point CP4, a current measurement on the vertical wire in the cockpit, was the chosen weak responder. The current probe was disconnected and a 50 load connected in its place at the cockpit-end of the data cable (T1C4) and the noise measurement was taken. It was immediately obvious in the DAS that resonances were in the data which were well above the noise floor. Figure 3-7 depicts the processed data of the response and, as can be seen, there are peaks which 
equate to something about 30 meters in length resonating. This was obviously not the 18 inch vertical wire; it was the response of one of the other instrumented points crossing over to the input of Test Point CP4. It was probably the aircraft resonant response as detected by one of the D-dot sensors located in the cockpit.

All other inputs to the Nanofast transmitter were disconnected and the noise measurement was repeated. Figure 3-8 is the response.

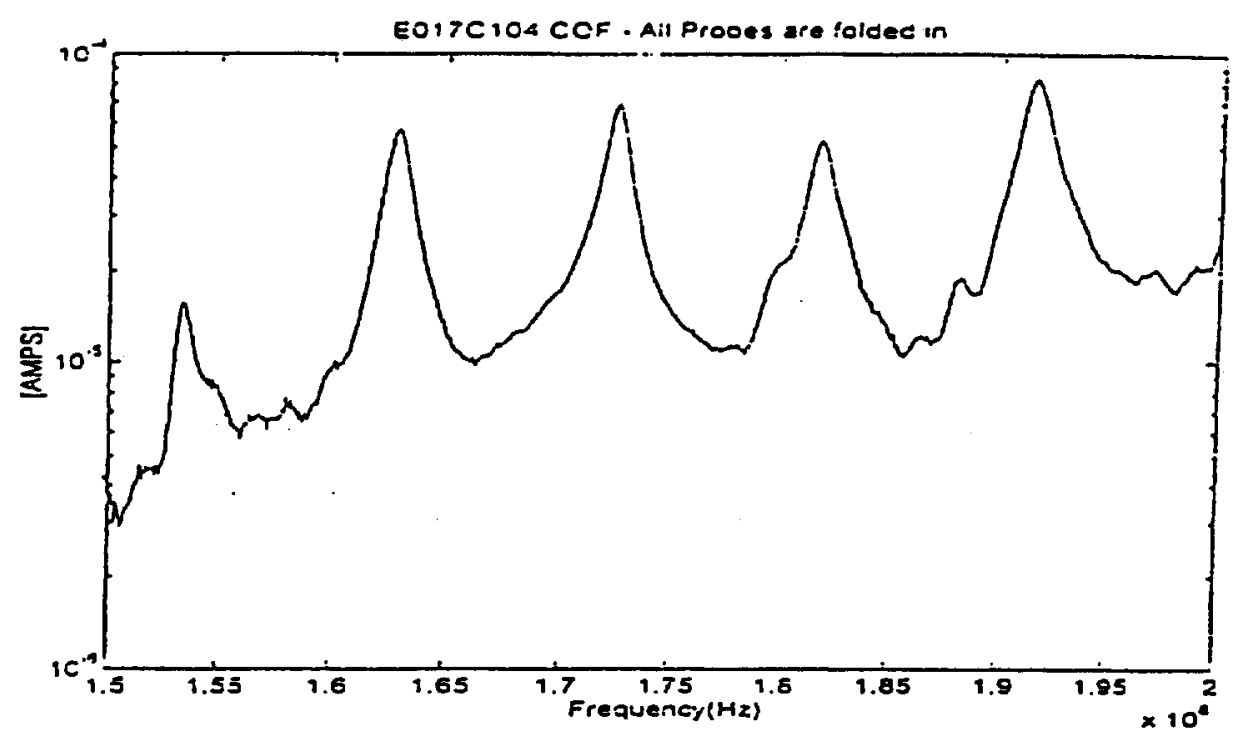

Figure 3-7. Test Polnt CP4 Instrumentation Noise: Other Inputs Connected.

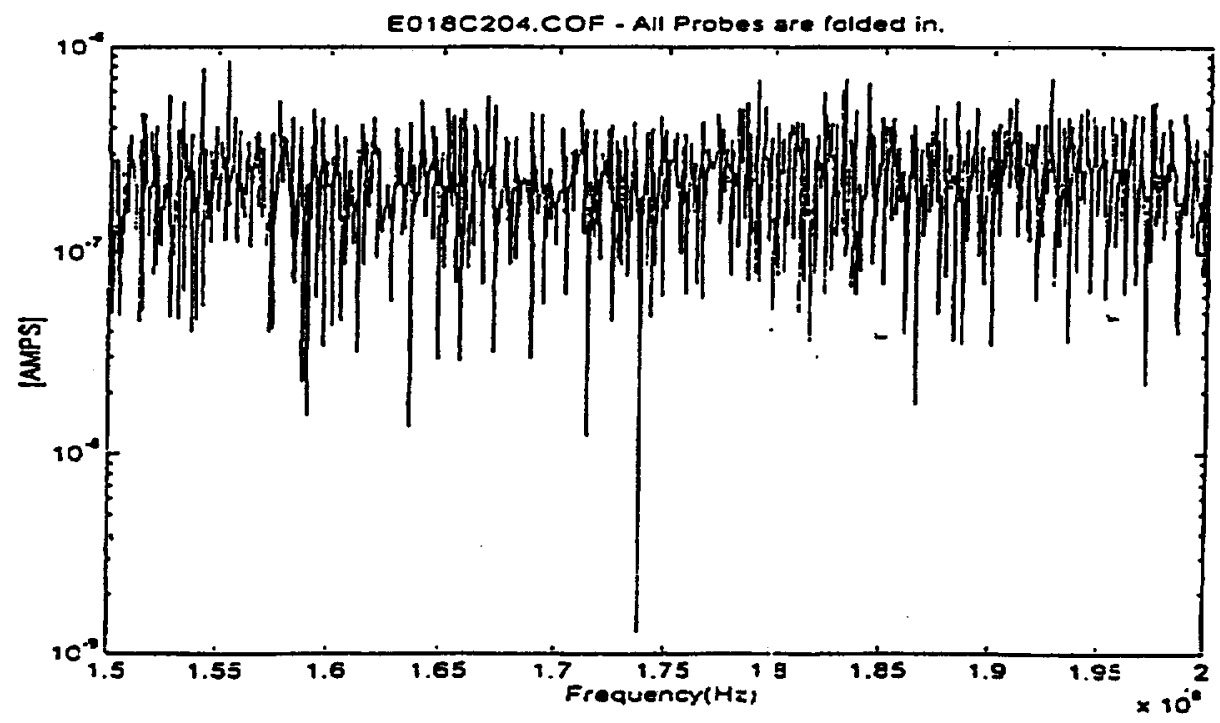

Figure 3-8. Test Point CP4 Instrumentution Noise: Other Inpute Dleconnected. 
It is $40 \mathrm{~dB}$ below the peak responses of Figure 3-7 and has the characteristic noise shape. Time did not permit a thorough investigation to determine which input(s) were causing the problem. Both $\mathrm{CP} 1$ and $\mathrm{CP} 3$ cockpit D-dot sensors were connected to the same transmitter (see Table 2-4), were strong responders, and exhibited the same resonances for many measurements.

For reasons discussed above, it was not feasible to instrument like-responders on the same transmitters. Data acquisition was repeated for Test Point CP4 in Test Series C with no other inputs connected to the transmitter. Data were subsequently acquired in this manner on all test points having a weak response in a particular test series. 


\section{REFERENCES}

1. Poggio, Andrew, et. al., "NASA FBL/PBW Program, NASA Boeing 757 HIRF Test Plan, Low Power On-the-Ground Tests," Lawrence Livermore National Laboratory, Livermore, CA, 7 October 1994.

2. Millard, J. W., et. al., "Large Electromagnetic System-Level Iluminator (LESLI) Facility Site Operating Procedures," UIE-TR-92-0017-R1, United International Engineering, Inc. Albuquerque, NM, 28 May 1993.

3. Randall, R. N., "CWDAS Version 6.4 User's Manual," UIE-TR-91-0012, R2, UIE, Inc., Albuquerque, New Mexico, September 1993. 


\title{
Appendix C
}

\section{The Determination of the Constitutive Parameters of a Medium with Application to a Reinforced Concrete Pad}

\author{
A. J. Poggio \\ G. J. Burke \\ S. T. Pennock \\ Lawrence Livermore National Laboratory \\ January 15, 1995
}




\section{CONTENTS}

Acknowledgment 3

Introduction 4

Proposed Approach 6

Solutions for a Wire Over Ground 6

Physical Layout $\quad 8$

Measurement Process $\quad 8$

Experiment and Data 14

Acquired Data 14

Extraction of Attenuation and Phase Constants 20

Determination of Relative Dielectric Constant and Conductivity

Analysis

29

Conclusions and Summary

50

General Comments

50

Data Summary

51

Concerns and Suggested Future Work 51

References 


\section{Acknowledgment}

The authors are grateful to a number of individuals for their support during the performance of the experimental program. The Test Director was Ly Dao of USAF Phillips Laboratory and the experimental operations team, led by John Millard of United International Engineering Inc., included James Tucker of UIE.

This effort was part of the NASA Low Power On-the-Ground Test portion of the NASA Boeing 757 HIRF Tests under Project Manager Charles Meissner. This, in turn, was part of the NASA Fly-By-Light/Power-by-Wire Program. 


\section{Introduction}

This report describes the experimental and analytical program performed to determine the constitutive parameters of the reinforced concrete pad in the test facility used during the Low Power On-the-Ground portion of the NASA Boeing 757 HIRF Tests [1]. These tests were conducted during the period September 20 to October 21, 1994 in the LESLI facility at the Phillips Laboratory, Kirtland AFB, NM.

The on-the-ground tests were designed to meet several objectives including support of a flight test series and the generation of data for the validation of codes and models that could be used to predict the electromagnetic environment in transport aircraft. To satisfy these objectives, tests were to be executed in a known environment and the data compared to modeling results. A critical feature of this testing was the "known environment" which implies knowledge of the parameters which are critical to an effective modeling activity and which could include, among many other things, definitions of the airplane and its physical and electrical configuration, the ground upon which it sits when stationary, the fields impinging on the aircraft, and the radiating or bounding structure in the simulator. We would want to specify the electromagnetic characteristics of the entire space that would likely enter into a mathematical modeling effort so that the model can be made as "close" to physical reality as desired prior to exercising computational algorithms which might introduce their own uncertainties.

Since we are evaluating codes used for determination of the electromagnetic environment in aircraft and since the on-the-ground test involved an airplane parked on a pad in the simulator facility, we would require a definition of the constitutive parameters of the pad. Initially, the test plan called for the pad to be partially covered by a highly conducting covering composed of interconnected aircraft landing mats with a total surface area in excess of $15,000 \mathrm{ft}^{2}$. Difficulties associated with timely delivery of the mats, installation of the panels on the pad, and uncertainties in the quality of the electrical interconnection of the individual panels prevented use of these panels and required situation of the airplane on the bare, reinforced concrete pad. As a result, the need for measurement of the constitutive parameters of the LELSI concrete pad arose. 
The evaluation of the constitutive parameters was performed under a number of significant constraints that served to restrict the available options. The constraints were:

- In order to limit costs, the measurement process was to be minimized in time extent without severely compromising the quality of the data

- The measurement process was limited by the availability of the experimental system including the RF sources and the data acquisition system. Ultimately, this limitation confined the pad characterization tests to a one-week period including assembly and disassembly of the test fixtures and equipment, testing and dry runs of the entire system, equipment down-time, and actual acquisition runs for the data.

- Construction details for the pad, constructed over 40 years ago, were not available. Thus, reinforcing rod location, concrete thickness and specifications were unknown.

- The measurement techniques were to be completely noninvasive, namely, the pad could not be cored or probes emplaced below the surface.

- The RF equipment and the data acquisition team's experience in performing ground parameter measurements were somewhat limited. Accurate reflection angle, scattering and polarization measurements as might be required in Brewster angle and wave tilt measurements were possibly beyond the capabilities of the available equipment.

Since the precise detailed structure of the pad is unknown and since a detailed spatial description of the constitutive parameters is not required for the purposes of the code validation exercise in view of the level of precision with which other environmental descriptors are known, a technique which averages material characteristics over some spatial extent has been deemed adequate. In view of these observations, the constraints above, and discussions with experts in this technical area such as Drs. Ray King and George Hagen, a method based on wave propagation along wires closely coupled to the ground structure was chosen. The remainder of this report describes the test and analyses involved in determining the constitutive parameters. In the analysis, arguments are made regarding the adequacy of the quality of the results insofar as their role in the code validation exercise is concerned. It would not be reasonable to expend inordinate resources to greatly reduce uncertainties in the parameters when the uncertainties might only be reflected as second order effects. But, realizing the potential value of this scheme for the determination of constitutive parameters in general situations, some concerns and suggestions for future work are presented. 


\section{Proposed Approach}

The method chosen for the determination of the constitutive parameters is based on the theoretical solution for current propagation on a wire above a homogeneous half-space with arbitrary permittivity $\varepsilon$, and conductivity $\sigma$. For the purposes of this study, the permeability $\mu$ of both half spaces (above and below the ground plane) will be assumed equal to that of free space $\mu_{0}$. A solution for the current distribution on an infinitely long wire over ground, with no limitations on the ground constants, has been developed by Chang, Olsen and Kuester $[2,3,4]$. Their analysis leads to a modal expansion of the current on the infinitely long wire in which they found two discrete modes and continuous spectrum components associated with radiation. One of the discrete modes is referred to as the structure attached or transmission line mode, is dominant near the source on a wire close to the ground, and is a slow wave in the case of conducting grounds. The other discrete mode exhibits fast wave characteristics, has substantially less attenuation along the direction of the wire than does the transmission line mode, and is mainly important for large heights above ground.

\subsection{Solutions for a Wire Over Ground}

In [2], with time variation exp[-i $\omega t$, an integral equation for the current on an infinite horizontal wire along the $\mathrm{x}$ axis, with radius $\mathrm{a}$ and height $\mathrm{z}=\mathrm{h}$ over a ground plane, is solved for a delta-function source located at $x=0$. The solution for the current is

$$
I(x)=\frac{2}{\pi \zeta_{0}} \int_{-\infty}^{\infty} \frac{\exp (i k x \gamma)}{\mathrm{M}(\gamma)} d \gamma
$$

where $M(\gamma)$ is the Fourier transform of the integral equation kernel and $k=\left(\mu_{0} \varepsilon_{0}\right)^{1 / 2}$, is the propagation constant in the upper half-space. For a perfectly conducting wire, $\mathrm{M}(\gamma)$ is [4]

$$
\mathrm{M}(\gamma)=\zeta^{2}\left[H_{o}^{(1)}(\zeta A)-J_{0}(\zeta A) H_{o}^{(1)}(2 \zeta H)\right]+P(\gamma, 2 H)-\gamma^{2} Q(\gamma, 2 H)
$$

where $\mathrm{H}=\mathrm{kh}$ and $\mathrm{A}=\mathrm{ka}$, and $\zeta=\left(1-\gamma^{2}\right)^{1 / 2}$ with $\operatorname{Im} \zeta \geq 0$. The functions $\mathrm{P}$ and $\mathrm{Q}$ are given by the integrals

$$
P(\gamma, Z)=\frac{2}{i \pi} \int_{-\infty}^{\infty} \frac{e^{-u_{1} Z}}{u_{1}+u_{2}} d \lambda \quad Q(\gamma, Z)=\frac{2}{i \pi} \int_{-\infty}^{\infty} \frac{e^{-u_{1} Z}}{n^{2} u_{1}+u_{2}} d \lambda
$$


where $n=\left(\varepsilon_{r}+i \sigma / \omega \varepsilon_{0}\right)^{1 / 2}$ for $\varepsilon_{\mathrm{r}}$ and $\sigma$ the relative dielectric constant and conductivity of the ground and

$$
\begin{array}{ll}
u_{1}=\left(\lambda^{2}-\zeta^{2}\right)^{1 / 2}, & u_{2}=\left(\lambda^{2}-\zeta_{n}^{2}\right)^{1 / 2}, \quad \operatorname{Re} u_{1}, u_{2} \geq 0 \\
\zeta_{n}=\left(n^{2}-\gamma^{2}\right)^{1 / 2}, & \operatorname{Im} \zeta_{n} \geq 0
\end{array}
$$

The current in Equation (1) is represented as a spectrum of exponential waves with a propagation constant $\mathrm{k} \gamma$. However, if the integration contour is deformed into the complex $\gamma$ plane, the current is found to be composed of one or two discrete modes at poles of the integrand where $M(\gamma)=0$ and three branch cut integrals [4]. For the wire height and frequencies considered here, the important components are a discrete mode with $\gamma=\gamma_{p}$, where $\mathrm{M}\left(\gamma_{\mathrm{p}}\right)=0$, and a branch cut integral from $\gamma=1$. This discrete mode has the character of an attenuating slow wave and is called the transmission line mode. The component of current in this mode is given by the residue at the pole as

$$
I_{p}(x)=4 i\left(\mu_{0} \varepsilon_{0}\right)^{-1 / 2} \exp \left(i k \gamma_{p} x\right) / M^{\prime}\left(\gamma_{p}\right)
$$

There can be a second discrete mode, known as the surface-attached mode, which has the nature of a fast wave, but it does not appear significant in the case of our measurements. The branch cut integral yields a component of current with asymptotic form $\exp (\mathrm{ikx}) / \mathrm{x}^{2}$, so it is important very near the source but it quickly drops below the transmission line mode. However, at some larger distance the exponential decay of the transmission line mode will always take it below the branch cut term which is decreasing algebraically. Hence, there is a window in $\mathrm{x}$ where the transmission line mode is dominant, and this region is of primary interest in our measurements since information about the ground parameters is most easily extracted from $\gamma_{p}$.

In the typical forward solution for the discrete modes, the ground constants $\varepsilon$ and $\sigma$ are substituted into Equation (2) and the solution for $M(\gamma)=0$ is found by numerical iteration. For the problem at hand, namely determination of $\varepsilon_{\mathrm{r}}$ and $\sigma$ given the attenuation and phase constant for a measured current distribution on the wire, the inverse problem must be solved. Formally, for a given frequency, wire radius, and height above ground, $\alpha$ and $\beta$ are determined by fitting the measured current $I(x)$. Then $\gamma_{p}=(\beta+i \alpha) / k$ is substituted for $\gamma$ in Equation (2) and the equation is solved numerically for $\varepsilon_{\mathrm{r}}$ and $\sigma$. The final results are then the constitutive parameters for the pad at a set of frequencies (as many as desired). 


\subsection{The Physical Layout}

The concrete pad that is the subject of this analysis is shown in Figure 1. The pad, which is approximately 94 meters in diameter, is composed of reinforced concrete with unknown structural characteristics. The source region is covered with an aluminum plate as shown, which measures approximately 20 meters from the edge of the pad to the triangular plates forming the transition region from aluminum to concrete. For the purposes of the characterization, one of the LESLI rhombic antenna wires was stretched, under some tension, across the pad and continued across the earth surroundings. The wire, with diameter $3 / 16$ inches (radius $=0.238 \mathrm{~cm}$ ), was approximately 200 meters in length with the portion beyond the 100 meter mark containing randomly placed loads in an attempt to suppress reflections when used in the rhombic configuration. In an attempt to isolate the wire from the pad over a considerable portion of its length, the wire was placed on a dielectric support. The closed cell Styrofoam support was composed of two strips each 1/4 inch thick and 8 inches wide to form a $1 / 2$ inch by 8 inch support structure. The support was secured to the concrete with duct tape. A cross section view of the arrangement of the wire, Styrofoam support, aluminum plane, and concrete pad is shown in Figure 2. The Styrofoam support material was characterized using a network analyzer and a stripline fixture, and its relative dielectric constant was measured to be approximately 1.03 over the range of frequencies from $50 \mathrm{MHz}$ to $1 \mathrm{GHz}$. The wire was tensioned with turnbuckles and secured to a stake beyond the edge of the concrete pad. The remainder of the wire, containing the randomly located loads, was allowed to remain on the ground in the random configuration it assumed when lowered to the ground. Cutouts were made in the Styrofoam support strip to accommodate the current probes. Ultimately, the axis of the wire was $5 / 8$ inches $(1.5875 \mathrm{~cm}$ ) with an uncertainty of $1 / 8$ inch above the ground plane. The current was measured with Prodyne I320 current probes that were connected to NanoFast high frequency fiber optic links by one-meter long coaxial cables that were orthogonal to the wire to minimize coupling and high frequency losses. The signals were routed to a fiber optic (F-O) receiver for optical to electrical conversion and then to an $\mathrm{HP}$ automatic network analyzer. Ultimately the network analyzer output was routed to a recording computer. The system is shown in Figure 3.

\subsection{The Measurement Process}

To execute the tests, the wire was driven against the aluminum ground plane and the current distribution was measured at selected points along the wire. From this measured 


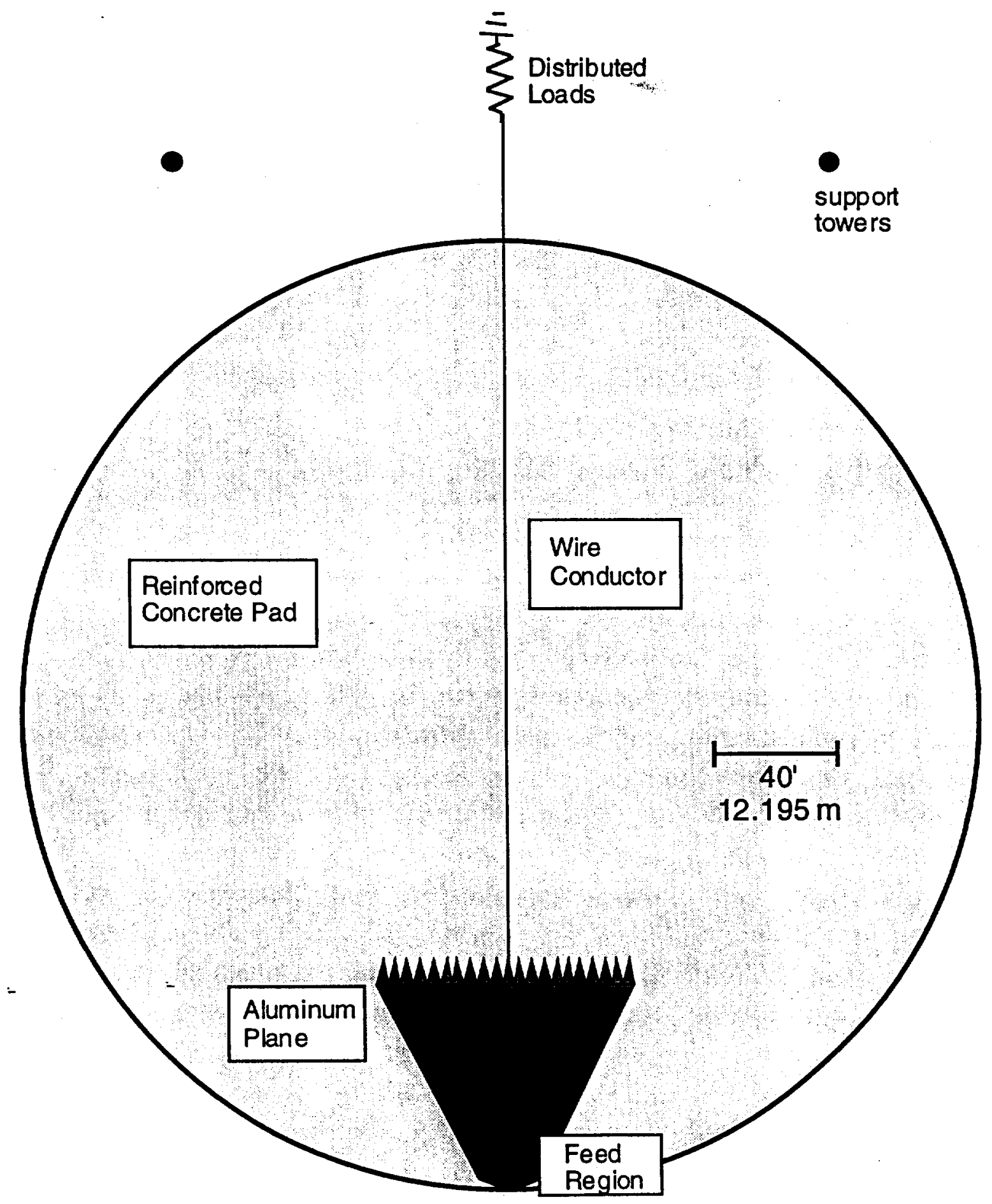

Figure 1. Pad characterization wire layout 
8

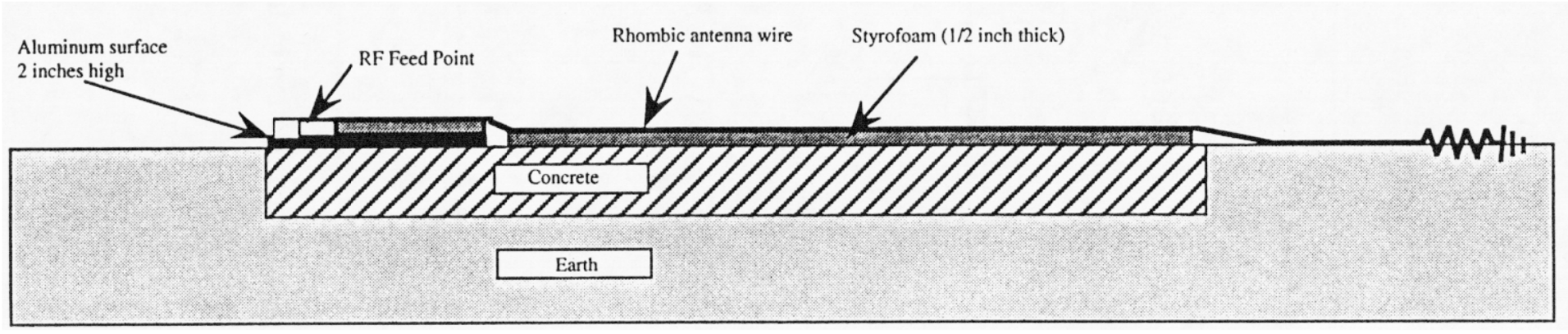

Figure 2. Pad Characterization Wire Layout - Cross Sectional View 


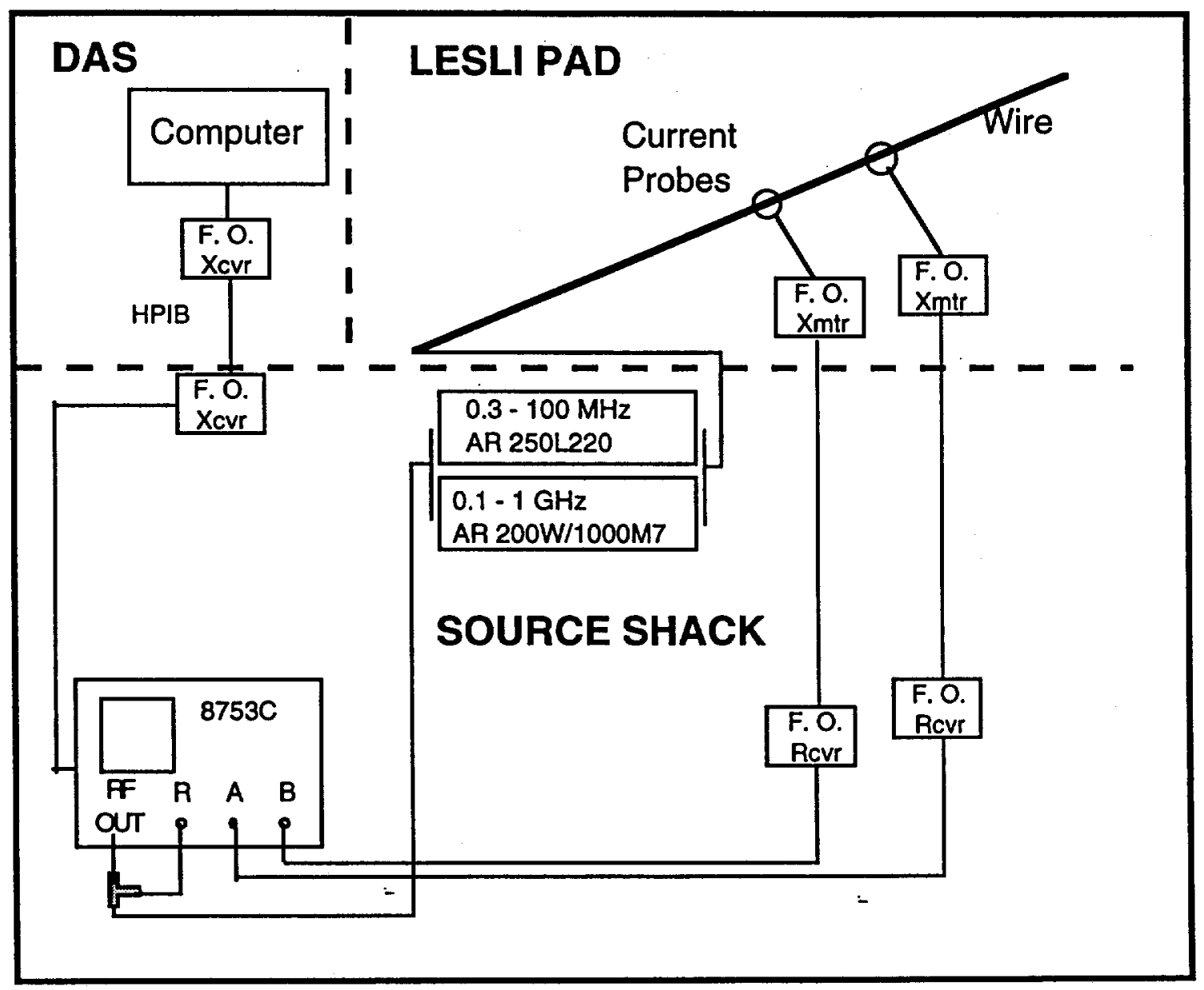

Figure 3. Ground characterization instrumentation 
current distribution the attenuation and phase constants were determined using methods described later. The method used in data acquisition was to simultaneously measure the current at two points along the structure over the entire range of frequencies with one point (Point 1) always located at the first measurement site closest to the source, viz., $x=22.5$ meters. For the purposes of this study, the frequency range was from approximately 1 $\mathrm{MHz}$ to approximately $1 \mathrm{GHz}$. Starting at a point 22.5 meters from the feed point on the aluminum plane that coincides with the rhombic apex, the current was measured at $30 \mathrm{~cm}$ intervals for a total of 90 points. The locations, with the reference probe at 22.5 meters (Point 1), are given by

$$
\mathrm{x}(\text { meters })=22.5+0.3(\mathrm{n}-1), \quad \mathrm{n}=\{1,90\}
$$

Based on the premise that the current distribution for a wire very close to the ground is dominated, especially near the source, by a complex exponential $\exp (\mathrm{ik} \gamma \mathrm{x})$ with $\gamma$ defined by zeroes of $\mathrm{M}(\gamma)$ in the complex plane, it becomes clear that relative measurements of current are adequate for evaluation of attenuation and phase constants. For example, the ratio of current at Point $\mathrm{n}$ to the current at Point 1 when the currents are measured simultaneously, or are the results of identical source conditions at the feedpoint, is simply

$$
I\left(x_{n}\right) /\left(x_{1}\right)=\exp \left(i k \gamma\left(x_{n}-x_{1}\right)\right)
$$

Clearly the reference signal strength is normalized out of the analysis and evaluation of the attenuation and phase constant becomes straightforward, in principle. The data was collected by stepping through all frequencies of interest in a spatial-pairwise scheme $\left[I\left(x_{n}\right)\right.$ and $\left.I\left(x_{1}\right)\right]$ for all $n$. Hence, we measure and store $I\left(x_{n}, \omega_{j}\right) / I\left(x_{1}, \omega_{j}\right), n=\{2,90\}$, and $j=\{1, J\}$, i.e., we record the normalized current (as well as each component, in reality) at fixed positions for stepped frequency sweeps. Ultimately, we use this data in a different format, namely, we use the normalized current at fixed frequency for stepped position to determine $\alpha$ and $\beta$. The structure of this data matrix is represented graphically in Figure 4 where $I\left(x_{i}, \omega_{j}\right)$ is represented by $I_{i j}$ and the data is acquired along columns (fixed position) and processed along rows (fixed frequency). This then allows the determination of the normalized attenuation and phase constants as a function of frequency. Using these, one then performs the inverse evaluation of the constitutive parameters as described later. Some uncertainties arise regarding this approach to the determination of the constitutive parameters. Namely, it is unknown, a priori, if this method is robust and unaffected by the presence of noise in the measured data. The literature abounds with non-robust methods for evaluation of the parameters in complex exponentials. In addition to the impact of measurement noise in the data, the impact of other uncertainties such as the importance of 


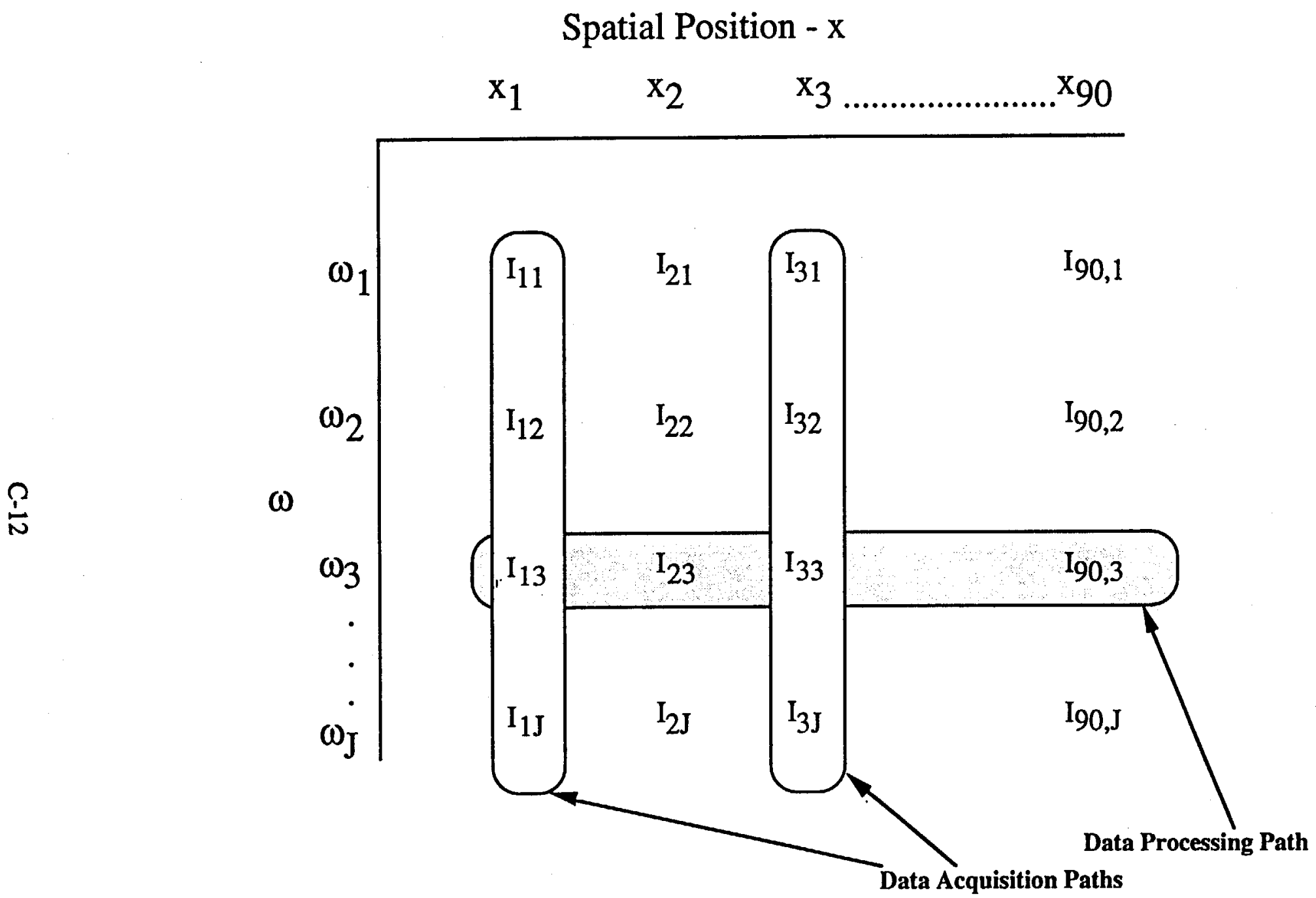

Figure 4. Data Acquisition and Processing Paths 
precision in the height of the wire above the ground plane, is unknown. All these uncertainties could be corrected with some further work in which numerical testing is offered as a potential fix to this dilemma.

\section{Experiment and Data}

\subsection{The Acquired Data}

An example of the data, as acquired, is shown in Figure 5 for two points along the wire, namely, Point 1 (the reference) and Point 20. For fixed position, the data represent the spectrum of the current and the plot is an example of the vertical strip in Figure 4. During the acquisition phase, ninety such data records were generated, i.e., current as a function of frequency for fixed position. In order to remove the source strength from the data, the current magnitude was normalized to the reference current at Point 1 and such a result is shown in Figure 6. The poor data behavior beyond $250 \mathrm{MHz}$ is due in large part to the signal level at Point 20 having fallen below the noise floor in the measurement system. In the vicinity of this anomalous behavior, the current at Point 20 was more than $60 \mathrm{~dB}$ below the current at Point 1 . And, in this frequency range, the current at Point 1 had already fallen more than an order of magnitude below its value at low frequencies. These combined effects could easily drive the signal below the system measuring capabilities. Also, there are instances where the current at Point 20 exceeds that at Point 1 . This can be due to uncertainties in the measurements or the presence of a standing wave on the structure.

For the purposes of data analysis, the records were processed to provide an additional set of records which portray the current as function of position for fixed frequency. This presentation is representative of the horizontal strip in Figure 4 and a visual example of the data for such a "strip" is shown in Figure 7. The data in Figure 7 exhibit a substantial amount of variation on a point to point basis thus leading to questions regarding the source of this structure in the current distribution. Observations were made that the peaks did not move with changes in frequency, thus ruling out a strong standing wave on the line as the cause for these peaks. An investigation was made of the possibility that perturbations and inclusions in the concrete in the vicinity of the peaks might cause the excursions. Visual inspection of the pad and a study of photographs of the pad with the wire in place did not provide an obvious clue as to the cause. The possibility of rebar-induced perturbations in 


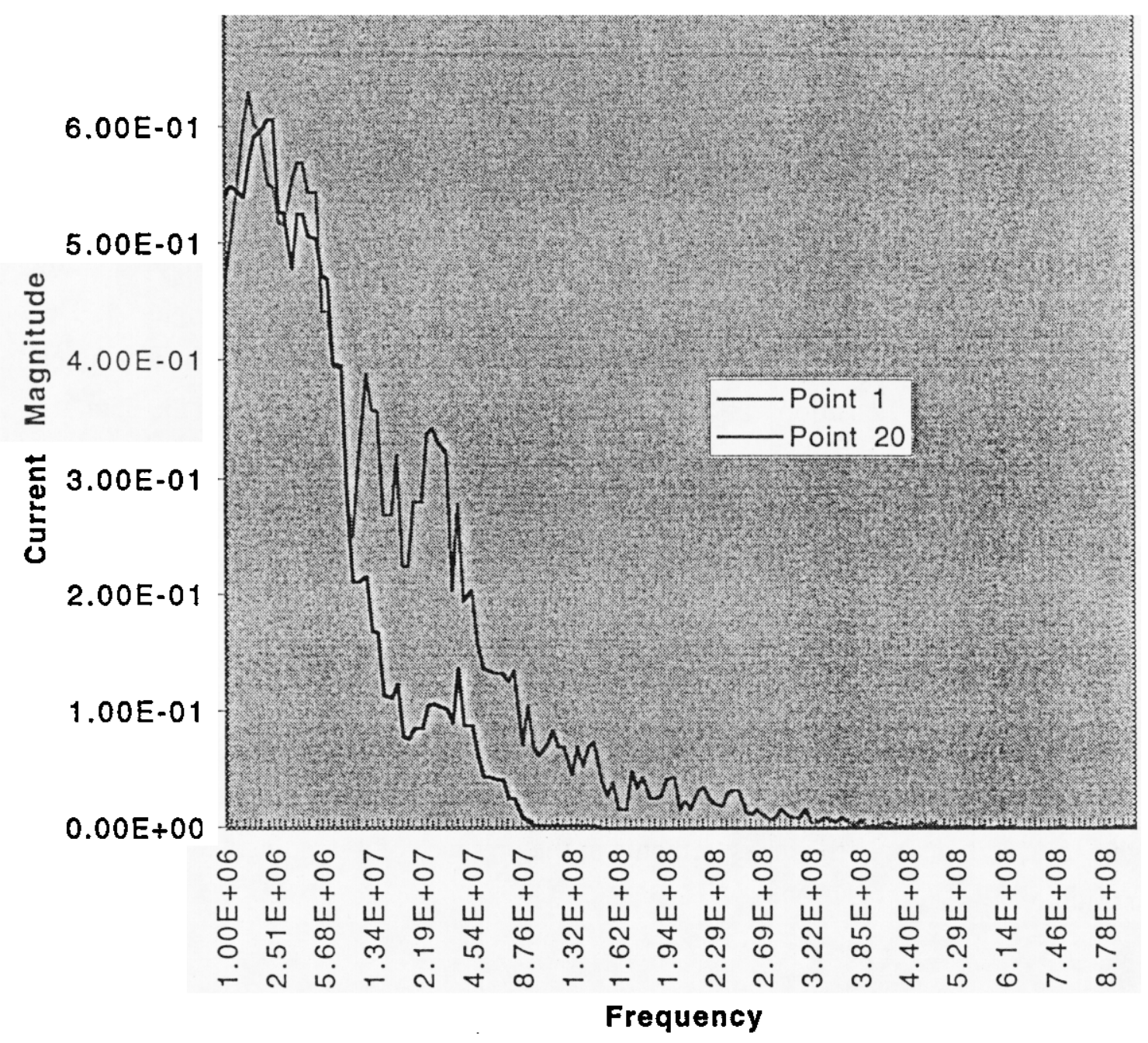

Figure 5. Current magnitude spectrum at Points 1 and 20 


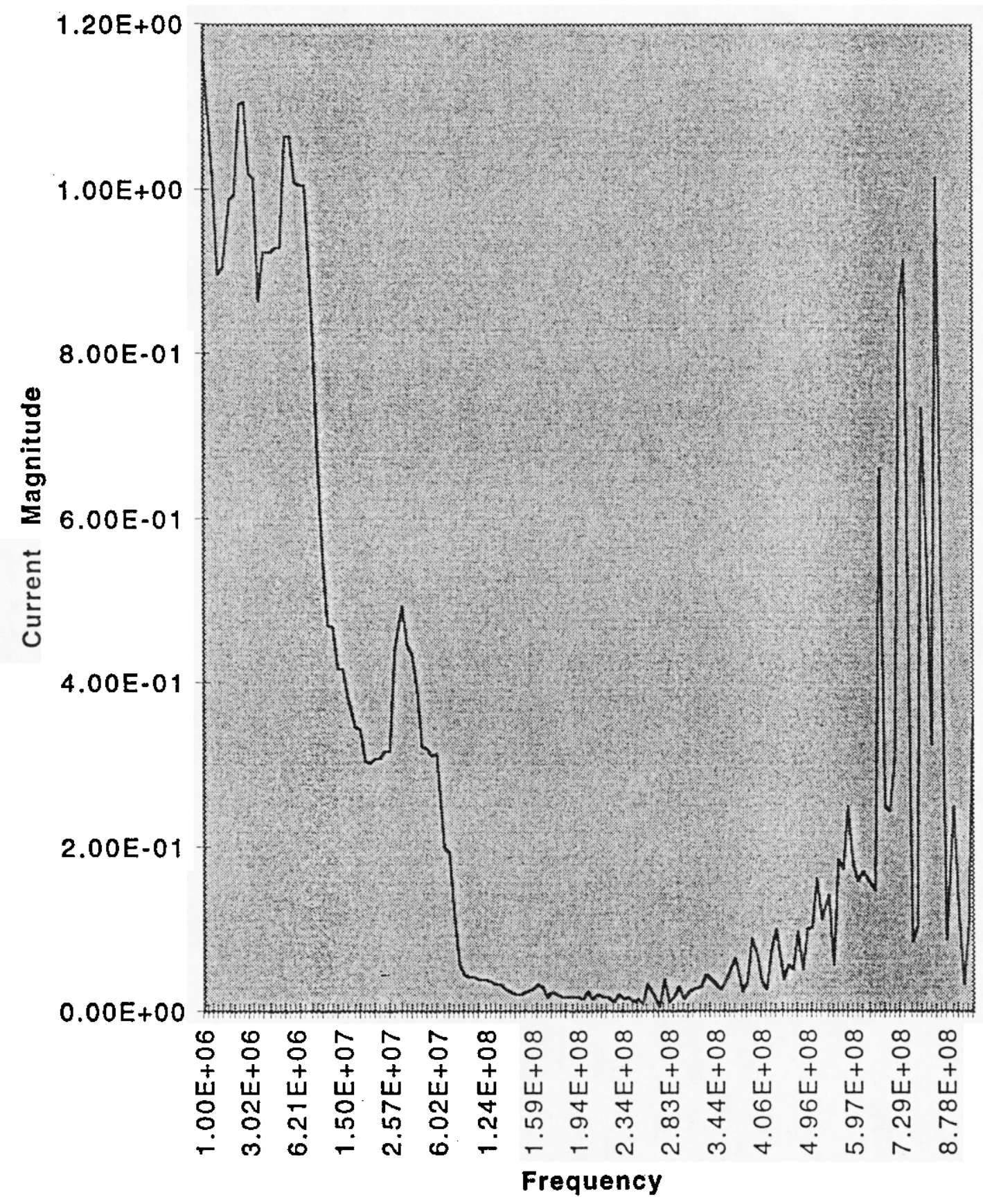

Figure 6. Normalized current magnitude spectrum at Point 20 


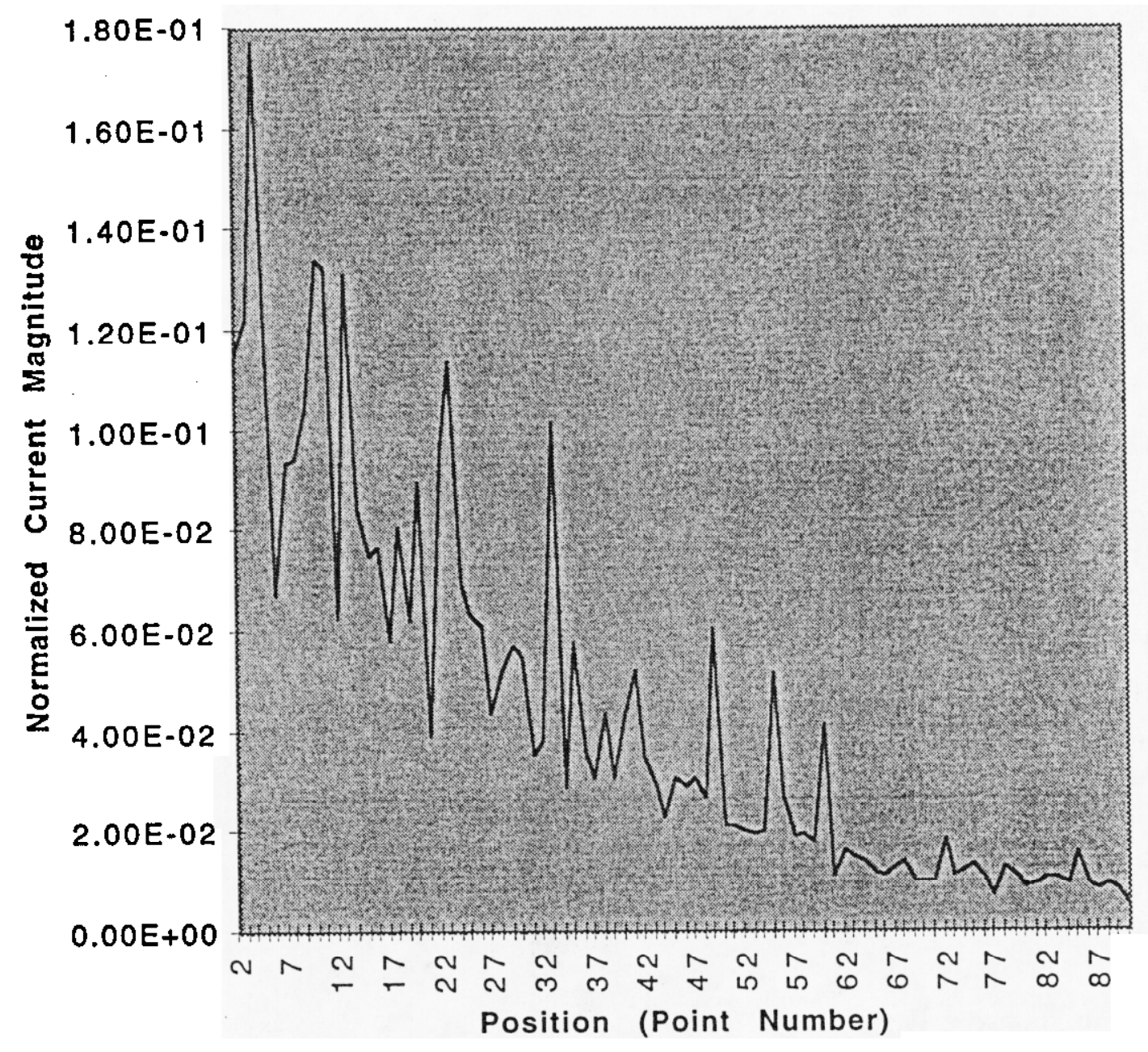

Figure 7 Normalized current as a function of position along the wire at $30 \mathrm{MHz}$ 
the fields and currents was also offered. However, modeling was performed using NEC [6] to analyze an end-driven wire very close ( $h=5 / 8$ inches) to a ground $\left(\varepsilon_{\mathrm{r}}=6, \sigma=0.006\right.$ $\mathrm{S} / \mathrm{m}$ ) with a single strand of perfectly conducting rebar that was buried at six inches. The effects on the wire current distribution were negligible except when the wire and rebar were parallel and aligned in the vertical plane. In the parallel case, the current showed perturbations in the average values of $\alpha$ and $\beta$ but nothing in its behavior as localized as seen in the measured data. Nothing could be detected which was capable of causing such a rapid change in current. Also, the pad was constructed of concrete squares and the wire was oriented diagonally across the squares. Given common concrete building practices, the rebar was most likely oriented parallel to the sides of the squares and orthogonal to edges so that it is unlikely that the rebar and wire were parallel. Other possibilities were considered, including measuring equipment malfunction.

A 3-D view of the data in the 10 to $275 \mathrm{MHz}$ range over the sampled extent of the wire allows a visualization of the previous discussions and is shown in Figure 8. The spectral behavior of the current (without normalization), its spatial behavior and its decay rates in these two variables is evident. The presence of the "spikes" or peaks in the current as a function of position mentioned earlier is also evident. Note that the "spikes" occur at identical positions independent of frequency and manifest themselves in this figure as ridges or striations at a constant position coordinate. Since the data acquisition for a single run was effected at a fixed position with frequency stepped, an offset or malfunction throughout a run could manifest itself as a frequency independent perturbation at a fixed location. A few sources of such error were considered. A malfunctioning or improperly placed or configured current probe could produce such perturbations. The current probes were of the "clothespin" type, that is, they were spring-loaded clamps that fit around the wire. These were-repositioned for each run with only the reference at Point 1 unperturbed during the data acquisition process. Another possibility was inadvertent or improperly applied attenuators in the fiber optic link.' Such a situation could be realized if the stepping attenuator system which was electronically controlled was sporadically "sticking" and reporting an incorrect value to the data acquisition system. At the present time, the identification of the source of the variability of the data has not been successful.

1 J. H. Millard, Private Communication 


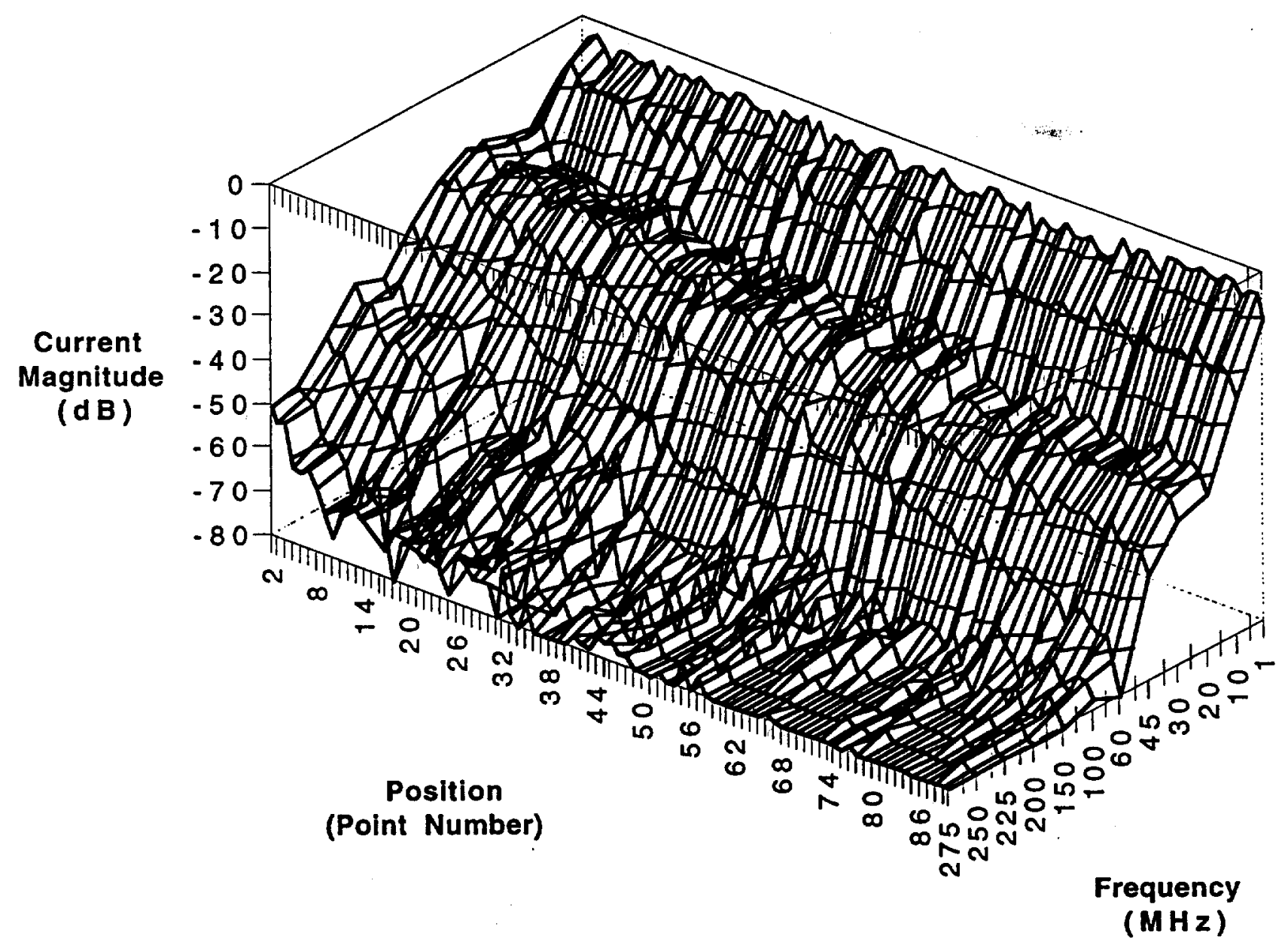

Figure 8. Magnitude of current on the wire as a function of position and frequency 


\subsection{Extraction of Attenuation and Phase Constants}

As explained in Section 2.1, the component of current of diagnostic value is the transmission line mode which is dominant in a window from the source to a point where it drops below the branch cut mode. Hence, we need to locate this region on the wire and estimate the attenuation and phase constants. Given that the current can be represented by

$$
I(x, \omega)=I_{0} \exp \left[i(\beta(\omega)+i \alpha(\omega))\left(x-x_{1}\right)\right], k \gamma=\beta+i \alpha
$$

an exponential fit to the acquired data (or a normalized version of the data) or a linear fit to the log of the data can provide values for the normalized attenuation and phase constants. Using the complex natural logarithm of the data, one has

$$
-[\alpha(\omega)-i \beta(\omega)]\left(x-x_{1}\right)+\operatorname{Ln}\left(I_{0}\right)=\operatorname{Ln}(I(x, \omega))
$$

so that, for two points along the structure at $x_{i}$ and $x_{j}$ at a fixed frequency $\omega_{n}$, one can solve for $\alpha\left(\omega_{n}\right)-i \beta\left(\omega_{n}\right)$

$$
\alpha\left(\omega_{n}\right)-i \beta\left(\omega_{n}\right)=\left[\operatorname{Ln}\left(I\left(x_{i}, \omega_{n}\right)\right)-\operatorname{Ln}\left(I\left(x_{j}, \omega_{n}\right)\right)\right] /\left(x_{j}-x_{i}\right)
$$

Keep in mind that these are complex natural logs. Using the real and imaginary parts of the equation with the current represented by $I I(x, \omega) \operatorname{lexp}(j \phi(x, \omega))$, with $\phi(x, \omega)$ the phase at $x$ and $\omega$, it is a simple matter to extract $\alpha\left(\omega_{n}\right)$ and $\beta\left(\omega_{n}\right)$ and present them in common log notation thus allowing easy conversion to a $\mathrm{dB}$ scale

$$
\begin{aligned}
& \left.\alpha\left(\omega_{n}\right)=2.3025\left[\log \left|I\left(x_{i}, \omega_{n}\right)\right|-\log \mid I\left(x_{j}, \omega_{n}\right)\right]\right] /\left(x_{i}-x_{j}\right) \\
& \beta\left(\omega_{v}\right)=\left[\phi\left(x_{i}, \omega_{n}\right)-\phi\left(x_{j}, \omega_{n}\right)\right] /\left(x_{i}-x_{j}\right)
\end{aligned}
$$

In the face of the variability of the data, a Least Squares (LSQ) fit to the data was performed. A linear fit was performed to the log magnitude and phase of the data, individually. Also, a complex exponential was LSQ fit to the complex data. The differences were minimal and the following relates to separate fits to the magnitude and phase information as represented in Equations (9) and (10).

The difficulty in performing LSQ fits to the data for the wire above ground arises from two sources: 1) the measurement system had its limitations and beyond a certain spatial point the data could become "noisy" as the noise floor was encountered and 2) contributions to the current other than the transmission line mode could become dominant at sufficiently large distances at high frequencies. Since the branch cut term decays as $\mathrm{x}^{-2}$, it always prevails over the transmission line mode at sufficiently large distances and manifests itself as a flattening of the current magnitude distribution with distance from the source. In fact, 
modeling of the physical configuration under consideration has confirmed such characteristics. But, the noise floor could provide a similar behavior. Since it does not matter that we identify the structure's cause but only avoid it in our LSQ fits, the initial data fitting involved some "eyeballing" of the data. This required inspection of the $\log$ (current magnitude) and of the phase to perform a fit to that part which looked "roughly linear". The danger here is that small errors in the resulting value of attenuation and phase constant could ultimately lead to large errors in the $\varepsilon_{\mathrm{r}}$ and $\sigma$ if the method of solution was not robust.

Examples of the process of evaluating $\alpha$ and $\beta$ are illustrated in Figures 9 through 12. In Figures 9 and 10 the magnitude and phase data for $30 \mathrm{MHz}$ are shown. Also shown in the Figures 9 and 10 are the linear fits to the log magnitude and phase over the entire range (including all points from 2 through 90 ), respectively. Figures 11 and 12 show the respective plots for a frequency of $100 \mathrm{MHz}$. There, the magnitude and phase deviate from linear beyond certain points along the wire. Over the linear portions in both cases one can see the rapidly attenuating slow wave characteristic of the transmission line mode. Of course, when the magnitude begins to decay at a slower rate we may be seeing either the surface attached mode, the branch cut contribution, or evidence of the noise floor. When the phase deviates from linear and slow wave, we begin to see a wave that is increasing in velocity and may eventually reach the velocity in the upper medium or become the fast wave whose existence is predicted theoretically. The orderly behavior of the phase suggests that the cause of the flattened current distribution is not the noise floor but rather the branch cut . Ultimately, however, this issue does not impact our analysis as it is the transmission line mode that provides attenuation and phase constants that are most important in the evaluation of the relative dielectric constant and conductivity.

\subsection{Determination of Relative Dielectric Constant and Conductivity}

The process of solving for $\varepsilon_{\mathrm{r}}$ and $\sigma$ has already been touched upon. Once values for $\alpha$ and $\beta$ in the transmission line mode are obtained for a given frequency, the value of $\gamma_{p}=\beta+i \alpha$ is determined. An iterative solution of the equation $M\left(\gamma_{p}\right)=0$ for $n$, the index of refraction for the medium, yields $\varepsilon_{r}$ and $\sigma$ since $n=\left(\varepsilon_{r}+i \sigma /\left(\omega \varepsilon_{0}\right)\right)^{1 / 2}$. The values of $\varepsilon_{\mathrm{r}}$ and $\sigma$ can range from 1 and 0 , respectively, to infinity, although real ground will have a much more limited range. For $\varepsilon_{\mathrm{r}}=1$ and $\sigma=0$, the values of $\alpha$ and $\beta$ will be nearly $\alpha=0$ and $\beta=k$. 


\section{Position (Point Number)}

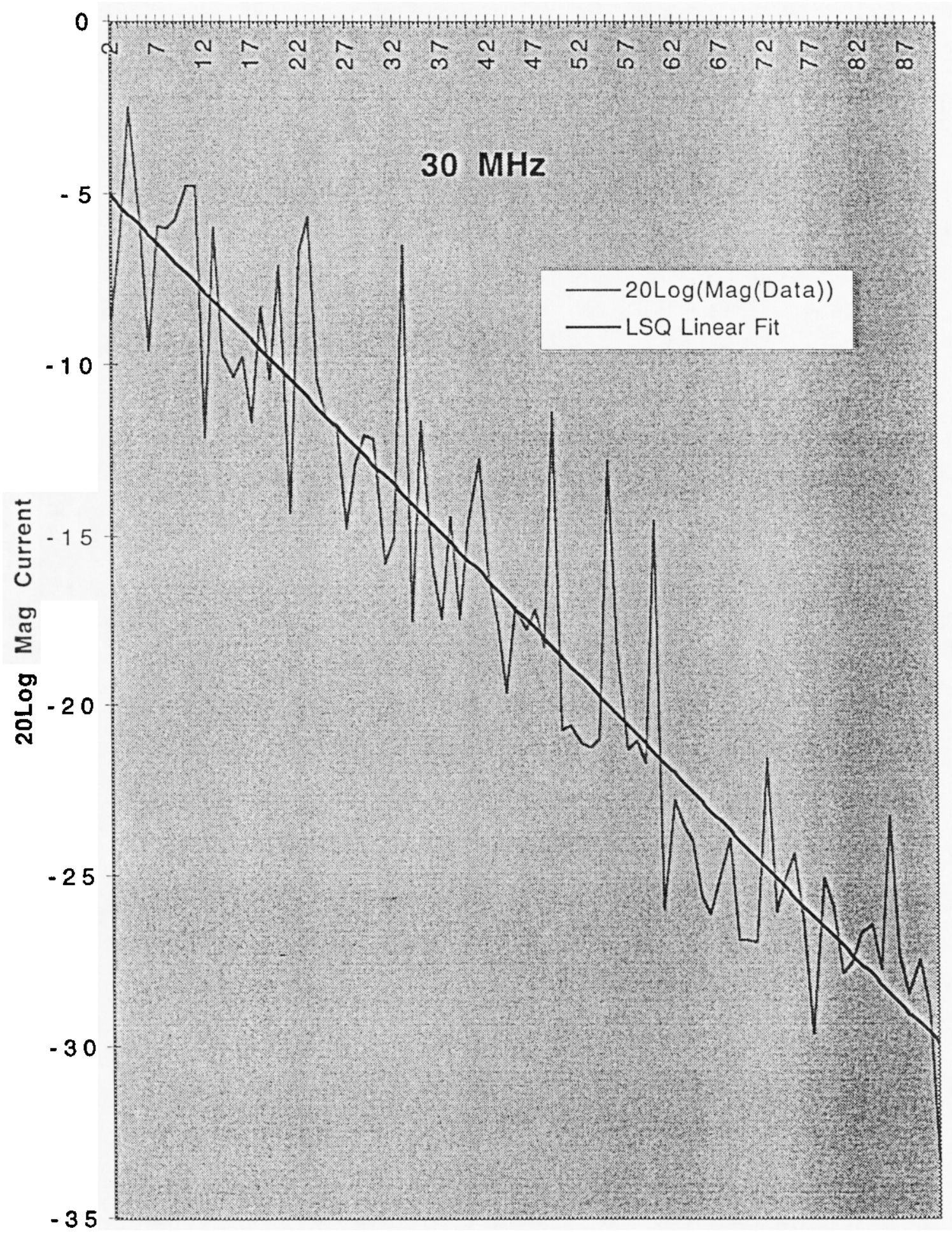

Figure 9. Log magnitude of normalized current and linear LSQ fit over Points 2 to 90 


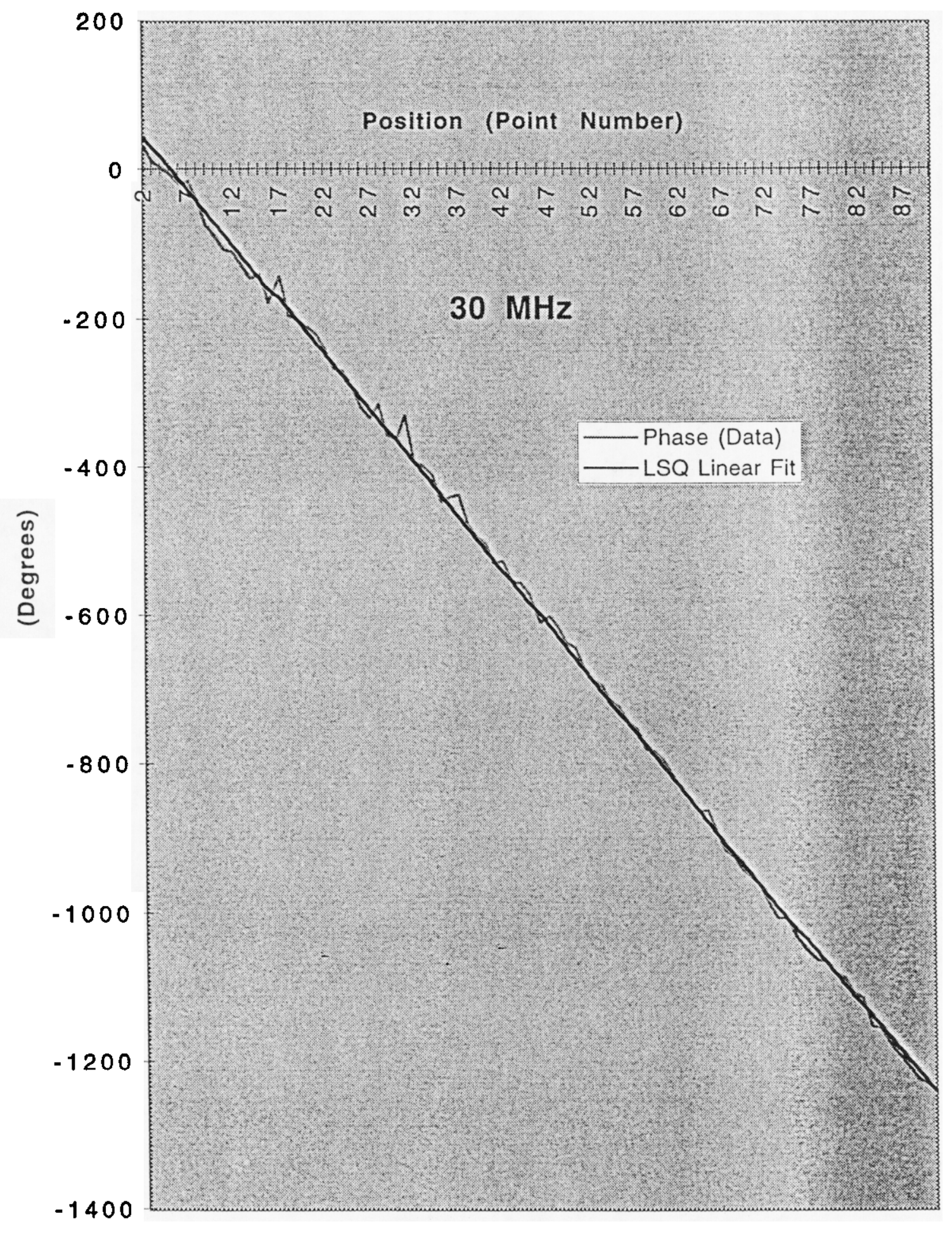

Figure 10. Phase of normalized current and linear LSQ fit over Points 2 to 90 


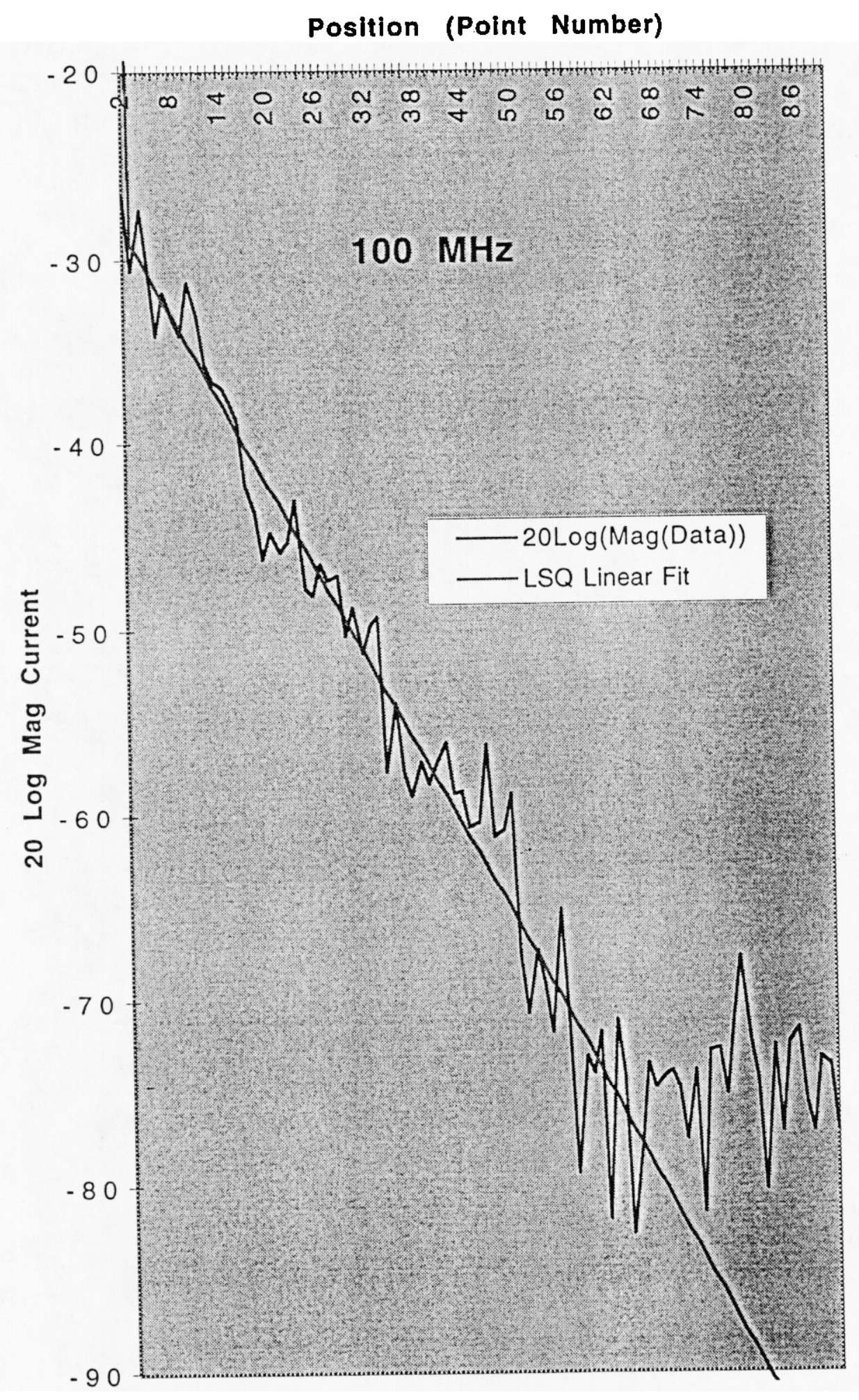

Figure 11. Log magnitude of normalized current and linear LSQ fit over Points 2 to 66 


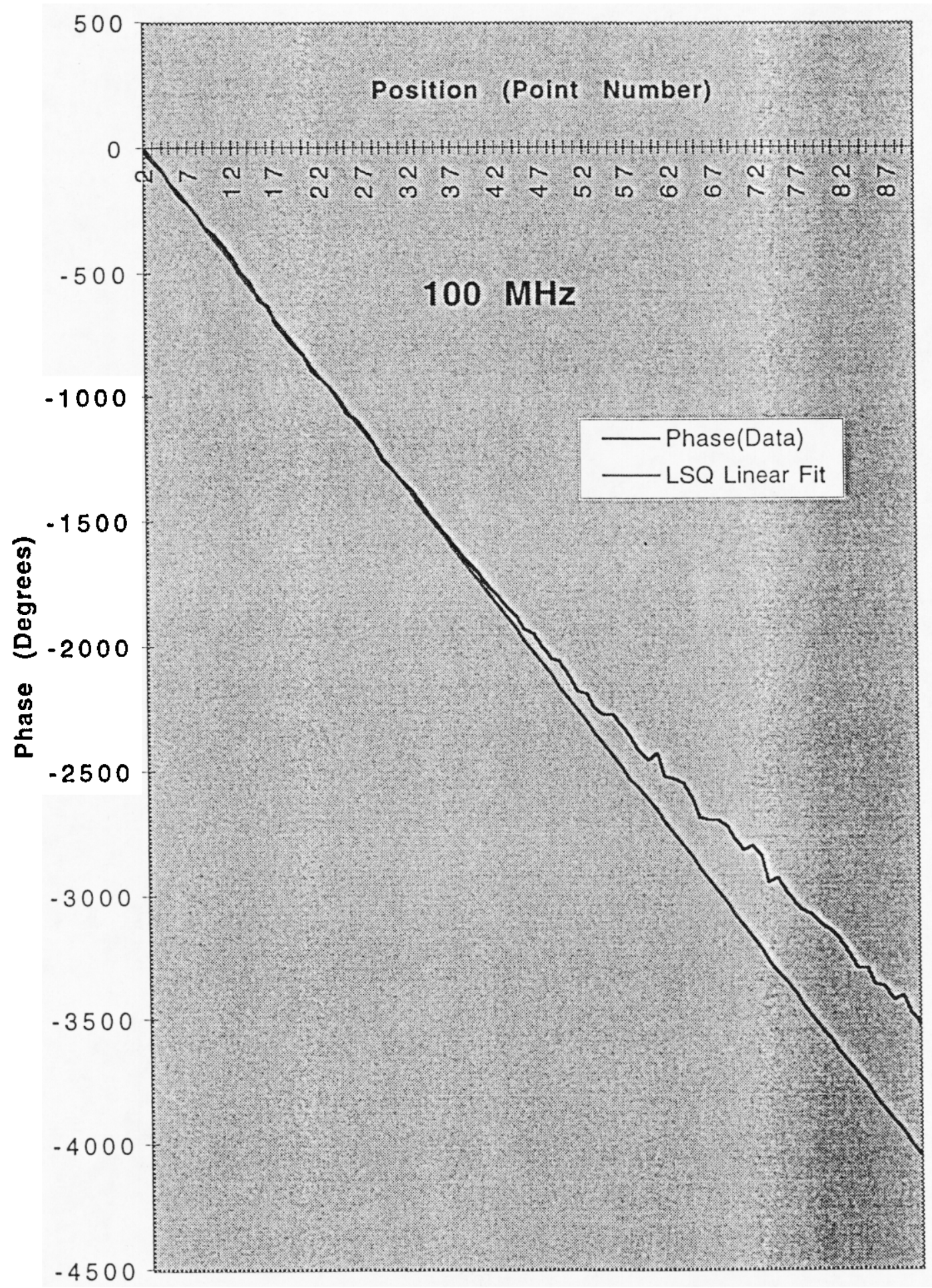

Figure 12. Phase of normalized current and linear LSQ fit over Points 2 to 30 
As $\varepsilon_{\mathrm{r}}$ and $\sigma$ are increased, $\alpha$ and $\beta$ will both start to increase for the attenuating slow wave of the transmission line mode. However, as $\varepsilon_{\mathrm{r}}$ and $\sigma$ continue to increase $\alpha$ and $\beta$ will eventually return to nearly $\alpha=0$ and $\beta=k$, which holds for a wire over perfectly conducting ground. Hence $\alpha$ and $\beta$ are limited to a range that can only be determined by solving $M\left(\gamma_{p}\right)=0$ for a given wire height.

A graphical illustration of the process of determining $\varepsilon_{\mathrm{r}}$ and $\sigma$ from $\alpha$ and $\beta$ is found in Figures 13 and 14. The curves were generated by solving the forward problem (given $\varepsilon_{\mathrm{r}}$ and $\sigma$, solve $M\left(\gamma_{p}\right)=0$ for $\gamma_{p}$ and therefore for $\alpha / k$ and $\beta / k$ ) with the results plotted as $\alpha / k$ and $\beta / \mathrm{k}$ versus $\sigma$ with $\varepsilon_{\mathrm{r}}$ as a parameter for wire diameter of $3 / 16$ inches and height of $5 / 8$ inches at $10 \mathrm{MHz}$. An experimentally determined pair $\alpha / \mathrm{k}$ and $\beta / \mathrm{k}$ determine horizontal lines on Figures 13 and 14, and values for $\varepsilon_{\mathrm{r}}$ and $\sigma$ representing a solution must fall on these lines in both figures. If no such consistent solution can be found, the cause must be error in the measurement or the experimental parameters. While this graphical approach yields insight into the solution process and its sensitivity to errors, the actual solutions for $\varepsilon_{r}$ and $\sigma$ were obtained by purely numerical solution of the equation $M\left(\gamma_{p}\right)=0$.

Since $\alpha$ and $\beta$ were determined by fitting noisy measured data, and since it was possible that inaccurate values of $\varepsilon_{\mathrm{r}}$ and $\sigma$ could be obtained that were out of the physically possible range, constraints were imposed during the solution process. This increased efficiency and ensured physical realizability of the results. For the cases of interest, the solutions were constrained to lie in a range considered reasonable for concrete materials even with dense rebar inclusions, namely, $1<\varepsilon_{\mathrm{r}}<40$ and $0.0001<\sigma<0.01$.

The frequency range over which an accurate determination of the attenuation and phase constants was possible was constrained due to several causes. First, the spatial extent over which the current was probed was limited (approximately 26.7 meters) and, at low frequencies, the attenuation in the current was small and difficult to measure accurately. At high frequencies the current decayed quite rapidly so that the linear regions in the log magnitude and phase plots were quite small. In both cases, difficulties were encountered in accurately evaluating attenuation constants and phase factors. In the high frequency case, the LSQ fit was strongly dependent on the chosen range of fit with attendant uncertainties in the constants that were obtained. The behavior seen in Figures 11 and 12 is illustrative of the problem of the shrinking linear region even though, at $100 \mathrm{MHz}$, it is quite mild. On 


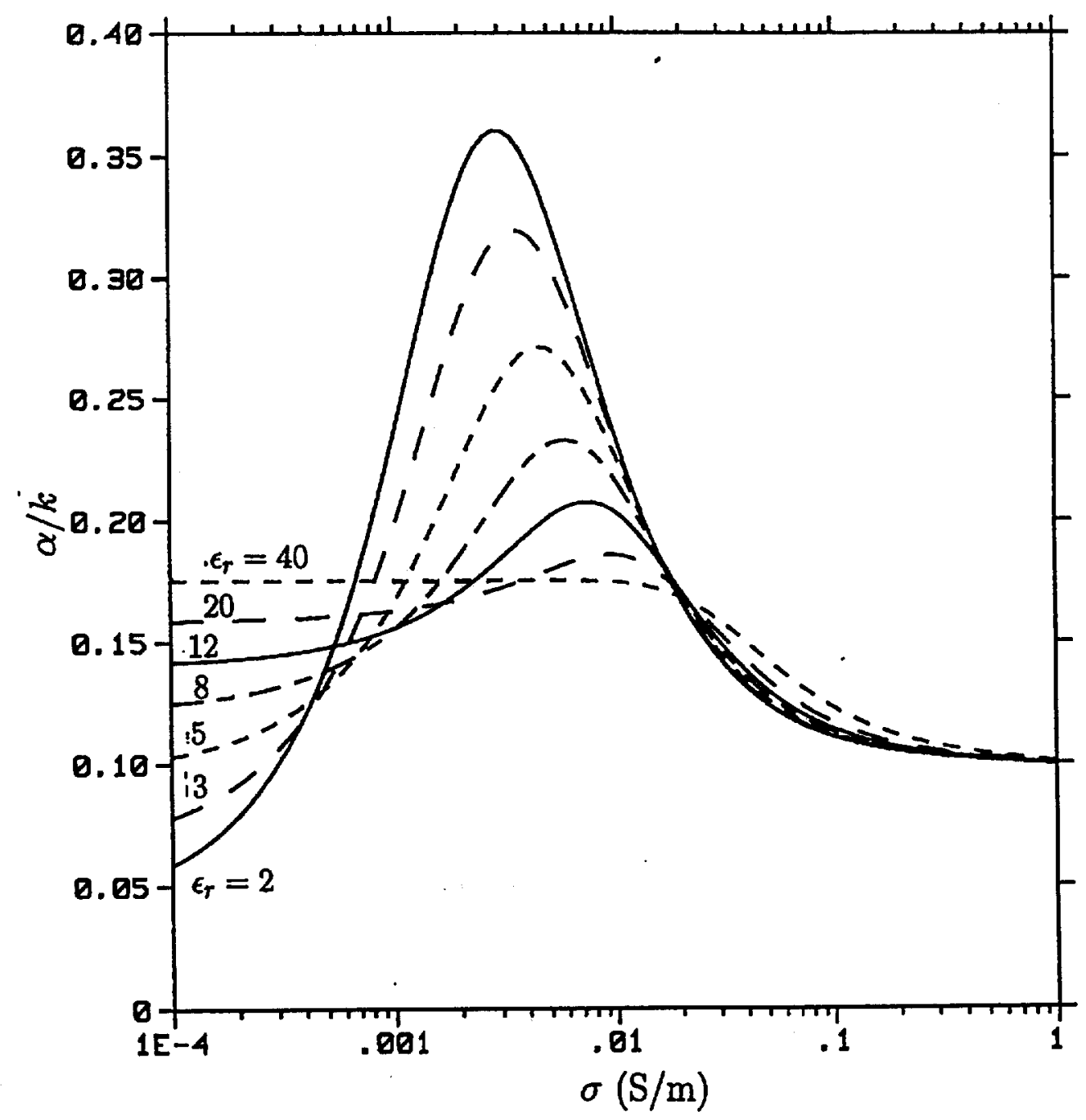

Figure 13. Normalized attenuation constant vs. conductivity for various $\varepsilon_{\mathrm{r}}$ at $10 \mathrm{MHz}$ 


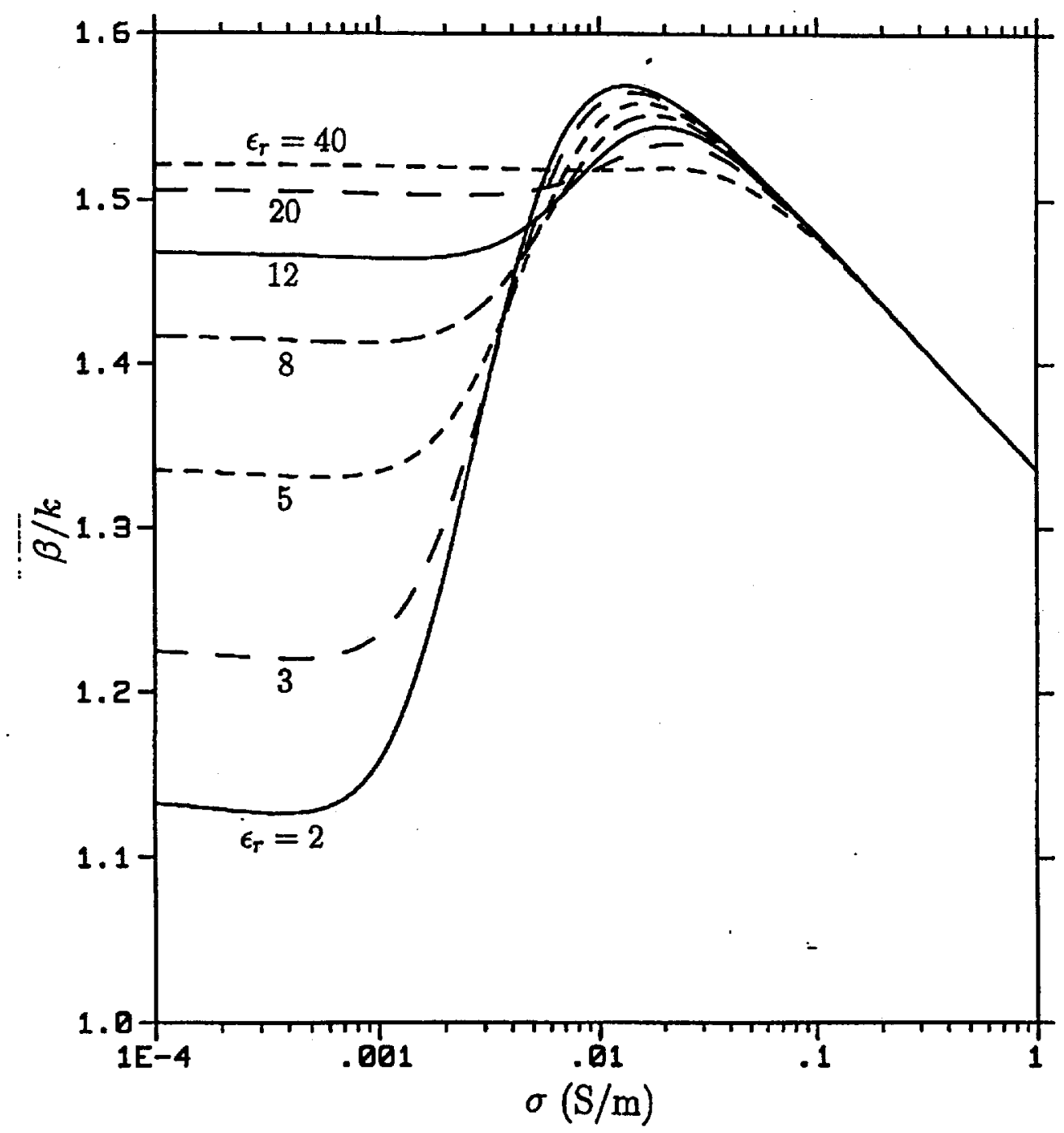

Figure 14. Normalized phase constant vs. conductivity for various $\varepsilon_{\mathrm{r}}$ at $10 \mathrm{MHz}$ 
the other hand, the behavior of the log magnitude of the current at $250 \mathrm{MHz}$ which is shown in Figure 15 leads to much greater difficulty in defining the linear region and thus leads to greater uncertainty in establishing the value for the attenuation constants. The phase constant determination is also difficult at high frequencies. Figure 16 is the phase plot over 17 points on the wire at $250 \mathrm{MHz}$. Clearly, the phase change per cell is quite large and errors are possible. Given the available data and the inability to redo portions of the experiment, a range was determined over which the uncertainties in the values for $\alpha$ and $\beta$ was considered small. For the purposes of our analysis this range was defined as approximately $5 \mathrm{MHz}<\mathrm{f}<150 \mathrm{MHz}$. The lower limit was also consistent with concerns of the experiment personnel regarding their ability to make accurate measurements on the pad in view of previous experience with adverse ground loops and other low frequency limitations. Figure 17 and 18 represent the normalized constants ( $\alpha / \mathrm{k}$ and $\beta / \mathrm{k}$ ) versus frequency as determined from the experimental data.

The data for $\alpha$ and $\beta$ were used to determine the effective relative dielectric constant and the conductivity of the half space beneath the wire. The process was reasonably robust in the area defined for confidence in estimates of attenuation and phase constants. Outside this range, the process might not return a solution ( values of $\varepsilon$ and $\sigma$ were not available which would simultaneously satisfy $M\left(\gamma_{p}\right)=0$ ). In some cases, "near" solutions were available in that a given value of $\varepsilon_{\mathrm{r}}$ would not yield a unique value of $\sigma$ but $\mathrm{M}(\gamma)$ was almost equal to zero for a range of $\sigma$. These data were not used in this analysis.

\section{Analysis}

In order to extend our estimates for $\varepsilon_{\mathrm{r}}$ and $\sigma$ beyond the range where reliable values of $\alpha$ and $\beta$ were determined, a theoretical model was used as the underpinning for the results. Such models have been developed in the literature and, for orientational-polarization effects, one has the Debye equations [7,8]

$$
\begin{aligned}
& \varepsilon_{\mathrm{r}}=\varepsilon_{\mathrm{rinf}}+\left(\varepsilon_{\mathrm{rs}}-\varepsilon_{\mathrm{rs}}\right) /\left(1+\omega^{2} \tau^{2}\right) \\
& \sigma=\omega \tau\left(\varepsilon_{\mathrm{rs}}-\varepsilon_{\mathrm{rs}}\right) /\left(1+\omega^{2} \tau^{2}\right)
\end{aligned}
$$

where $\varepsilon_{\text {rinf }}$ is the optical limit for the permittivity, $\varepsilon_{\mathrm{rs}}$ is the dc value, and $\tau$ is the orientational relaxation time for this model. Plots versus frequency of $\varepsilon_{\mathrm{r}}$ and $\sigma$ extracted from the 


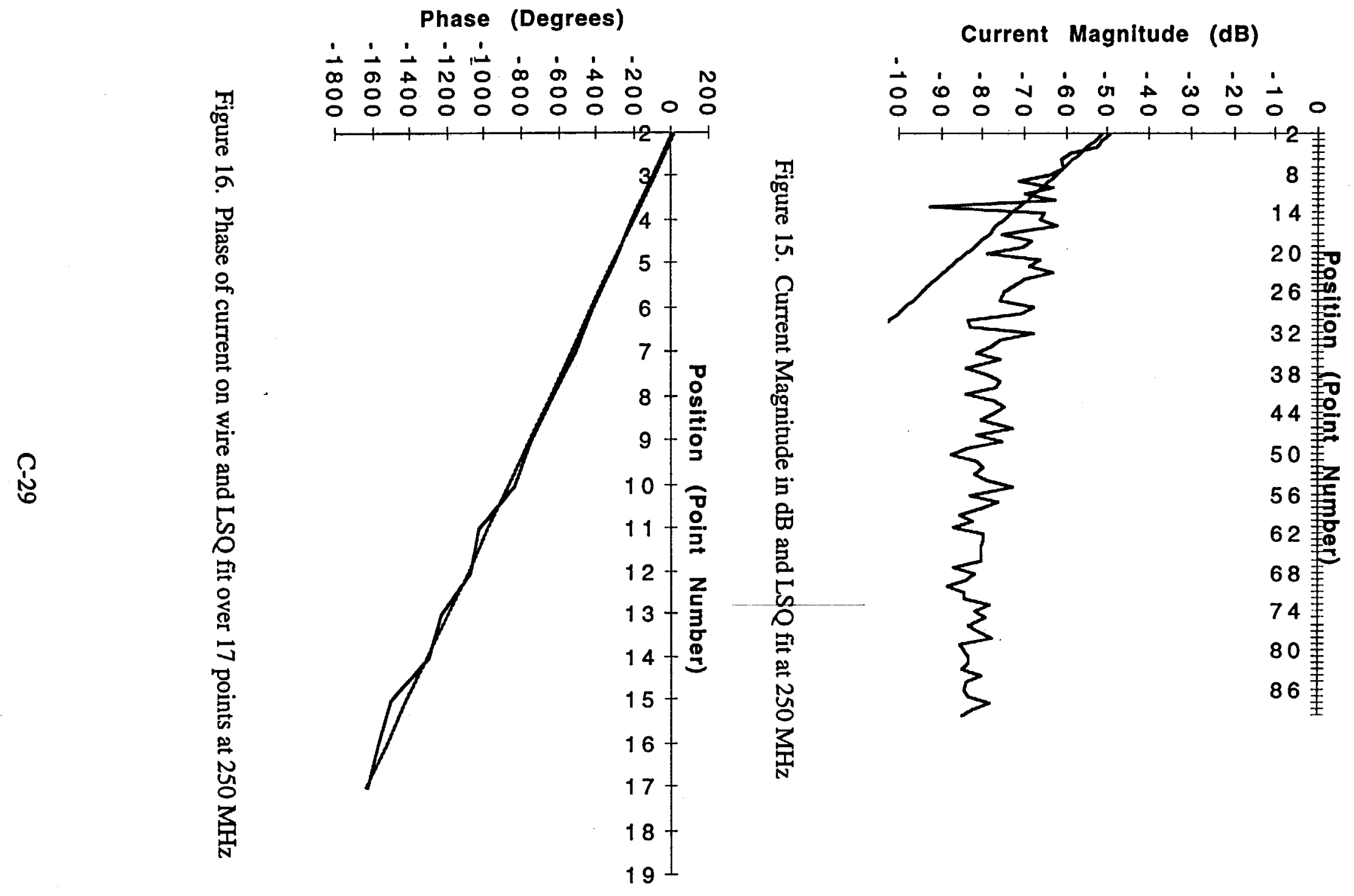




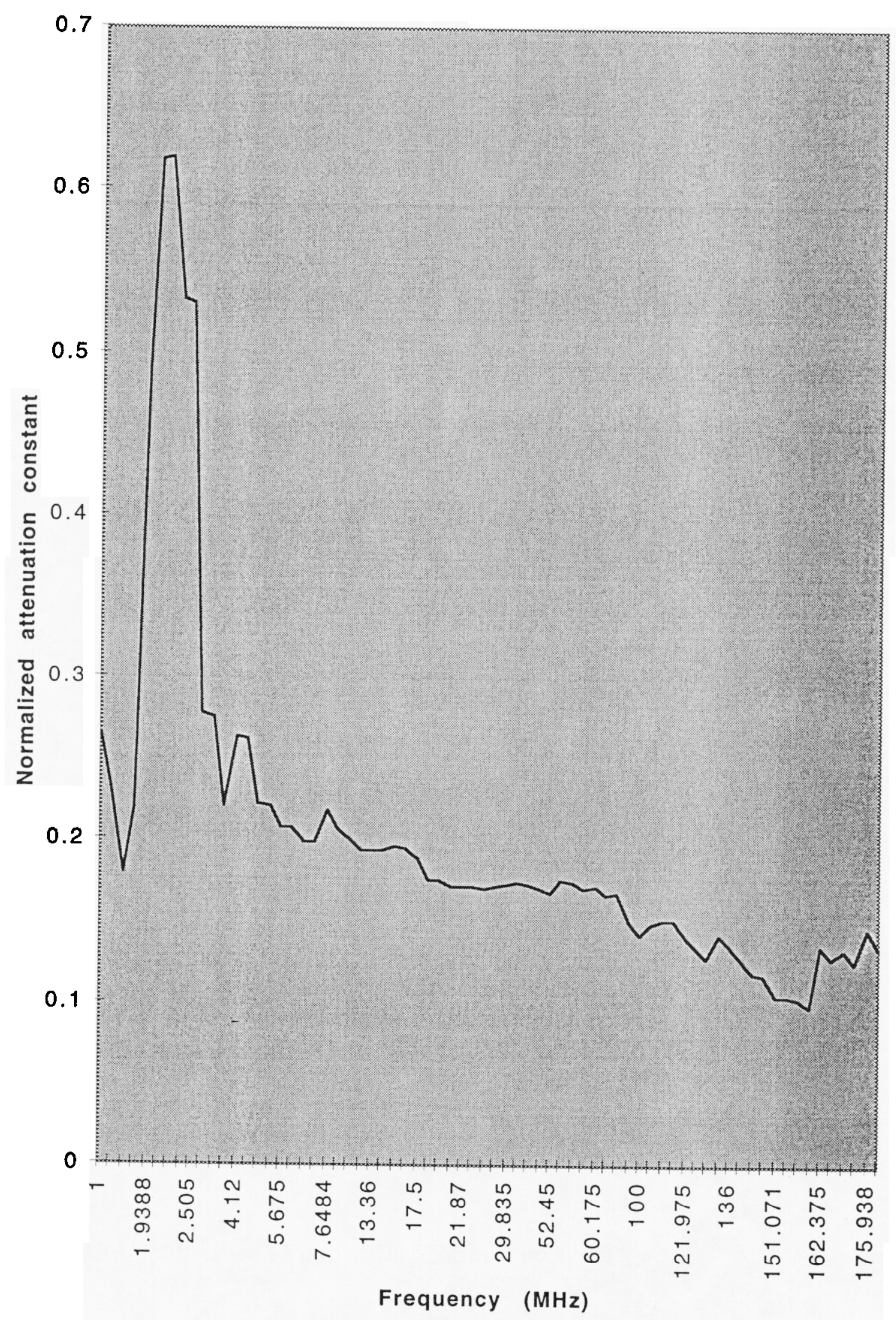

Figure 17. Normalized attenuation constant versus frequency 


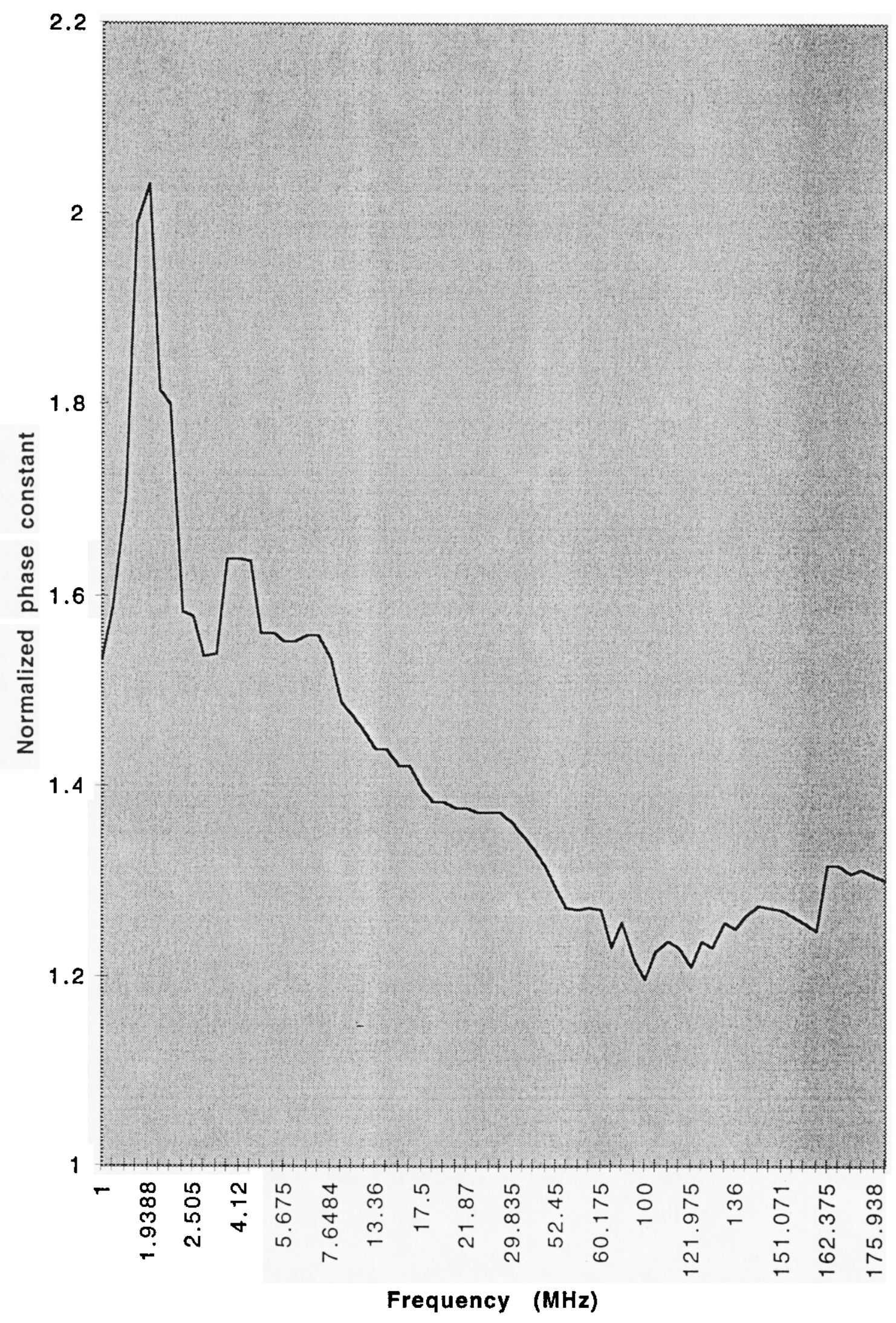

Figure 18. Normalized phase constant versus frequency 
measured current are shown in Figures 19 and 20, respectively. The anomalous behavior of $\sigma$ in the range 60 to $80 \mathrm{MHz}$ is not well understood. Also shown in each figure is a least squares fit to the model for the behavior of the constitutive parameters versus frequency. In the LSQ fits performed for the data in hand, the values of $\varepsilon_{\mathrm{rs}}, \varepsilon_{\mathrm{rinf}}$ and $\tau$ were not the same for the fits of the two sets of data. Clearly, such agreement for the two models would be expected for a perfect model as would $\varepsilon_{\mathrm{r}}$ and $\sigma$ pairs in agreement with the Kronig-Kramers relationships [9]. For the curves shown, however,

$$
\begin{aligned}
& \varepsilon_{\mathrm{r}}=4.2418+6.1943 /\left(1+1.129 \cdot 10^{-17} \cdot \omega^{2}\right) \\
& \sigma=4.416 \cdot 10^{-11} \cdot \omega /\left(1+1.354 \cdot 10^{-17} \cdot \omega^{2}\right) \text { for the full range of data } \\
& \sigma=3.6385 \cdot 10^{-11} \cdot \omega /\left(1+1.135 \cdot 10^{-17} \cdot \omega^{2}\right) \text { for the restricted data }
\end{aligned}
$$

The restricted data set is composed of the full range of data with frequencies below $18 \mathrm{MHz}$ excluded. The behavior of the permittivity over the frequency range shown is orderly and constrained while that of the conductivity is more variable. Also, the models seem to fit the behavior of the permittivity better than the conductivity. The impact of these issues on propagation in the upper half space is adequately reflected in the variation of the reflection coefficients with variations in the lower medium's wavenumber (or index of refraction, $\mathrm{n}$ ) which depends on $\varepsilon_{\mathrm{r}}$ and $\sigma$. This wavenumber arises repeatedly in the analyses and is a key determinant of the reflected and transmitted waves at the interface of the materials when a plane wave, for instance, is incident. The wavenumbers in the pad medium and free space, respectively, are given by

$$
\begin{aligned}
& k_{1}=k_{0} n=k_{0}\left[\varepsilon_{r}\left(1+i \sigma /\left(\omega \varepsilon_{0} \varepsilon_{r}\right)\right]^{1 / 2}\right. \\
& k=k_{0}=\omega\left[\mu_{0} \varepsilon_{0}\right]^{1 / 2}
\end{aligned}
$$

Two limiting types of propagation in the lower half space are possible, i.e., the conditions under which propagation is conduction or displacement eurrent dominated. Conduction current domination will occur when $\sigma \gg \omega \varepsilon_{0} \varepsilon_{\mathrm{r}}$ and will result in a propagation constant that is essentially independent of the relative dielectric constant in the ground, $\varepsilon_{\mathrm{r}}$. On the other hand, displacement current will dominate when $\sigma<\omega \varepsilon_{0} \varepsilon_{\mathrm{r}}$ and will result in propagation characteristics relatively insensitive to conductivity, $\sigma$, and frequency, $\omega$. The "boundary" between these two regions can be said to be approximately

$$
\sigma=\omega \varepsilon_{0} \varepsilon_{\mathrm{r}}
$$

Figure 21 shows such boundaries for frequencies of $f=10,20,50,100,400$, and 1000 $\mathrm{MHz}$. Also shown in the figure is a contour connecting elements of the set of $\{\varepsilon, \sigma\}$ pairs that have been determined experimentally. The sequencing, which is in ascending values 


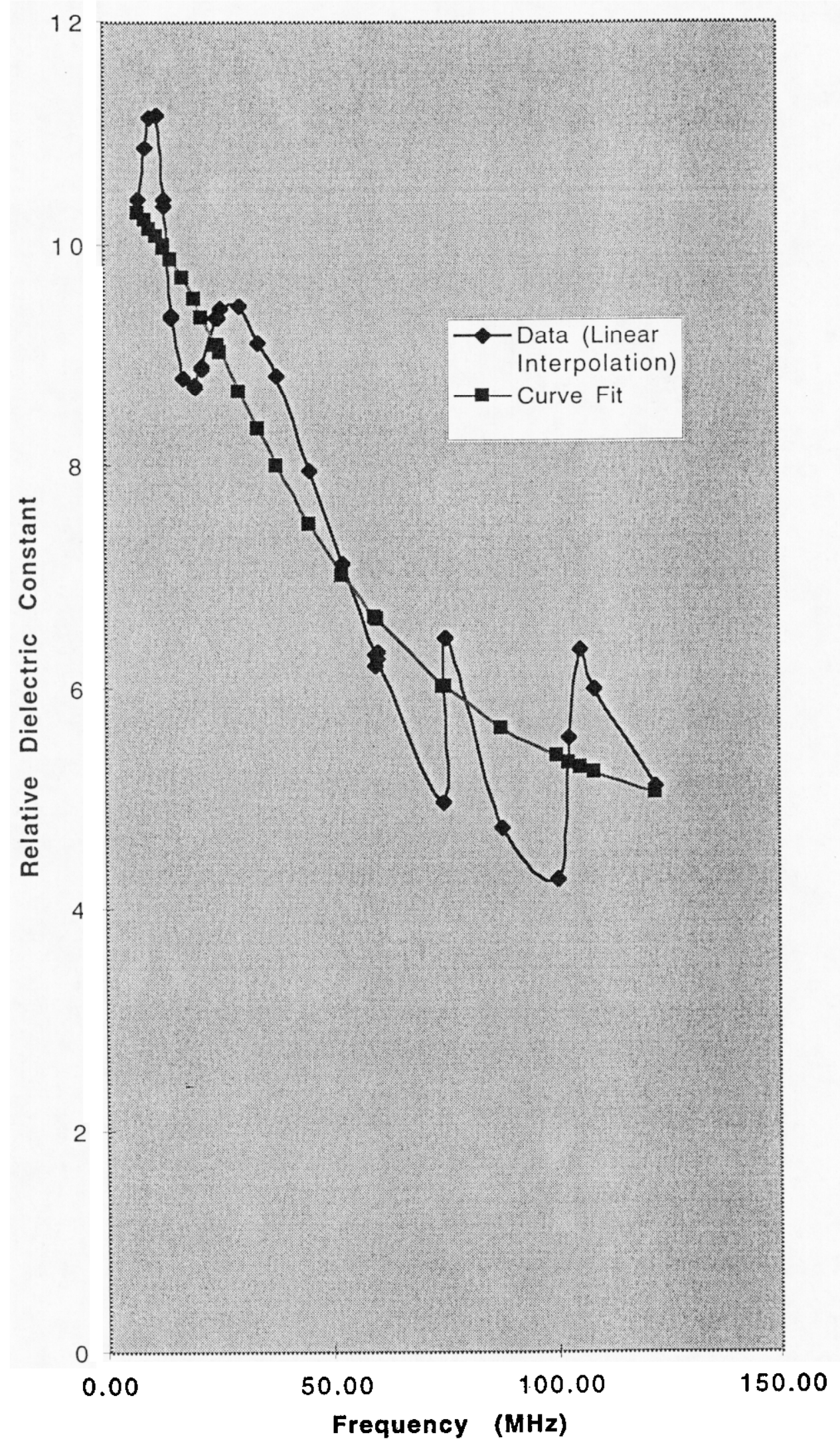

Figure 19. Relative dielectric constant versus frequency - experimental data and curve fit 


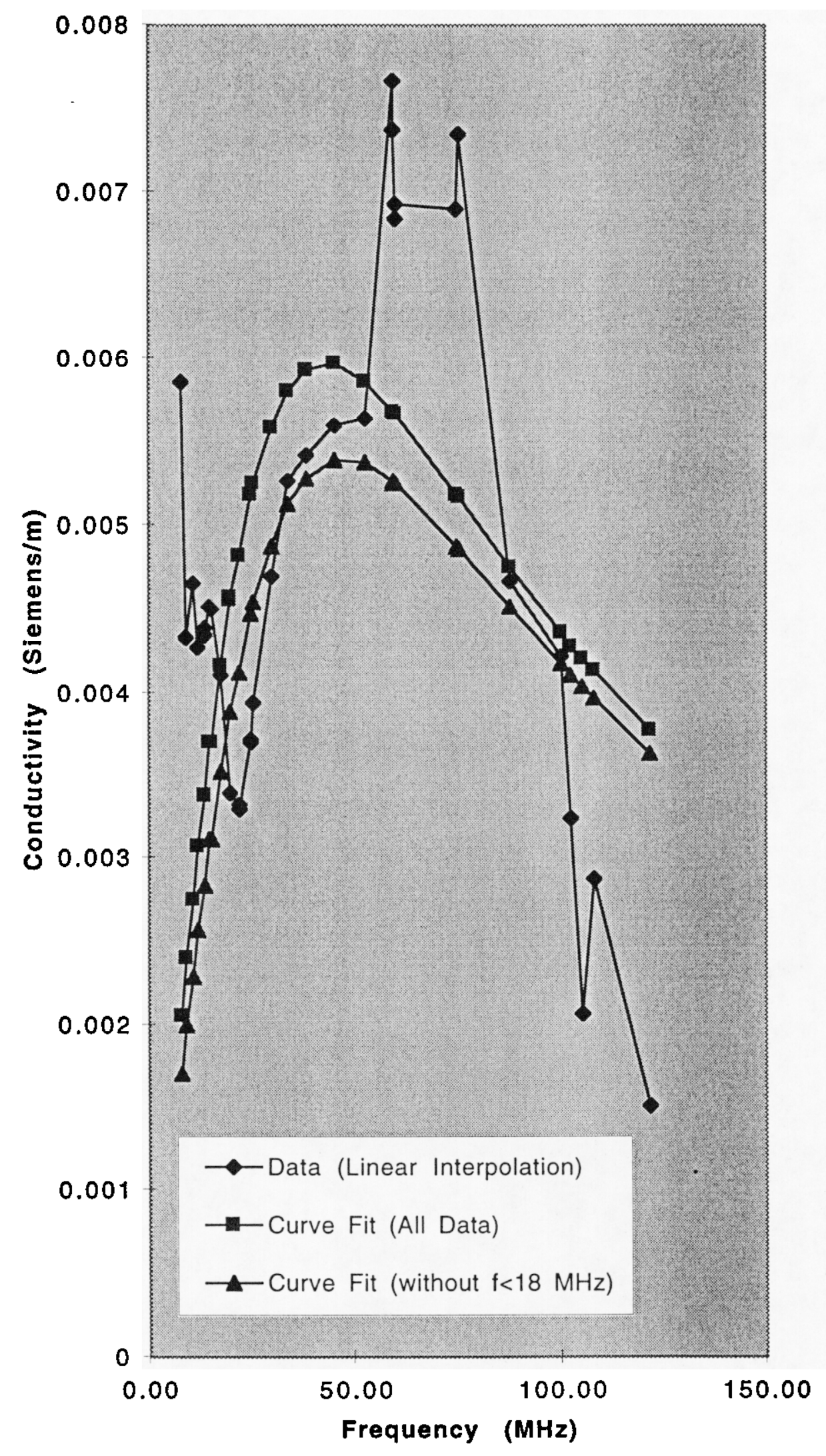

Figure 20. Conductivity versus frequency - experimental data and curve fits 


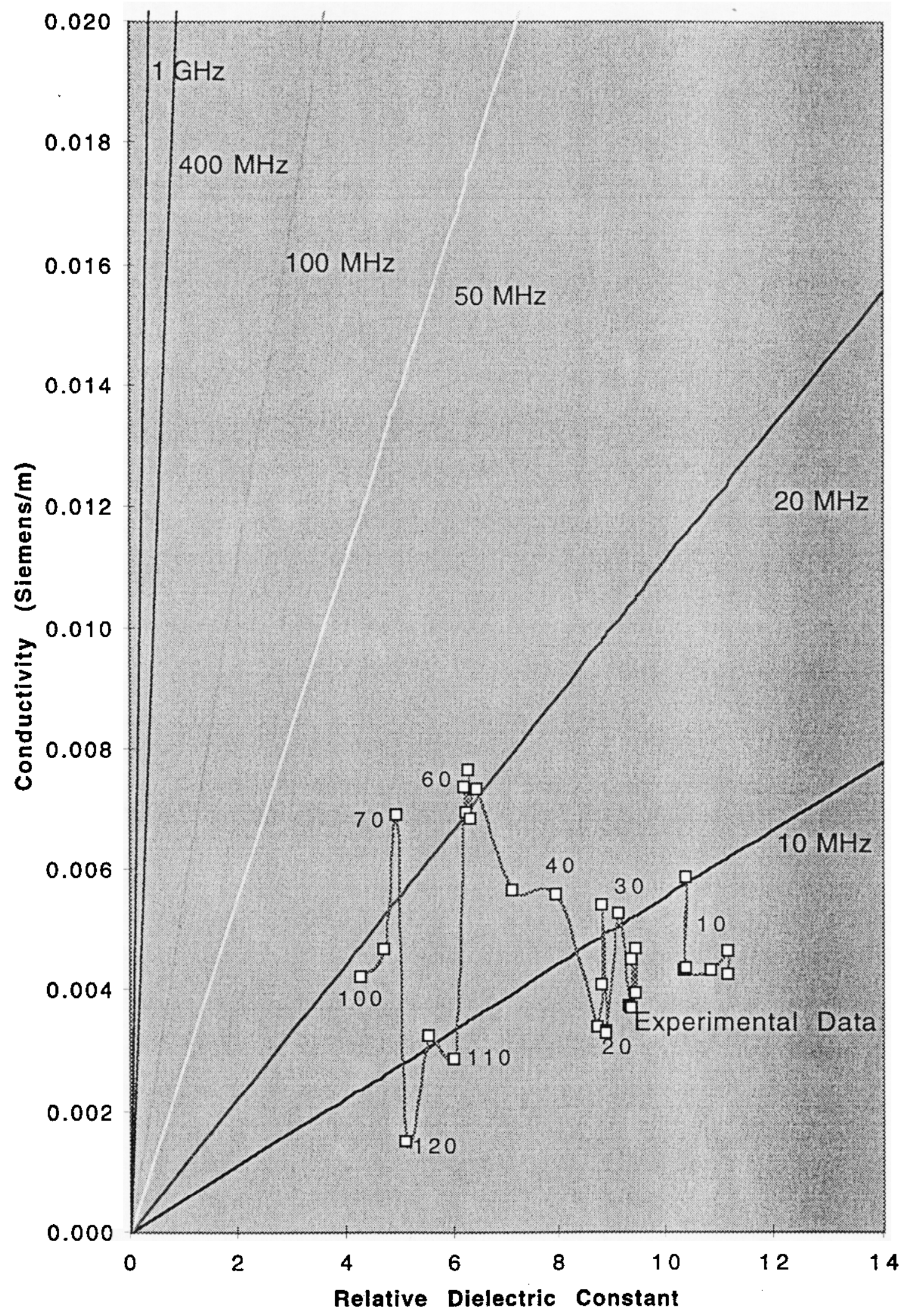

Figure 21. Displacement/Conduction boundaries and experimental $\left\{\varepsilon_{r}, \sigma\right\}$ with frequencies 
of conductivity, does not convey any physical information. The important feature to be observed in the figure is the range of the $\{\varepsilon, \sigma]$ set and the fact that the propagation is displacement current dominated at frequencies above about 20 to $50 \mathrm{MHz}$. Another feature is that for the material under study, using average values over the frequency range $\left(f_{1}, f_{2}\right)$ of Equations (13) and (14),

$$
\begin{aligned}
& <\varepsilon_{r}\left(f_{1}, f_{2}\right)>=4.24+2.93 \cdot 10^{8} \cdot\left[\tan ^{-1}\left(2.11 \cdot 10^{8} \cdot f_{2}\right)-\tan ^{-1}\left(2.11 \cdot 10^{8} \cdot f_{1}\right)\right] /\left(f_{2}-f_{1}\right) \\
& <\sigma\left(f_{1}, f_{2}\right)>=2.595 \cdot 10^{5} \cdot \ln \left[\left(1+5.29 \cdot 10^{-16} \cdot f_{2}{ }^{2}\right) /\left(1+5.29 \cdot 10^{-16} \cdot f_{1}{ }^{2}\right)\right] /\left(f_{2}-f_{1}\right)
\end{aligned}
$$

one finds that average values of $\varepsilon_{\mathrm{r}} \sim 7$ and $\sigma \sim 0.005$ appear to be reasonable over the 10 to $100 \mathrm{MHz}$ range. Of course, for frequencies above $50 \mathrm{MHz}$ the values of the conductivity may be of secondary importance.

To further support the observations based on the behavior of the propagation constant or wavenumber in the pad material, attention was directed towards the Fresnel reflection coefficients at the pad interface. These reflection coefficients are given by

$$
\begin{aligned}
& R_{H}=\left[\mu_{1} k_{0} \cos \theta-\mu_{0}\left(k_{1}{ }^{2}-k_{0}{ }^{2} \sin ^{2} \theta\right)^{1 / 2}\right] /\left[\mu_{1} k_{0} \cos \theta+\mu_{0}\left(k_{1}{ }^{2}-k_{0}{ }^{2} \sin ^{2} \theta\right)^{1 / 2}\right] \\
& R_{E}=\left[\mu_{0} k_{1}{ }^{2} \cos \theta-\mu_{1} k_{0}\left(k_{1}{ }^{2}-k_{0}{ }^{2} \sin ^{2} \theta\right)^{1 / 2}\right] /\left[\mu_{0} k_{1}{ }^{2} \cos \theta+\mu_{1} k_{0}\left(k_{1}{ }^{2}-k_{0}{ }^{2} \sin ^{2} \theta\right)^{1 / 2}\right]
\end{aligned}
$$

with $\theta$ measured from the normal to the surface, the subscript $E$ denoting the electric field vector in the plane of incidence, subscript $\mathrm{H}$ denoting the electric field vector perpendicular to the plane of incidence, and the subscript 1 denoting the subsurface material (concrete, steel, soil, etc.). Figures 22 to 26 are contour plots of the reflection coefficient magnitude in $\varepsilon_{\mathrm{r}}-\sigma$ space for select frequencies in the range of interest. The values of the contours (magnitude of $\mathrm{R}$ ) are not as important and are not shown. Rather, the curves are meant to convey that the reflection coefficients (both $R_{E}$ and $R_{H}$ ) for the range of incidence angles are mainly functions of $\varepsilon_{R}$ and are therefore displacement current dominated. The implication is that for frequencies greater than about $32 \mathrm{MHz}$ (or thereabouts), the precise definition of $\sigma$ is not as important as an accurate definition of $\varepsilon_{R}$.

The expressions used in the LSQ fits (Equations (13) and (14)) were plotted parametrically over the range of $0 \mathrm{MHz}$ to $1 \mathrm{GHz}$. A version of Figure 21 with the LSQ-fit curve is shown in Figure 27. The fit is reasonable for the purposes of understanding the impact of $\left\{\varepsilon_{r}, \sigma\right\}$. Clearly, the constitutive parameter values lead to strong displacement current 
$16 \mathrm{MHz}$

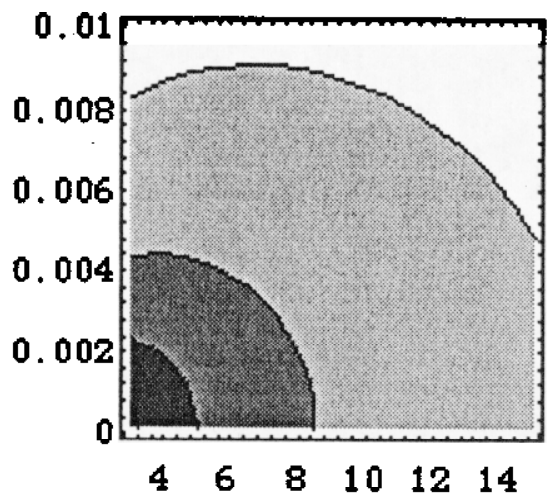

$64 \mathrm{MHz}$

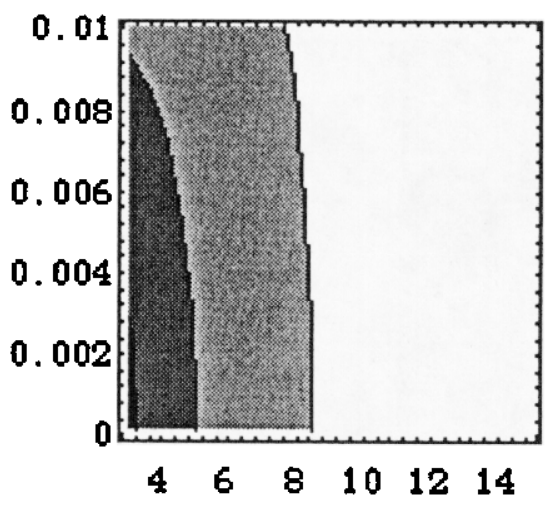

$256 \mathrm{MHz}$

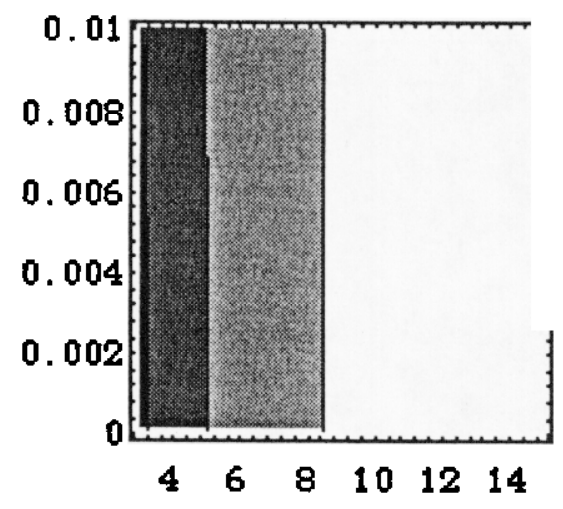

$32 \mathrm{MHz}$

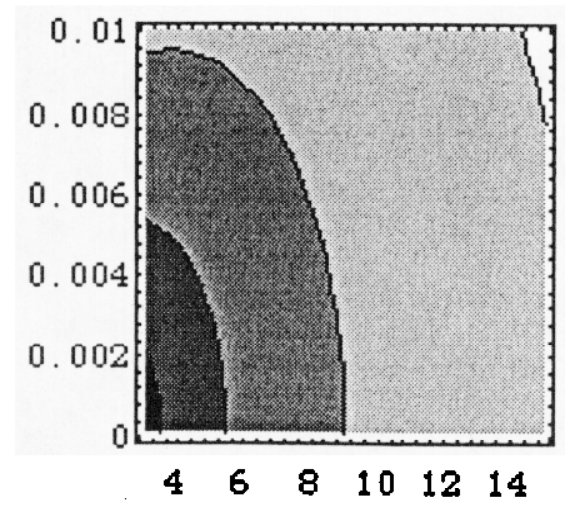

$128 \mathrm{MHz}$

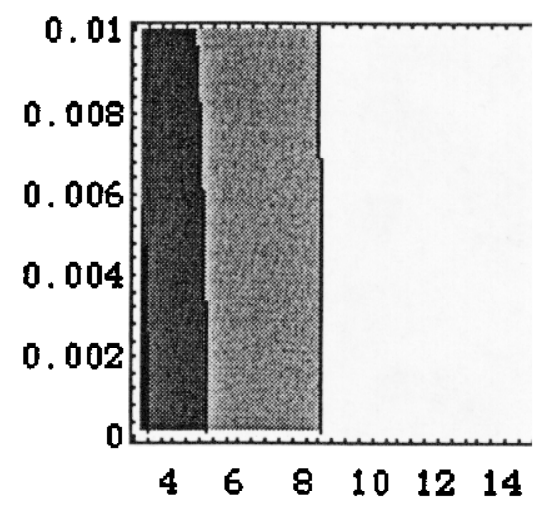

$512 \mathrm{MHz}$

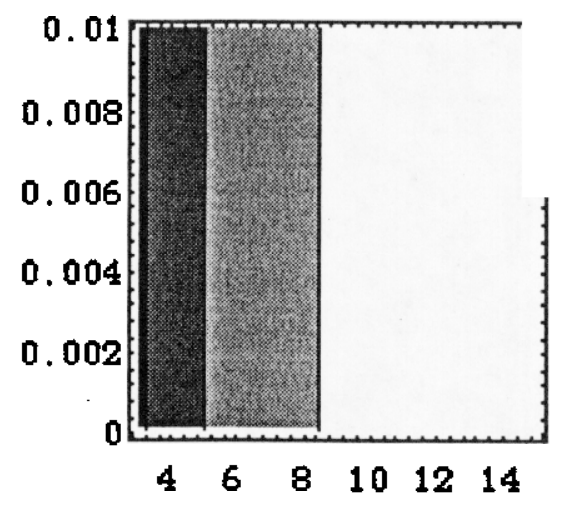

Figure 22. $R_{E}=R_{H}$ for normal incidence $(\theta=0)$ (Vertical axes are conductivity in Siemens/m and horizontal axes are relative dielectric constant) 
$16 \mathrm{MHz}$

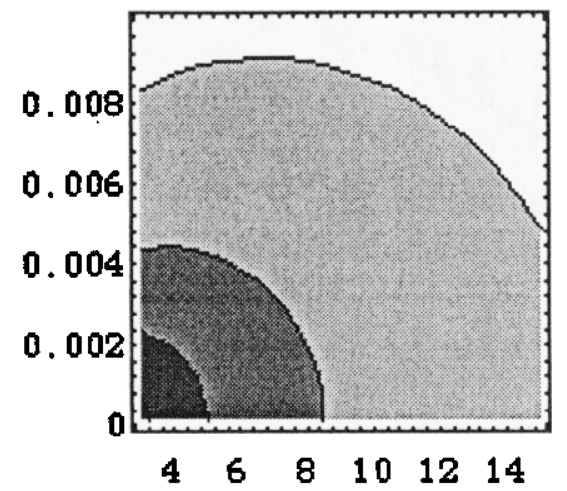

$64 \mathrm{MHz}$

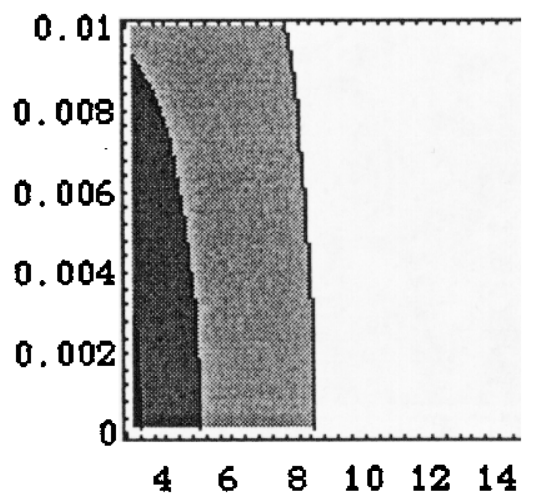

$256 \mathrm{MHz}$

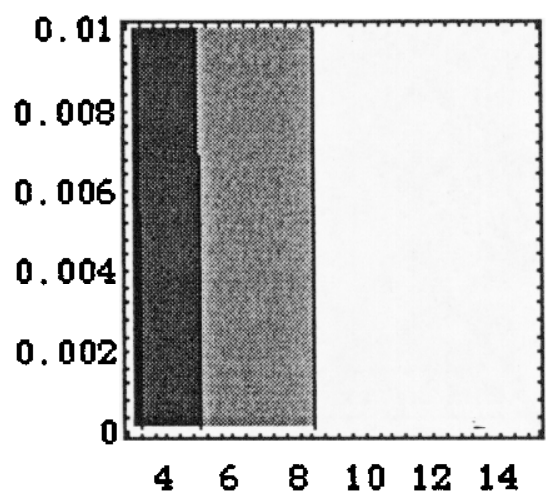

$32 \mathrm{MHz}$

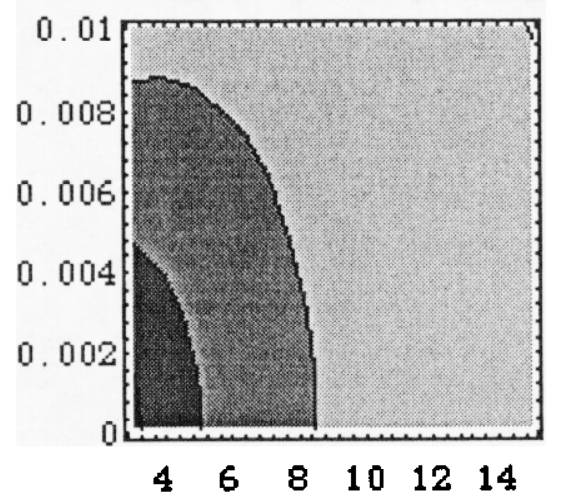

$128 \mathrm{MHz}$

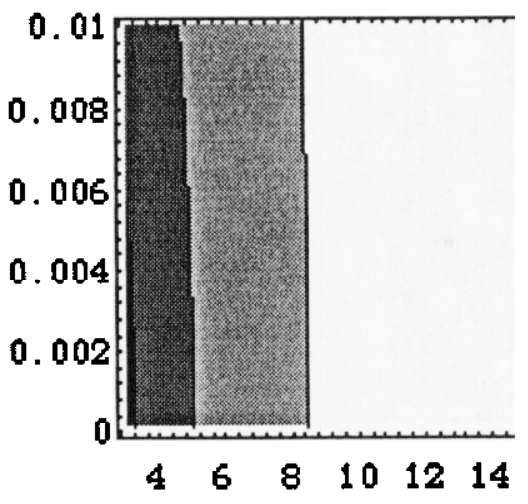

$512 \mathrm{MHz}$

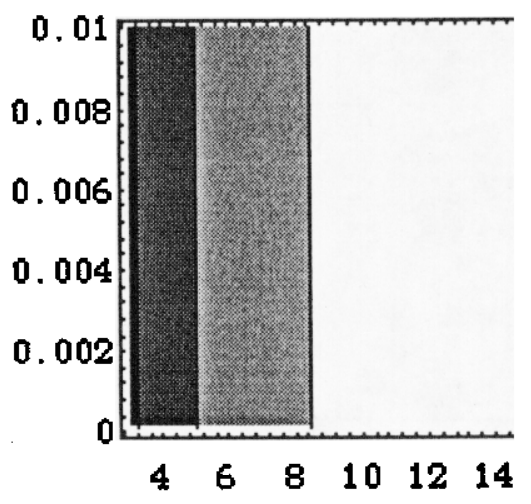

Figure 23. $R_{\mathrm{H}}$ for incidence at 45 degrees (Vertical axes are conductivity in Siemens/m and horizontal axes are relative dielectric constant) 
$16 \mathrm{MHz}$

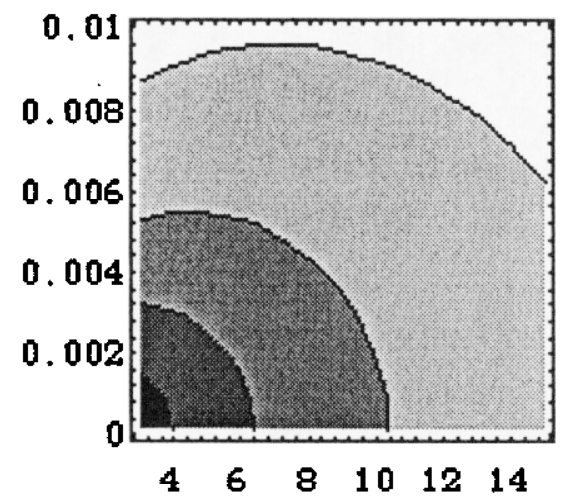

$64 \mathrm{MHz}$

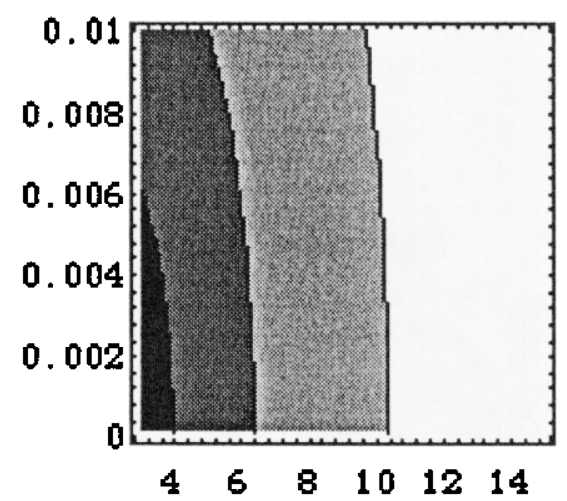

$256 \mathrm{MHz}$

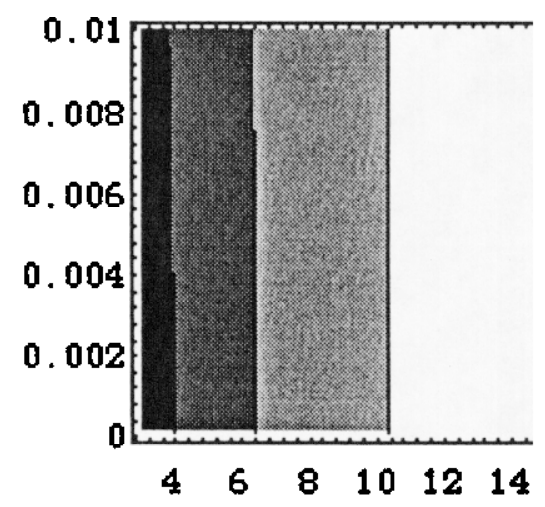

$32 \mathrm{MHz}$

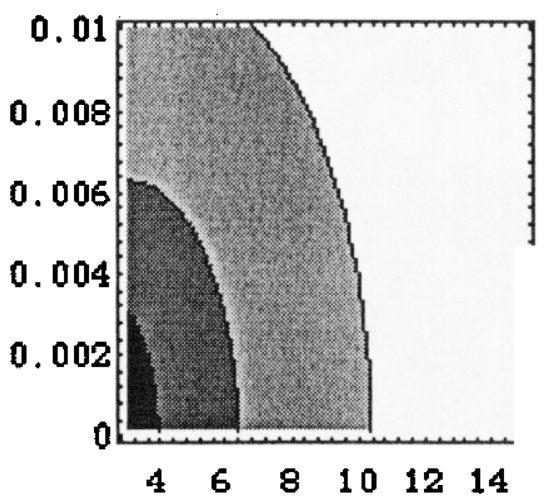

$128 \mathrm{MHz}$

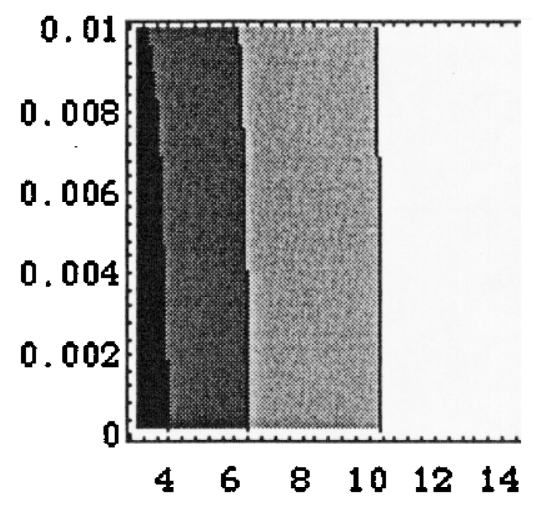

$512 \mathrm{MHz}$

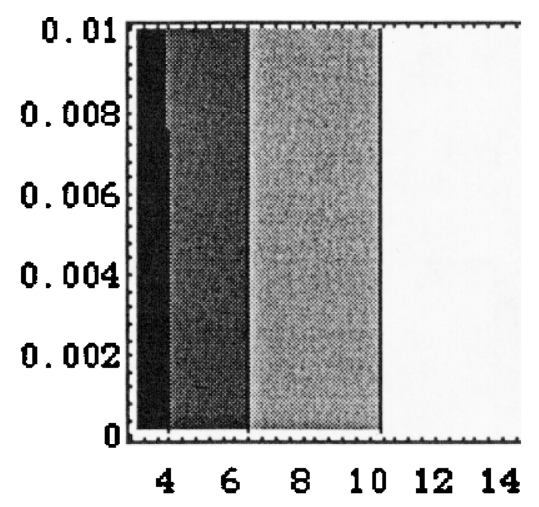

Figure 24. $R_{E}$ for incidence at 45 degrees (Vertical axes are conductivity in Siemens/m and horizontal axes are relative dielectric constant) 
$16 \mathrm{MHz}$

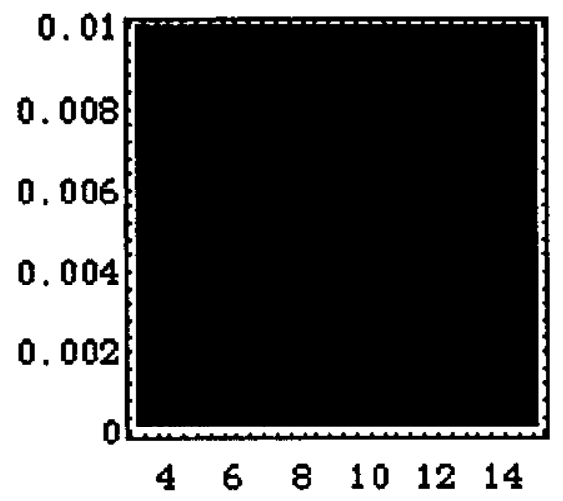

$64 \mathrm{MHz}$

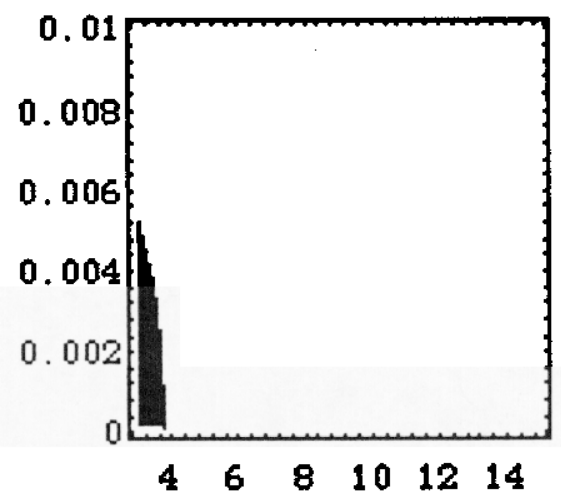

$256 \mathrm{MHz}$

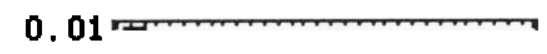

0.008

0.006

0.004

0.002

$\begin{array}{llllll}4 & 6 & 8 & 10 & 12 & 14\end{array}$
$32 \mathrm{MHz}$

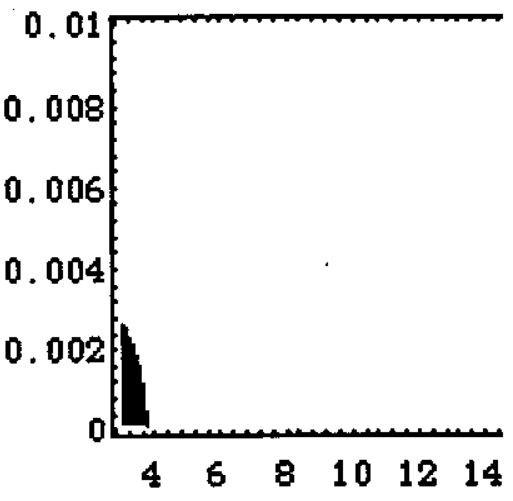

$128 \mathrm{MHz}$

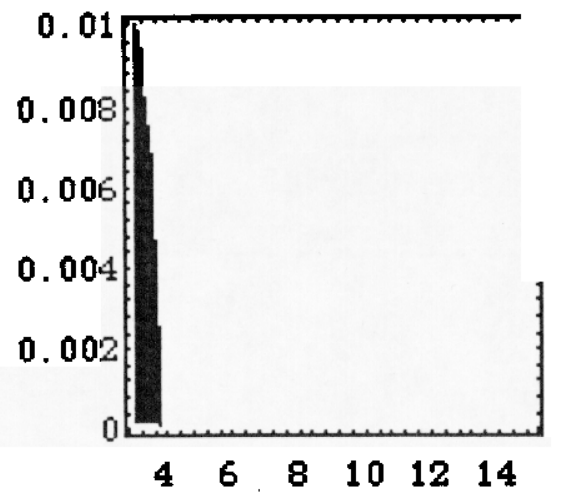

$512 \mathrm{MHz}$

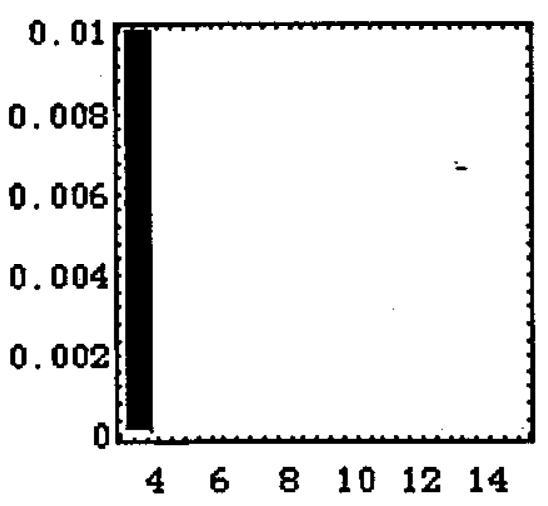

Figure 25. $R_{H}$ for grazing angle of incidence ( 85 degrees from normal incidence) (Vertical axes are conductivity in Siemens/m and horizontal axes are relative dielectric constant) 
$16 \mathrm{MHz}$

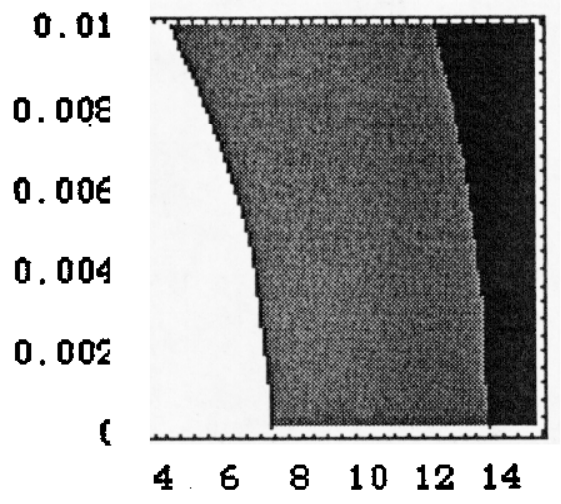

$64 \mathrm{MHz}$

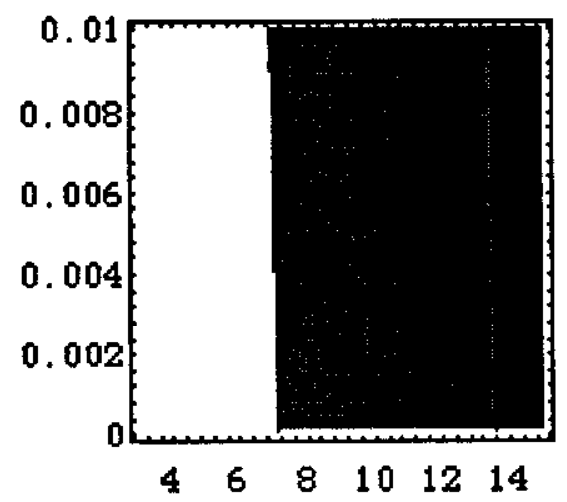

$256 \mathrm{MHz}$

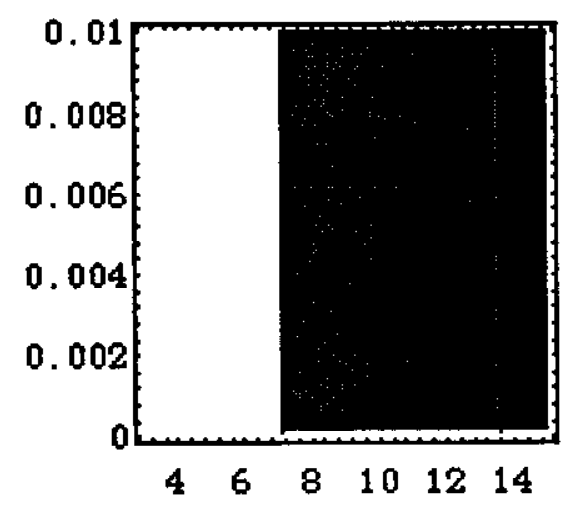

$32 \mathrm{MHz}$

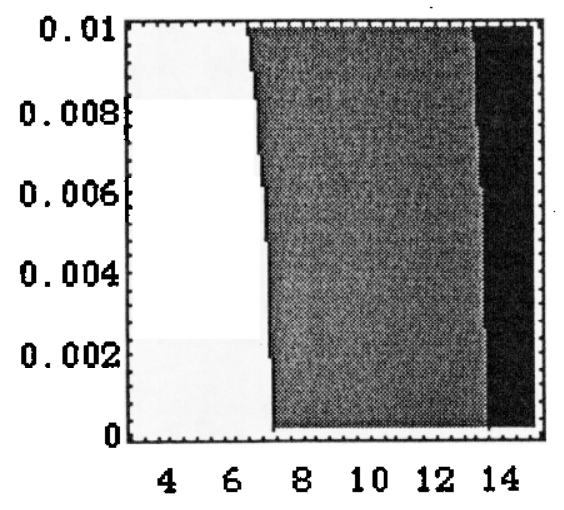

$128 \mathrm{MHz}$

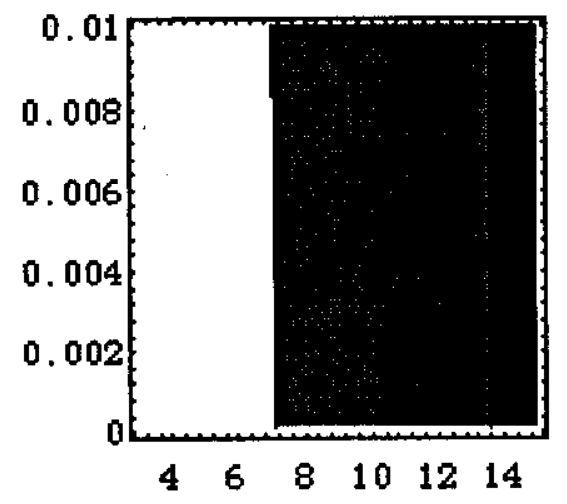

$512 \mathrm{MHz}$

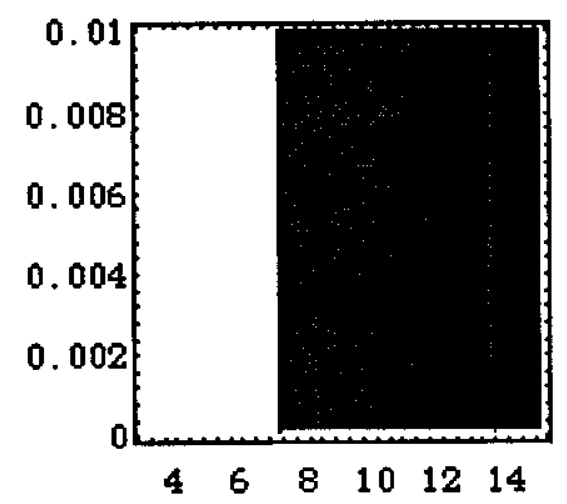

Figure 26. $R_{E}$ for grazing angle of incidence ( 85 degrees from normal incidence) (Vertical axes are conductivity in Siemens/m and horizontal axes are relative dielectric constant) 


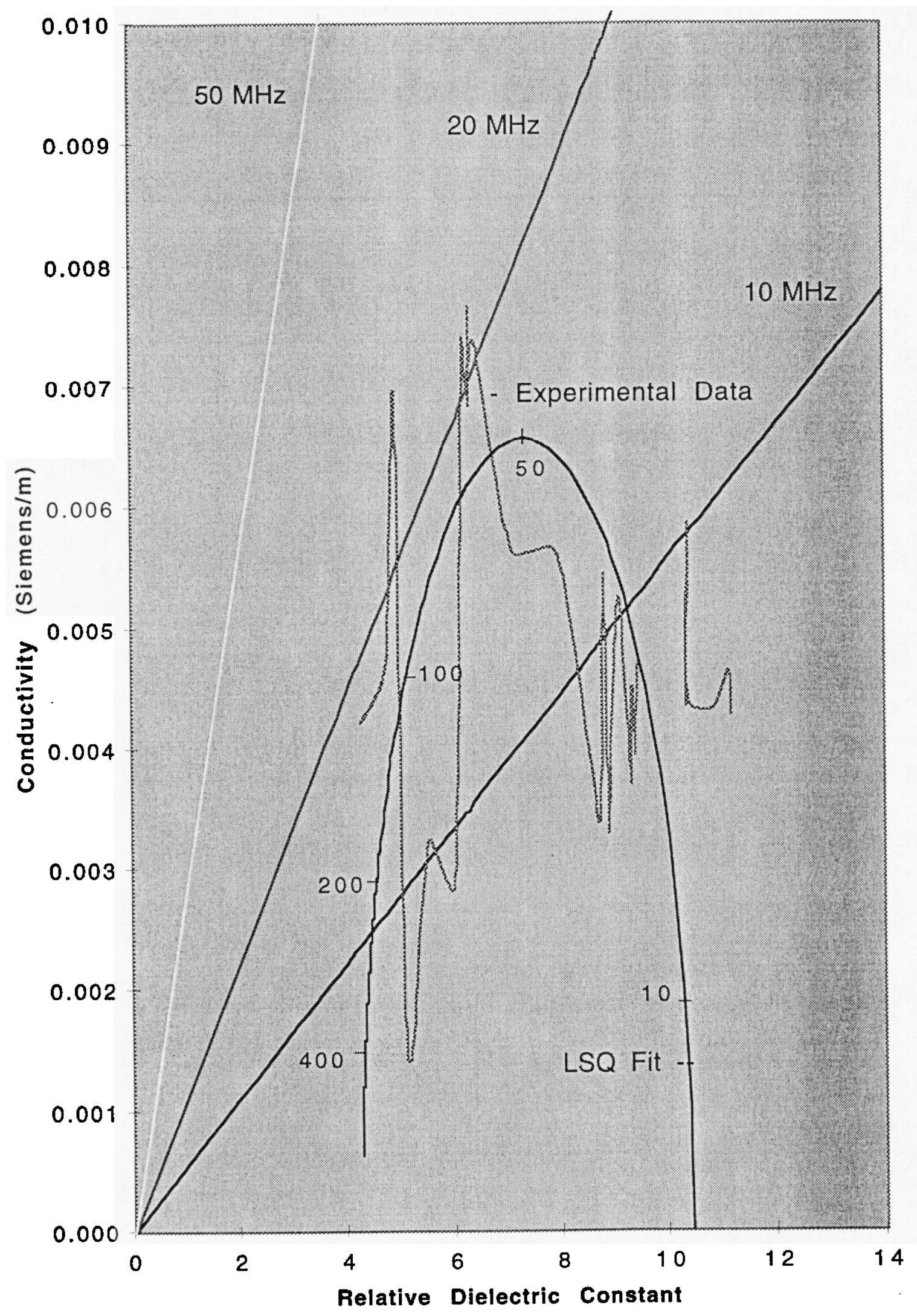

Figure 27. Experimental data and LSQ fit for the constitutive parameters 
effects and the system is generally displacement current dominated. Plots of the relative dielectric constant and conductivity are shown in Figures 28 and 29 for frequencies up to about $300 \mathrm{MHz}$ to illustrate that the region of variability of the parameters is rather confined.

Another indicator of the importance of conduction or displacement current effects is the index of refraction for the medium, i.e.,

$$
\mathrm{n}=\left[\varepsilon_{\mathrm{r}}+\mathrm{i} \sigma /\left(\omega \mathrm{e}_{0}\right)\right]^{1 / 2}
$$

Clearly, $\sigma$ becomes unimportant when $\mathrm{n}$ can be adequately represented by

$$
\mathrm{n} \sim\left[\varepsilon_{\mathrm{r}}\right]^{1 / 2}
$$

Figure 30 shows curves representing the magnitude of Equations (19) and (20) and illustrates that above about $40 \mathrm{MHz}$, the differences are less than 3\%. Above about 150 $\mathrm{MHz}$, the index of refraction has effectively lost its dependence on frequency, and above $100 \mathrm{MHz}$, it has lost its strong dependency on $\sigma$. The index of refraction is, however, strongly affected by the values used for the relative dielectric constant throughout the entire frequency range.

Finally, the magnitude of the reflection coefficient was considered as a measure of the importance of $\sigma$. For our purposes, only the normal incidence coefficient was evaluated using the index of refraction in Equation (19) and the approximation $(\sigma=0)$ given by Equation (20). Figure 31 is a plot of the two representations for $R_{E}=R_{H}$ for normal incidence. The deviation in the magnitude is less than about $5 \%$ for all frequencies. The reflection coefficient is effectively independent of frequency above $150 \mathrm{MHz}$ and nearly so above $80 \mathrm{MHz}$. It is nearly independent of $\sigma$ above $50 \mathrm{MHz}$.

The previous discussions have considered only the relative importance of $\varepsilon_{\mathrm{r}}$ and $\sigma$ in the determination of parameters defining wave propagation in the medium. In the following we consider the magnitude of the index of refraction evaluated from the constitutive parameters resulting from our measurements, and look at its comparison to the magnitude of the index obtained from our models for the constitutive parameters based on LSQ fits to our data. Figure 32 is a plot of the magnitude of $n$ versus frequency using the model for $\varepsilon_{\mathrm{r}}$ and $\sigma$ as compared to the magnitude as determined from $\varepsilon_{\mathrm{r}}$ and $\sigma$ obtained from processing 


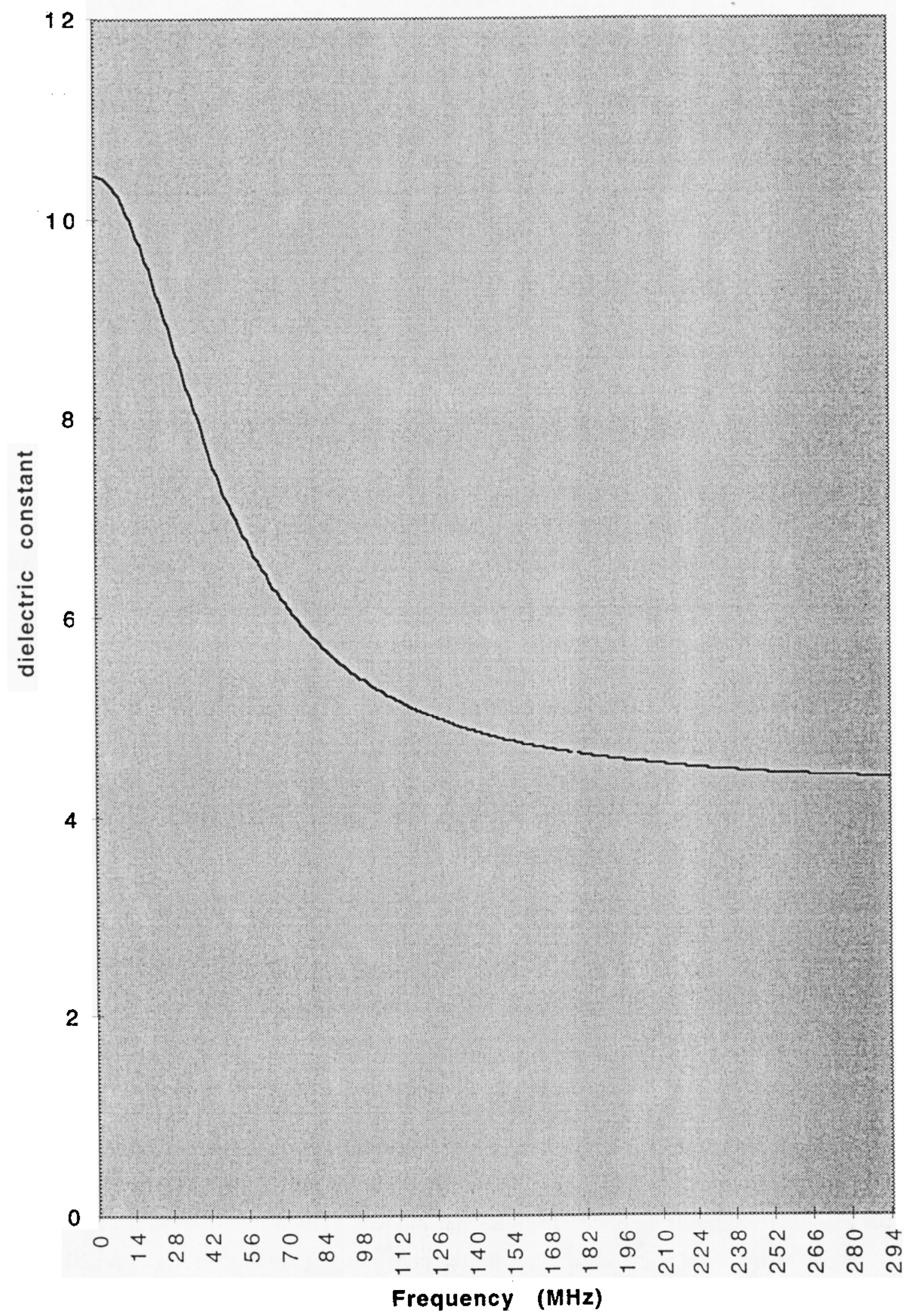

Figure 28. Relative dielectric constant versus frequency per Equation (13) 


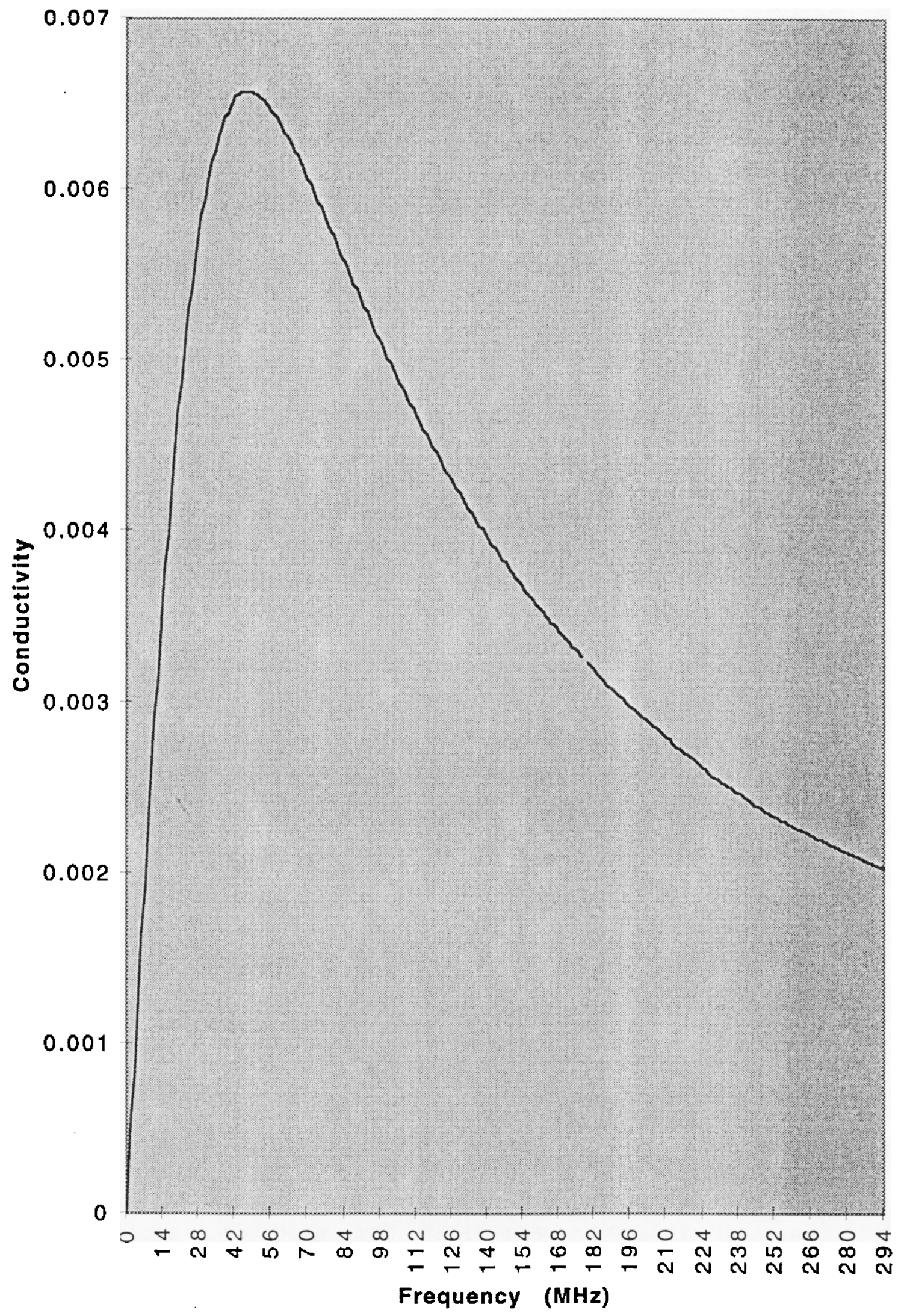

Figure 29. Conductivity (Siemens/m) versus frequency per Equation (14) 


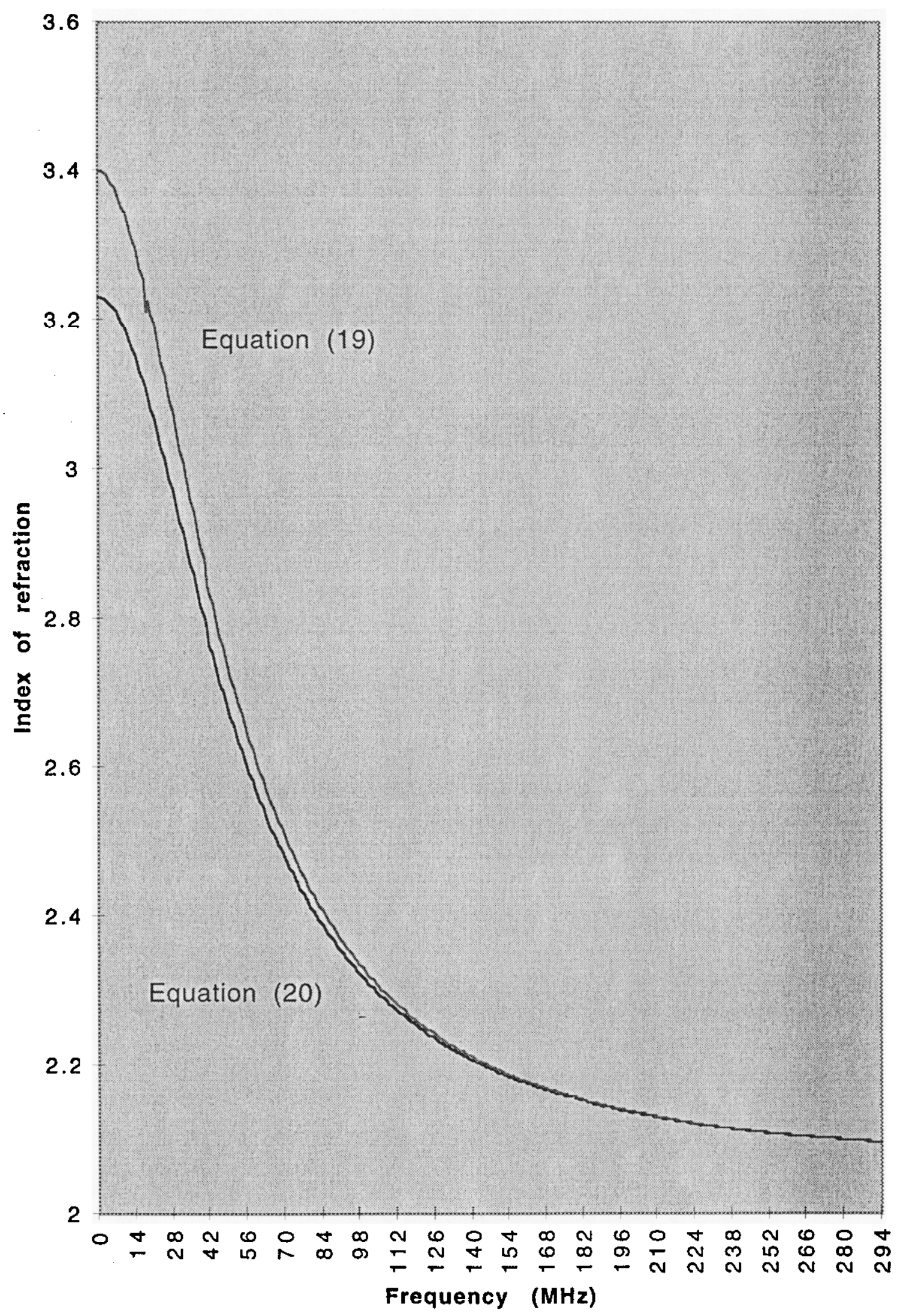

Figure 30. Two representations for the index of refraction versus frequency 


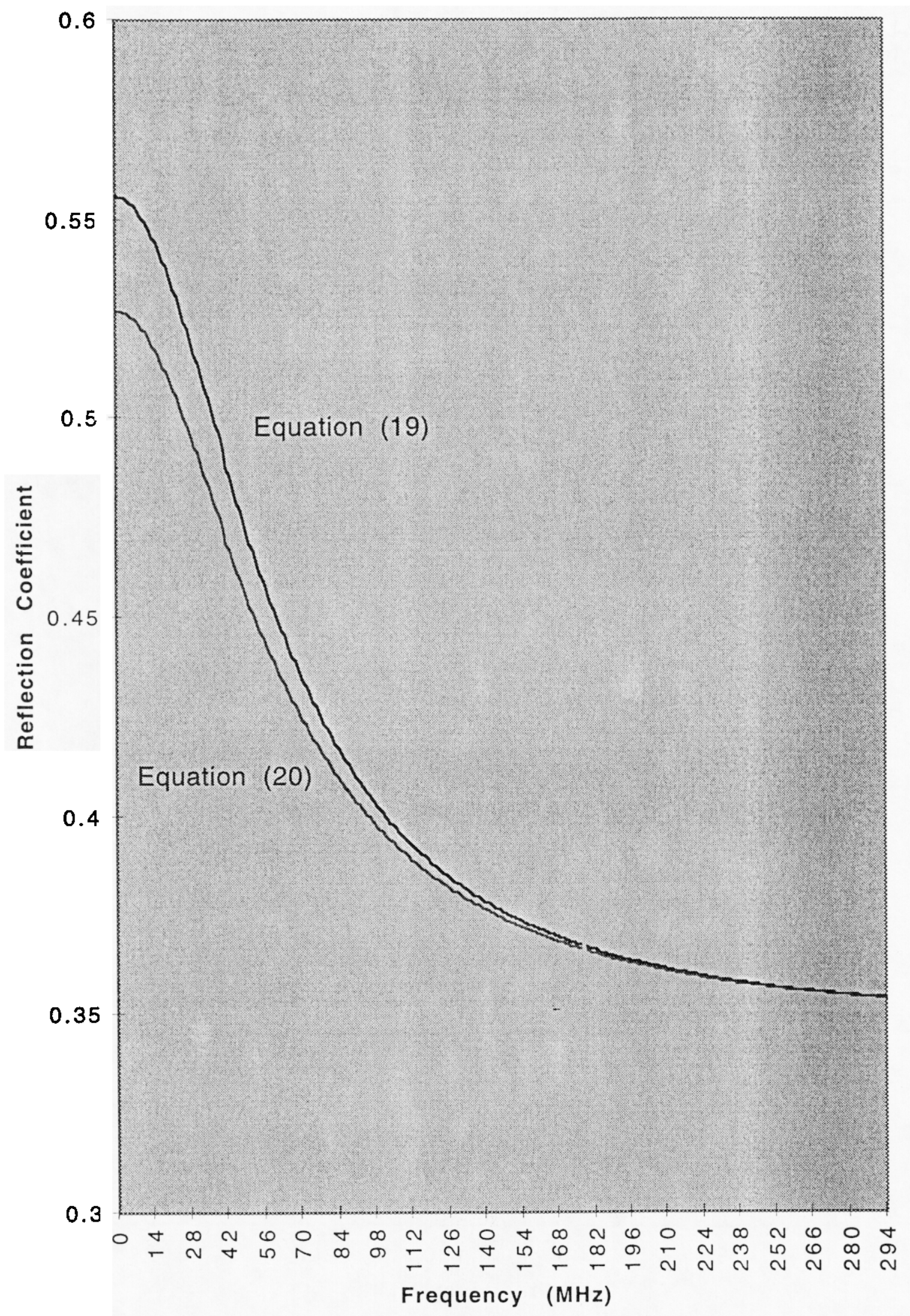

Figure 31. Two representations for the normal incidence reflection coefficient vs. frequency 


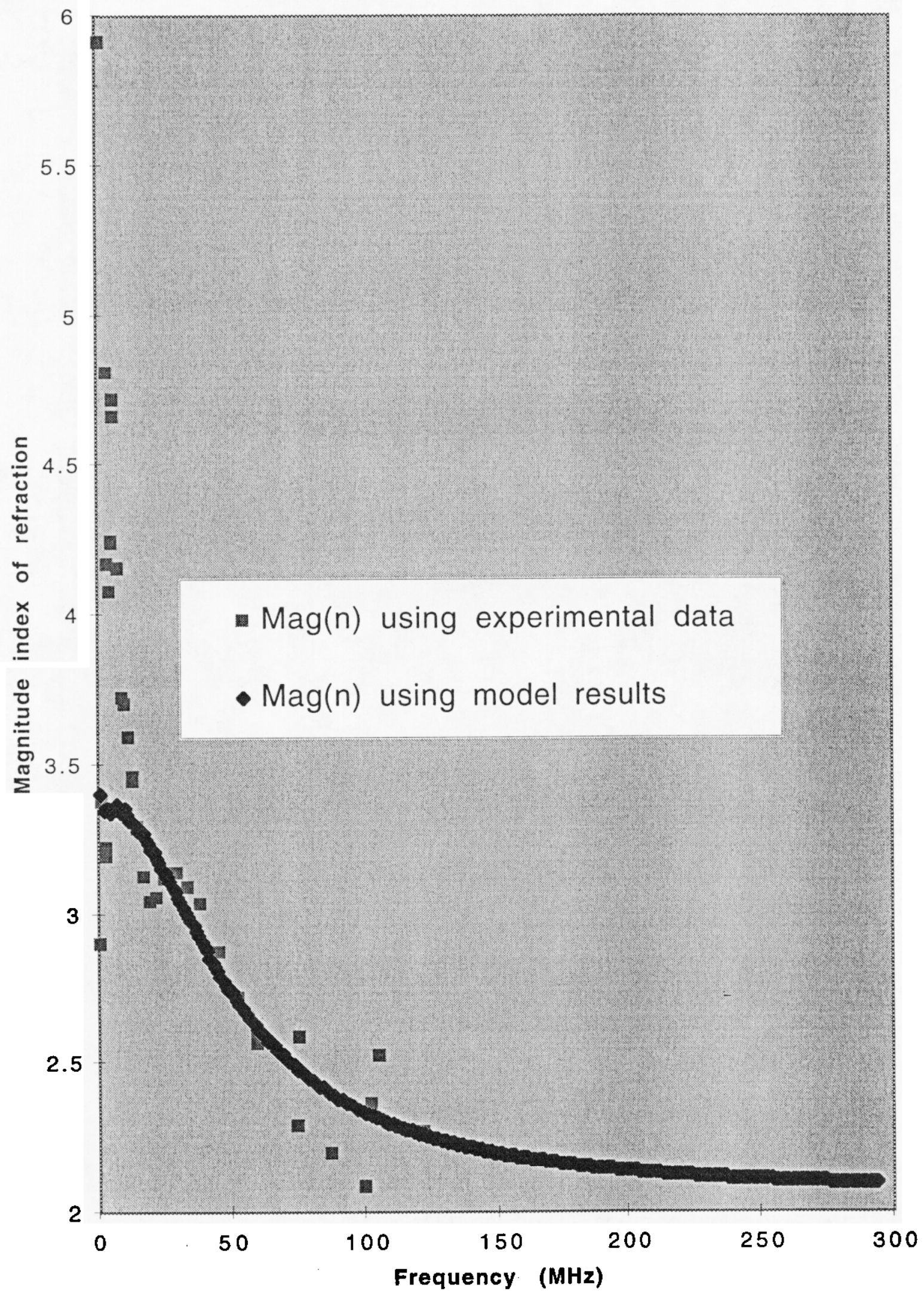

Figure 32. Magnitude of index of refraction as obtained from raw data and from models 
our experimental data. The agreement over the range from $18 \mathrm{MHz}$ or so through 126 $\mathrm{MHz}$ is quite reasonable and provides some confidence in using the models for prediction at higher frequencies. The apparent discrepancy between the model and the experimentallybased results may be in part due to the difficulty in determining $\alpha$ and $\beta$, and ultimately $\varepsilon_{\mathrm{r}}$ and $\sigma$, from the experimental data.

The relative sensitivity of the reflection coefficient to variations in the index of refraction for the special condition where $\mu_{1}=\mu_{0}$ will be considered to provide some indication of the effects of errors in the process. A full error analysis is beyond the scope of this report. With

$$
\begin{aligned}
& R_{H}=\left[\cos \theta-\left(n^{2}-\sin ^{2} \theta\right)^{1 / 2}\right] /\left[\cos \theta+\left(n^{2}-\sin ^{2} \theta\right)^{1 / 2}\right] \\
& R_{E}=\left[n^{2} \cos \theta-\left(n^{2}-\sin ^{2} \theta\right)^{1 / 2}\right] /\left[n^{2} \cos \theta+\left(n^{2}-\sin ^{2} \theta\right)^{1 / 2}\right]
\end{aligned}
$$

consideration will be given to

$\left(1 / R_{\mathrm{H}}\right)\left(\partial \mathrm{R}_{\mathrm{H}} / \partial \mathrm{n}\right)=2 \mathrm{n} \cos \theta\left(\mathrm{n}^{2}-1\right)^{-1}\left(\mathrm{n}^{2}-\sin ^{2} \theta\right)^{-1 / 2}$

$\left(1 / R_{E}\right)\left(\partial R_{E} / \partial n\right)=2 n \cos \theta\left(n^{2}-\sin ^{2} \theta\right)^{-1 / 2}\left(n^{2}-2 \sin ^{2} \theta\right) /\left(n^{4} \cos ^{2} \theta-n^{2}+\sin ^{2} \theta\right)$

An approximation for the fractional change due to changes in $\mathbf{n}$ is

$$
\begin{aligned}
& \left|\Delta R_{H} / R_{H}\right|=2\left|n \cos \theta\left(n^{2}-1\right)^{-1}\left(n^{2}-\sin ^{2} \theta\right)^{-1 / 2} \Delta n\right| \\
& \left|\Delta R_{E} / R_{E}\right|=2\left|n \cos \theta\left(n^{2}-\sin ^{2} \theta\right)^{-1 / 2}\left(n^{2}-2 \sin ^{2} \theta\right) /\left(n^{4} \cos ^{2} \theta-n^{2}+\sin ^{2} \theta\right) \Delta n\right|
\end{aligned}
$$

Over the range from 20 to $120 \mathrm{MHz}$ (the range of the experimental data in Figure 32), we find that $\left|\Delta R_{H} / R_{H}\right| \leq 0.6|\Delta n|$ and $\left|\Delta R_{E} / R_{E}\right| \leq 1.5|\Delta n|$. The maximum value in the latter result is an overestimated bound except in the vicinity of the Brewster angle. Removed from the Brewster angle, the result for $R_{E}$ is in keeping with the result for $R_{H}$.

In view of the observations made in this section, the constitutive parameters determined from experimental data over a limited frequency range and the models based on these limited data appear to yield reliable values outside the range of the useable experimental data. 


\section{Conclusions and Summary}

\subsection{General Comments}

The approach taken herein has proven to be a viable method to determine the constitutive parameters of a medium using noninvasive techniques. There have not been reports in the literature of attempts to perform such constitutive parameter extraction other than in the form of passing comments [10]. The method presented here is based on sound theoretical bases. Some approximations and assumptions have been made in the analysis, e.g., that $\mu_{1}=\mu_{2}=\mu_{0}, \sigma_{\text {wire }}=\infty$, homogeneity of the ground, absence of reflected waves on the wire (effective infinite length), perturbations of the measured current distributions by the measuring system are minimal, and the calibration factors for the measuring system are accurate. The method, while not necessarily robust, has yielded valuable information regarding the characteristics of the ground pad and has uncovered some of the capabilities and shortcomings of the approach. Some comments will be included in a later section.

Since the test program was conducted in a one-week period which did not allow for repeats of the data acquisition/analysis process or even for modifications of the process given observations based on analysis of the data, improvements in the experimental setup to permit improved estimates of the constitutive parameters have not been possible Nonetheless, a reasonable representation of the constitutive parameters of the reinforced concrete pad in the LESLI facility at the Phillips Lab has been achieved.

The constitutive parameters determined by this process are to be used in a program where the realistic ground is to be part of a computer model used in code validation. The test object, a Boeing 757 parked on a reinforced concrete pad, has a fuselage which is seven or more feet above the ground and the test points, except for a top-mounted external blade antenna, are located internal to the fuselage. It has always been expected that the ground will have its most pronounced effect on the structure of the field illuminating the airplane and will most likely have little effect on secondary scattering where the illuminating wave impinges on the airplane, scatters, impinges on the ground, scatters, and impinges on the airplane (thus "perturbing" the illuminating field). In a full wave analysis, this separation or consideration of the order of scattering is not an issue. We only make these comments to reflect on the relative importance of the ground characteristics in the ultimate solution. 


\subsection{Data Summary}

The experimental data in the form of current distributions on a wire over ground were collected over a frequency range from approximately $1 \mathrm{MHz}$ to $1 \mathrm{GHz}$ at 90 spatial points spaced at $30 \mathrm{~cm}$ intervals. Reasonably accurate estimates of attenuation and phase constants were obtained for frequencies between $10 \mathrm{MHz}$ and $126 \mathrm{MHz}$. Below this range, the spatial extent of sampling did not permit accurate estimates of the attenuation constant while beyond this frequency range, the sampling density and the limited support of the transmission line mode did not permit accurate estimates of the phase and attenuation constants, respectively. Over the frequency range, the constants led to estimates of $\varepsilon_{\mathrm{r}}$ and $\sigma$ which were "reasonable" and within the range of physical realizability. Outside the range, the estimates for the constitutive parameters were decidedly less reliable. The estimates from the reliable range were used in a least squares fit to the Debye equations to yield expressions for the constitutive parameters and their average values over range $\left(f_{1}, f_{2}\right)$ :

$$
\begin{aligned}
& \varepsilon_{r}=4.2418+6.1943 /\left(1+1.129 \cdot 10^{-17} \cdot \omega^{2}\right) \\
& \sigma=4.416 \cdot 10^{-11} \cdot \omega /\left(1+1.354 \cdot 10^{-17} \cdot \omega^{2}\right) \\
& <\varepsilon_{r}\left(f_{1}, f_{2}\right)>=4.24+2.93 \cdot 10^{8} \cdot\left[\tan ^{-1}\left(2.11 \cdot 10^{8} \cdot f_{2}\right)-\tan ^{-1}\left(2.11 \cdot 10^{8} \cdot f_{1}\right)\right] /\left(f_{2}-f_{1}\right) \\
& <\sigma\left(f_{1}, f_{2}\right)>=2.595 \cdot 10^{5} \cdot \operatorname{Ln}\left[\left(1+5.29 \cdot 10^{-16} \cdot f_{2}^{2}\right) /\left(1+5.29 \cdot 10^{-16} \cdot f_{1}^{2}\right)\right] /\left(f_{2}-f_{1}\right)
\end{aligned}
$$

Analysis of the experimental data allowed the identification of areas where the displacement current effects dominated. Certainly, displacement current effects were dominant above 50 $\mathrm{MHz}$ and it was evident from the reflection coefficient analysis that it was effectively independent of conductivity above $50 \mathrm{MHz}$ and effectively independent of frequency above $100 \mathrm{MHz}$. Thus concern regarding the conductivity was secondary as its impact would be felt only below $50 \mathrm{MHz}$.

\subsection{Concerns and Suggested Future Work on the Process}

As mentioned previously, time and operational constraints did not permit a more in-depth study of the constitutive parameters and the technique used for their determination. In the future, this technique deserves an extended investigation as it represents a viable method for noninvasive determination of $\varepsilon_{\mathrm{r}}$ and $\sigma$. Specifically, several issues should be carefully studied to assess their impact on accuracy. In the following, several suggestions are made for future uses of the method and several areas are recommended for study. 


\section{Spatial Sampling}

Prior to testing, an analysis was executed to bound the sampling density required to get reliable data over the interval $10 \mathrm{MHz}$ to $400 \mathrm{MHz}$ subject to the operational constraints of time and equipment availability. At the low frequencies it was anticipated that the difficulties would arise from the total attenuation that would be experienced over the entire sampling range. At the high frequencies it was expected that the difficulty would arise from the length of the interval over which the transmission line mode was dominant and the number of samples in this interval. In both cases the accuracy of the measurement system was a concern as was its dynamic range. While relative measurements $\left(\mathrm{I}(\mathrm{x}) / \mathrm{I}\left(\mathrm{x}_{0}\right)\right)$ were adequate for our purposes, the system was limited by its inability to make accurate measurements of either component below $-80 \mathrm{dBm}$ or thereabouts. It was originally felt that sampling at four samples per wavelength was adequate so that the method could produce high quality results at $250 \mathrm{MHz}$ at a sample spacing of $30 \mathrm{~cm}$. Also, the total sampling range was 26.7 meters and it was estimated that sampling over a wavelength at low frequencies (approximately $11 \mathrm{MHz}$ ) would be adequate to assess the attenuation constant. In the presence of noise, reflections, and other fluctuations in the current distribution, the determination of attenuation constant at low frequencies was difficult. The attenuation observed over the entire sampling range was small. At high frequencies, these problems were compounded by the small interval over which the transmission line mode was dominant, the dynamic range of the measurement system, and the small number of samples per wavelength .

In future exercises, it would be beneficial to start the sampling closer to the source to increase the reference signal level and to maximize the length over which the transmission line mode dominates. This will help to extend the high frequency limit. Also, the sampling density should be increased in the high frequency regime in order to generate more samples per wavelength. For the low frequency range, the length over which sampling takes place should be made as large as possible so that the current is sampled over several wavelengths. In the face of limited resources, rather than having uniform sampling over the entire measurement range, it will be beneficial to sample more densely near the source for high frequency coverage and less densely for low frequency coverage. This suggests a nonuniform sampling scheme and may imply a logarithmic sampling rate with more density near the source and less farther away. 


\section{Reflections}

The presence of reflections on the line must be minimized. The current distribution must be entirely that of a transmission line mode on an infinite line. The extraction of reflected waves from unknown locations could make the process difficult if not impossible. Proper loading of finite length lines or sufficient length could make this issue insignificant.

\section{Height}

At present the impact of uncertainties on the accuracy of parameter estimates is unknown, in detail. Certainly the uncertainties here (plus or minus $1 / 8$ " at a height of $5 / 8$ ") have not caused major difficulties but the overall impact should be studied.

\section{System Effects}

The effects of the measurement probes, e.g., the current probes and their cables, on the accuracy of the current distribution measurement should be looked at in detail.

\section{Mathematical Issues}

The process, which is a classical inversion problem, may not be robust. Small errors in $\alpha$ and $\beta$ could lead to significant errors in $\varepsilon_{\mathrm{r}}$ and $\sigma$ although such problems were not overwhelming in this case. This aspect should be studied to lend a measure of usefulness to this technique. In the range of displacement current domination, when a consistent solution may not be available because of great uncertainties in $\sigma$ due to its limited impact on the current distribution, are there methods to obtain reasonable estimates of $\varepsilon_{\mathrm{r}}$ which would suffice? Are alternate methods available? Likewise, similar questions must be answered for the case when conduction current dominates. 


\section{References}

1) A. J. Poggio, R. A . Zacharias, S. T. Pennock, C. Avalle, R. Sharpe, K. Kunz, and C. Meissner, "NASA FBL/PBW Program NASA Boeing 757 HIRF Test Plan, Low Power On-the-Ground Tests," October, 1994

2) D. C. Chang and R. G. Olsen, "Excitation of an Infinite Antenna Above a Dissipative Earth," Radio Science, Vol. 10, No. 8,9, pp. 823-831, August-September 1975.

3) E. F. Kuester, D. C. Chang and R. G. Olsen, "Modal Theory of Long Horizontal Wire Structures Above the Earth, 2, Properties of Discrete Modes," Radio Science, Vol. 13, No. 4, pp. 615-623, July-August 1978.

4) R. G. Olsen, E. F. Kuester and D. C. Chang, "Modal Theory of Long Horizontal Wire Structures Above the Earth, 1, Excitation," Radio Science, Vol. 13, No. 4, pp. 605613, July-August 1978.

5) G. J. Burke, "A Comparison of Solutions for Wires Over Ground", 8th Annual Review of Progress in Applied Computational Electromagnetics, 16-20 March, 1992.

6) G. J. Burke and A. J. Poggio, "Numerical Elecromagnetics Code (NEC) - Method of Moments" Lawrence Livermore National Laboratory, Rept. UCID - 18834, January 1981.

7) R. W. P. King and G. S. Smith, Antennas in Matter, MIT Press, 1981

8) R. S. Elliott, Electromagnetics, McGraw-Hill, 1966

9) C. Kittel, Quantum Theory of Solids, John Wiley, 1963

10) B. Rama Rao and D. N. Jones, "Characterization of a High Frequency Beverage Antenna Using a Fiber-Optic Measurement Technique", Antennas and Propagation Symposium, June 24 - 28, 1991, London, Ontario 
C-55 



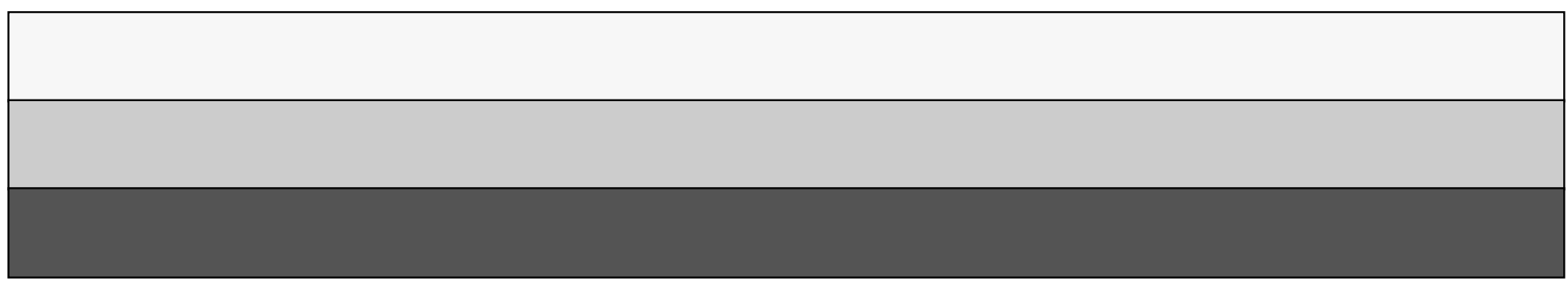

